
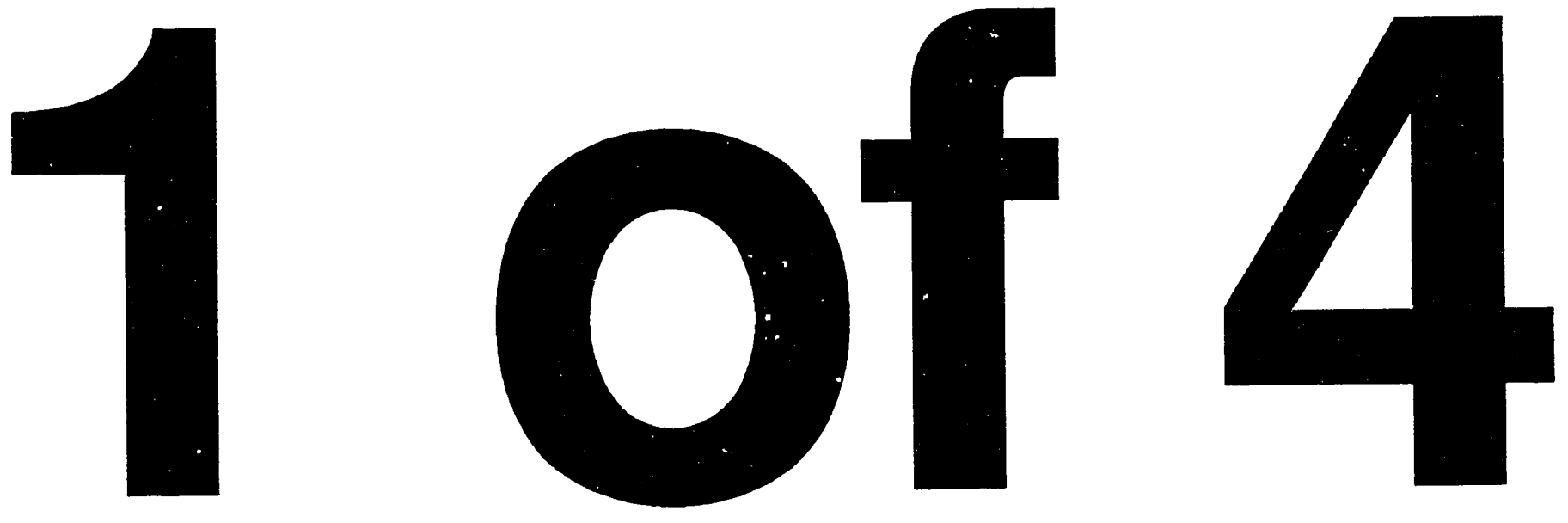


\title{
Site-SPECIFIC ANALYSIS OF RADIOLOGICAL AND PHYSICAL PaRAMETERS FOR Cobbly SOILS AT THE GunNison, Colorado, Processing Site
}

\section{January 1994}

\author{
RECTVE \\ FEB 071994 \\ OSTI
}

\section{DISCLAIMER}

This report was prepared as an account of work sponsored by an agency of the United States Government. Neither the United States Government nor any agency thereof, nor any of their employees, makes any warranty, express or implied, or assumes any legal liability or responsibility for the accuracy, completeness, or usefulness of any information, apparatus, product, or process disclosed, or represents that its use would not infringe privately owned rights. Reference herein to any specific commercial product, process, or service by trade name, trademark, manufacturer, or otherwise does not necessarily constitute or imply its endorsement, recommendation, or favoring by the United States Government or any agency thereof. The views and opinions of authors expressed herein do not necessarily state or reflect those of the United States Government or any agency thereof. 


\section{INTENDED FOR PUBLIC RELEASE}

This report has been reproduced from the best available copy. Available in paper copy and microfiche.

Number of pages in this report: 362

DOE and DOE contractors can obtain copies of this report from:

Office of Scientific and Technical Information

P.O. Box 62

Oak Ridge, TN 37831

(615) 576-8401

This report is publicly available from:

National Technical Information Service

Department of Commerce

5285 Port Royal Road

Springfield, VA 22161

(703) 487-4650 
SITE-SPECIFIC ANALYSIS OF RADIOLOGICAL AND PHYSICAL PARAMETERS FOR COBBLY SOILS AT THE GUNNISON, COLORADO, PROCESSING SITE

January 1994

Prepared for U.S. Department of Energy UMTRA Project Office Albuquerque, New Mexico

Prepared by Jacobs Engineering Group Inc.

Albuquerque, New Mexico 
TABLE OF CONTENTS

Section

Page

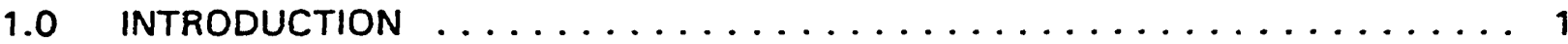

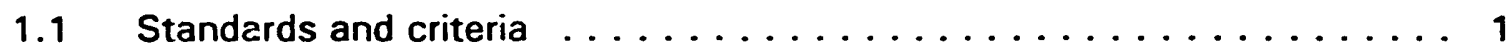

1.2 Remedial action ........................ 3

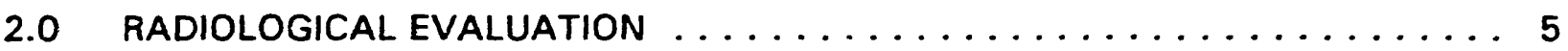

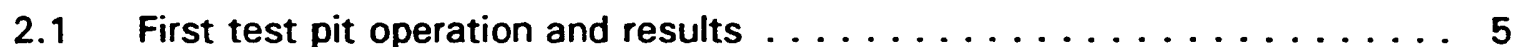

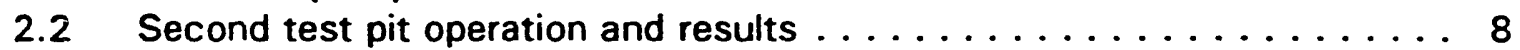

2.3 Statistical parameters for cobbly soils: combined analysis . . . . . . . 11

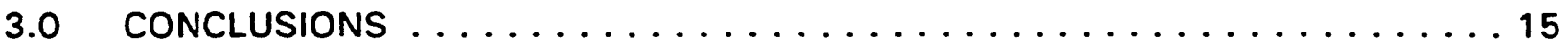

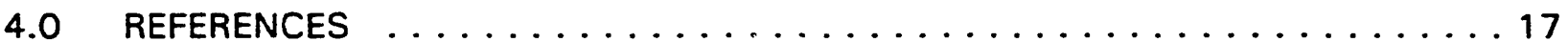

APPENDIX A GENERIC PROTOCOL FOR THORIUM-230 CLEANUPNERIFICATION AT UMTRA PROJECT PROCESSING SITES

APPENDIX B U.S. DEPARTMENT OF ENERGYIU.S. NUCLEAR REGULATORY COMMISSION CORRESPONDENCE PROCEDURE FOR BULK RADIONUCLIDE DETERMINATION, EXCAVATION CONTROL, AND SITE VERIFICATION FOR SOILS CONTAINING COBBLES

APPENDIX C TEST PIT LITHOLOGICAL LOGS AND RADIOLOGICAL DATA COBBLY SUBSOIL INVESTIGATION JUNE 1990

APPENDIX D TEST PIT LITHOLOG!CAL LOGS AND RADIOLOGICAL DATA COBBLY SUBSOIL PIT INVESTIGATION NOVEMBER 1992 


\section{LIST OF FIGURES}

Figure

Page

2.1 Test pit locations for first cobbly subsoil investigation: June $1990 \ldots \ldots 6$

2.2 Test pit locations for second cobbly subsoil investigation: November $1992 \ldots .9$

\section{LIST OF TABLES}

Table

Page

2.1 Range and average radionuclide concentrations as a function of size partitioning relation to $\# 4$ mesh sieve for composite test pit samples: June 1990 characterization .......................

2.2 Range and average radionuclide concentrations as a function of size partitioning relative to $\# 4$ mesh sieve for composite test pit samples:

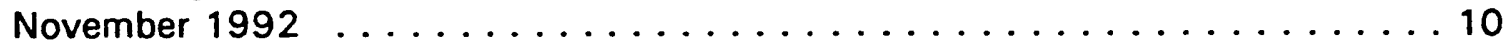

2.3 Summary of mass partition functions and radionuclide concentrations for composite soil samples retained on a $\# 4$ mesh sieve: June 1990 and November 1992 investigations . . . . . . . . . . . . . . . . 12

2.4 Calculated radionuclide mean concentrations and mean concentrations at the upper 95 percent confidence interval for the larger soil size fraction, as a function of subpile, offpile, and background locations . . . . . . . . 14 


\section{LIST OF ACRONYMS AND ABBREVIATIONS}

\section{Acronym}

ALARA

cm

DOE

EPA

$f$

ft

$\mathrm{m}^{2}$

NRC

$\mathrm{pCi} / \mathrm{g}$

$\mathrm{pCi} / \mathrm{L}$

ppm

$\mathrm{Ra}-226$

Th-230

UMTRCA

$U_{\text {total }}$

$\mu \mathrm{R} / \mathrm{hr}$

\section{Definition}

as low as reasonably achievable

centimeters

U.S. Department of Energy

U.S. Environmental Protection Agency

function

feet

square meter

U.S. Nuclear Regulatory Commission

picocuries per gram

picocuries per liter

parts per million

radium-226

thorium-230

Uranium Mill Tailings Radiation Control Act

total uranium

microroentgens per hour 


\subsection{INTRODUCTION}

The remedial action at the Gunnison, Colorado, processing site is being performed under the Uranium Mill Tailings Radiation Control Act (UMTRCA) of 1978. Public Law 95-604 (42 USC $\$ 7901$ et seq.) Under UMTRCA, the U.S. Environmental Protection Agency (EPA) is charged with the responsibility of developing appropriate and applicable standards for the cleanup of radiologically contaminated land and buildings at 24 designated sites, including the Gunnison, Colorado, inactive processing site. Section 108 of Public Law 95-604 states that the U.S. Department of Energy (DOE) shall "select and perform remedial actions at the designated processing sites and disposal sites in accordance with the general standards" prescribed by the EPA. Regulations governing the required remedial action at inactive uranium processing sites were promulgated by the EPA in 1983 and are contained in 40 CFR Part 192 (1993), Health and Environmental Protection Standards for Uranium and Thorium Mill Tailings.

Subpart B of 40 CFR Part 192 consists of standards for the cleanup of land and buildings. The standards applicable to land cleanup activities are as follows:

"Remedial actions shall be conducted so as to provide reasonable assurance that, as a result of residual radioactive materials from any designated processing site, the concentration of Radium-226 in land averaged over an area of 100 square meters $\left(\mathrm{m}^{2}\right)$ shall not exceed the background level by more than --

1) Five picocuries per gram ( $\mathrm{pCi} / \mathrm{g}$ ), averaged over the first 15 centimeters $(\mathrm{cm})$ of soil below the surface, and

2) Fifteen $\mathrm{pCi} / \mathrm{g}$, averaged over $15-\mathrm{cm}$-thick layers of soils more than $15 \mathrm{~cm}$ below the surface."

In addition, 40 CFR Part 192 provides criteria for applying supplemental standards for the cleanup and stabilization of other radionuclides that present a hazard commensurate with radium-226 (Ra-226) (40 CFR \$192.21 [1993]), such as thorium-230 (Th-230). A Generic Protocol for Thorium-230 Cleanup/Nerification at UMTRA Project sites under development by the DOE UMTRA Project Office (Appendix A) has received U.S. Nuclear Regulatory Commission (NRC) verbal approval for implementation. Fundamental provisions of the protocol are as follows:

- Th-230 concentrations exceeding Ra-226 concentrations will be remediated such that Ra-226 concentrations one thousand years in the future, including both in situ Ra-226 and Ra-226 produced by natural decay of Th-230 over a 1000 -year period, when averaged over $100 \mathrm{~m}^{2}$, will not exceed either $5 \mathrm{pCi} / \mathrm{g}$ in the first $15-\mathrm{cm}$ surface soil layer, or $15 \mathrm{pCi} / \mathrm{g}$ in successive 15-cm subsurface layers, above background (Generic Protocol). 
- For deeply buried material, stop excavations when the RAECOM computer code, using site-specific parameters, calculates a Rn-222 flux of $3.9 \mathrm{pCi} / \mathrm{m}^{2} \mathrm{~s}$ and expected long-term conditions are appropriate, or when construction safety or feasibility becomes a concern.

- Excavation of elevated Th-230 encountered below the water table in the saturated zone will be assessed relative to the practicality with which dewatering can be performed. An as low as reasonably achievable (ALARA) analysis using pathway techniques will be performed in cases where a major portion of the site contains Th-230 which extends into the saturated zone, and excavation into the zone is impractical.

There are no promulgated standards for the cleanup of residual soil concentrations of total uranium $\left(U_{\text {total }}\right)$. A separate Special Study is being conducted to assess potential impacts of residual uranium soil contamination on groundwater, and to evaluate the need for developing supplemental remediation standards based on pathway analysis and ALARA considerations.

The EPA standards were originally based on an understanding of radiologically contaminated, fine-grained, tailings-like soil. However, a recent NRC evaluation of the soil cleanup standards for cobbly soil has led to the conclusion that standards should have a universal interpretation of bulk soil concentrations, where bulk is defined as the total activity in picocuries divided by the total sample mass in grams, independent of the radionuclide distribution as a function of soil size fraction (Appendix B). Recognizing that UNTRA Project site cleanup activities will entail the cleanup of radiologically contaminated cobbly soil, the NRC also has concurred on a procedure developed by the DOE's TAC for excavation control and verification of cobbly subsoil (Appendix B). The accepted protocol is based on establishing a representative mass partition function ( $f$ ) that is the ratio of the mass of the soil fraction retained on a \#4 sieve to the mass of the soil fraction passing a $\# 4$ sieve, and the characteristic radionuclide concentration on the larger soil size fraction retained on a \#4 mesh sieve. The mass partition function can be developed and applied for the entire site (Soil Verification Alternative 2 [Statistical]). Section 5.9 of the cobbly soil cleanup procedure or for each $100 \mathrm{~m}^{2}$ grid (Site Verification Alternative 1 [Grid Specificl). Section 5.8 of the cobbly soil cleanup procedure. Bulk radionuclide concentration can be determined using the mass partition function, the radionuclide concentration on the larger size soil fraction, and radiometric/radiochemical measurements of only the finer soil fraction passing a \#4 sieve.

The standards given atove are based on bulk Ra-226 concentrations elevated above background level. Measurements of background radioactivity near the Gunnison, Colorado, processing site, have resulted in the following determinations (DOE, 1992):

- Background gamma exposure rates at one meter above the earth average 16.6 microroentgens per hour $(\mu \mathrm{R} / \mathrm{hr})$. 
- Background Ra-226 concentrations near the processing site average $1.7 \mathrm{pCi} / \mathrm{g}$.

- Background Rn-222 concentrations in air at various locations near the processing site average approximately 1.0 picocuries per liter (pCi/L).

\section{REMEDIAL ACTION}

The remedial action at the processing site will be conducted to remove the tailings and contaminated materials to meet the above-delineated EPA bulk soil cleanup standards for surface and subsurface soils. The site areas disturbed by remedial action excavation will be either contoured or backfilled with radiologically uncontaminated soil and contoured to restore the site. The final contours will produce a final surface grade that will create positive drainage from the site. 


\subsection{RADIOLOGICAL EVALUATION}

Two site characterizations were conducted (June 1990 and November 1992) to more accurately determine the cobbly nature of the subsoil underlying the Gunnison, Colorado. processing site, and to ascertain: 1) the mass partitioning of the subpile soil relative to a \#4 mesh sieve, and 2) the radiological contamination associated with the soil size fractions passing and retained on a $\# 4$ mesh sieve. The purpose of this investigation was to obtain the necessary parameters to characterize the bulk radionuclide concentrations for the site foundation soil, particularly the subpile area. Earlier investigations were based on either samples containing only the finer soil fraction, or, in the event that sampies were unavailable, analytical modeling assumptions that the subsoil was composed of only finer grained material. With these more recent site characterization data, an appropriate excavation depth for remediating radiologically contaminated cobbly subsoil may be determined that complies with the recent DOE bulk interpretation of the Ra-226 and Th-230 soil cleanup standards that NRC concurred on. It also provides necessary information regarding the way in which excavation control and verification should be performed.

\section{$2.1 \quad$ FIRST TEST PIT OPERATION AND RESULTS}

The first test pit operation (June 1990) consisted in constructing, with a backhoe, 28 test pits distributed over the site, as well as in the near off-site environment, and obtaining cobbly soil samples. Test pit locations are shown in Figure 2.1. As seen in the figure, 10 test pits were dug in the pile area, nine in the mill yard/ore storage area, five along East Gold Basin Road berms, and four near the tailings pile perimeter. The depth excavated into the cobbly foundation soil ranged from $1.5 \mathrm{ft}$ to $7 \mathrm{ft}(0.5 \mathrm{~m}$ to $2 \mathrm{~m})$, and averaged $3.2 \mathrm{ft}(1.0 \mathrm{~m})$. Soil samples of the finer scil size fraction passing a \#4 mesh sieve were collected from approximately every $1-\mathrm{ft}(0.3-\mathrm{m})$ depth increment, and analyzed for Ra-226 and Th-230 (a total of 97 samples). In addition, 14 bulk samples of cobbly soil were collected from individual $1 \mathrm{ft}(0.3 \mathrm{~m})$ depth increments for 10 selected test pits, and were portioned relative to a \#4 sieve to assess the radionuclide distributions relative to this soil size division: one sample each for eight test pits, and two samples each for three test pits. Soil size fractions retained and passing a \#4 mesh sieve were analyzed for Ra-226, Th-230, Th-232, and total uranium. A mass partition function, the ratio of the mass of large size soil sample fraction retained on a \#4 mesh sieve to the mass of the finer size soil fraction passing a \#4 mesh sieve, was also determined for each of the 10 test pits, as well. Table 2.1 summarizes the range and average radionuclide concentrations as a function of soil size partitioning relative to a \#4 mesh sieve. The mass partition function, $f$, for the cobbly soil ranged from 0.17 to 6.26 , and had an average and standard deviation 3.29 and 1.87, respectively.

The field lithological logs and off-site laboratory and on-site measurement results are contained in Appendix $C$. On analysis of the individual test pit radionuclide depth profiles, one notes that this data indicates that differential migration of Th-230 relative to Ra-226 has occurred, particularly in the cobbly subpile soil. 

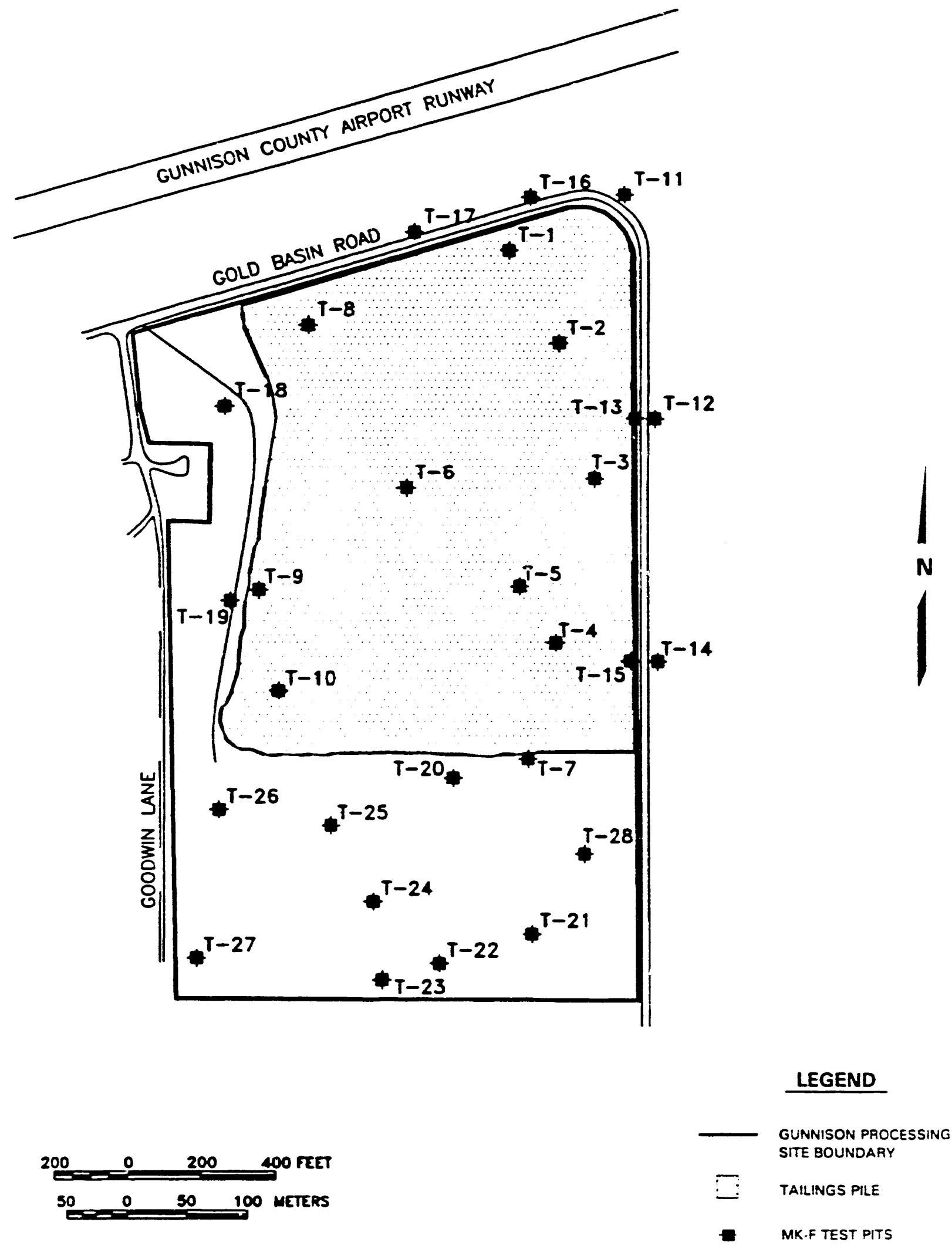

FIGUFE 2.1

TEST PIT LOCATIONS FOR FIRST COBBLY SUBSOIL INVESTIGATION: JUNE 1990 GUNNISON PROCESSING SITE, COLORADO 
Table 2.1 Range and average radiont:clide concentrations as a function of size partitioning relative to \#4 mesh sieve for composite test pit samples: June 1990 characterization

\begin{tabular}{|c|c|c|c|c|c|c|}
\hline \multirow[b]{3}{*}{ Soil size -action } & \multicolumn{6}{|c|}{ Test pit location } \\
\hline & \multicolumn{2}{|c|}{$\begin{array}{c}\text { Subpile plus offpile } \\
\text { (10 pits) }\end{array}$} & \multicolumn{2}{|c|}{$\begin{array}{l}\text { Subpile } \\
\text { (5 pits) }\end{array}$} & \multicolumn{2}{|c|}{$\begin{array}{l}\text { Uffpile } \\
\text { (5 pits) }\end{array}$} \\
\hline & Range & Mean & Range & Mean & Range & Mean \\
\hline \multicolumn{7}{|c|}{ Cobbles > \#4 Mesh Sieve } \\
\hline $\mathrm{Ra}-226(\mathrm{pCi} / \mathrm{g})^{\mathrm{a}}$ & $1.2-3.2$ & 1.74 & $1.2-3.2$ & 1.74 & $1.0-3.2$ & 1.74 \\
\hline Th-230 (pCi/g) & $0.7-19$ & 6.0 & $1.3-19$ & 11 & $0.5-1.5$ & 0.96 \\
\hline Th-232 (pCi/g) & $0.5-i .5$ & 1.0 & $0.5-1.5$ & 1.1 & $0.6-1.1$ & 0.9 \\
\hline$U_{\text {total }}(\mathrm{ppm})^{b}$ & $1.3-40$ & 8.8 & $4.8-40$ & 16.2 & $1.3-1.6$ & 1.4 \\
\hline \multicolumn{7}{|c|}{ Fines < \#4 Mesh Sieve } \\
\hline $\mathrm{Ra}-226(\mathrm{pCi} / \mathrm{g})$ & $0.8-14$ & 3.7 & $3.0-4.6$ & 3.4 & $0.8-14$ & 4.0 \\
\hline Th-230 (pCi/g) & $1.1-180$ & 58.1 & $1.1-180$ & 111.8 & $1.0-15$ & 4.4 \\
\hline Th-232 (pCi/g) & $0.8-1.9$ & 1.2 & $0.9-1.9$ & 1.3 & $0.8-1.4$ & 1.1 \\
\hline$U_{\text {total }}(\mathrm{ppm})$ & $1.5 \cdot 87$ & 28.4 & $34-87$ & 54.2 & $1.5-6 . i$ & 2.6 \\
\hline
\end{tabular}

${ }^{a} \mathrm{pCi} / \mathrm{g}=$ picocuries per gram.

${ }^{b p m}=$ parts per million. 
Although typically only a $1-\mathrm{ft}(0.3 \mathrm{~m})$ deep excavation would be required to comply with the EPA bulk Ra-226 cleanup standard, an additional $1.5 \mathrm{ft}(0.5 \mathrm{~m})$ of soil removal would be required to ensure that the total bulk Ra-226 ingrowth from residual Th-230 in 1000 years does not exceed $15 \mathrm{pCi} / \mathrm{g}$ above background.

\section{SECOND TEST PIT OPERATION AND RESULTS}

To more closely delineate subpile radiological concentrations and to develop the necessary statistical parameters to apply the NRC-approved excavation and verification protocol for cobbly soil (Soil Verification Alternative 2 [Statistical], Section 5.9 of the cobbly soil cleanup procedure), a second test pit operation was conducted in November 1992. Since the protocol was being designed when the first test pit (June 1990) investigation was being performed, the second test pit operation was necessary to compliment the initial cobbly soil data base.

Twenty additional test pits were excavated into the pile subsoil to an average depth of $5 \mathrm{ft}(1.5 \mathrm{~m})$, and three background pits were constructed to the same depth in the off-site area. Figure 2.2 shows the test pit locations. Test pits were excavated and the cobbly soil sampled in $1 \mathrm{ft}(0.3 \mathrm{~m})$ increments in accordance with the NRC protocol (Appendix B). At the time of the test pit operation. groundwater ranged from 2 to $8 \mathrm{ft}(0.6$ to $2.4 \mathrm{~m})$ beiow the tailings/foundation soil interface, and was encountered in 17 of the 23 test pits. The cobbly soil was sieved using a \#4 mesh sieve, and composite soil samples for each test pit were obtained for the soil fractions retained on, and passing the sieve. Both soil fractions were weighed, and analyzed for Ra-226, Th-230, Th-232, and total uranium. An aliquot of only the fine soil size fraci in was also obtained from each $1 \mathrm{ft}(0.3 \mathrm{~m})$ test pit depth increment from 19 test pits, and separately analyzed for these radionuclides. Table 2.2 summarizes the range and average radionuclide concentrations as a function of soil size partitioning relative to a $\# 4$ mesh sieve for the composite soil samples from each test pit. The mass partition functions, $f$, for the 23 test pits ranged from 0.94 to 4.59 , and averaged 3.31 with a standard deviation of 0.80 .

Field lithological logs, and off-site laboratory and on-site measurement results are given in Appendix $D$. The results obtained from both test pit operations are compatible. The radiological analysis of the finer soil size fraction for $1-\mathrm{ft}$ $(0.3-\mathrm{m})$ depth increment samples from 19 test pits (data in Appendix D) clearly indicate that Ra-226 is readily adsorbed/precipitated within the first foot to 1.5 $\mathrm{ft}(0.5 \mathrm{~m})$ of subpile soil. However, differential migration of Th-230 relative to Ra-226 has produced significantly elevated concentrations of Th-230 at depth. During test pit operations, moderate to heavy rust staining of the cobbly soil and groundwater was observed. This staining is presumably due to the formation of ferric hydroxide precipitates, and elevated Th-230/uranium concentrations are generally associated with this staining. 


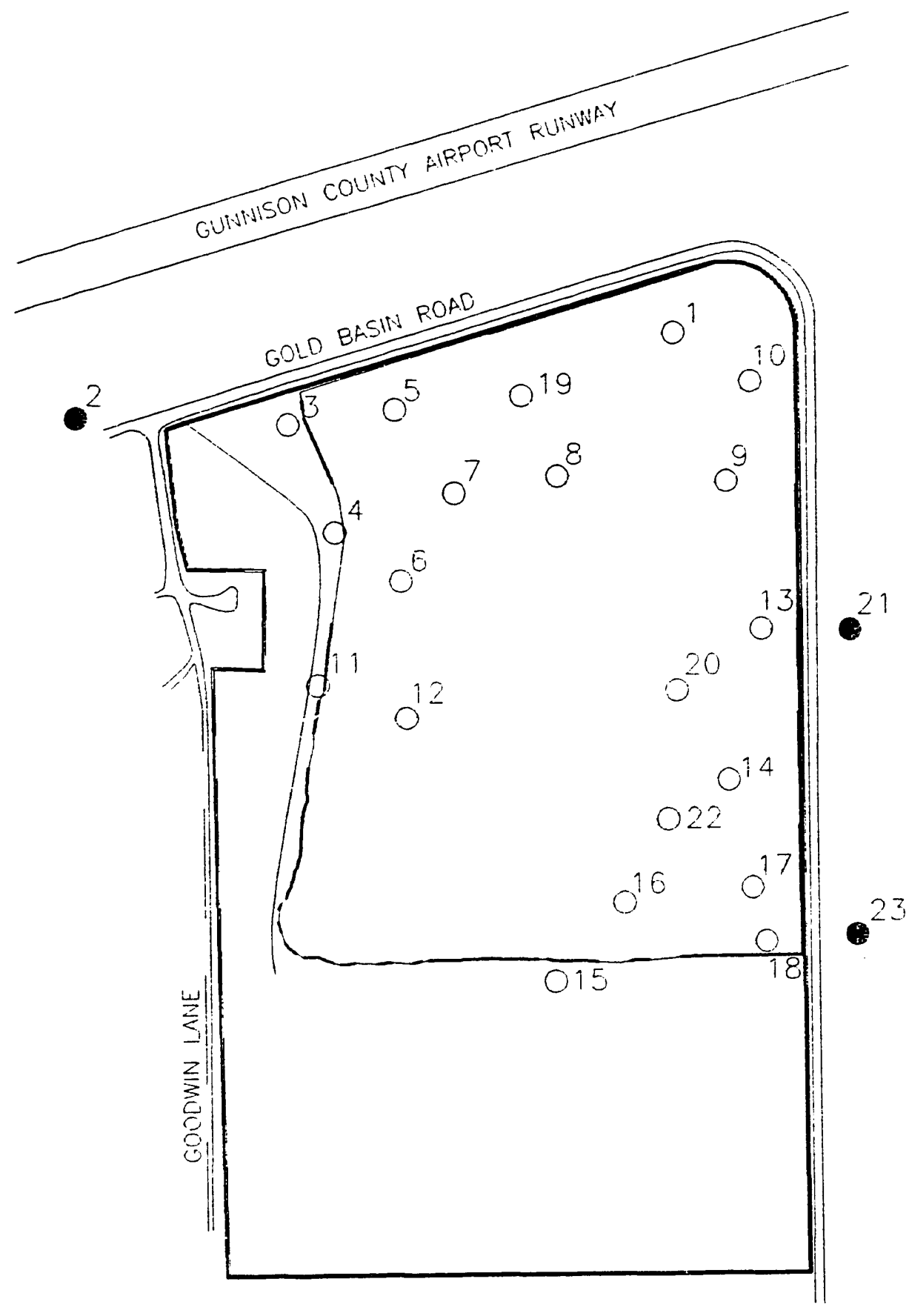

LEGEND

\begin{tabular}{ll}
\multicolumn{1}{c}{ LEGEND } \\
\hline & $\begin{array}{l}\text { GUNNISON PROCESSING } \\
\text { SITE BOUNDARY }\end{array}$ \\
$\square \quad$ TAILINGS PILE \\
MK-F TEST PITS \\
BACKGROUND TEST PITS
\end{tabular}

FIGURE 2.2

TEST PIT LOCATIONS FOR SECOND COBBLY SUBSOIL INVESTIGATION: NOVEMBER 1992, GUNNISON PROCESSING SITE, COLORADO 
Table 2.2 Range and average radionuclide concentrations as a function of size partitioning relative to \#4 mesh sieve for composite test pit samples: November 1992

\begin{tabular}{|c|c|c|c|c|}
\hline \multirow{3}{*}{$\begin{array}{l}\text { Soil size } \\
\text { fraction }\end{array}$} & \multicolumn{4}{|c|}{ Test pit location } \\
\hline & \multicolumn{2}{|c|}{ Subpile plus offpile (20 test pits) } & \multicolumn{2}{|c|}{ Background (3 pits) } \\
\hline & Range & Mean & Range & Mean \\
\hline \multicolumn{5}{|c|}{ Cobbles > \#4 Mesh Sieve } \\
\hline $\operatorname{Ra}-226(p C i / g)^{a}$ & $1.2-4.0$ & 2.2 & $1.6-2.5$ & 1.9 \\
\hline Th-230 (pCi/g) $)^{b}$ & $0.8-69$ & 20.8 & $1.9-3.2$ & 2.7 \\
\hline Th-232 (pCi/g) & $0.6-3.6$ & 1.6 & $1.2-1.7$ & 1.5 \\
\hline$U_{\text {total }}(p p m)^{b}$ & $2.2-20$ & 11.2 & $1.3-2.2$ & 1.8 \\
\hline \multicolumn{5}{|c|}{ Fines $\lessdot \# 4$ Mesh Sieve } \\
\hline $\mathrm{Ra}-226(\mathrm{pCi} / \mathrm{g})$ & $1.7-15$ & 4.1 & $1.1-2.5$ & 1.7 \\
\hline Th-230 (pCi/g) & $3.8-660$ & 140 & $1.3-16$ & 7.1 \\
\hline Th-232 (pCi/g) & $0.8-20$ & 5.9 & $1.2-1.3$ & 1.25 \\
\hline$U_{\text {total }}(p p m)$ & $2.4-86$ & 30 & $1.9-2.0$ & 2.0 \\
\hline
\end{tabular}

${ }^{a} \mathrm{pCi} / \mathrm{g}=$ picocuries per gram.

${ }_{\mathrm{ppm}}=$ parts per million. 


\subsection{STATISTICAL PARAMETERS FOR COBBLY SOILS: COMBINED ANALYSIS}

To obtain the necessary statistical parameters to appropriately perform site characterization, excavation control, and verification for the observed cobbly site soil (Soil Verification Alternative 2 [Statistical]. Section 5.9 of the cobbly soil cleanup procedure in Appendix B), the results of the two separate test pit investigations are combined to yield a average mass partition function of 3.30 . with a standard deviation of 1.20 for the 33 test pits considered. At the lower 95 percent confidence limit, the site statistical mass partition function, $f_{L}$, to be used for site verification is 2.96 (from 33 test pits' data). Therefore, approximately 25 percent $\left(1 /\left[1+f_{L}\right]\right)$ of the cobbly subsoil, by weight, is less than a $\# 4$ mesh sieve, and 75 percent $\left(f_{L} /\left[1+f_{L}\right]\right)$ of the cobbly subsoil, by weight, is greater than a \#4 mesh sieve, at the lower 95 percent confidence limit.

As noted in Tables 2.1 and 2.2, the Ra-226 and Th-232 concentrations for the larger size soil fraction retaired on a \#4 mesh sieve are approximately equal for subpile and offpile/background areas, Th-230 and total uranium concentrations are compatible for offpile and background areas, and Th-230 and total uranium concentrations are elevated in the subpile relative to corresponding offpile and background values. Therefore, to provide representatively conservative radionuclide concentrations for the larger size soil fraction, it is appropriate to construct separate average concentrations for the offpile and subpile regimes. The average mass partition functions and radionuclide concentrations (larger size soil fraction) and corresponding standard deviations for the subpile, offpile, and background areas are summarized in Table 2.3, and were determined by combining the data used to develop Table 2.1 (June 1990) and Table 2.2 (November 1992). The site background bulk Ra-226 concentration was determined to be $1.8 \mathrm{pCi} / \mathrm{g}$ from the mass-weighted average of the data for the background pits in Table 2.2 ( $f=2.96$ was used for weighting).

The calculated mean and statistical upper 95 percent confidence limit radionuclide concentrations are presented in Table 2.4 for the subpile, offpile. and background areas. 
Table 2.3 Summary of mass partition functions and radionuclide concentrations for composite soil samples retained on a \#4 mesh sieve: June 1990 and November 1992 investigations

\begin{tabular}{|c|c|c|c|c|c|c|}
\hline \multirow{2}{*}{$\begin{array}{l}\text { Test rit ID } \\
\text { and } \\
\text { statistical } \\
\text { summary }\end{array}$} & \multirow[b]{2}{*}{ Location } & \multirow{2}{*}{$\begin{array}{c}\text { Mass partition } \\
\text { function } \\
1=(M>\# 4) \mid \\
(M<\# 4)\end{array}$} & \multicolumn{4}{|c|}{$\begin{array}{l}\text { Radionuclide concentration } \\
\text { mass fraction }>\# 4 \text { sieve }\end{array}$} \\
\hline & & & $\begin{array}{l}\mathrm{Ra}-226 \\
\text { (pCi/g) }\end{array}$ & $\begin{array}{l}\text { Th-230 } \\
\text { (pCi/g) }\end{array}$ & $\begin{array}{l}\text { Th-232 } \\
\text { (pCi/g) }\end{array}$ & $\begin{array}{l}U_{\text {totol }} \\
\text { (ppm) }\end{array}$ \\
\hline $1^{a}$ & Subpile & 3.87 & 2.5 & 15 & 1.7 & 2.2 \\
\hline 2 & Background & 4.59 & 2.5 & 3.2 & 1.2 & 1.9 \\
\hline 3 & Subpile & 3.73 & 1.3 & 29 & 2.3 & 2.6 \\
\hline 4 & Subpile & 3.10 & 1.2 & 3.7 & 0.3 & 4.1 \\
\hline 5 & Subpile & 2.15 & 1.9 & 29 & 2.5 & 8.6 \\
\hline 6 & Subpile & 3.65 & 1.8 & 1.6 & 0.6 & $1+$ \\
\hline 7 & Subpile & 2.66 & 1.9 & 7.5 & 0.7 & 20 \\
\hline 8 & Subpile & 2.70 & 2.0 & 0.8 & 0.5 & 20 \\
\hline 9 & Subpile & 4.42 & 3.0 & 47 & 2.9 & 7.0 \\
\hline 10 & Subpile & 3.38 & 3.1 & 21 & 1.2 & 5.1 \\
\hline 11 & Subpile & 3.28 & 2.0 & 12 & 0.9 & 1.9 \\
\hline 12 & Subpile & 2.39 & 1.3 & 3.1 & 0.6 & 23 \\
\hline 13 & Subpile & 3.31 & 2.5 & 41 & 3.6 & 6.3 \\
\hline 14 & Subpile & 0.94 & 1.5 & 13 & 1.2 & 20 \\
\hline 15 & Subpile & 3.43 & 2.8 & 1.6 & 0.9 & 1.6 \\
\hline 16 & Subpile & 3.22 & 1.6 & 10 & 0.7 & 32 \\
\hline 17 & Subpile & 3.68 & 2.8 & 7.1 & 1.6 & 13 \\
\hline 18 & Subpile & 3.34 & 2.1 & 41 & 2.0 & 6.0 \\
\hline 19 & Subpile & 4.04 & 2.2 & 22 & 2.4 & 13 \\
\hline 20 & Subpile & 3.12 & 2.7 & 42 & 2.3 & 11 \\
\hline 21 & Background & 3.93 & 1.6 & 1.9 & 1.5 & 2.2 \\
\hline 22 & Subpile & 4.22 & 4.0 & 69 & 2.2 & 11 \\
\hline 23 & Background & 2.93 & 1.7 & 3.1 & 1.7 & 2.2 \\
\hline $\mathrm{TP} 2^{\mathrm{b}}$ & Subpile & 2.93 & 1.2 & 19 & 0.5 & 9.8 \\
\hline TP4 & Subpile & 4.22 & 3.2 & 6.2 & 1.1 & 40 \\
\hline TP6 & Subpile & 3.80 & 1.3 & 1.3 & 1.0 & 20 \\
\hline TP8 & Subpile & 1.32 & 1.5 & 19 & 1.5 & 4.8 \\
\hline
\end{tabular}


Table 2.3 Summary of mass partition functions and radionuclide concentrations for composite soil samples retained on a \#4 mesh sieve: June 1990 and November 1992 investigations (concluded)

\begin{tabular}{|c|c|c|c|c|c|c|}
\hline \multirow{2}{*}{$\begin{array}{l}\text { Test pit ID } \\
\text { and } \\
\text { statistical } \\
\text { summary }\end{array}$} & \multirow[b]{2}{*}{ Location } & \multirow{2}{*}{$\begin{array}{c}\text { Miass partition } \\
\text { function } \\
f=(M>\# 4) / \\
(M<\# 4)\end{array}$} & \multicolumn{4}{|c|}{$\begin{array}{l}\text { Radionuclide concentration } \\
\text { mass fraction }>\# 4 \text { sieve }\end{array}$} \\
\hline & & & $\begin{array}{l}\mathrm{Ra}-226 \\
\text { (pCi/g) }\end{array}$ & $\begin{array}{l}\text { Th-230 } \\
\text { (pCi/g) }\end{array}$ & $\begin{array}{l}\text { Th-232 } \\
\text { (pCi/g) }\end{array}$ & $\begin{array}{l}U_{\text {sotel }} \\
\text { (ppm) }\end{array}$ \\
\hline TP10 & Subpile & 3.00 & 1.5 & 10 & 1.4 & 6.3 \\
\hline TP15 & Gffpile & 2.94 & 1.2 & 0.7 & 1.1 & 1.4 \\
\hline TP17 & Offpile & 2.34 & 2.0 & 1.2 & 1.2 & 1.5 \\
\hline TP20 & Offpile & 6.26 & 1.0 & 0.5 & 0.7 & 1.6 \\
\hline TP23 & Offpile & 0.17 & 1.3 & 0.9 & 0.9 & 1.3 \\
\hline TP26 & Offpile & 5.89 & 3.2 & 1.5 & 0.6 & 1.4 \\
\hline \multirow[t]{4}{*}{ Average } & $\begin{array}{l}\text { Subpile/ } \\
\text { Offpile/Bkg }\end{array}$ & 3.30 & 2.0 & 15 & 1.4 & 9.6 \\
\hline & Subpile & 3.20 & 2.1 & 19 & 1.5 & 12 \\
\hline & Offpile & 3.52 & 1.7 & 1.0 & 0.9 & 1.4 \\
\hline & Background & 3.82 & 1.9 & 2.7 & 1.5 & 2.1 \\
\hline \multirow[t]{4}{*}{$\begin{array}{l}\text { Standard } \\
\text { Deviation }\end{array}$} & $\begin{array}{l}\text { Subpile/ } \\
\text { Offpile/Bkg }\end{array}$ & 1.20 & 0.7 & 17 & 0.8 & 9.6 \\
\hline & Subpile & 0.84 & 0.8 & 18 & 0.9 & 9.7 \\
\hline & Offpile & 2.55 & 0.9 & 0.4 & 0.3 & 0.1 \\
\hline & Background & 0.84 & 0.5 & 0.7 & 0.3 & 0.2 \\
\hline
\end{tabular}

${ }^{a}$ Test pit numbers 1-23, November 1992 investigation.

b Test pit with TP designations, June 1990 investigation.

${ }^{{ }^{c}} \mathrm{Bkg}=$ background locations. 
Table 2.4 Calculated radionuclide mean concentrations and mean concentrations at the upper 95 percent confidence interval for the larger soil size fraction, as a function of subpile, offpile, and background locations

\begin{tabular}{|c|c|c|c|}
\hline \multirow[b]{2}{*}{ Radionuclide } & \multicolumn{3}{|c|}{$\begin{array}{l}\text { Mean/mean concentration at upper } 95 \% \text { confidence limit } \\
\text { (pCi/g) }\end{array}$} \\
\hline & Subpile & Offpile & Background \\
\hline $\mathrm{Ra}-226$ & $2.1 / 2.4$ & $1.7 / 2.6$ & $1.9 / 2.7$ \\
\hline Th-230 & $19 / 25$ & $1.0 / 1.4$ & $2.7 / 3.9$ \\
\hline Th-232 & $1.5 / 1.8$ & $0.90 / 1.2$ & $1.5 / 2.0$ \\
\hline$U_{\text {total }}^{a}$ & $12 / 15$ & $1.4 / 1.5$ & $2.1 / 2.4$ \\
\hline
\end{tabular}

aranium concentrations in parts per million (ppm). 


\subsection{CONCLUSIONS}

For site characterization, bulk radiological concentrations in the offpile and subpile regions will be calculated using the following:

$$
C_{B}=C_{<\# 4}[1 /(1+f)]+C_{>\# 4}[f /(1+f)],
$$

where

$\mathrm{C}_{<\# 4}=$ the site measured radionuclide concentration of the finer soil size fraction passing a \#4 mesh sieve measured in the on-site labor tory,

$C_{>\# 4}=$ the site average radionuclide concentration of the larger soil size fraction retained on a $\# 4$ mesh sieve for the area in question from Table 2.4, and

$f=$ the site average mass partition fraction, 3.30, from Table 2.3.

However, for site verification, the statistical average values at the upper and lower 95 percent confidence limits are used in the relation above for $C_{>\# 4}$, as a function of radionuclide, and $f$, respectively, to separately calculate bulk concentrations for the offpile and subpile areas.

On this basis, for cobbly subsoil to comply with the bulk cleanup standards (total Ra-226 concentrations from residual Ra-226 and Th-230 to not exceed $15 \mathrm{pCi} / \mathrm{g}$ above background, $1.8 \mathrm{pCi} / \mathrm{g}$ bulk Ra-226 concentration), the corresponding concentrations in the finer soil size fraction (passing a \#4 mesh sieve) in the offpile areas could vary as follows:

1) $C_{<\# 4 R a}=58.8 \mathrm{pCi} / \mathrm{g} \mathrm{Ra}-226$, when the Th-230 concentrations on the finer fraction, $\mathrm{C}_{<\# 4 \mathrm{Th}}$, are less than or equal to $62.2 \mathrm{pCi} / \mathrm{g}$;

2) $C_{<\# 4 T h}=168.4 \mathrm{pCi} / \mathrm{g} \mathrm{Th}-230$, if there is evidence that the Th-230 has differentially migrated relative to Ra-226, and the residual bulk Ra-226 concentration is $1.8 \mathrm{pCi} / \mathrm{g}\left(\mathrm{C}_{<\# 4 \mathrm{Ra}}=1.7 \mathrm{pCi} / \mathrm{g}\right)$, corresponding to average background concentrations; or

3) Th-230 concentrations ( $\mathrm{pCi} / \mathrm{g}$ ) determined by the following relation for residual Ra-226 concentrations measured on the finer soil size fraction, $\left(C_{<\# 4 \mathrm{Ra}}\right)$, in the range of 1.7 to $58.8 \mathrm{pCi} / \mathrm{g}$,

$$
C_{<\# 4 \text { Th }}=171.6-1.86 \times C_{<\# 4 R a}
$$

As noted earlier, Ra-226 is readily adsorbed or precipitated within the first 1 to $1.5 \mathrm{ft}(0.3$ to $0.5 \mathrm{~m}$ ) of the subpile cobbly soil, and available data indicates that differential migration of Th-230 has occurred to depths below 3.0 to $4.0 \mathrm{ft}(0.9$ to $1.2 \mathrm{~m})$ beneath the tailings/subpile interface over about 30 percent of the subpile area. In areas where differential migration has occurred, residual bulk Ra-226 concentrations are within the 
range of background. Therefore, excavation control and verification in a large percent of the subpile area will depend on Th-230 concentrations. Accordingly, for the subpile area, the maximum residual Ra-226 and Th-230 concentrations on the finer soil size fraction that would comply with the bulk Ra-226/Th-230 standards $(15 \mathrm{pCi} / \mathrm{g}$ total residual Ra-226 above background, $1.8 \mathrm{pCi} / \mathrm{g}$ bulk Ra-226 concentration) are:

1) $99.7 \mathrm{pCi} / \mathrm{g}$ Th-230, if there is evidence that the Th-230 has differentially migrated relative to $\mathrm{Ra}-226$, and the residual bulk $\mathrm{Ra}-226$ concentration is $1.8 \mathrm{pCi} / \mathrm{g}$, corresponding to average background concentrations $\mathrm{IC}_{<\# 4 \mathrm{Ra}}=$ $1.7 \mathrm{pCi} / \mathrm{g})$;

2) Th-230 concentrations ( $\mathrm{pCi} / \mathrm{g}$ ) are determined from the residual $\mathrm{Ra}-226$ concentrations measured un the finer soil size fraction, $\left(C_{<\# 4 R a}\right)$, in the range of 1.7 to $55 \mathrm{pCi} / \mathrm{g}$ by the relation

$$
C_{<\# 4 T h}=102.9-1.86 \times C_{<\# 4 R a}
$$

Composite and individual $1.0 \mathrm{ft}(0.3 \mathrm{~m})$ depth increment measurements of Th-232 concentrations indicate the remedial action for elevated Th-232 will not be a consideration on the Gunnison processing site.

If subsequent site characterization to determine excavation limits complying with the bulk concentration standards (total Ra-226 not exceeding $15 \mathrm{pCi} / \mathrm{g}$ ) indicates that shallow groundwater is expected to be encountered, the provisions of the Generic Protocol for Thorium Cleanuprvíification at UMTRA Project Sites (Appendix A, Protocol for Contamination in the Saturated Zone) will be applied.

Uranium contamination of the subpile cobbly soil, as noted in the Introduction, is being investigated as a separate Special Study, and any cleanup requirements will be addressed in that investigation. 


\subsection{REFERENCES}

DOE (U.S. Department of Energy), 1992. Environmental Assessment of Remedial Action at the Gunnison Uranium Mill Tailings Site Near Gunnison, Colorado, final, DOE/EA-0372, DOE UMTRA Project Office, Albuquerque Operations Office, Albuquerque, New Mexico.

\section{CODE OF FEDERAL REGULATIONS}

40 CFR Part 192, "Health and Environmental Protection Standards for Uranium and Thorium Mill Tailings," U.S. Environmental Protection Agency (1993).

\section{UNITED STATES CODE}

42 USC $\$ 7901$ et seq., Uranium Mill Tailings Radiation Control Act. November 8, 1978. 
APFENDIX A

GENERIC PROTOCOL FOR THORIUM-230

CLEANUP/VERIFICATION AT UMTRA PROJECT

PROCESSING SITES 


\section{Generic Protocol for Thorium-230 Cleanup/Verification at UMTRA Project Sites}

The excavation of materials contaminated with thorium-230 iTh ${ }^{230}$ ) at one or more UMTRA Project sites may require extensive, deep removal of soil materials to ensure that the radium$226\left(\mathrm{Ra}^{226}\right)$ concentrations will comply with EPA's surface and subsurface soil cleanup standards (i.e., 40 CFR 192). The following discussion presents a unified approach for the future application of standards for $T^{230}$ at the UMTRA Sites.

\section{Introduction}

The cleanup of radiologically contaminated soils on UMTRA Project sites provides explicit requirements for the remediation of soils contaminated with Radium-226 $\left(R a^{226}\right)$, which include limits of 5 and 15 picocuries/gram $(\mathrm{pCi} / \mathrm{g}$ ) for the initial and successive 15 centimeter $(\mathrm{cm})$ deep layers, respectively, averaged over an area of $100 \mathrm{~m}^{2}$. If other radionuclides are encountered in sufficient quantities and concentrations to constitute a significant radiolc.gical hazard, the supplemental standards provisions of 40 CFR 192.21 and 40 CFR 192.22 provide guidance for performing remedial action for these radionuclides in a manner that comes as close to meeting the applicable standard as is reasonable under the circumstances.

Thorium-230 ( $\mathrm{Th}^{230}$ ), which naturally decays, with a half-life of 77.000 years, to form $\mathrm{Ra}^{226}$ is also present in uranium mill tailings and contaminated soils. Therefore, it may be readily shown that for soils containing initial $R^{225}$ and $T^{230}$ concentrations, at time $t=0$, of $\operatorname{Ra}^{225}(t=0)$ and $\operatorname{Th}^{230}(t=0)$, respectively, the $\operatorname{Ra}^{226}$ concentration at any later time, $t$, is:

$$
R a^{225}(t)=R a^{226}(t=0) e^{-\lambda t}+\operatorname{Th}^{230}(t=0)\left(1-e^{-\lambda t}\right) \text {. }
$$

where $\lambda$ is the decay constant for $\mathrm{Ra}^{225}$, or $4.32 \times 10^{.4} \mathrm{yrs}^{-1}$.

Furthermore, the geochemical behavior of $\mathrm{Ra}^{226}$ and $\mathrm{Th}^{230}$ in typical UMTRA site environments have been observed to be significantly different. Under neutral or basic soil conditions, neither $\mathrm{Ra}^{226}$ nor $\mathrm{Th}^{230}$ are preferentially mobile geochemically (i.e., both radionuclides will form chemical compounds that have similar potential for migrating into soils). However, under acidic conditions, the chemical forms taken by these radionuclides are significantly different in their potential for depth migration in soil, with $\mathrm{Th}^{230}$ being more mobile than $\mathrm{Ra}^{226}$.

In windblown tailings areas, mill yards, and ore storage areas of UMTRA sites, it has been observed that: 1) the surface and subsurface soils are normally at neutral $\mathrm{pH}$; 2) the radiological material does not contain abundant quantities of free acid; and 3) the $R^{226}$ and $\mathrm{Th}^{230}$ concentrations are in near secular equilibrium (their activities are approximately equal). The near secular equilibrium for the radiological contamination in these areas results from the fact that most of the uranium ores processed were in near equilibrium. Therefore, the application of soil cleanup procedures for $\mathrm{Ra}^{226}$ according to EPA standards would also reduce the $\mathrm{Th}^{230}$ concentrations to acceptable levels by default, and the total $\mathrm{Ra}^{226}$ as a function of time will not exceed 5 or $15 \mathrm{pCi} / \mathrm{g}$, for surface and subsurface soil respectively.

However, under acidic soil conditions that may prevail in the foundation soil under uranium mill tailings, the subpile region, or in surface and subsurface soils of raffinate or evaporation 
pond, the different geochemical interactions of $\mathrm{Ra}^{226}$ and $\mathrm{Th}^{230}$ will cause these radionuclides to differentially migrate. Generally, under these conditions, $\mathrm{Ra}^{226}$ is adsorbed or coprecipitated on soil within a depth of one to two feet, and $\mathrm{Th}^{230}$ migrates deeper into the subsoil until neutralization of the transporting pore water occurs, where it is removed from solution by the formation of insoluble precipitates or co-precipitates (thorium or thcro-ferro hydroxides, for example). For example, at the Spook, Wyoming site. $T^{230}$ differentially migrated as deep as 20 feet below the raffınate pond before being stabilized by neutralization. In order to be in harmony with the supplemental standards provisions to reduce $\mathrm{Th}^{230}$ concentrations to as low as raasonably achievable (ALARA), and to come as close to meeting otherwise applicable standards as is reasonable under the circumstances, an excavation depth less than 20 feet was selected as a viable solution for this site.

It may be concluded that the cleanup of the initial $\mathrm{Ra}^{226}$ contamination according to standards does not necessarily mitigate against the ultimate ingrowth of residual $\mathrm{Ra}^{226}$ with time due to the radioactive decay of residual $\mathrm{Th}^{230}$ in all areas within a site. As a consequence, residual $\mathrm{Ra}^{226}$ concentrations at a later date, due to ingrowth from $\mathrm{Th}^{230}$ contamination, may pose an undesirable health hazard. Therefore, thic supplemental standards provision of 4C CFR 192 requires the development of a cleanup criterion for $T h^{230}$. which is health protective by reducing exposures to levels that are ALARA, keeping in consideration the measures necessary to implement the remedial actions under the circumstances that exist at: the site. The following procedure establishes appropriate remedial action concentration limits for $\mathrm{Th}^{230}$, and is proposed to be implemented at UMTRA Project sites after concurrence from all governing agencies involved with activities at each site agree to its implementation.

\section{Generic Protocoi}

As can be seen from the equation presented in the introduction, the overall 1000-year maximum concentration of $\mathrm{Ra}^{225}$ in the soils will either be equal to the present $\mathrm{Ra}^{225}$ inventory (if $\mathrm{Th}^{230}$ concentrations are equal to or less than $\mathrm{Ra}^{225}$ concentrations), or the total $\mathrm{Ra}^{226}$ inventory one thousand years in the future (if $T^{230}$ concentrations exceed $R^{226}$ concentrations). If $\mathrm{Ra}^{225}$ concentrations are equal to or exceed $\mathrm{Th}^{230}$ concentrations, the site will already meet the $\mathrm{Th}^{230}$ supplemental standard by default when the site is remediated to the 40 CFR 192 standards for $\mathrm{Ra}^{22 E}$.

Therefore, the supplemental standard chosen for $\mathrm{Th}^{230}$ needs only to ensure that the overall $\mathrm{Ra}^{226}$ concentration one thousand years in the future, when averaged over 100 square-meter areas, will not exceed either $5 \mathrm{pCi} / \mathrm{g}$ in the first $15 \mathrm{~cm}$ layer or $15 \mathrm{pCi} / \mathrm{g}$ in successive $15 \mathrm{~cm}$ layers.

It should be noted that, in keeping with the NRC interpretation of the EPA's soil cleanup standards, the $\mathrm{Ra}^{226}$ concentrations are considered to be bulk concentrations, as determined by the recently developed, NRC-approved protocol for excavation control and soil verification of cobbly subsoils.

\subsection{Protocol for Contamination at Depth}

As the depths of excavations become deeper to remove elevated $\mathrm{Th}^{230}$, the thickness of overiying fill material that is eventually used to remediate the site will increase. As a result, 
attenuation of radon-222 $\left(\mathrm{Rn}^{2 ? 2}\right)$ diffusing through the overlying fill material will also increase. Therefore, as the overlying clean fill material thickness increases, the resultant attenuation of the radon generated from the associated ingrowth of $\mathrm{Ra}^{226}$ will allow higher residual concentrations of $\mathrm{Th}^{230}$ to be left in place, while still attaining a level of protection equivalent to the intent of the $\mathrm{Ra}^{226}$ soil cleanup standards. To determine this concentration, the NRC model (presented in the Draft Generic Environmental Impact Statement on Uranium Milling; NUREG-0511; April 1979) can be used to determine the radon-222 $\left(\mathrm{Rn}^{222}\right)$ flux that would produce 0.02 Working Levels $(W L)$ in a hypothetical structure built on a 100 square-meter $\left(\mathrm{m}^{2}\right)$ grid. The following equation was used:

$$
\begin{aligned}
& C=F A B N R * 1000, \text { where: } \\
& \qquad \begin{aligned}
C & =R n^{222} \text { concentration }(\mathrm{pCi} / \mathrm{l}) \\
F & =R n^{222} \mathrm{fl}\left(\mathrm{A} \cdot \mathrm{i}-\mathrm{Ci} / \mathrm{m}^{2}-\mathrm{s}\right) \\
A & =\text { Area over which the flux enters }\left(\mathrm{m}^{2}\right) \\
B & =\text { Flux reduction factor for entering structure (unitless) } \\
V & =\text { Volume of the structure }\left(\mathrm{m}^{3}\right) \\
R & =\text { Effective } R n^{222} \text { removal rate }\left(\mathrm{s}^{-1}\right) \\
1000 & =\text { conversion factor }\left(1 / \mathrm{m}^{3}\right)
\end{aligned}
\end{aligned}
$$

In areas where basements are feasible (based on local construction practices and deep groundwater table), it should be assumed that the thickness of fill material is eight feet less than the depth of the excavation. Using $A=103 \mathrm{~m}^{2}, B=0.5, V=250 \mathrm{~m}^{3}$, and $R=$ $1.98 \times \mathrm{i}^{-4} \mathrm{~s}^{-1}$, a flux of $3.9 \mathrm{pCi} / \mathrm{m}^{2}-\mathrm{s}$ would produce indoor air concentrations of approximately $4.0 \mathrm{pCi} / / \mathrm{Rn}^{222}$. Assuming radon daughters are present at $50 \%$ equilibrium, this would correspond to $0.02 \mathrm{WL}$.

(2) Thus, the RAECOM computer code can be used to calculate the resultant flux from higher concentrations at depth in order to determine if further excavation is warranted. As long as the calculated flux is less than $3.9 \mathrm{pCi} / \mathrm{m}^{2}-\mathrm{s}$, it can be assumed that equivalent protection is provided. The calculations shall use site-specific parameters when available. Reasonably conservative parameters that consider the expected site conditions shall be used when site-specific data are unavailable.

\subsection{Protocol for Contamination in the Saturated Zone}

Another scenario potentially impacting excavations to remove elevated $\mathrm{Th}^{230}$ concentrations is when groundwater is encountered at shallow depths. Since the $\mathrm{Th}^{230}$ contamination has been present within the saturated zone long enough for soluble constituents to have been mobilized, it is reasonable to assume that any remaining $\mathrm{Th}^{<30}$ that may be encountered within a saturated zone will not be appreciably mobilized by pH neutral groundwater. Furthermore, it is known that the diffusion coefficient decreases dramatically as soils approach full saturation until it reaches values typical of water (Radon Attenuation Handbook for Uranium Mill Tailings Cover Design; NUREG/CR-3533; April 1984). It is therefore reasonable to assume that $\mathrm{Rn}^{222}$ generated within a saturated zone generally will not diffuse to the surface. Finally, it is very difficult to perform deep, cost effective excavations within a saturated zone. 
(3) Therefore, whenever shallow groundwater is encountered, the following options will be considered:

(a) Excavation into the saturated zone will be considered when water pumping or other controls are reasonable and when high concentrations of $\mathrm{Th}^{230}$ extend only a short distance into the saturated zone.

(b) An ALARA analysis will be performed in cases where a major portion of the site contains Th-230 which extends into the saturated zone, and excavation into the zone is impractical. The ALARA analysis will use reasonably conservative assumptions to project future doses.

(c) If water pumping or other controls are not reasonable, excavation will halt at the level of the saturated zone.

\section{Verification Sampling}

Under typical site conditions, verification of the $\operatorname{Th}^{230}$ supplemental standard is to be achieved by a three-tiered sampling approach.

(4) In areas within an UMTRA processing site that are suspected of preferentially mobilizing thorium contamination over radium contamination (e.g. . under raffinate pits), based upon process knowledge or other sources such as previous sampling data, $100 \%$ of the grids are to be sampled and analyzed for $\mathrm{Th}^{230}$.

(5) In subpile areas, $10 \%$ of the grids will be sampled.

(6) In areas where process knowledge and characterization data indicates no potential for preferential mobilization (e.g., windblown tailings and ore storage areas), grids will not be sampled for $\mathrm{Th}^{230}$.

An analysis of verification data from the Tuba City, Arizona, UMTRA site, which has completed remediation and used this strategy, found no instances in the area sampled at the rate of 1 out of 25 grids where $\mathrm{Th}^{230}$ concentrations would cause future (i.e., at $t=1000$ years) expected $\mathrm{Ra}^{226}$ concentrations to exceed 40 CFR 192 standards for $\mathrm{Ra}^{226}$. Furthermore, preliminary results confirm the expectation that $\mathrm{Th}^{230}$ concentrations are generally equal to or less than $\mathrm{Ra}^{226}$ concentrations in areas other than beneath the raffinate pits, and $\mathrm{Ra}^{226}$ concentrations are well correlated to $\mathrm{Th}^{230}$ concentrations in these areas.

If any verification samples exceed the $\mathrm{Th}^{230}$ criteria of this protocol, the surrounding eight grids will be examined to determine whether or not these grids also exceed the criteria. If sample results have not been generated for the surrounding grids already, archived samples of such grids will be analyzed. If any of the surrounding grids also exceed the $T^{230}$ criteria, the surrounding eight grids around such grids will also be examined. This process will continue until no more of the surrounding grids exceed the $\mathrm{Th}^{230}$ criteria. All grids that exceed the criteria will undergo further remediation unless there is sufficient justification and concurring parties agreement to do otherwise. 


\section{Conclusion}

Based upon the above discussions, the following generic protocol shall be used for the excavation of $\mathrm{Th}^{230}$ at all $\mathrm{fl}$.Jre UMTRA Project sites:

(1) Excavate bulk $\mathrm{Th}^{230}$ to a 1000-year corrected bulk Ra ${ }^{226}$ concentration of 5 or $15 \mathrm{pCi} / \mathrm{g}$ (as appropriate) in 15-cm layers;

(2) For deeply buried material, stop excavations when the RAECOM computer code, using site-specific parameters, calculates a $R^{222}$ flux of $3.9 \mathrm{pCi} / \mathrm{m}^{2}-\mathrm{s}$;

(3) Consider the following options whenever shallow groundwater is encountered:

(a) Excavate into the saturated zone when water pumping or other controls are reasonable, especially when high concentrations of $\mathrm{Th}^{230}$ extend only a short distance into the saturated zone.

(b) An ALARA analysis will be performed in cases where a major portion of the site contains Th-230 which extends into the saturated zone, and excavation into the zone is impractical. The ALARA analysis will use reasonably conservative assumptions to project future doses.

(c) Halt excavations at the level of the saturated zone when water pumping or other controls are not reasonable.

(4) Perform verification sampling for bulk $\mathrm{Th}^{230}$ in all grids underneath raffinate pits or other areas suspected of having a mechanism to preferentially mobilize $\mathrm{Th}^{230}$ over $\mathrm{Ra}^{226}$;

(5) Perform verification sampling for bulk $\mathrm{Th}^{230}$ in $10 \%$ of the grids underneath sub-pile areas; and

(6) Do not perform verification sampling for bulk $\mathrm{Th}^{230}$ in grids for which process knowledge and characterization data indicates no potential for preferential migration. 


\begin{abstract}
APPENDIX B
U.S. DEPARTMENT OF ENERGYIU.S. NUCLEAR REGULATORY COMMISSION CORRESPONDENCE

PROCEDURE FOR BULK RADIONUCLIDE DETERMINATION, EXCAVATION CONTROL, AND SITE VERIFICATION FOR SOILS CONTAINING COBBLES
\end{abstract}




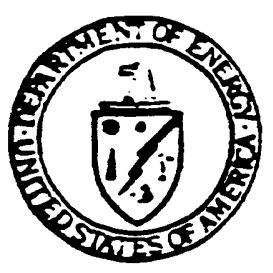

Department of Energy

Nouquerque Operations Ottice

P.O. Box 5400

Abuquerque New Mexico 87115

\section{GEP O :}

\section{FIDIRAT EXPRTSS}

yr. John Sumeier

Crief, Uranium Recovery Bramh

Division of Iow-Ievel Baste

Managenent \& Decomissioning

office of Muclear Materials Safety

and Safeguards

0.S. Nuclear Regulatory Comission

Mail Stop 5-E-2

1 White Flint North

12555 Rockville Pike

Pockville, ID 20852

Dear Mr. Sumeier:

Historically, the majority of Ra-226 contaminated materials being cleaned IP at Uranium Mill Tailings Renedial Action (UMRA) Project sites consisted of tailings, ore spoils, tailings intermixed with fine-grained soils (winoblown and vicinity properties) and fine-grained soils under residual milling waste (tailings and raffinate ponds). Hovever, in 1988, conditions excountered wille renediating in-230 contamination which persisted ance Ra-226 had been removed at both the Riverton, Wyoming, and Durango, colorado, sites were substantially different.

At both sites, which were located on alluvial flootplains, subsriface soils consisted of a large percentage of cobbles and gravels which were retained on a $\$ 4$ sieve $(4.76 \mathrm{~mm})$. In addition, it was determined that approximately 95 percent of the total cancentration of radioactivity was deposited an the fines (those soils passing a 14 sieve).

Based on these considerations and the fact that cleardp verification sampling rartinely enployed by the Remedial Action Contractors did not readily accomodate sampling and analysis of materials greater than \$4 sieve, the following protocol for $1 \mathrm{th}-230$ cleamp/verification to a bulk cubsurface condentration of $35 \mathrm{pCi} / \mathrm{g}$ was adopted and apsioved by the U.S. Department of Energy (DOE) and the U.S. Nuclear Reglatory Comission (NRC) in modifications to the Remedial Action Plans for those sites.

1. Determine the "fires ass fraction" by collecting several (10) representative bulk field samples, and separating fractions greater than and less than a 14 sieve:

fires mass fraction $=$ mass fines/mass total $=$ unitless fraction 
12. John sumeier

2. Determine Ra-226 (or In-230) cancentration (passing the 14 sieve) in verification samples collected and analyzed ascording to standard methodology described in MIK-Ferguson Cupany (MR-F) Procedure RAC-015, "Verification Procedures for Vicinity Properties and Tailings Sites."

3. Determine "bulle" Ra-226 (or In-230) cancentration drring clearp and verification by correcting the Ra-226 (or Th-230) cancentration on fines as follars:"

bulk Pa-226 cancentration = (fines Pa-226 cancentration) $X$ (fines mass fraction) or, bulk $\mathbf{I n - 2 3 0}$ cancentration $=$ (fines $\mathbf{I n - 2 3 0}$ concentratian) $X$ (fines rass saction)

The DOE proposes to adopt this basic protocol, an a site epecific basis, as a standard coerating practice for cleans and verification of both resichal Ra-226 and In-230 contamiration at UMIRA sites yet to be remediated. The bulk cancentrations of Ra-226 ax In-230 determined by the above procedure will comply with the remediation standard for Pa-226, and suplemental cleardp requirements for Th-230, as specified in $40 \mathrm{CrR}$ 192. Bulk Ra-226 concentrations will not exceed 5 and $15 \mathrm{pCi} / \mathrm{g}$ for respective $15 \mathrm{col}$ deep suface and subsurface layes averaged over 100 III. Similarly, the bulk cancentration of subsurface $\mathrm{Th}-230$ in $15 \mathrm{~cm}$ depth increments will not exceed $35 \mathrm{pCi} / \mathrm{g}$ averaged over $100 \mathrm{~m}^{2}$. The details of the protoosl will be more campletely described in an added section to Procedre RAC-015 which will address then the protool wall be implemented; the numer of eamples needed to provide the initial value for the fines mass fraction; the frequency of updatixe/verifying the fines mass fraction as work progresses across a site; how the fines mass fraction used will be logged on field data sheets, etc. It will also be included in enture updates of the Tectrical Appreact Doament and referenced as appropiciate in Remedial Action Plars for sites where it vill be applied.

The sites where this protool could be applied waild likely include Grand Juntion, Grmison, Rifle, Slick Rock, and Naturita, colorado, sine they are all located on alluvial Iloodilains possessing characteristios similar to those described above. Therefore, the DDE requests the NRX's conarrence that axd a protocol is consistent with the requirements of 40 CFR 192 and that its cantractors should be directed to formally incorporate the protsool into standard operating procedures and address its potential appliation in site Remedial Action Plans. 
Mr. John Surmeier

$$
-3 \text { - SEF } 061991
$$

If you should have any further questions regarding this request, please call Mr. Dan Hetzler of wy staff at (FIS) 845-5657.

sincerely,

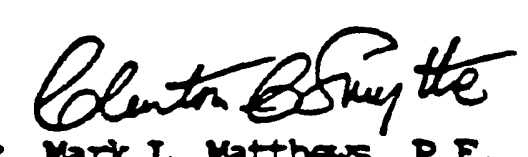

Mark L. Matthews, P.E.

Project Yarager

Uranilu Mill Tailings Remedial Action Project office

C:

F. Bosiljevac, L.MrA

S. Hill, TAC

M. Miller, Tac

D. Canzales, Tac

J. Oldham, MK-F

D. Carlson, IR-F

M. Madeen, Or-Geotech 


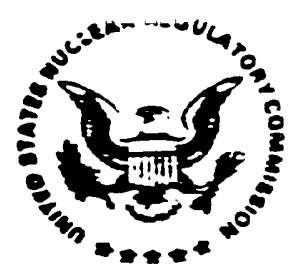

UNITED STATES

NUCLEAR REGULATORY COMMISSION

WASHINGTON, D. C. 20555

SEP 171991

Mr. Mark L. Matthews P.E.

Project Manager

Uranium Mill Tailings Remedial Action

Project Office

Department of Energy

Albuquerque Operations Office

P.0. Box 5400

A ibuquerque, New Mexico 87115

Dear Mr. Matthews:

Your letter of September 6, 1991, requested U.S. Nuclear Regulatory Commission (NRC) concurrence in the use of a procedure for determining and verifying radium-226 concentrations at locations with large quantities of cobbly material. Your letter states that at several Title I sites, DOE has encountered large quantities of radium-226 or thorium-23C contaminated material with a high content of cobbly material (greater than a No. 4 sieve size). Your tests show that the contained radioactivity is concentrated in the finer fraction with the coarse fraction containing negligible quantities (less then 5 percent). Procedures presently in use by your contractors for sampling and analyses are designed for relatively fine grained homogeneous soils with a minimum of larger material and are not adequate to characterize the radioactive concentrations in the heterogeneous size material being encountered. Your proposed approach would rely on measurement of the radium-226 or thorium-230 content in the finer fraction to obta in an average concentration for the entire sample.

We agree that determining an average radium-226 or thorium-230 content over an entire sample would be consistent with the Environmer:al Protection Agency (EPA) standards in 40 CFR 192 if the radium-226 content of the two size fractions and the percentage of each size fraction are properly factored. Part 192.12 states that the concentration of radium-226 in land can be averaged over an area of 100 square meters to meet the standards of not exceeding background level by more than $5 \mathrm{pCi} / \mathrm{g}$ averaged over the first $15 \mathrm{~cm}$ of soil below the surface and $15 \mathrm{pCi} / \mathrm{g}$ averaged over $15 \mathrm{G}$ thick layers of soil more than 15 cm be low the surface.

You plan to address the details of the procedure in a section to be added to Procedure RAC-015 which will define when the procedure would be used, the numer and distribution of samples to be taken, the determination of the radium-226 distribution and size fractions, and other appropriate details.

We agree that the proposed approach has the potential for maintaining compliance with EPA's standards while avoiding over excavation of contaminated 
Mark Matthews

-2 -

material. However, for final concurrence, we will have to review the details of your revised Procedure RAC-015 and the effects of its implementation on a site-specific basis. Any questions can be addressed to Allan Mullins of my staff at FTS-492-0578.

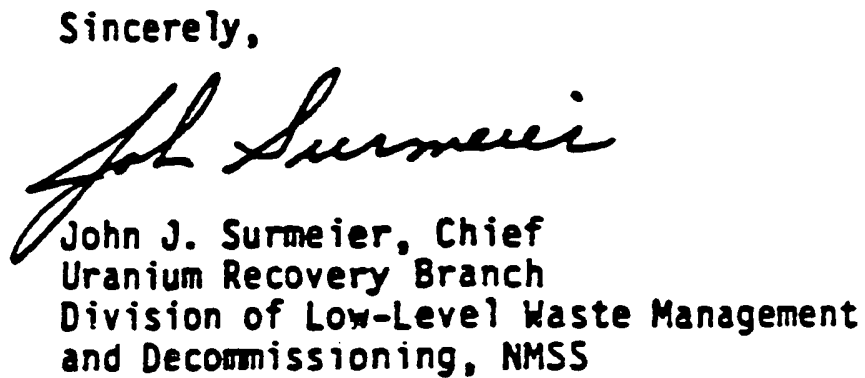

cc: D. Metzler, DOE, Alb. 


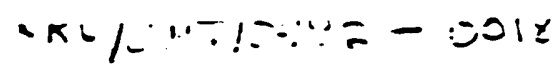

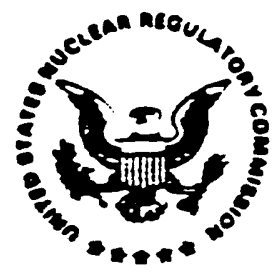

UNITED STATES

NUCLEAR REGULATORY COMMISSION

MASHINGTON. D. C. 20555

\section{$A P R+1292$}

Mr. Albert R. Chernoff, Project Manager

Uranium Mill Tallings Remedial Action

Project Office

U.S. Department of Energy.

Albuquerque Operations Office

P.0. Box 5400

Albuquerque, Nex Mexico 87115

Dear Mr. Chernoff:

We have reviewed the procedure on "Bulk Radionuclide Determination, Excavation Control, and Site Verification For Cobbly Solis" sent with your letter of March 26, 1992, and supplemented by a revised Page 12 sent by facsirile on March 30, 1992. He hereby concur with its use on U.S. Department of Energy Uranfum Mill Tailings Remedial Action Project sites containing a high percentage of cobbly subsoil. This procedure, designated RAC-0P-003, should be referenced in the specific Renedial Action plans for those sites where it will be used.

One item of note concerns the section of the procedure discussing authority (Section 1.3). This section should be revised to reference this letter of concurrence rather than the September 17, 1991, letter cited, which agreed with the concept but did not concur with the procedure.

Any questions stould be addressed to Allan Mullins of to staff at FTS 964-2578.

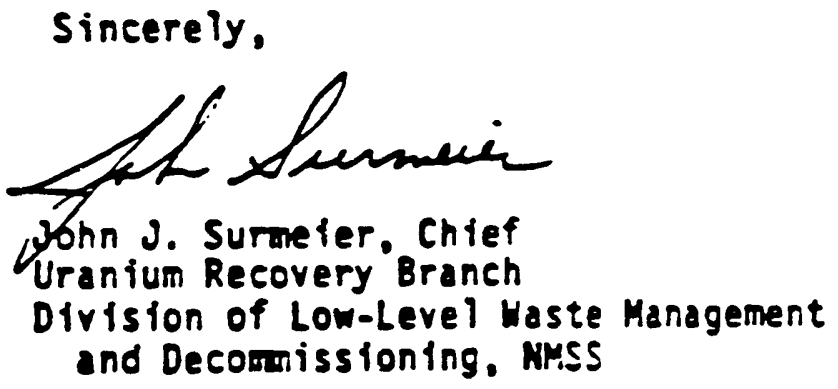

ce: D. Metzler, DOE Alb

P. Mann, DOE Alb

D. Gonzalez, TAC 


\section{Soil Verification Using Cobbles-To-Fines Correction}

\subsection{SCOPE}

\subsection{Purpose}

This procedure will be used for determining and verifying average bulk radionuclide concentrations for radium-226 (Ra-226), thorium-230 (Th-230), and, if necessary, thorium-232 (Th-232) at locations where the subsoil consists of a percentage of cobbles in the bulk sample sufficient to affect measurement of the total radionuclide concentration. Excavation control and verification will be based on bulk concentrations determined by this procedure.

1.2

Applicability

This procedure may be applied in areas designated for routine soil excavation and verification (see RAC-OP-003) where the subsoil media contains a high percentage of cobbles.

\subsection{Authority}

Letter to A.R. Chernoff, DOE/UMTRA from J.J. Surmeier, NRC dated April 4, 1992.

\subsection{REFERENCES}

2.1 RAC Health Physics Procedure RAC-RP-005 - Radiological Instrumentation.

2.2 RAC Health Physics Procedure RAC-OP-002 - Excavation Control Procedure

2.3 RAC Health Physics Procedure RAC-OP-003

\subsection{DEFINITIONS}

3.1 Cobbles - The portion of a composite soil sample which will not pass through a \$4 mesh sieve.

3.2 Fines - The portion of a composite soil sample which will pass through a $\# 4$ mesh sieve.

3.3 Mass partition function, f, of a cobbly soil sample - the ratio of the dry mass of the cobbles ( $M_{20.4}$, to the dry mass of the fines ( $M_{\text {cod }}$ ):

$f=M_{\text {sod }} / M_{\text {cod }}$ (cobble to fine ratio). 
where the total dry mass of the sample, $M_{7}$, is

$M_{T}=M_{>04}+M_{\text {cos. }}$

3.4 Radiological concentration (Ra-226, Th-230, or Th-232), in picocuries per gram (pCi/g), for the fines or cobbles, are designated by $C_{\text {sme }}$ and $C_{\text {ses, }}$ respectively.

3.5 Bulk radionuclidé concentration (Ra-226, Th-230, or Th-232), in pCi/g, is designated by $C_{n}$, and calculated using

$C_{.}=$Total Sample Radioactivity (pCi) / Total Dry Mass of Sample (g)

$C_{8}=\left(C_{\text {ese }} \times M_{\text {ces }}+C_{\text {sed }} \times M_{\text {sed }}\right)\left(M_{\text {ces }}+M_{\text {sed }}\right)$

$C_{1}=C_{\text {sod }}(1 /(1+f))+C_{\text {sod }}(f /(1+f))$.

3.6 Student $t$ distribution $(t)$ - the mathematical quantity used to define the distribution of test statistics for small sample populations; used herein to determine the lower and upper 95 percent confidence values for mass partition function and cobble radionuclide concentrations respectively.

3.7 Running Average - The determination of statistical quantities based on the available data and recalculated as more data becomes available.

3.8 Statistical Mass Partition Function $\left(f_{l}\right)$ - The mass partition function $(f)$, at the lower 95 percent confidence value calculated from test pit or running average data.

3.9 Statistical Cobble Radionuclide Concentration $\left(C_{>u}\right)$ - The cobble radionuclide concentration $\left(C_{>}, 4\right)$ at the upper 95 percent confidence value calculated from test pit or running average data.

\subsection{REQUIREMENTS}

\subsection{Prerequisites}

4.1.1 All instruments used under this procedure shall have valid calibration.

4.1 .2

Backup data (correlations, etc.) must be acquired, retained onsite, and made available for audit, on all methods and analyses used for excavation control and verification measurements. 


\subsection{Tools, Material, Equipment}

4.2.1 Buckets, wheelbarrows, mesh sieves or screens 14.8 millimeter), shovels or backhoe, weighing scale up to 200 lbs.. drying oven, and other materials, as necessary. to obtain representative bulk soil samples. It should be noted that a 1/4 inch hardware cloth is approximately equivalent to a $\$ 4$ mesh sieve, and may be used in lieu of a $\$ 4$ mesh sieve.

\subsection{Precautions/Limits}

N/A

\subsection{Acceptance Criteria}

N/A

\subsection{PROCEDURE}

\subsection{Site Evaluation}

5.1.1 This guidance applies to processing sites and vicinity property areas. If the work area under consideration is less than 0.5 acre, the mass partition function $(f)$ will be based on soil sampling from one centrally located test pit.

5.1.2 The statistical mass partition function $\left(f_{L}\right)$ and statistical cobble radionuclide concentration $\left(C_{2 u}\right)$ may be determined by analysis of samples collected from test pits prior to construction. The purpose for developing a statistical mass partition function is enly to obtain an estimate of the excavation depth required for compliance with radiological cleanup standards.

5.1.3 Approximately 30 uniformly distributed sampling locations (test pits) will be used for the entire site. Fewer test pits may be used on small sites with prior approval from the HP \& E Manager.

5.1.4 If test pit excavation activities are performed during remedial action, the statistical mass partition function $\left(f_{L}\right)$ shall be obtained by calculating a running average of the corresponding parameters obtained as test pit work progresses across the site. 


\subsection{Test Pit Soil Sampling and Analysis}

5.2.1 Dig each test pit to the estimated depth of contamination at the location, and record the test pit surface elevation and maximum depth of each test pit.

5.2.2 Collect one composite sample from each test pit. The composite soil sample shall be comprised of all the material contained in a standard shovel from each one foot increment (no material sha'l be discarded). Sampling shall begin at the cobbly soil surface or tailings/cobbly soil interface and continue through a minimum of 5 feet of cobbly material or all the cobbly material (whichever is less).

5.2.3 Sieve the composite sample through a $\$ 4$ mesh sieve, collecting the fines and cobbles in separate buckets of known weight. Weigh both fractions separately. Thoroughly mix the fine fraction and extract two (2) representative 500 gram can samples.

5.2.4 Determine the percent moisiure content by weight of one of the can samples and calculate the adjusted dry weight of the fines. Calculate the adjusted dry weight of the cobbles assuming a moisture content of 1.5 percent. Record all pertinent information on the Cobbles to Fines Calculation Sheet (Attachment 1). Calculate the mass partition function $(f)$ for the test pit using the adjusted dry weight of the fines and cobbles in the relation defined in Definition 3.3.

5.2.5 The second can shall be analyzed by the site laboratory for Ra-226 (and Th-232 if necessary) and sent to the vendor laboratory for Ra-226 and Th-230 analysis (and Th-232 if necessary). Record on-site and vendor analysis results on Attachment 1.

Note: Analysis for Th-232 shall only be performed if the site characterization indicates Th-232 is present.

5.2.5.1 Using the initial on-site analysis results and the vendc, analysis results for all test pit or running average samples. a Ra-226 correction factor shall be established using the following equation:

Ra-226 C.F. $=\frac{R_{1}+R_{2}+\ldots R_{n}}{n}$ 


$$
\begin{aligned}
& \text { where, C.F. }=\text { correction factor, } \\
& R_{n}=\text { Vendor results }(\mathrm{pCi} / \mathrm{g}) \text { divided by } \\
& \text { initial OCS, results ( } \mathrm{pCi} / \mathrm{g} \text { ) fo: the } \\
& n^{\text {th }} \text { sample (i.e., } 1,2, \ldots n \text { ), and } \\
& n=\text { number of satios. }
\end{aligned}
$$

5.2.5.2 The correction factor shall be updated using the pertinent - analyses results from QA samples (section 5.10).

5.2.6 Ship the fraction retained on the $\# 4$ sieve from each test pit to the vendor laboratory, in the 5-gallon bucket. This material shall be cleaned, crushed, and analyzed for Ra-226, Th-230 and, if necessary, Th-232. Record vendor analysis results on Attachment 1.

5.2.7 Calculate the bulk radionuclide concentration $\left(C_{B}\right)$ for the test pit using the mass partition function (f) for the test pit (section 5.2.4), the vendor radiological concentration of the fines $\left|C_{\text {cas }}\right|$, and the radiological concentration of the cobbles $\left(C_{2, a 4}\right)$ in the equation defined in Definition 3.5. Bulk radionuclide concentrations should be calculated for Ra-226, Th-230, and Th-232 (if necessary).

5.3 Establish background bulk radionuclide concentrations for Ra-226, Th-230, and, if necessary, Th-232, by the sampling and analyses detailed in steps 5.2.1 through 5.2.6 at three uncontaminated background locations containing cobbly subsoil.

5.4 Statistical Mass Partition Function - Alternative 1 (Test Pits)

5.4.1 Upon completion of test pit sampling, pertinent data shall be compiled on the Statistical Data Sheet (Attachment 2), and the statistical mass partition function shall be calculated using the following equation:

$$
t_{L}=\bar{f}-t(s / \sqrt{n})
$$

where, $f_{L}=$ statistical average mass partition function at the lower 95 percent confidence value.

$\bar{f}=$ mean mass partition functions of $n$ samples,

$s=$ sample standard deviation for the $n$ samples.

$t=t$ from Attachment 3, and

$n=$ number of observations. 
5.4.2 The mean of the sample population is determined as follows:

$$
\bar{f}=\frac{f_{1}+f_{2}+\ldots f_{n}}{n}
$$

where, $f_{1.2 \times n}=$ the value of $f$ for sample 1, 2, or $n$; and $n=$ the number of samples.

5.4.3 The standard deviation is calculated as follows:

$$
s=\frac{\sqrt{\sum_{n=1}^{n}\left(f_{n}-T\right)^{2}}}{n-1}
$$

where, $\mathbf{S}=$ sample standard deviation,

$f_{n}=$ value of $f$ for sample $n$,

$\bar{f}=$ mean of $f$ for $n$ samples, and

$n=$ number of samples.

5.4.4 The statistical cobble radionuclide concentration for Ra-226, Th-230 and Th-232 (if necessaryl shall be calculated using the following equation:

$$
C_{>u}=\bar{C}+t(s / \sqrt{n})
$$

where, $C_{>u}=$ The statistical cobble radionuclide concentration at the upper 95 percent confidence value,

$\overline{\mathbf{C}}=$ mean radionuclide concentration of the $n$ samples,

$s=$ standard deviation of the 30 samples,

t $=$ from Attachment 3, and

$n=$ number of samples.

5.5 Statistical Mass Partition Function - Alternative 2 (Running Average)

5.5.1 The statistical mass partition function $\left(f_{b}\right)$ and the statistical cobble radionuclide concentration ( $C_{>u}$ ) may be obtained by calculating a running average of the corresponding parameters using the equations 
in sections 5.4.1 and 5.4.4 respectively. Compile data on the Statistical Data Sheet and recalculate $f_{L}$ and $C_{>u}$ as data is obtained from each new test pit.

5.6 The statistical mass partition function and statistical cobble radionuclide concentration for alternative 1 or 2 , will only be used for excavation control to obtain an estimate of the final excavation depth to comply with EPA's radiological cleanup standards. Final excavation depths and verification will be determined as described in section 5.7 through 5.9 .

5.6.1 By solving the equation in section 3.5 for $C_{c \infty}$ and using the statistical mass partition function $\left(f_{L}\right)$ and the statistical cobble radionuclide concentration $\left(C_{>}\right)$) an estimate of the allowable fines radionuclide concentration may be obtained as follows:

$$
c_{\text {e*14 }}=\frac{c_{B}-c_{2 U}\left[f_{L} /\left(1+f_{L}\right)\right]}{\left[1 /\left(1+f_{L}\right)\right]}
$$

where, $C_{<e a}=$ The estimated fines radionuclide concentration,

$C_{B}=$ the applicable limit (i.e., 5 or $15 \mathrm{pCi} / \mathrm{g}$ for Ra-226 or Th-232 or $35 \mathrm{pCi} / \mathrm{g}$ for Th-2301,

$f_{L}=$ statistical mass partition function, and

$C_{>u}=$ statistical cobble radionuclide concentration.

\subsection{Verification Soil Sampling \& Analysis}

5.7.1 Grid the entire site into squares of $100 \mathrm{yd}^{2}\left(-100 \mathrm{~m}^{2}\right)$. Grids shall be uniformly distributed over the site so as to obtain representative data. Record location and elevation for each $100 \mathrm{yd}^{2}$ grid.

5.7.2 Further subdivide each grid where excavation control is being performed into approximately $10 \times 10$ foot squares (see below). Subdividing grids may normally be done visually by the technician performing the survey. Soil sample extraction will be performed at each of the nine $10 \times 10$ foot squares within the grid. Each soil plug should consist of all the material contained in a standard shovel. This will include soil, rock, and small/large gravel. No material is to be discarded. The soil plug will be taken to a depth of 15 centimeters (cm). Sample collection shall be random (non-biased). 


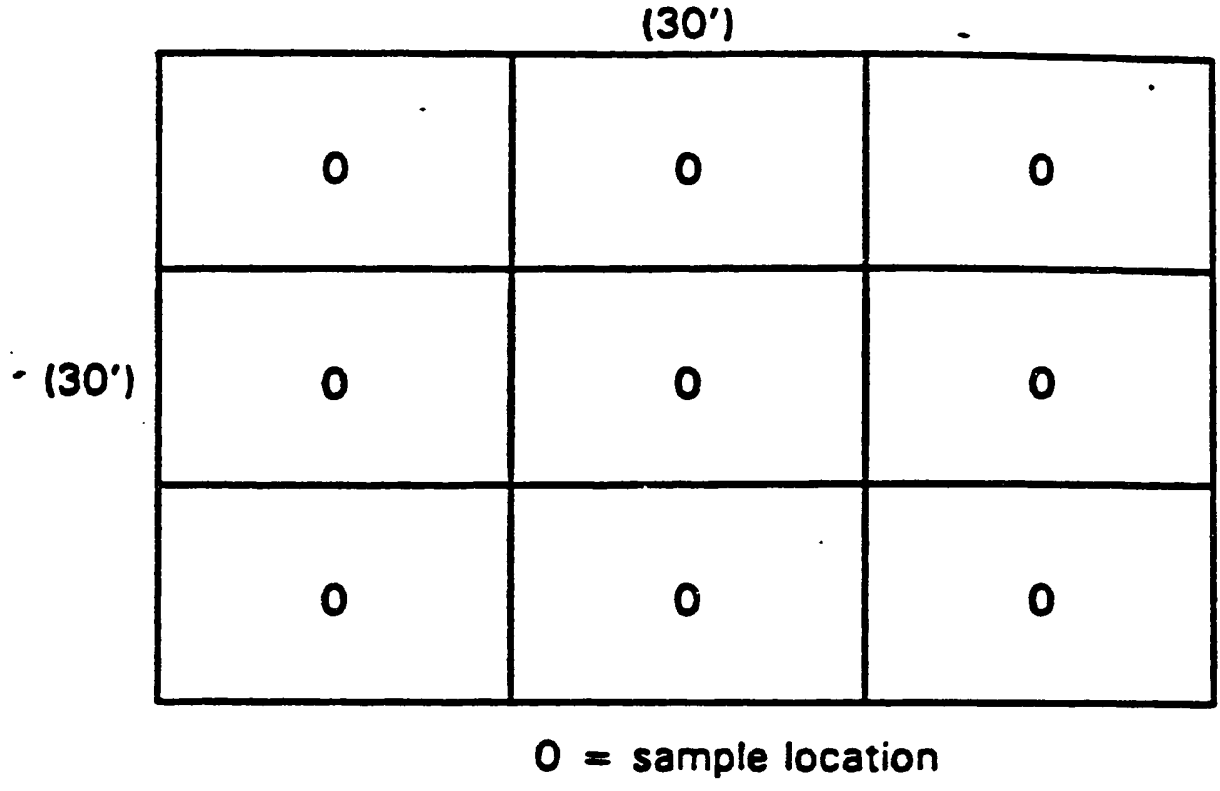

5.7.3 The nine soil plugs $(80-100$ lbs combined weight) comprise a composite sample.

5.7.4 Sieve the composite sample through a \#4 mesh sieve, collecting the fines and cobbles in separate buckets of known weight. Thoroughly mix the fine fraction.

\subsection{Site Verification Alternative 1 (Grid Specific)}

5.8.1 For verification alternative 1, weigh both fractions separately and record information on Attachment 1.

5.8.2 Extract two (2) $500 \mathrm{~g}$ can samples of the fine fraction for moisture content and radiological analysis.

5.8.3 Determine the moisture content of the fines and calculate the adjusted dry weight. Calculate the adjusted dry weight of cobbles assuming a moisture content of 1.5 percent. Calculate and record the mass partition function for the grid (Attachment 1 ).

5.8.4 Analyze the second can sample of fines for Ra-226 and, if necessary, Th-232 with the on-site OCS. Calculate the corrected radionuclide concentration using the site Ra-226 correction factor determined in section 5.2.5.1.

5.8.5 Calculate the bulk radionuclide concentration $C_{B}$ :or the grid using the grid specific mass partition function, radionuclide concentration of the fines (the initial corrected concentration shall be used for calculating the Ra-226 bulk radionuclide concentration) and the statistical cobbles 
radionuclide concentration $\left(C_{>4}\right)$ in the formula in section 3.5. All data shall be recorded on Form F1-OP-003-4.

5.8.6 Dry, seal and store the sample for 20 day on-site analysis.

5.8.7 After a minimum of 20 days, reanalyze the sample and calculate the final bulk radionuclide concentration $\left(C_{B}\right)$ for the grid.

5.9 Site Verification Alternative 2 (Statistical)

5.9.1 For each $100 \mathrm{~m}^{2}$ grid, a 9-plug composite soil sample of the fines soil will be obtained following the technique outlined in Section 5.7.

5.9.2 Extract a $500 \mathrm{~g}$ can sample of the fine fraction for radiometric analysis. Discard the larger size soil fraction.

5.9.3 Analyze the can sample for Ra-226 and, if necessary. Th-232 with the on-site OCS. Calculate the corrected radionuclide concentration using the site correction factor determined in section 5.2.5.1.

5.9.4 Calculate the bulk radionuclide concentration for the grid using the radionuclide concentration of the fines (the initial corrected concentration shall be used for calculating the Ra-226 bulk radionuclide concentration), the statistical mass partition function $\left(f_{L}\right)$ and the statistical cobble radionuclide concentration $\left(C_{>u}\right)$ in the formula from section 3.5 as modified below.

$$
C_{B}=C_{<+4}\left[1 /\left(1+f_{L}\right)\right]+C_{24}\left[i_{L} /\left(1+f_{L}\right)\right]
$$

Record the pertinent data on the Alternative 2 Bulk Concentration Data Sheet (Attachment 4).

5.8.6 Dry, seal and store the sample for 20 day on-site analysis.

5.8.7 After a minimum of 20 days, reanalyze the sample and calculate the final bulk radionuclide concentration $\left(C_{8}\right)$ for the grid.

5.10 The verification sample from the 25 th $100 \mathrm{~m}^{2}$ grid of each block will be sent to an outside Vendor laboratory for independent Ra-226, Th-230, and, if necessary. Th-232 analyses in accordance with Quality Assurance requirements. 


\subsection{RECORDS/REPORTS/NOTIFICATIONS}

6.1 All data shall be recorded on the appropriate data sheet.

\subsection{ATTACHMENTS}

7.1 Attachment 1 - Cobble to Fines Calculation Sheet (FI-OP-003-4)

7.2 Attachment 2 - Statistical Data Sheet (F2-OP-003-4)

7.3 Attachment 3 - Critical Values of $t$.

7.4 Attachment 4 - Alternative 2 Bulk Concentration Data Sheet (F4-OP-003-4) 


\section{COBBLES TO FINES CALCULATION SHEET}

Size:

Sample/Grid I.D.

\section{Fines}

Wr. Sample

Bucket

Wi. Bucker

Wr. Sample

Adjusted

Diy Wr. $\left(M_{c o e}\right)^{\bullet}$
Cobbles

Wi. Sample

- Bucker

Wr. Bucket

Wr. Sumple

Adjusted

Diy Wr. $\left(M_{\text {sod }}\right)^{\cdots}$
Sheet of

Date:

\section{Moisture Content Fines}

Wet Wi. SampletPan Dry Wr. Sample/Pan Tare Wi. Pan:

Wr. Water"

Wr. Soil"

Perceni Water $(\%, \bullet)$

- Adjusted Dry Wr. of the Fines =

Wi Sample $\times 11$ - $\$$ water

- Adjusted Dry Wr. of the Cobbles =

Wi. Sample $\times 11 \cdot .0151$

Mass Partition fuhetion (f) $=$. $\quad M_{204} / M_{\text {cad }}=$

\begin{tabular}{|c|c|c|c|c|}
\hline $\begin{array}{c}\text { Radiological } \\
\text { Analysis }\end{array}$ & $\begin{array}{l}{ }^{220} R_{a} \\
\left(D C_{1} / g\right)\end{array}$ & $\begin{array}{l}230 \mathrm{Th} \\
(\mathrm{DC} / \mathrm{g})\end{array}$ & $\begin{array}{l}23 \times T h \\
(p C i / g)\end{array}$ & COMMENTS \\
\hline $\begin{array}{l}\text { Cobbies } \\
\left(C_{204}\right)\end{array}$ & & & & \\
\hline $\begin{array}{l}\text { On-Size Analysis } \\
\text { Fines } \\
\text { (C coal }\end{array}$ & $\frac{\bullet}{\bullet}$ & $N / A$ & & \\
\hline $\begin{array}{l}\text { Vendor Analysis } \\
\text { Fines } \\
\text { IC ceal }\end{array}$ & & & & \\
\hline $\begin{array}{l}\text { Bulk Radionuelide } \\
\text { Concentration } \\
\text { (C) }\end{array}$ & $\frac{\pi}{\pi t}$ & & & \\
\hline
\end{tabular}

- Inal: $\quad \bullet$ inival Correcreo.

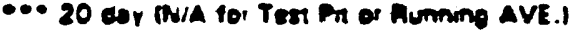

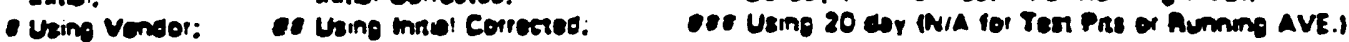

2Na Correction Factor:

Reviewed By:

Date:

F1.0P.003.4 


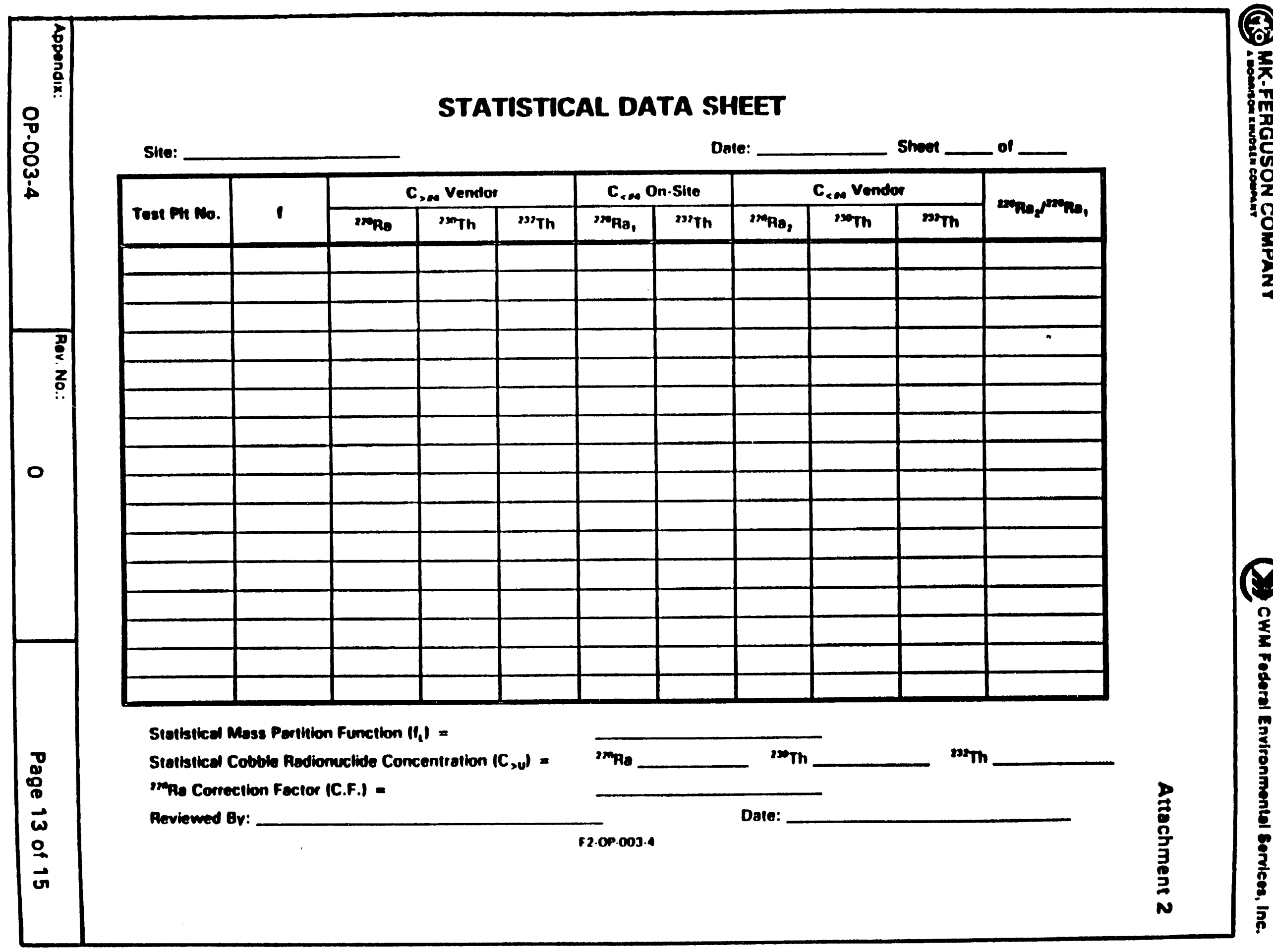




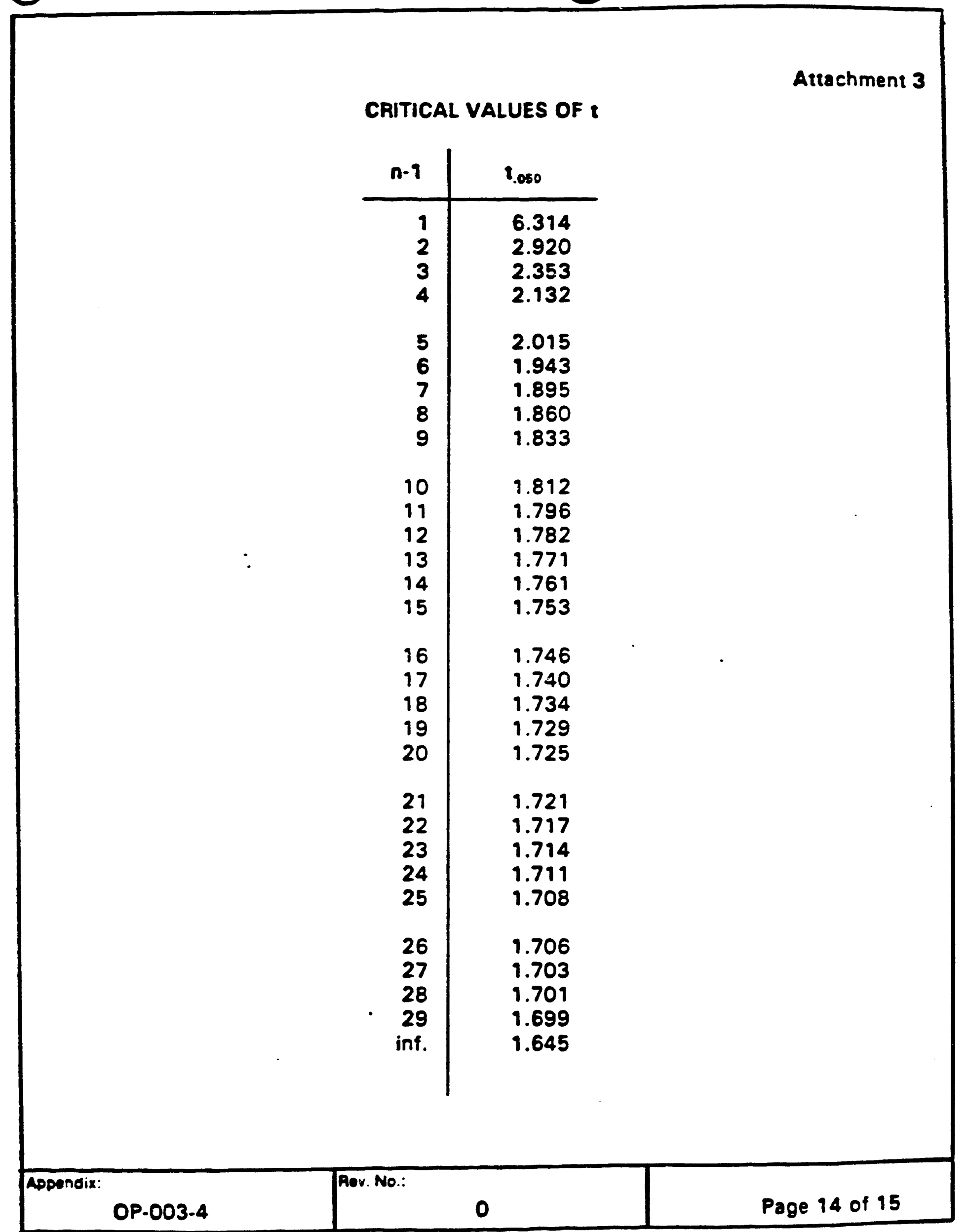




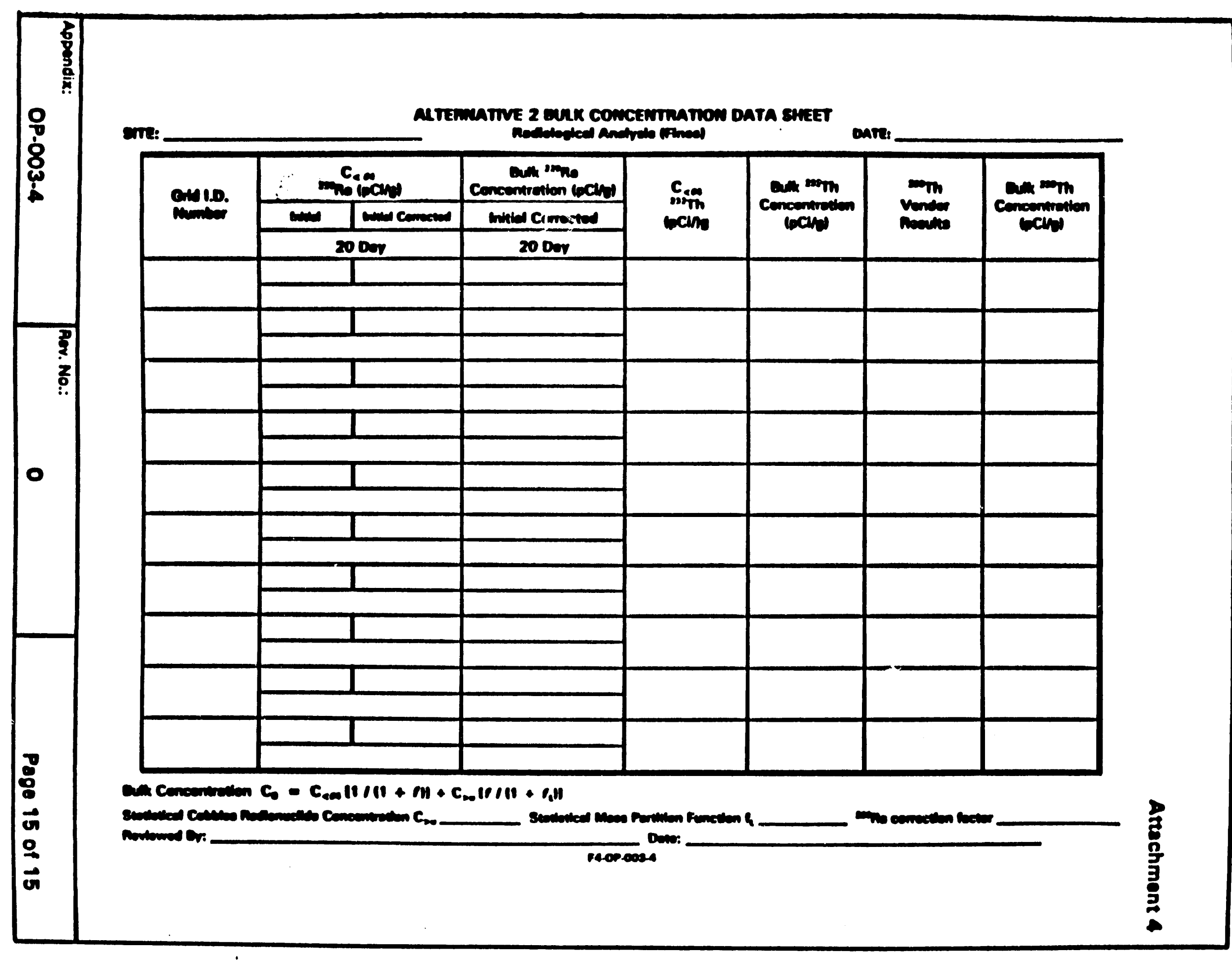


APPENDIX C

TEST PIT LITHOLOGICAL LOGS AND RADIOLOGICAL

DATA COBBLY SUBSOIL INVESTIGATION

JUNE 1990 
LITHOLOGICAL LOGS

COBBLY SUBSOIL INVESTIGATION JUNE 1990 


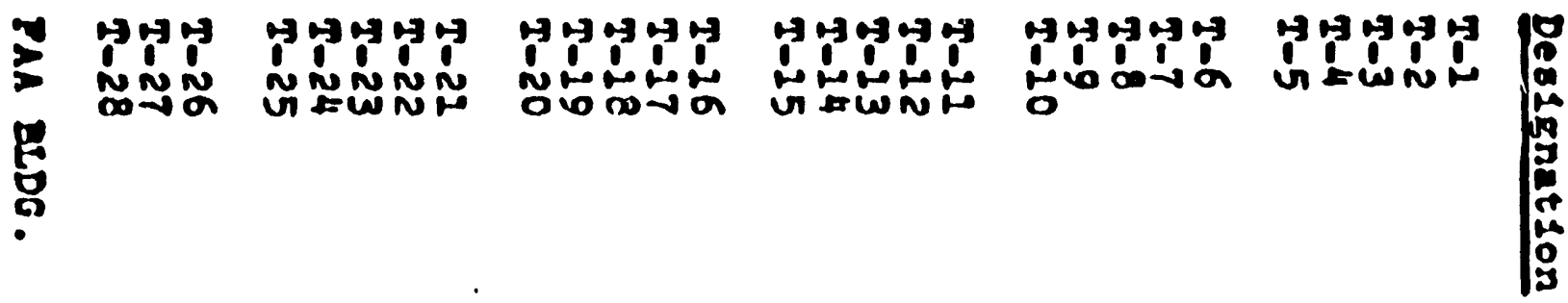
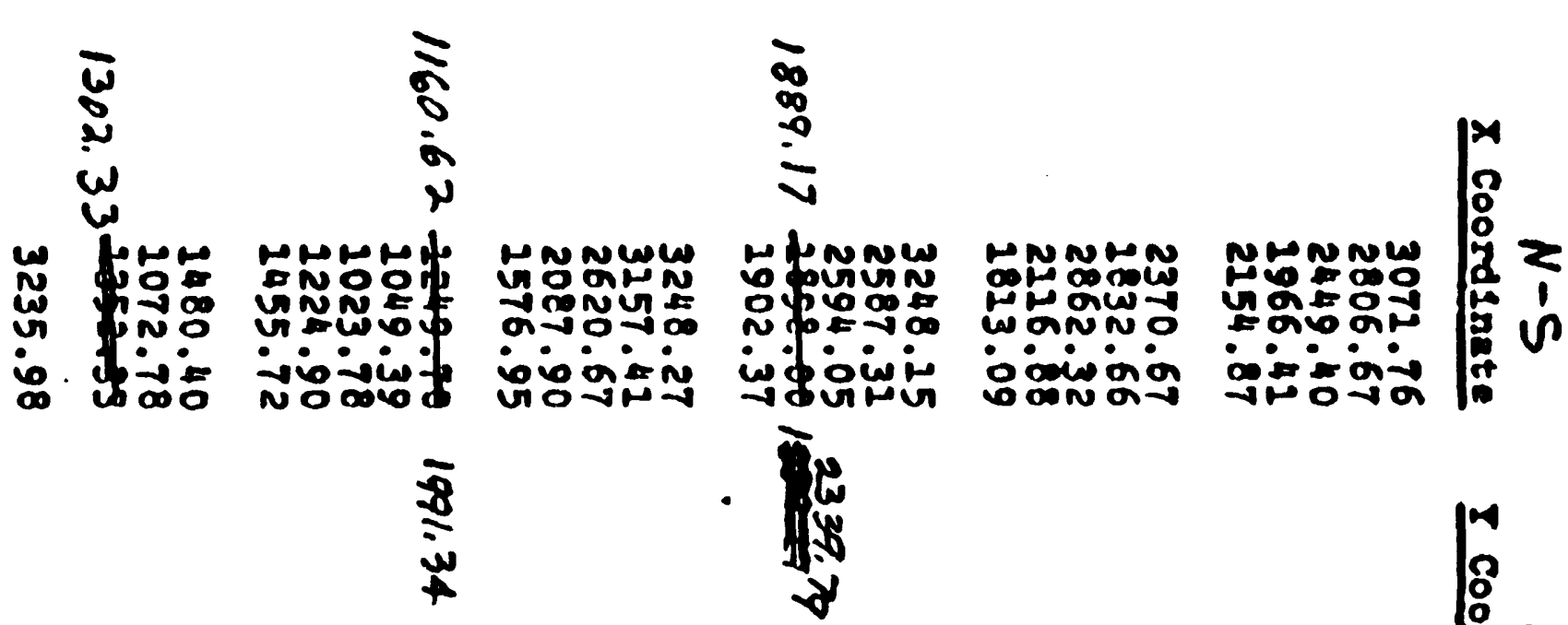

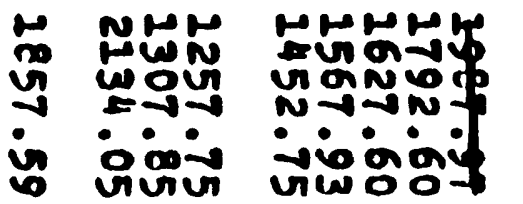

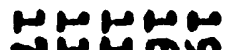
ज्ञovico no: gn:

NMNN F⿻上丨 $6-66=$

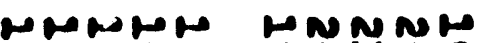
nNwon bopos

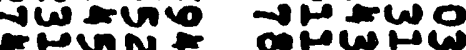
-
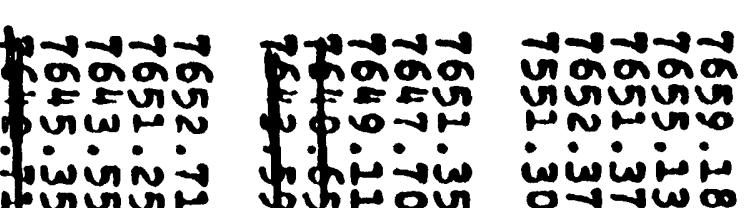
ํํํำ
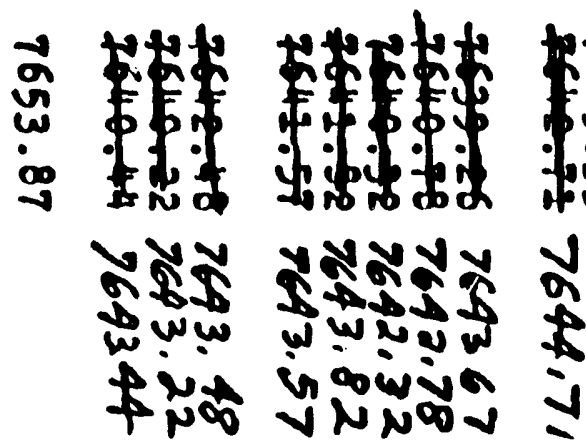

xid

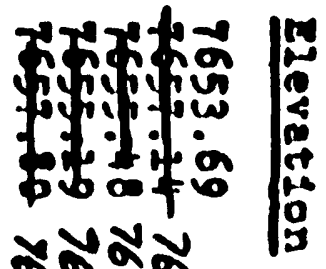


"LI: 1. - TEST. PIT LOG

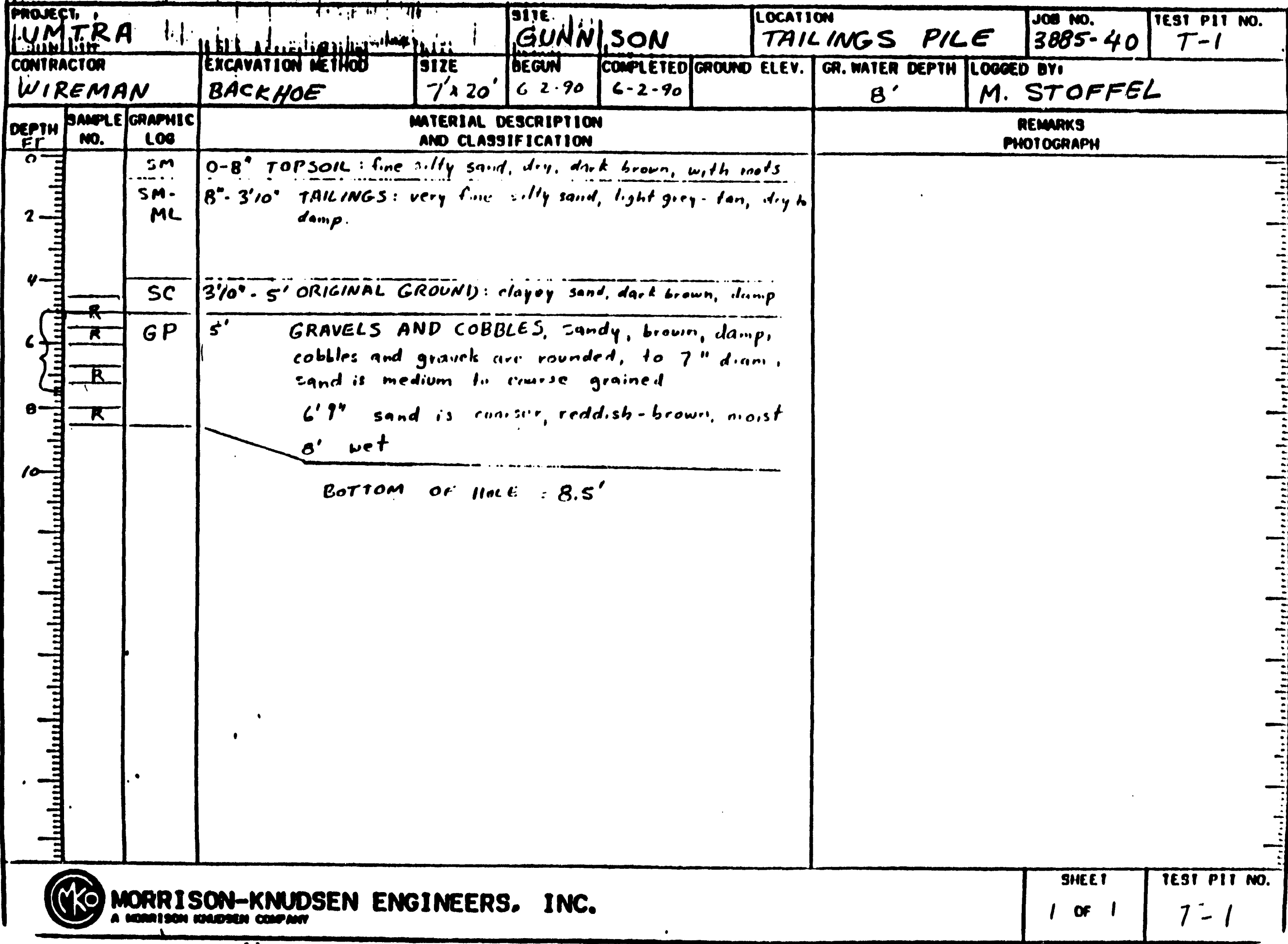




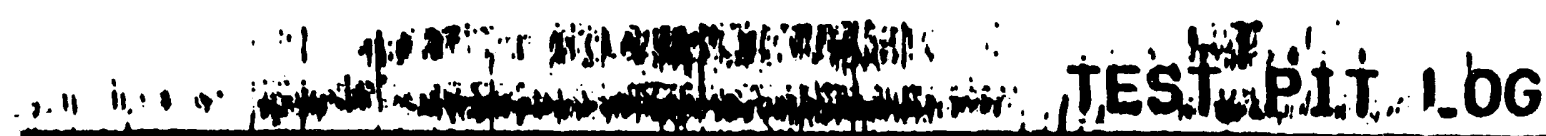

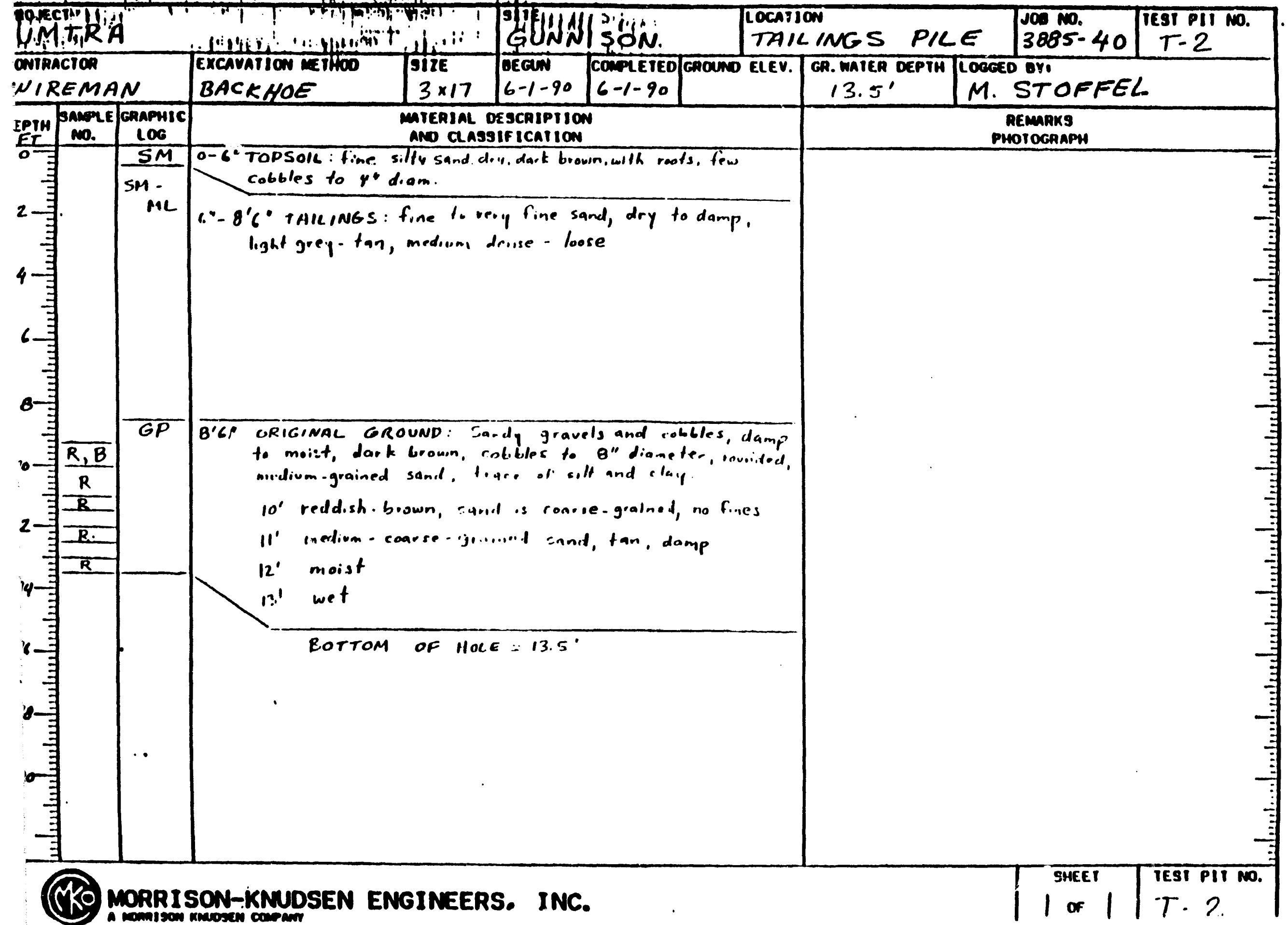




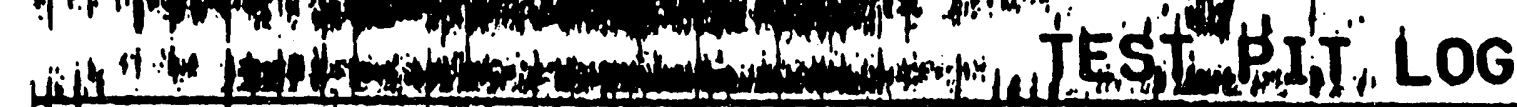

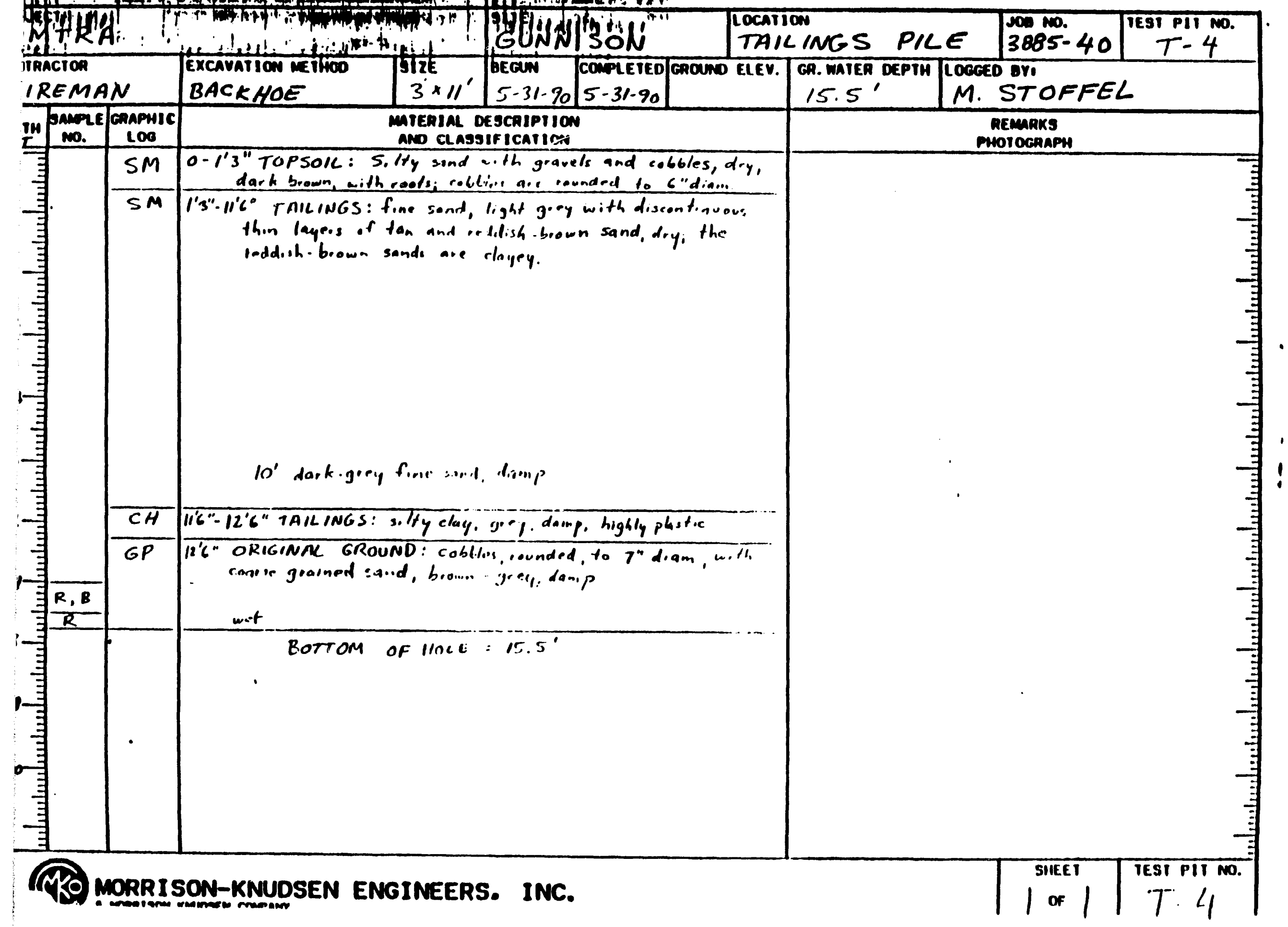




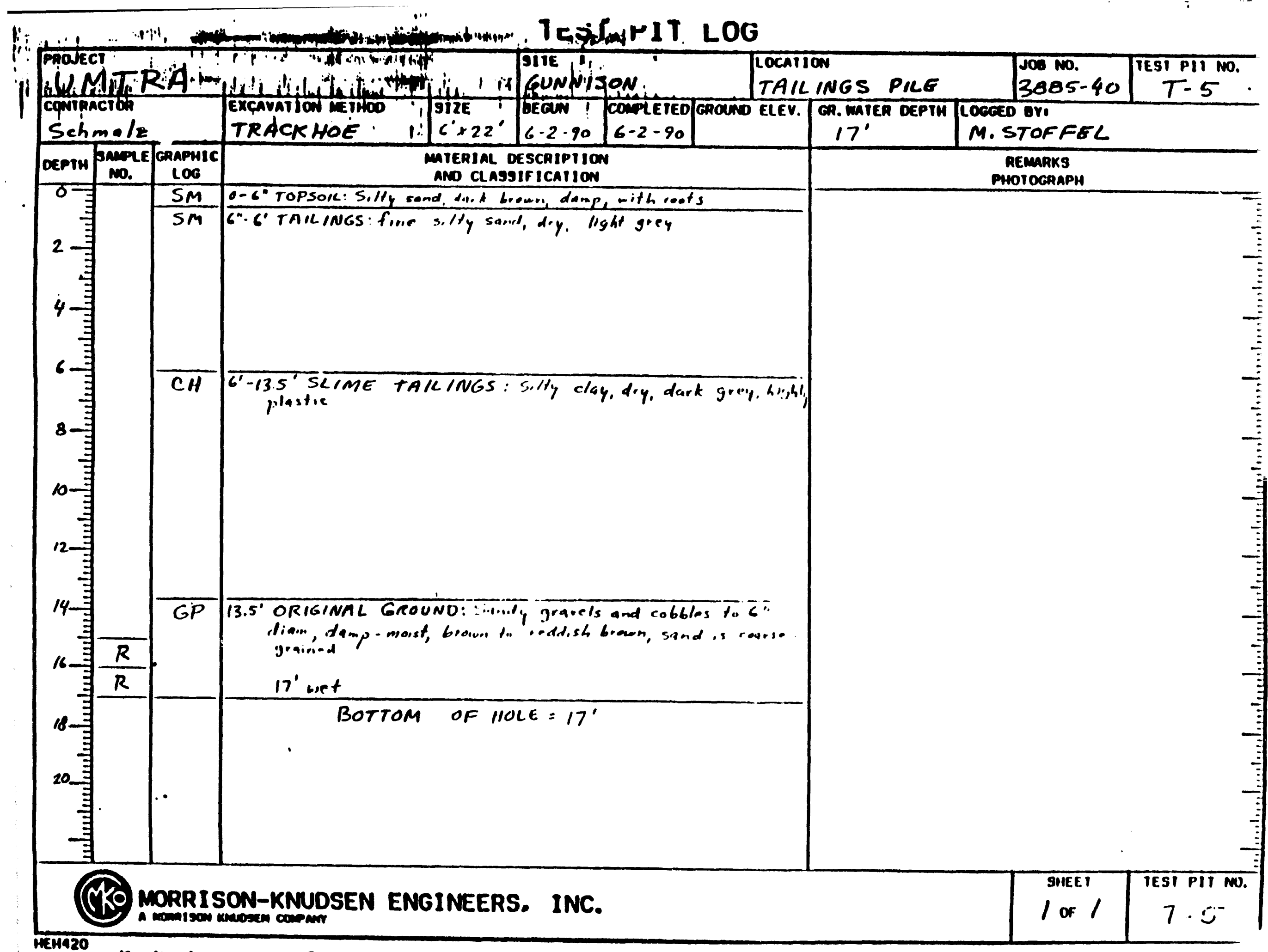

r.: $, 0,1$, 


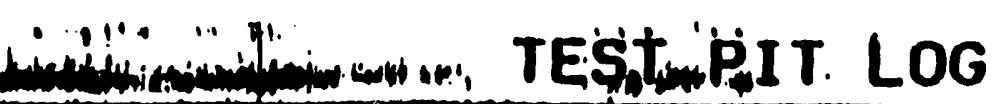

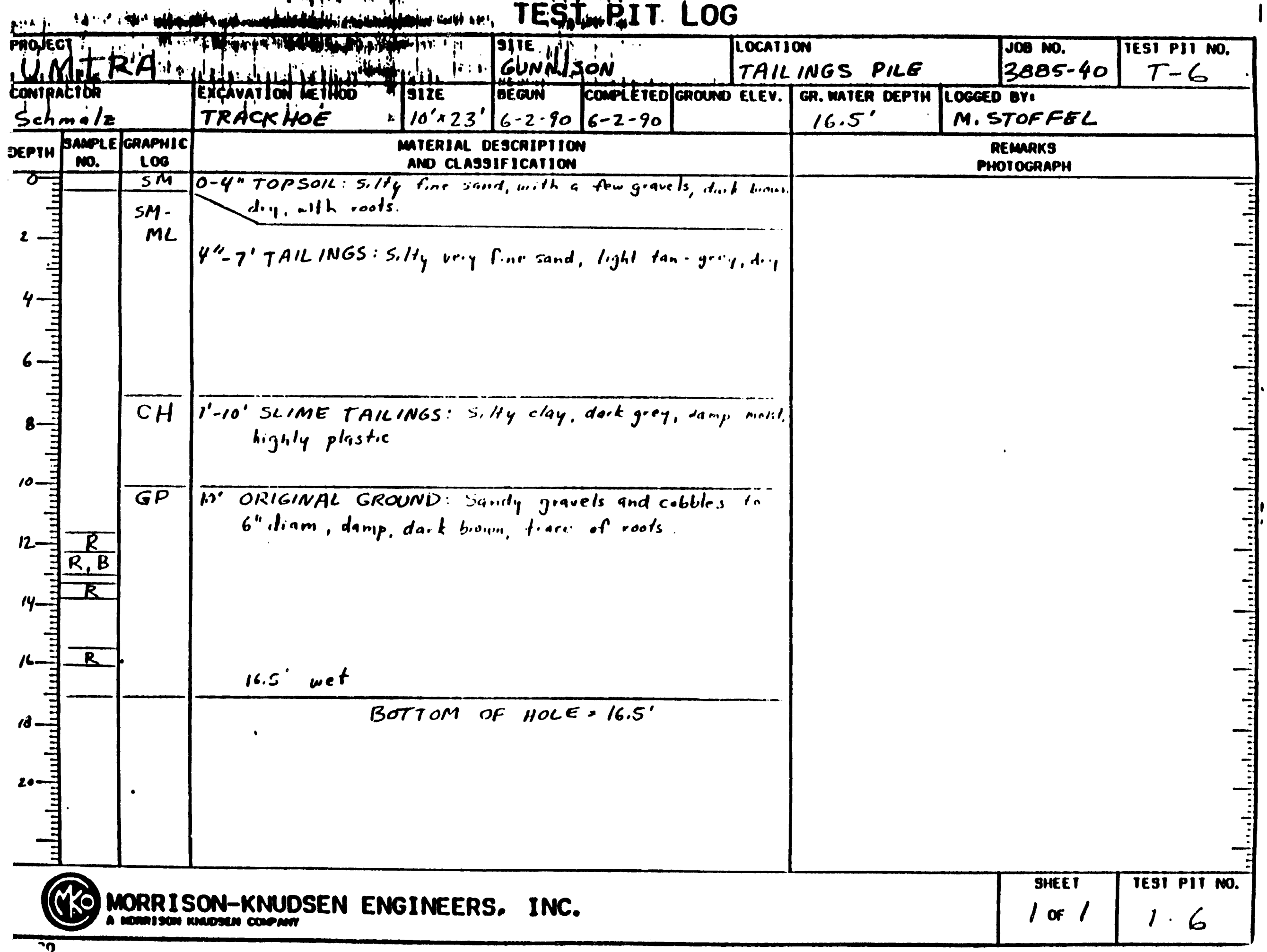




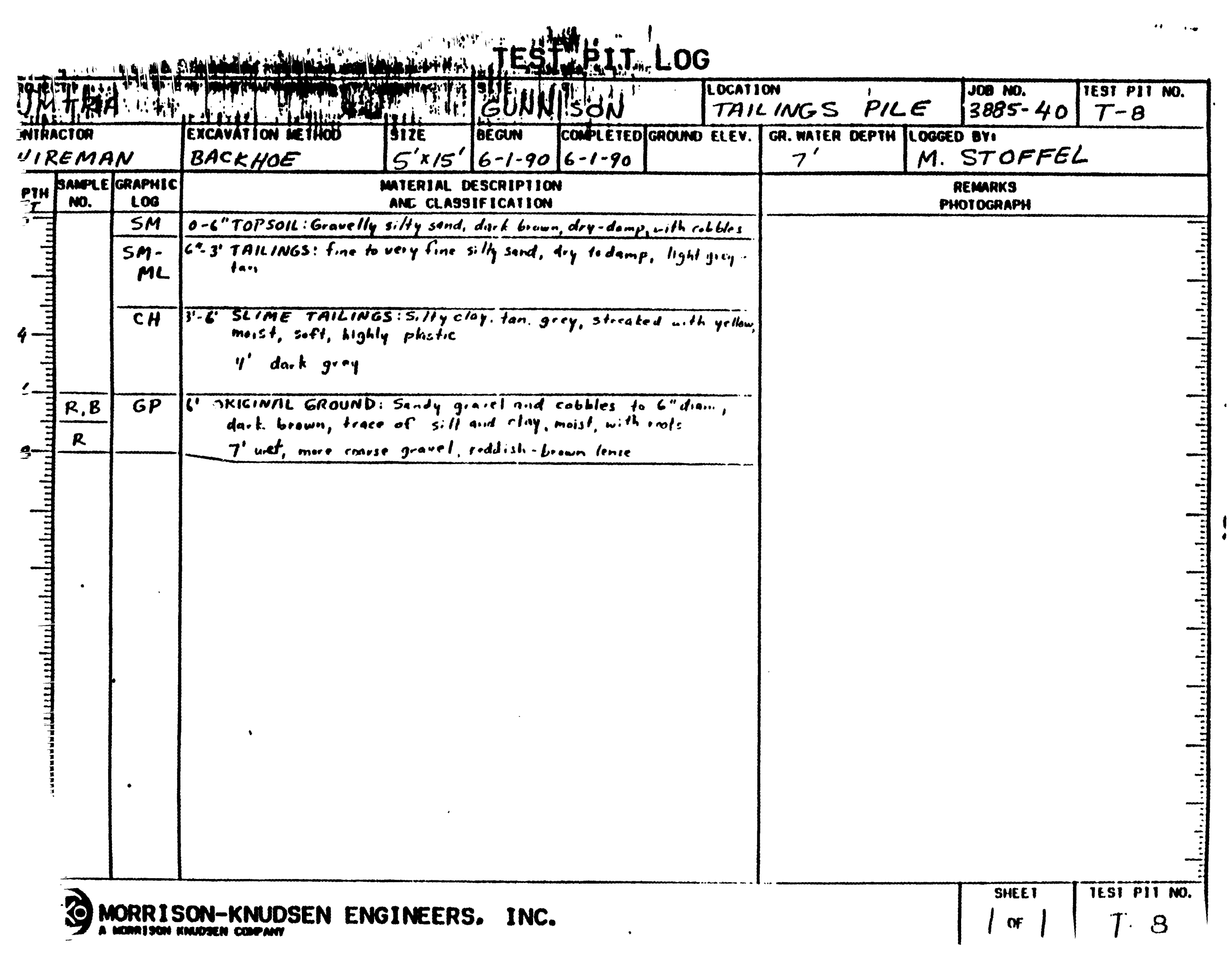




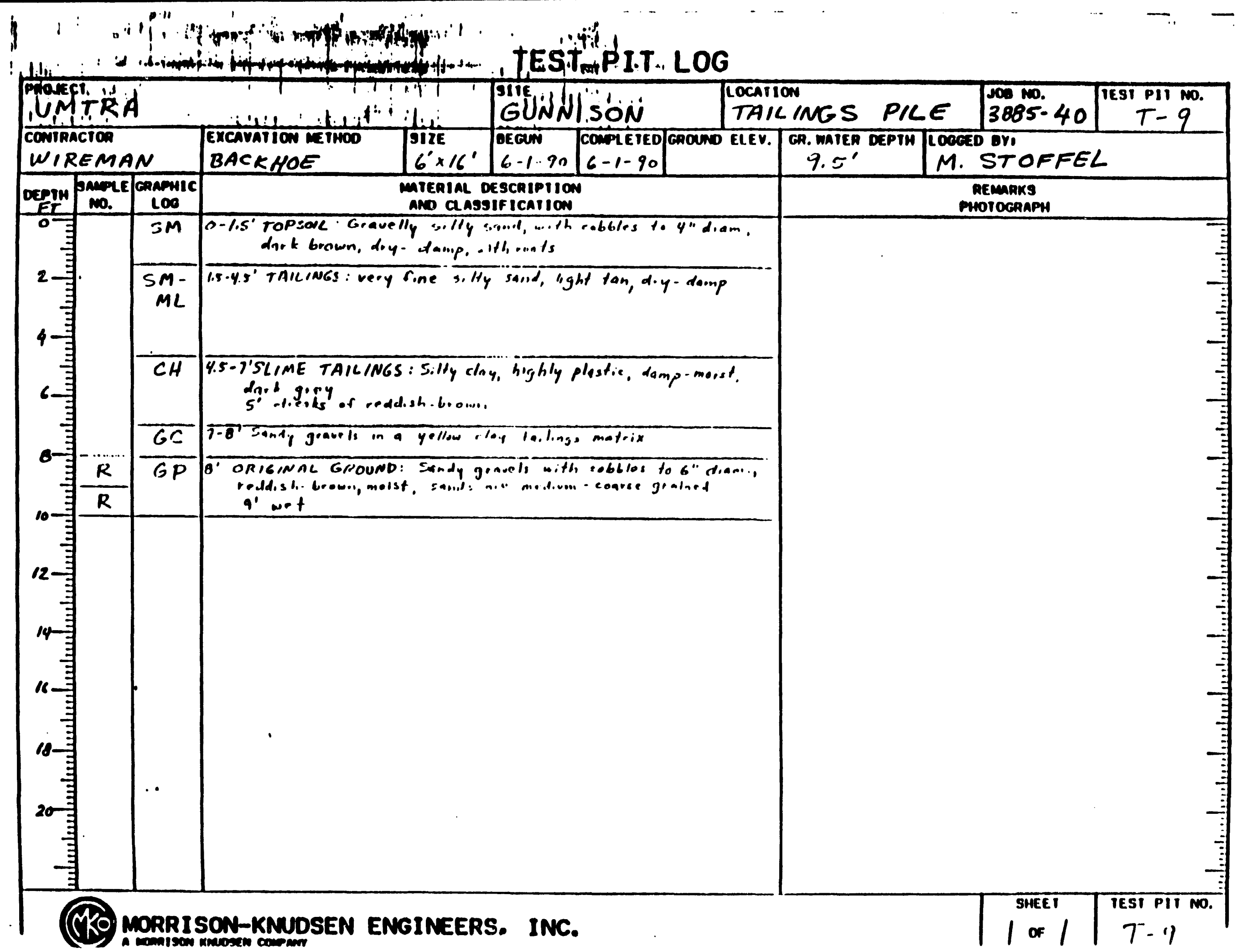




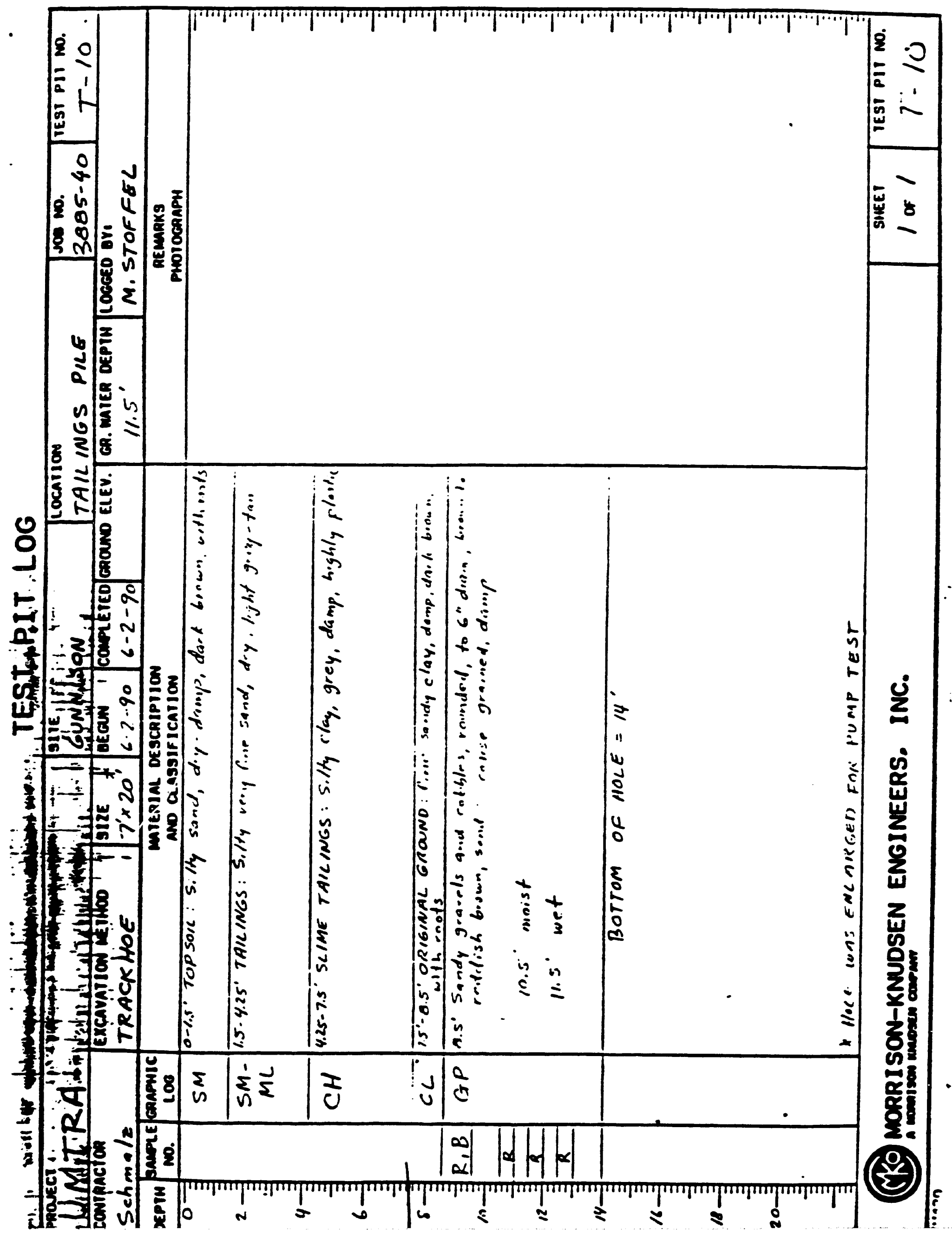




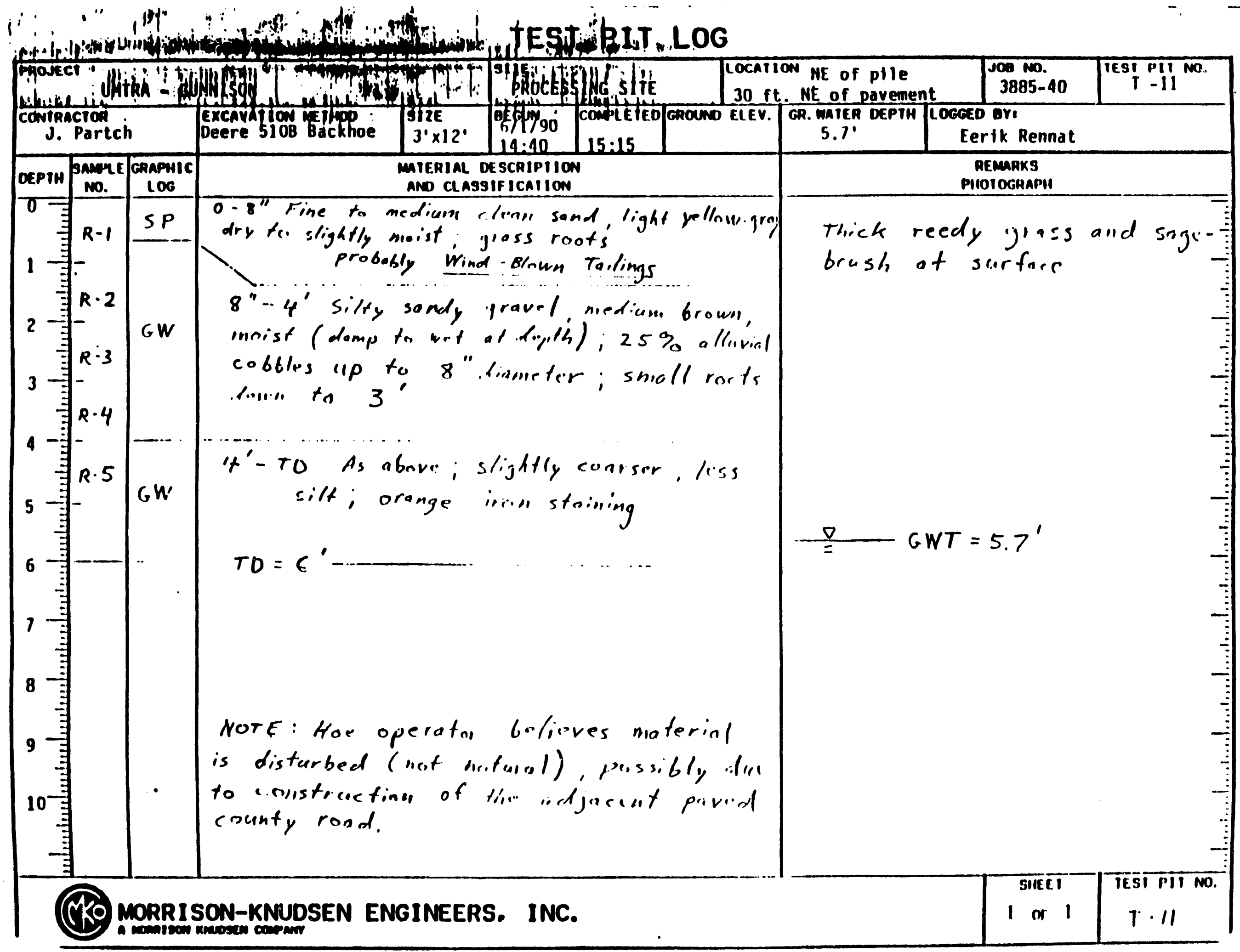




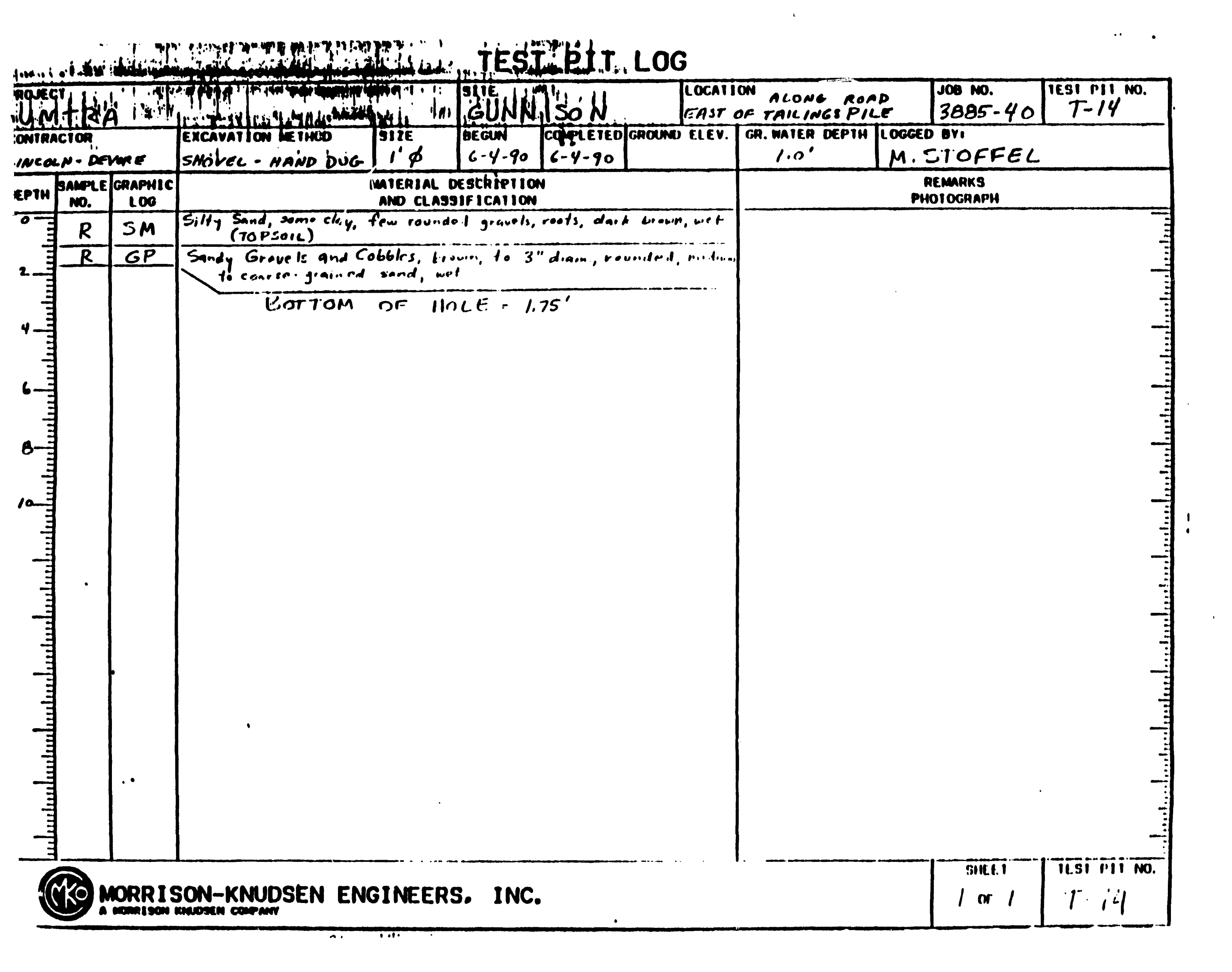




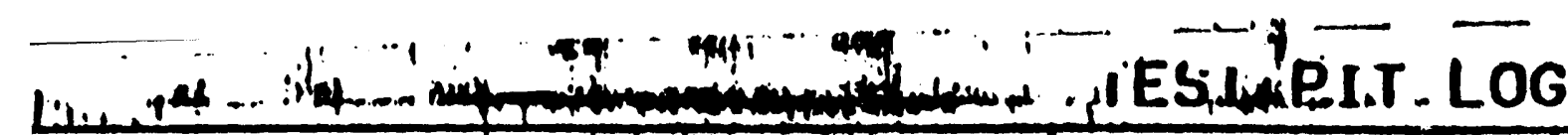

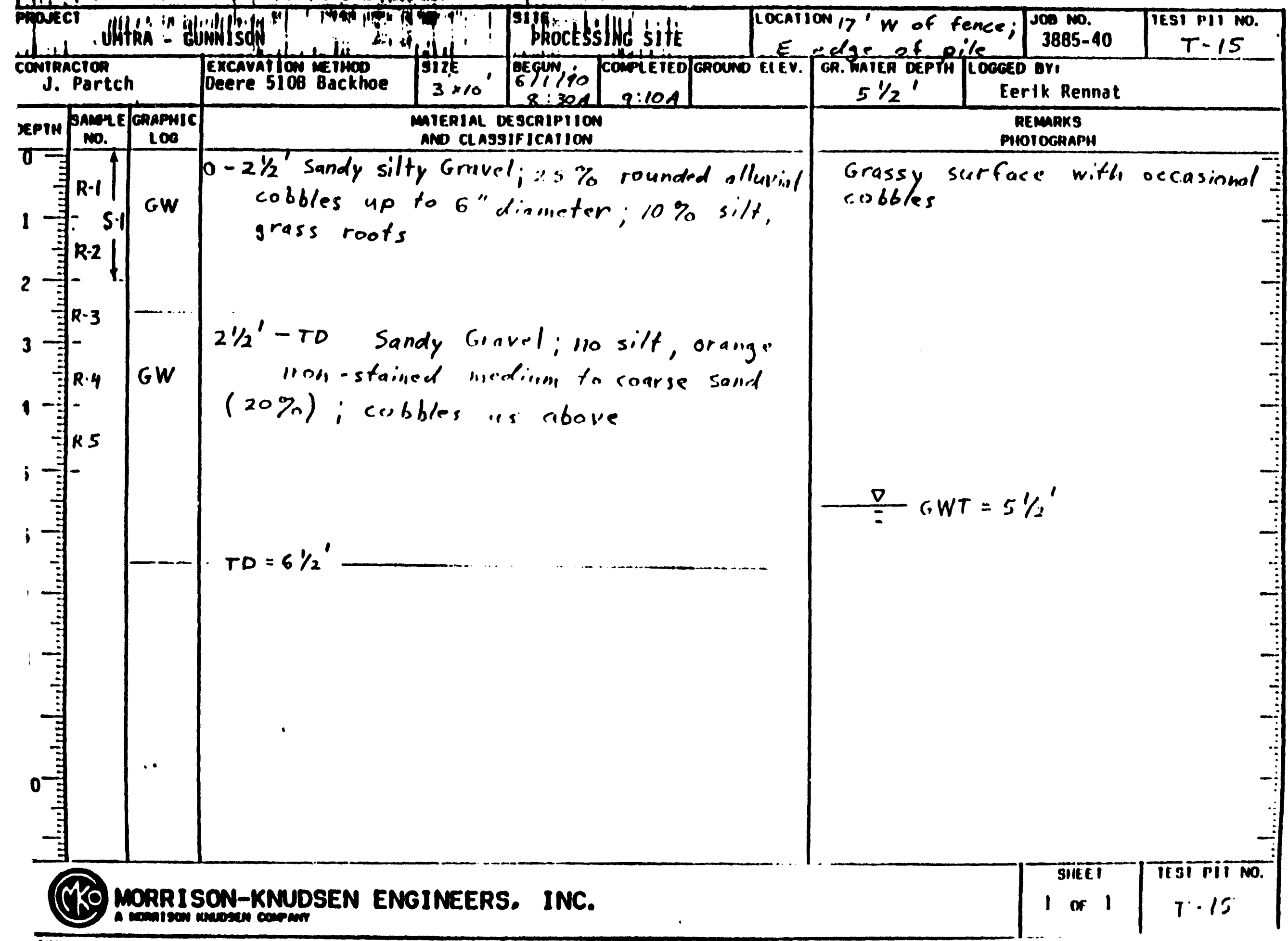




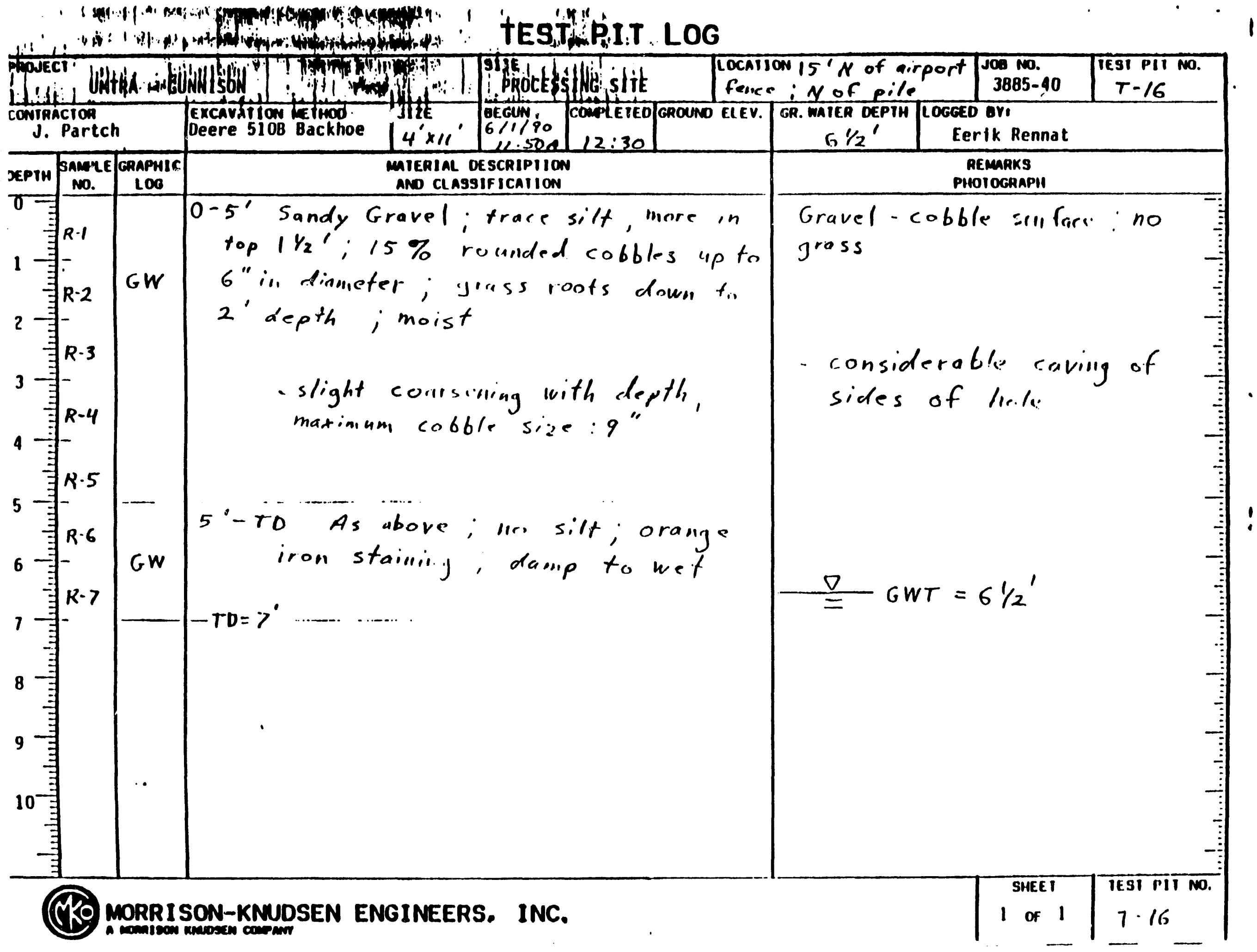




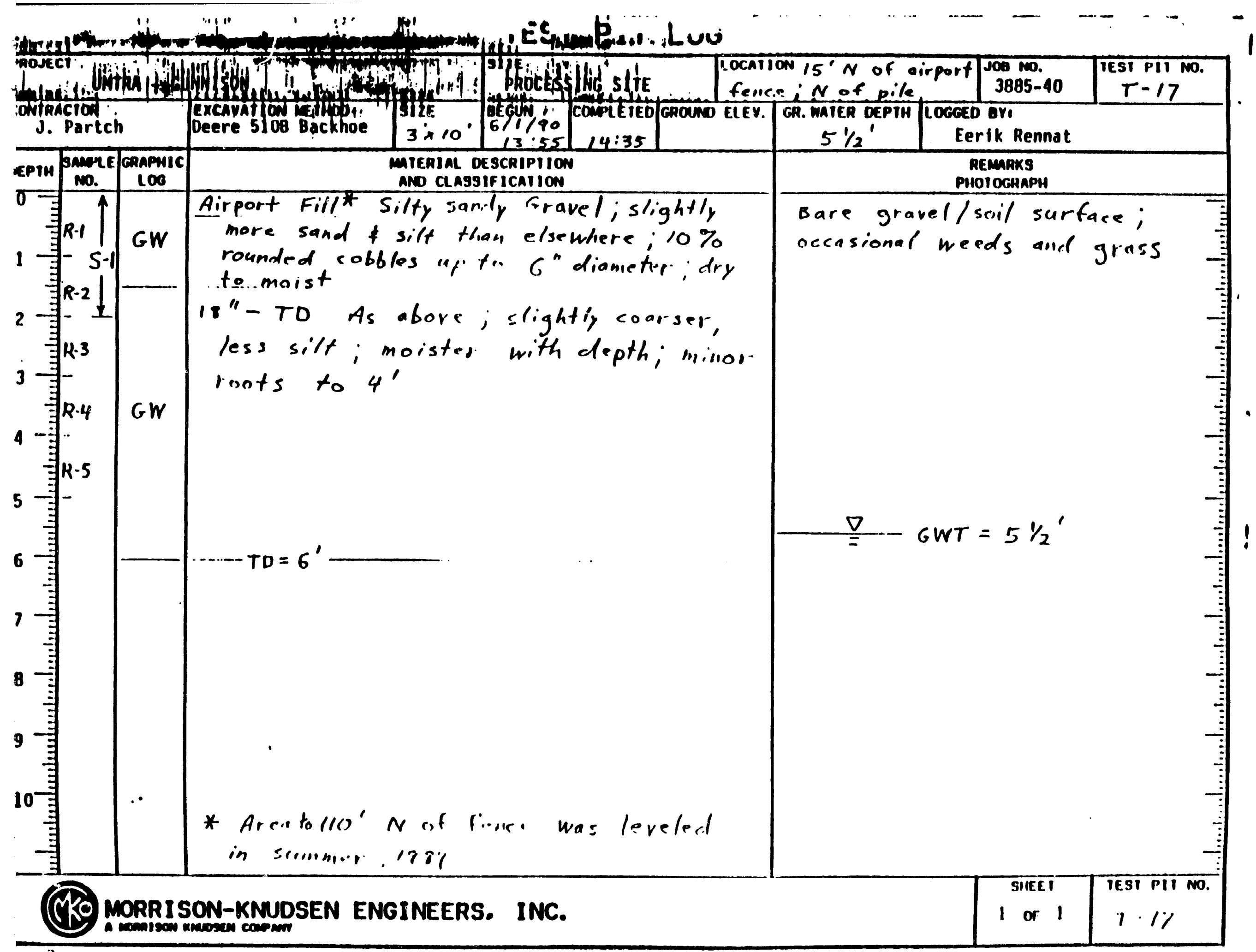




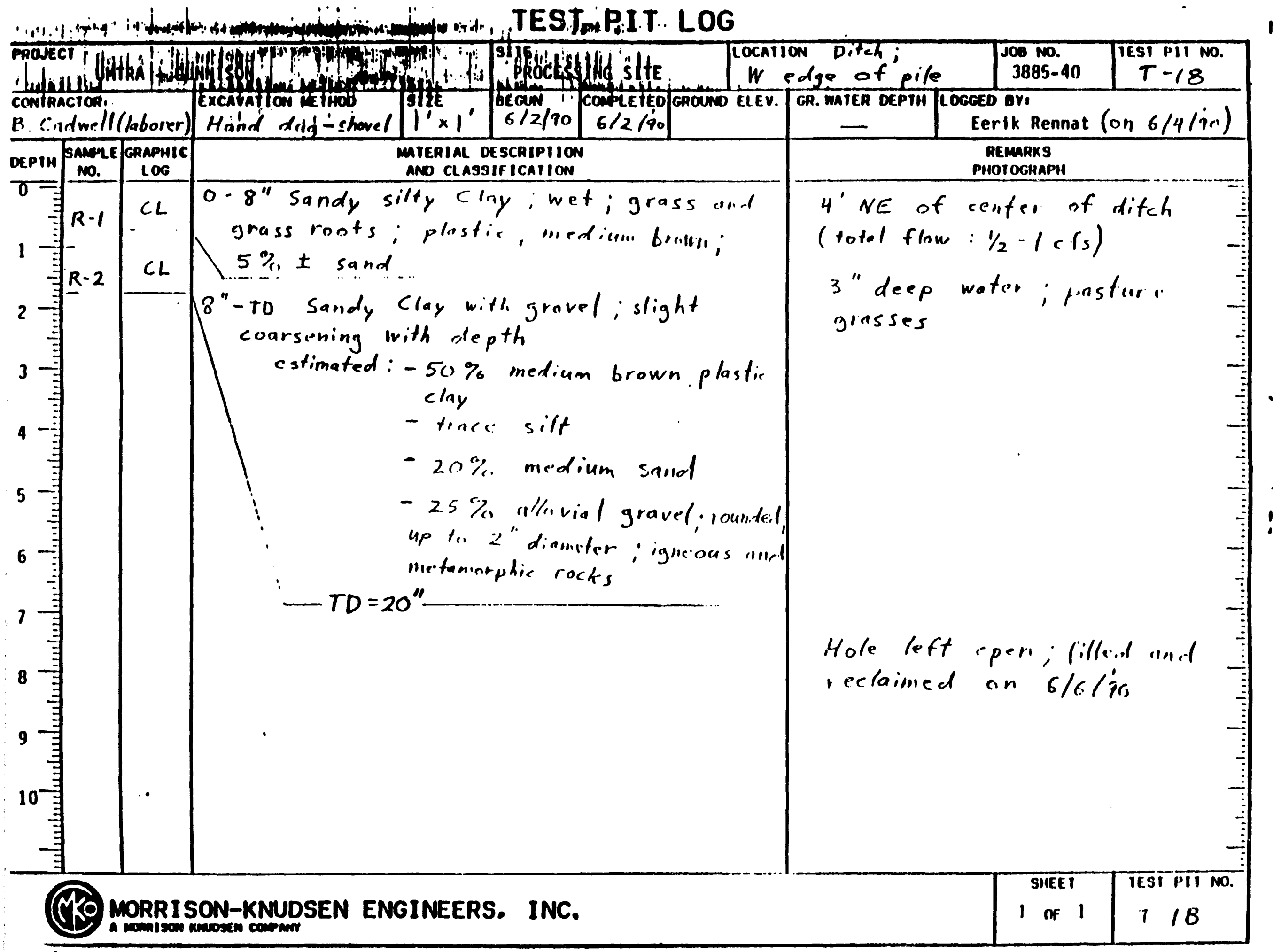


Fu :

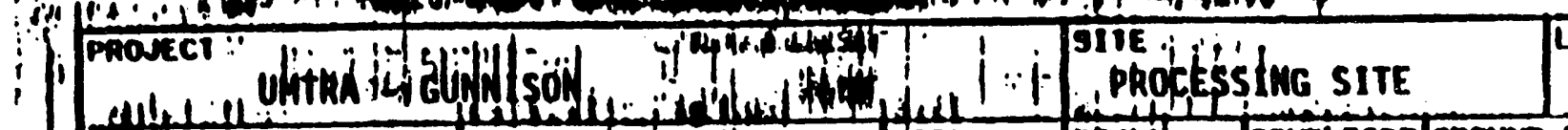

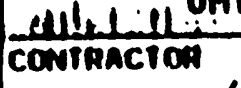
Excavartion RE 11000 STize LOCATION Ditch.

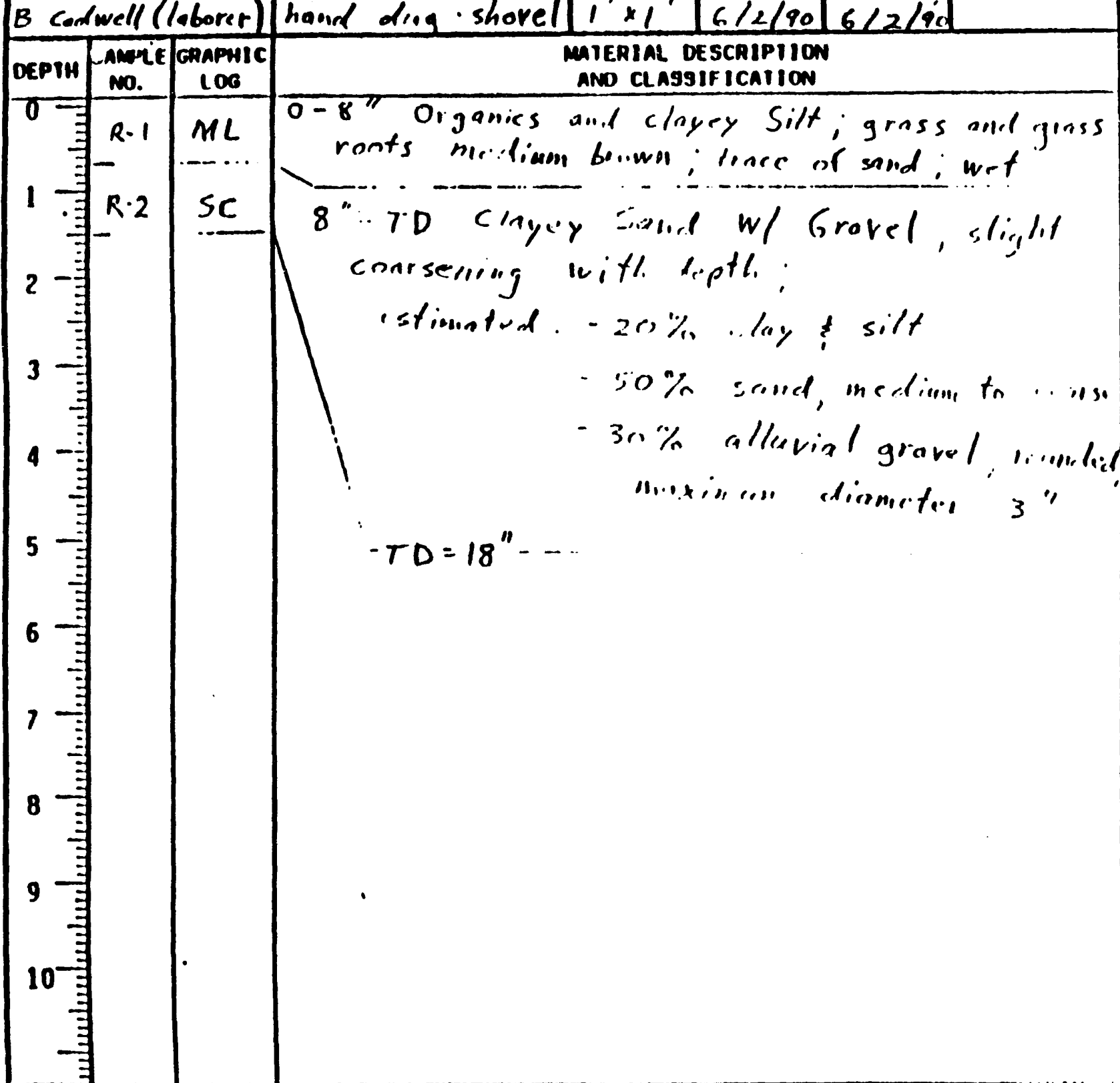

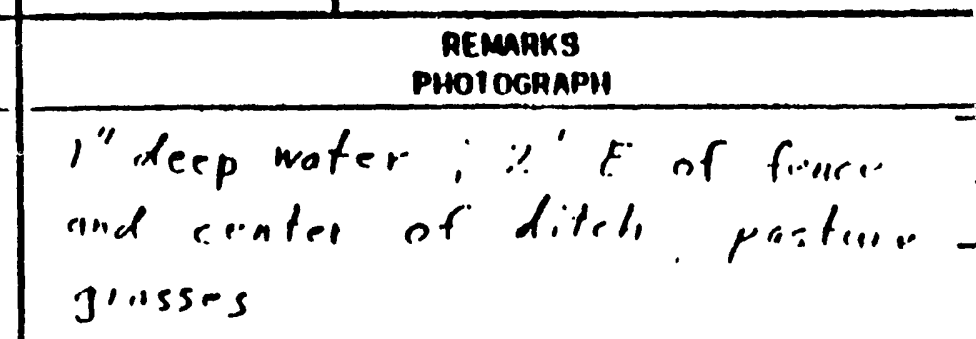

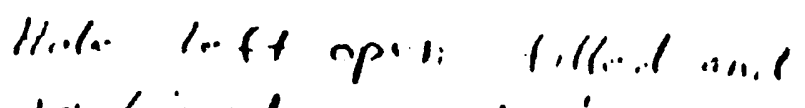
1. laimed nis 6/6/7is 


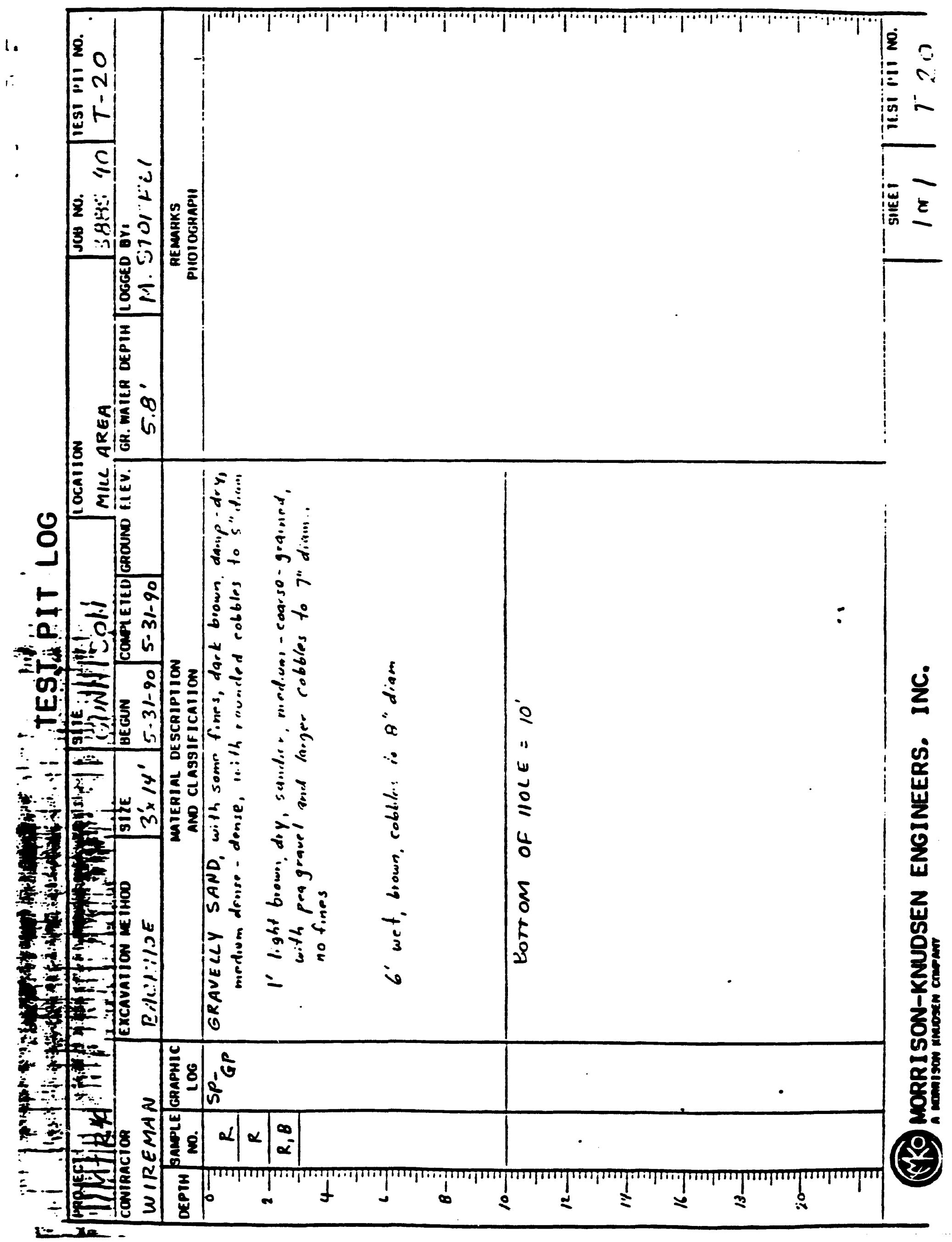




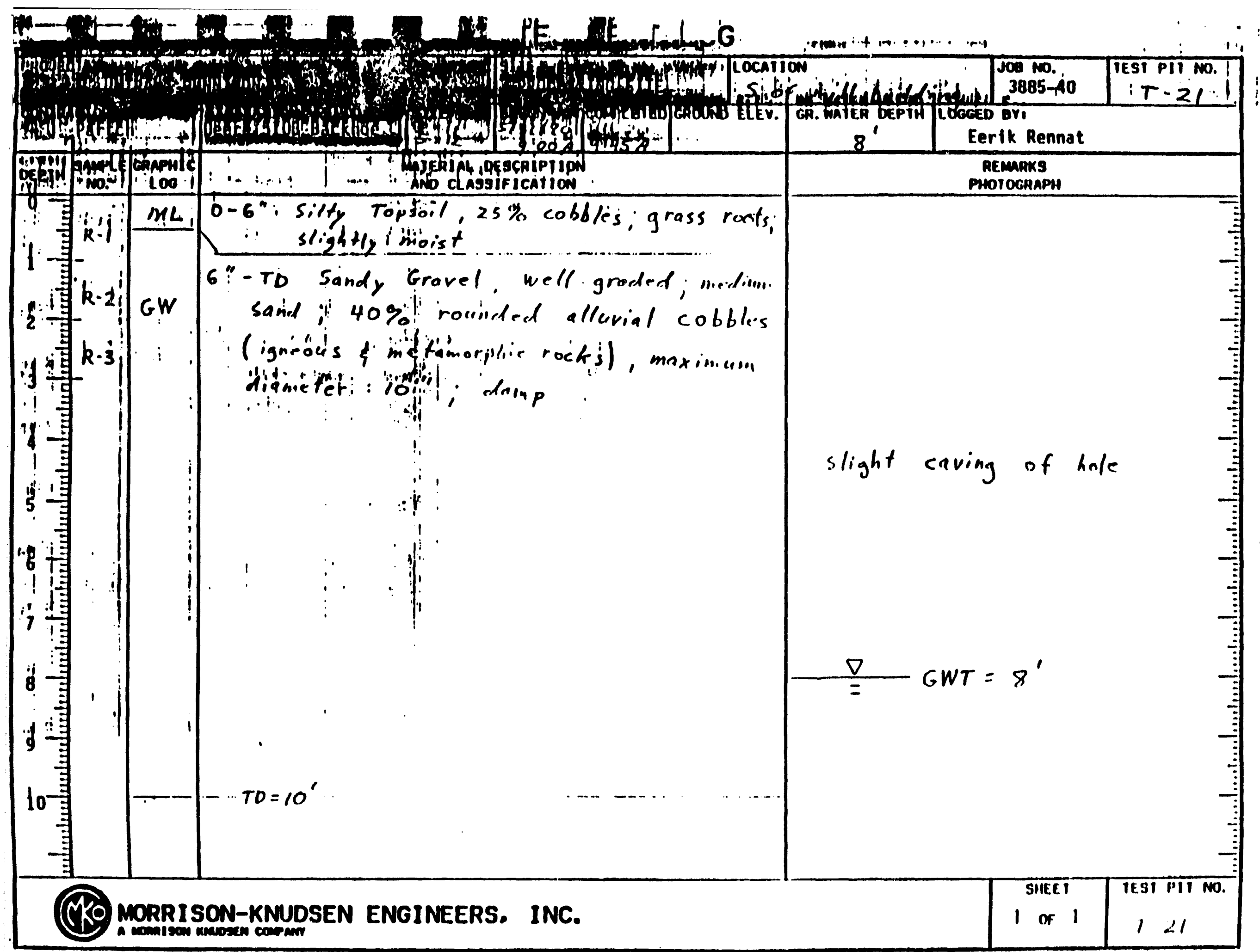

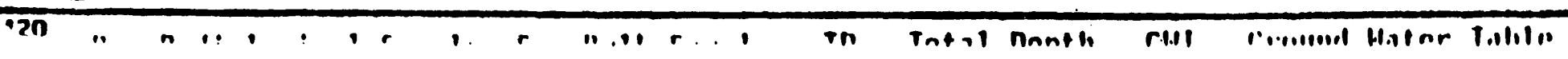





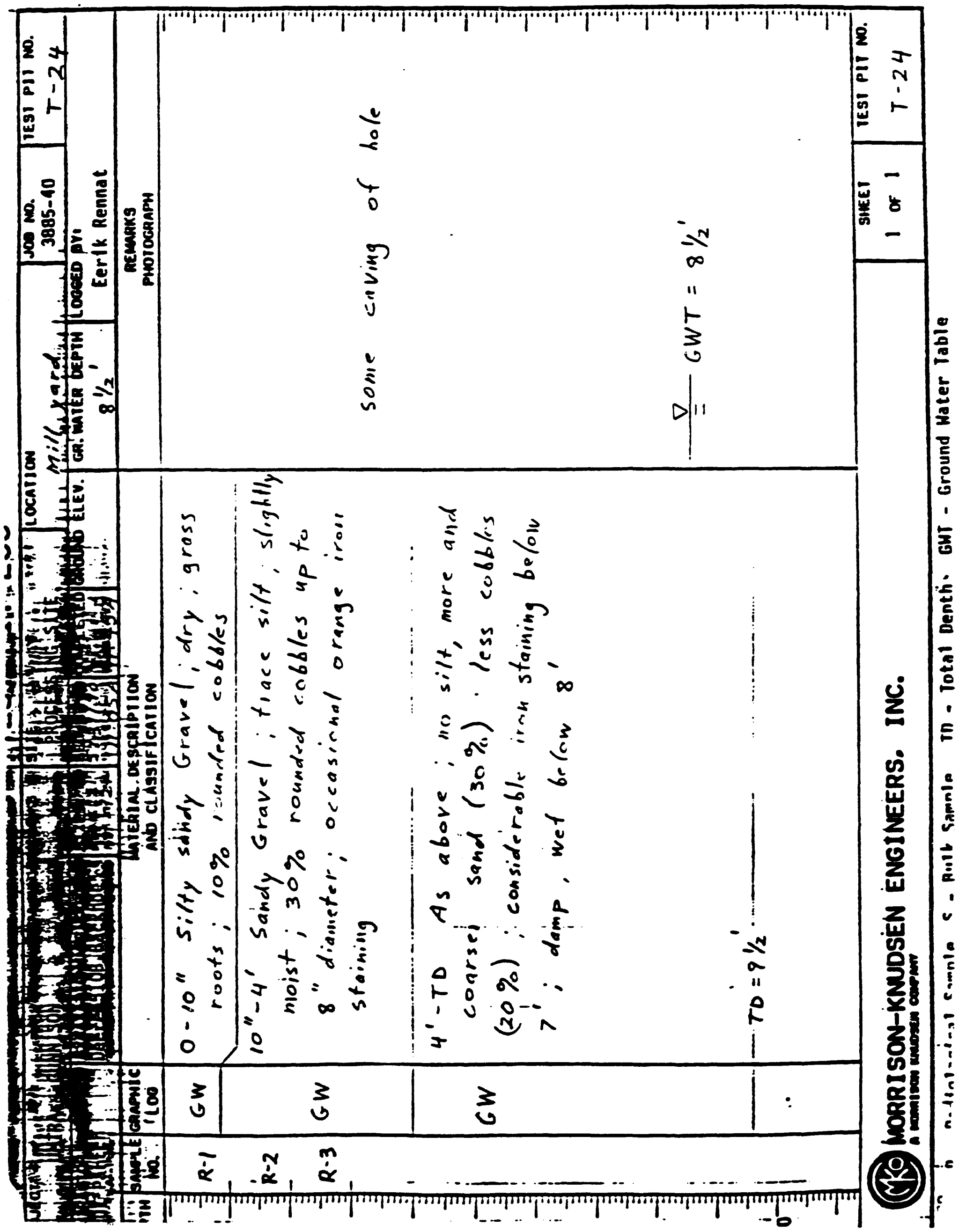




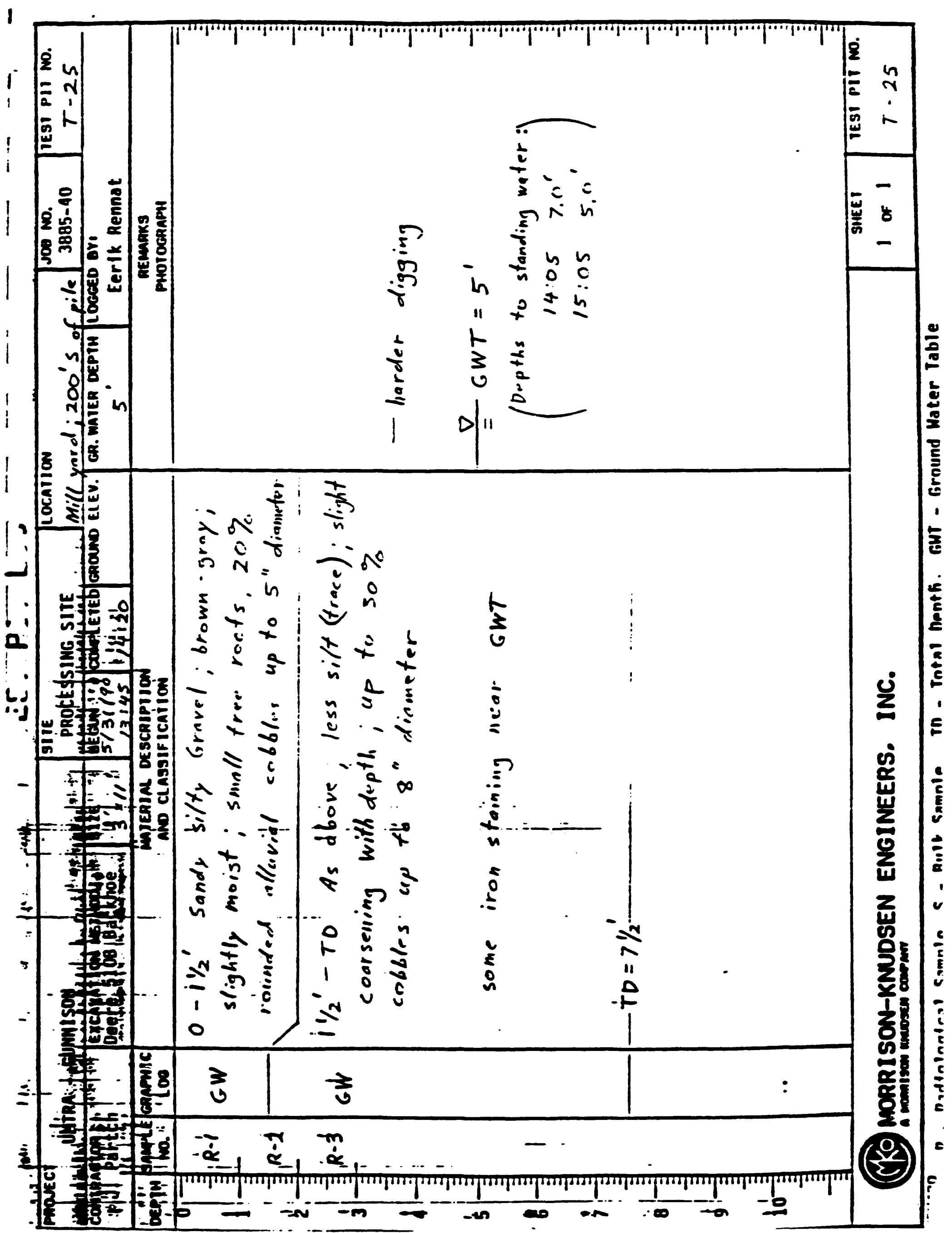




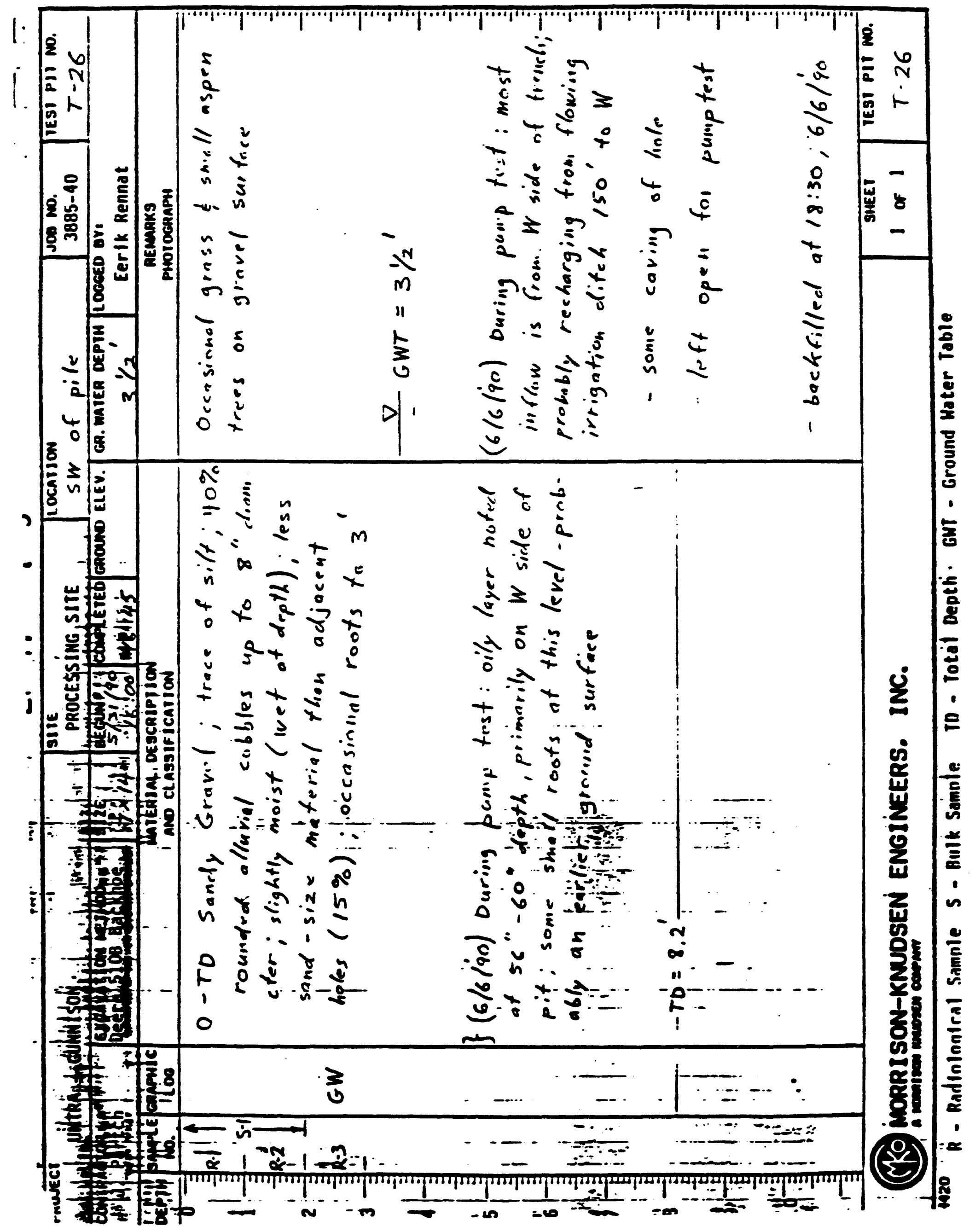


VENDCIR LABORATORY COMPOSITE SOIL SAMPLE MIEASUREMENTS: SIEVE ANALYSIS AND

RADIOLOGICAL MEASUREMENTS

FOR COMPOSITE SOIL SAMPLES <\#4 AND > \#4 MESH

SIEVE COBBLY SUBSOIL INVESTIGATION

JUNE 1990 
ENGINEERS

AND

CONSTRUCTORS

MK-FERGUSON COMPANY

A MORALON XNUDSEN COMPANY

a EADOUAATERS OFFICE

ONE EREENEW PUAZA

C EvELAND OMOUSA Mirs

CLEVE. (216) 523-56001TELEX: 985542

AEPLY TO. MK-FERGUSON COMPANY

AEMEDIAL ACTIONS

CONTRACTOR-UMTRA PROJECT

CO BOX 9135

NLBUOUEROUE. NEW MEXICO U.SR 87119

August 9, 1990

90-3050-548

Mr. Mark L. Matthews

Project Manager

U.S. Department of Energy

Uranium Mill Tailings Remedial Action Project Office

First National Bank Building

5301 Central Avenue N.E.

Suice 1700

Albuquerque, New Mexico 87108

SUBJECT: Information on Gunnison requested by TAC

REFERENCE: Contract NO. DE-ACO4-83AL18796

Dear Mr. Matthews:

This letter is provided to transmit information that has recently been requested by various persons at the TAC.

Attachment 1 contains laboratory test results for gradations, water content, specific gravity and absorption for the Disposal Site and gradations for the pile and subpile area of the Processing site which were requested by Paul Darr and Jim Crain.

Attachment 2 contains laboratory test results on Ra-226 and Th-230 for the Processing Site which were requester by Doug Conzales.

Attachment 3 contains Estimates of employee numbers which was prepared by MKES and was requested by Sandy Beranich.

If you have any questions, please contact John Briggs or Rob Cooney of my staff at 766-3093.

Sincerely,

MK-Ferguson Company

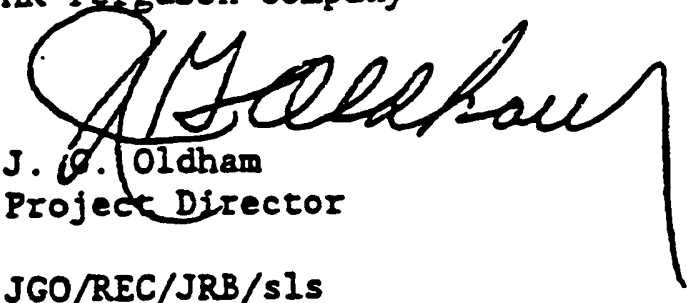

Enclosure

CC: P. Mann - DOE/NMTRA (w/enc.)

C. Smythe - DOE/UITRA (w/o enc.)

bcc: R. E. Cooney (w/enc.) D PiorfeY - TAC/UMTRA (w/enc.)

D. R. Sanders - MRES (w/o enc.)

J. R.: Briggs (w/enc.)

$4513 \mathrm{~K}$.

File - EDT (w/enc.)

File (w/0 enc.) 
$1 \div \div$

RIB

IKE DOC.3885-GUN-L-09-01094-00

$\because \quad$

Gootochnical Consultants

M41 Motor St.

Grand Junction. CO 81505

(303) $242-8968$

$=$

July 9, 1990

Mr. Erik A. Rennat

Morrison-knudsen Environmental Services, Inc.

180 howard Street

San Francisco, CA 94105

Re: Sieve Analysis

Grunison, $\infty$

Dear Mr. Rennet:

Enclosed please find two copies of corrected Seive Analysis regarding above captioned job.

If we can be of any further assistance to you, please do not hesitate to contact this office at any time.

Respectfully submitted,

LINCOLN DEVORE, InC.

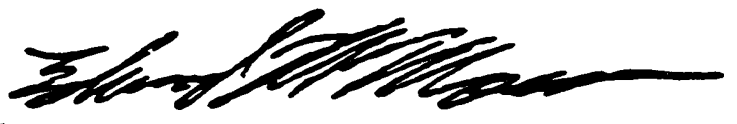

By:

Edward M. Morris

Branch Manager

Io Job No.: 72199-J 
PROJECT LOCATION: Gundison Processing Site SAHPLE LOCATION: T-2 $91 / 2^{\circ}-10^{\circ}$

\begin{tabular}{|c|c|c|c|}
\hline $\begin{array}{l}\text { SIEVB } \\
\text { NO. } \\
\text { E=E= } \\
8 \% \\
7 \%\end{array}$ & $\begin{array}{l}\text { solt } \\
\text { Wt. gD } \\
==2== \pm=2\end{array}$ & $\begin{array}{l}\mathbf{X} \\
\text { Retained } \\
0=m==m= \\
0 \\
0\end{array}$ & 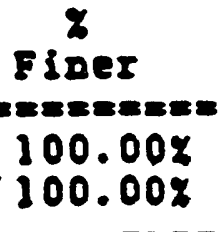 \\
\hline $\begin{array}{l}6 \% \\
5 *\end{array}$ & & $\begin{array}{l}0 \\
0\end{array}$ & $\begin{array}{l}100.008 \\
100.008\end{array}$ \\
\hline 3". & $\begin{array}{l}7363 \\
9603\end{array}$ & $\begin{array}{l}10.88 \\
52.80\end{array}$ & $\begin{array}{l}59.528 \\
47.208\end{array}$ \\
\hline $\begin{array}{l}2 * \\
1-1 / 2 *\end{array}$ & $\begin{array}{l}10568 \\
10649\end{array}$ & $\begin{array}{l}58.10 \\
58.55\end{array}$ & $\begin{array}{l}41.90 \% \\
11.458\end{array}$ \\
\hline $\begin{array}{l}1 * \\
3 / 4 *\end{array}$ & $\begin{array}{l}11523 \\
11906\end{array}$ & $\begin{array}{l}63.35 \\
65.46\end{array}$ & $\begin{array}{l}36.658 \\
34.548\end{array}$ \\
\hline $\begin{array}{l}1 / 2 \% \\
3 / 8 *\end{array}$ & $\begin{array}{l}12631 \\
13048\end{array}$ & $\begin{array}{l}69.45 \\
71.74\end{array}$ & $\begin{array}{l}30.55 \% \\
28.268\end{array}$ \\
\hline 10 & $\begin{array}{l}13562 \\
14233\end{array}$ & $\begin{array}{l}74.57 \\
78.25\end{array}$ & $\begin{array}{l}25.438 \\
21.758\end{array}$ \\
\hline $\begin{array}{l}\quad 20 \\
* 40\end{array}$ & $\begin{array}{l}15091 \\
16107\end{array}$ & $\begin{array}{l}82.97 \\
88.56\end{array}$ & $\begin{array}{l}17.038 \\
11.448\end{array}$ \\
\hline $\begin{array}{l}100 \\
1200\end{array}$ & $\begin{array}{l}17138 \\
17516\end{array}$ & $\begin{array}{l}94.23 \\
96.31\end{array}$ & $\begin{array}{l}5.778 \\
3.698\end{array}$ \\
\hline
\end{tabular}

BIEVE NAALYSIS

$\because$ Soll wt. gm 18188.00

- $=2=E=$ 
PROJECT LOCATION: Gunnison Frocessing Site

SAMPLE LOCATION: T-4 $14^{\circ}$ Slightly Cemented

\begin{tabular}{|c|c|c|c|}
\hline $\begin{array}{l}\text { SIEVE } \\
\text { NO. } \\
\text { =E=E= }=8\end{array}$ & $\begin{array}{l}\text { SOIL } \\
\text { wt. gm } \\
======2\end{array}$ & 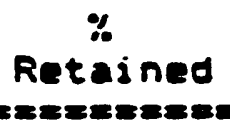 & $\begin{array}{c}\% \\
\text { Finer } \\
\text { meseme=m }\end{array}$ \\
\hline $8^{\infty}$ & $\begin{array}{l}0 \\
0\end{array}$ & $\begin{array}{l}0 \\
0\end{array}$ & $\begin{array}{l}100.00 \% \\
100.00 \%\end{array}$ \\
\hline $\begin{array}{l}6^{*} \\
5 \times\end{array}$ & $\begin{array}{l}6100 \\
6100\end{array}$ & $\begin{array}{l}34.42 \\
34.42\end{array}$ & $\begin{array}{l}65.58 \% \\
65.58 \%\end{array}$ \\
\hline $\begin{array}{l}\text { 4" } \\
3 "\end{array}$ & $\begin{array}{l}6100 \\
6100\end{array}$ & $\begin{array}{l}34.42 \\
\$ 4.42\end{array}$ & $\begin{array}{l}65.58 \% \\
65.58 \%\end{array}$ \\
\hline $\begin{array}{l}2^{\prime \prime} \\
1-1 / 2 " 1\end{array}$ & $\begin{array}{l}7360 \\
8850\end{array}$ & $\begin{array}{l}41.53 \\
49.93\end{array}$ & $\begin{array}{l}58.47 \% \\
50.07 \%\end{array}$ \\
\hline $\begin{array}{l}1 \% \\
3 / 4 n\end{array}$ & $\begin{array}{l}10930 \\
11980\end{array}$ & $\begin{array}{l}61.67 \\
67.59\end{array}$ & $\begin{array}{l}38.33 \% \\
32.41 \%\end{array}$ \\
\hline $\begin{array}{l}1 / 2 " 1 \\
3 / 8^{\prime \prime}\end{array}$ & $\begin{array}{l}12870 \\
13500\end{array}$ & $\begin{array}{l}72.61 \\
76.17\end{array}$ & $\begin{array}{l}27.39 \% \\
23.83 \%\end{array}$ \\
\hline $\begin{array}{l}* 4 \\
110\end{array}$ & $\begin{array}{l}14332 \\
14710\end{array}$ & $\begin{array}{l}80.86 \\
82.99\end{array}$ & $\begin{array}{l}19.14 \% \\
17.01 \%\end{array}$ \\
\hline $\begin{array}{l}120 \\
140\end{array}$ & $\begin{array}{l}\text { 15JUZ } \\
16139\end{array}$ & $\begin{array}{l}86.33 \\
91.06\end{array}$ & $\begin{array}{l}13.67 \% \\
8.94 \%\end{array}$ \\
\hline $\begin{array}{l}100 \\
200\end{array}$ & $\begin{array}{l}17124 \\
17489\end{array}$ & $\begin{array}{l}96.61 \\
98.64\end{array}$ & $\begin{array}{l}3.39 \% \\
1.36 \%\end{array}$ \\
\hline
\end{tabular}

$\because$ SIEVE ANALYSIS

Soil Wt. $\mathrm{gm}$

$17724.0 \mathrm{C}$

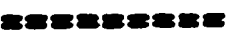




$$
1 . \div
$$

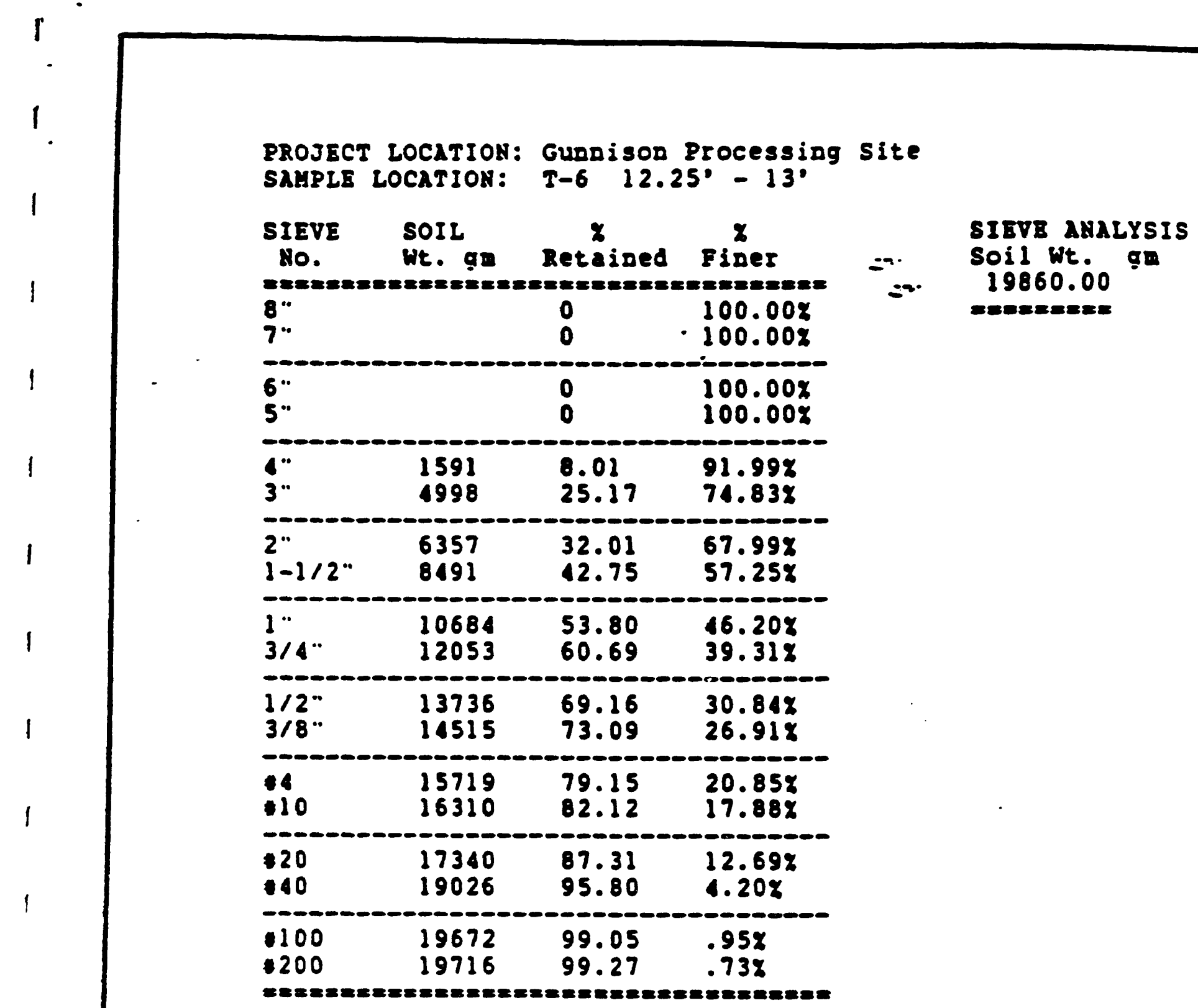


PROJECT LOCATION, Gunnison Frocessing Site SAMFLE LOCATION: T-8 $6^{\circ}-7^{\circ}$ Interface

\begin{tabular}{|c|c|c|c|}
\hline $\begin{array}{l}\text { SIEVE } \\
\text { NO. } \\
== \pm==2\end{array}$ & $\begin{array}{l}\text { SOIL } \\
\text { Wt. gm } \\
=====\end{array}$ & $\begin{array}{c}\% \\
\text { Retained }\end{array}$ & $\begin{array}{l}\% \\
\text { Finer } \\
\text { s=me=s=as }\end{array}$ \\
\hline $\begin{array}{l}8^{\prime \prime} \\
7 "\end{array}$ & & $\begin{array}{l}0 \\
0\end{array}$ & $\begin{array}{l}100.00 \% \\
100.00 \%\end{array}$ \\
\hline $\begin{array}{l}6 " 1 \\
5 " 1\end{array}$ & & $\begin{array}{l}0 \\
0\end{array}$ & $\begin{array}{l}100.00 \% \\
100.00 \%\end{array}$ \\
\hline $\begin{array}{l}\text { 4" } \\
3 "\end{array}$ & 0 & $\begin{array}{l}0 \\
0\end{array}$ & $\begin{array}{l}100.00 \% \\
100.00 \%\end{array}$ \\
\hline $\begin{array}{l}2 " 1 \\
1-1 / 2 "\end{array}$ & $\begin{array}{l}3700 \\
4450\end{array}$ & $\begin{array}{l}24.18 \\
29.08\end{array}$ & $\begin{array}{l}75.82 \% \\
70.92 \%\end{array}$ \\
\hline $\begin{array}{l}11 " \\
3 / 4 "\end{array}$ & $\begin{array}{l}5600 \\
6665\end{array}$ & $\begin{array}{l}36.60 \\
43.56\end{array}$ & $\begin{array}{l}63.40 \% \\
56.44 \%\end{array}$ \\
\hline $\begin{array}{l}1 / 2 " 1 \\
3 / 8 "\end{array}$ & $\begin{array}{l}7420 \\
7910\end{array}$ & $\begin{array}{l}48.50 \\
51.70\end{array}$ & $\begin{array}{l}51.50 \% \\
48.30 \%\end{array}$ \\
\hline $\begin{array}{l}\$ 4 \\
110\end{array}$ & $\begin{array}{l}8710 \\
9250\end{array}$ & $\begin{array}{l}56.93 \\
60.46\end{array}$ & $\begin{array}{l}43.07 \% \\
39.54 \%\end{array}$ \\
\hline $\begin{array}{l}\quad 20 \\
140\end{array}$ & $\begin{array}{l}10176 \\
11299\end{array}$ & $\begin{array}{l}66.51 \\
73.85\end{array}$ & $\begin{array}{l}33.49 \% \\
26.15 \%\end{array}$ \\
\hline $\begin{array}{l}1100 \\
1200\end{array}$ & $\begin{array}{l}12897 \\
133044\end{array}$ & $\begin{array}{l}84.29 \\
86.95\end{array}$ & $\begin{array}{l}15.71 \% \\
13.05 \%\end{array}$ \\
\hline
\end{tabular}

¿- SIEVE ANALYSIS Soil Wt. gm 15300000

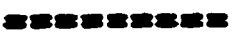


PROJECT LOCATION: GuDRisOn Processing Site SAMPLE LOCATIOA: $T-1081 / 2^{\circ}-91 / 2^{\circ}$ Slightly cemented

SIEVB SOIL
Ho.
Wt. ga Retained Finer

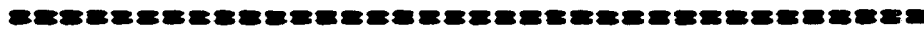

$8 *$

$7^{-0}$

)

6

$5^{-}$

000000

3- $2632 \quad 22.39 \quad 77.618$

3457 29.41 $30.59 \%$

$1-1 / 2 \% \quad 5586 \quad 47.52 \quad 52.48 \%$

-

$\begin{array}{llll}1- & 6208 & 52.81 & 47.198\end{array}$

$3 / 44^{\circ} \quad 7069 \quad 60.13 \quad 39.878$

$-0-0-0-0-0-0-0-0-0-0-0-0-0$

$1 / 2{ }^{*} \quad 7682 \quad 65.35 \quad 34.65 \%$

$3 / 80^{\circ} \quad 8134 \quad 69.19 \cdot 30.818$

$148817 \quad 75.00 \quad \frac{25.008}{98.98}$

$1.0 \quad 9285 \quad 78.98 \quad 21.02 \%$

$1200-9835 \quad 03.66 \quad 16.348$

$\begin{array}{llll}40 & 10586 \quad 90.05 \quad 9.958\end{array}$

$\begin{array}{llll}1100 & 11185 & 95.14 & 4.868 \\ 200 & 11296 & 96.09 & 3.91 \%\end{array}$


PROJsCT LOCATIOH: Gundison Processing Site SAHPLE LOCATIOH: T-IS

\begin{tabular}{|c|c|c|c|c|c|}
\hline 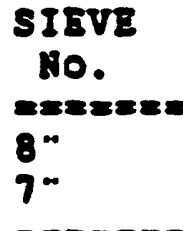 & $\begin{array}{l}\text { SOIL } \\
\text { Wt. gD } \\
\text { =E=E==E= }\end{array}$ & 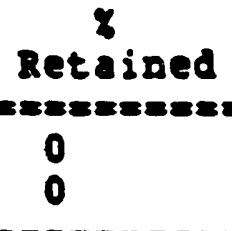 & $\begin{array}{c}y \\
\text { Einer } \\
==0=0=0= \\
100.008 \\
100.008\end{array}$ & $=29$ & 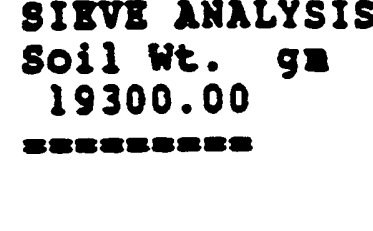 \\
\hline $\begin{array}{l}6 \% \\
5 *\end{array}$ & & $\begin{array}{l}0 \\
0\end{array}$ & $\begin{array}{l}100.008 \\
100.008\end{array}$ & & \\
\hline 3"* & 2090 & $\begin{array}{l}0 \\
10.83\end{array}$ & $\begin{array}{l}100.008 \\
89.178\end{array}$ & & \\
\hline $\begin{array}{l}2 * \\
1-1 / 2 *\end{array}$ & $\begin{array}{l}2560 \\
6570\end{array}$ & $\begin{array}{l}13.26 \\
34.04\end{array}$ & $\begin{array}{l}86.748 \\
65.968\end{array}$ & & \\
\hline$\frac{1}{3 / 4}$ & $\begin{array}{l}8720 \\
10010\end{array}$ & $\begin{array}{l}45.18 \\
51.87\end{array}$ & $\begin{array}{l}54.828 \\
48.138\end{array}$ & & \\
\hline $\begin{array}{l}1 / 2 * \\
3 / 8 .\end{array}$ & $\begin{array}{l}11600 \\
12580\end{array}$ & $\begin{array}{l}60.10 \\
65.18\end{array}$ & $\begin{array}{l}39.908 \\
34.828\end{array}$ & & \\
\hline 110 & $\begin{array}{l}14400 \\
15450\end{array}$ & $\begin{array}{l}74.61 \\
80.05\end{array}$ & $\begin{array}{l}25.398 \\
19.958\end{array}$ & & \\
\hline $\begin{array}{r}20 \\
.40\end{array}$ & $\begin{array}{l}16595 \\
17840\end{array}$ & $\begin{array}{l}85.98 \\
92.41\end{array}$ & $\begin{array}{l}14.028 \\
7.568\end{array}$ & & \\
\hline $\begin{array}{l}100 \\
1200\end{array}$ & $\begin{array}{l}18771 \\
18953\end{array}$ & $\begin{array}{l}97.26 \\
98.20\end{array}$ & $\begin{array}{l}2.74 \% \\
1.808\end{array}$ & & \\
\hline
\end{tabular}


PROJECT LOCATIOH: Gundison Processing site

SAHPLE LOCATIOH: T-17 $0^{\circ}-2^{\circ}$

\begin{tabular}{|c|c|c|c|}
\hline $\begin{array}{l}\text { SIEVE } \\
\text { HO. } \\
\text { E=E= } \\
8 * \\
7 *\end{array}$ & $\begin{array}{l}\text { SOIL } \\
\text { Wt. gD } \\
\text { =E==e= }\end{array}$ & $\begin{array}{l}\text { Z } \\
\text { Retained } \\
===0=a= \\
0 \\
0\end{array}$ & $\begin{array}{c}x \\
\text { Finer } \\
100.008 \\
100.008\end{array}$ \\
\hline $\begin{array}{l}6 \% \\
5 *\end{array}$ & & $\begin{array}{l}0 \\
0\end{array}$ & $\begin{array}{l}100.008 \\
100.008\end{array}$ \\
\hline 3 & 2210 & $\begin{array}{l}0 \\
15.90\end{array}$ & $\begin{array}{l}100.008 \\
84.108\end{array}$ \\
\hline $\begin{array}{l}2 * \\
1-1 / 2 *\end{array}$ & $\begin{array}{l}4770 \\
5650\end{array}$ & $\begin{array}{l}34.32 \\
40.65\end{array}$ & $\begin{array}{l}65.688 \\
59.358\end{array}$ \\
\hline $\begin{array}{l}1 \% \\
3 / 4\end{array}$ & $\begin{array}{l}6680 \\
7380\end{array}$ & $\begin{array}{l}18.06 \\
53.09\end{array}$ & $\begin{array}{l}51.948 \\
46.918\end{array}$ \\
\hline $\begin{array}{l}1 / 2 * \\
3 / 8 *\end{array}$ & $\begin{array}{l}8120 \\
8560\end{array}$ & $\begin{array}{l}58.42 \\
61.58\end{array}$ & $\begin{array}{l}11.58 \% \\
38.428\end{array}$ \\
\hline 10 & $\begin{array}{l}9734 \\
10250\end{array}$ & $\begin{array}{l}70.03 \\
73.74\end{array}$ & $\begin{array}{l}29.978 \\
26.268\end{array}$ \\
\hline $\begin{array}{r}20 \\
140\end{array}$ & $\begin{array}{l}10849 \\
11599\end{array}$ & $\begin{array}{l}78.05 \\
83.45\end{array}$ & $\begin{array}{l}21.958 \\
16.558\end{array}$ \\
\hline $\begin{array}{l}100 \\
200\end{array}$ & $\begin{array}{l}12633 \\
13070\end{array}$ & $\begin{array}{l}90.88 . \\
94.03\end{array}$ & $\begin{array}{l}9.128 \\
5.97 x\end{array}$ \\
\hline
\end{tabular}

SIEVE AHALYSIS

$=$ Soil wt. gm

$0=0=0=0$

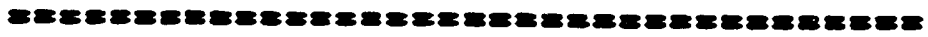


PROJECT LOCATIOA: Gunnison Processing Site

SAHPLE LOCATION: T-20 3'

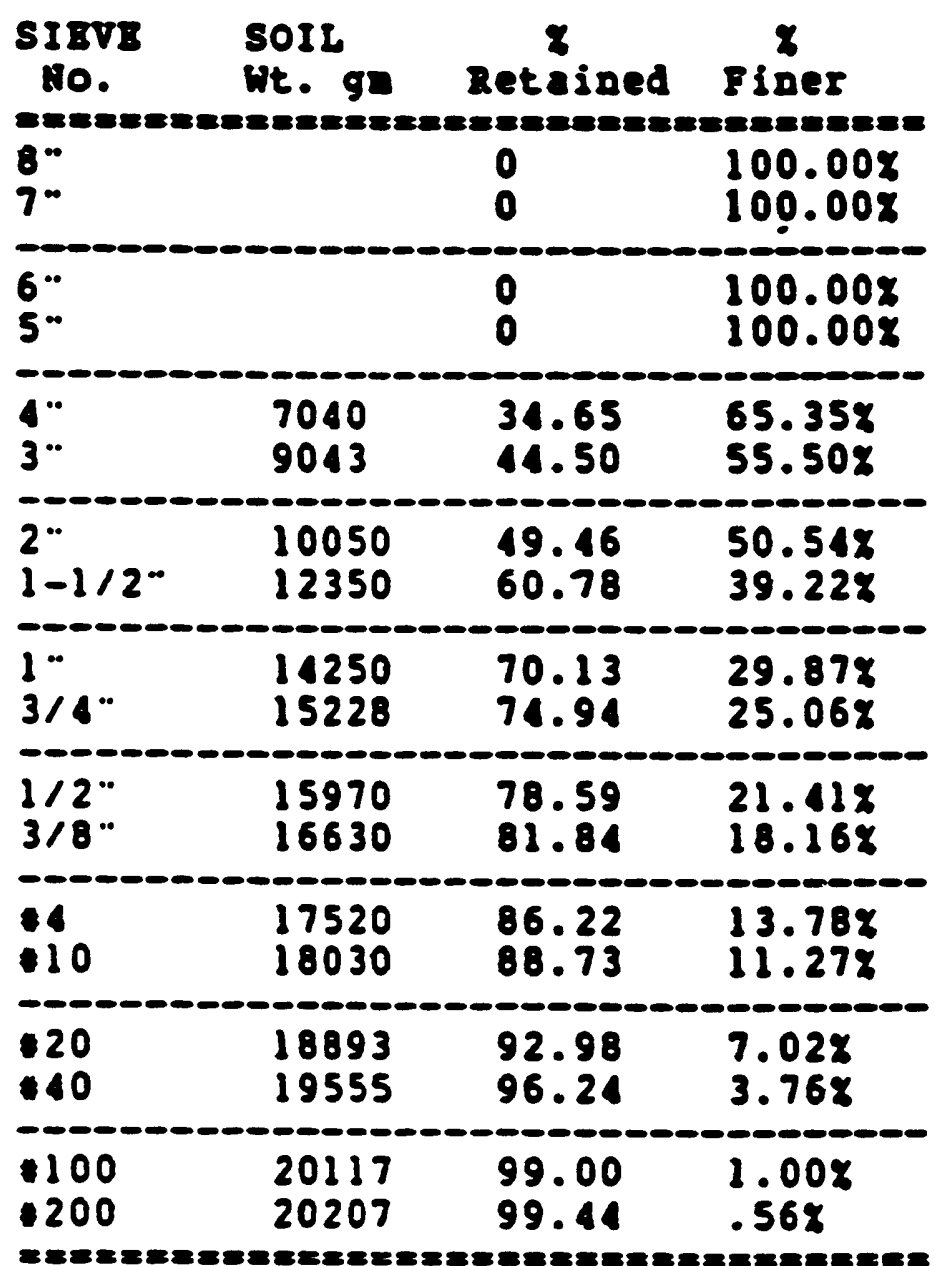


PROJECT LOCATION: Gunnison Frocessing Site SAMFLE LOCATION: T-23 $2^{\circ}-21 / 2^{\circ}$

SIEVE SOIL $\%$ \%

No. Wt. gn Retained Finer

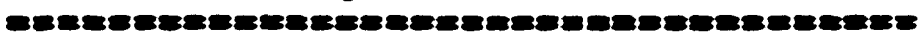

8

7"

6"

5" $100.00 \%$

$4^{\prime \prime}(0.00 \%$

3" $\quad 0^{\circ} \quad 100.00 \%$

$2^{11} 1000.00 \%$

$-$

\begin{tabular}{lccc}
$11 / 20$ & 0 & 0 & $100.00 \%$ \\
\hline 11 & 55 & 3.02 & $96.98 \%$ \\
$3 / 4 "$ & 82 & 4.55 & $95.45 \%$ \\
\hline $1 / 2 "$ & 158 & 8.71 & $91.29 \%$ \\
$318 "$ & 209 & 11.53 & $88.47 \%$ \\
\hline 14 & 258 & 14.23 & $85.77 \%$ \\
110 & 315 & 17.39 & $82.61 \%$ \\
\hline 120 & 470 & 25.92 & $74.08 \%$ \\
140 & 824 & 45.47 & $54.53 \%$ \\
\hline 100 & 1299 & 71.09 & $28.31 \%$ \\
1200 & 1420 & 78.35 & $21.65 \%$
\end{tabular}

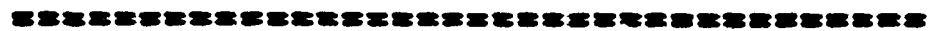

SIEVE ANALYSIS Soil Wt. ga 1811.90

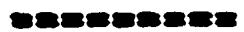




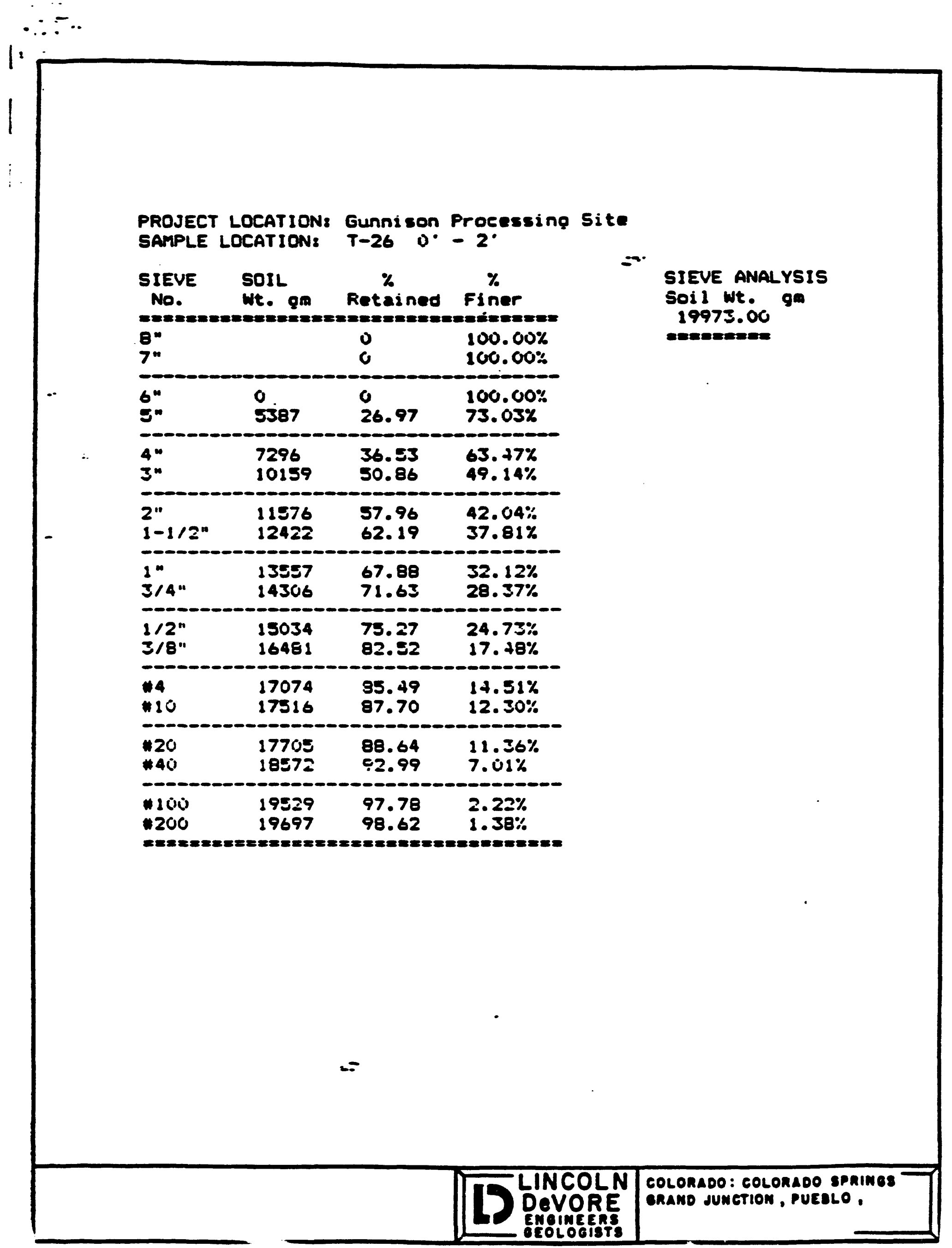


Helene ranglois MR-FERGUSON (Gunnison) P.O. BOX 9136 AIbuquareque, NH 87119

Aetn:

Profect: Gunnison

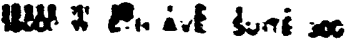
cology colonace cosi

luss beving wat swrte Janks neval an nowi 23-Ju1-90

Page: 1 Copy: 2 of 3 set : 1

Job: $002621=$

Received: 19-Jun-90 $15: 19$ PO : 3050-511-10004 1006

status: Final

enopis sypes cosl Method 705

Ra-226 Error Th-230 Error
Total

sample To

oc1/2 20. Des/1020*

GUN-5S-0002-T-3-CELL

GUH-55-0002-T-3-CELL

GUN-5S-0003-T-3-CELI

CUIF-35-0004-T-4-CEIL

GUN-35-0005-T-4-CELL

GUN-55-0006-T-13-CELL

OUN-5S-0007-T-13-CELL

GUN-55-0008-T-13-CELL

GUN-SS-0009-T-13-CELL

GUN-5S-0010-I-13-CELL

$54 \pm 4$

$200 \pm 20$

$27 \pm 3$

$25 \pm 2$

10314

$1216 \pm$

$20 \pm 2$

$230 \pm 10$

9124

$3.7 \geq 0.9$

$16 \pm 2$

$7.5 \pm 1.3$

$62 \pm 3$

$0.6 \pm 1.5$

$19 \pm 2$

$5.4 \pm 2.2$

$16 \pm 2$

GUN-SS-0021-T-15-CELL

GUN-5S-0012-T-15-CELL

GUN-SS-0013-T-15-CELL

$2.8 \pm 0.9$

$17 \pm 2$

GUN-SS-0014-T-25-CEIL

$4.2 \pm 2.0$

$30 \pm 2$

$2.4 \pm 0.8$

$202 \pm 4$

$2.7 \pm 0.8$

$88 \pm 4$

GUN-S5-0015-T-15-CELL

GUN-55-0016-T-20-CELL

GUN-SS-0017-T-20-CELL

GUN-SS-0018-T-20-CELL

GUN-S5-0019-T-21-CELL

GUN-5S-0020-T-21-CELL

$3.8 \pm 2.0$

$120 \geq 10$

$105 \pm 4$

$8.7 \pm 2.4$

$210 \pm 10$

$4.5 \pm 2.1$

$62 \pm 3$

$7.8 \pm 2.4$

$29 \pm 2$

$7.6 \pm 1.2$

$3.4 \geq 0.8$

$6.5 \pm 2.1$

$21 \pm 2$

GUN-SS-0021-T-21-CELL

$1.9 \pm 0.7$

$4.6 \pm 0.9$

GUN-5S-0022-T-22-CEIL

GUN-S8-0023-T-22-CELL

$3.8 \pm 0.9$

$2.3 \geq 0.7$

$2.1 \pm 0.7$

$0.9 \pm 0.5$

GUN-SS-0024-T-22-CELL

OUI-83-0025-T-23-CELL

$4.6 \pm 2.0$

$2.1 \pm 0.5$

$2.0 \pm 0.7$

$1.4 \pm 0.5$

$2.3 \pm 0.6$

$1.2 \pm 0.5$

cUY $-88-0027-T-24-C E L L$

2.420 .6

$2.5 \pm 0.6$

CUN-35-0028-T-24-CELL

$1.3 \pm 0.6$

$1.4 \pm 0.5$

OUN-55-0025-T-24-CELL

GUN-5S-0030-T-25-CELL

$2.5 \pm 0.6$

$0.7 \pm 0.4$

3. $4 \pm 2.0$

$2.5 \pm 0.7$ 


\section{BARRINGER LABORATORIES INC.}

nes a con a.1 \&. 1820

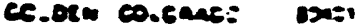

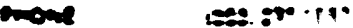

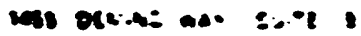

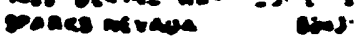
mons ins areses $6-3 u d-30$

Helene Langlols MK-FERGUSON (GurnLEON) 8.0. Box 9136

Albuguereque, NM 87129

Page: Copy: 2 of 2
Recelved: 29-Jun-5C i8:46 po : $3050-512-10004$ 1005
Aetr:

jroject: Gunnsson
SPATHE:

Eingl compd 39008 0012

sleve Analyado

$$
t_{n-1(9)}=2.262
$$

\section{Sangle it}

\section{I of sotulite}

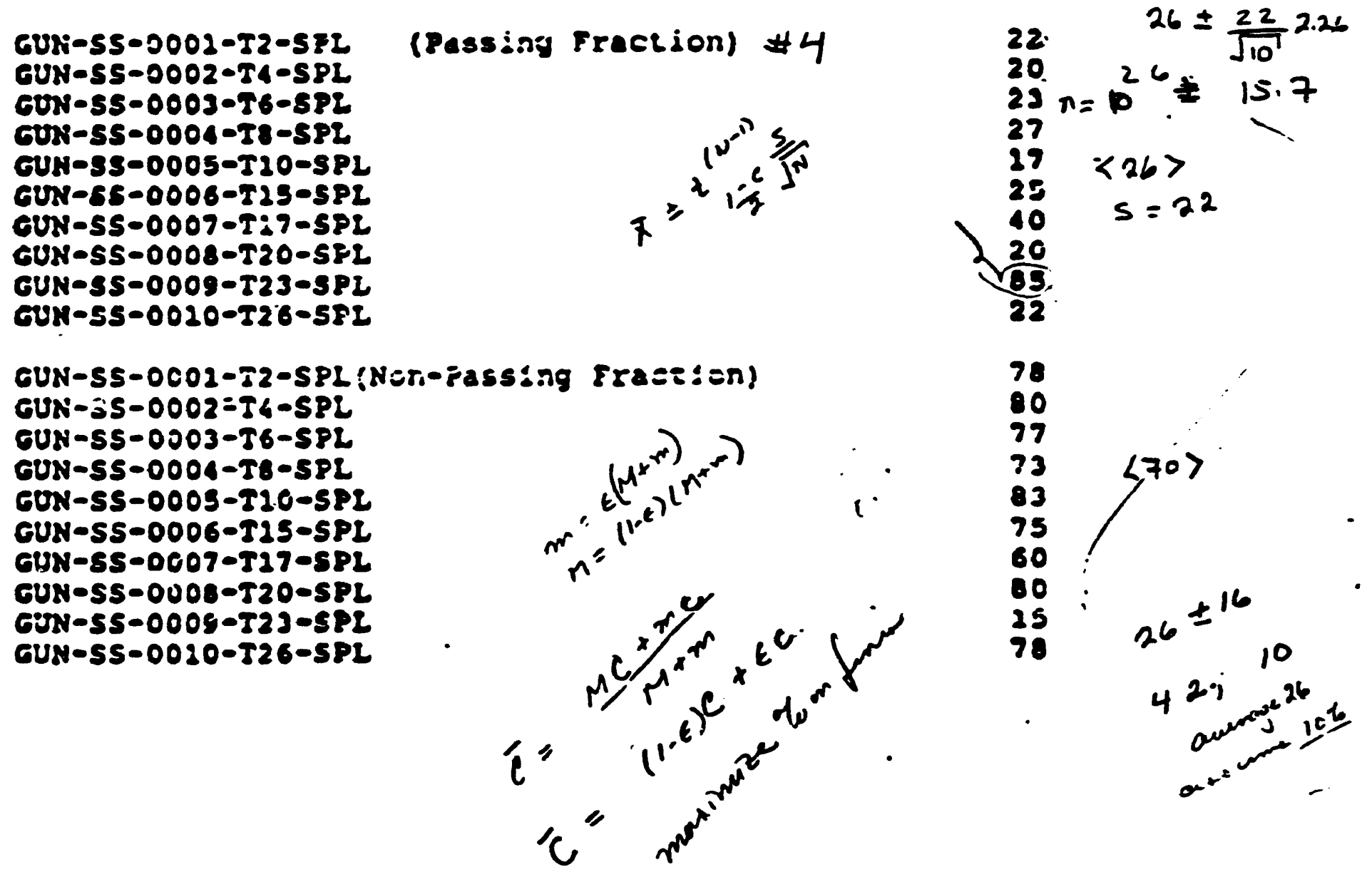



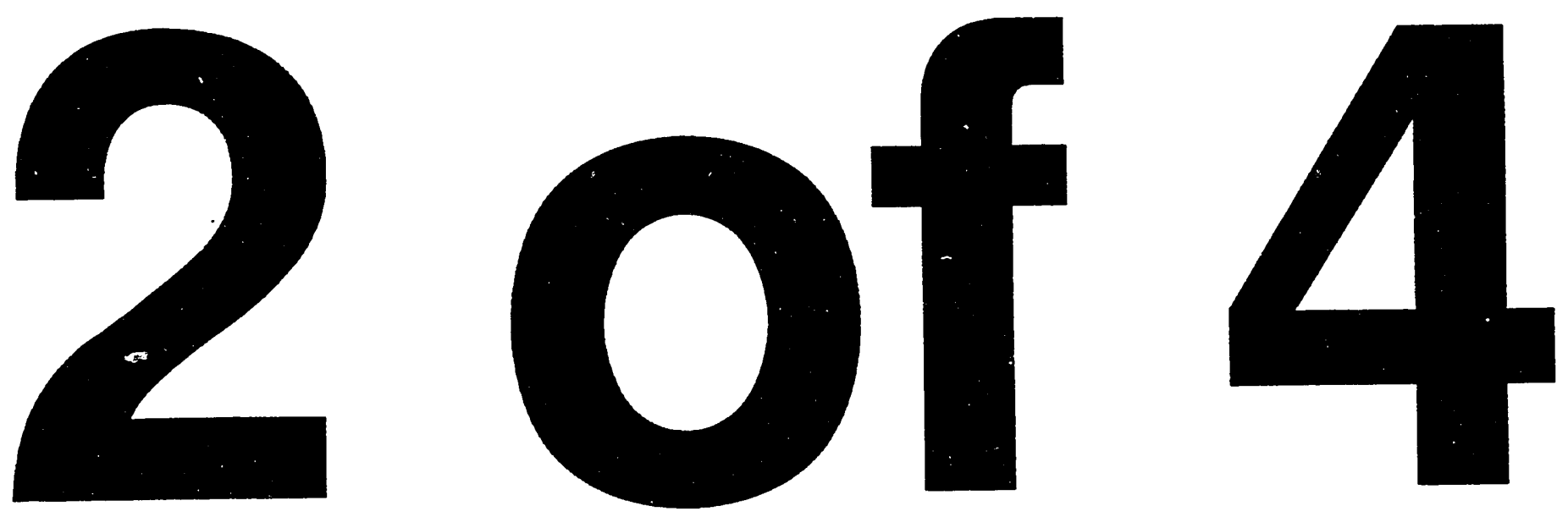


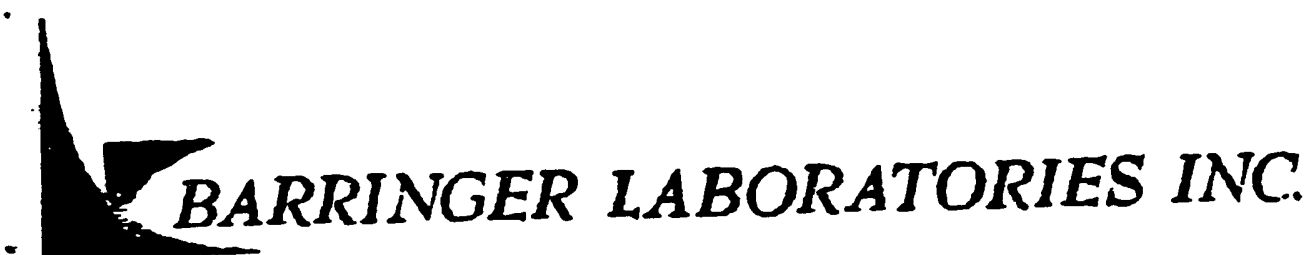

Helene Ianglo1s MX-FERGUSON (Gunnison) P.0. Box 9136

Albuquerque, NM 87219

Attn:

Project: Gunnison

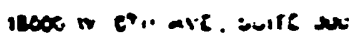

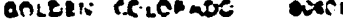
PaOAl 7201, 387 ise?

lajes Deminc war. Suite is parks vevaba om.jus mions. 23-Jul-90

Page: copy: 2 of 3 Set :

Recefred: 19-Jun-90 15:19 PO : 3050-511-20004 005

Iob: 9026215

\section{Eapie IYPO 8011}

Ra-226 Error Th-230 Error
Total Total
pej/g 20t rel/a 20k

sansle Id

GUN-SS-0031-T-25-CELI GUN-SS-0032-T-25-CELL GUN-SS-0033-T-26-CEIIL OUN-85-0034-T-20-CELL GUN-SS-0035-T-26-CELL GUN-5S-0036-T-27-CELL GUN-BS-0037-T-27-CELL OUN-SS-0038-T-28-CELL GUN-S5-0039-P-28-CELL GUN-5S-0040-T-28-CELL

GUN-SS-0041-T-1-CEI_L GUN-55-0042-T-1-CELL GUN-SS-0043-T-2-CELL OUN-SS-0044-T-1-CELI GUN-55-0045-T-2-CELL CUX-SS-0046-T-2-CELI GU1R-S5-0047-T-2-CELL GUN-55-0048-T-2-CELL CUH-55-0049-T-2-CEIL GUN-SS-0050-T-5-CELI

GUN-S5-0051-T-5-CELL CUR-5S-0052-T-6-CELL OUN-83-0053-T-6-CEII GUN-SS-0054-T-6-CEIL GUN-35-0055-T-6-CELL GUH-5S-0056-T-7-CELL GUN-55-0057-T-7-CELI CUN-SS-0058-T-7-CELL GUN-55-0059-T-7-CELL OUN-SS-0060-T-8-CELL
$1.2 \pm 0.6$

$2.3 \pm 0.6$

$0.9 \pm 0.5$

$1.3 \pm 0.6$

$1.5 \pm 0.6$

$2.0 \pm 0.5$

$2.3 \pm 0.6$

$3.6 \pm 1.0$

$=.3 \pm 0.9$

$2.9 \pm 0.9$

$1.7 \pm 0.7$

$3.1 \pm 0.7$

$1.3 \pm 0.6$

$3.8 \pm 1.0$

$2.5=0.8$

$0.6 \pm 1.3$

$4.0 \pm 1.0$

$6.4 \pm 2.2$

$5.4 \pm 2.2$

$4.6 \pm 1.0$

$8.3 \pm 2.4$

$1.0 \pm 0.5$

$1.4 \pm 0.6$

$2.0 \pm 0.5$

$1.5 \pm 0.6$

$2.2 \pm 0.7$

4.421 .0

$4.2 \pm 1.0$

$2.5 \pm 0.8$

$3.2 \pm 0.9$
$2.2 \pm 0.5$

$0.8 \pm 0.4$

$0.6 \pm 0.4$

$0.8 \pm 0.4$

$0.8 \pm 0.4$

$0.8 \pm 0.4$

$1.3 \pm 0.5$

$4.3 \pm 0.9$

$1.4 \pm 0.5$

$2.2 \pm 0.6$

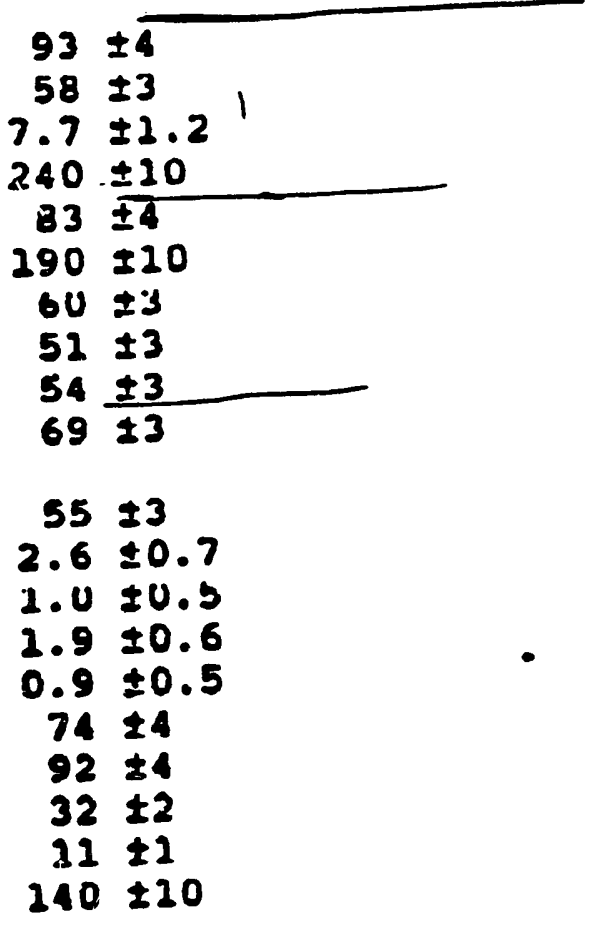




\section{BARRINGER LABORATORIES INC. -}

Helene rangloi:

MK-FERGUSON (GunnIEOn)

P.O. Box 9136

Albuquerque, NM 87219

Attn:

Project: Gunnison

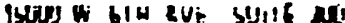

Coien: ceirshas soso: inerse :205! 21:-1009

14SB DEWINE waV. SUite is epankis weviga eassi inome $23-341-y 0$

Page: 3 Copy: 2 of 3 Set : 1

Iob: 9026212

Status:

Enal

Aample Iypor Bo11

Ra-226 Error Th-230 Error
Tnta]
Tntinl

Sample Ia

GUN-S5-0061-T-9-CELL GUN-SS-0062-T-9-CELL GUN-SS-0063-T-10-CELI GUN-SS-0064-I-10-CELL GUN-S5-0065-T-10-CELL GUN-SS-0066-T-10-CELL GUN-SS-0067-T-11-CELL GUN-SS-0068-T-11-CELL GUN-S5-0069-T-11-CELL GUN-SS-0070-T-11-CELL

GUN-SS-0071-T-11-CELL GUN-SS-0072-T-12-CELL. GUN-SS-0073-T-22-CELL GUN-SS-0074-T-12-CELL GUN-S5-0075-T-14-CELL GUN-SS-0076-T-14-CELL GUN-SS-0077-T-16-CELL GUN-SS-0078-T-16-CELL GUN-SS-0079-T-16-CELL GUN-SS-0080-T-16-CELL

GUN-SS-0082-T-16-CELL GUN-SS-0082-T-16-CELL GUN-S3-0083-T-16-CELL GUN-SS-0084-T-17-CELL GUN-35-0083-T-27-CELL GUN-SS-0086-T-17-CELL GUN-S5-0087-T-27-CELL GUN-S5-0088-T-17-CELL GUN-85-0089-T-28-CELL GUN-SS-0090-T-18-CELI ors 1/4 20: ocila 20:

$2.3 \pm 0.8 \quad 84 \pm 4$

$2.1 \pm 0.8 \quad 73 \pm 4$

$2.3 \pm 0.8 \quad 99 \pm 4$

$2.9 \pm 0.9 \quad 103 \pm 4$

$8.4 \pm 1.4 \quad 22 \pm 2$

$0.4 \pm 0.4 \quad 5.2 \pm 2.0$

$21 \pm 2 \quad 21 \pm 2$

$3.6 \pm 0.9 \quad 3.0 \pm 0.8$

$2.5 \pm 0.8 \quad 2.1 \pm 0.5$

$3.7 \pm 2.1 \quad 68 \pm 3$

$3.6 \pm 1.0 \quad 130 \pm 10$

$29 \pm 3 \quad 24 \pm 2$

$25 . \pm 2 \quad 12 \pm 1$

$4.6 \pm 1.0 \quad 1.7 \pm 0.6$

$2.0 \pm 0.8 \quad 2.2 \pm 0.7$

$0.8 \pm 0.5 \quad 0.7 \pm 0.4$

$2.8 \pm 0.8 \quad 2.2 \pm 0.7$

$2.4 \pm 0.6 \quad 1.0 \pm 0.5$

$1.0 \pm 0.5 \quad 2.5 \pm 0.6$

$0.8 \pm 0.5 \quad 0.7 \pm 0.4$

$1.6 \pm 0.6 \quad 7.7 \pm 1.2$

$2.2 \pm 0.7 \quad 80 \pm 4$

$2.7 \pm 0.8 \quad 58 \pm 3$

$2.1 \pm 0.7 \quad 1.5 \pm 0.6$

$19 \pm 2 \quad \cdot 12 \pm 1$

$2.2 \pm 0.7 \quad 2.3 \pm 0.7$

$1.5 \pm 0.6 \quad 0.9 \pm 0.4$

$1.4 \pm 0.6 \quad 1.8 \pm 0.6$

$2.8 \pm 0.6 \quad 2.7 \pm 0.6$

$4.9 \pm 1.1 \quad 1.0 \pm 0.5$
Recelved: 19-Tun-90 15:19 $\$ 006$ 


\section{BARRINGER LABORATORIES INC'}

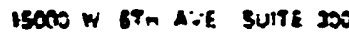

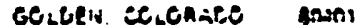
piking iso. 2:?.100?

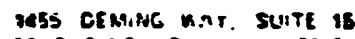

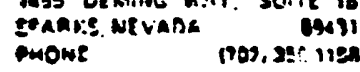
23-Ju1-90

Helene Langlois

MR-PERGUSON (Cunnleon;

P.O. Box 9136

Albuquerque, NH 87119

Page: 4

copy: 2 of 3

set: 2

Attn:

Broject: Gunnison

Received: 19-Jun-90 15:19 Iob: 9026212

\section{saplo zype: 0012}

\begin{tabular}{|c|c|c|c|c|}
\hline Samole Id & $\begin{array}{r}\text { Ra-226 } \\
\text { Total } \\
\text { Del/a }\end{array}$ & $\begin{array}{l}\text { Ertor } \\
200\end{array}$ & $\begin{array}{r}\text { Th-230 } \\
\text { Total } \\
\text { pcisa }\end{array}$ & $\begin{array}{l}\text { Ertor } \\
20 *\end{array}$ \\
\hline GUN-SS-0091-T- & 8.3 & \pm 2.4 & 37 & \pm 3 \\
\hline
\end{tabular}


Helene Langlole

MK-TERGUSON (GUNRIson)

P.O. Box 9136

Albuguerque, IM 87119

Page:

Copy:

see :
Received: 21-Jun-90 10:20 PO : 3050-512-10004\$007
Attn:

Project: Gunnison

Iob: : $902644 \mathrm{E}$ status:

Final

\section{Demplo zype: 0011}

Ra-226 Exror Th-230 Error Totel

Total

$08 i / 020 *$

peila 20*

GUN-SS-0092-T- 19

GUN-55-0093-T-8

$\begin{array}{ll}23 & \pm 2 \\ 2.4 & 10.8\end{array}$

$130 \pm 10$

$200 \pm 10$ 
ENGINEERS

AND

CONSTRUCTORS

NADOUAFTERS OFFICE

ONE ERIEVIEU PLAZA

CLEVELAND OMIOUSA MAi1

PHONE (216) 5233600 . TELEX 985542

AEPLY TO MK.FEAGUSON COMPANY

AEMEDIAL ACTIONS

CONTAACTOA UMTRA PROJECT

PO BOX 9136

October 9, 1990

$90-3050-719$

Mr. Mark I. Matthews

Project Manager

U.S. Department of Energy

Uranium Mill Tailings Remedial Action Project Office

First National Bank Building

5301 Central Avenue N.E.

Suite 1700

Albuquerque, New Mexico 87108

SUBJECT: Requested Data for Gunnison Site

REFERENCE: Contract No. DE-AC04-83AL18796

Dear Mr. Matthews:

As per request from the Gunnison Design Planning Meeting on September 19, 1990, please forward the amended Barringer Report and test pit logs T-1 through T-28 to Doug Gonzales, and the test pit logs L-1 through $1-14$ to Paul Darr.

If you have any questions, please contact John Briggs or Rob Cooney of my staff at $766-1667$.

Sincerely,

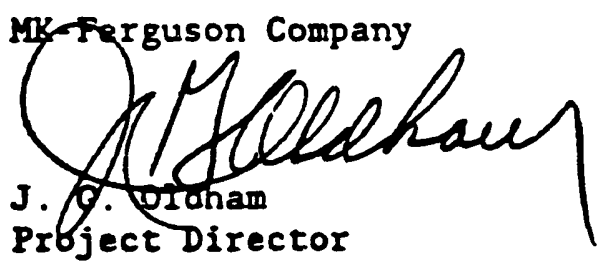

$\mathrm{JGO} / \mathrm{REC} / \mathrm{JRB} / \mathrm{sls}$

Enclosures

cc: D. Leske - GRJ (w/o enc.)

R. Rager - TAC/NMTRA (w/enc.)

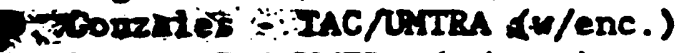

P. Dart - TAC/UMTRA (w/enc.) 
MKE DOC. 3885-GUN-R-09-01202-00

BARRINGER LABORATORIES INC.

PAS6 "i. 8th dive. celden, Coboredo 00401 Phons: poss z77.1607 Eax: (205) 277-1009

TELECOPY TRANSMITTAL

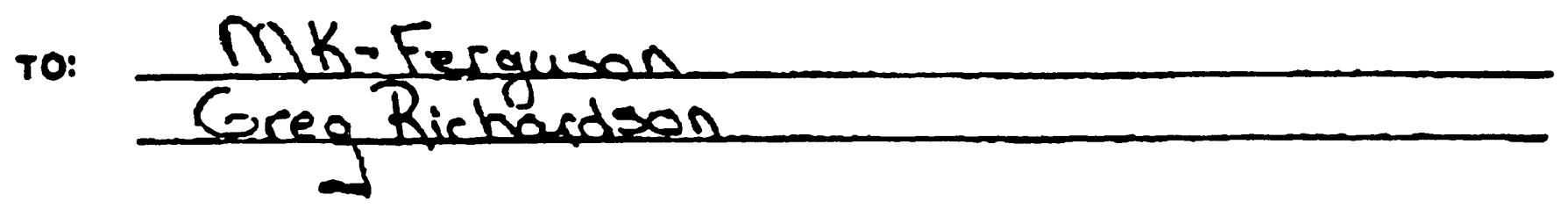

FROM: Dave basher, Gasy -2ito

DATE: $7 \cdot 17$

we are sendino 4 pages.

Fr:-

ond inzales 
$f^{\prime 7 / 17 / 90} \quad 15: 09 \quad 83032771689$

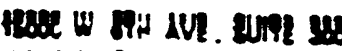
ciscuten colunaso conor

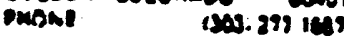

\section{BARRINGER LABORATORIES INC.}

chs Demue war. ents ve parks mivaba Nowe: 16-Jul-90

Helene Langlole

Idx-Exheuson (Gunnison)

P.0. Box 9136

Albuquerque, Ny 87129

Page: 2

Copy: 1 of 2

Attn:

Project: Gunnison

Sob: 2026235
Recelved: 19-Jun-90 $08: 46$ PO: $3050-512-10004 \quad 005$

NTENDED REPORT

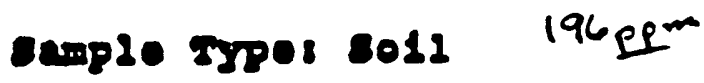

searus: Final

a. $\mathbf{P b}$

sample Io mele $n e / 9$

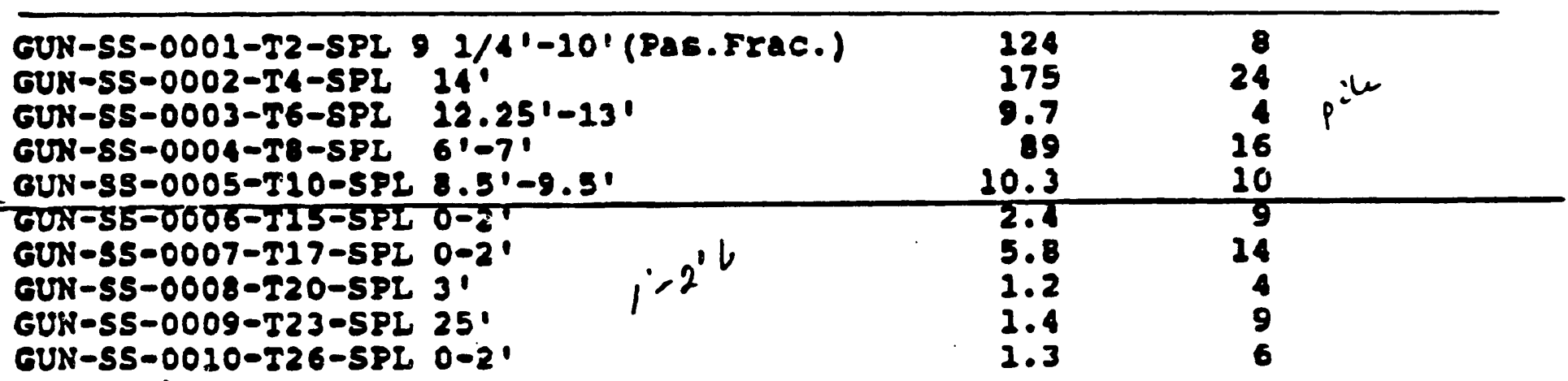

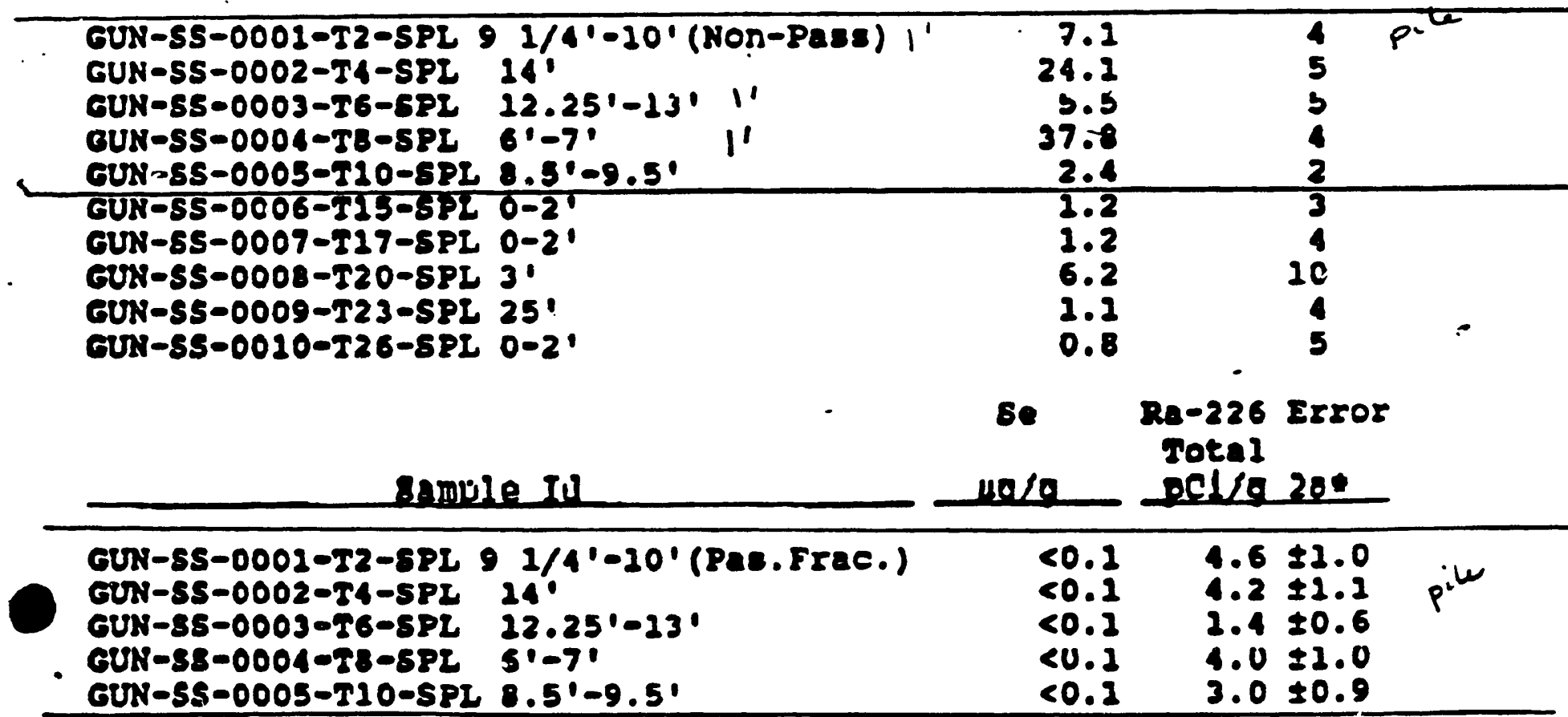


Helene ranglols

ud-FERGuson (Gunnison)

P.O. Box 9136

Albuquergua, and 87119

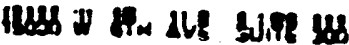
colocy. colonuos eswor
meme

Leas bemew war. sirte is

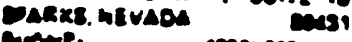
crenes.

\section{0-รบ1-90}

Page:

Copy:

1 of 2

Aten:

Project: Gunnluun

Recelved: 19-Jun-90 08:46 PO : 3050-512-10004 1005

Sob: $\quad 0026235$

starus: Final

AYENDED REPORT

Anple Iype: 2011

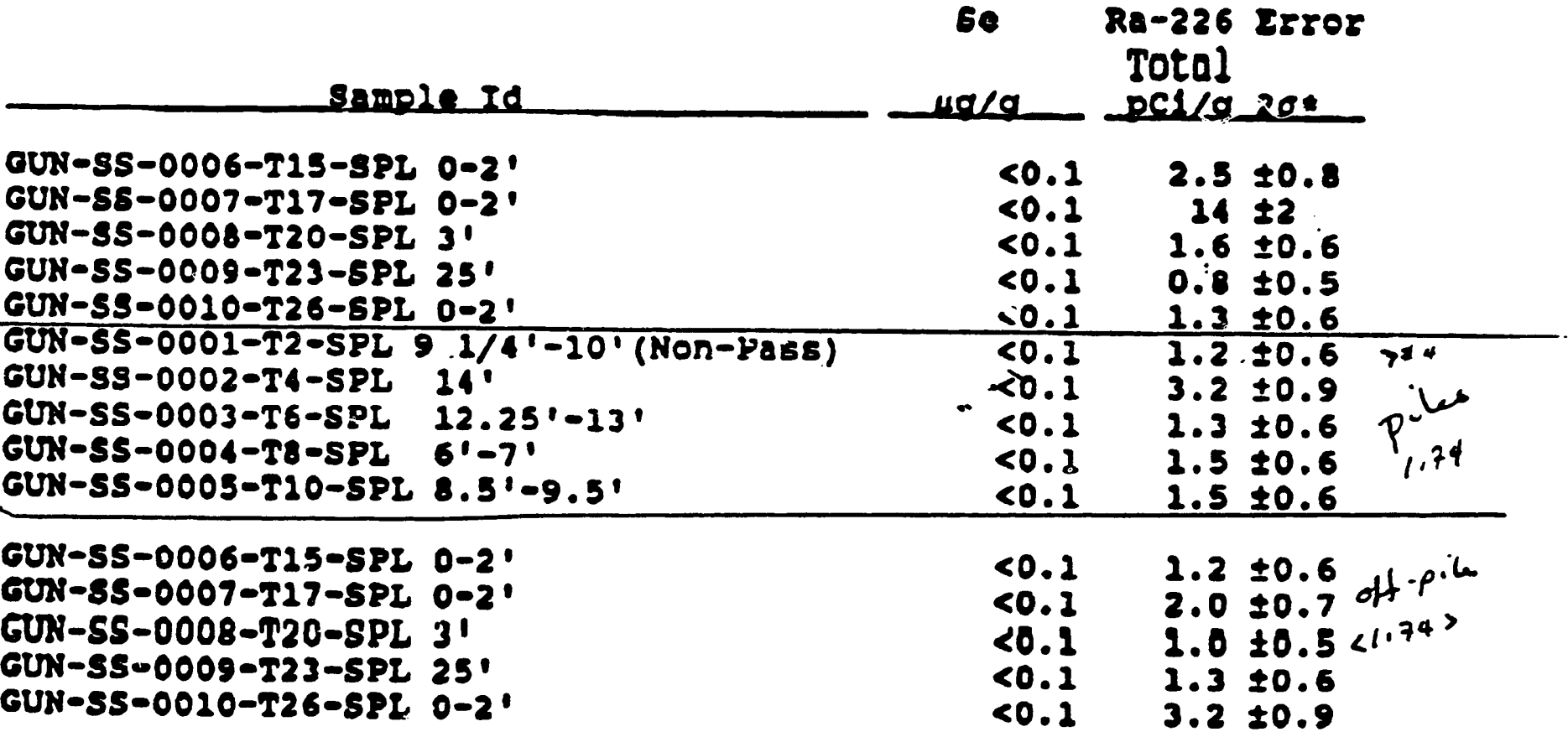

Sangle Id

Ih-230 Exrcr Th-232 Exror

Total Total

oc1/a 20* Dei/a 20\%

\begin{tabular}{|c|c|c|}
\hline 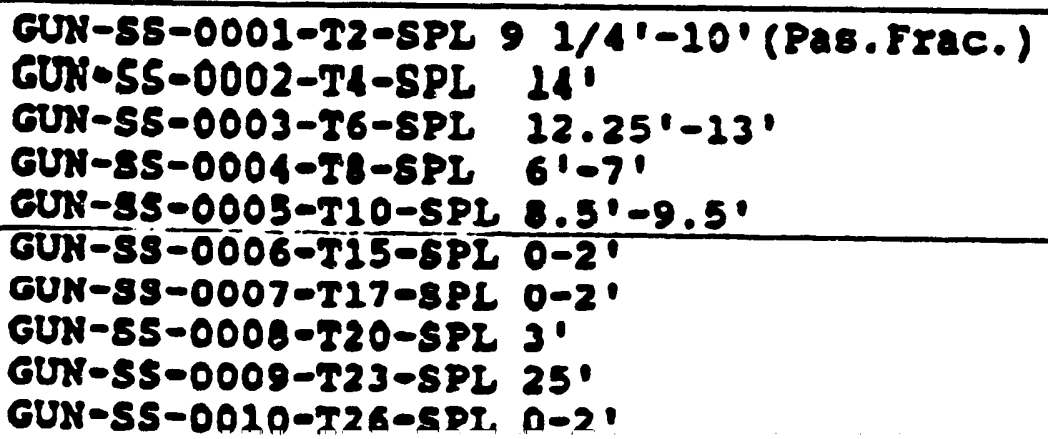 & $\begin{aligned} 180 & \pm 10 \\
78 & \pm 1 \\
2.1 & \pm 0.5 \\
180 & \pm 10 \\
120 & \pm 20 \\
3.7 \pm 0.8 & \\
15 & \pm 3 \\
1.1 & \pm 0.5 \\
1.3 & \pm 0.5 \\
1 & \pm 0.5\end{aligned}$ & $\begin{array}{ll}1.2 & \pm 0.6 \\
0.9 & \pm 0.5 \\
1.1 & \pm 0.5 \\
1.5 & \pm 0.6 \\
1.9 & \pm 0.7 \\
1.9 & \pm 0.6 \\
0.1 & \pm 0.5 \\
0.9 & \pm 0.5 \\
1.2 & \pm 0.6 \\
1.2 & \end{array}$ \\
\hline
\end{tabular}




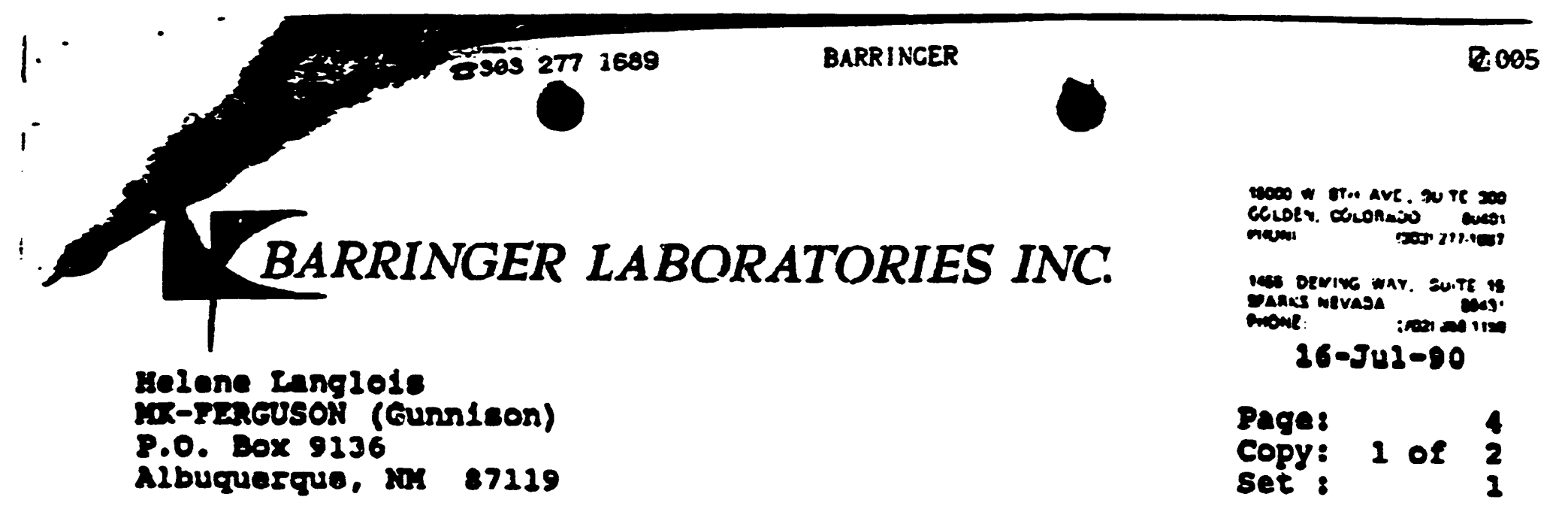

Attn:

Projece! Gunnisos

Recelved: 19-Jun-90 $08: 46$ Fo : $3050-511-10004 \$ 005$

Tob: 2026235:

ATNDED REORT

Dangle spes 0012

\begin{tabular}{|c|c|c|c|c|}
\hline snmple Io & $\begin{array}{l}0 \\
\text { Tota } 1 \\
\text { no }\end{array}$ & $\begin{array}{l}x-40 \\
\text { Total } \\
\text { gei } / 9(1)\end{array}$ & $\begin{array}{l}52502 \\
200\end{array}$ & $\begin{array}{c}x-40 \\
\text { rotal } \\
2 c 1 / 20(2)\end{array}$ \\
\hline $\begin{array}{l}\text { GUN-S8-0006-T2S-SPI } 0-2^{\prime} \\
\text { GUN-SS-0007-T27-SPI } 0-2^{\prime} \\
\text { GUN-SS-0008-T20-SPL } 3^{\prime} \\
\text { GUN-S5-0009-T23-SPL } 25^{\prime} \\
\text { GUN-SS-0010-T26-5PL } 0-2^{\prime}\end{array}$ & $\begin{array}{l}1.4 \\
1.5 \\
1.6 \\
1.3 \\
1.4\end{array}$ & $\begin{array}{l}31 \\
36 \\
33 \\
38 \\
3 y\end{array}$ & $\begin{array}{l} \pm 3 \\
\pm 3 \\
\pm 3 \\
\pm 3 \\
\pm 3\end{array}$ & $\begin{array}{l}1.6 \\
2.0 \\
1.9 \\
1.6 \\
1.6\end{array}$ \\
\hline
\end{tabular}

(2) Note: As determined by gamna spectroccopy. Although an attempt was made to deteraine the extent of interference from Ra-226/Radon 222 within the potassium-40 region of Interest, problems were encoutered with these sanples.

These resulte are the best availoble from our detection equipment and existing procedure. There is some doubt as to thelr accuragy.

(2) Note: As Aetermined by UCP and the ratio $K-40 / K=0.0118$. 


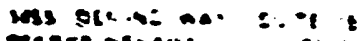

concestrana sim.

$\rightarrow 0$ l

$6-301-30$

Page:

Copy:

Bet:

2 or 2

Aren:

groject: Gunnsson
Recelved: 19-Jun-SC CE:SE Po : 3050-521-20004 1005

Sob: 2026235

STARHA: [1ng]

80mp10 5yper 8011

sleve Analyalo

sasole id sor sotwite

GU1-55-2002-I2-5FI GUN-S5-0002-T4-SPL GUN-55-0003-T6-5PL GUN-S5-0004-TB-5PL GUN-35-0005-T20-5PL GUN-56-0006-T25-5PL GUN-55-0007-T27-5PL CUN-55-0008-T20-5FL GUN-S5-0009-T23-SPL CUN-S5-0020-T26-5PL

(Passing Fraction)

GUN-55-0001-52-5PL (NCn-Fassing Fraston! 78

SUN-SS-0002-T4-SPL

GUN-5S-0003-T6-5PL

GUN-S5-0004-T8-5PL

GUN-55-000S-T20-5P2

GUN-S5-0006-T25-5PL

GUN-55-0007-T27-SPL

GUN-SS-0008-T20-5PL 
ON-SITE RADIOLOGICAL MEASUREMENTS OF 1-FT DEPTH INCREMENT SOIL SAMPLES < \#4 MESH SIEVE COBBLY SUBSOIL INVESTIGATION JUNE 1990 


\section{CELL EMANATION SAMPLING LOG}

PROJECT/SITE NAME: LinITADP/ECQ aVi=CA

GRID COORDINATES:

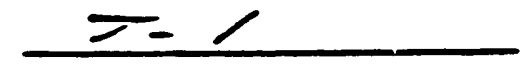

DATE TIME - FUNCTION PCI/gM COLNTED COUNTED COUNTED FUNCTIOH PCI/gT COITED ELEVATION MET $\frac{7-7.7}{7-13-90} \frac{1122}{1201} \frac{1274.6}{957.5} \frac{2.0}{1.5} \frac{\text { SEW }}{\text { SELQ }}$

SAMPLE ID: $\because \because \%=0-C .0 \%$ EMANATION 0.25

RSO REVIEW

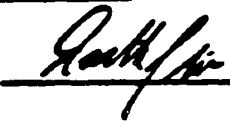

TH-230

93

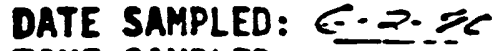
TIME SAMPLED: DATE SEALED: TIME SEALED: $\angle>C 0$ UET HEIGHT: C. $2 \% 3$ DATE OPENED: $>-.3 .>1$ TIME OPENED: $\angle \overline{i c}$ DATE RESEALED: $2-13-70$ TIME RESEALED: $\frac{1132}{63}$ DRY HEIGHT:

623

\section{DATE TIME FUNCTION PCI/gm COUNTED COUNTED COUNTED}

ELEVATION WET $\frac{7-1-2.9}{7-13.90} \frac{1123}{1202} \frac{11735}{1239.7} \frac{2.1}{2.3}$ SEW UNEQUILIBRATED

GUA. $=50 \%=$ EMANATION 0.06

RSO REVIEW
Ena

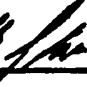

TH-230

61
DATE SAMPLED: $\leqslant-=-.-\mathrm{C}$ DATE SEALED: $4 \cdot 4-9 C$. UET HEIGHT: = DATE OPENED: $=6$ TIME OPENED: $=36$ DATE RESEALED: $7-13.96$ TIME RESEALED: DRY MEIGHT:

\section{DATE COUNTED COUNTED \\ FUNCTION $\mathrm{PCl} / \mathrm{gm}$ \\ COUNTED BY}

ELEVATION HET $\frac{7-9.4}{7-13-40} \frac{1130}{1259} \frac{646.5}{13 \% 3} \frac{1.1}{0.3}$ SEW UNEQUILIBRATED

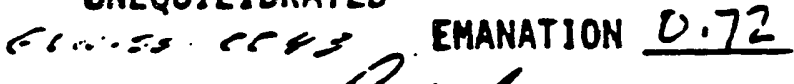

RSO REVIEH

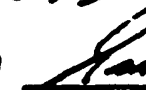

DATE SAMPLED: $=\because-\vec{z})$ DATE SEALED: $6-4 \cdot 9()$ MET HEIGHT: $=\cdots-\cdots$ DATE OPENED: TIME OPENED: $=\frac{15}{7-13}$ DATE RESEALED: $7-13-90$ TIME RESEALED: 1132 DRY HEIGHT: 538

\section{DATE TIME FUNCTION PCI/gM COUNTED COUNTED COUNTED}

ELEVATION HET E.8.5.

MET

DRY $7-13-90$ UNEQUILIBRATED GuN-SD-cios: EMANATION 0.78 .

RSO REVIEH

TH-230

240

DATE SAMPLED: $6 \cdot 3-9 C$ DATE SEALED: $6-4 \cdot 9 \mathrm{C}$ MET MEIGHT: $G=\because 2$ DATE OPENED: $=-Z T$ TIME OPENED: $\angle=-$ DATE RESEALED: $7 \cdot 13.4 \%$ TIME RESEALED: 1132 DRY MEIGHT: $5 \times 3$
1332B
$04 / 25 / 89$
HEALTH PHYSICS PROCEDURES

\begin{tabular}{|c|c|}
\hline REV NO. & 1 \\
\hline PAGE NO. & $019-4$ \\
\hline
\end{tabular}


CELL EMAMATION SAMPLING LOG

PROJECT/SITE MAME: UMTRA /GUNNISON

GRID COORDIMATES:

$T=2$

DATE TIME - FUNCTION PCI/gm COUNTED DATE SAMPLED: COUNTED COUNTED RUMCTION PCI/gI COUTED TIME SAMPLED:

DATE SEALED:

ELEVATION, MET $\frac{7-12-90}{7-13-90} \frac{1139}{1218} \frac{1038.8}{6521} \frac{1.8}{1.2} \frac{\text { SEW }}{\text { QEEW }}$ TIME SEALED: UNEQUILIBRATED

MET MEIGHT:

DATE OPENED:

SAMPLE ID: GWHSS-0045 EMANATION 0.37

RSO REVIEH

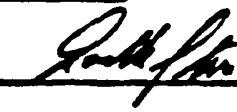

TH-230

82

TIME OPENED:

DATE RESEALED:

TIME RESEALED:

DRY MEIGHT:

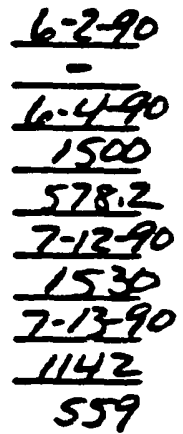

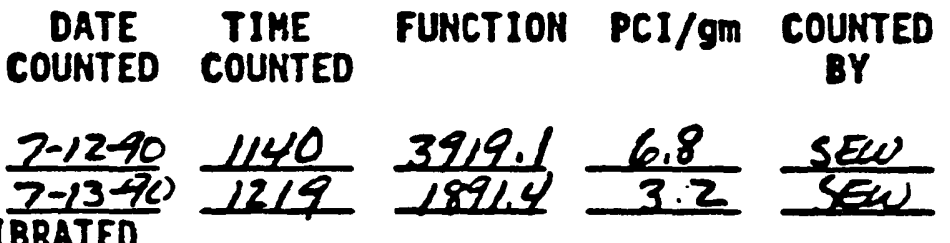

DATE SAMPLED:

DATE SEALED:

MET WEIGHT:

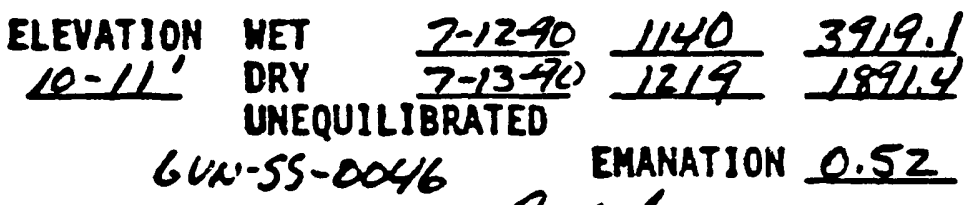

RSO REVIEH THe TH-230 190

DATE OPENED:

TIME OPENED:

DATE RESEALED:

TIME RESEALED:

DRY MEIGHT:

$6-1-90$

6.490

$580.4 *$

7-1290

1530

$7-13-90$

1142

592

DATE TIME FUNCTION PCI/GM COUNTED
COUNTED COUNTED

ELEVATION MET $\frac{7-12-90}{2-13-90} \frac{1148}{1251} \frac{2414.0}{9281} \frac{4.2}{1.8} \frac{\text { SEW }}{5 E U}$ UNEQUILIBRATED GUN-SS-0047. ERANATION 0.62

RSO REVIEH Sedafle TH-230 60

DATE TIME FUNCTION PCI/gI COUNTED
COUNTED COUNTED

DATE SAMPLED:

DATE SEALED:

MET MEIGHT:

ELEVATION MET $\frac{2-12-90}{2-13-90} \frac{1150}{1252} \frac{3346,0}{2221.3} \frac{6.1}{4.0}=$ SEW - UNEQUILIBRATED GUN-SS-0048 EMANATION 2.34

DATE OPENED:

TIME OPENED:

DATE RESEALED:

TIME RESEALED:

RSO REVIEN

TH-230

DRY MEIGHT:

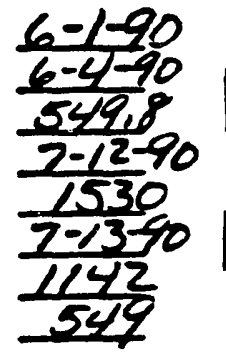

13328

$04 / 25 / 89$

HEALTH PHYSICS PROCEDURES

\begin{tabular}{|lc}
\hline REV NO. & 1 \\
\hline PAGE NO. & $019-4$ \\
\hline
\end{tabular}




\section{CELL EIANATION SAHPLING LOG}

PROJECT/SITE MAME: GRID COORDINATES:

$\frac{\text { Unpteolevwnison }}{2.2}$
$\therefore$

DATE TIHE - FUNCTION
COUNTED COUNTED

$\mathrm{PCI} / \mathrm{gm}$

COUNTED DATE SAMPLED: $\leq->$ PC COUNTED COUNTED

BY UNEQUILIBRATED

SAMPLE ID: EVN-SS-COP EMANATION 0.32

RSO REVIEH

TH-230

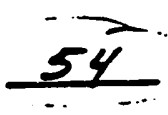
TIME SAMPLED: DATE SEALED: $\overline{6-\%-90}$ TIME SEALED: 1500 WET WEIGHT: 609.6, DATE OPENED: TIME OPENED: 1.530 DATE RESEALED: $z-13-90$ TIME RESEALED: $\frac{1248}{548}$ DRY HEIGHT: 542

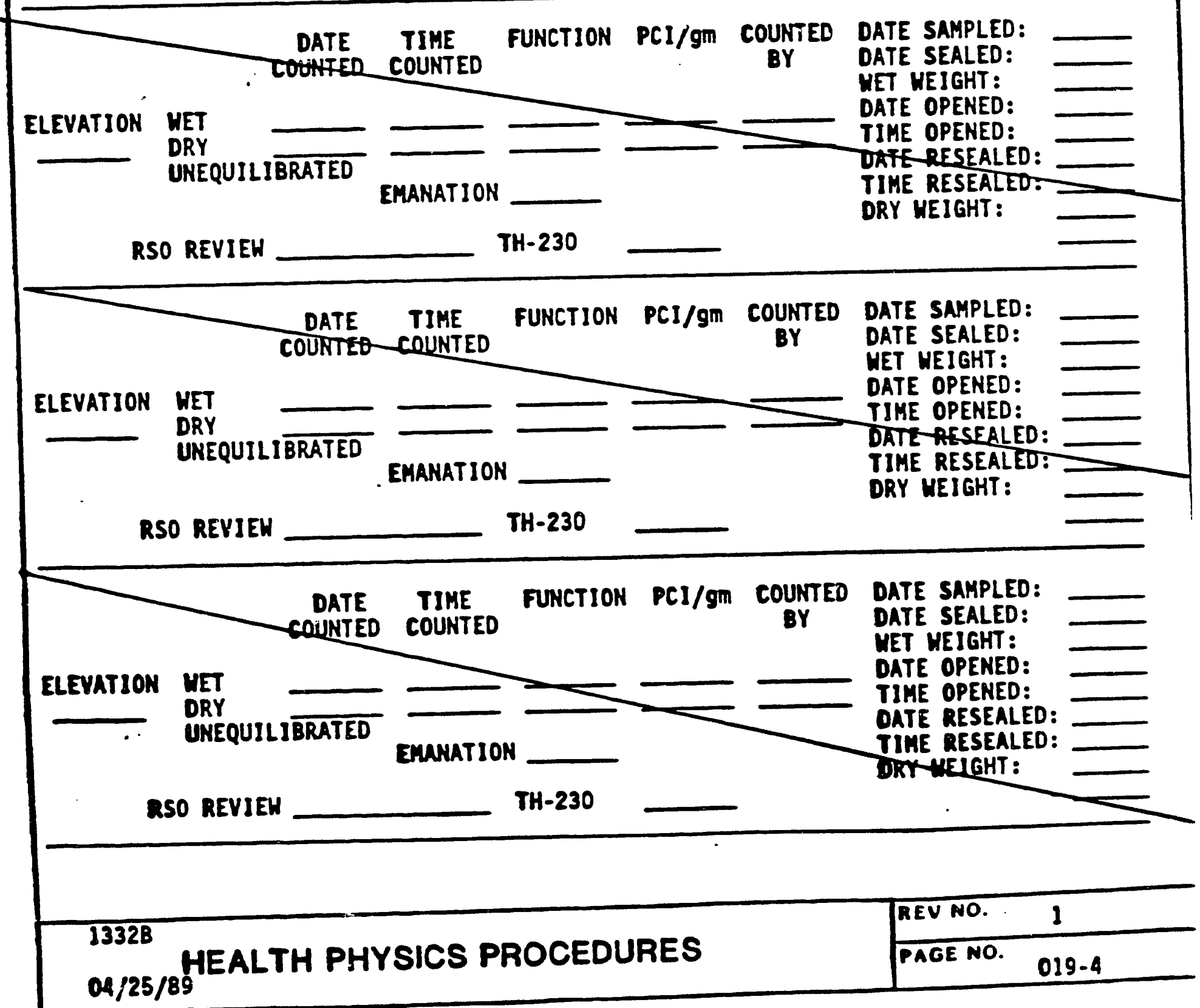


CELL EMAKATION SAMPLING LOG

PROJECT/SITE MAME: UMTTA /GUNNISON

GRID COORDINATES: $T=3$

$$
-
$$

DATE TIME - FUNCTION PCI/gII COUNTED COUNTED COUNTED

BY

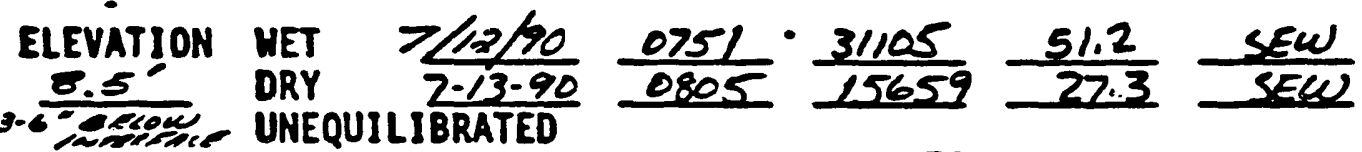

SAMPLE 1D: 6UN.55-0001

EMANATION 0.50

RSO REVIEH

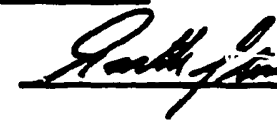

TH-230

200

$\begin{array}{cccc}\text { DATE } & \text { TIME } & \text { FUNCTION PCI/gM } & \text { COUNTED } \\ \text { COUNTED } & \text { COUNTED }\end{array}$

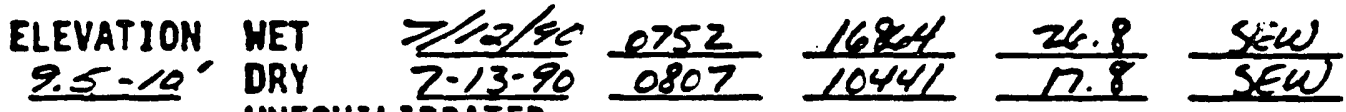
UNEQUILIBRATED GUN-SS-0002 EMANATION 0.38

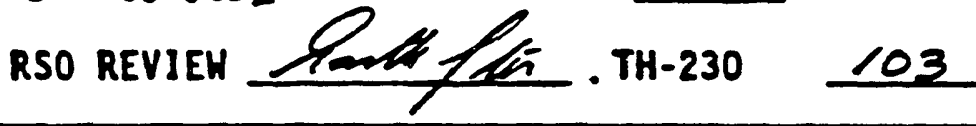

$\begin{array}{cccc}\text { DATE } & \text { TIME } \\ \text { COUNTED COUNTED } & \text { PUNION PCI/gM COUNTED } \\ \text { BY }\end{array}$

ELEVATION MET $\frac{7 / 3 / 9 C}{7-13-90} \frac{0800}{0814} \frac{11587}{7188} \frac{15.6}{11.7} \frac{\text { SEW }}{5 E W}$

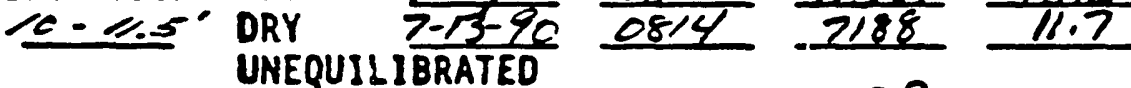
6UU'-55-0003 EMANATION 0.38

RSO REVIEH

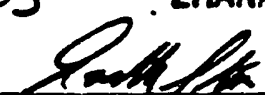

TH-230

116

\section{DA}

DATE SAMPLED: 6 /80 TIME SAMPLED: DATE SEALED: TIME SEALED: WET WEIGHT: DATE OPENED: TIME OPENED: DATE RESEALED: TIME RESEALED: DRY HEIGHT:

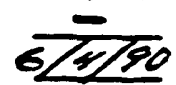
1500 Ecom $143 / 80$ 0805 $7-13^{-90}$ $\frac{2745}{573}$ 573

DATE SAMPLED: DATE SEALED: MET VEIGHT: DATE OPENED: TIME OPENED: DATE RESEALED: TIME RESEALED: DRY MEIGHT:

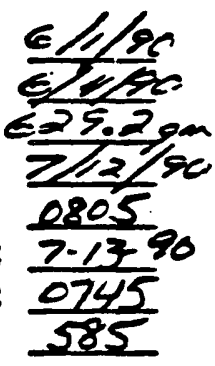

DATE SAMPLED: DATE SEALED: HET HEIGHT: DATE OPENED: TIME OPENED: elise
Elyle
$\frac{14450}{212 / 80}$
$\frac{0512}{7-13-9}$ TIME RESEALED: DRY MEIGHT:
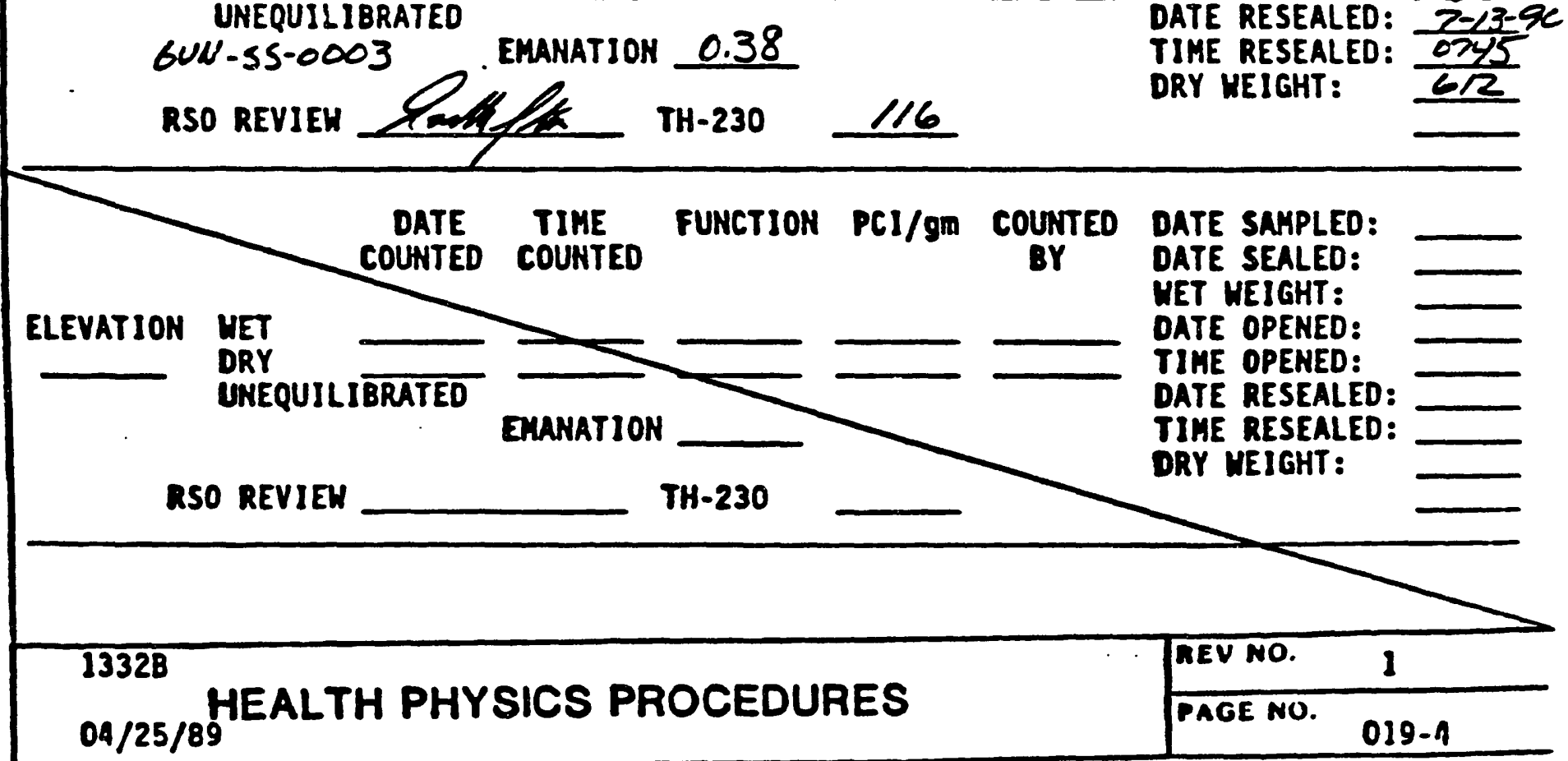

COUNTEO DATE SAMPLEO: DATE SEALED: WET HEIGHT: DATE OPENED: TIME OPENED: DATE RESEALED: TIME RESEALED: DRY MEIGHT:

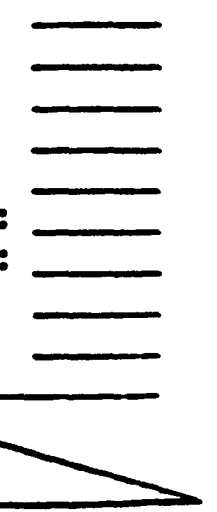

\begin{tabular}{|lc}
\hline REV NO. & 1 \\
\hline PAGE NO. & $019-1$ \\
\hline
\end{tabular}




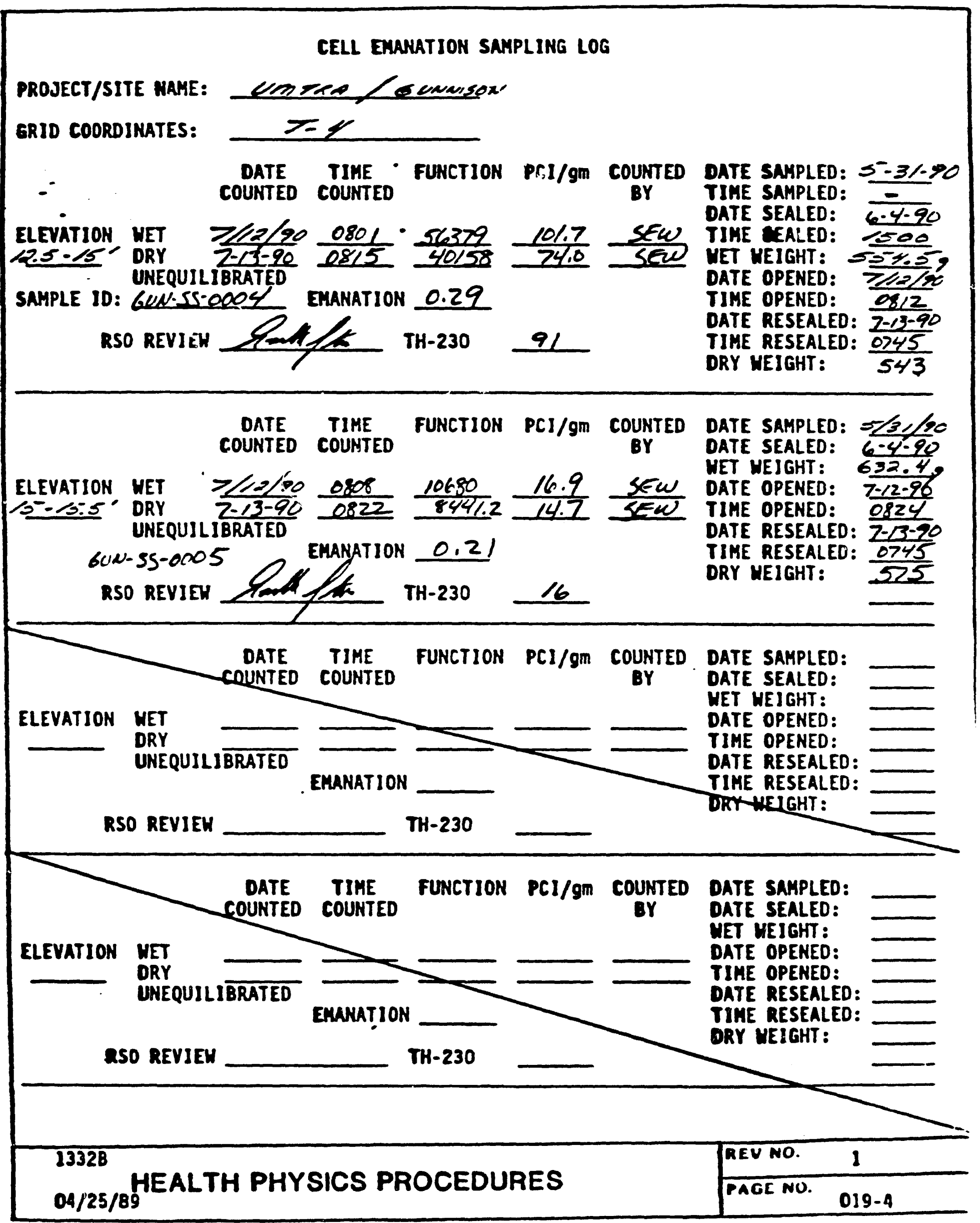


CELL EMANATION SNMPLING LOG

PROJECT/SITE MAME: UMTTA LGUACESON

GRID COORDINRTES:

$7=5$

\section{Taimecter at

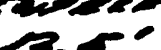 \\ ELET \\ ELEVATION HET CS-CE DRY \\ SAMPLE ID: UHEQUILIBRATED DATE TIME
COUNTED COUNTED \\ UNCTION PCI/gm COUNTED

$$
\frac{1159}{1301} \cdot \frac{3171.4}{2410.5}
$$ $\frac{5.6}{4.3}$ SEW} RSO REVIEH

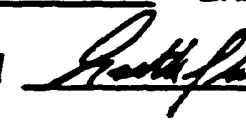

EMar

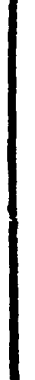

\section{E!}

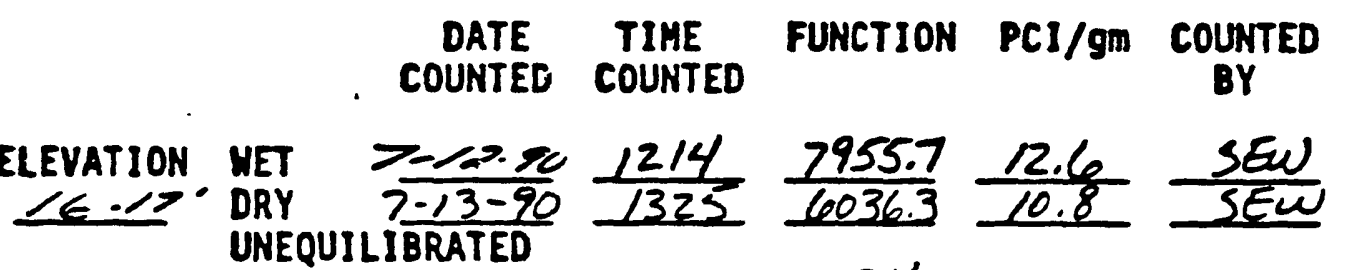
UNEQUILIBRATED

GUN-SS-COF' EMANATION 0.24

RSO REVIEH

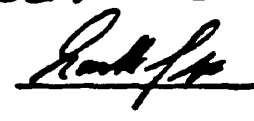

TH-230

5
DATE SAMPLED: $6-2->0$ TIME SAMPLED:

DATE SEALED:

TIME SEALED:

TIME SEALED: $1=00$

NET WEIGHT: 5639

DATE OPENED: $\frac{63.18}{-12.80}$

TIME OPENED: 1530

DATE RESEALED: $7.13-90$

TIME RESEALED: DRY MEIGHT:
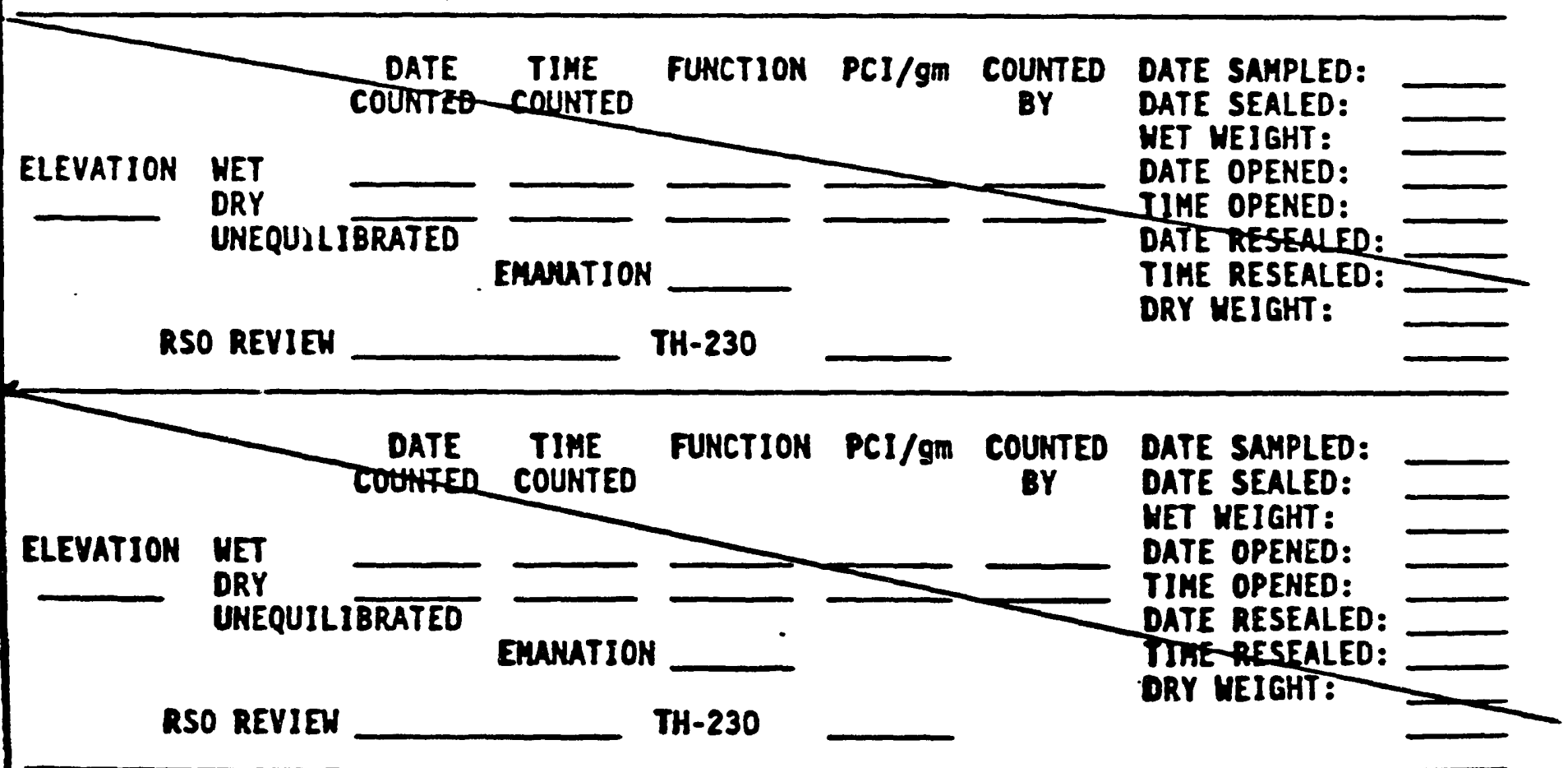

13328 $04 / 25 / 89$

HEALTH PHYSICS PROCEDURES

\begin{tabular}{l} 
REV NO. \\
\hline PAGE NO. \\
\hline
\end{tabular}


CELL EMAHATION SAMPLING LOG

PROJECT/SITE NAME: UMTRA/GUNNISON

GRID COORDINATES:

$$
T-6
$$$$
\begin{array}{cc}
\text { DATE } & \text { TIME } \\
\text { COUNTED } & \text { COUNTED }
\end{array}
$$

ELEVATION MET

$11.25-12.25^{\circ}$ DRY UNEQUILIBRATED

$\frac{2-12-90}{2-13-90}$

$\frac{1223}{1335}$

$\frac{4585}{489.6}$

$\frac{0.8}{0.9} \frac{S E w}{S E w}$

SAMPLE 10: GUN-SS-0053 EMANATION -0.07

RSO REVIEH

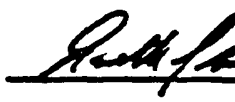

\section{DATE TIME COUNTED COUNTED}

TH-230

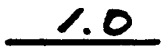

COUNTED

BY
COUNTED DATE SAMPLED:

TIME SAMPLED:

DATE SEALED:

TIME SEALED:

NET HEIGHT:

DATE OPENED:

TIME OPENED:

DATE RESEALED:

TIME RESEALED:

DRY HEIGHT:
$6 \cdot 2 \cdot 90$

$\frac{-}{1-40}$

1500

$565: 1$

2-12-90

1530

7-13-90

$\frac{1323}{524}$

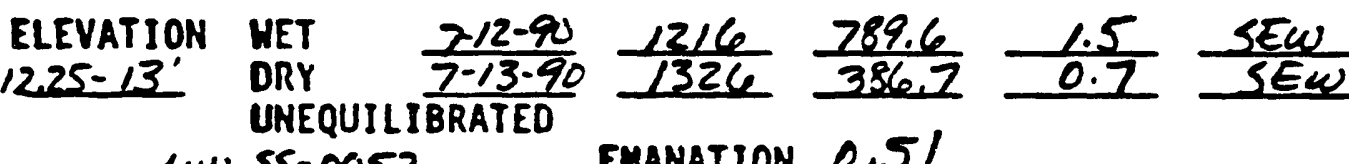
CUN-SS-0052

RSO REVIEH

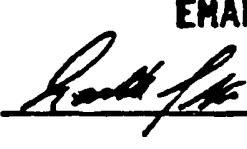

TH-230 2.6

DATE SAMPLED:

DATE SEALED:

$6-2-90$

UET HEIGHT: 538.3

DRTE OPENED: $\quad z-12-90$

TIME OPENED: 1530

DATE RESEALED: $7-13-90$

TIME RESEALED: $\angle 310$

DRY WEIGHT:

\section{DATE TIME FUNCTION PCI/gm COUNTED COUNTED COUNTED}

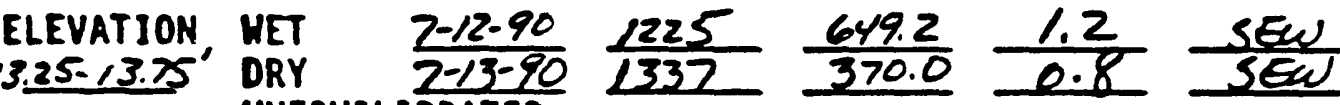 UNEQUILIBRATED GUNSS-0054 EMANATIOH 0.43}

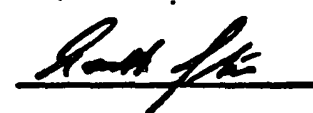
TH-230 1.9

DATE SAMPLED: $\quad 6-2-90$ DATE SEALEO: $6-4-90$ HET HEIGHT: $53 / 2$ DATE OPENED: $z-12-90$ TIME OPENED: -530 DATE RESEALED: $\overline{7 \cdot 13}-10$ TIME RESEALED: 1323 DRY MEIGHT: 490.

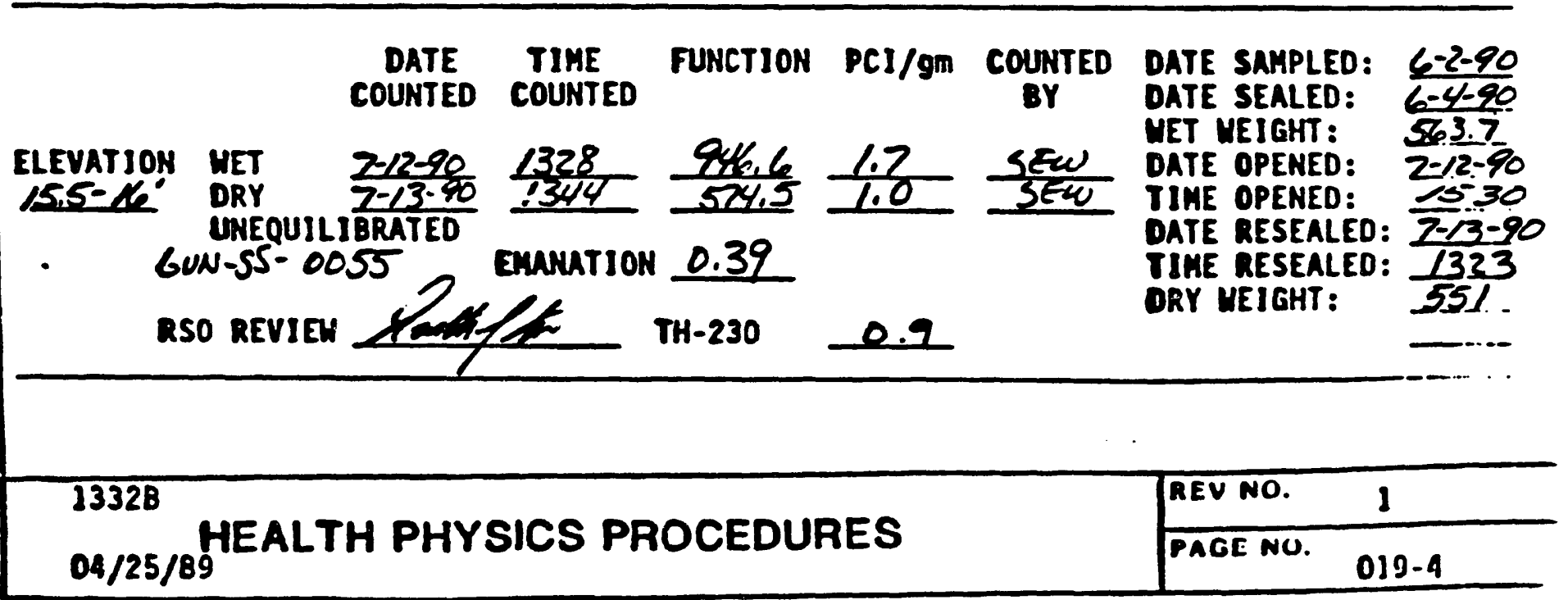




\section{CELL EMHATION SAMLIMG LOG}

PROJECT/SITE NAME: UMTRA /GUNNISON

GRID COORDINTES:

$T-7$

\section{DATE TIME - FUNCTION PCI/gI COUNTED COUNTED COUNTED}

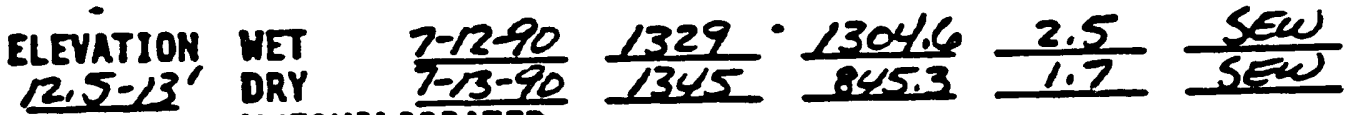

UNEQUILIBRATEO

SAMPLE TIT: GUN-SS-0.56 ENANTION 0.35

RSO REYIEH

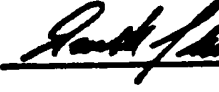

TH-230
69
DATE SAMPLED: $6-2-90$ TIME SAMPLED: DATE SEALED: TIME SEALED: UET NEIGHT: DATE OPENET: TIME OPENED: DATE RESEALED: TIME RESEALED: DRY WEIGHT: $\frac{-1-90}{6-4 / 20}$

1500 $\frac{1523.8}{523}$ $7-12-90$ 1530 $2-13-90$ 1323

\section{DATE TIME FUNCTIOH PCI/gm COUNTED COUNTED COUNTED BY} ELEVATION MET $\frac{7-12-90}{7-13-93.5} \frac{1337}{1352} \frac{3689.6}{2272.0} \frac{6.9}{4.1} \frac{\text { SEW }}{\text { SEW }}$ UNEQUILIBRATED EMANATION 0.38 GUN-SS-0OST Eranate RSO REVIEH

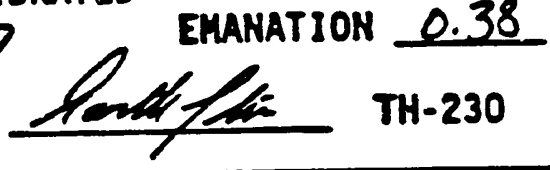

92

\section{DATE TIME COUNTED COUNTED \\ COUNTED BY}

ELEVATIOH MET $13,5-14,5^{\circ}$ DRY UNEQUILIBRATED

GUN-SS-0058 RSO REVIEH

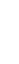
TH-230

\section{Emanation 0.34} $\frac{1338}{1352}$ 32 SEU

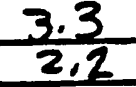
2,2

\section{DATE TIME FUNCTION PCI/gM COUNTED
COUNTED COUNTED}

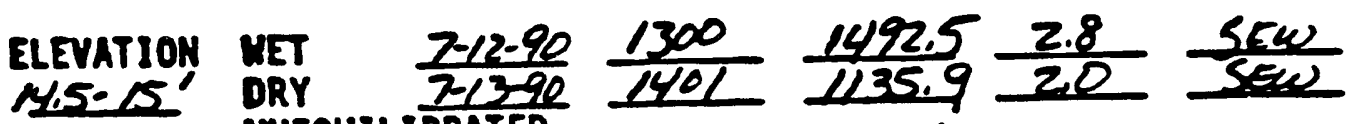
GUN-SS-0059 EMHATION 0.24 
CELL EMNATIOH SAMPLING LOG

PROJECT/SITE HAHE:

GRID COORDINATES:

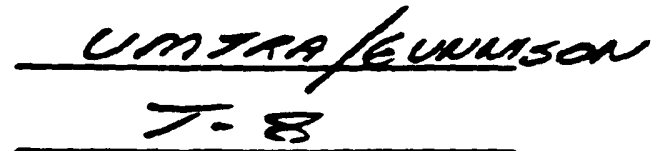

$-$

DATE TIME
COUNTED COUNTED

ELEVATION MET

$G->$ ORY $\frac{7-292}{2-13-90} \frac{1302}{1402} \frac{19192}{1168.2} \frac{2.8}{1.8} \frac{5 E W}{5 E W}$

SAMPLE ID: ONW-ES-0OSO ENANATION 0.39

RSO REVIEU

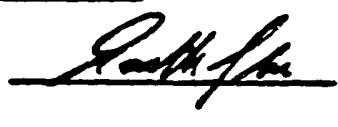

TH-230

140

DATE SAMPLED: $6-\angle-50$

TIME SAMPLED:

DATE SEALED:

TIME SEALED:

UET NEIGH: :

DATE OPENED:

TIME OPENED:

$\overline{E-1-96}$

DATE RESEA' T: $\frac{2-13-90}{2334}$

TIME RESEALCD: 1334 DRY MEIGHT:

\section{DATE TIME FUNCTION PCI/gM COUNTED COUNTED COUNTED \\ BY}

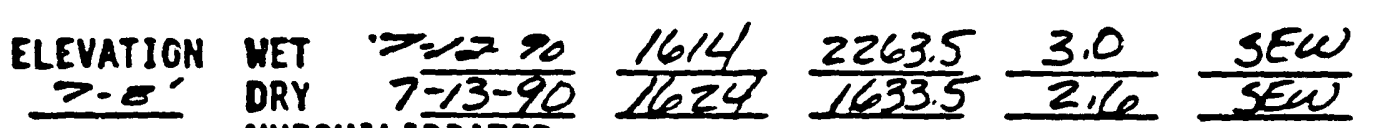
UNEQUILIBRATED GUN.S5-00\%3 EMANATION 0.28

RSO REVIEH
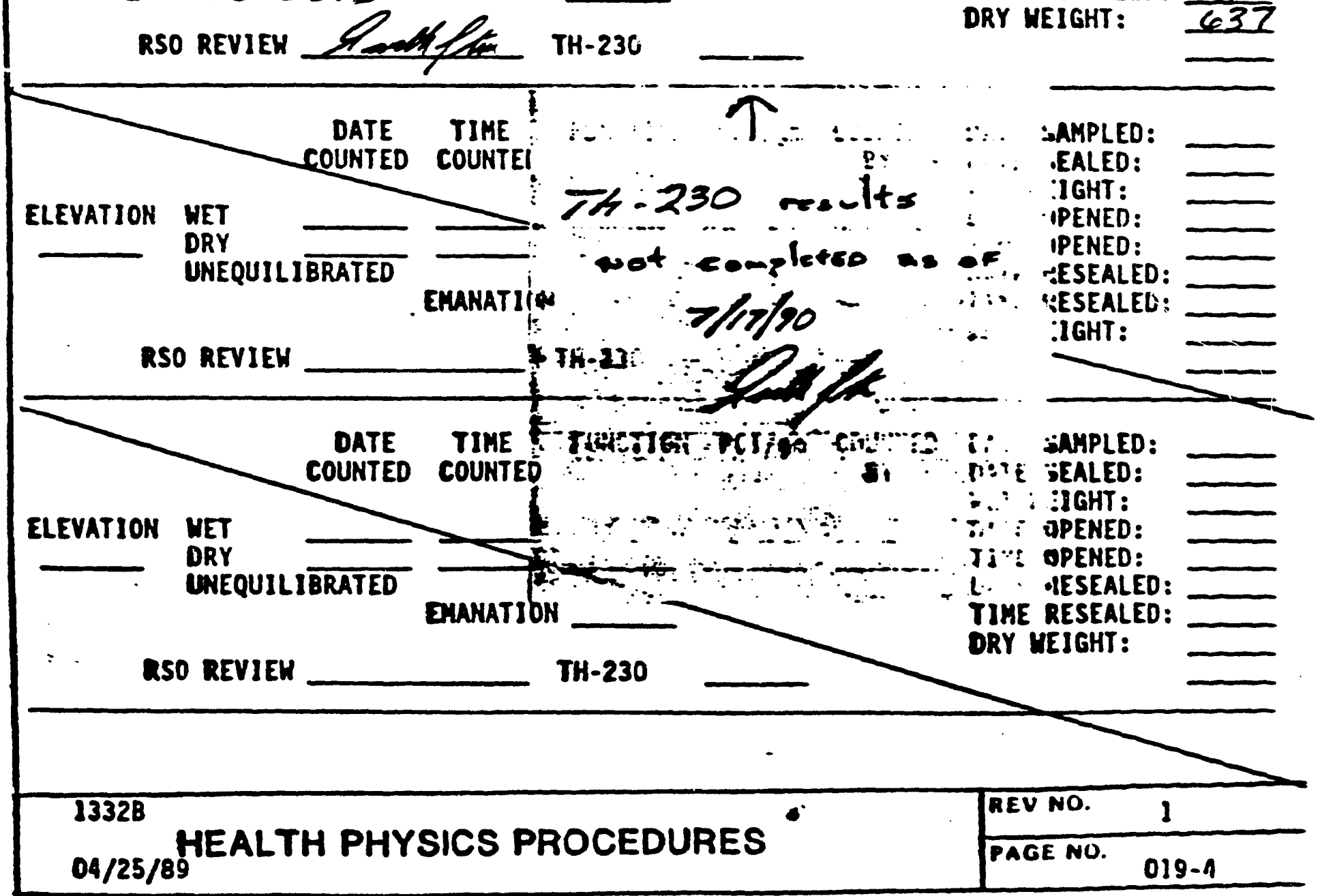

DATE SAMPLED: $\frac{6-1-90}{6 \cdot 15-90}$ HET MEIGHT: 765.35 DATE OPENED: TIME OPENED: $\angle 545$ DATE RESEALED: $7-13-90$ TIME RESEALED: 1502 DRY MEIGHT: 1232 


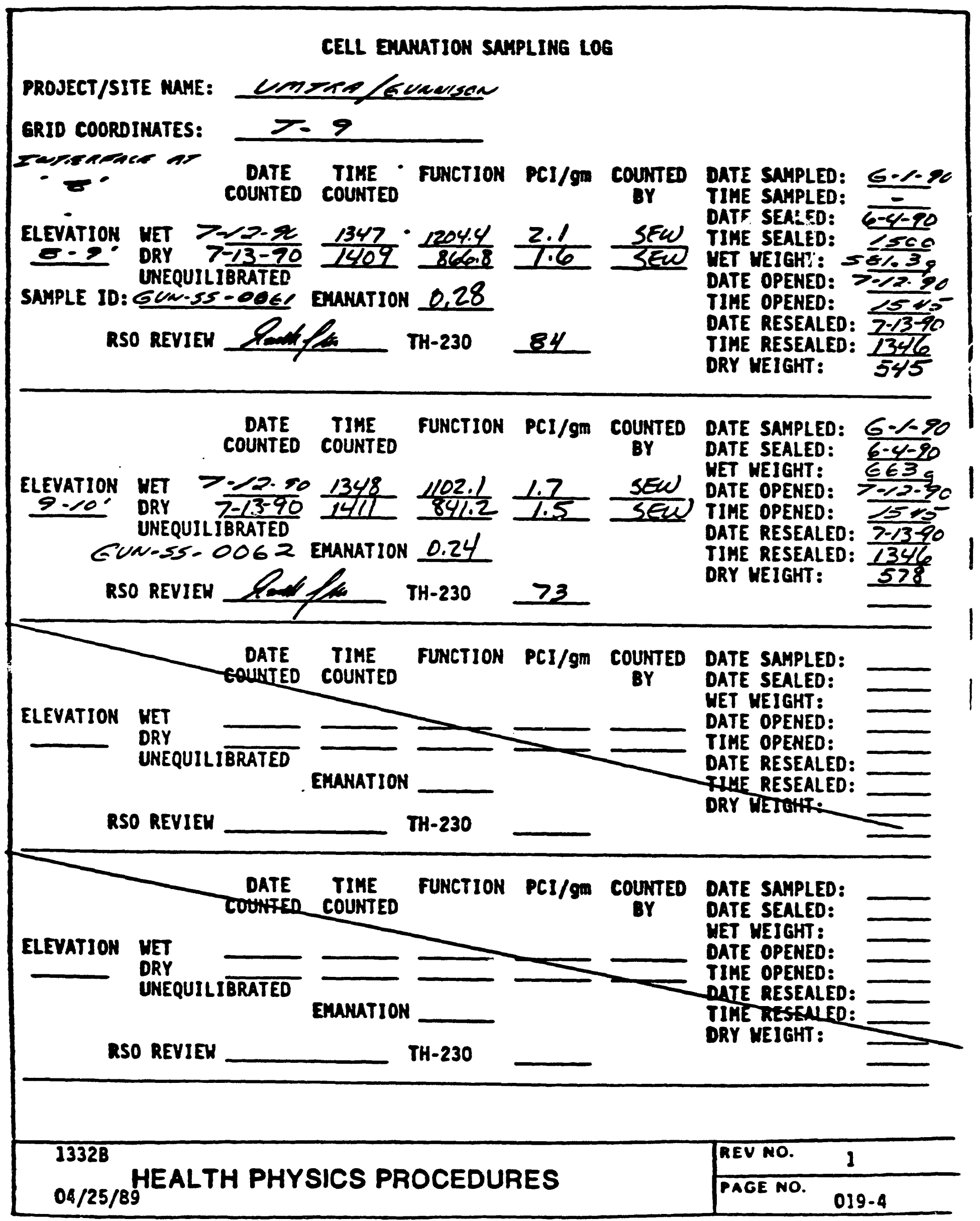




\section{CELL EMHATION SAMPLING 106}

PROJECT/SITE MNE: UNTTP/EuNerSON

GRID COORDINATES: $\quad Z=$

Zycorater at DATE TIME

$r=-1$ COUNTED COUNTED

ELEVATION WET $7 \frac{-17.80}{7-13-90} \frac{1404}{1478} \frac{1487.7}{1035.0} \frac{2.7}{1.7} \frac{\text { SEW }}{\text { SEW }}$

UNEQUILIBRATED

EMANATION 0.30

RSO REVIEH Rearfas TH-230 99

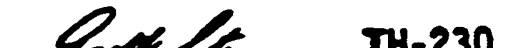

RSO REVIEU Sompla TH-230

COUNTED OATE SAMPLED: $6 \cdot 2.70$

TIME SAMPLED:

DATE SEALED: $\overline{6-4-90}$

TIHE SEALE: 1500

SAMPLE ID: EGN-S5-OCG EMAHATIOH

MET MEIGHT: $5-7.6$ *

DATE OPENED: $>-1-2-20$

TIME OPENED: $15 \% 5-$

DATE RESEALED: $7-13-9$

TIME RESEALED: $\frac{1346}{108}$

DRY MEIGHT:

\section{DATE TIME FUNCTION PCI/gI COUNTED COUNTED COUNTED} ELEVATION WET $\frac{7-20}{7-13-90} \frac{1406}{1419} \frac{1845.8}{1098.5} \frac{2.9}{1.7} \frac{\text { SEW }}{\text { SEW }}$ UNEQUILIBRATED

GUN-SS-OOEY EMANATION 0.41

RSO REVIEH

\section{TH-230}

103

\section{DATE TIME FUNCTION PCI/gI COUNTED COUNTED COUNTED}

ELEVATION MET $\frac{7-2.8}{7-13.90} \frac{14 / 3}{1427} \frac{425.2}{542.0} \frac{0.7}{0.9} \frac{\text { SEW }}{\text { SEW }}$ G UN-SS.0065 EMANATION -0.28

RSO REVIEH

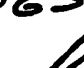

\section{7}

\author{
Lat
}

FUMCTION PCI/gm

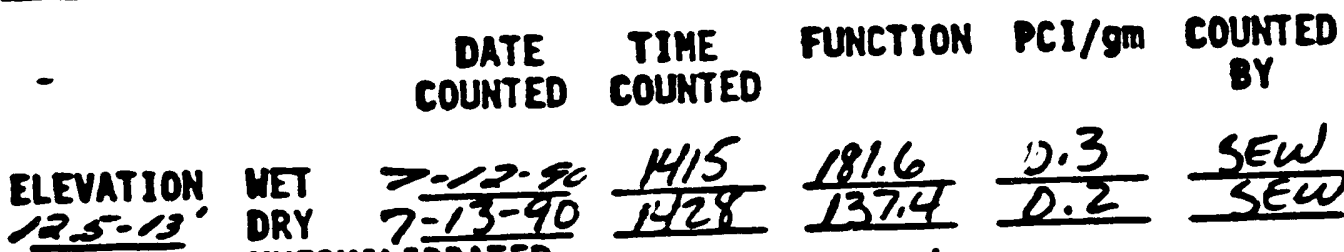

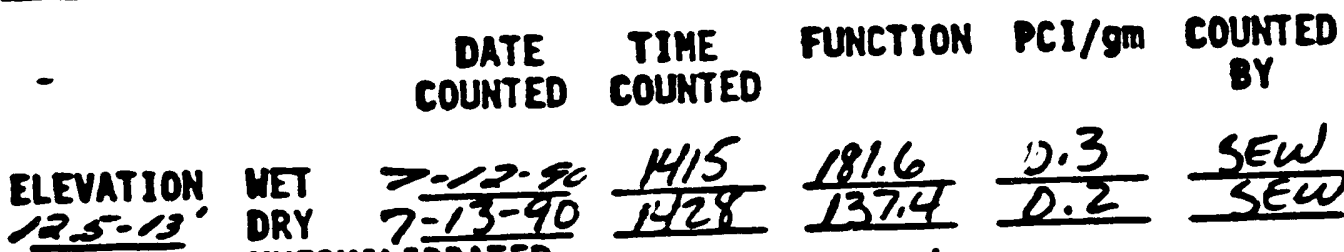
UNEQUILIBRATED

Gun-s5-0066

EMNATION 0,24

RSO REVIEH

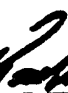

TH-230
5.2
DATE SAMPLED: $6-2-20$ DATE SEALED: $6-4-90$ MET VEIGHT: 633.3 . DATE OPENED: TTIME OPENED: $15<5$ DATE RESEALED: $7 / 3-90$ TIME RESEALED: DRY MEISHT:
DATE SAMPLED: $6-2.20$ DATE SEALED: $\frac{1-4-90}{6}$ MET UEIGHT: 600.79 DATE OPENED: 72.40 TIME OPENED: DATE RESEALED: $z \cdot 13-90$ TIME RESEALED: DRY UEIGHT:
04/25/89 ${ }_{\text {1332B }}^{\text {HEALTH PHYSICS PROCEDURES }}$
DATE SAMPLED: $\angle-2.80$ DATE SEALED: $6-5-20$ MET MEIGHT: E\$2, DATE OPENED: $\frac{1}{2-12.90}$ TIHE OPENED: $\frac{15-15}{7-13-90}$ OATE RESEALED: $\frac{7-13-90}{1359}$ TIME RESEALED: ORY NEIGHT: 
CELL EMUTIOH SAPLING LOG

PROJECT/SITE MME: UMTRA/GUNNISON

GRID COORDINATES: $T-1 /$

$=$

DATE TIHE - FLNCTION PCI/gm COUNTED COUTTE COUNTED BY

DITE SNMPLED:

DATE SEALED:

ELEYATION WET

$\frac{7-17-90}{\frac{7-13-90}{1422}} \cdot \frac{5934.7}{14349}-\frac{9.4}{7.2}$ SEW TIME SEALED:

D-L̈ DRY

UNEQUILIBRATED

SEW

UET NEIGHT:

DATE OPENED:

TIME OPENED:

DATE RESEALED:

RSO REVIEH

2

TH-230

21

TIME RESEALED:

DRY UEIGHT:

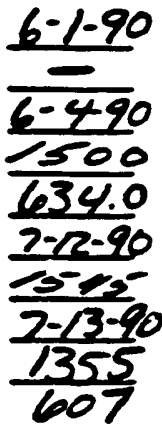

\section{DATE TIME FUNCTION PCI/gm COUNTED COUNTED COUNTED \\ BY}

ELEVATION MET $1-2^{\prime}$

\section{$\frac{7-12-90}{7-13-40} \frac{1423}{1437}$}

$\frac{1944.9}{1699.2}$

$\frac{3.3}{3.1}$ UNEQUILIBRATED GUN-SS-0068 EMNATION 0.13

RSO REVIEY

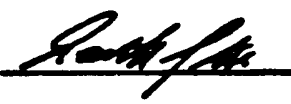
TH-230

3.0
DATE SAMPLED: $\quad 6-190$

DATE SEALED: $6-4.90$ HET MEIGHT: 592.2

DATE O:ENED: $7-12.90$ TIME OPENED: $\frac{2-129}{7-135}$ DATE RESEALED: $7-13-20$ TIME RESEALED: DRY MEIGHT:

$\begin{array}{cccc}\text { DATE } & \text { TIME } \\ \text { COUNTED COUNTED } & \text { FUNCTION PCI/gM COUNTED } \\ B Y & \end{array}$

ELEVATION HET $2-3^{\prime}$ $\frac{7-12-90}{7-13-90} \frac{1431}{1440}$ UNEQUILIBRATED GUN-SS-0069 EMANTION 0.22

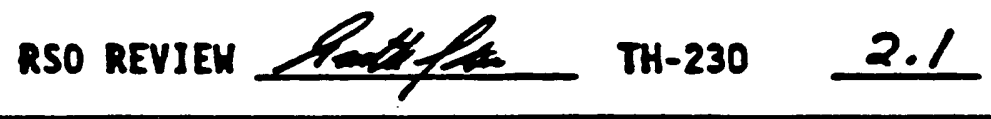

\section{DATE TIME COUNTED COUNTEO \\ FUNCTION PCI/gm \\ $\operatorname{MRT}_{\text {DRY }} \frac{7-12-90}{7-13-90} \frac{1432}{1445} \frac{2525.8}{1731.6} \frac{4.0}{2.6} \frac{\text { SEW }}{\text { SEW }}$}

3LY UNEQUILIBRATED GUN-SS-0070 Euntion 0.31 RSO REVIEX TH-230 68
DATE SAMPLED: $\quad 6-1-.70$ DATE SEALED: $6.4 \cdot 90$ WET HEIGHT: $\frac{614.9}{7 \%}$ DATE OPENED: $7-12-90$ TIME OPENED: $\frac{7}{5 / 25}$ DATE RESEALED: $\overline{z-13-90}$ TIHE RESEALED: DRY MEIGHT $\frac{1403}{1655}$ 
CELL ENHTION SNMPLING 106

PROJECT/SITE HAE: UMTPA/LUNNISON

CRID COOROINATES:

$T-11$

DATE TIME - FHCTION COUNTED COUNTED

PCI/gin $\frac{7-12-99}{2-13-90} \frac{1439}{1452} \cdot \frac{165.6}{811.0} \frac{2.5}{1.2} \frac{\text { sew }}{\text { sen }}$ $4=5$

WET DRY ONEQUILIBRATED SAMPLE ID: GUN-SS-0OZL EMAMATION 0.50

RSO REVIEH

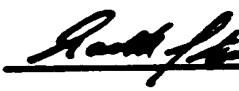

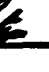
TH-230

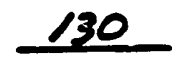

COUNTED BY
DATE SIYPLED: $6-1-90$ DATE SEALED: TIHE SEALED: 1500 NET NEIGHT: 639.9 is DATE OPENED: $\quad 7-12-90$ TIME OPENED: $\mathrm{C}$ DATE RESEALED: $7-13-0$ TIME RESEALED: 14103 DRY NEIGHT: 676

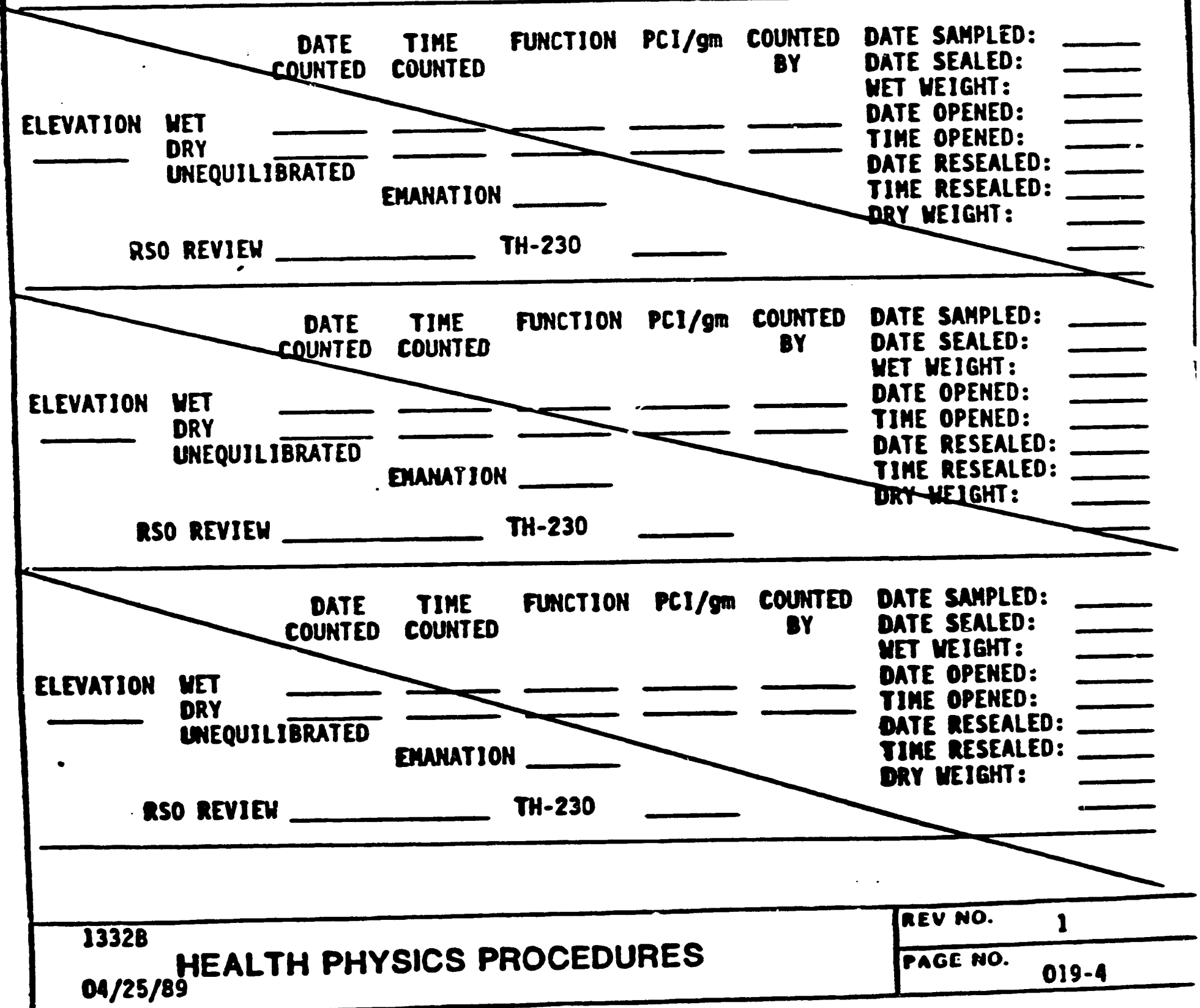


PROJECT/SITE MAME: UMTRA / GUNNISON

GRID COORDINATES:

$T-12$

DATE TIHE - FUNCTION PCI/gm COUNTED DATE SAMPLED: COUNTED COUNTED TI TIME SAMPLED: ELEVATION MET $\frac{7-12-90}{2-13-90} \frac{1441}{1454} \frac{13880}{97349} \frac{24.0}{18.1}$ SEW DATE SEALED: UNEQUILIBRATED TIME SEALED: MET MEIGHT: DATE OPENED: SAMPLE ID: GUN-S5-0072 EMANATION 0.30 RSO REVIEW Rewfla TH-230 14 DIME OPEHED: $\frac{1-4 s}{7-13-90}$ TIME RESEALED: 1403 DRY MEIGHT:

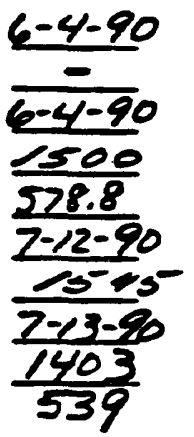

\section{DATE TIME FUNCTION PCI/gm COUNTED COUNTED COUNTED \\ BY}

ELEVATION, MET $1-1,25$ $\frac{7-12-90}{7-13-90} \frac{1448}{1501}$ UNEQUILIBRATED 6UN-5S.0073 ERANATION 0,28

RSO REVIEW

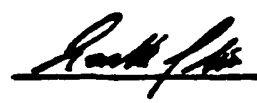

TH-230 II
DATE SAMPLED: DATE SEALED: MET MEIGHT: DATE OPENED: TIME OPENED: DATE RESEALED: TIME RESEALED: DRY MEIGHT:

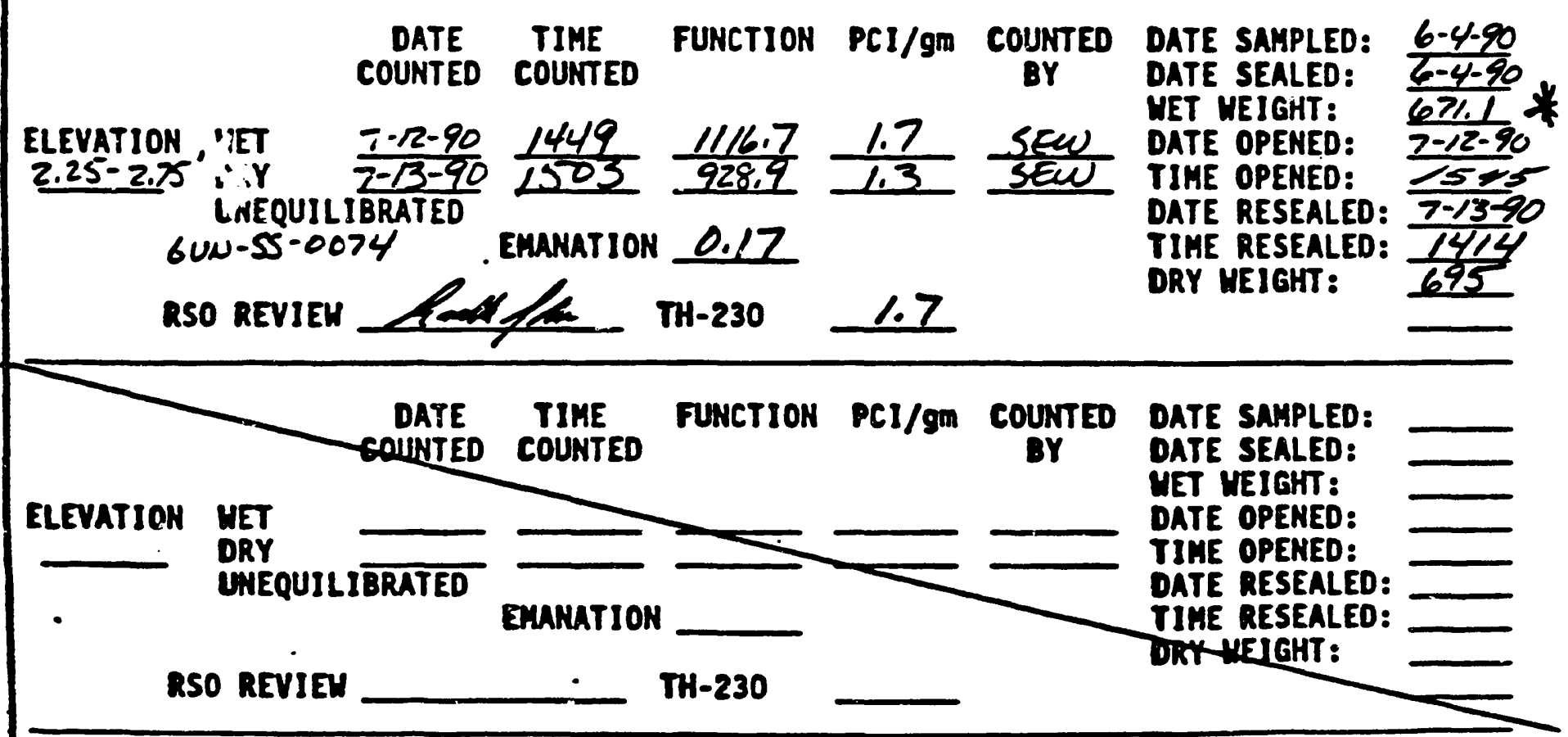

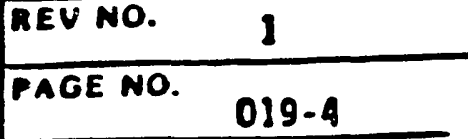


CELL EHWAT:SN SAMPLIHG LOG

PROJECT/SITE MHE: UMTRA/LUNNISON

GRID COORDINATES:

$$
T-13
$$$$
=
$$

DATE TIME - FUHCTION PCI/gI COUNTED COUNTED ELEVATION MET $\frac{7-12-90}{7-1340} \frac{0810}{0824} \cdot \frac{63629}{44157} \frac{121.2}{79.9} \frac{\text { SEW }}{3520}$ UNEQUILIBRATED

SAMPLE ID: GUN-SS-0006 EWATATION 0.31

COUATED or
Rour tas

DATE TIME FUNCTION COUNTED COUNTED

ELEVATION MET $1-2^{\prime}$ RSO REVIEW

\section{7-12-90}

TH-230

$6^{2}$ 
CELL EMAHATION SAMPLING LOG

PROJECT/ ITE MANE:

GRID COORDINATES:

$\frac{\text { Unotare / Gunarson }}{7-13}$

$=$

DATE TIME
COUNTED COUNTED

\section{ELEYATION UET \\ $y-5^{\prime}$ \\ DRY}

\section{Teles 0828.}

$\frac{212 / 40}{7-13-90}$

$\frac{0828}{0841}$

$\frac{2691.7}{2340.6}$

UNEQUILIBRATED

IMANATION 0.13

SAMPLE ID: $6 U N-35-\infty 010$

RSO REVIEH

Rompere

TH-230

17

DATE SAMPLED:

TIME SAKPLED:

DRTE SEALED:

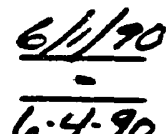

SEW TIME SEALED:

UET MEIGHT:

DATE OPENED:

TIME OPENED:

$\frac{6.4-20}{19-20}$ SEw

$\bullet$ DATE RESEALED: TIME RESEALED: ORY HEIGHT:
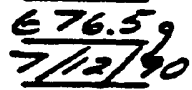

$15 \equiv 0$

$2-3-90$

$08, \overline{5}$

638

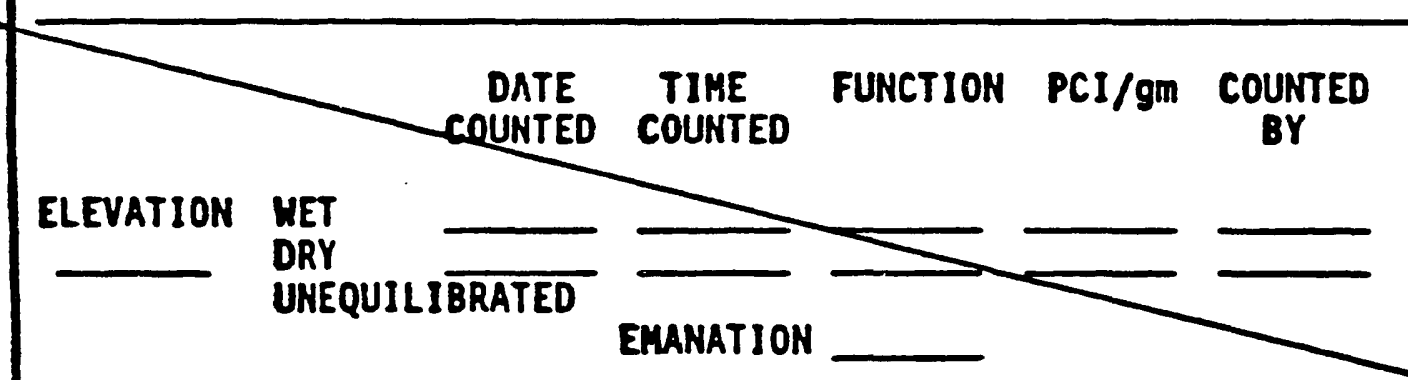

RSO REVIEU

TH-230

DATE SAMPLED:

DATE SEALED:

MET WEIGHT:

DATE OPENED:

TIME OPENED:

DATE RESEALED:

TIME RESEALED:

OAK NEIGHT:
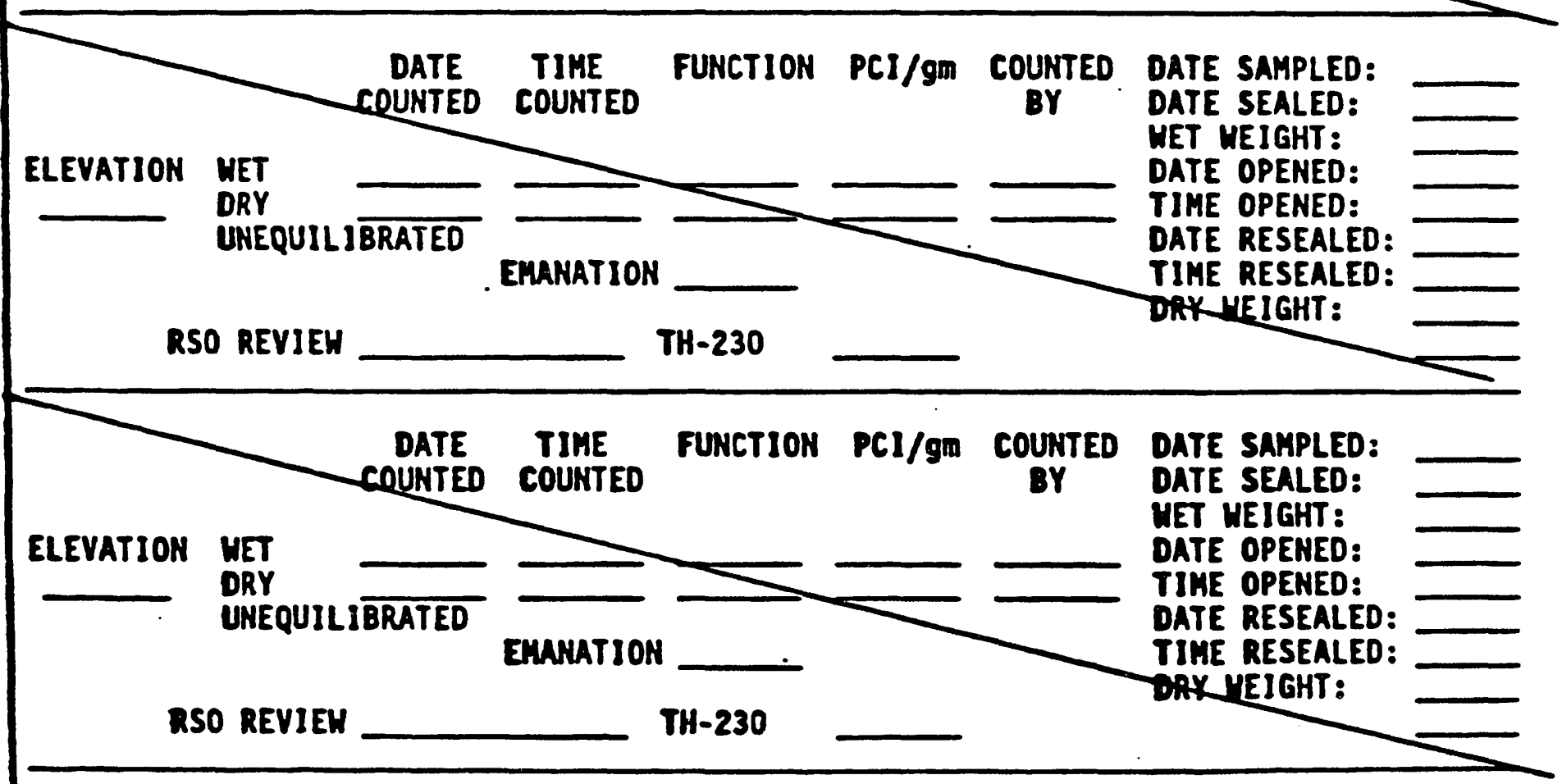

1332B

HEALTH PHYSICS PROCEDURES

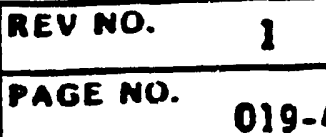


CELL EMAKATIOH SAMPLING LOG

PROJECT/SITE MAME: UMTRA/GUNNESON

GRIO COORDIKATES:

$T-14$

DATE TIME - FUNCTION PCI/gm COUNTED DATE SAMPLED: 6-4-90 COUNTED COUNTED BY TIME SAMPLED:

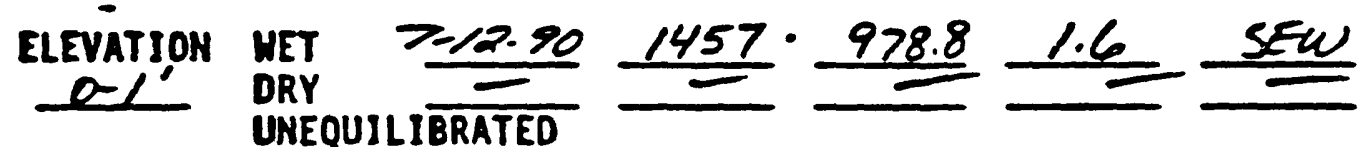

SAMPLE 10: GUN-SS-0075

EMANATION -

DATE SEALED:

TIME SEALED:

WET MEIGIT:

DATE OPENED:

TIME OPENED:

$\frac{-}{6-4-90}$

1500.

614

$7-12.90$

DATE RESEALED: $7-1390$

RSO REVIEH LOTH TH-230 2.0

* Failed leak test

TIME RESEALED:

DRY MEIGHT:

DATE
COUNTED COUNTED FUNCTION PCI/gm $\underset{B Y}{\text { COUNTED }}$

DATE SAMPLED: $6-490$ DATE SEALED: $\frac{6-4-90}{9-9}$

ELEVATION MET
$1-117$ DRY $\frac{-12-90}{7-13-90} \frac{1458}{1510} \frac{547.7}{762.0} \frac{0.7}{1.1} \frac{\text { SEW }}{5 E W}$ WET MEIGHT:

TIME OPENED: Y- 2.90

DATE RESEALED: $\frac{1540}{213-90}$

UNEQUILIBRATED
GUN-SS-0O>6 EMANATION -0.39

TIME RESEALED:

RSO REVIEH Hepte TH-230 0.7 DRY MEIGHT:
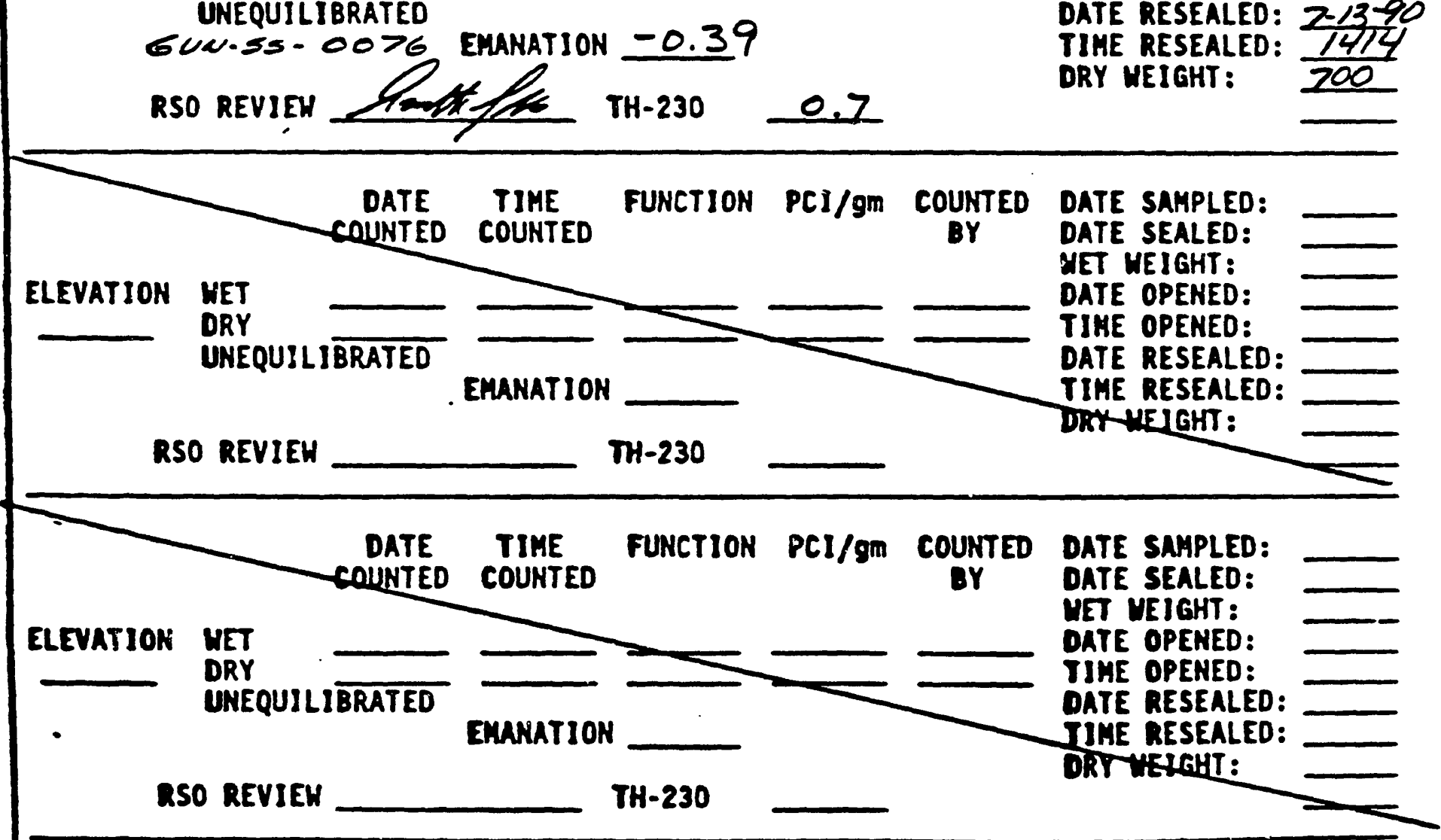

13328

04/25/89

HEALTH PHYSICS PROCEDURES

REV NO. 1

PAGE No. O19-4 


\section{CELL EMANATION SAMPLING LOG}

PROJECT/SITE NAME:

GRID COORDINATES:

$\frac{\text { Um ene / Gunwison }}{2=13}$

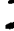

DATE TIME - FUHCTION PCI/gM COUNTED COUNTED COUNTED BY

ELEYATIO o-r

\section{MET} $7-12-90$ UNEQUILIBRATED

SAMPLE 10: GUN-SS-00/1 EMANATION 0.36 RSO REVIEH

SEW SEW

\section{DATE TIME COUNTED COUNTED}

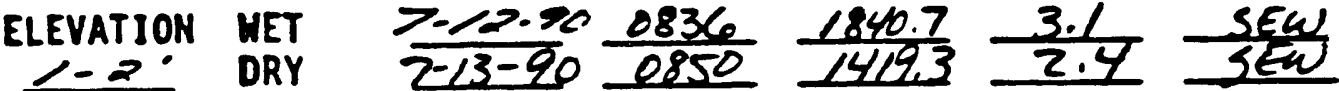

\section{UNEQUILIBRATED} GUN-SS-0012 EMANATION 0.23

RSO REVIEH

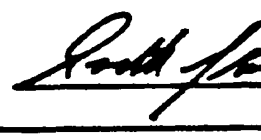

COUNTED BY

\section{DATE TIME COUNTED COUNTED}

ELEVATION WET $\frac{7-2.90}{7-13.90} \frac{0853}{0858} \frac{1079.6}{438.6} \frac{2.0}{0.8} \frac{\text { SEW }}{\text { SEW }}$ UNEQUILIBRATED GUN-5S-0013 EMANATION 0.6

\begin{abstract}
3
\end{abstract}

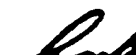

RSO REVIEH

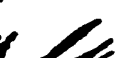
TH-230

86 
CELL EMAKATION SAMPLING LOG
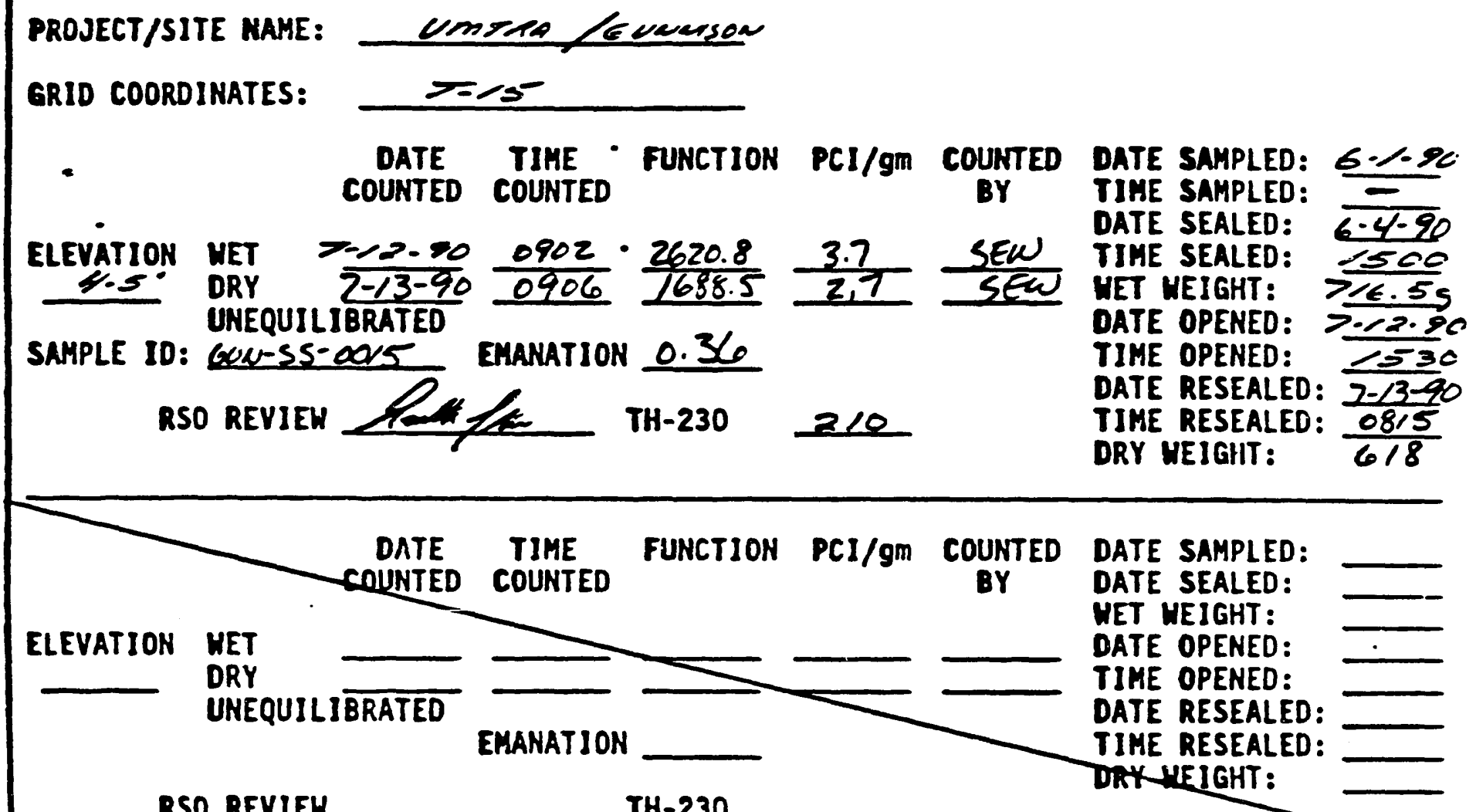

RSO REVIEH

TH-230

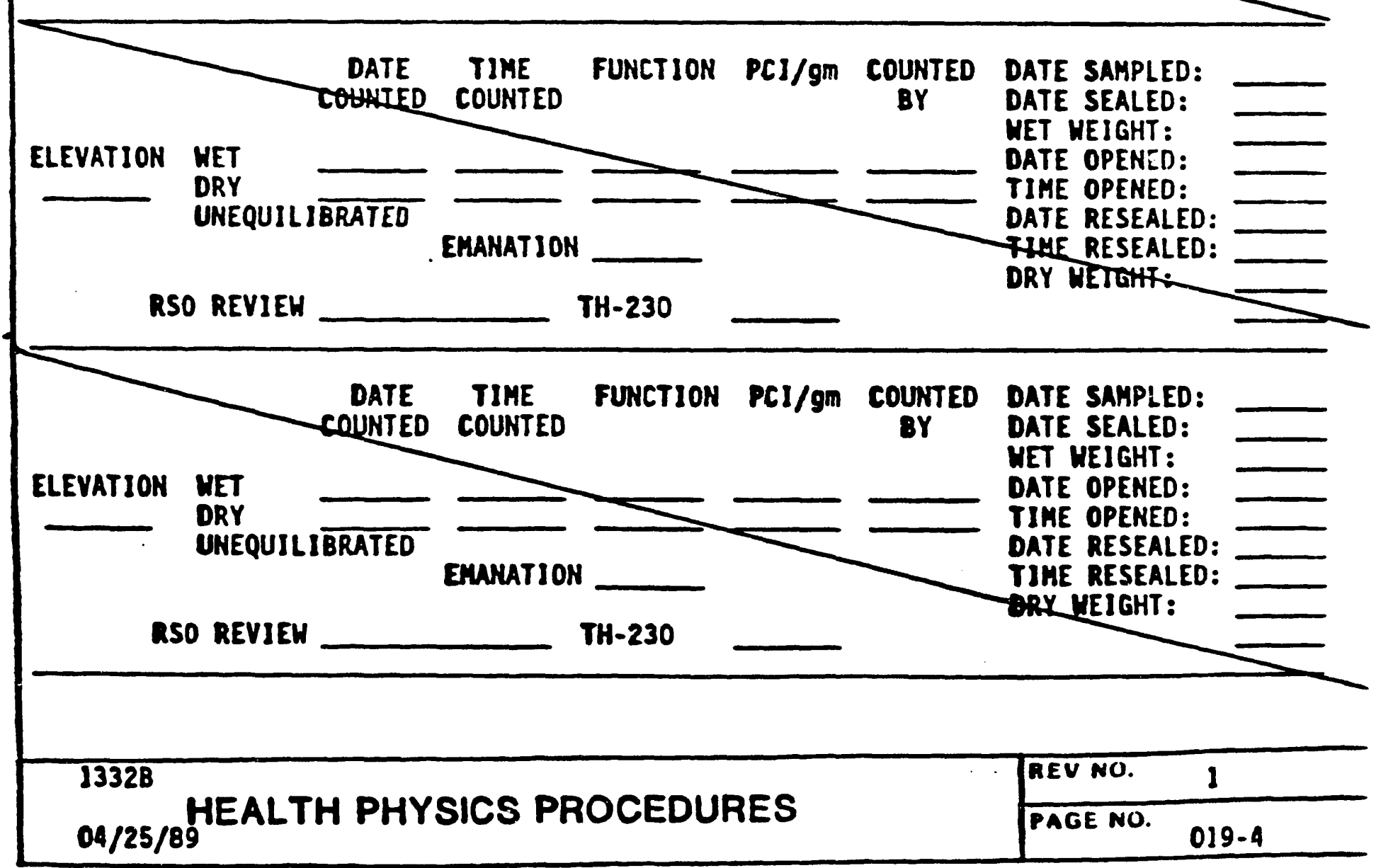




\section{PROJECT/SITE MAME: OMTTA /EUAUSON \\ GRID COORDIHATES:} $=$

\section{DATE TIME - FUNCTION PCI/gm COUNTED COUNTED COUNTED $7=13-90$ BY \\ 2.0 7.9 $\frac{\text { SEW }}{\text { SEW }}$}

\section{ELEVATION} MET $7-12.90$ DRY UNEQUILIBRATED

SAMPLE ID: GUN-SS-007T EMANATION 0,13 RSO REVIEH

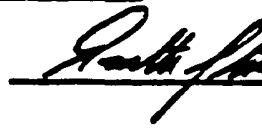
TH-230

2.2

\section{DATE TIME COUNTED COUNTED}

ELEVATION HET $1-2=$ UNEQUILIBRATED RSO REVIEH

\section{Sore Elase \\ .} $\frac{1506}{1519} \frac{771.6}{505.1} \frac{1.2}{0.8} \frac{\text { SEW }}{\text { SEW }}$

0.35
COUNTED BY

TH-230

1.0
DATE SAMPLED: E- $1-90$

TIME SAMPLED:

DATE SEALED:

TIME SEALED:

DATE OPEMED 767.8

TIME OPENED: $15-45$

DATE RESEALED: $7 \cdot 13-90$

TIME RESEALED:

DRY MEIGHT:

$\frac{1414}{538}$

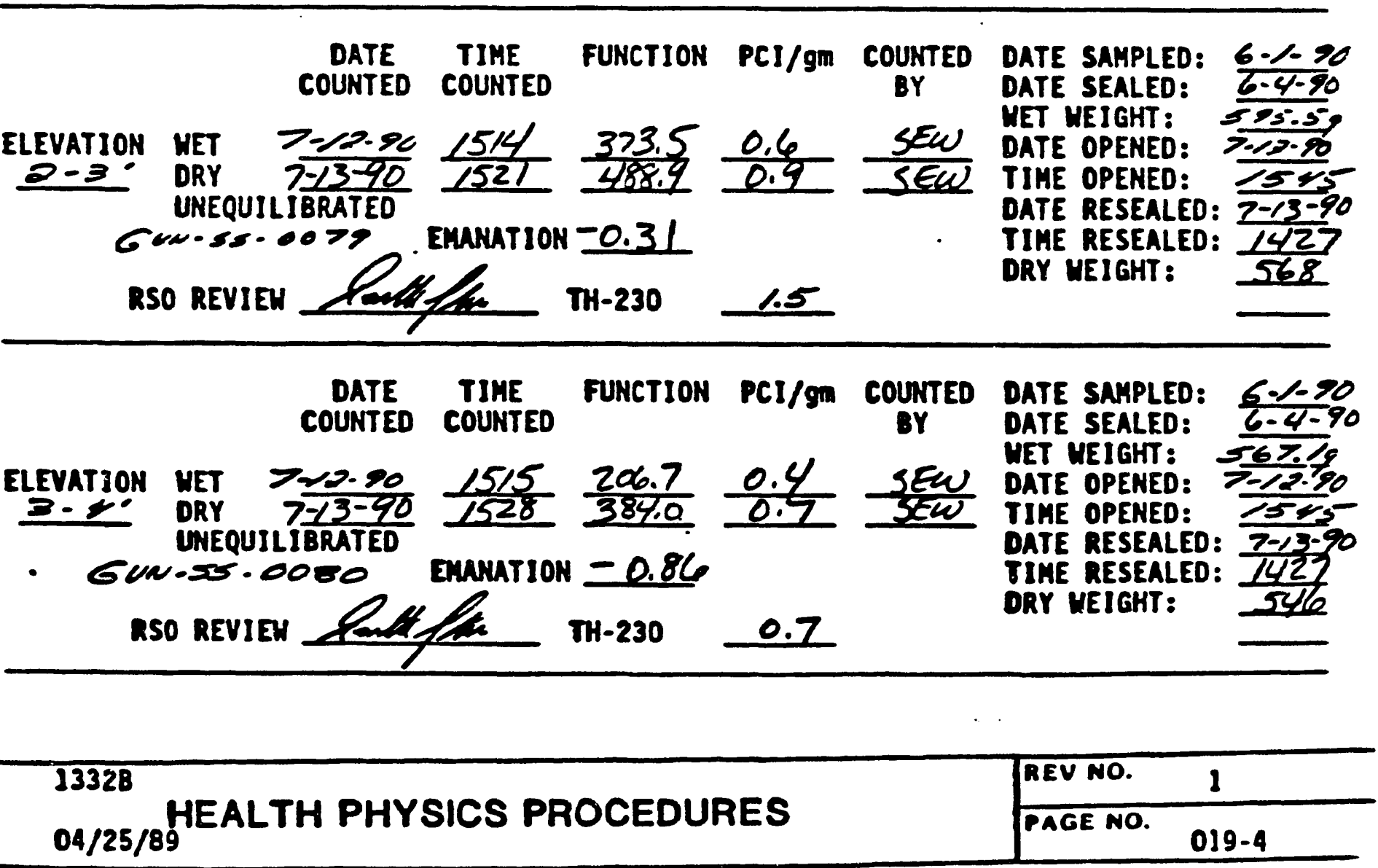


CELL EMANATION SAMPLIHG LOG

PROJECT/SITE MAME: CMERA/GUNNISON

GRID COORDINATES:

$7=16$

DATE TIME - FUNCTION PCI/gm COUNTED DATE SAHPLED: $6-\angle 90$
COUNTED COUNTED

ELEVATION HET $722.90 \frac{1522}{150} \cdot 688.31 .2$ SEC TINE SEALED: 1500 Y-5 ORY $7 \overline{13-90} \frac{1522}{1529} 642.1$ 1.1 SEW MET HEIGIT:

UNEQUILIBRATED

DATE OPENED:

TIME OPENED:

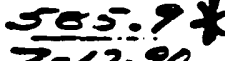

SAMPLE ID: EUN-S5-00EI EMANATION 0.07

RSO REVIEW

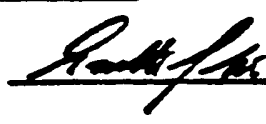

TH-230

7.7

DATE RESEALED: $z-13-90$

$=345$ DRY WEIGHT:

$\frac{1427}{610}$

\section{DATE TIME FUNCTION PCI/gM COUNTED COUNTED COUNTED}

ELEVATION WET $7-\frac{12.90}{7-13-90} \frac{1524}{1536} \frac{1023.8}{423.1} \frac{1.8}{0.7} \frac{\text { SEW }}{\text { SEW }}$ UNEQUILIBRATED

GUN.S5-00E2 EMANATION 0.59

RSO REVIEH

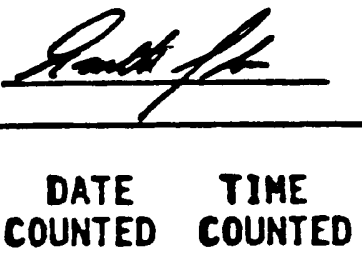

TH-230

77
DATE SAMPLED: 6.1 .90 DATE SEALED: UET WEIGHT: DATE OPENED:

TIHE OPENED:

DATE RESEALED: TIHE RESEALED: DRY MEIGHT:

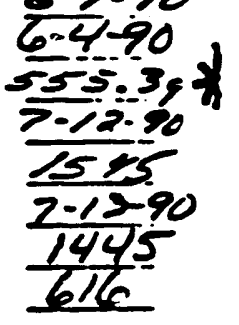

$6=490$ (5. 1585 $2-13-90$ 616

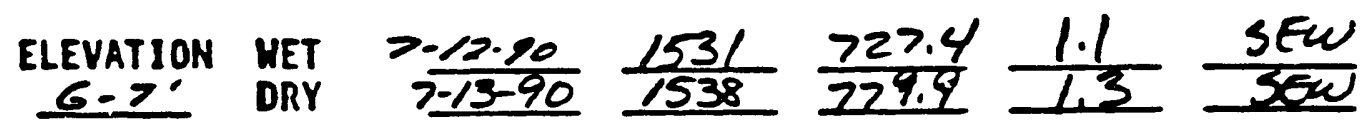
UNEQUILIBRATED GUN-SS-0083 EMANATION $=0.07$

RSO REVIEH fentefe TH-230 58

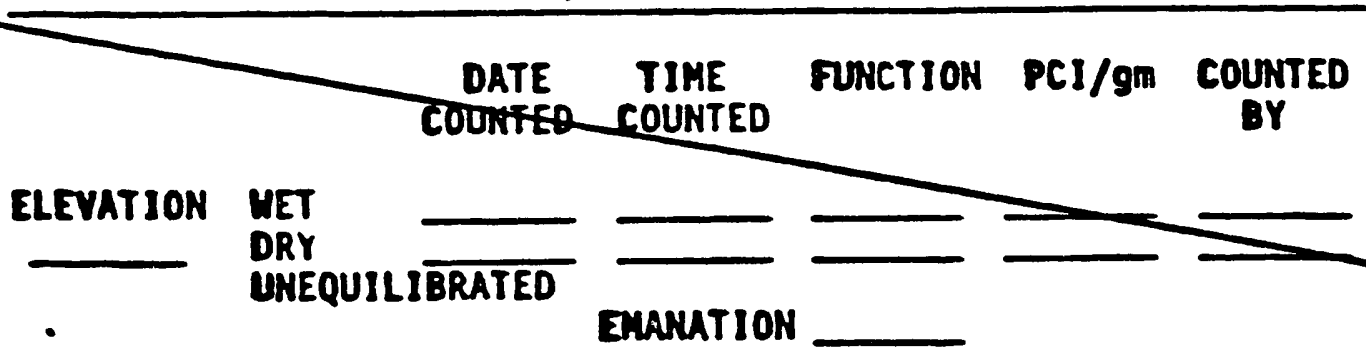

RSO REVIEH

TH-230
DATE SAMPLED:

DATE SEALED:

MET MEIGHT:

DATE OPENED:

TIME OPENED:

DAFE RESEALED:

TIME RESEALED.

DRY MEIGHT:
13328

$04 / 25 / 89$
HEALTH PHY

S PROCEDURES

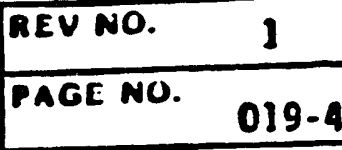


CELL ENAMATION SNMPLING LOG

PROJECT/SITE WAE: UMTRA /GUNALSON

GRID COORDINATES:

$\tau-17$

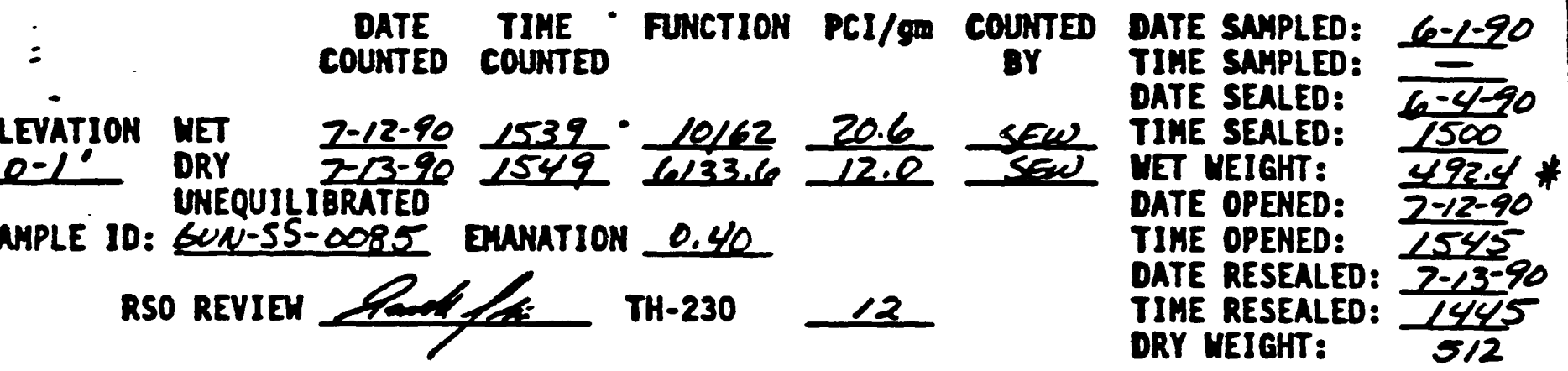

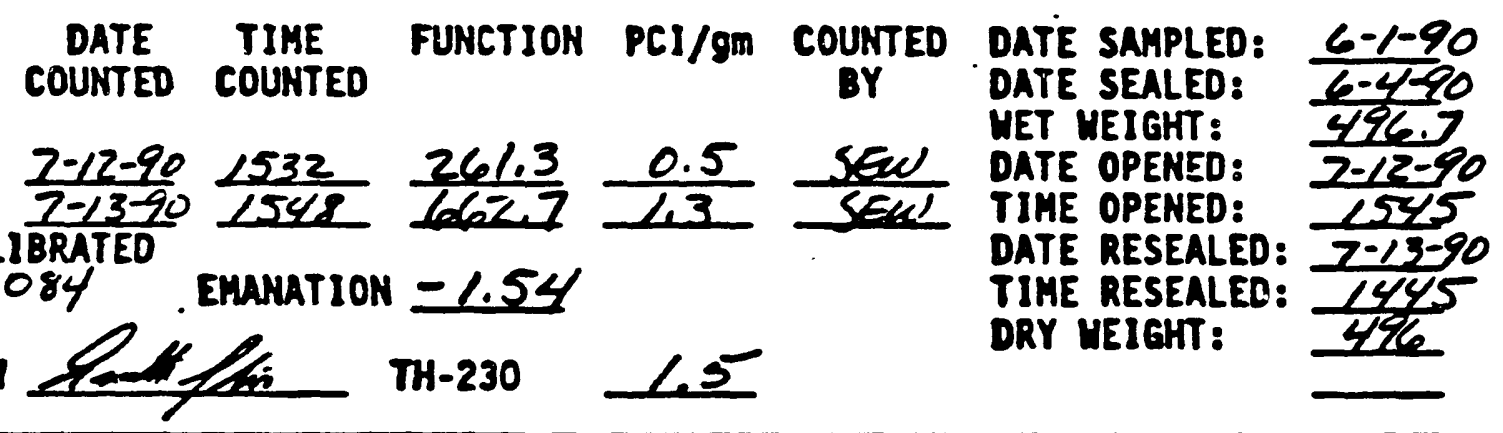

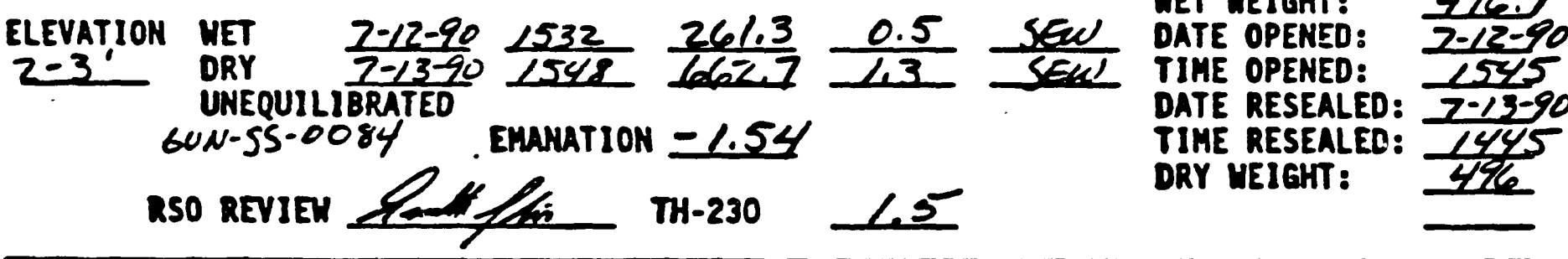

\section{DATE THME FUHCTION PCI/gM COUNTED COUNTED COUNTED}

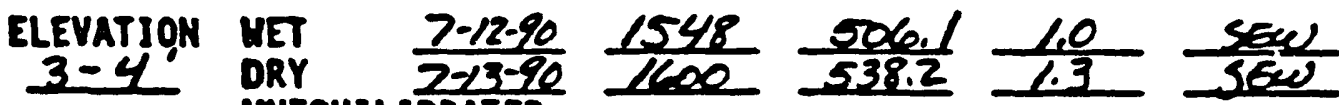
WEQUILIBRATED 6UN-SS-0087 EMHATION $=0.06$ RSO REVIEN

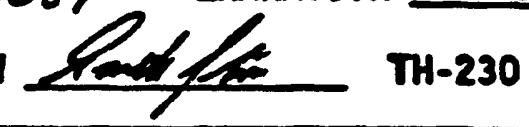
0.9
DATE SAMPLED: $6-1-90$ DATE SEALED: $6-C-q O$ WET VEIGHT: 5E5.6 DATE OPENED: $\quad z-12-90$ TIME OPENED: 1545 DATE RESEALED: $7-13-90$ TIME RESEALED: ORY UEIGHT: I/S 


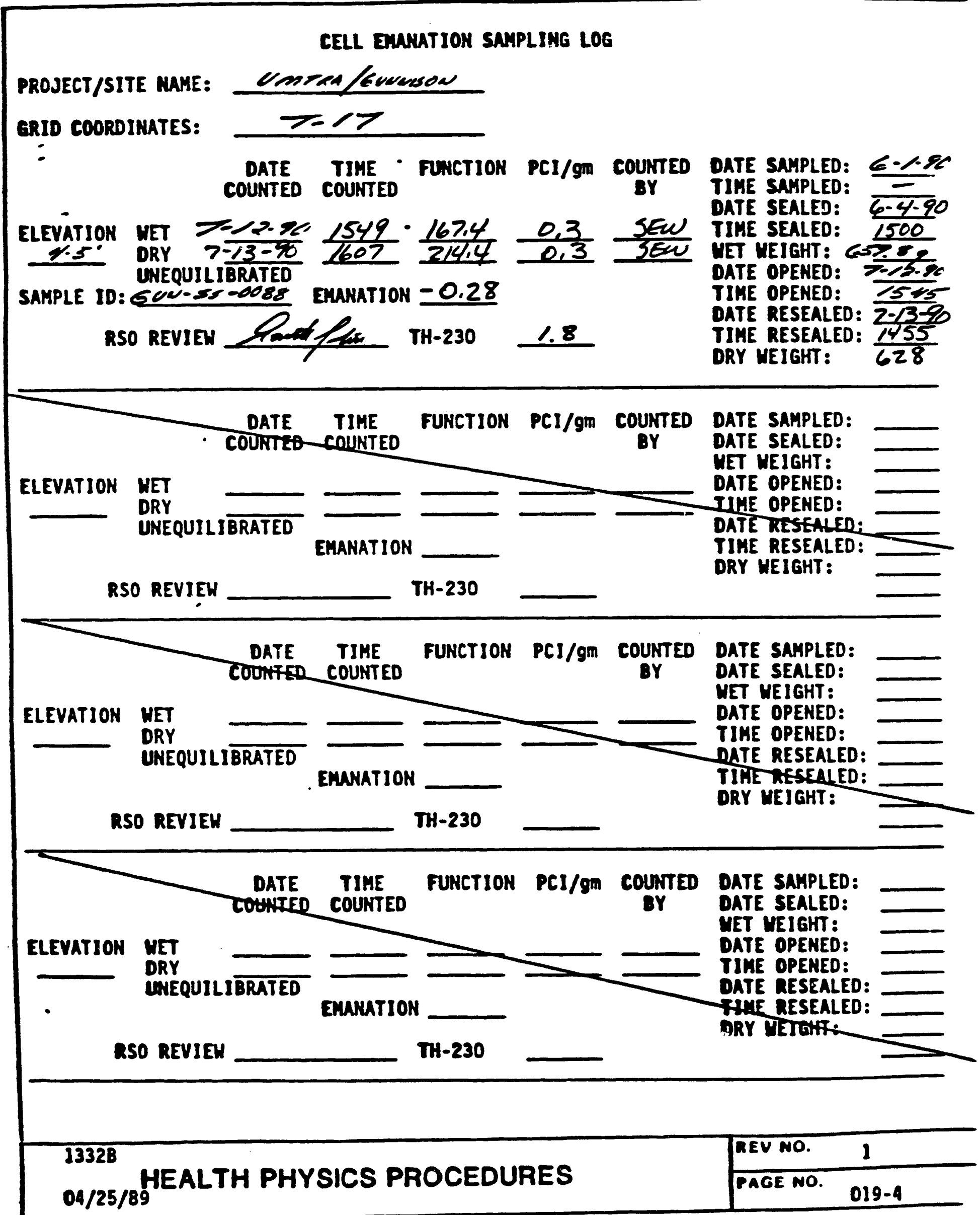




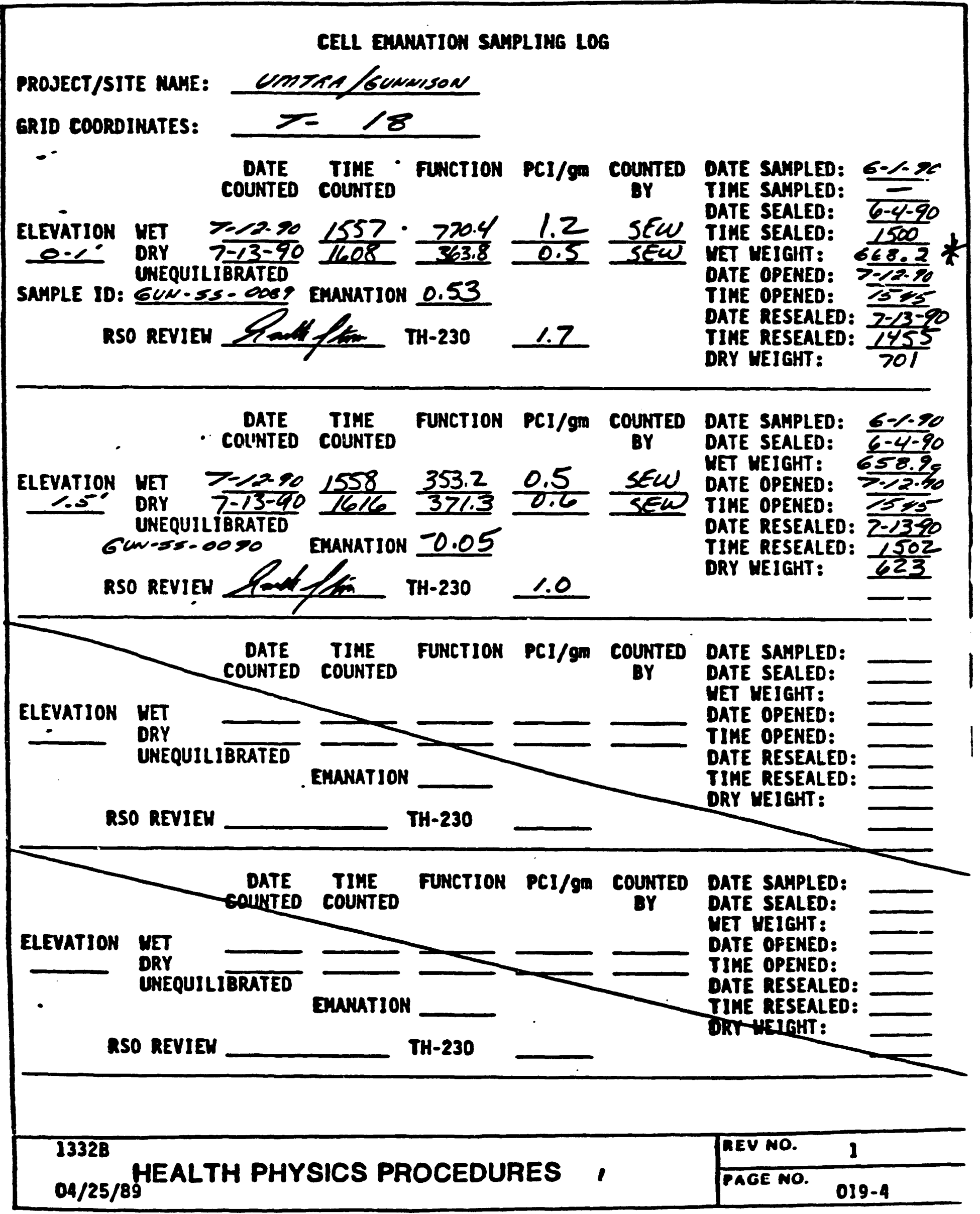




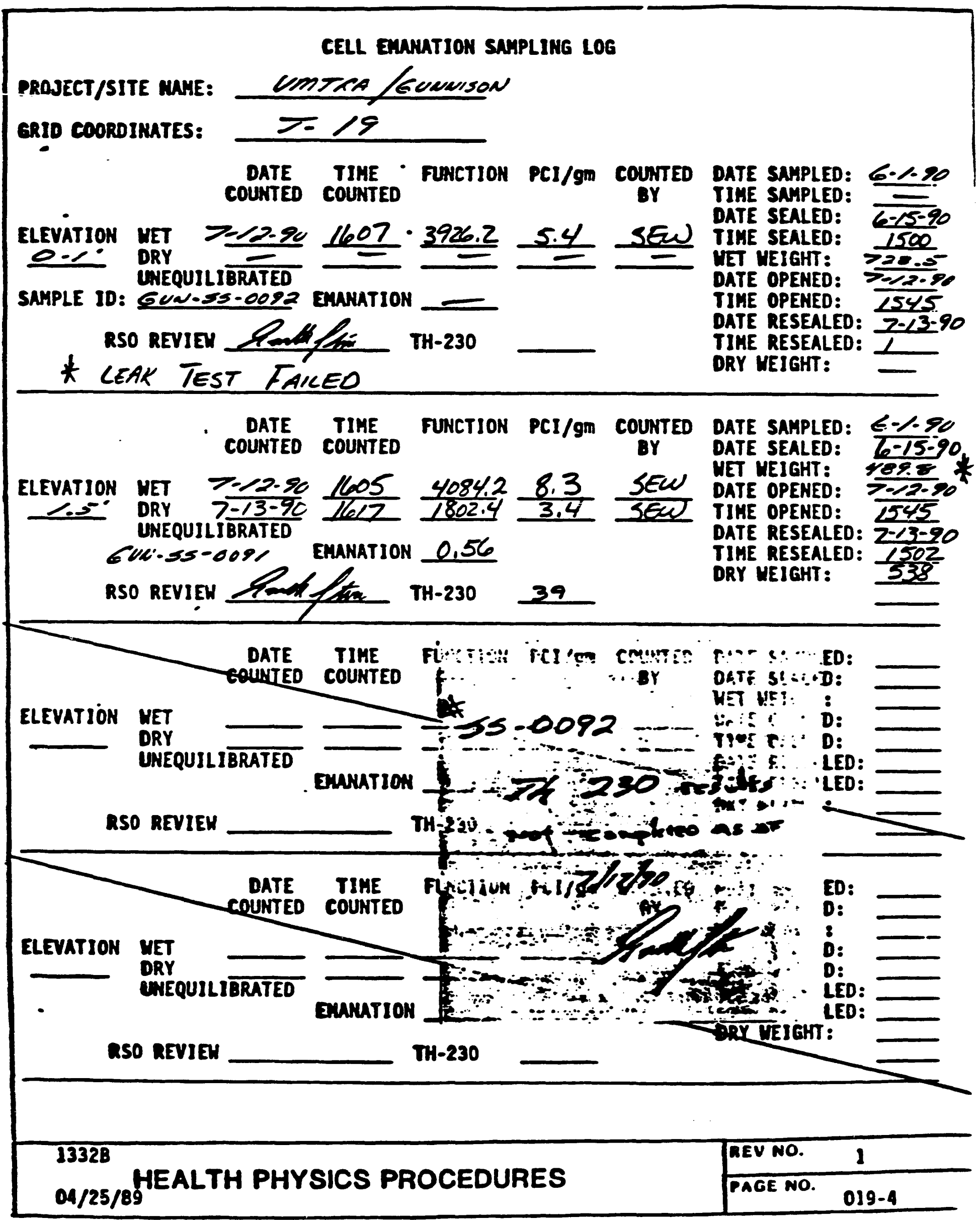




\section{CELL ENHUTION SAMPING LOG}

PROJECT/SITE MAME:

ensten/evenson
$y=20$

ERID COORDIMTES:

DATE TIME - FUNCTION PCI/SM COUNTED
COUNTED COUTED

:

ELEYATION WET $\frac{7-12.90}{7-13-90} \frac{0903}{0908} \cdot \frac{397.3}{678.2} \frac{0.6}{1.1} \frac{\mathrm{sEW}}{\mathrm{SEW}}$

- WEQUILIBRATED

SAMPLE ID: GUN-S5-00/6 EMANATION-0.71

RSO REVIEU

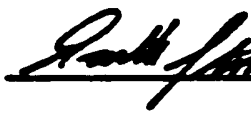

1
62
DATE SNMPLED: $=-3 / 90$ TIME SAMPLED: DATE SEALED: TIME SEALED: NET NEIGHT: DATE OPENED: TIME OPENED: DATE RESEALED: TIME RESEALED: DRY UEIGHT:

\section{DATE TIME FUNCTION PCI/gI COUNTED COUNTED COUNTED EMANATION 0.49}

ELEVATION MET $7-10.90 \frac{0910}{7-13.90} \frac{3973.1}{0915} \frac{6.5}{2036.2} \frac{3.4}{\text { SEW }}$ UNEQUILIBRATED GUN-5S-0017 RSO REVIEH

TH-230

7.8

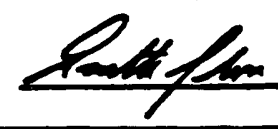

DATE SAMPLED: $=-2 /:=0$ DATE SEALED: 6-4-90 MET MEIGHT: COS. $\mathrm{C}$ DATE OPENED: $=2-20$ TIME OPENED: DATE RESEALED: TIME RESEALED: DRY MEIGHT:
1530 $7-13-90$ 0.857 $\cos 4$

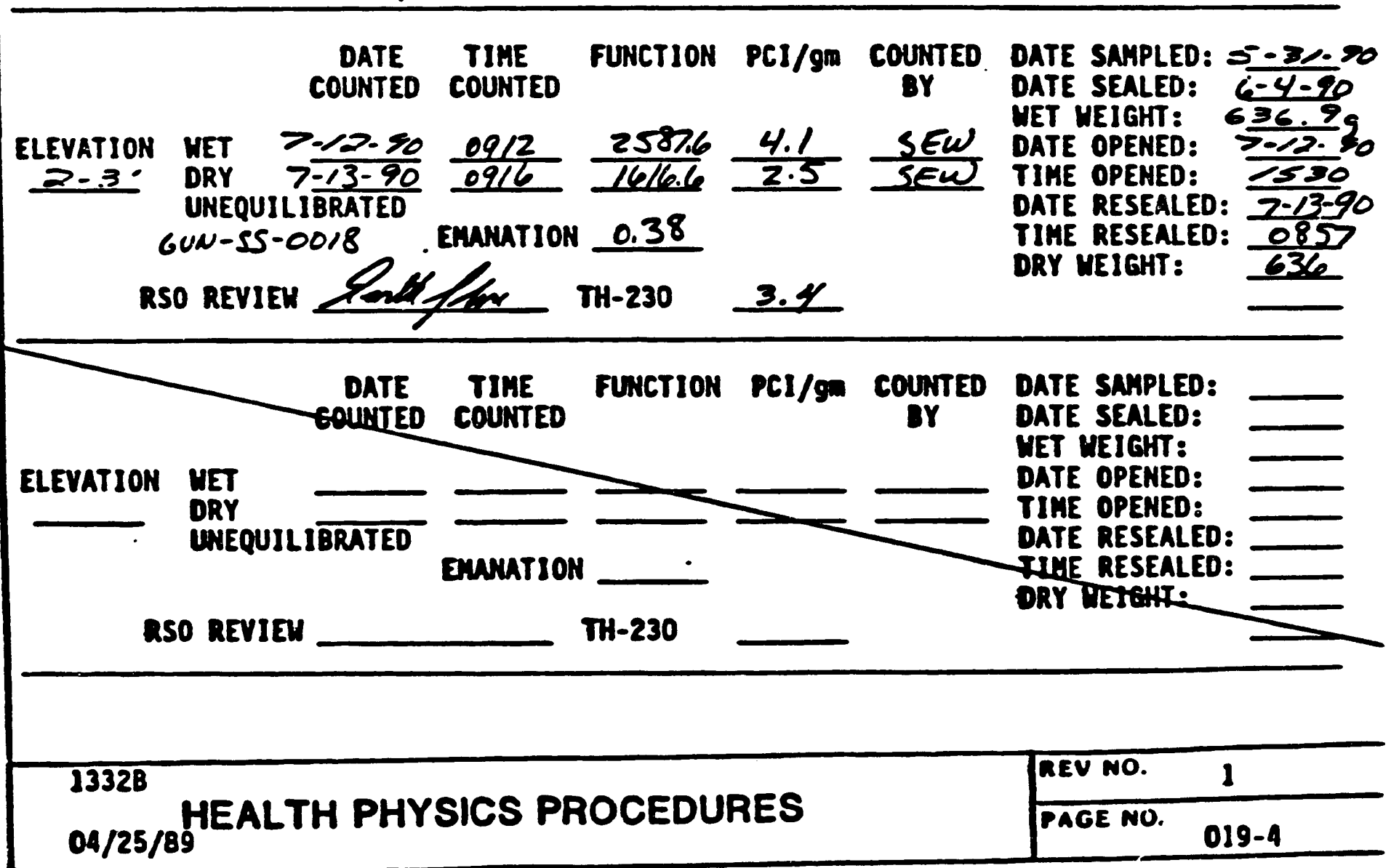




\section{CELL EMHTION SAYPLING LOG}

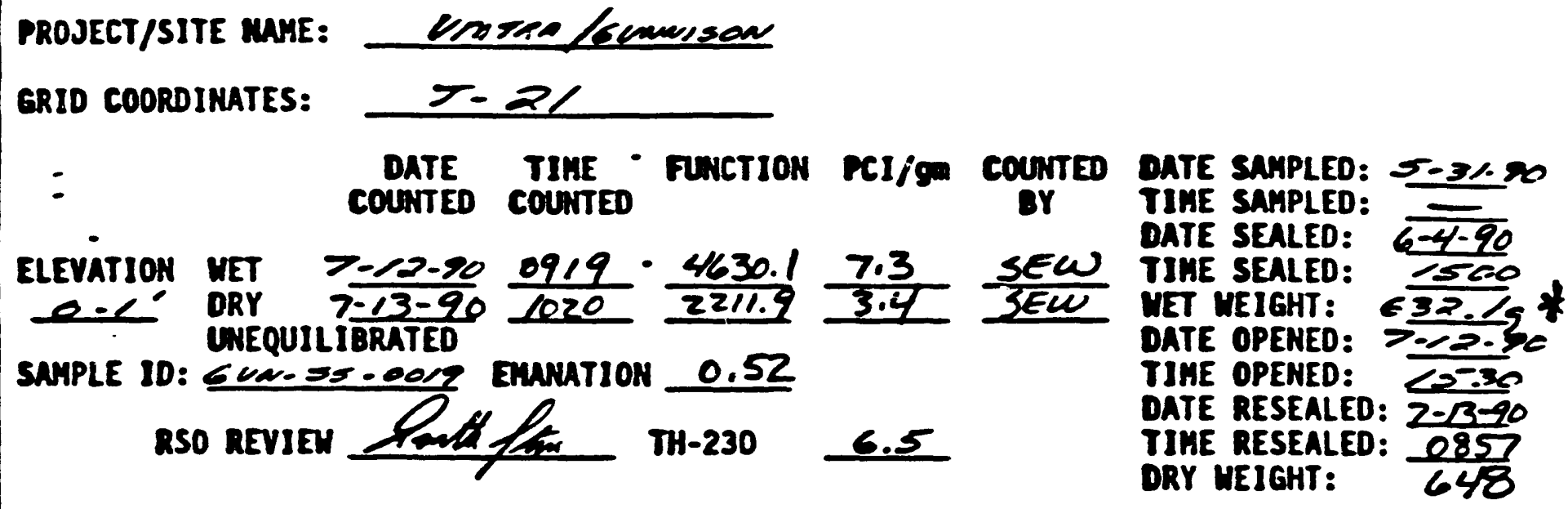

ELEVATION WET $\frac{7-12-90}{7-13-90} \frac{0919}{1020} \cdot \frac{4630.1}{2211.9} \frac{7.3}{3.4} \frac{\text { SEW }}{\text { SEW }}$ UWEQUILIBRATED

SAMPLE 1D: Gua-s5-00/ EMNATIOH 0.52

RSO REVIEH

TH-230 $\quad 6.5$

DATE SAMPLED: $5-3 / 20$ TIME SAMPLED: DATE SEALED:

$6-4-90$ WET MEIGHT: $\frac{-5<0}{32.15}$ * DATE OPEKED: $=-12-30$ TIME OPENED: $\angle 3-30$ DATE RESEALED: $2-13-90$ TIME RESEALED: 085 DRY MEIGHT: $\frac{648}{64}$

DATE
COUNTED COUNE FUNCTION PCI/gm COUNTED

ELEVATION UET $>\frac{7-12-80}{7-13-90} \frac{0920}{1021} \frac{7520.2}{4155.9} \frac{12.1}{7.4} \frac{5 E W}{5 E W}$ UNEQUILIBRATED

GWW-5\$-0020 EMANATION 0.45

RSO REVIEH

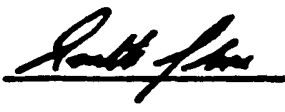

TH-230

2

DATE
COUNTED TIME
COUNTED

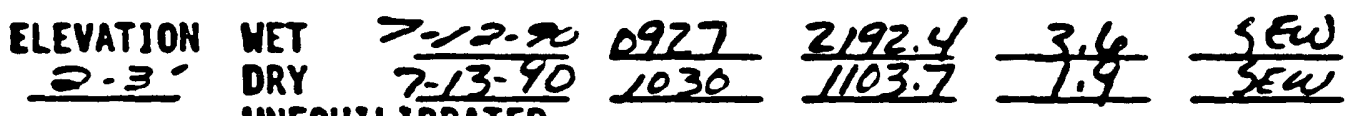
UNEQUILIBRATED

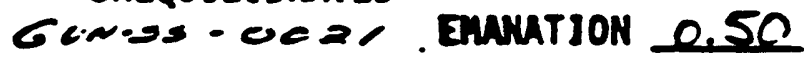

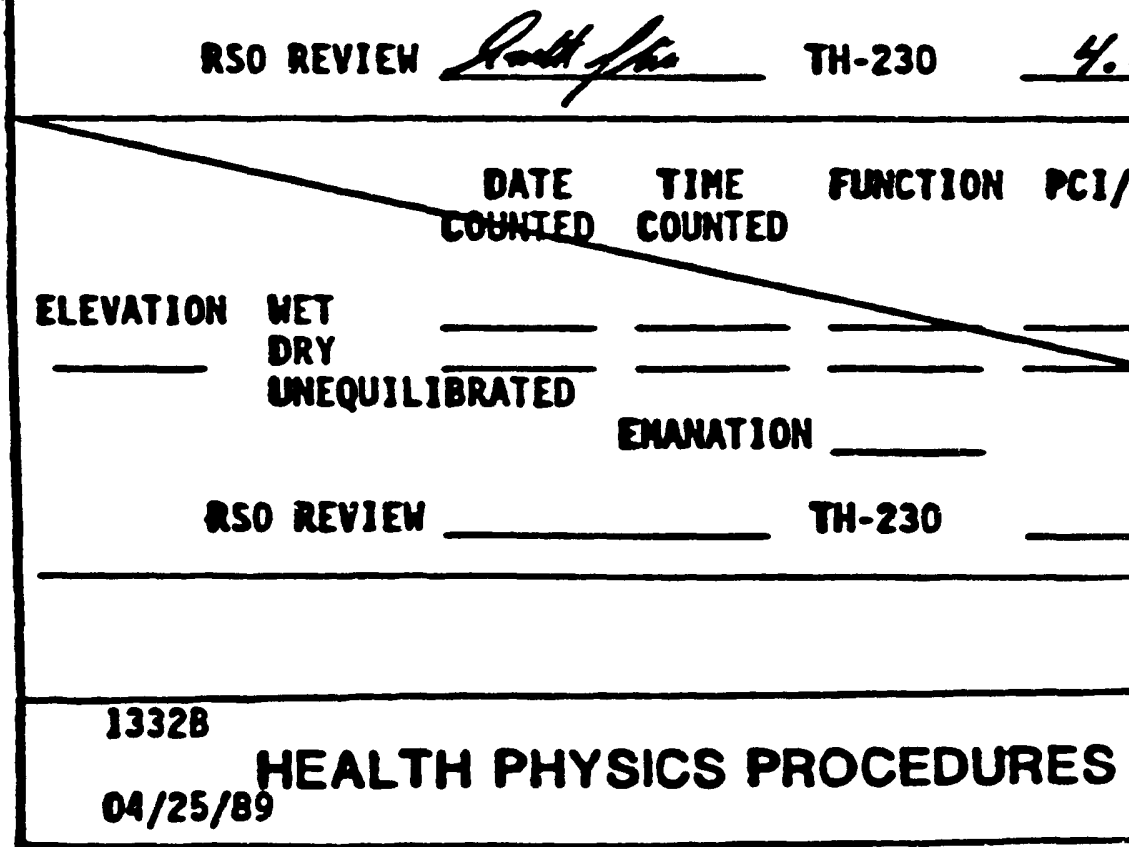

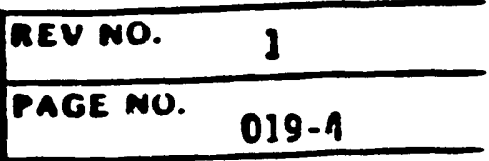

DATE 'SAMPLED: $5-3 /-26$ DATE SEALED: $6-4-90$ MET MEIGHT: 62/.79 DATE OPENED: >-12-s0 TIME OPENED: 15.30 DATE RESEALED: $7-13-91$ TIME RESEALED: 0.87 DRY MEIGHT: 564

DATE SAMPLED: $s=3 /=>0$ DATE SEALED: $6-4-90$ MET MEIGHT: $6>7.3$ DATE OPENED: -12.50 TIME OPENED: 1530 DATE RESEALED: $7-13-90$ TIME RESEALED: 0902 DRY MEIGHT: 5 T.9

DATE SAMPLED: DATE SEALED: UET UEIGHT: DATE OPENED: TIME OPENED: DATE RESEALED: TAME RESEALED: ORY VEIOTF'

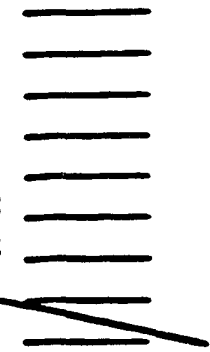




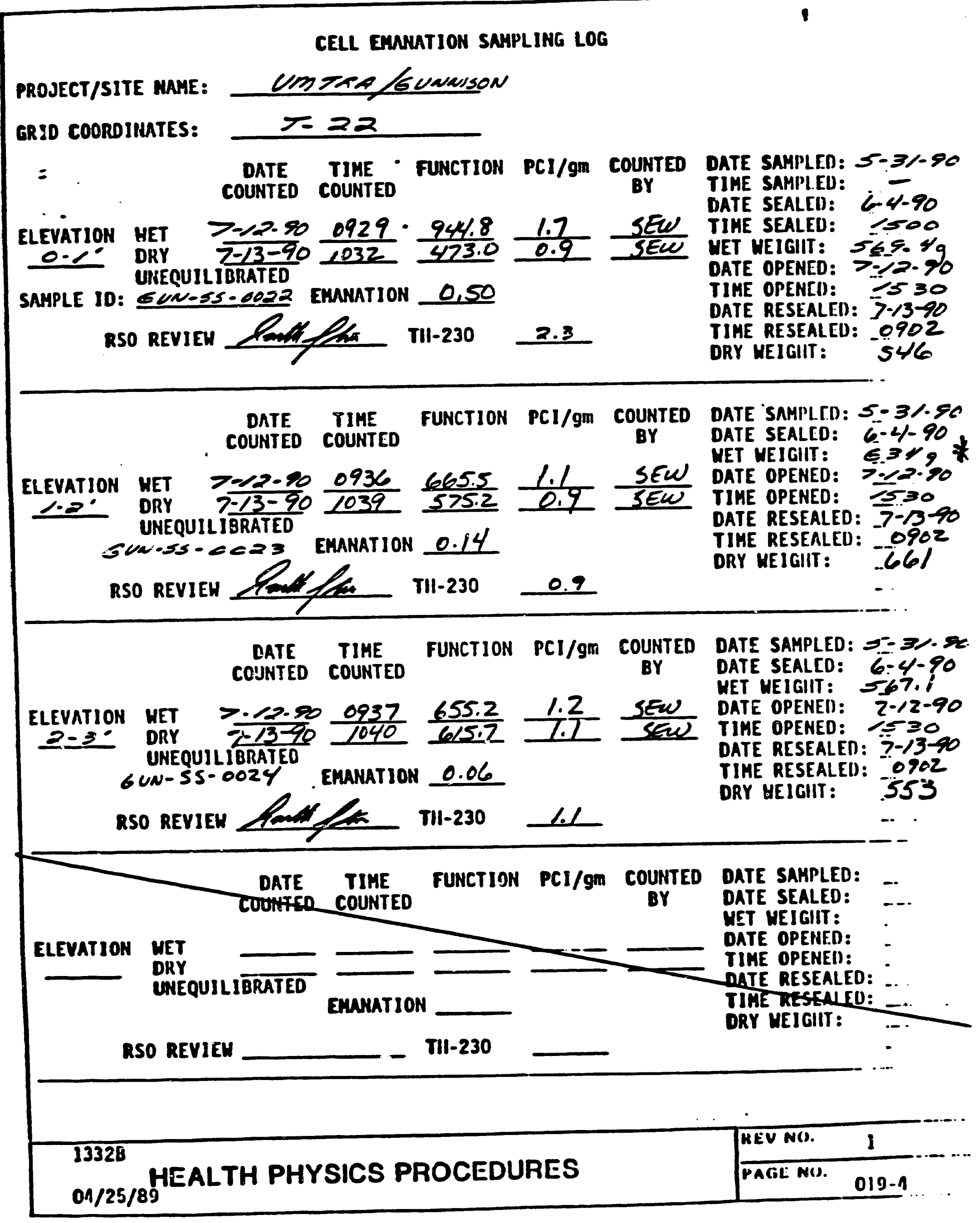


CELL EMANATION SAHPLING LOG

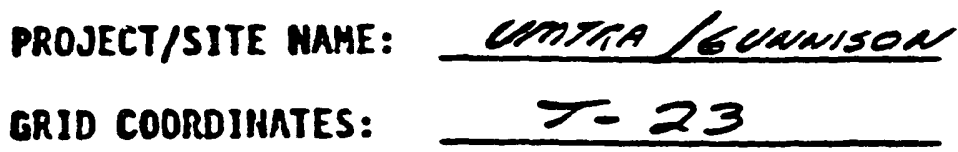

$$
=
$$

DATE TIME - FUNCTION PCI/gm COUNTED COUNTED

ELEVATION WET $\frac{72.90}{7-13.90} \frac{0945}{1048} \frac{74 / .1}{137.6} \frac{1.7}{1.6}$ UNEQUILIBRATED

SAMPLE ID: GUN-SS-0025 EMANATION 0.14

RSO REVIEW

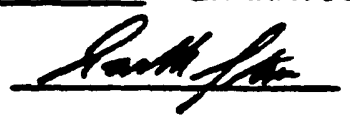

TII-230

1.4
COUNTED DATE SAHPLED: $3=3 \%-80$ BY TIHE SAMPIED:

DATE SEALED: 6.4 .90 SEW TIME SEALID: 1500 SEW MET HEIGIIT: $\$ \$ .1$ DATE OPENED: $>-2>-80$ TIME OPENED: 7.50 DATE RESEALEI): $7-13-90$ TIME RESEALED: 0908 DRY UEIGIIT: $\overline{3} 88$

\section{DATE TIME COUNTED COUNTED \\ FUNCTION PCI/gM COUNTED}

ELEVATION WET $\frac{7-2.90}{1-13-90} \frac{0946}{1049} \frac{346.1}{485.6} \frac{0.6}{0.8} \frac{\text { SFW }}{5 E W}$ UNEQUILIBRATEO EMANATION -0.4

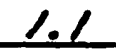

\section{$\frac{\text { COUNTEO }}{\text { DLEVATION REVIEH }}$}

\section{EMANATION}

RSO REVIEH

TIME TII-230
DATE SAMPLED: $5 \cdot 3 / .90$ DATE SEALCD: $\ddot{6}-4-90$ WET HEIGIIT: G:2.59 DATE OPENED: >->2. TIME OPENED: $\equiv$ DATE RESEALED: $7-13-90$ TIME RESEALEU: 0908 DRY HEIGIIT:

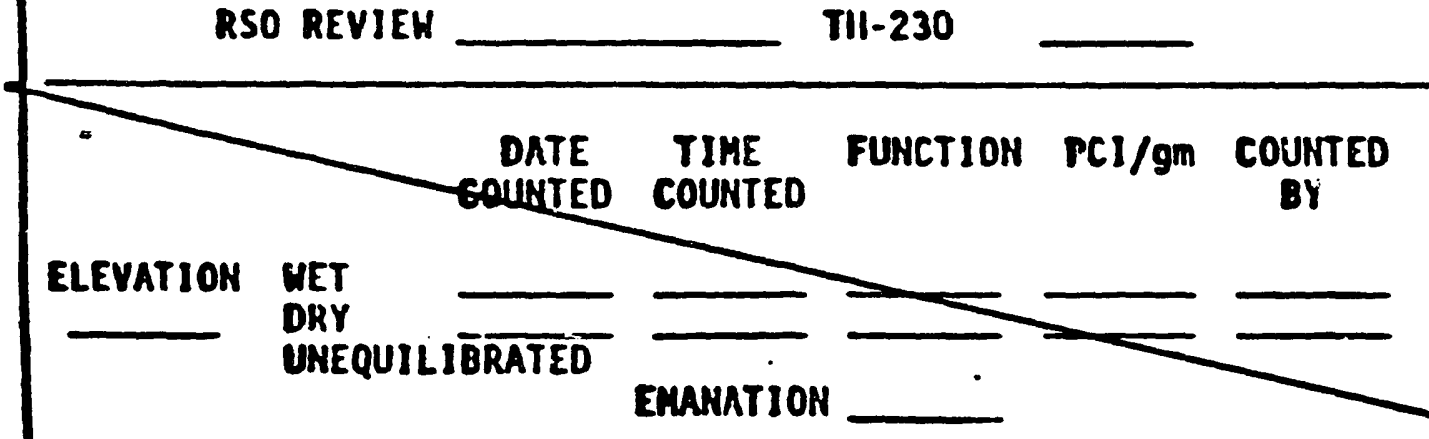

RSO REVIEW

TII-230
DATE SAMPLED: -

DATE SEALED: -

MET MEIGIIT:

DATE OPENED:

TIME OPEHED:

DATE RESEALED:

IIME RESEALEU:

DRT HELGUIT:
$1332 B$
91/25/89
HEALTH PHYSICS PROCEDURES

MEV NIS. $\quad 1$




\section{PROJECT/SITE MAME: UMTRA / GUNULSON}

GRID COORDIMATES:

$T=24$

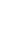

\section{DATE TIME - FUNCTION PCI/gI COUNTED COUNTED COUNTED BY}

ELEVATION WET $\frac{7-12-90}{2-13-90} \frac{0954}{1056} \frac{1041.3}{182.2} \frac{1.8}{1.3} \frac{\text { SEW }}{\text { SEL }}$ SAMPLE ID UHEQUILIBRATED

SAMPLE ID: GUN-SS-0027 EUNATION 0.35

RSO REVIEH

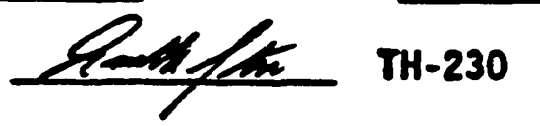

1.4

DATE TIME FUNCTION PCI/gM COUNTED
COUNTED COUNTED

ELEVATIOH MET

L-2: DRY UNEQUILIBRATED

GUN-SS-DO28

RSO REVIEW

COUNTED COUNTED

$\frac{483.1}{1.42 .1} \frac{0.8}{1.1}$ EMANATION $=0.33$

Romzen TH-230

1.4
SET HEIGHT:

DATE OPENED:

TIME OPENED:

DATE RESEALED:

TIME RESEALED:

DRY MEIGHT:

SEW

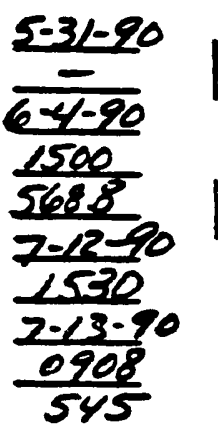

$5-31-90$

TIME SAMPLED:

SEALED:

MET MEIGHT:

DATE RESEALED:

TIME RESEALED: DRY MEIGHT:

\section{DATE COUNTED COUNTED \\ TIME \\ FUNCTION}

$\mathrm{PCl} / \mathrm{gm}$

COUR

ELEVATION $z-3 i$

WET

$\frac{7-1290}{7-13-90}$

$\frac{1002}{1105} \frac{389.2}{480.0}$

0.6

DRY $7-13$ UNEQUILIBRATED

GUN-SS-0029

EMANATIOH -0.23

RSO REVIEW
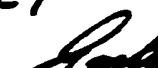

1

5 TH-230

0.7

SEC SES

\section{BY}

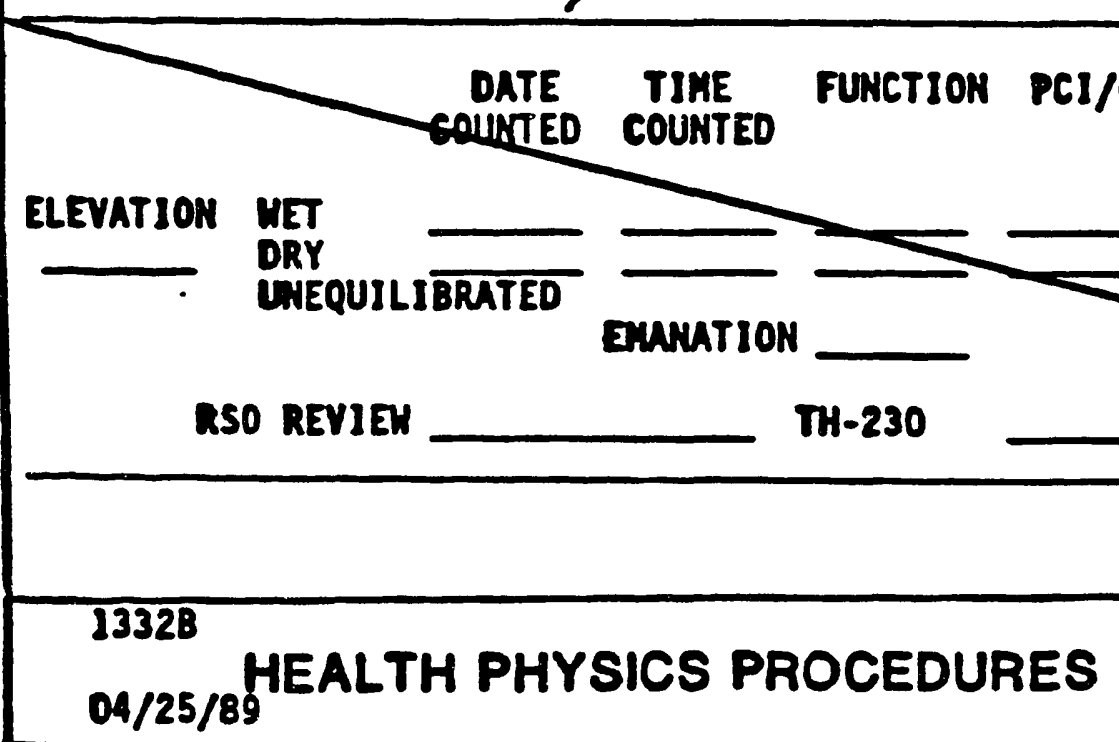

DATE SAMPLED: 5-31-90 DATE SEALED: $6-4-90$ MET MEIGHT: DATE OPENED: TIME OPENED: DATE RESEALED: TIME RESEALED: O2/5 DRY MEIGHT:

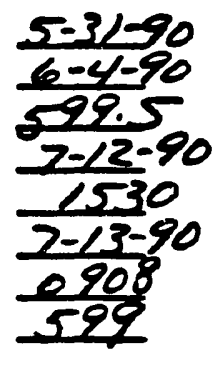

$5-31-90$ 99.5 7-12-90 1530 6129: $7-1290$ 1530 635 
CELL EMARATION SAMPLING LOG

PROJECT/SITE MAME: UMTRA /GUNNISON

GRID COORDIMATES:

$T-25$

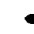
DATE TIME FUNCTIOH PCI/gm COUNTED
COUNTED COUNTED ELEVATIOK WET $\frac{7-12-90}{Z-13-90} \frac{1003}{1106} \cdot \frac{2857.3}{1887.8} \frac{5.4}{3.6} \frac{\text { SEW }}{\text { SEW }}$ UNEQUILIBRATED

SAMPLE ID: GUN-SS-0030 EMANATION 0.34 RSO REVIEW

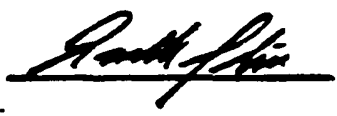

TH-230 2.5

DATE TIME FUNCTION PCI/gm COUNTED
COUNTED COUNTED

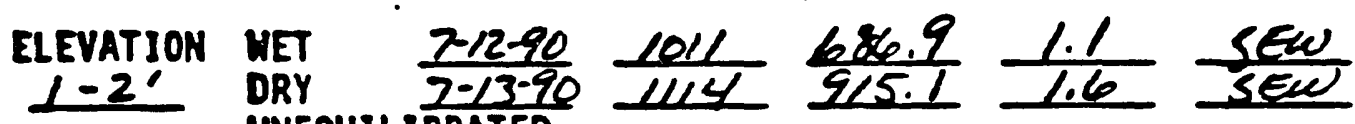
UNEQUILIBRATED

GUN-SS-0031 EMANATION $=0,33$

RSO REVIEH Sfoff le TH-230

DATE TIME FUNCTION PCI/gN COUNTED COUNTED

ELEVATION MET
$2-3$ DRY $\frac{7-12-90}{7-13-90} \frac{1012}{1115} \frac{1307.5}{999.7} \frac{2.1}{1.9} \frac{\text { SEW }}{\text { SEU }}$ UNEQUILIBRATED GuNi-55-0032 EMANATION 0.24 RSO REVIEN

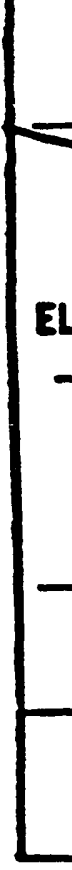
1332B $04 / 25 / 89$
DATE SAMPLED: TIME SAMPLED: DATE SEALED: TIME SEALED: MET MEIGHT: DATE OPENED: TIME OPENED: DATE RESEALED: TIME RESEALED: DRY MEIGHT:

$5-31-90$ $\frac{-19-90}{6-450}$ 1500 5291 z-12-90 $\frac{1532}{2-1350}$ 7-13-90 $\frac{0915}{529}$ 529
DATE SAMPLED: DATE SEALED: MET MEIGHT: DATE OPENED: TIME OPENED: DATE RESEALED: TIME RESEALED: DRY MEIGKT:
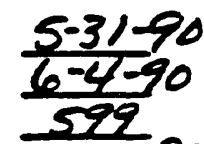
$7-12.90$ 1530 $7-13-90$ 0915 588

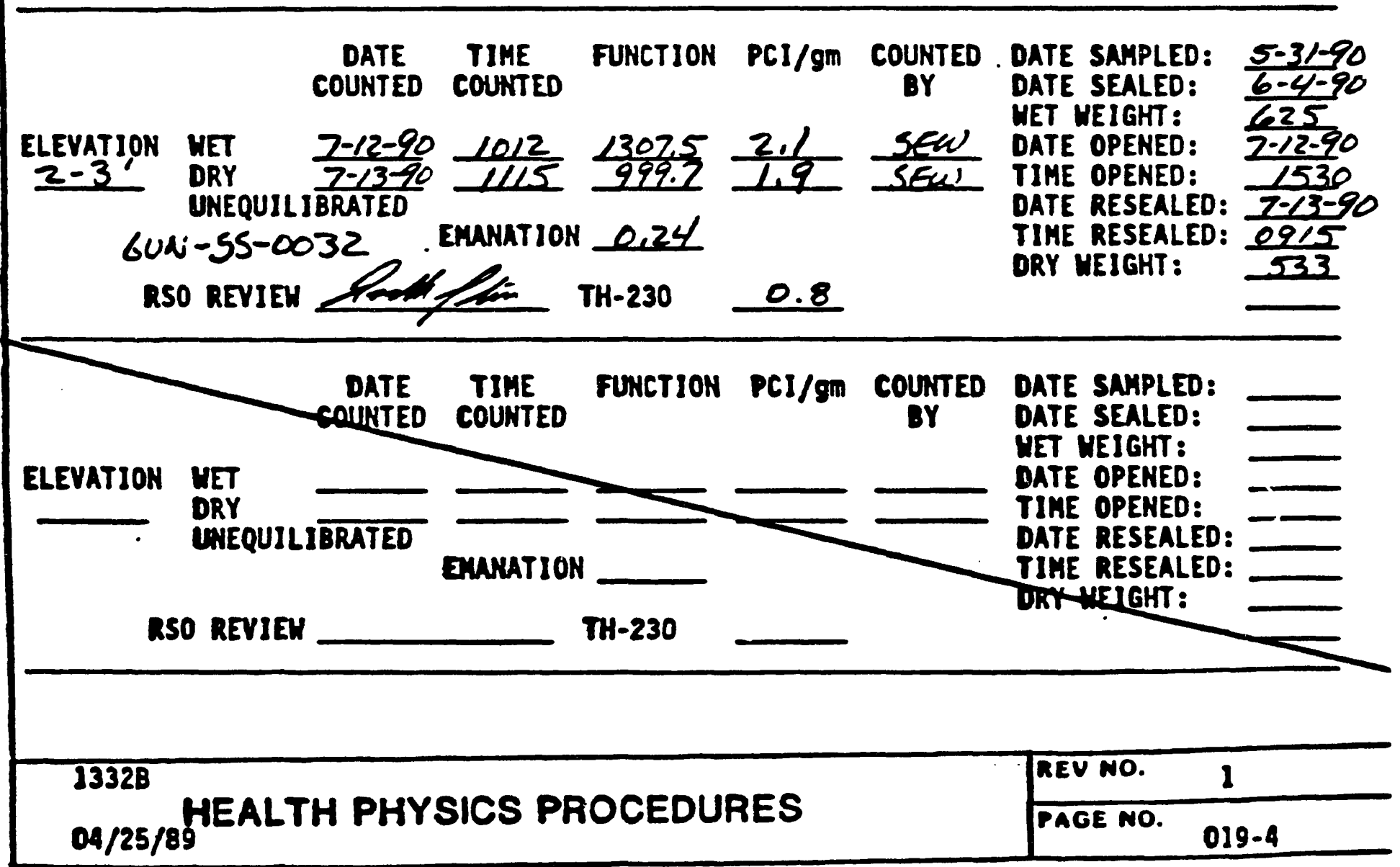




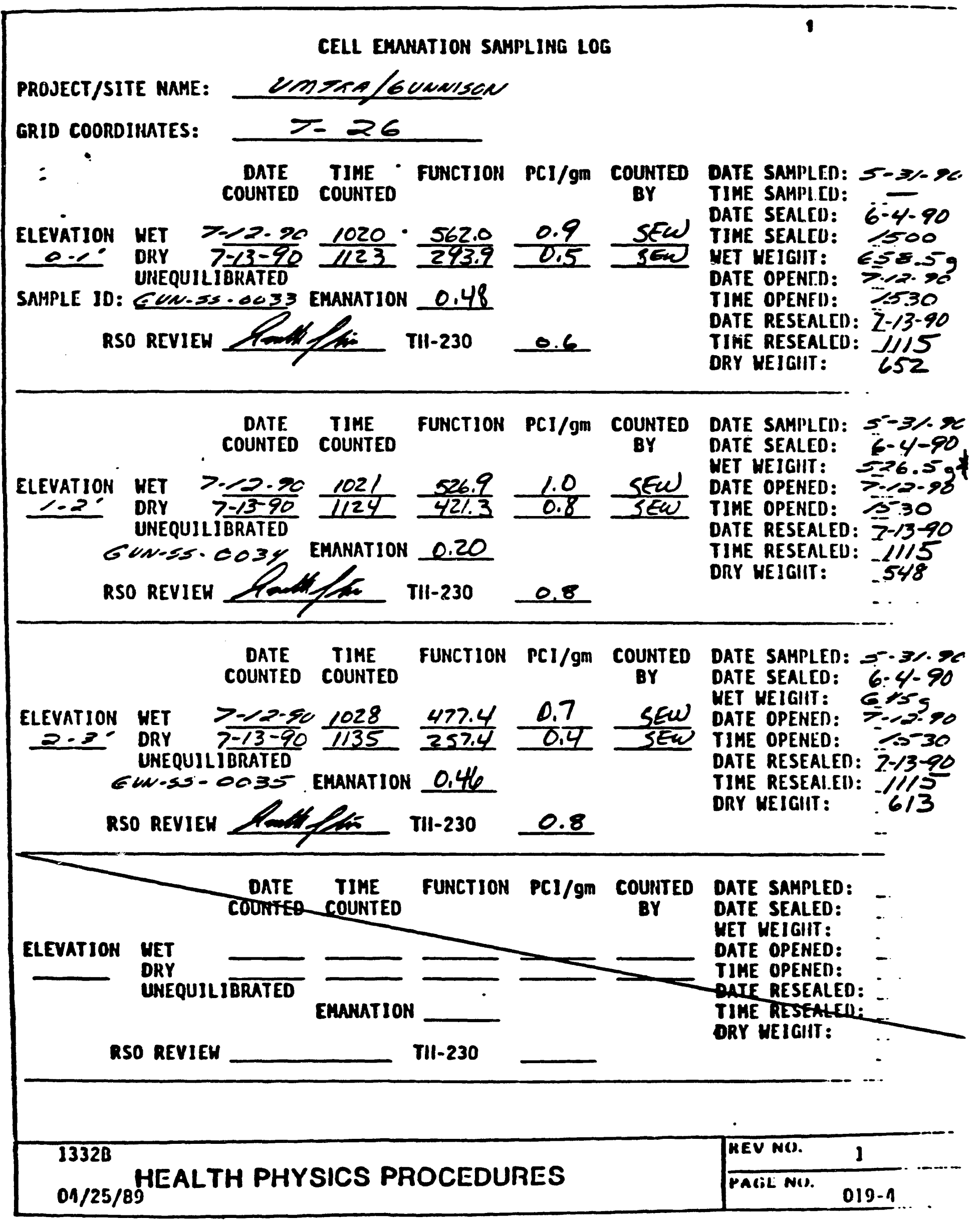




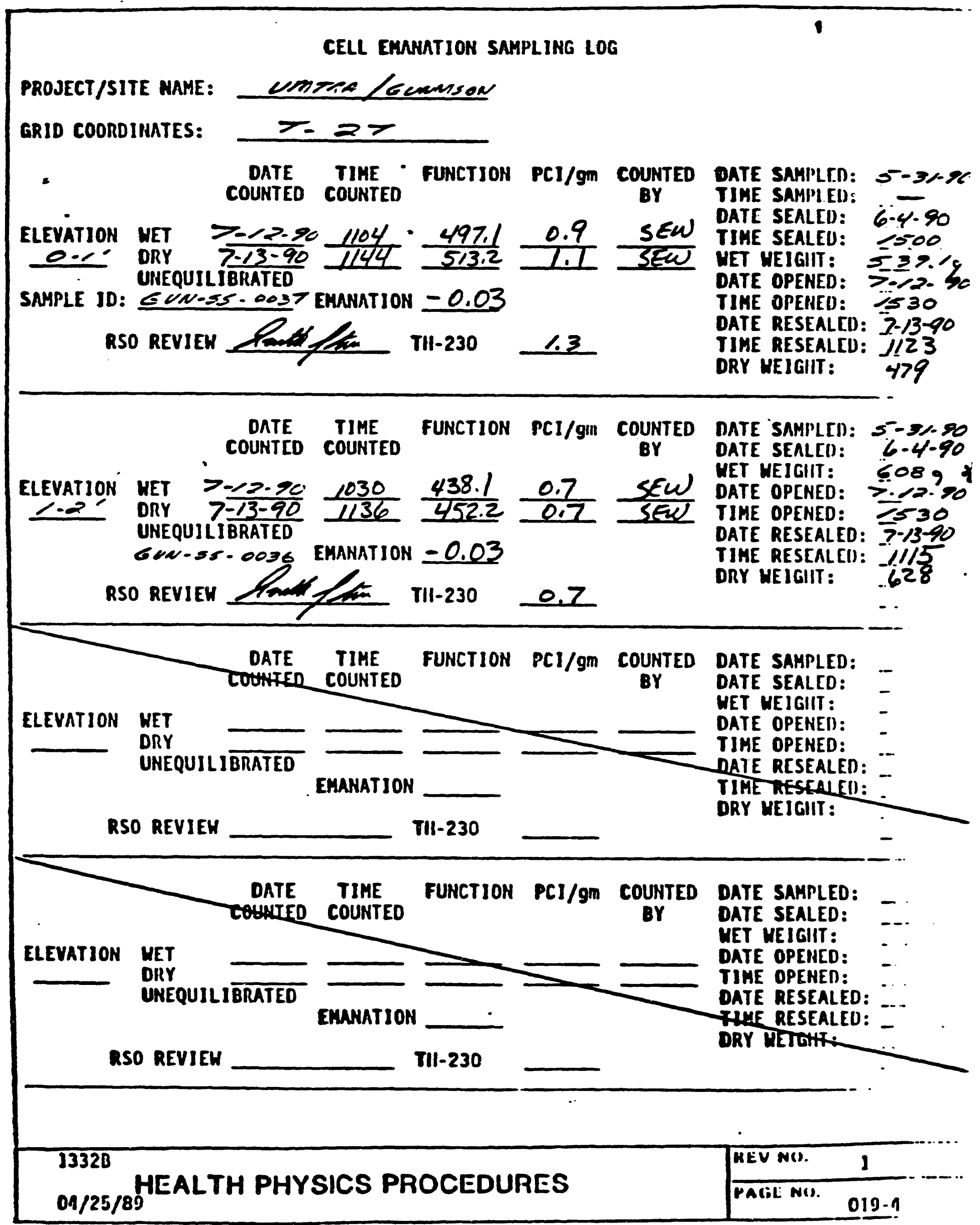


CELL ENATION SAMPLIMG LOG

PROJECT/SITE WHE: UMTRA/GUNNLSON

GRID COORDINATES:

$T-28$

$=$

DATE TIME - FUNCTION PCI/gm COUNTED COUNTED COUNTED BY

DATE SAMPLED: TINE SAMPLED: DATE SEALED:

ELEVATIOH WET $\frac{7-1290}{7-13-90} \frac{1106}{1145} \frac{11.00}{942.2} \frac{3.2}{1.9} \frac{\text { SEW }}{5 E W}$ SAMPLE ID: LUN-SS-CO38 EMNATION 0.41

TIHE SEALED:

UET MEIGHT:

DATE OPENED:

TIME OPENED:

$5-31-20$

DATE RESEALED: $\overline{1-4-80}$

TIME RESEALED: DRY NEIGHT:

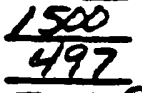

$\frac{492}{7-1290}$

1530

RSO REVIEH

TH-230

4.3

$\begin{array}{cc}\text { DATE } & \text { TIME } \\ \text { COUNTED } & \text { FOUNTED }\end{array}$

ELEVATIOH MET
$1-2$ DRY $\frac{7-12-90}{7-13-90} \frac{1113}{1152} \frac{371.3}{673.0} \frac{0.7}{1.3} \frac{5 E W}{3 E W}$ UNEQUILIBRATED

LUN-SS-0039 EMANATION $=0.81$

RSO REVIEH

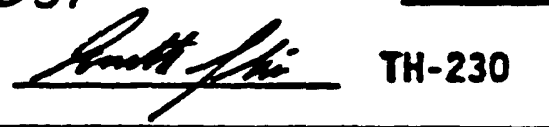

1.4

DATE SAMPLED:

DATE SEALED:

MET MEIGHT:

DATE OPENED:

TIME OPENED:

DATE RESEALED:

TIME RESEALED:

DRY MEIGHT:

$2-13-90$

1123

496

\begin{tabular}{|c|c|c|c|}
\hline $\begin{array}{l}\text { DATE } \\
\text { COUNTED }\end{array}$ & $\begin{array}{l}\text { TIME } \\
\text { COUNTE }\end{array}$ & FUNCTIO & \\
\hline
\end{tabular}

ELEVATION WET

$2-3$

WET

$\frac{7-12-90}{7-13-90}$

$\frac{1114}{1154}$

$\frac{773.3}{59.2}$

$\frac{1.2}{1.2} \frac{5 E(x)}{5(2)}$

UNEQUILIBRATED

Guess-0040

EMANATIOH 0.23

$5-32-90$

$6 \cdot 4-90$

526.5

$7 \cdot 12-90$

1530

$7-13-90$

RSO REVIEY

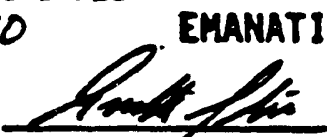

TH-230

2.1

DATE SAMPLED: $6-2-90$

DATE SEALED: $6-4-90$

WET MEIGHT:

DATE OPENED:

TIME OPENED:

DATE RESEALED:

TIME RESEALED:

DRY MEIGHT:

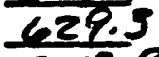

$\frac{629.9}{7-12-90}$

1530

$7-13-90$

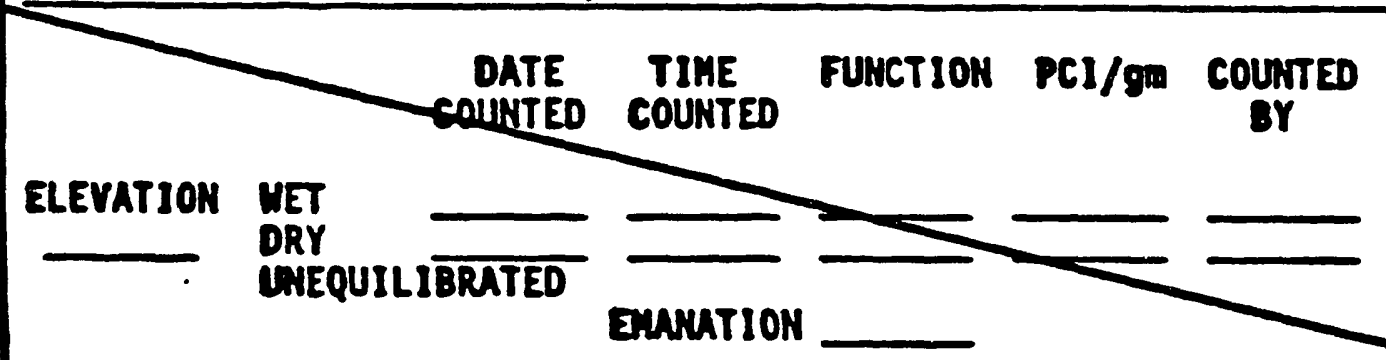

ASO REVIEY

TH-230

DATE SAMPLED:

DATE SEALED:

UET VEIGHT:

DATE OPENED:

TIME OPENED:

DATE RESEALED:

TIME RESEALED:

ORT-WEIFHT:

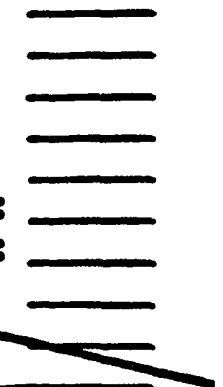

13328

$04 / 25 / 89$

HEALTH PHYSICS PROCEDURES

\begin{tabular}{|c|c|}
\hline REV NO. & 1 \\
\hline PAGE NO. & $019-4$ \\
\hline
\end{tabular}




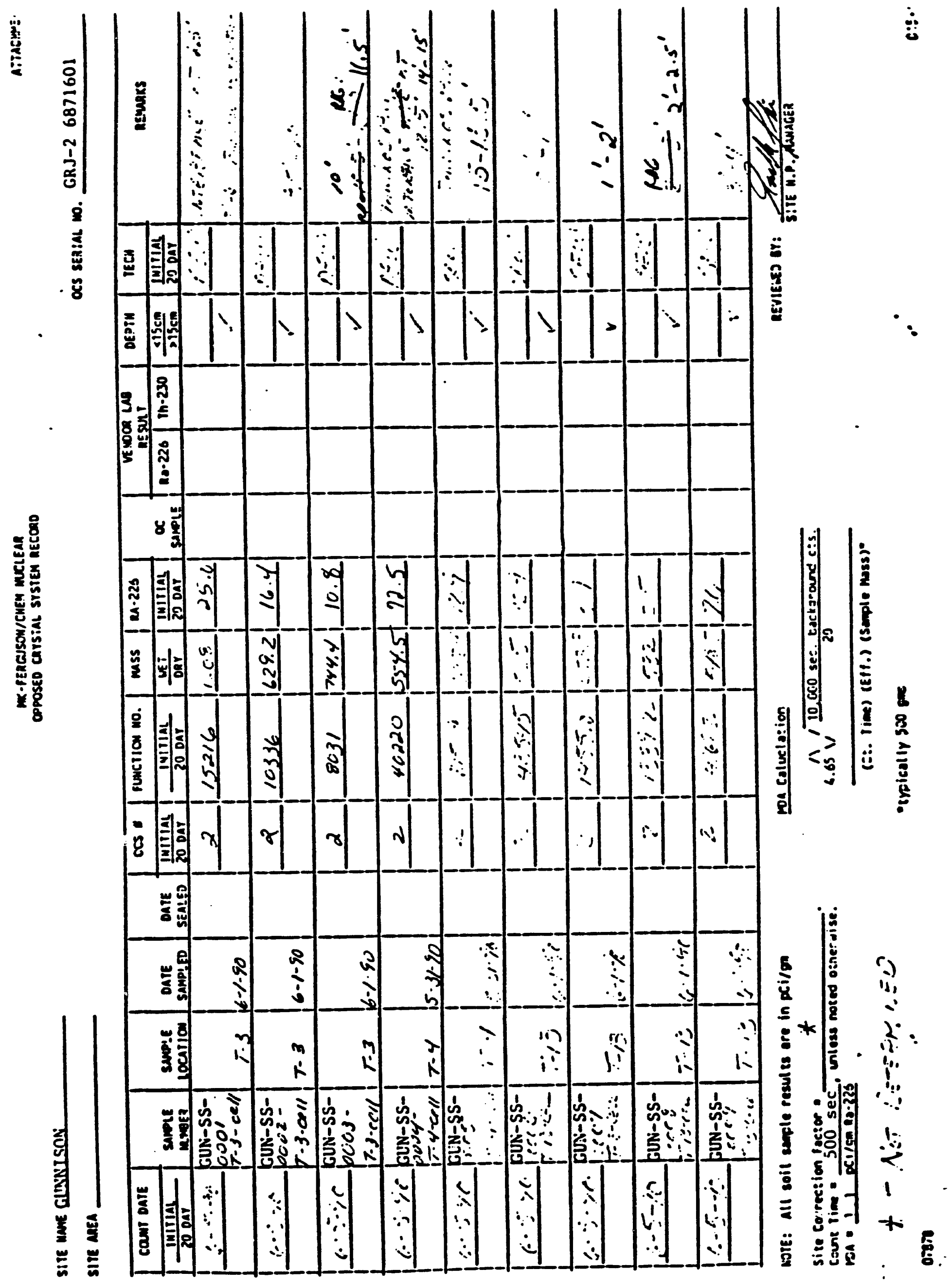




\section{SIIE Area}

ocs serilal wo. GRJ-2 6871601

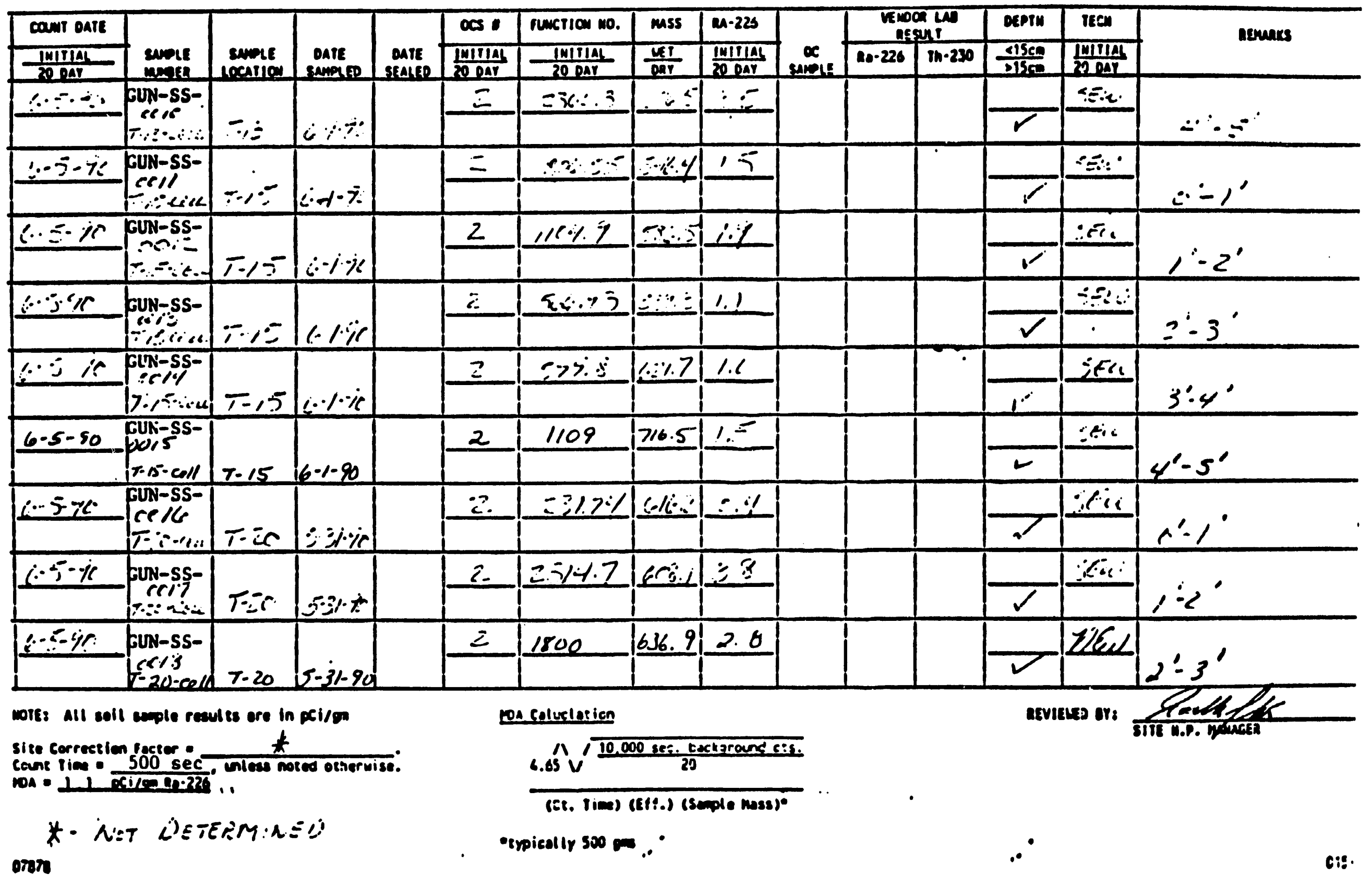




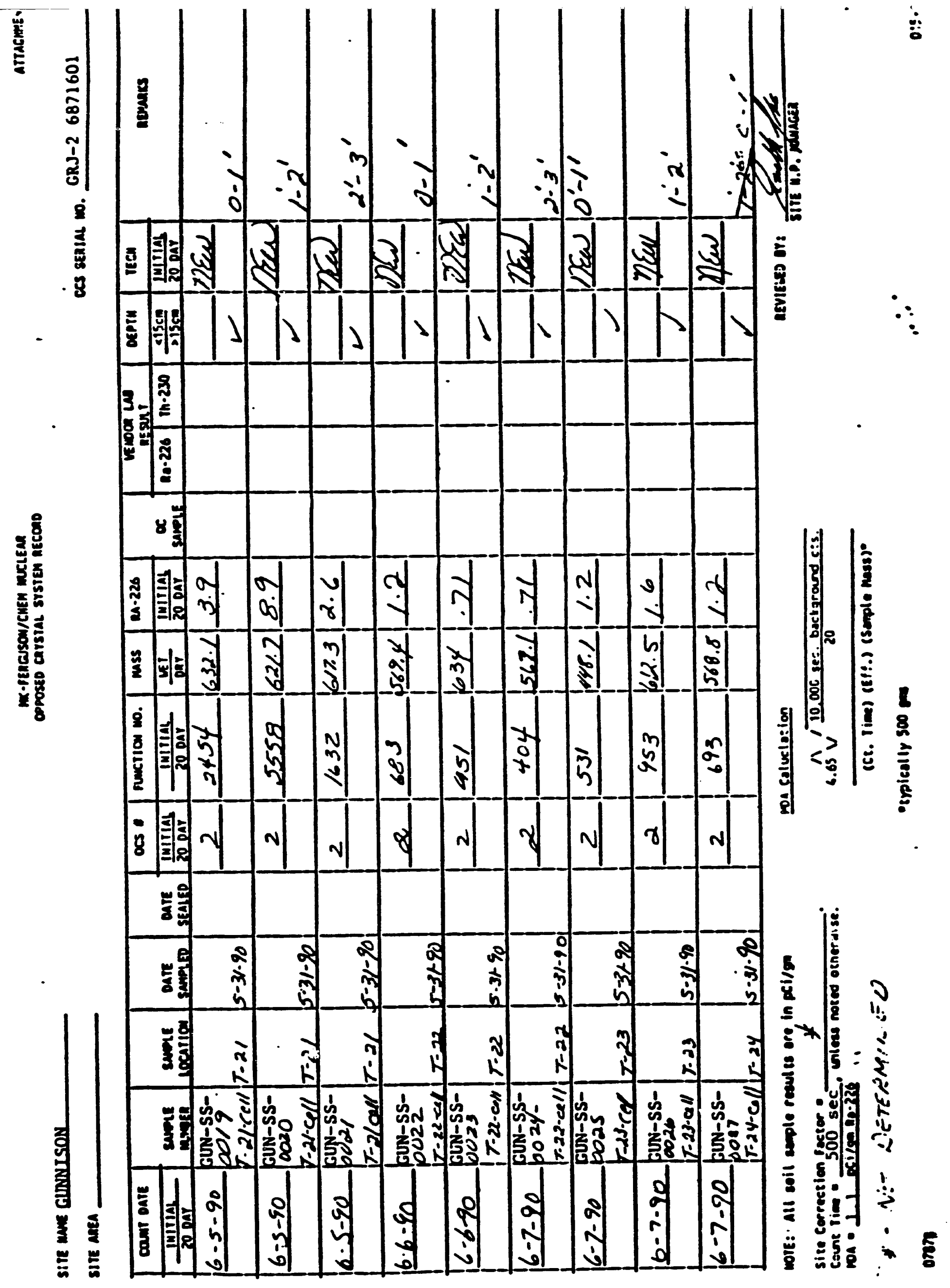




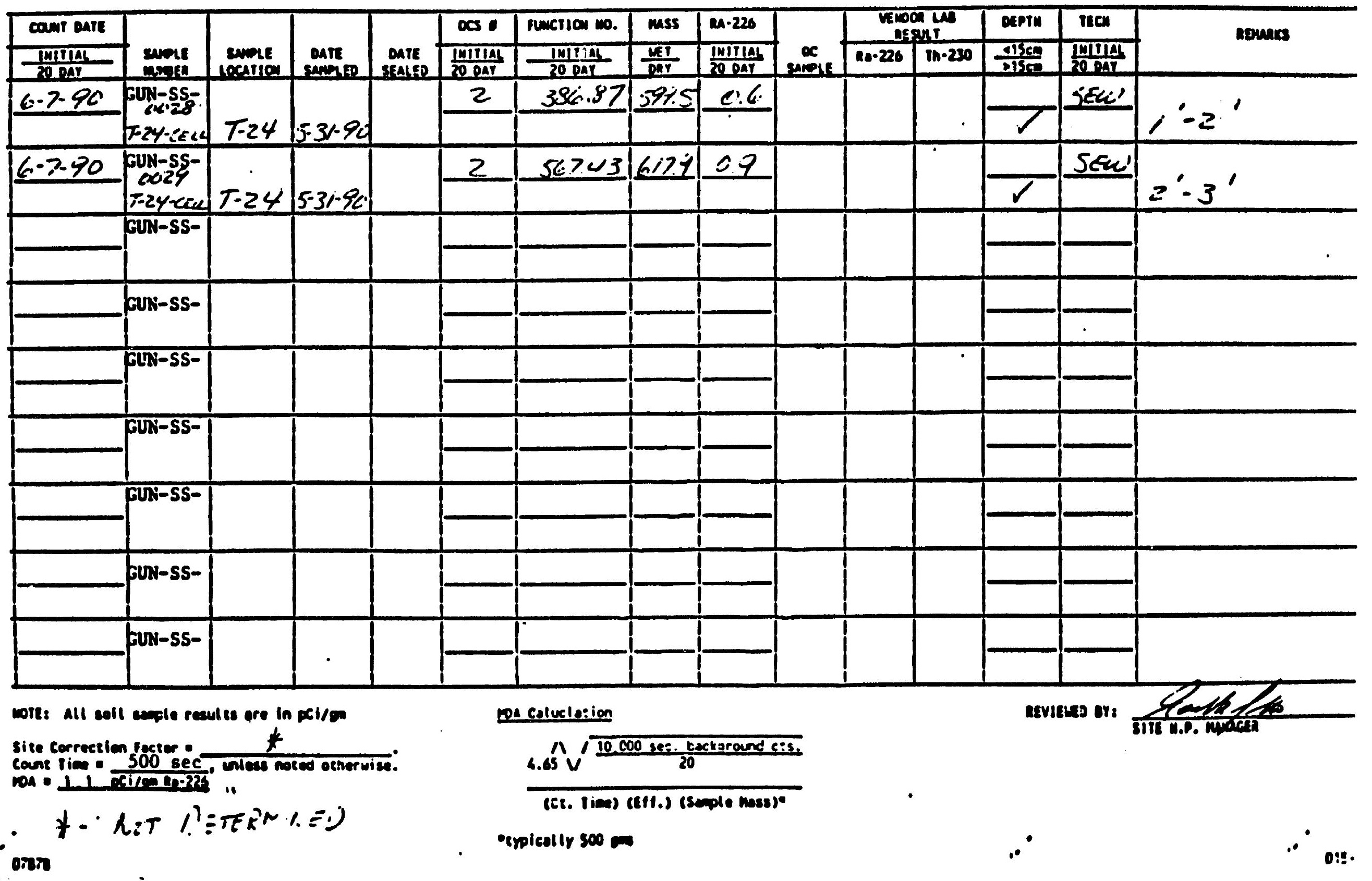




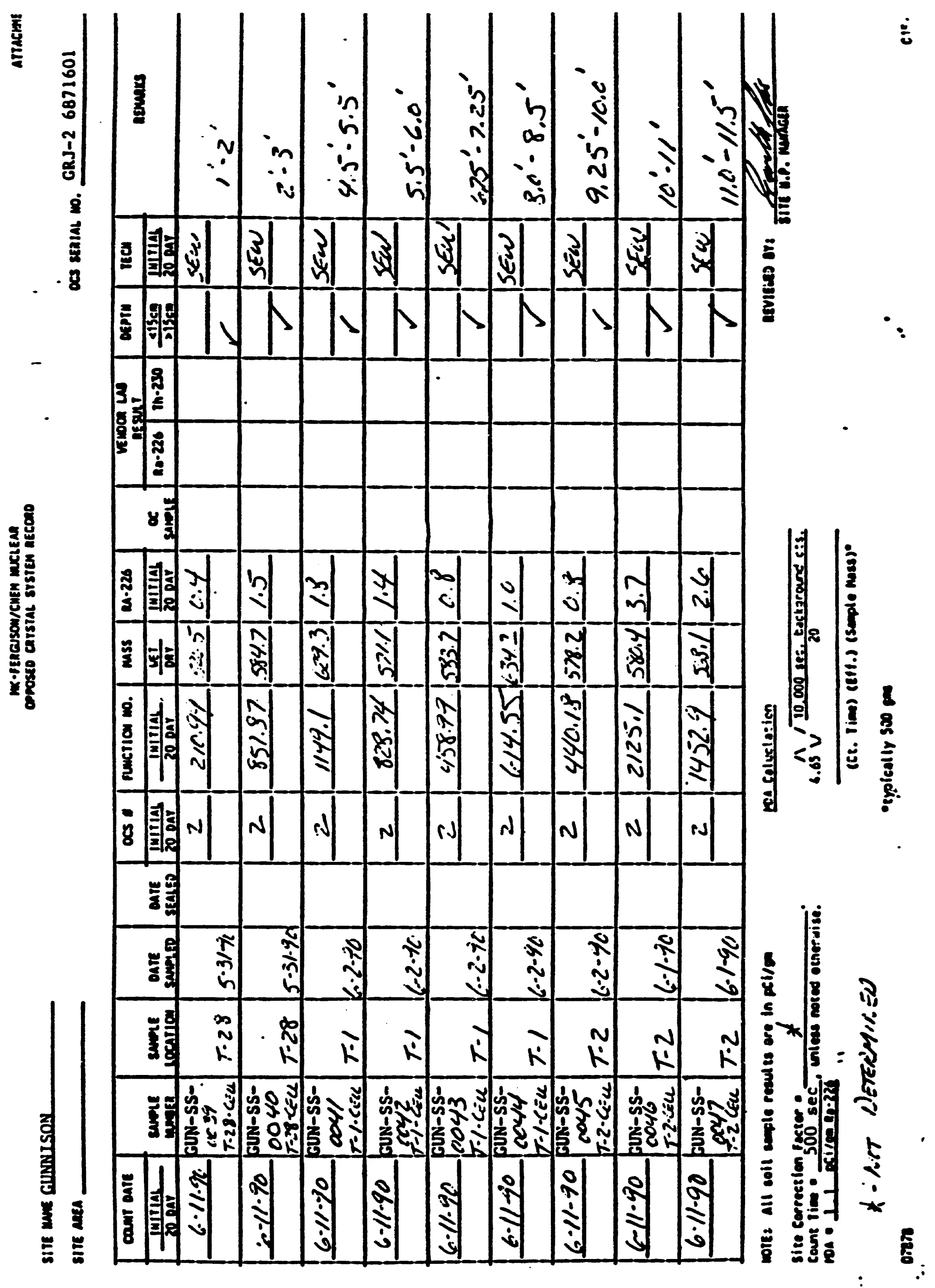


SITE Mare GIDNISON

sire amen des stalal wo. GRJ-2 6871601

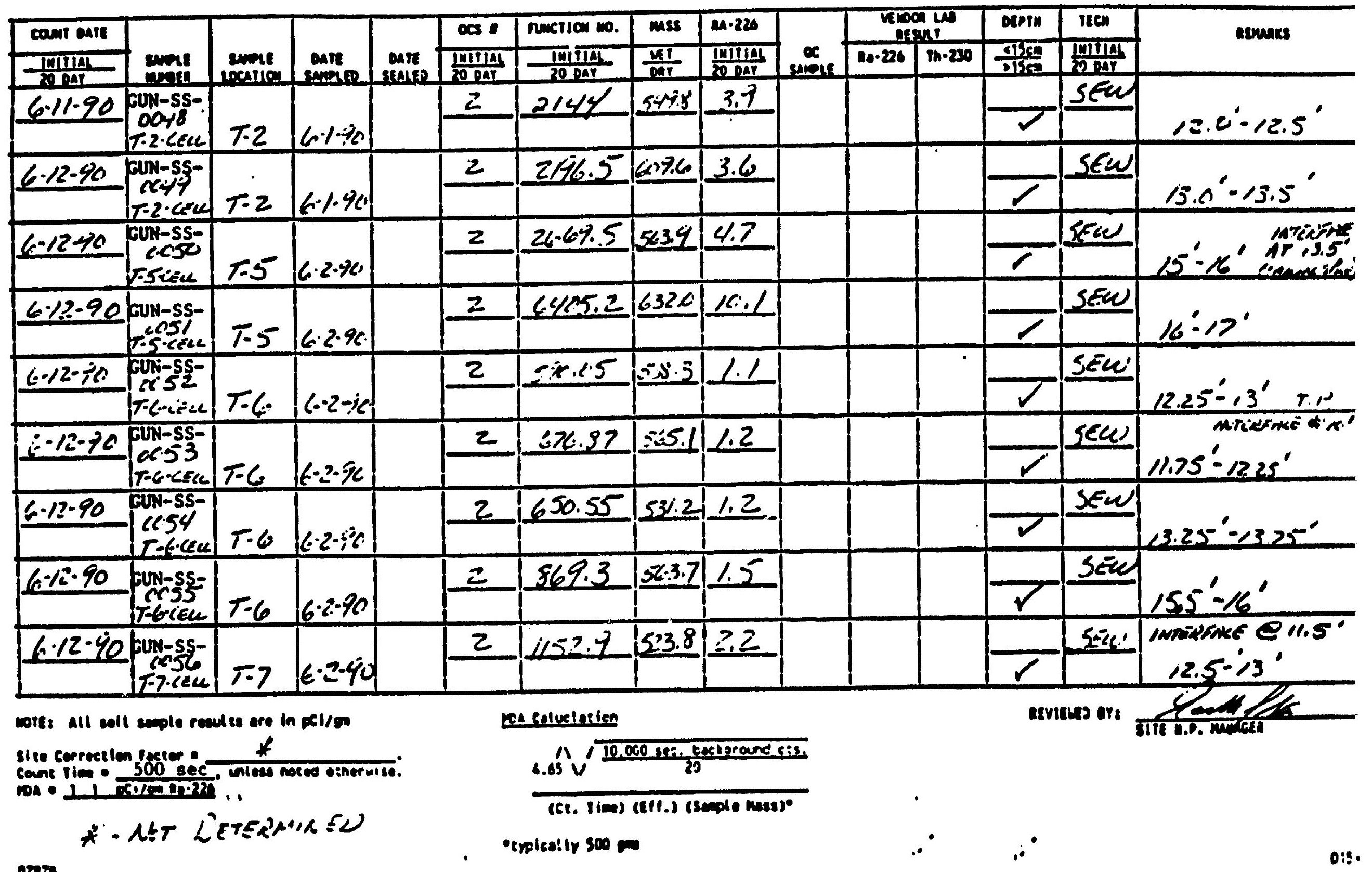


des seatal wo. GRJ-2 6871601

sire auta

\begin{tabular}{|c|c|c|c|c|c|c|c|c|c|c|c|c|c|c|}
\hline cant oAre & & & & & ocs. & function wo. & mass & ma-226 & & & Tis & OEPTW & HECW & nemeres \\
\hline$\frac{\operatorname{lmIINA}}{200 A 1}$ & sum: & $\begin{array}{l}\text { sure } \\
\text { section }\end{array}$ & $\begin{array}{l}\text { OARE } \\
\text { SMMPIPP }\end{array}$ & $\begin{array}{l}\text { OAIE } \\
\text { SEALEP }\end{array}$ & 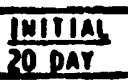 & $\frac{\text { IIIIIAY }}{30 \text { DAY }}$ & $\frac{\text { LEI }}{\text { Der }}$ & $\frac{\lim (\operatorname{IIMA}}{30 \operatorname{loAr}}$ & $\begin{array}{l}\alpha \\
\text { Sateif }\end{array}$ & 20.226 & $m \cdot 230$ & $\frac{415 \mathrm{~cm}}{315 \mathrm{~cm}}$ & $\frac{\frac{1111141}{300 \Delta 1}}{3001}$ & \\
\hline 6.12 .90 & GUN-SS- & & & & 2 & $=5 \times 0.4$ & $5 \dot{x-2}$ & $4 . \overline{2}$ & & & & & SFies & \\
\hline & $E=7$ récl & $T=7$ & $6 \cdot 2 \cdot 90$ & & & & & & & & & $V$ & & $13.0-13$ \\
\hline$\therefore 12.90$ & GUN-SS- & & & & $\Xi$ & $\because 35.7$ & $\bar{x} 4.7$ & $\therefore .1$ & & & & & SES & \\
\hline & $7 \div 7-c_{\text {ceses }}$ & $T=7$ & $\dot{0}-276$ & & & & & & & & & $v$ & & $13.5-1$ \\
\hline$(\cdots, \bar{c}-90$ & $\begin{array}{l}\text { GUN-SS- } \\
-6.5 \%\end{array}$ & & & & 2 & $102 \dot{Y} .4$ & 20.7 & 1.2 & & & & & SEu & \\
\hline & & $T=?$ & $(-2-2,0.0$ & & & & & & & & & $\checkmark$ & & $14.5-15$. \\
\hline$\therefore-17-972$ & GUN-SS- & & & & 2 & 1215.6 & 1.586 & 1.8 & & & & & Séru & iwiereface $\subseteq C^{\prime}$ \\
\hline & 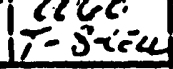 & $T-8$ & {$[-1 .-4 c$} & & & & & & & & & $\checkmark$ & & $6^{\circ}-7^{\circ}$ \\
\hline$\dot{s}-12.96$ & GUN-SS- & & & & $z$ & .91 .24 & $\pi^{2}<3$ & 1.7 & & & & & SEnd & 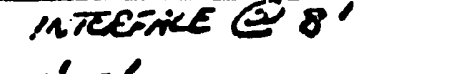 \\
\hline & $\mid r \cdot y \cdot c_{i} \mathrm{~s} s$ & $T=\bar{Y}$ & $\% \%-\dot{y c}$ & & & & & & & & & 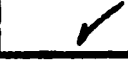 & & $8 \cdot 7^{\prime}$ \\
\hline$\therefore \cdots \cdot c^{2}-c^{2} c^{2}$ & $\begin{array}{l}\text { FUN-SS- } \\
\text { (C6-Z. }\end{array}$ & & & & 2 & $56 .+3$ & $(-6-3 n$ & 0.5 & & & & & jËí & \\
\hline & |T.q.G.cia & $T=4$ & $16 \cdot 1.96$ & & & & & & & & & $r$ & & $2 \cdot 1 c$ \\
\hline $1 .-12 \cdot 9 \%$ & $\begin{array}{l}\text { CUN-SS- } \\
c c c 3\end{array}$ & & & & $z$ & 535.6 .5 & 0.57 .6 & 1.6 & & & & & & A:EREACE, S: $7.5^{\circ}$ \\
\hline & Ticeliu & $T-1 C$ & $k \cdot 2.90$ & & & & & & & & & v & & $3.5-9.5$ \\
\hline $6 \cdot 12-9 c$ & Jun-ss- & & & & 2 & $3 \div-5.74$ & $(.53 .3$ & 1.4 & & & & & & \\
\hline & Frcices & $T-16$ & $6:-2 \cdot \pi c$ & & & & & & & & & 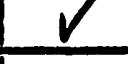 & & $16.5^{\circ}$ \\
\hline $1-12-90$ & funi-sS- & & & & 2 & 232.12 & an? & 0.4 & & & & & $\therefore$ & \\
\hline & 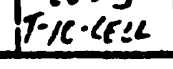 & $T-16$ & $6-2.92$ & & & & & & & & & $r$ & & $11.5 \cdot$ \\
\hline more: - All soll & I mople rear & ults ore 1 & pxi/gn & & & coluctusion & & & & & & & EUED ar: & IIE D.p. Mn \\
\hline $\begin{array}{l}\text { site correction } \\
\text { come tive }\end{array}$ & $\begin{array}{l}n \text { rector } \\
3000 \text { sec } \\
\end{array}$ & $\frac{*}{\text {. nntoes } n}$ & ined other & & & $6.65 v^{\prime \sqrt{10 x}}$ & $\frac{00 \mathrm{sec} \cdot \mathrm{t}_{1}}{20}$ & ectgrand & & & & & & \\
\hline$x$ & A:T & $1=\pi 5 \mathrm{~A}$ & $=0$ & & & ece & it1.) (s) & 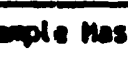 & & & & & & \\
\hline & & & & & & pically 300 & & & & & & . & & \\
\hline
\end{tabular}




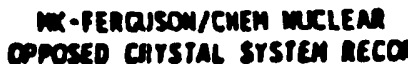

stre mue GINRIISON

ics serial no. GRJ-2 6871601

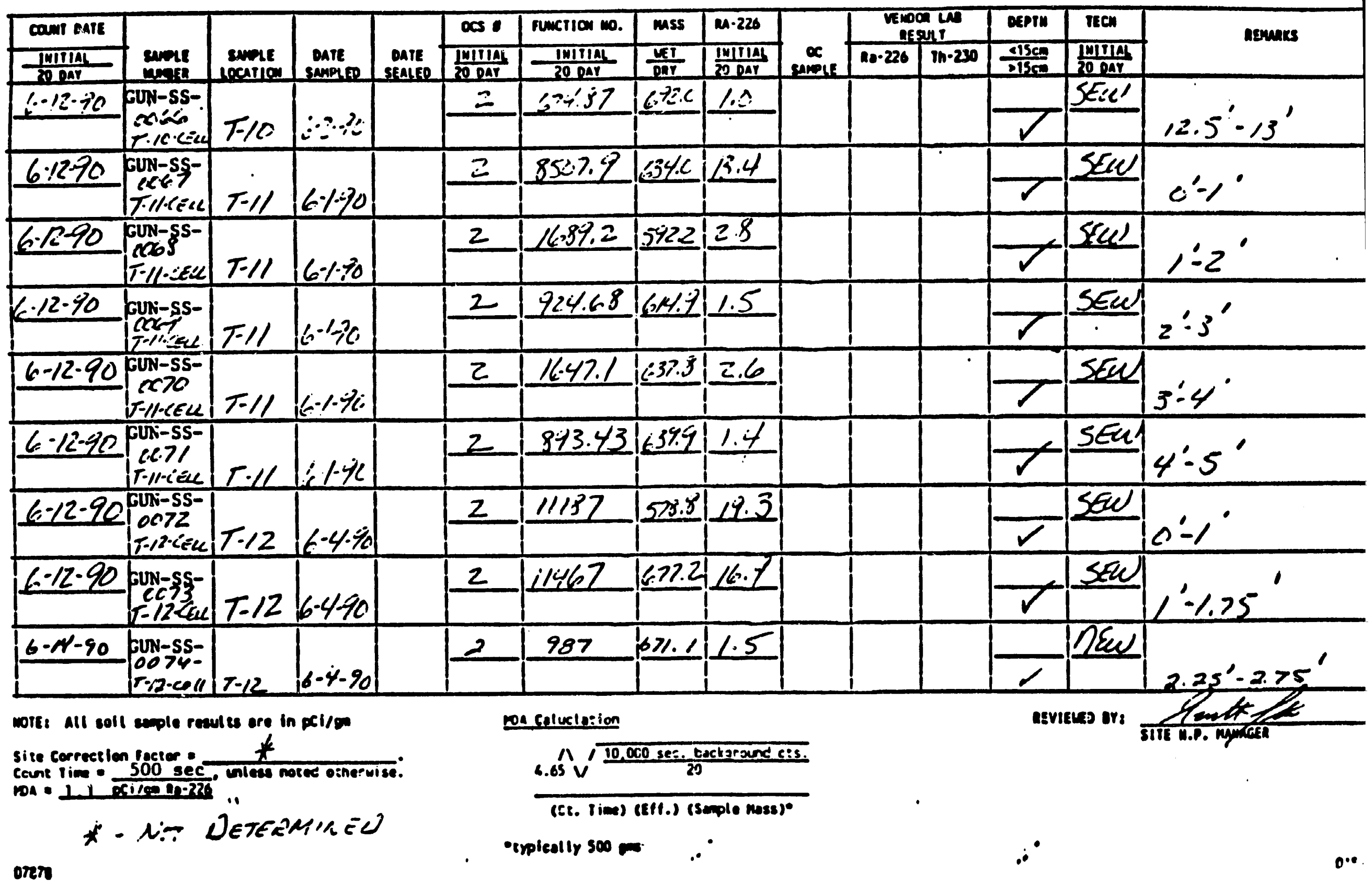


SITE Wure GINNALSON

SIre AnEA
Ces senial wo. GRJ-2 6871601

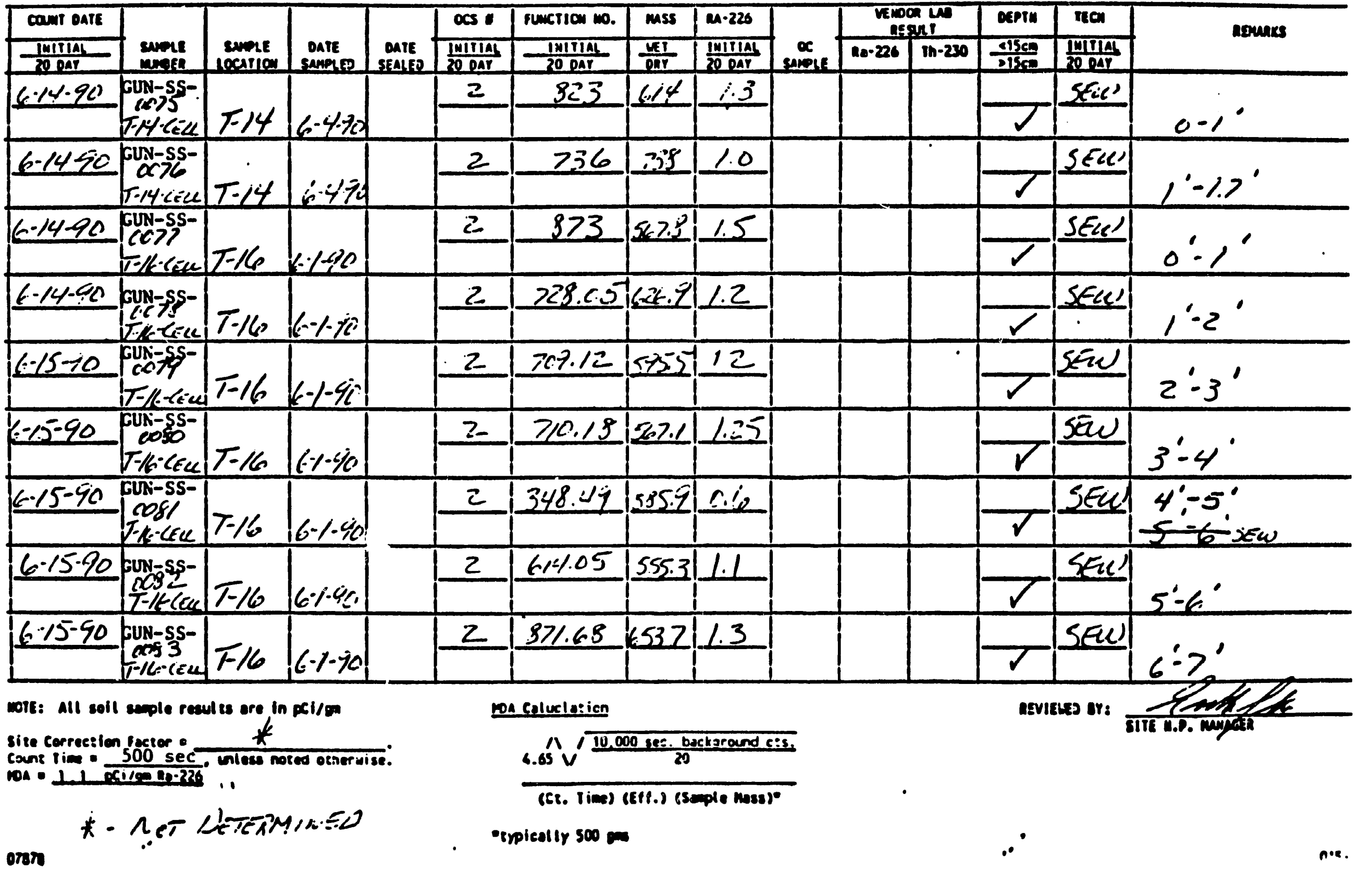


SITE Maten

\begin{tabular}{|c|c|c|c|c|c|c|c|c|c|c|c|c|c|c|}
\hline comit oale & & & & & cos. & rucriou mo. & mss & $m-226$ & & $\begin{array}{c}\text { VENOO } \\
\text { AFS }\end{array}$ & 紧 & oEPTIK & TECW & newars \\
\hline 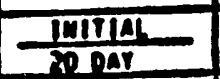 & super & $\begin{array}{c}\text { sume } \\
\text { secitios }\end{array}$ & $\begin{array}{l}\text { OATE } \\
\text { SAMPAP }\end{array}$ & $\begin{array}{c}\text { OARE } \\
\text { SEALIFD } \\
\end{array}$ & 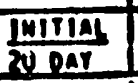 & $\frac{\text { InIIIAL }}{30 \text { PAI }}$ & $\frac{\text { QET }}{\text { DET }}$ & 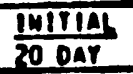 & $\begin{array}{c}\alpha \\
\sin 21:\end{array}$ & $100-226$ & $m n-250$ & $\frac{815 c m}{215 c=}$ & $\frac{1011 / 41}{30027}$ & \\
\hline $6.15 \cdots \mathrm{n}$ & SUN-SS- & & & & 2 & 6.17 .87 & $4 \times 7.7$ & 1.2 & & & & & SEQC & \\
\hline & $5=7$ iskes & 7.17 & $6-10 \div 9$ & & & & & & & & & $\checkmark$ & & \\
\hline $6-15=90$ & GUN-SS- & & & & 2 & 2322.1 & ilít & $1 \% .7$ & & & & & SEud & \\
\hline & $t=17$ tan & $T-17$ & $1,-1-8$ & & & & & & & & & $r$ & & \\
\hline $6-15-90$ & \begin{tabular}{|c|} 
cuN-SSS- \\
0.036
\end{tabular} & & & & $=$ & 223.3 & 517.2 & 1.4 & & & & & SESU & \\
\hline & T-iDiaed & $T-17$ & $1=9-9,0$ & & & & & & & & & & & \\
\hline $6-15-120$ & 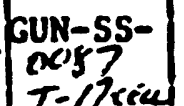 & $T-17$ & & & 2 & 470.24 & 505.4 & $1.0^{\circ}$ & & & & 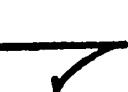 & $S E(c)$ & $3: 4^{\circ}$ \\
\hline $6-15-10$ & $\frac{1-1}{\text { Futiss- }}$ & & $(0.7 .3(2)$ & & $z$ & 432.121 & 657.3 & 0.6 & & & & & SELU & \\
\hline & $\mid x-17<<<<<$ & $T-17$ & $10.1-i c$ & & & & & & & & & 7 & & \\
\hline $6.15-40$ & CuN-SS- & & & & 2 & 547.45 & $66-9 .=$ & 0.8 & & & & & SF(e) & \\
\hline & $T=D^{2}-\mathrm{Ez}$ & $T=18$ & $16-1-i c$ & & & & & & & & & & & \\
\hline $6-15-40$ & GUN-SS- & & & & 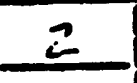 & 674.72 & 658.7 & 1.0 & & & & & sEul & \\
\hline $6-15-40$ & T-1Briece & $T=13$ & $60-1-70$ & & 7 & 11055,1 & 1,000 & 3 & & & & $\boldsymbol{V}$ & SE11) & \\
\hline & ins & $T-19$ & $1<-1.40$ & & & $(4) 2.7$ & & & & & & $\checkmark$ & & $1.5^{\prime}$ \\
\hline $6-15-90$ & fun-s & $1-19$ & $-1-9 d$ & & 2 & 4435,3 & $3^{2} 55$ & 6 & & & & & SEO & \\
\hline wres all, & 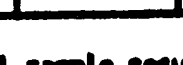 & & & & & & & & & & & & nes & \\
\hline & & wes ore & & & & Colecloien & & & & & & & & (x) \\
\hline Cod tive & & Gnloses n & & & & $6.65 v$ & & & & & & & & \\
\hline 犬 & $t_{G}^{\prime} T$ & AESTEN & Mits=2 & & & picille 300 - & & & & & & 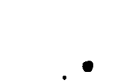 & & \\
\hline
\end{tabular}


SITE WaTE GIINNISON

SIIE MREA ces seasal wo. GRJ-2 6871601

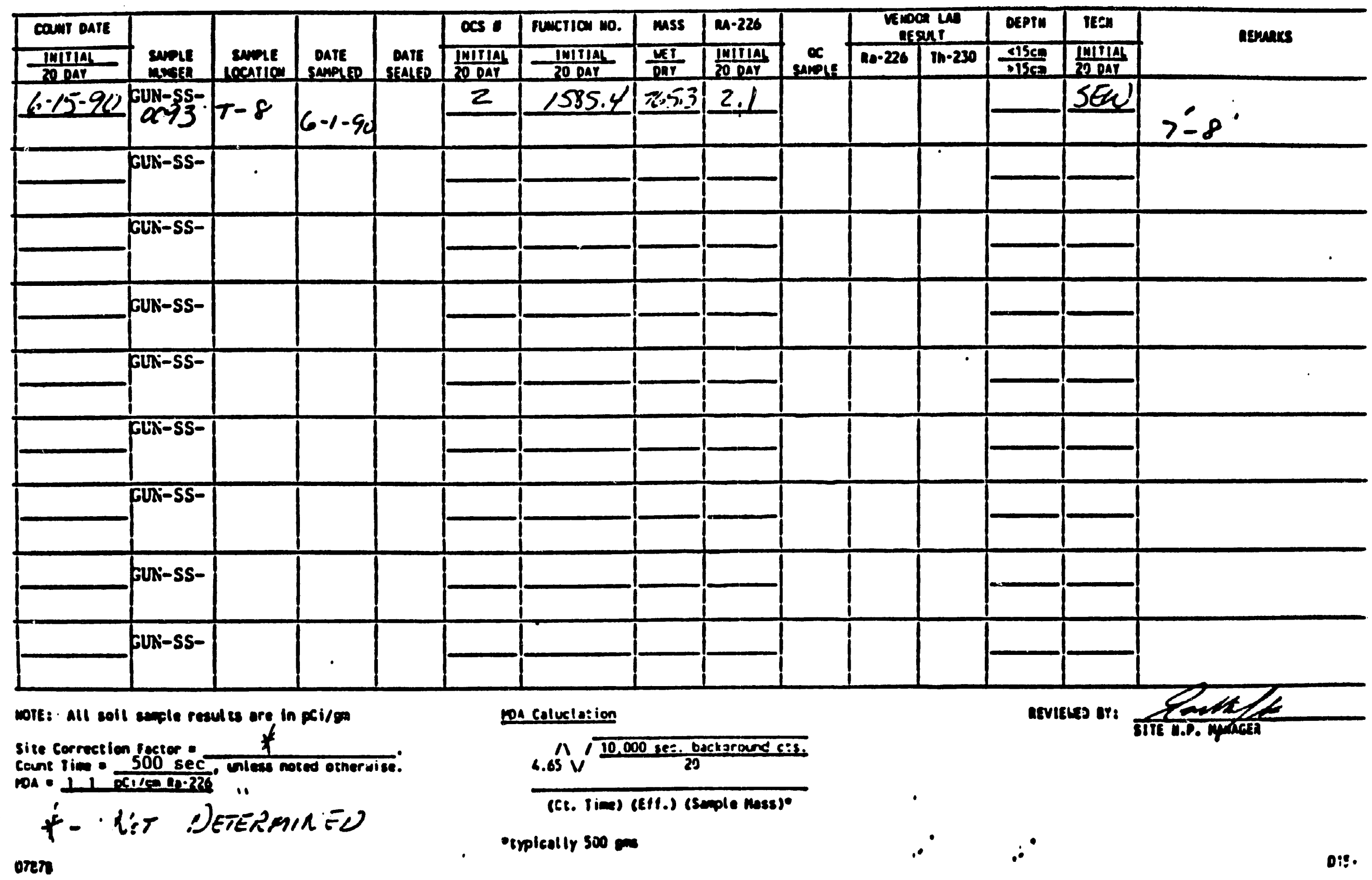


VENDOR LABORATORY TH-230 MEASUREMENTS OF

1-FT DEPTH INCREMENT SOIL SAMPLES < 4 MESH SIEVE COBBLY SUBSOIL INVESTIGATION

JUNE 1990 


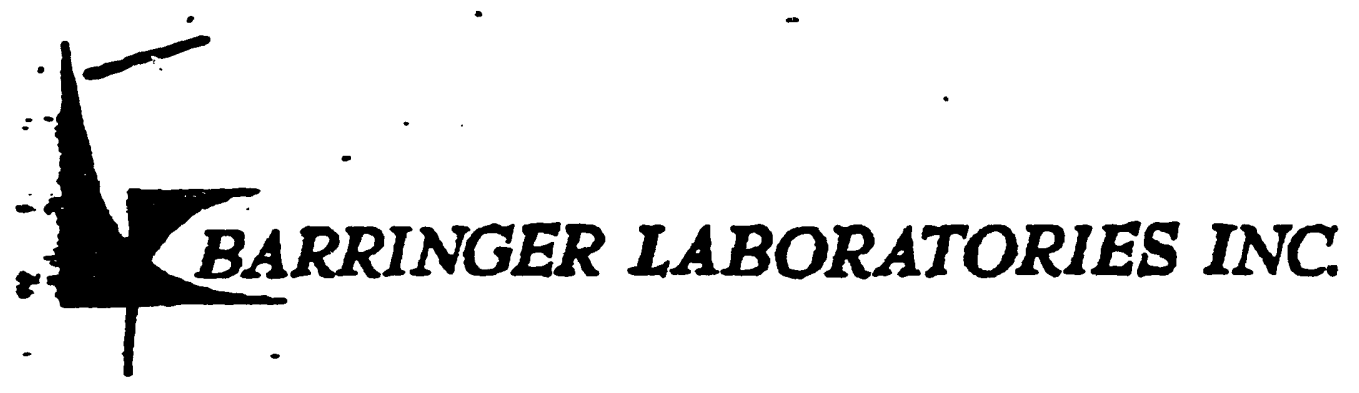

$$
\div
$$

\section{TELECOPY TRANSMITTAL}

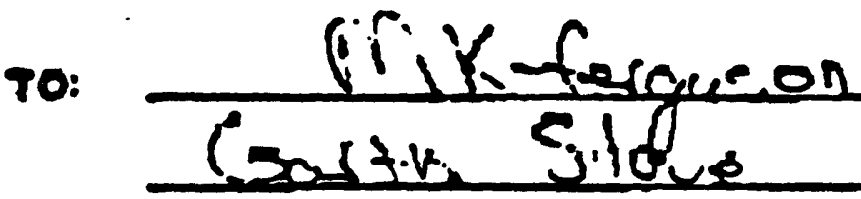

FPOM: - J vane Lasba.

SATE: $2-16$

we ARE SENDING 9 PAOES.

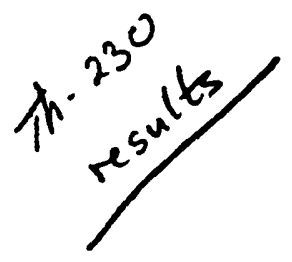




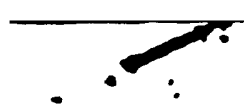

amalysess

$\operatorname{Th} 230$

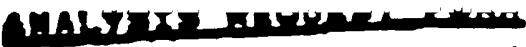

DATe mezivis: $6-19.90$

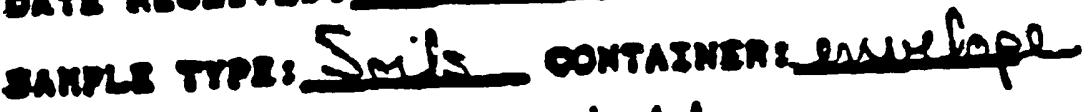

EAMPLE LOCATIOK: anabyers

catr gTaktros $6-2 \beta 20$

OATR ConpLrito:Z.rag

cunzu or ougtoors res

\begin{tabular}{|c|c|c|c|c|c|c|}
\hline LOC NUTSER & $\begin{array}{l}\text { PRAG- } \\
\text { SION }\end{array}$ & RESULT & REJULT & AVERAG & $\begin{array}{l}\text { DELo OEV. } \\
\text { Thom MEAN \& }\end{array}$ & COKMLNI \\
\hline $2621-1$ & - & -20020 & & & & \\
\hline 2 & & -10324 & & & & \\
\hline 3 & & 11624 & & & & \\
\hline 4 & & 9124 & & & & \\
\hline 5 & & $=16^{2}$ & & & & \\
\hline .6 & & $62 \pm 3$ &.$\cdot$ & & & \\
\hline 7 & & $=19^{7} 2$ & & & & \\
\hline 8 & & -3913 & & & & \\
\hline 9 & & $=15 \pm 2$ & $17 \pm 2$ & $16 \pm 2$ & 6.3 & \\
\hline 10 & & 1713 & & & & \\
\hline 11 & & $=30 \pm 2$ & & & & \\
\hline 12 & & $E 102 \pm 4$ & & & & \\
\hline 13 & & $=88 \pm 4$ & & & & \\
\hline 14 & & $E \cos 2 y$ & & & & \\
\hline 15 & & $-210 t 10$ & & & & \\
\hline 16 & & $62 \pm 3$ & & & & \\
\hline 17 & & $-7.8^{t} 1.2$ & $2.4 \pm 1.1$ & $7.6 \pm 1.2$ & 2.6 & \\
\hline$\therefore \quad 18$ & & $=3.4^{5} 0.8$ & & & & \\
\hline 19 & & $E 6.5^{2} 1.1$ & & - & & \\
\hline 20 & & $21+2$ & & & & \\
\hline 21 & & $\angle 4.0^{10.9}$ & & & & \\
\hline 22 & & $-2.3^{5} 0.7$ & & & & \\
\hline
\end{tabular}

Q.c. ctambaro nesults $23 \pm 5$

Q.e. known VALUS:

Q.c. etrzaszon mean.m

Q. C. Tangrt anmers $-3-10$ ?

oprke Recoviny, $x$

- Pscoesvar Fraction

- Eucpended freesion

T Toced Froet

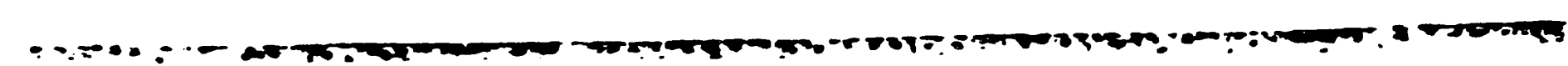




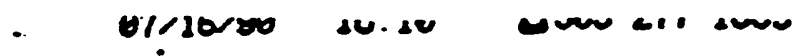

arer.

4 nenswos

ames Trese

contasmanz

cnnpler Loonszons.

ansyers

ente orantcos 2.2692

eare emuruter $2.5-10$

eindy or eugroors 129

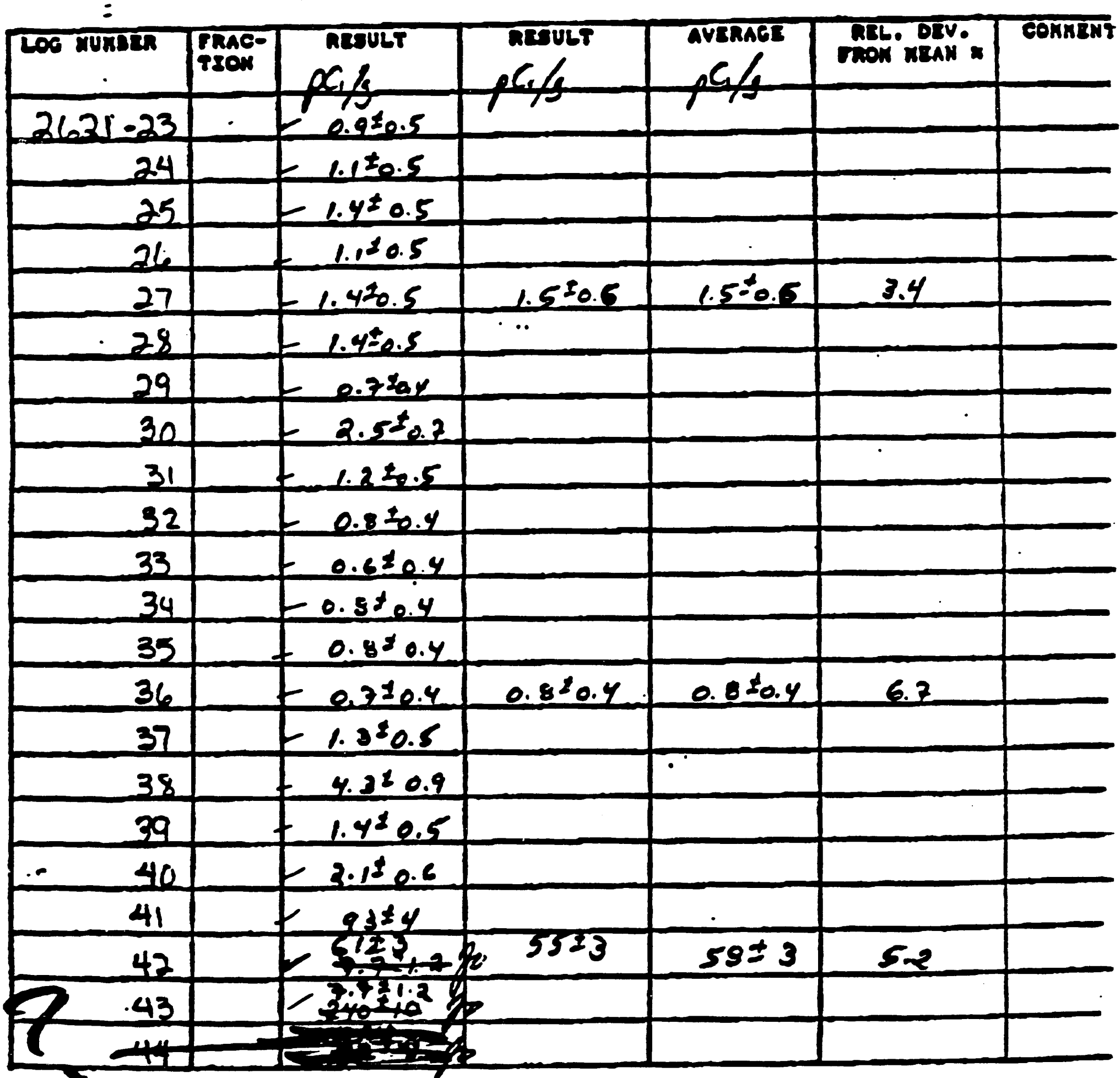

0.0. ctandand negult8

O.e. known values

Q.e. ranger namets

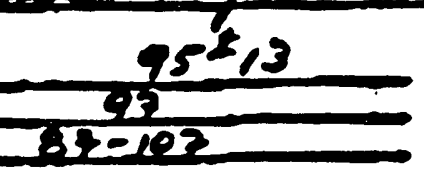

e.g. ezviatzon mean, 2.1 opike negoviny, m. 
-

por.

increses

mins stres.

canple cocatzens contaznims. arabrots 20

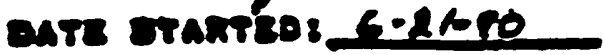

entr comprtises 2. E- 10.

endy or everoors ros

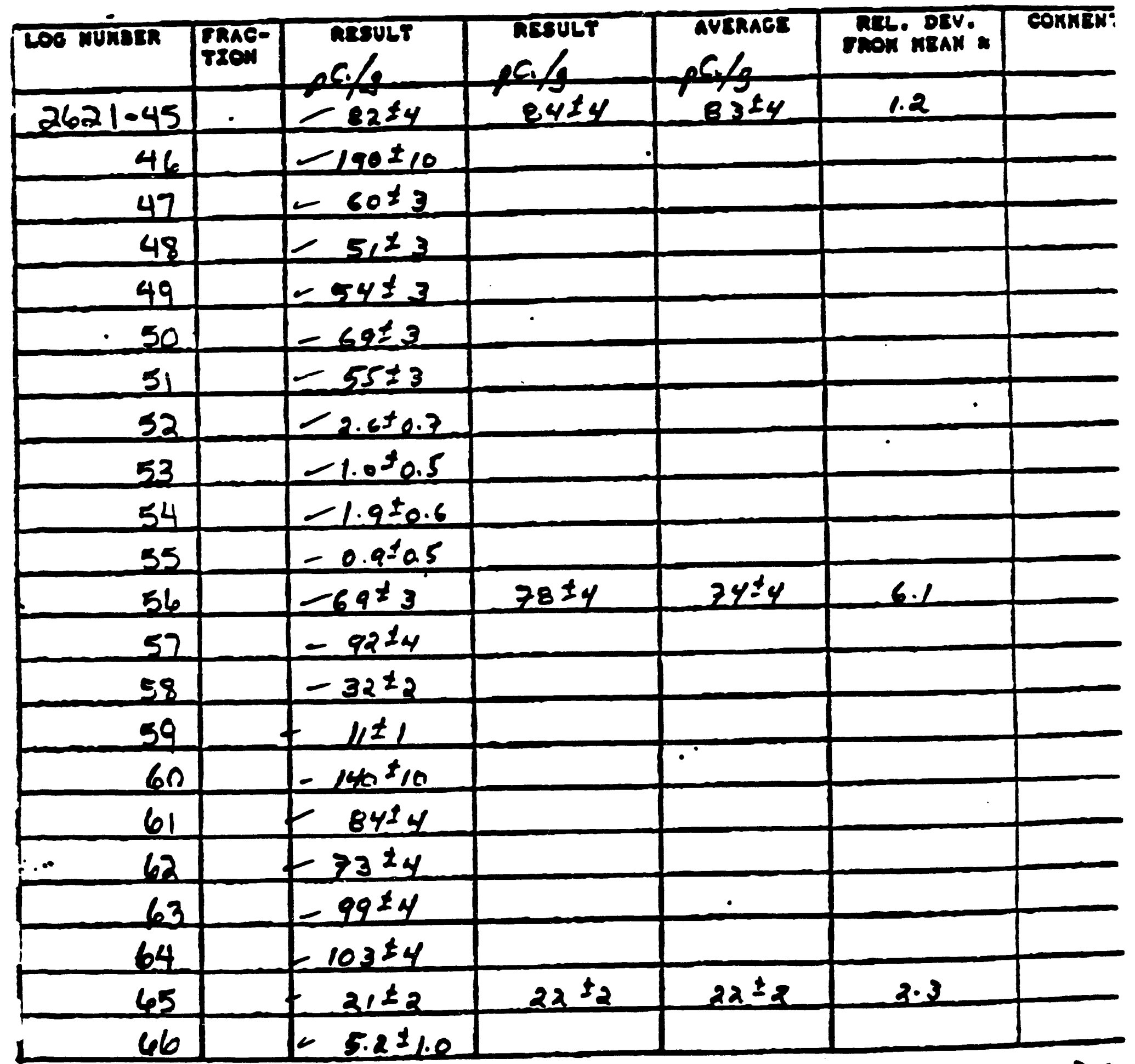

Q.e. etambaro azouls.

Q.e. known valuc:

-.5. Tarest hamers

- Oscoelved Fracrson

$\bullet$

reon MEAN *

3 


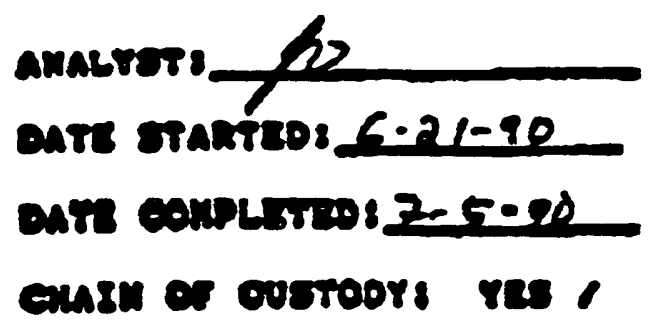

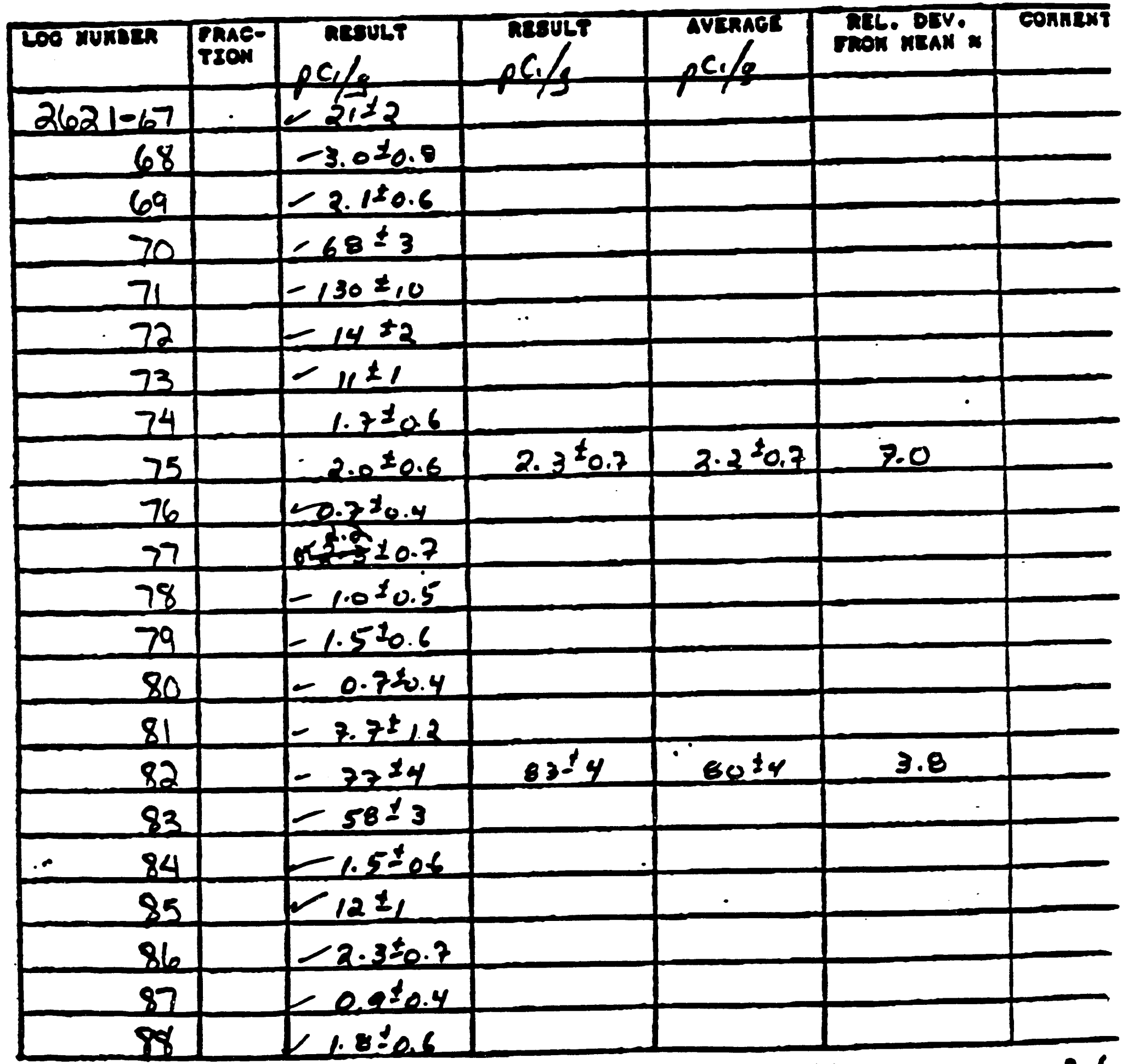

0.0. rtanoand nesult8_ g5 2 ?

-.c. kmown values 12

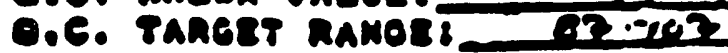

- Pscosivo Precison -.e. izvzatzon nean.m 2./

opike nceoviny, 


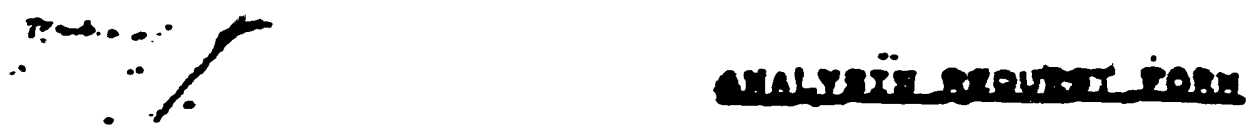

$-$ ares.

fic nesivio:

canors srosi. comrazmens

anakYTI $R_{2}$

eatre eranteol $6-2 /-10$

onte cavirtest 2.5 ro

EAMPLE Locatron:

exazk or eustoovi ves

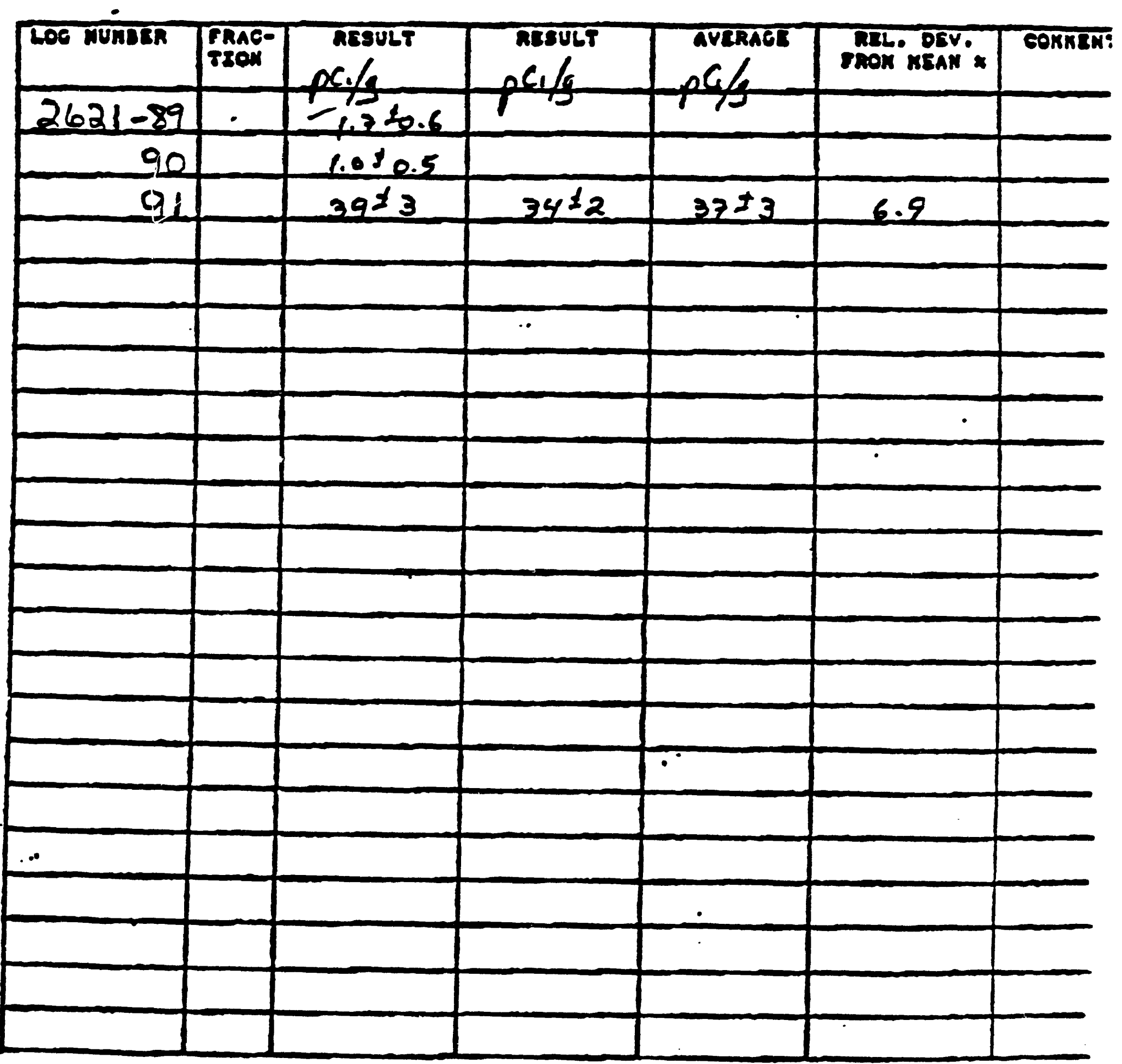

Q.e. manoano neoult8.

-.c. kmown valus

Q.e. Taneer namoes

$92 \pm, 3$ $-3-102$ -.e. Devzatzon mean.m_ Gi:

opike necovery, $x$

- - cesedvar froetion

- Bueponced Frackion

t Poted freet 


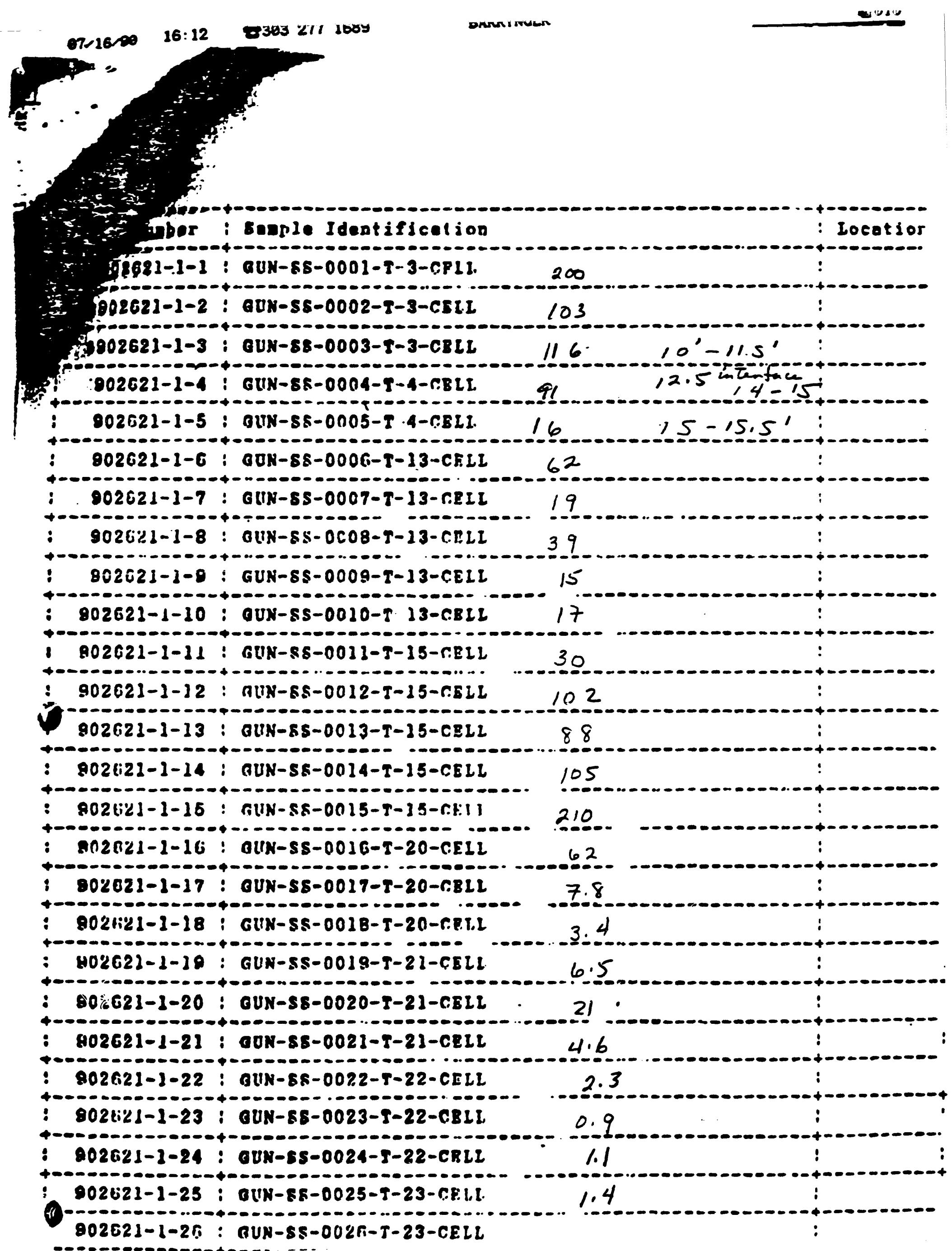




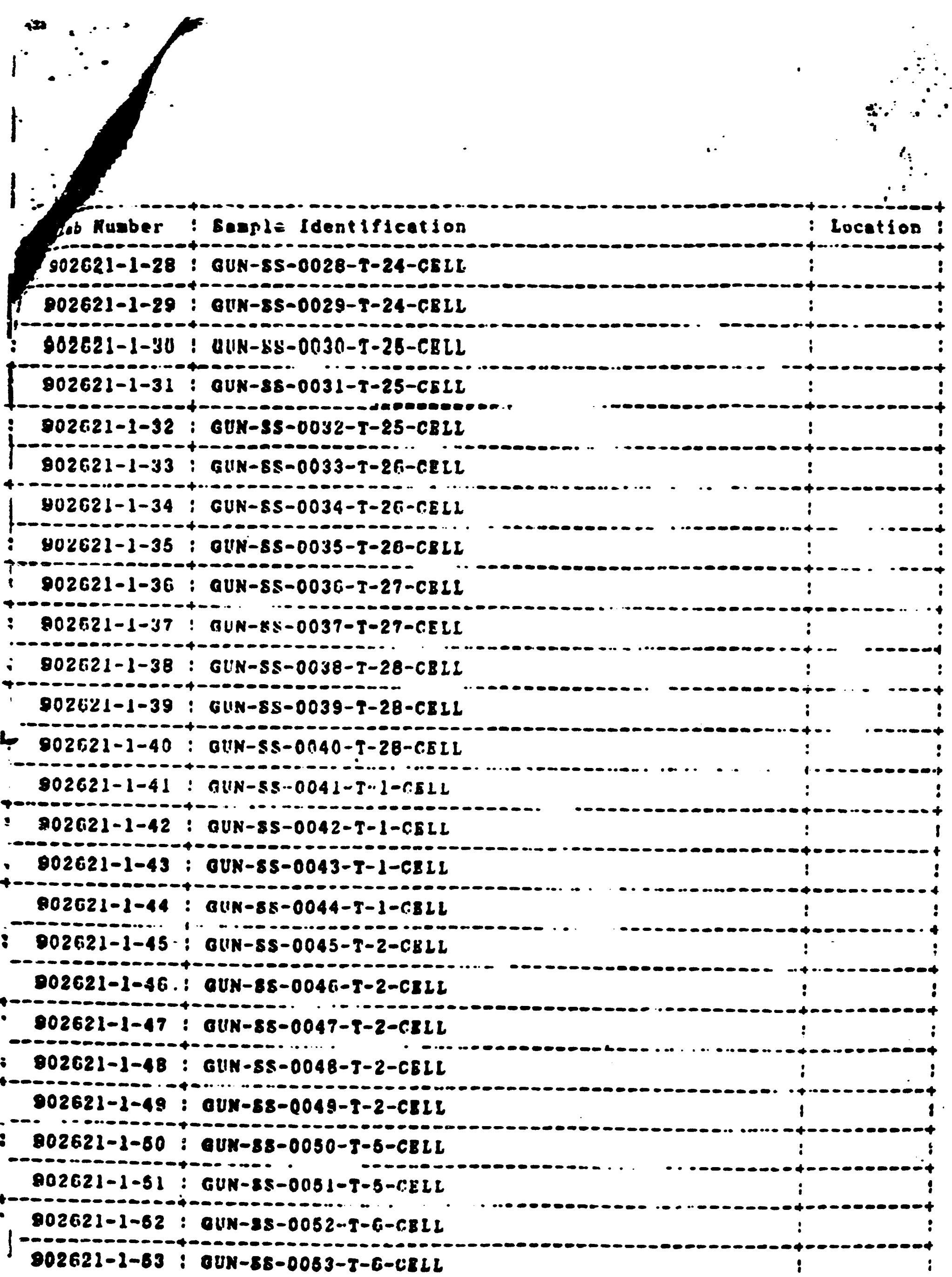




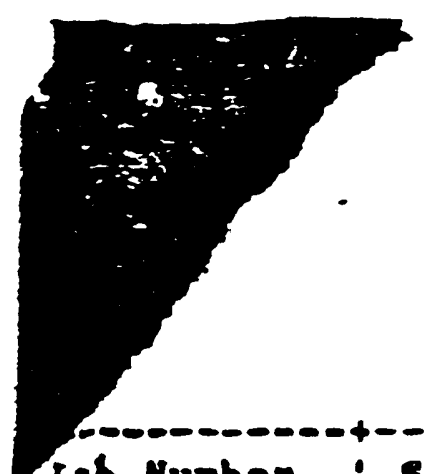

Leb Nuaber : Bapple Identificetion

902621-1-85: GUN-8S-00158-T-G-CKLL

-0-

902621-1-66: OUN-8E-0056-T-7-CEIL

902621-1-67: OUN-58-0057-T-7-CEII

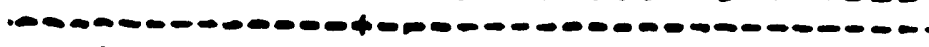

902.621-1-68: GUN-8S-0058-T-7-CBLL

\$02621-1-59: 6IIN-5s-0059-1-7-r.ELL

902621-1-60: GUN-SS-0060-T-8-CELL

\$025i2I-1-6I: GUN-SS-006I-T-Q-CBLL

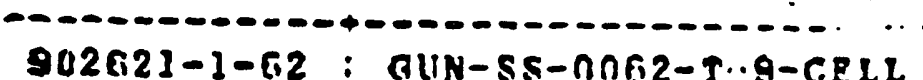

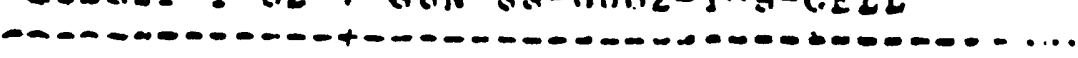

902621-1-6.3 : OUN-SS-n063-T-10-r.BLL

902621-1-64: OUN-SS-0064-T-10-CELL

$8025.21-1-6.5$ ! GUN-ES-006.5-7-10-C.8LL

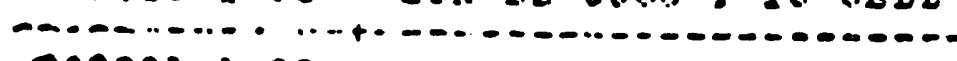

802621-1-66: CUN-55-006ri-5-10-C.ELL

$002621-1-67$ ! GUN-85-0067-T-11-CELI

$002621-1-68 ; 00 N-58-0068-T-11-6821$

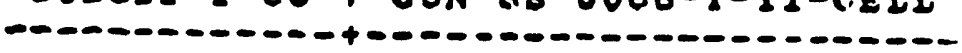

902521-1-69: CIN-8S-110R9-T-11-CELL

$802021-1-70 ;$ GUN-SE-007n-T-11-CELL

-

902621-1-71: OUA-88-0071-T-11-CELI

902521-1-73! 6(1X-88-0072-T-12-C.BLL

-

902621-1-73 :- GUN-5K-0073-T-12-C.5LL

802621-1-74: OUN-85-0074-T-12-CBLL

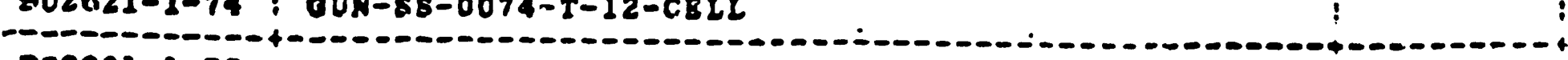

002621-1-75: OUN-8S-0075-T-14-CELL



902621-1-76: 6UN-SS-007G-T-J4-C.E.LL.

$802621-1-77$ : OUN-85-0077-T-16-CELL

-

D0282L-1-78: BUN-8E-0078-T-18-CBLL

802621-1-78: CUN-85-0079-9-16-6.ELL

- 

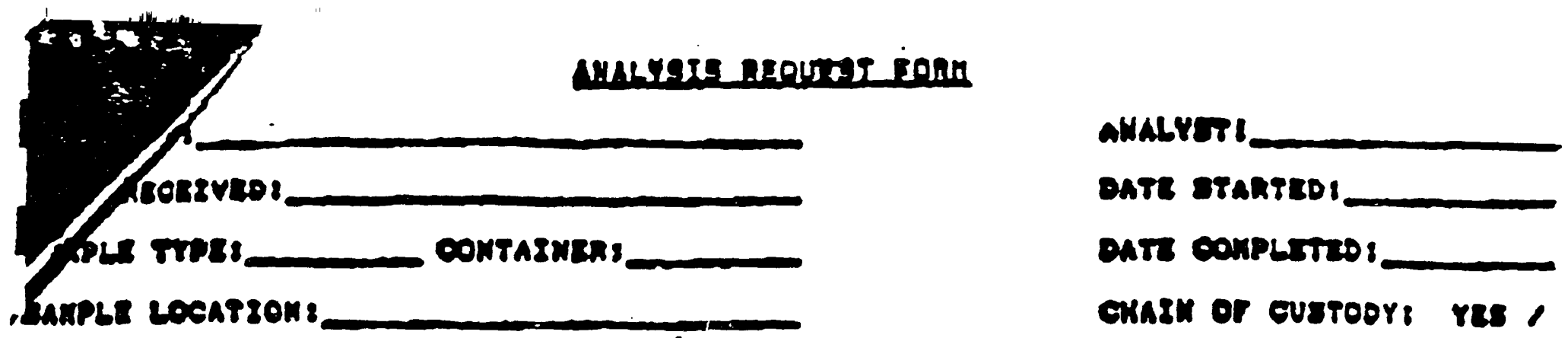

\begin{tabular}{|c|c|c|c|c|c|c|}
\hline 206 MUกDER & $\begin{array}{l}\text { Pance } \\
\text { Tson }\end{array}$ & aggutr & AESULT & AVERAGS & $\begin{array}{l}\text { REL. OEV. } \\
\text { IROK MEAK * }\end{array}$ & EOKKENG \\
\hline 2621 & - & $2.5 \pm 0.8$ & & & & \\
\hline$y^{+1}=2$ & & $6.6 \div 1.3$ & & & & \\
\hline 47 & & $4.0=1.0$ & & & & \\
\hline 48 & & $6 .+=1.2$ & & & & \\
\hline 49 & & $5.1 \pm 1.1$ & & & & \\
\hline $0,54.50$ & & $4.6+1.0$ & $\cdot \cdot$ & & & \\
\hline 5 & & $x 3 \pm 1.4$ & & & & \\
\hline 5 & & $1.0 \pm 0.5$ & & & • & \\
\hline 53 & & $1.4 \times 0.6$ & & & & \\
\hline & & $1.0=05$ & & & & \\
\hline & & $1.5 \pm 0.6$ & & & & \\
\hline 56 & & $2.2 \geq 0.7$ & & & & \\
\hline 5 & & $4.4+1.0$ & & & & \\
\hline $0.55 \quad 58$ & & $4.1 \pm 10$ & & & & \\
\hline & & $2.5=08$ & & & & \\
\hline & & $3.2 \pm 0.9$ & & & & \\
\hline 61 & & 2. $1 \pm 0.8$ & & & & \\
\hline 62 & & $2.1 \geq 0.8$ & & & - & \\
\hline 63 & & $0.3 \leq 0.8$ & & $\cdot$ & & \\
\hline 64 & & $3.4=0.9$ & $2.1=0.8$ & $29 \neq 0.9$ & $19 \%$ & \\
\hline 65 & & $8 y=1.4$ & & & & \\
\hline 66 & & $0.4 \pm 0.4$ & & & & \\
\hline
\end{tabular}

O.e. Etandano ncaulti_ Y8I6

Q.c. Kmown values

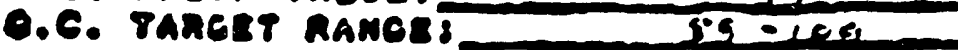

- Dscoedrac froesson o.c. orvzapzon mean.m j.o opike aceoverr, 
Lin....

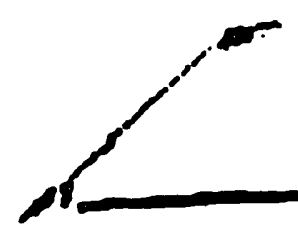

- messvior

cimpes rets.

COMYAEHER!

anPLS Locarzon 8

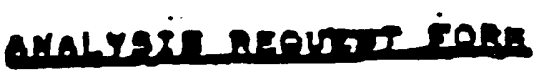

amabyors.

cati etantcos

cats conpletrot

cuazn or ouetooys res .

\begin{tabular}{|c|c|c|c|c|c|c|}
\hline LOS nUTDER & $\begin{array}{l}\text { PRAC- } \\
\text { TSON }\end{array}$ & $\begin{array}{l}\text { acsurt } \\
\rho h \% g\end{array}$ & RESULT & AVCRAGE & $\begin{array}{l}\text { REL DE DE. } \\
\text { TROA MLAN " }\end{array}$ & CONREN \\
\hline $2 \operatorname{lo} 21=$ & - & $2.1=0.7$ & & & & \\
\hline 24 & & $4.6 \pm 1.0$ & & & & \\
\hline & & $2.0=0.7$ & & & & \\
\hline & & $1.3=0.6$ & & & & \\
\hline & & $1.4 \pm 06$ & & & & • \\
\hline & & $1.3: 0.6$ & . & & & \\
\hline & & $1.5 \pm 0.6$ & & & & \\
\hline & & $3.4 \div 01.0$ & & & & \\
\hline & & $1.2=0.6$ & & & & \\
\hline & & $1.3=0.6$ & & & & $\because$ \\
\hline & & $0.9+0.5$ & & & & \\
\hline & & $1.3=0.6$ & & & & \\
\hline 35 & & $1.5 \div 0.6$ & & & & \\
\hline & & $1.0 \div 0.5$ & & & & \\
\hline - & & $1.3=0.6$ & & & & \\
\hline & & $3.6 \div 1.0$ & & & • & \\
\hline & & $3.3: 0.9$ & & & & \\
\hline 40 & & $2.9 \pm 09$ & & & & \\
\hline 41 & & $1.7+0.7$ & $\cdot$ & $\dot{-}$ & & \\
\hline 42 & & $3.1 \pm 0.9$ & & & & \\
\hline 43 & & $1.3 \pm 0.6$ & & & & \\
\hline 44 & & $3.8 \div 1.0$ & & & & \\
\hline
\end{tabular}

-.e. otanoano nesults

$113 \div 2$

-.e. known vabues

O.6. Panger namori o.c. oevzatzon mean,m.

sezke accoveny, $x$ 


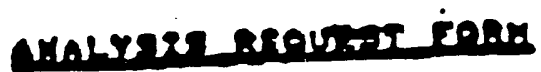

anameres. Ra 226

anr meprutor $\quad 6.19 .90$

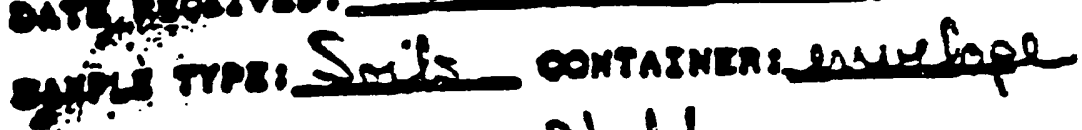

inisis Leatsons

shat

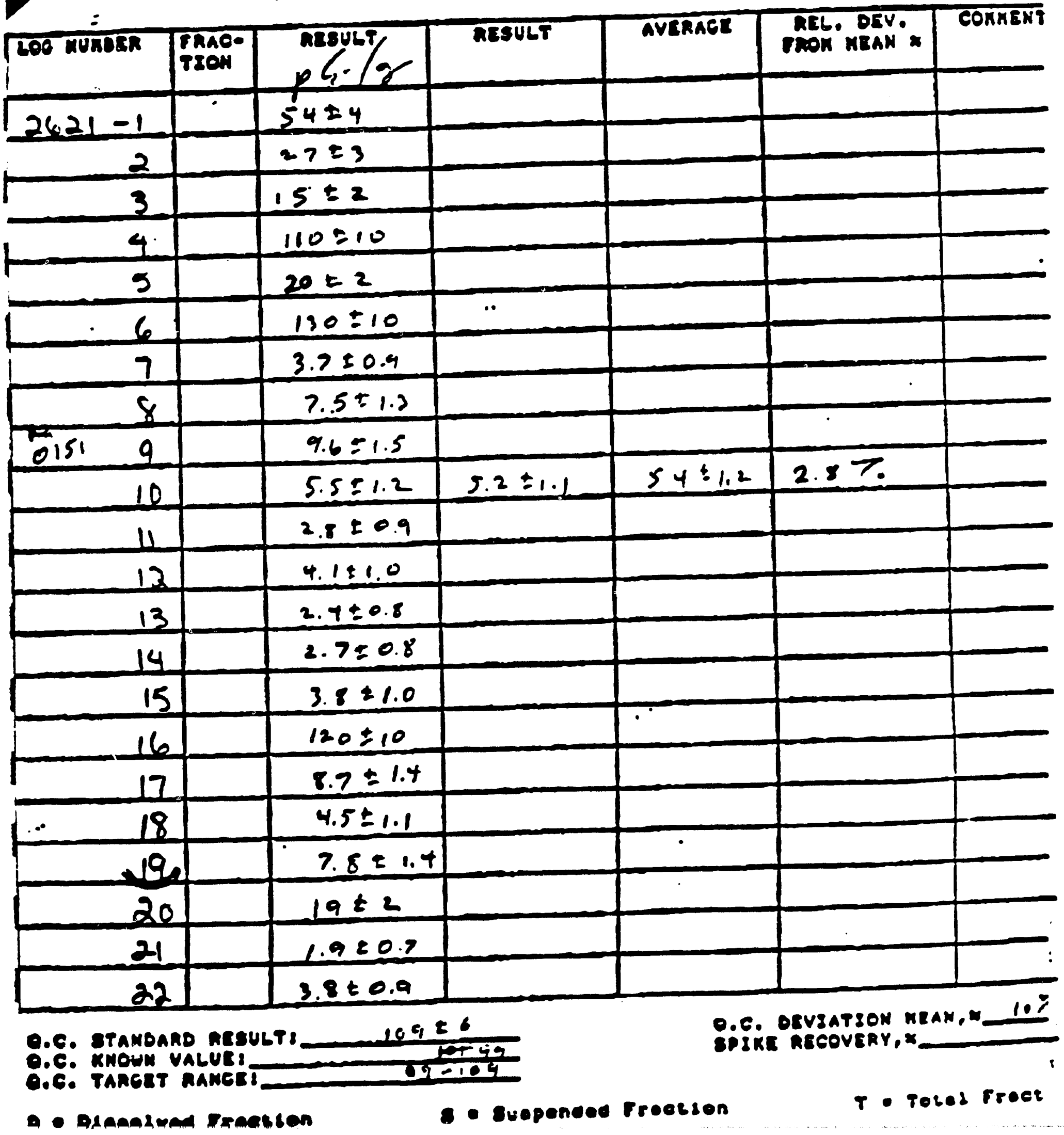

awabrers innot

onte etanteos

6.25

Datr complustor $7-12$

enazn of custoor, yes 


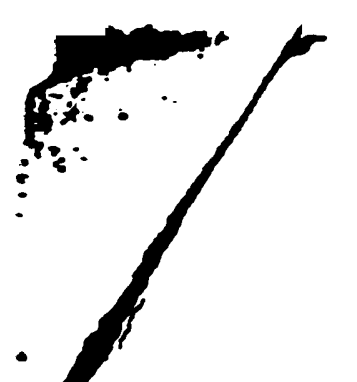

Lab Number : Sample Identification Locaition:

\$0262 I-J-55: (BUA-5S-0055-T-6-C.EL.L C-D.-. 802621-1-86: OLN-SS-005r-T-7-CEIL $9021: 21-1-39:$ (HUN-SS-0057-T-7-TELLL 907.621-1-58: 6IN-SS-0058-T-7-C:ELU. $902621-1-59$ : 6IIN-5S-0059-9-7-CELI

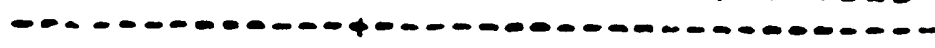
- $9025.21-1-60$ : GUN-SS-0กEOO-I-8-CEIL

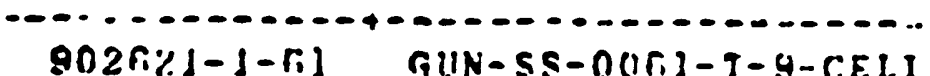
-

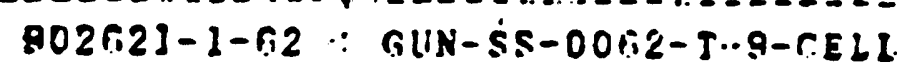
$80 \% 621-1-6.5$ : RiLs-s5-00R3-T-10-r.5LL

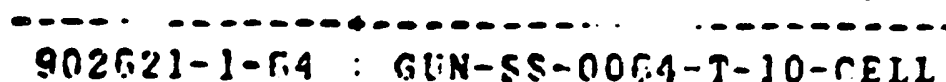

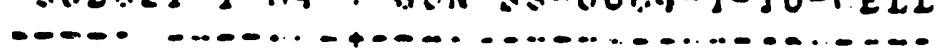
902621-1-1:5: G1!N-ss-n(16.5-T-10-1:L12

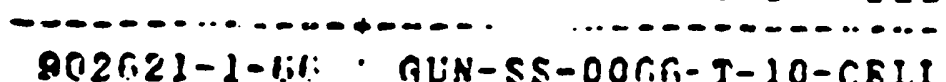

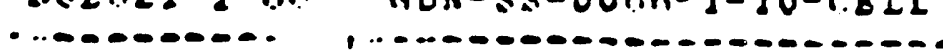
902 T2l-1-67 : RUN-SS-0067-T-11-CELL

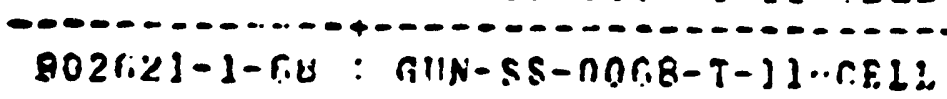
S021.21-1-6.

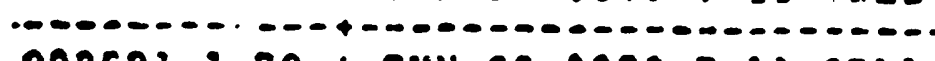
90262l-1-70: 6IIN-SS-0070-T-11-CELL

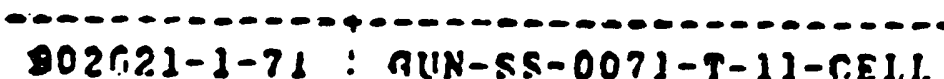

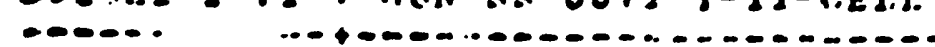

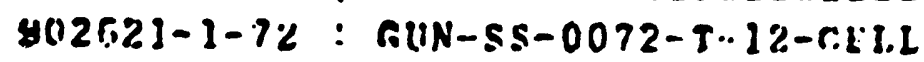
$902621-1-73: 061-5 s-0073-9-12-5.5 L 2$

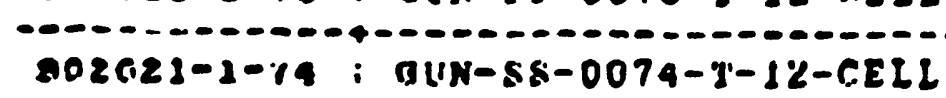
-0.- - 90262J-1-75: RIIN-SS-11075-T-14-CELLL $302621-1-76:$ r

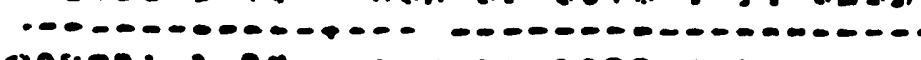
902621-1-77: QUN-SS-0077-T-16-CELL

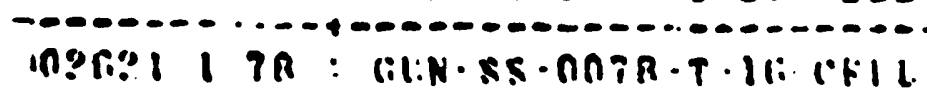

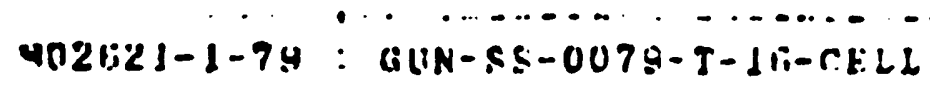

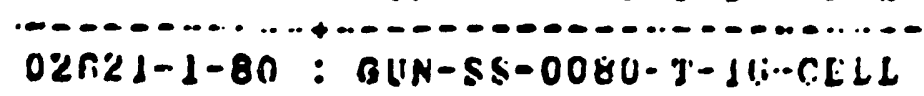

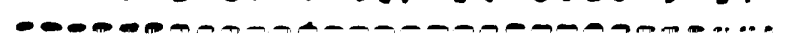




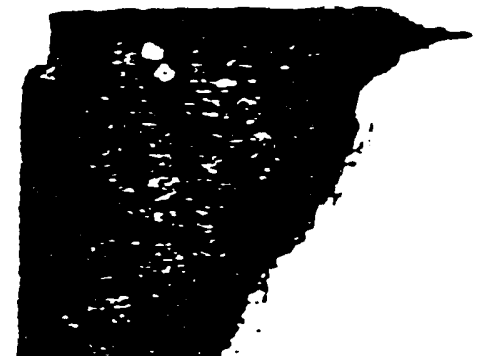

$-z^{2}$

$\because$ Lubet I stwple Idexelflation

T2621-1-28 ! OUN-SS-OU28-T-24-CELL

$002621-1-29$ : GUN-5S-0029.. T.24-CELL

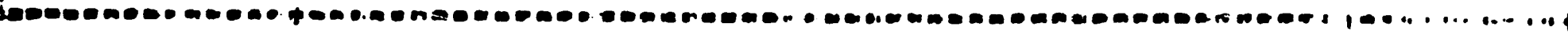

90262J-1-30: GLN-SS-0030-T-25-C.ELL

$002621-1-31$ : GUA-S3-0031-T-25-c4bL

$902621-1-32$ : CUN-SS-0032.-T-25-CELL

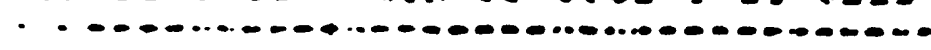

$9026.21-1-33: 54 A-5 s-0033-1-26-1: k L 1$

$902621-1-34:$ GUN-SS-0034-T-20-CELL

$902621-1-35$ : GUN-SS-0035-T-26-RELL

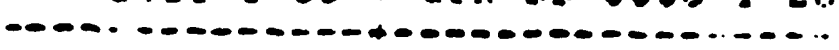

$9021.21-1-36:$ : GIN-SS.0031i-T-27--1:ELL.

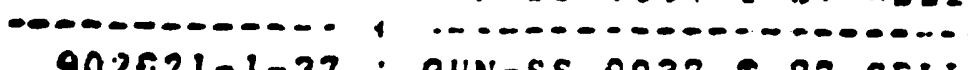

902621-1-37: RUN-SS-0037-T-27-6.51L

$902621-1-38: 6 U N-5 S-0038-8-28-C 8 L L$

$902521-1-34$ : GUN-SS-0039-T-28.1:ELL

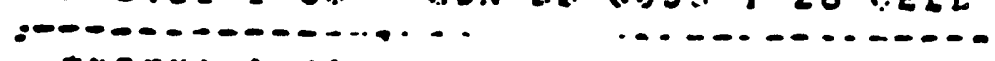

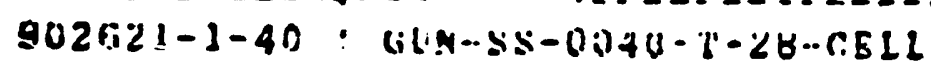

$902821-1-41:$ : GUN-SS-0041-T-1-CELL

$902621-1-42$ : GUN-SS-0042-T-1-CELL

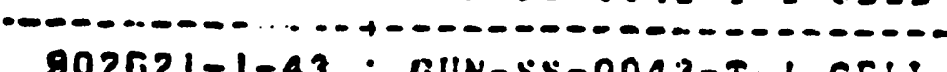

$902 \pi 21-1-43: G(1 N-S S-0043-T-1-C . E L L$

9026121-1-44: Blin-55-0044-T-1-LiblL

$902621-1-45$; UUN-SS-UU45-T-2-CELL

-

902621-1-46: OUN-SS-COU4R-T-2-CELL

902621-1-47:GLN-SS-0047-T-Z-RELL

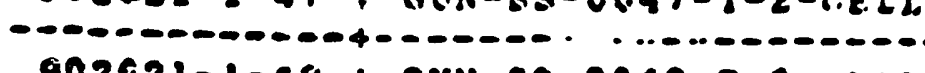

90262L-1-48: 6 IIN-ES-0048-T-2-CELL

$802621-1-49: 011 N-58-0049-T-2-C E L L$

-OC.......... . . .

9026.21-1-5n: aUn-SS-005n-T.5.C.ELL

$80 \% 621-1-51: 011 N-5 S-0051-7-5-C E L L$

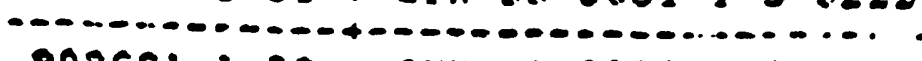

902521-1-52: 6UN-SS-0052-I-G-CELI.

-

902R.21-1-53: RUN-SS-0053-T-R.R-RELI. 


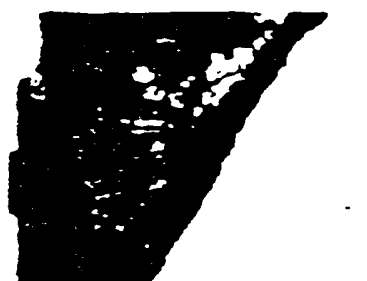

ab Number : Sample Identification

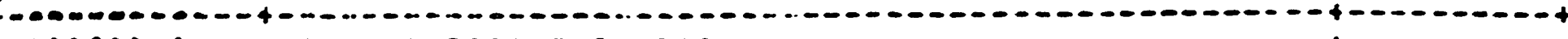

902021-1-1: OL1N-55-0001-T-3-CELI : $\quad 402021-1-2$ : GUN-55-0002-I-3-10ELI $902621-1-3 \cdot$ GUN-SS-0ON3-T-3-RELL : $902621-1-4$ QLN-5S-0004-T-4-C.ELL -.. - $902221-1-5$ : $902051-1-6:$ : 6UN-5S-0006-I-13.0.8LI

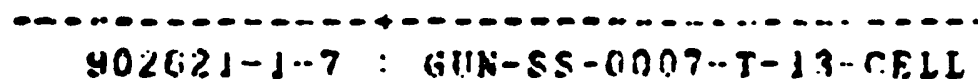

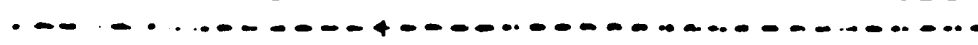

902621-1.8 : GUN-SS-0008-T-13-CELLL $902621-1-9$ : GUN-SS-0009-T-13-C.5LL . $902621-1-10: 0(1 N-55-002 n-T-13 \cdot C .5 L L$ S02021-1-11 : 611N-ss.0011-1-15-1ELi. \$02021-1-12: GLN-SS-0012-T-15-CELI Y02n:1-1-13 : GLN-5S-0013-T-15-C:BLL

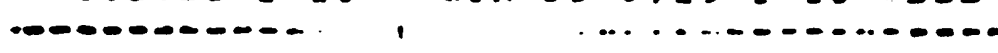

$9026 \% 1-1-14 \cdot$ rillN-SS-0014-1-1-25-1:EL1

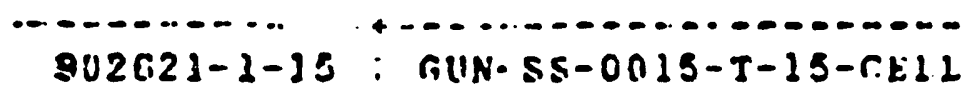

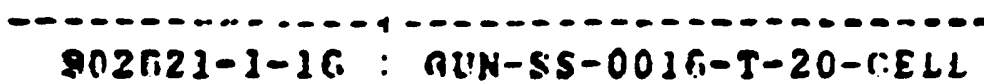

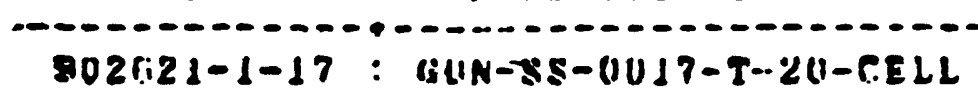

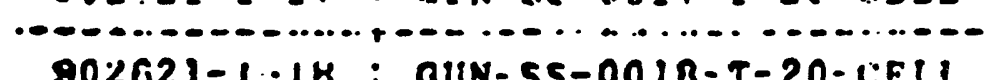
902 त21-1-16: OUN-5S-001B-T-20-1:EL.2

902021-1-19: GU1R-ES-0019-T-21-1:ELL 802621-1-20 : RUN-SE-0020-T-21-rELl, C.........._$802021-1 \cdot 21:$ HillN-SS-00\%1-T-21-C.812 P02621-1-22 : 6U1N-85-0022-T-22-CELL 80262L1-1-23 : RUN-SS-0023-T-22-rEII - $02021-1-24:$ QUN-58-0024-T-22-6.511 ans $9025.21-1.25:$ BUN-\$S-01025-g-23-C1:11 $00252 j-1-2 r_{0}:$ aUN-Ss-0026.

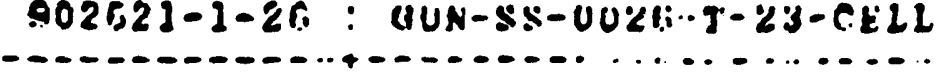

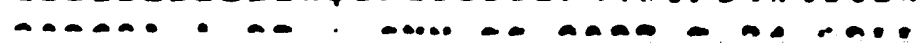




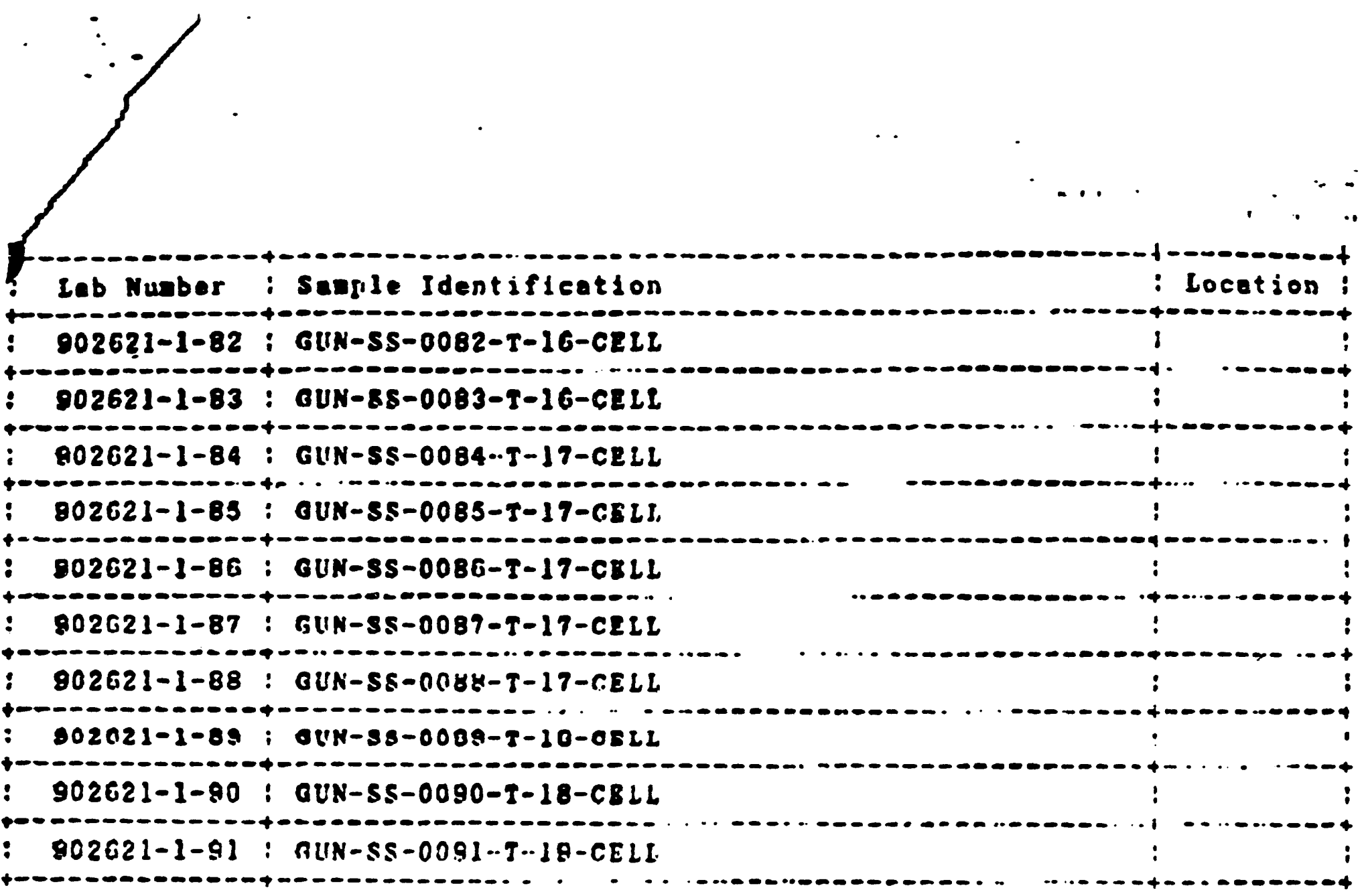




\section{APPENDIX D}

TEST PIT LITHOLOGICAL LOGS AND RADIOLOGICAL DATA COBBLY SUBSOIL PIT INVESTIGATION NOVEMBER 1992 
LITHOLOGICAL LOGS

COBBLY SUBSOIL INVESTIGATION

NOVEMBER 1992 
ENGINEERS

AND

CONSTRUCTOAS

Ro MK-FERGUSON COMPANY

a womison xmussen comparr

Isnocuntritens office

OnE ERievizu Rava

arveran om usa cin

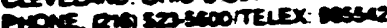

nen, to uxfenguson compan

nEM idul actions

contanctoout ponect

contractom

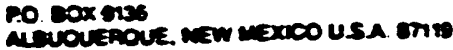

Jamuary 18, 1993

$33-3050-046$

MI. Steve Hamp

Site Manager

0.S. Department of Energy

Oranium Mill Tailings Remedial Action Project Office

First Nationel Bank Building

5301 Central Avenue M.E.

Suite 1700

Albuguerque. New Mexico 87108

SUBJECT: Cobbies-To-Fines Test Pit logs

Gunnison, Colorado

REFERENCE: Contract No. DE-ACO4-83ALI8796

Dear Mr. Hamp:

Enclosed please find the subject logs for your informstion. CWMFESI Iesults will te forwarded when they become arailable.

If you have any questions, please contact Bob Hindman at 246-2557.

sincerely.

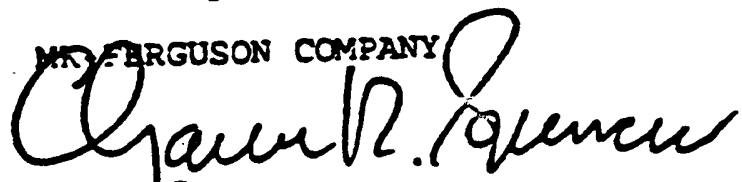

C. R.I Spencer

Construction Engineering Manager

CRS/RCH/mo

Attachment

ce: wartachments:

:J. MCBEe, TAC/OMTRA

w. Naugle. CDH 


\section{Cobbles-To-Fine Test Pit Locations}

MK-F Test Pits

\begin{tabular}{|c|c|c|}
\hline & $\begin{array}{l}N 3075 \\
N 22900 \\
N 22880 \\
N 2640 \\
N 2910 \\
N 2530 \\
N 2725 \\
N 2760 \\
N 2745 \\
N 2965 \\
N 2300 \\
N 2225 \\
N 2415 \\
N 2080 \\
N+650 \\
N 1810 \\
N 1840 \\
N 1720\end{array}$ & 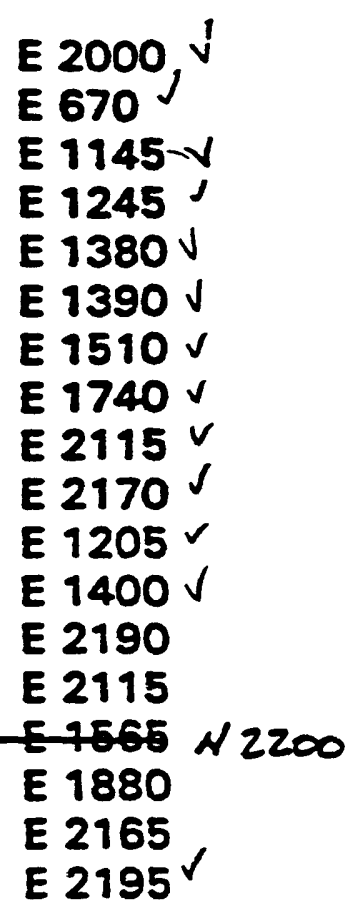 \\
\hline
\end{tabular}

IAC Test Pits

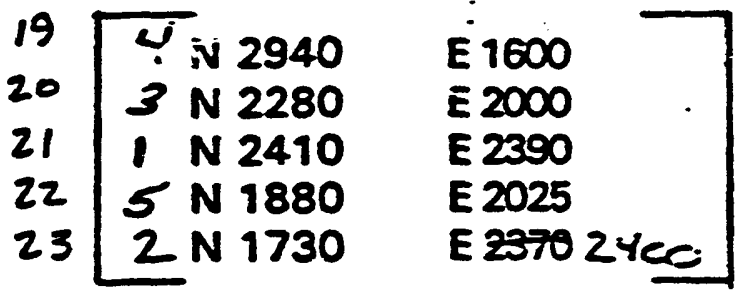



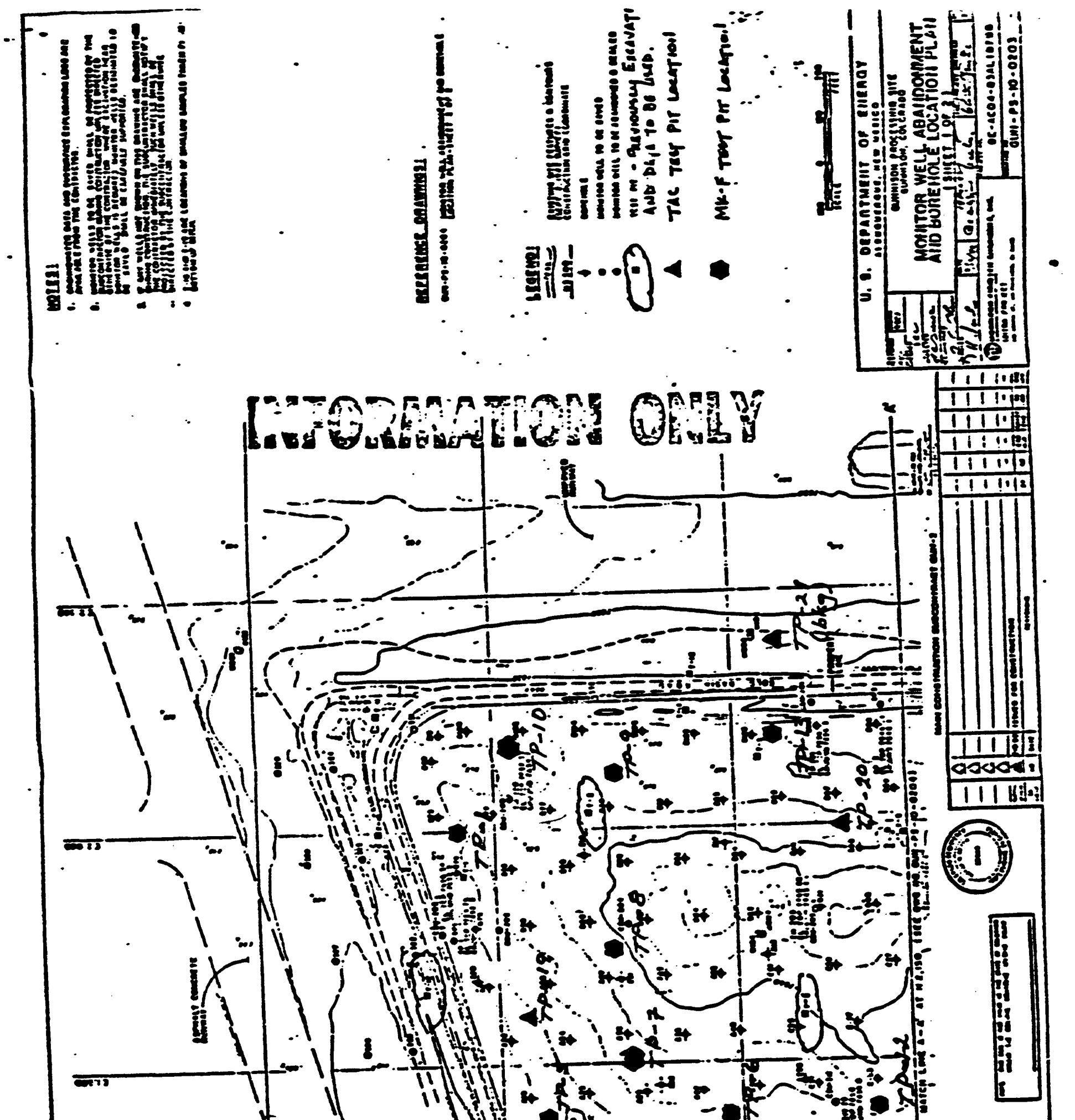

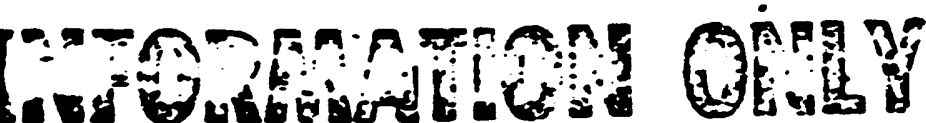

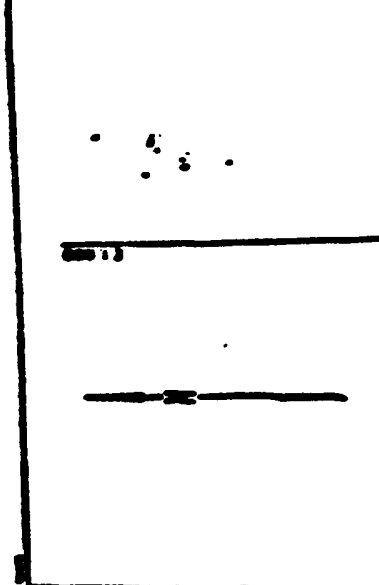




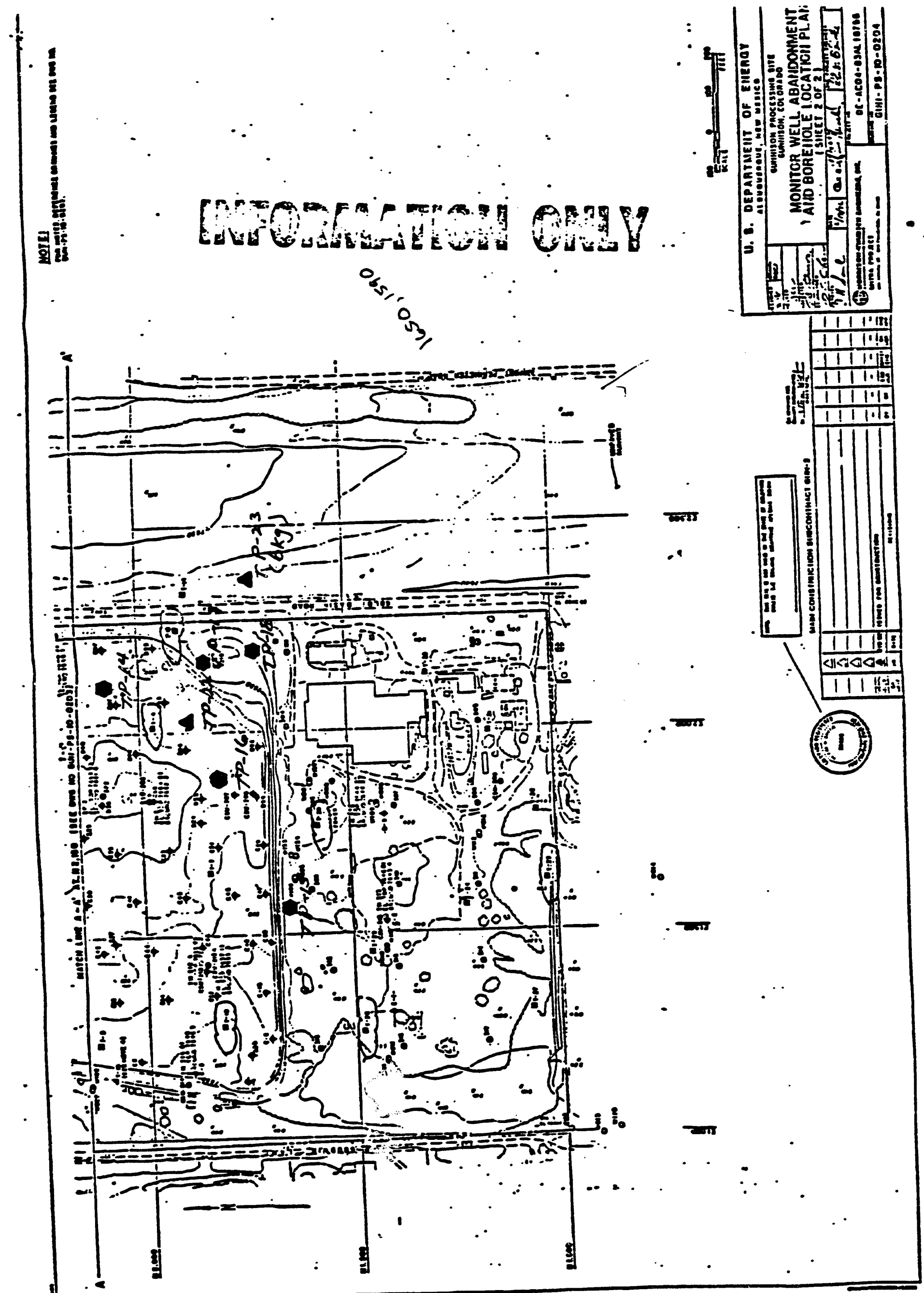




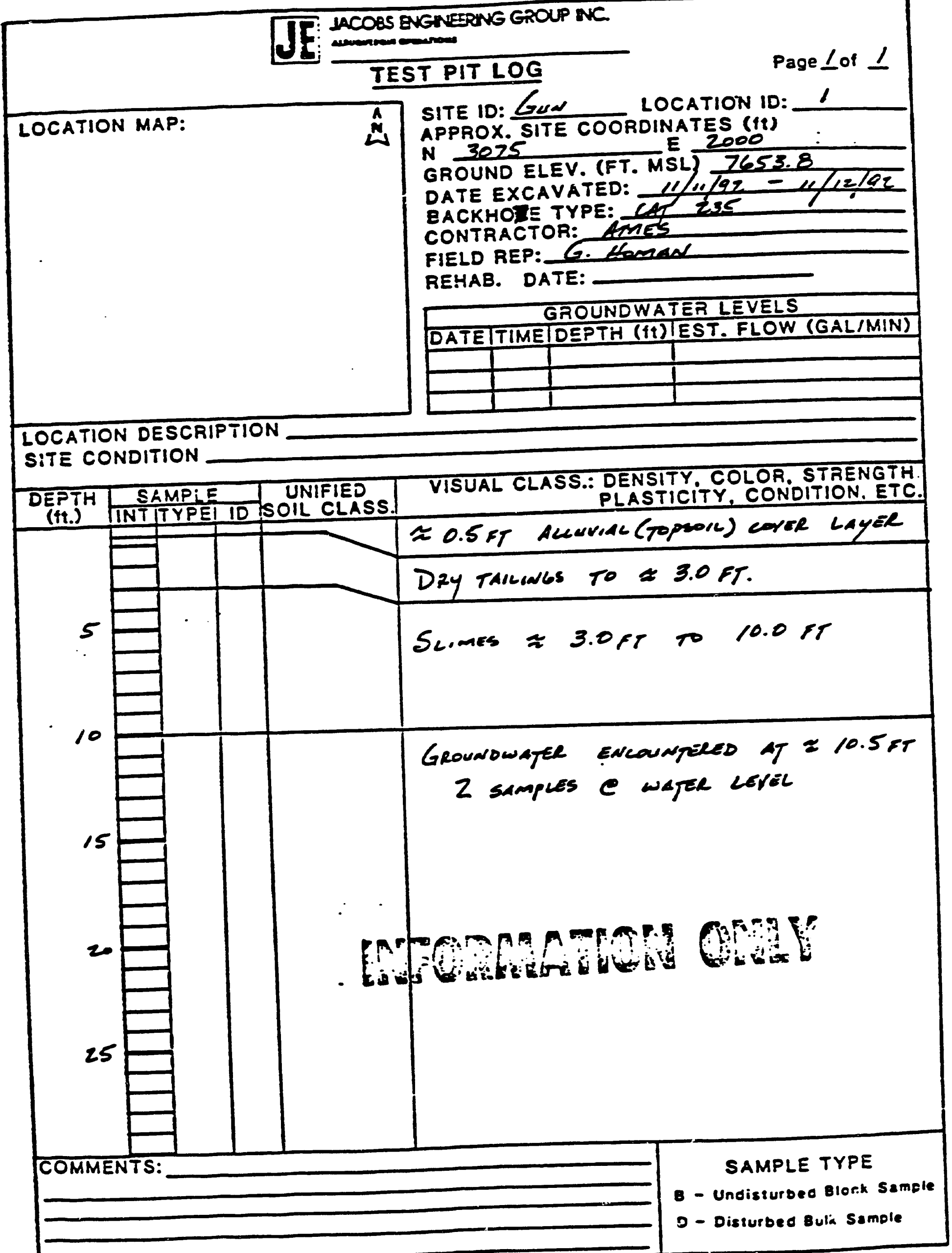


COBBLES-TO-FINE TEST PIT DATA

TEST PIT *:

LOCATION: $\mathbf{N} 3075$

E 2000

IAC TEST PIT: YES No $x$

CDH SAMPLES TO BE TAKEN BY MK-F/CWMFES: YES $\_$NO

SURFACE EIEVATION: 7653.8

* DEPTH OF TAIIINGS:

$10^{\prime}$

DEPTH OF GROUNDWATER:

$10.5^{\circ}$

SAMPLE INTERVAL: 2 sAmedes fINEN $e$ WATER LEVEL

COMMIENTS:

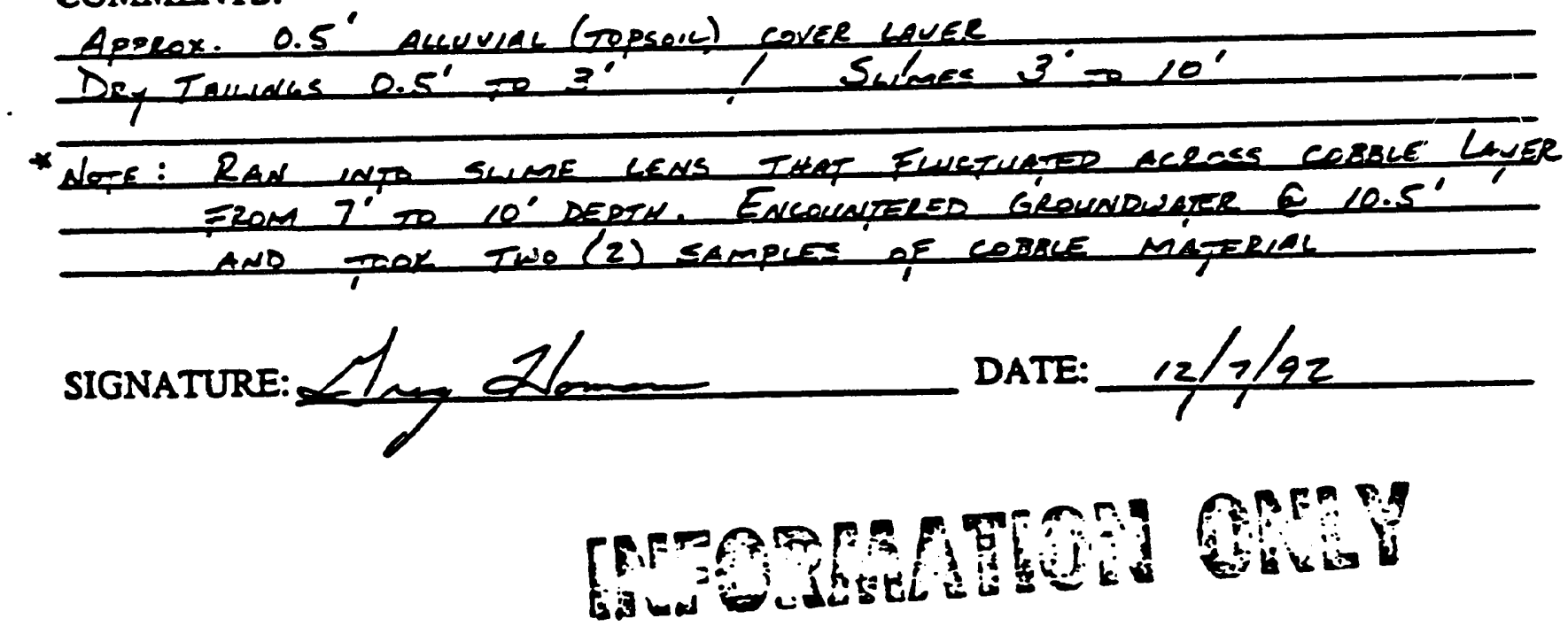




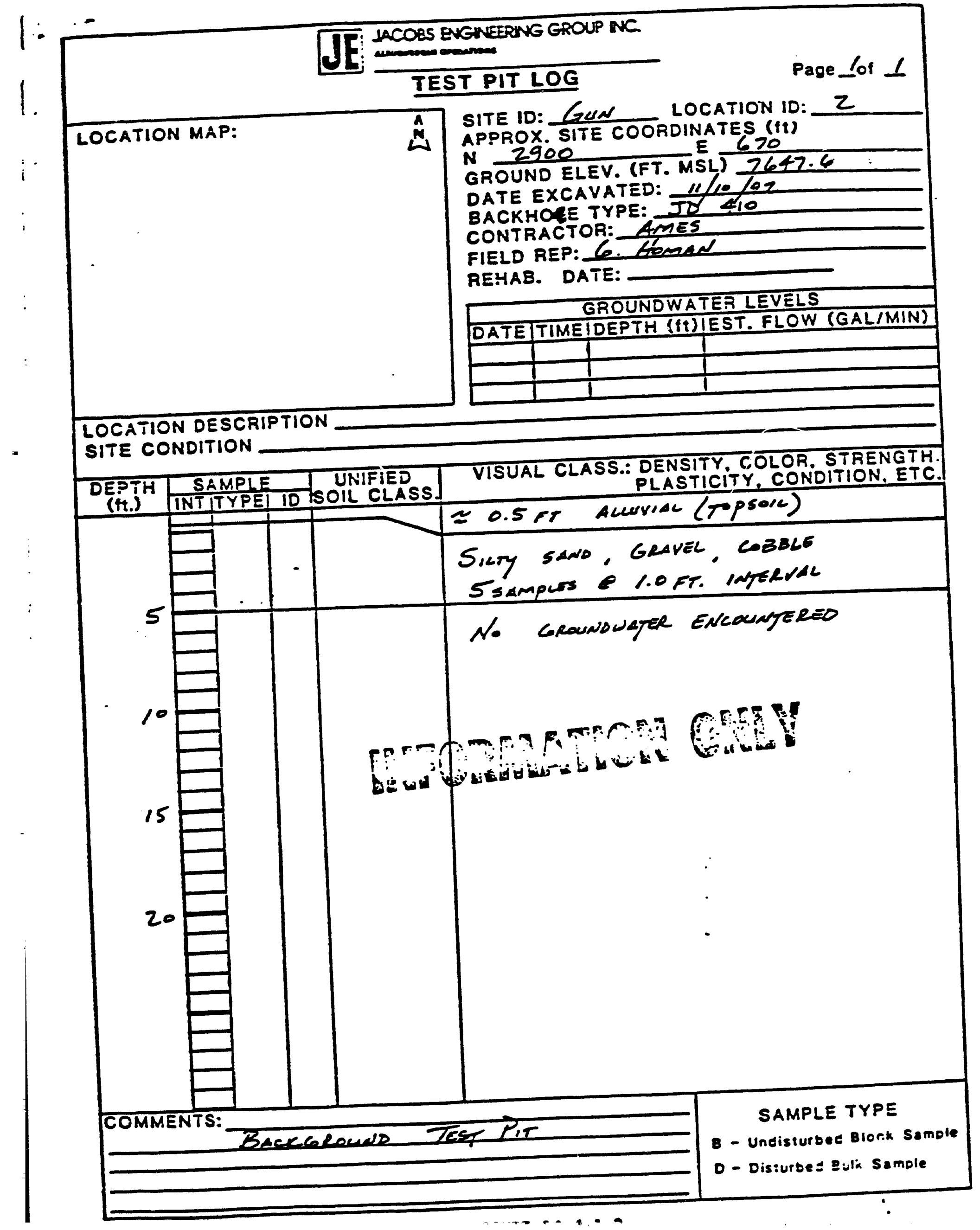


COBBLES-TO-FINE TEST PIT DATA

TEST PIT : 2

LOCATION: N_2900 E 670

TIC TEST PIT: YES No $X$

CDR SAMPLES TO BE TAKEN BY MKK-F/CWMFES: YES No $x$ SURFACE ELEVATION: 7647.6

DEPTH OF TAIIINGS/COBBLES INTERFACE: N/A (BACKGRouND TEST $p, \tau$ ) DEPTH OF GROUNDWATER: ReNE ENCOUNGLED

SAMPLE INTERVAL: 5 samples e 1.0 fT inferNAL

COMMENTS:

Bacralouxd Test Pit

SIGNATURE: DATE: $12 / 7 / 92$

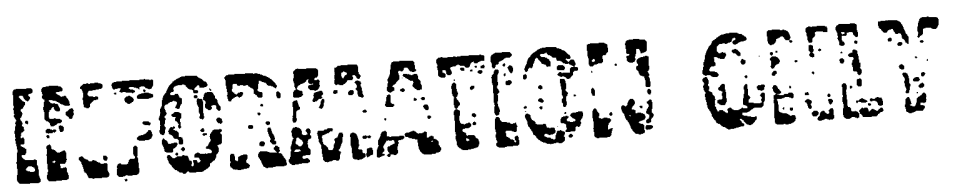




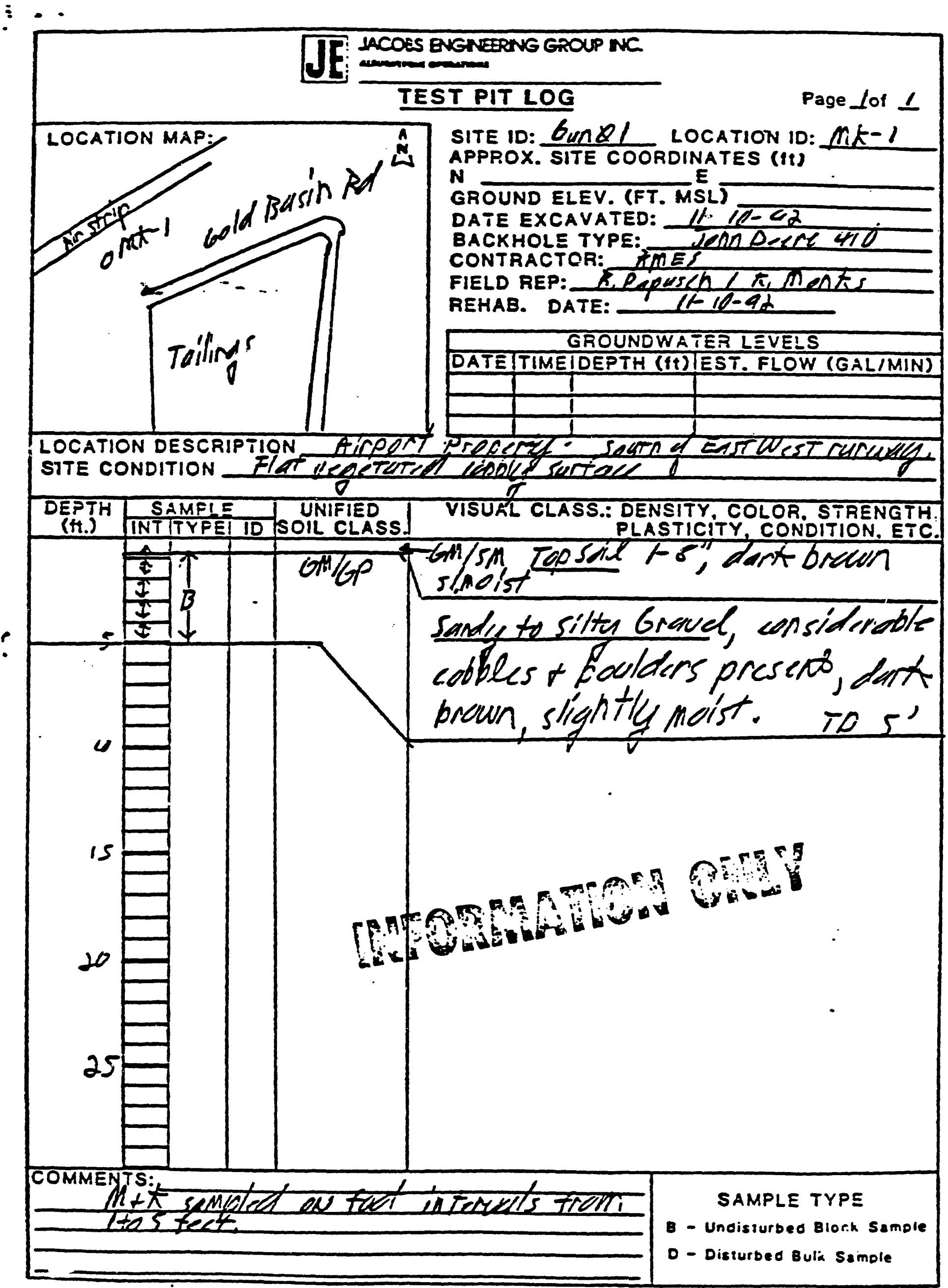




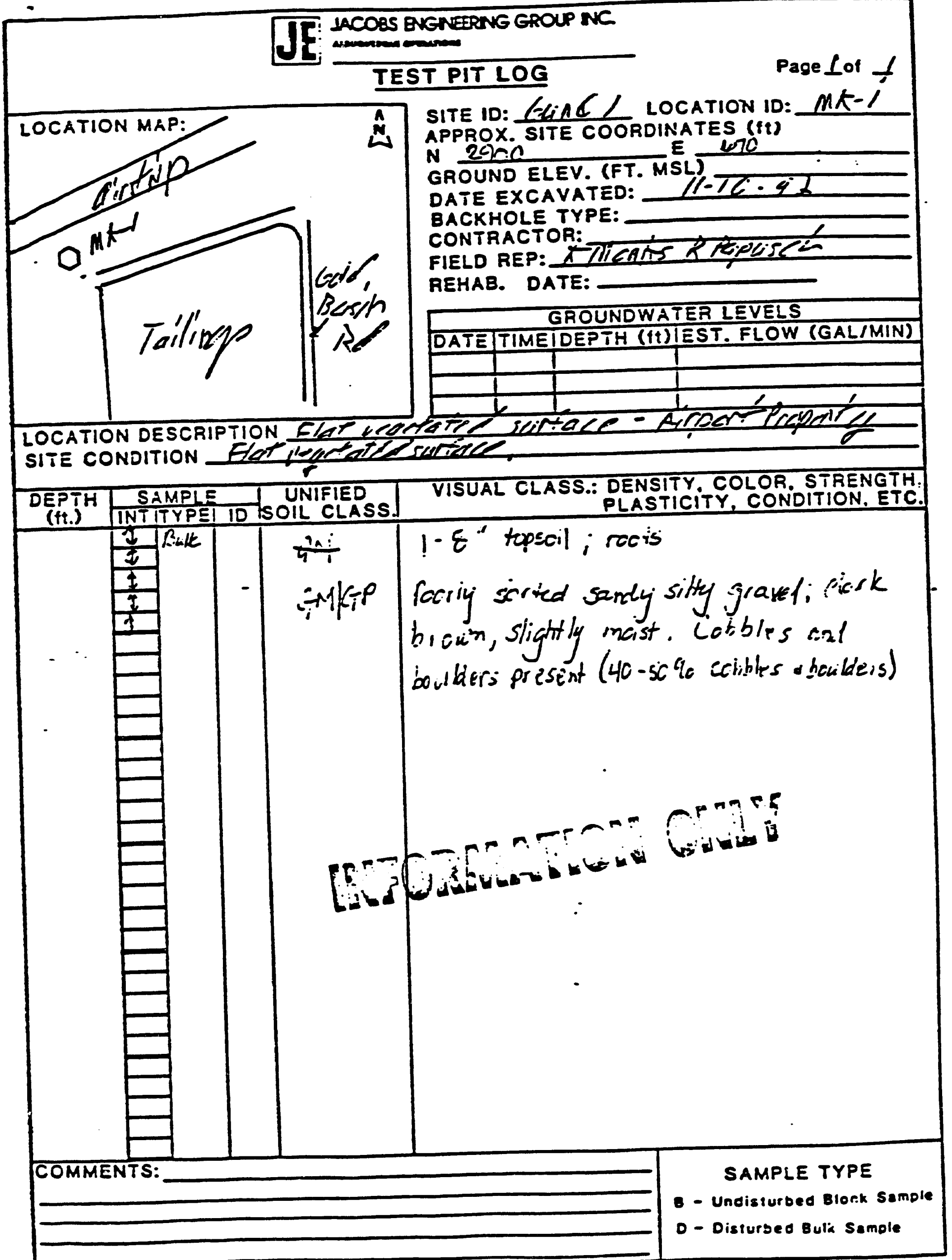




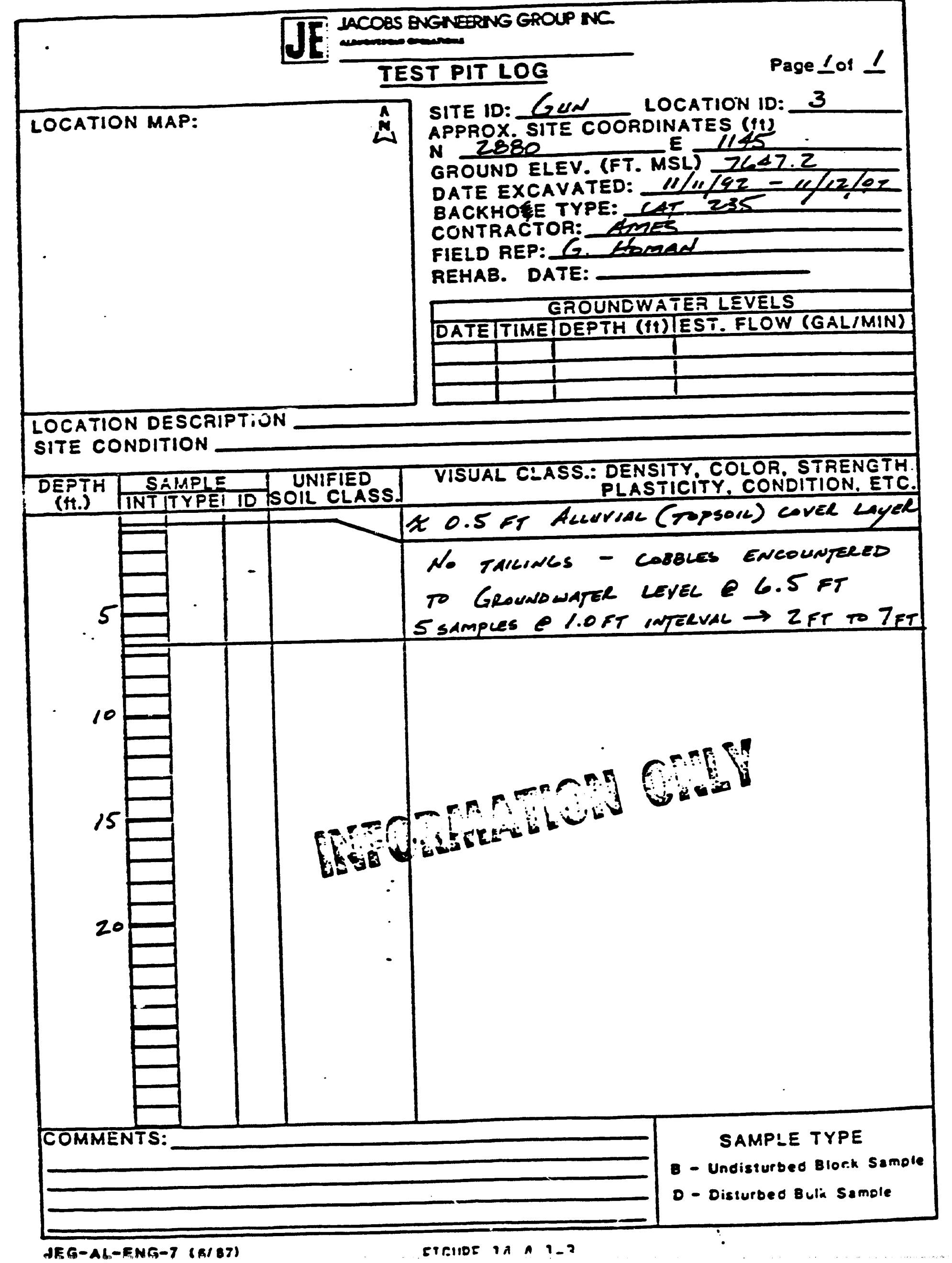




\section{COBBLES-TO-FINE TEST PIT DATA}

IEST PIT * $\quad 3$

IOCATION: N 2880 E_ $11 / 5$

TAC TEST PIT: YES No $x$

CDH SAMPLES TO BE TAKEN BY MIK-F/CWMFES: YES No $\quad X$ SURFACE ELEVATION: 7647.2

DEPTH OF TAIIINGS/COBBLES INTERFACE: No RILANGS ENCOUNGReD DEPTH OF GROUNDWATER: $6.5 \mathrm{ET}$

SAMPLE INTERVAI: 5 samples $e 1.0 \mathrm{fT}$ inferval $\rightarrow$ ZFT T0 $7 \mathrm{FT}$ COMAMENTS:

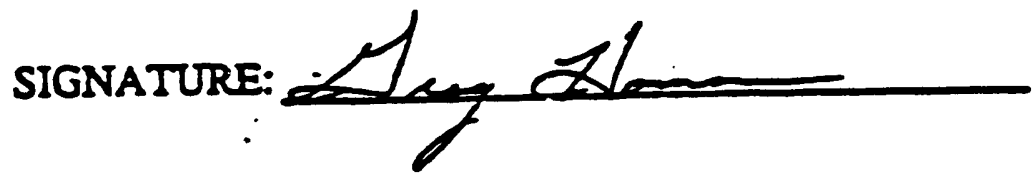

DATE: $12 / 7 / 92$ 


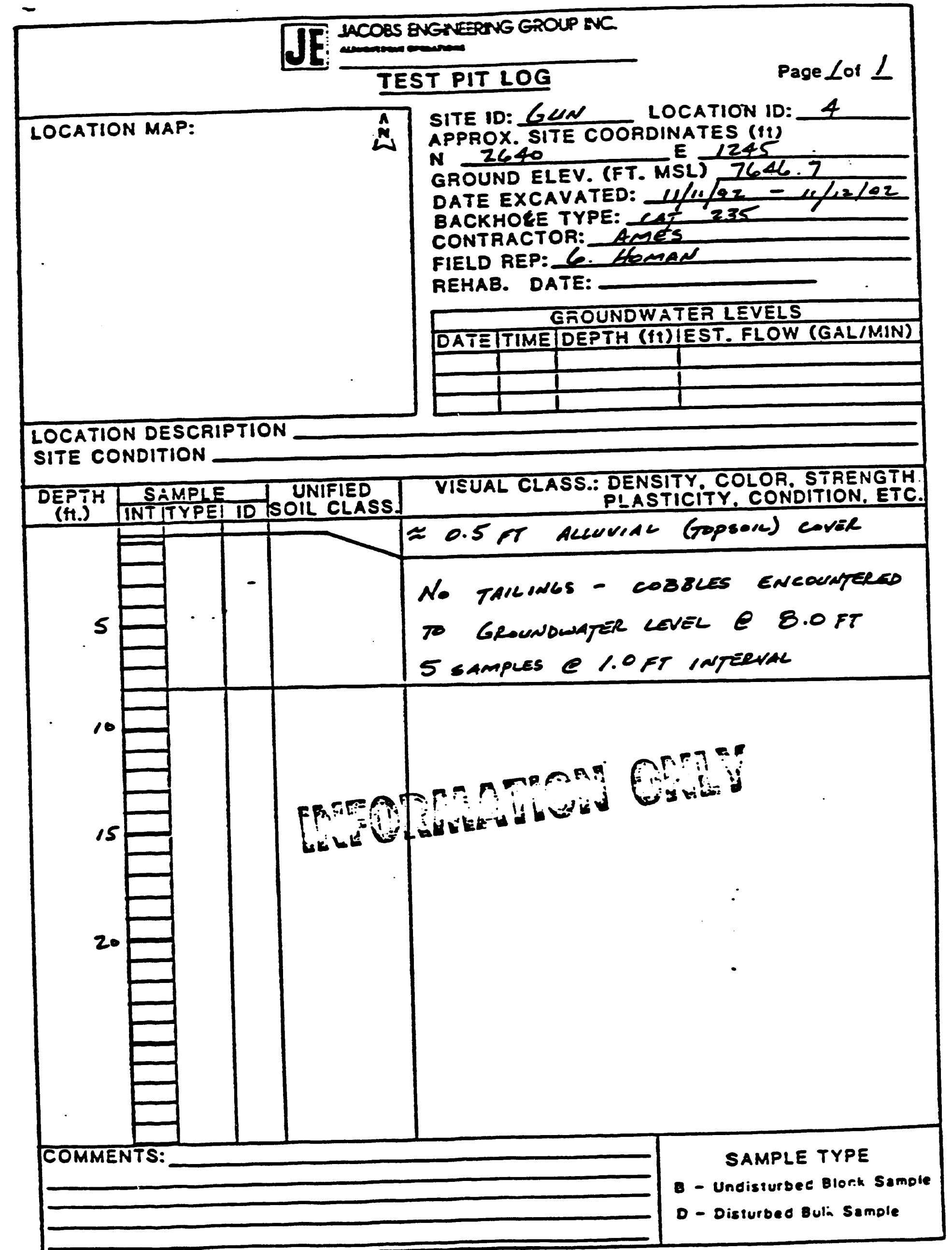


COBBLES-TO-FINE TEST PIT DATA

TEST PIT : 4

LOCATION: N 2640

E 1245

TAC TEST PIT: YES

No $x$

CDH SAMPLES TO BE TAKEN BY MK-F/CWMFES: YES No $x$

SURFACE EIEVATION: 7646.7

DEPTH OF TAIIINGS/COBBLES INTERFACE: No Jallendes ENCOUNGalso DEPTH OF GROUNDWATER:

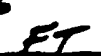

SAMPLE INTERVAI: 5 samples $0 \approx 1.0 \mathrm{FT}$ inferyal

COMDEENTS:

SIGNATURE:

DATE: $12 / 2 / a z$

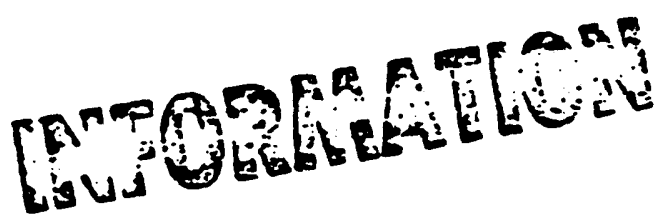

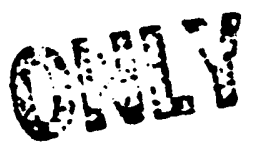




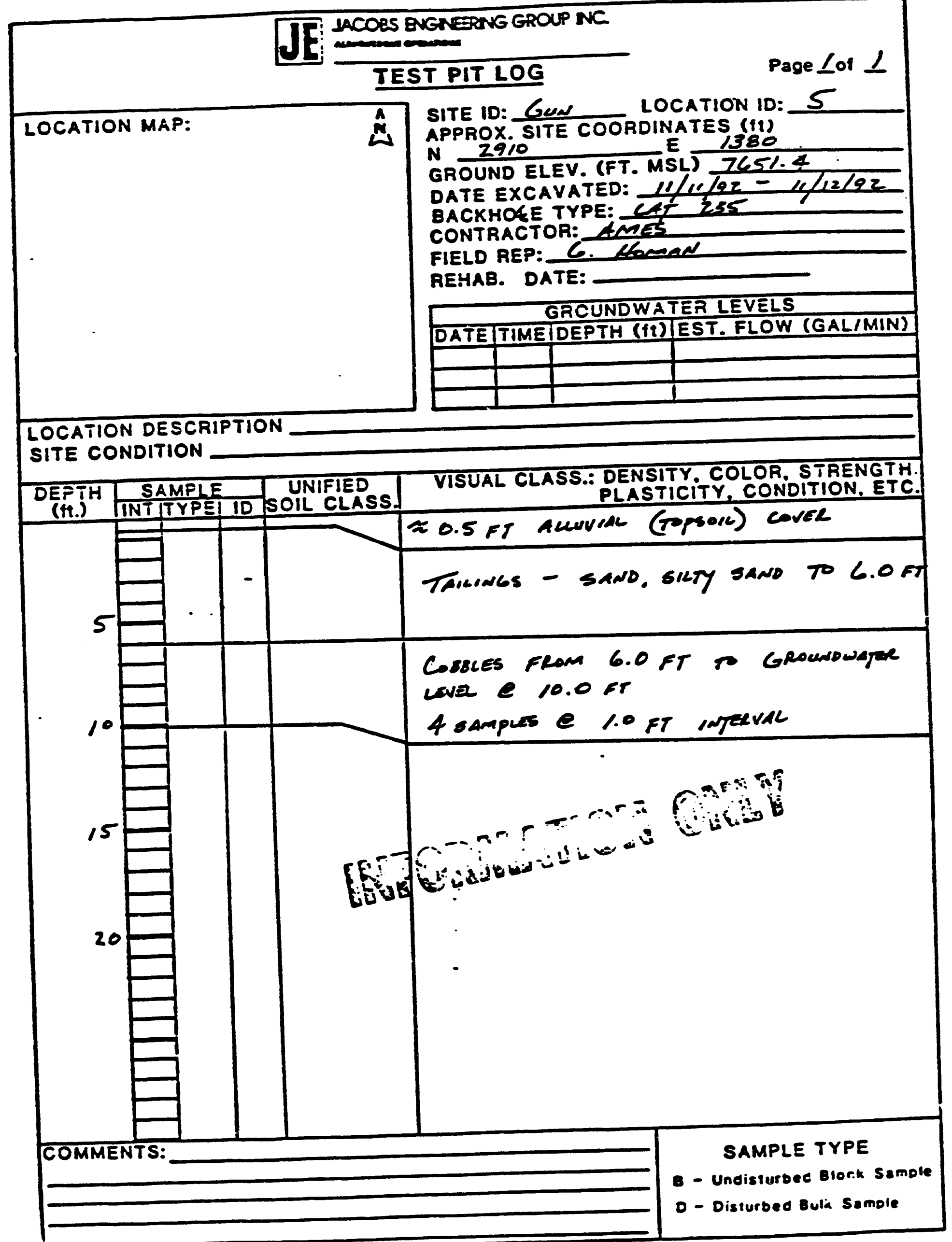


COBBLES-TO-FINE TEST PIT DATA

TEST PIT : 5

LOCATION: N 2910 E 1380

FAC TEST PIT: YES No $x$

CDR SAMPLES TO BE TAKEN BY MK-F/CWMFES: YES $\not \subset$ NO SURFACE ELEVATION: 7651.4

DEPTH OF TAIIINGS/COBBLES INTERFACE: $6: 0 \mathrm{FT}$

DEPTH OF GROUNDWATER: 10.0 ET

SAMPLE INTERVAI: A stapes e $1.0 \mathrm{FT}$ interval

COMMENTS:

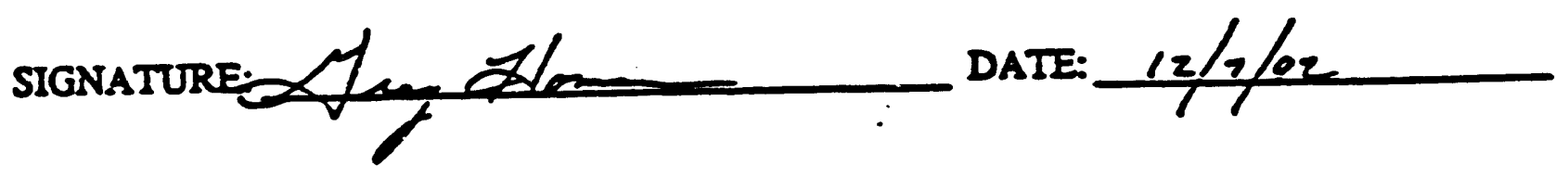




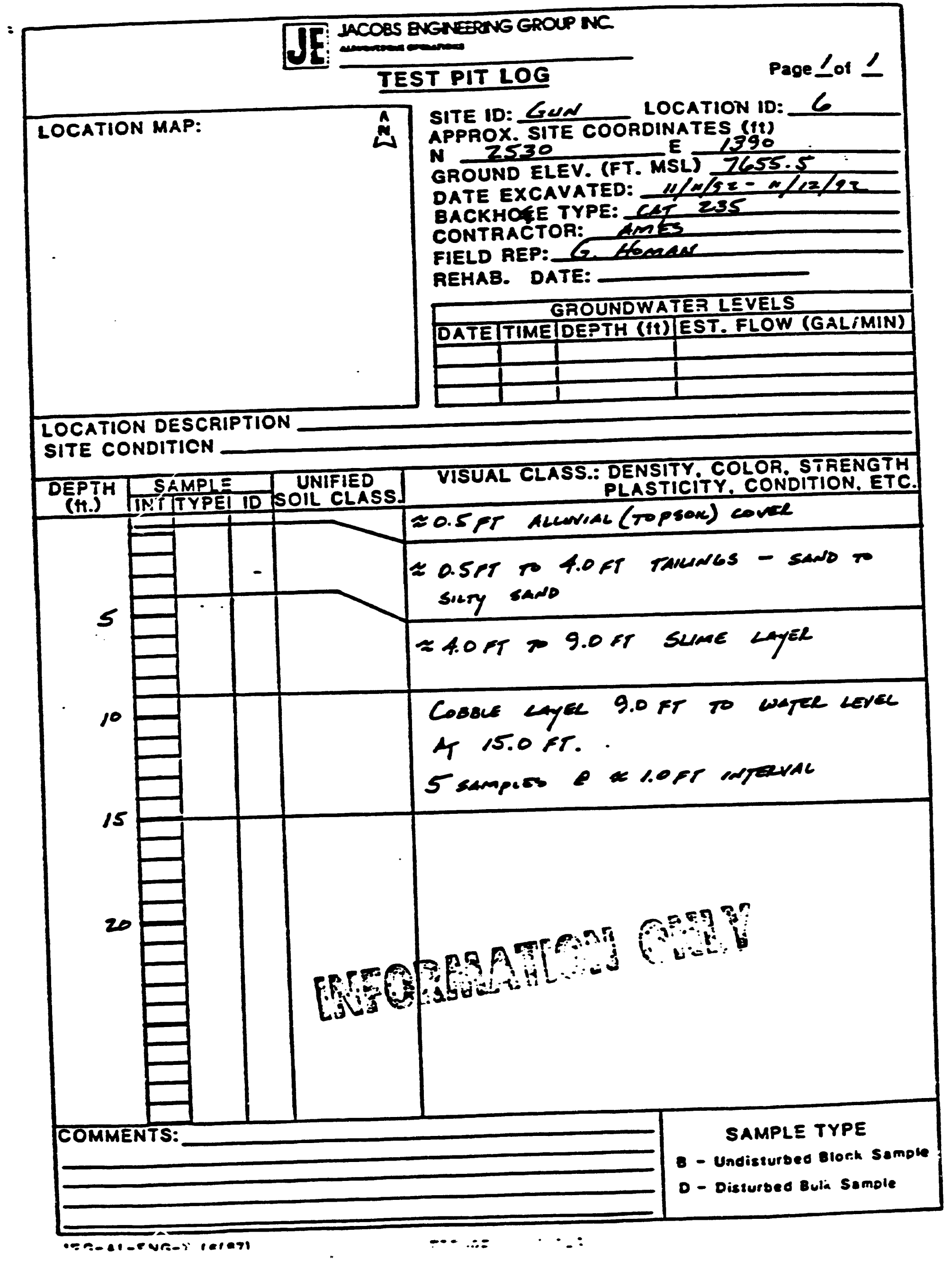



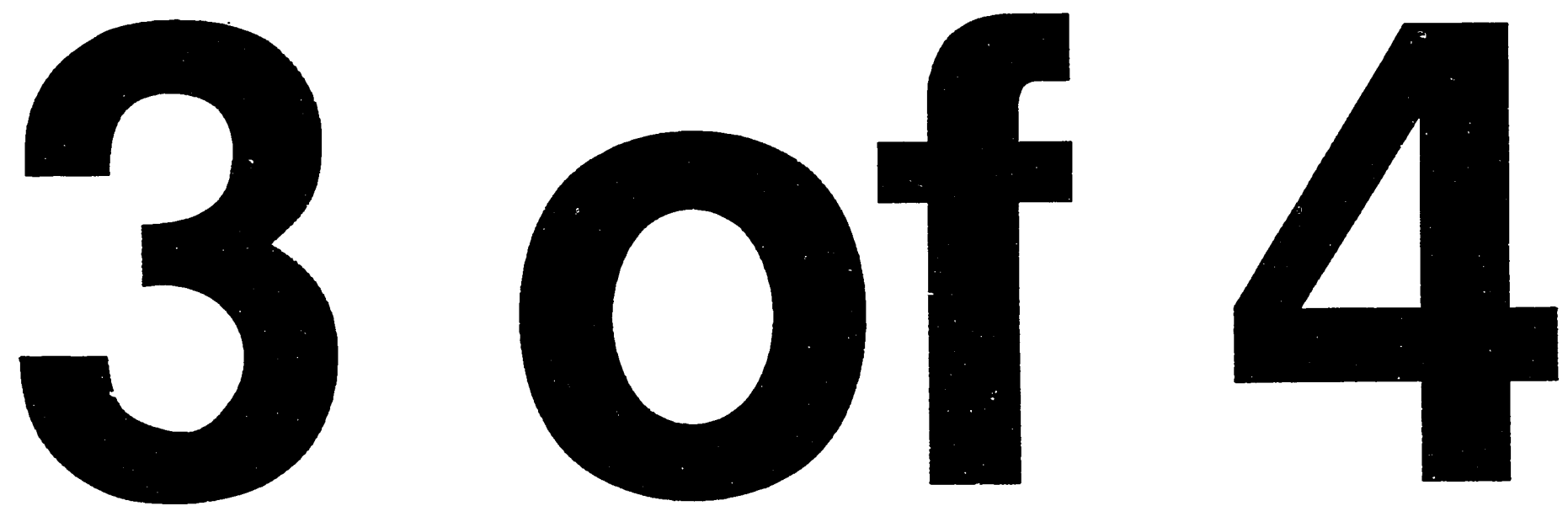


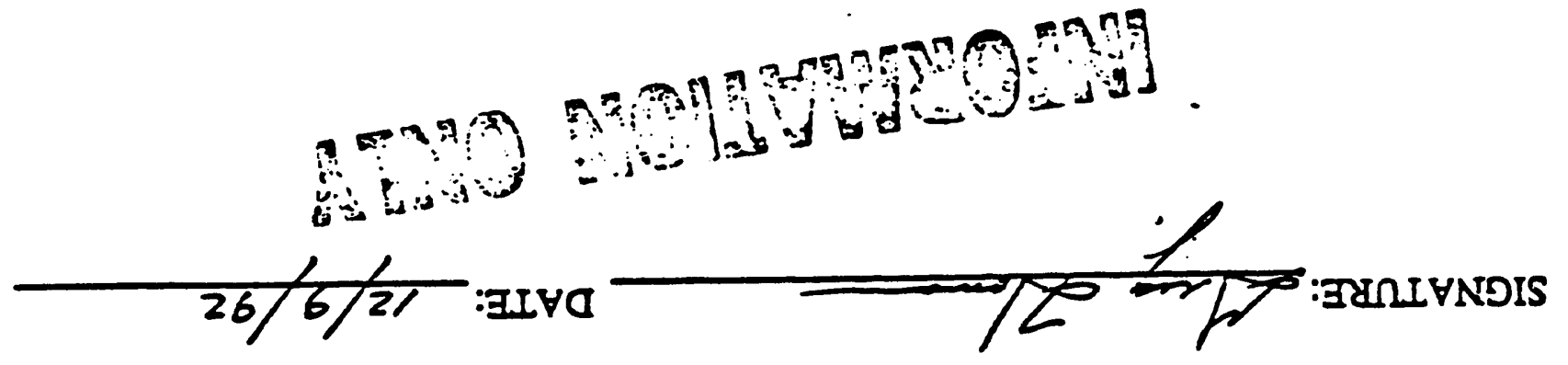

:SINEINTOO.

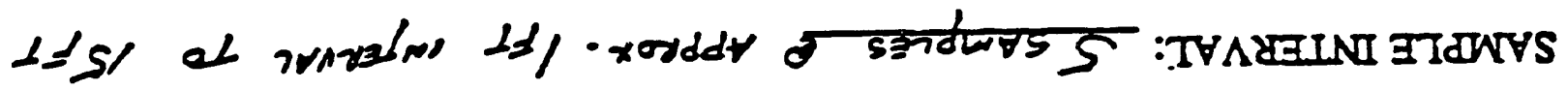

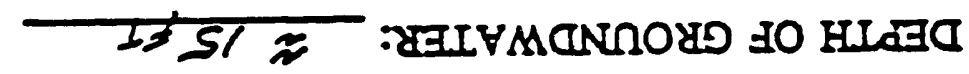

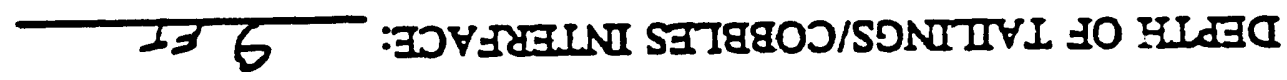
S.SSTL :NOLVAETH BOVIRIS

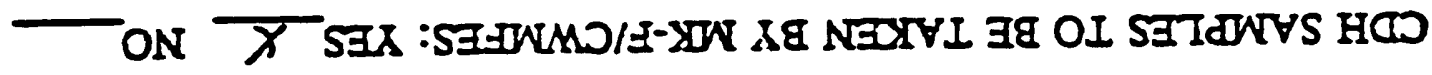
$\bar{X}$ ON T SEX :IId ISEL JYI

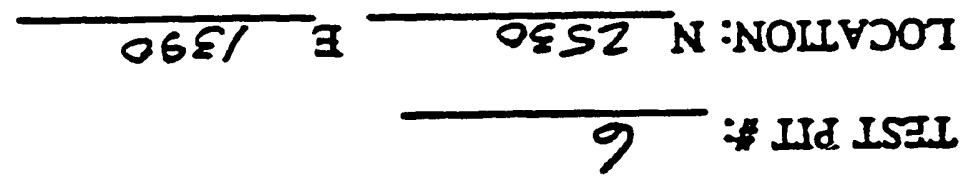

VIVA IId ISTI INIH-OI-ST7RgOD 


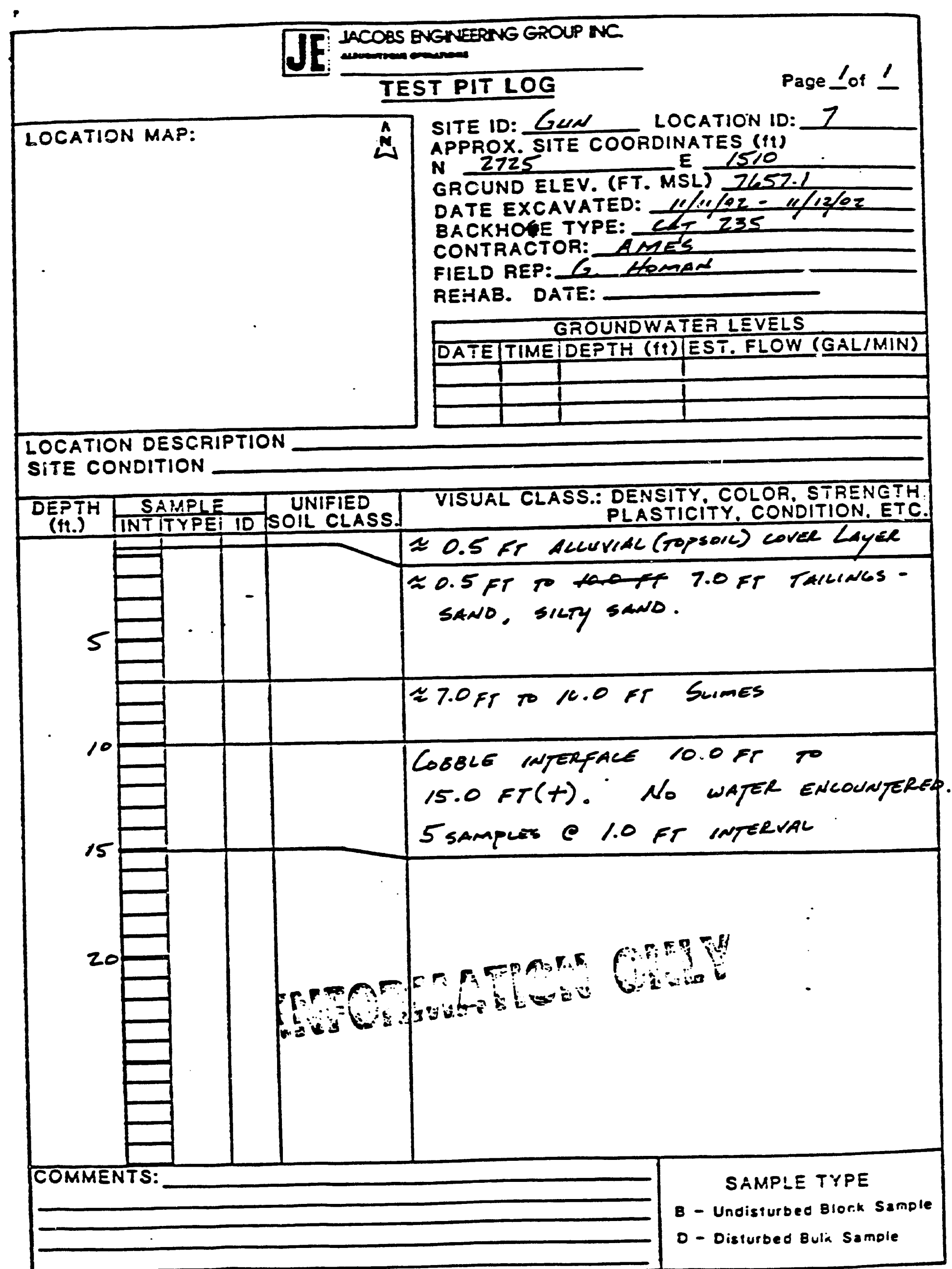


COBBLES-TO-FINE TEST PIT DATA

TEST PIT *

LOCATION: N_Z725 E 1510

TAC TEST PIT · YES No $x$

DH SAMPLES TO BE TAKEN BY NKK-F/CWMFES: YES No $x$ SURFACE ELEVATION: 7657.1

DEPTH OF TAIIINGS/COBBLES INTERFACE: $10 \mathrm{ET}$

DEPTH OF GROUNDWATER: No wAFER ENCOUNFECO

SAMPLE INTERVAL: 5 SAMPLES $1.0 \mathrm{FT}$ INTERVAL TO $15.0 \mathrm{FT}$

COMMENTS:

SIGNATURE: DATE:

$12 / 9 / a r$ 


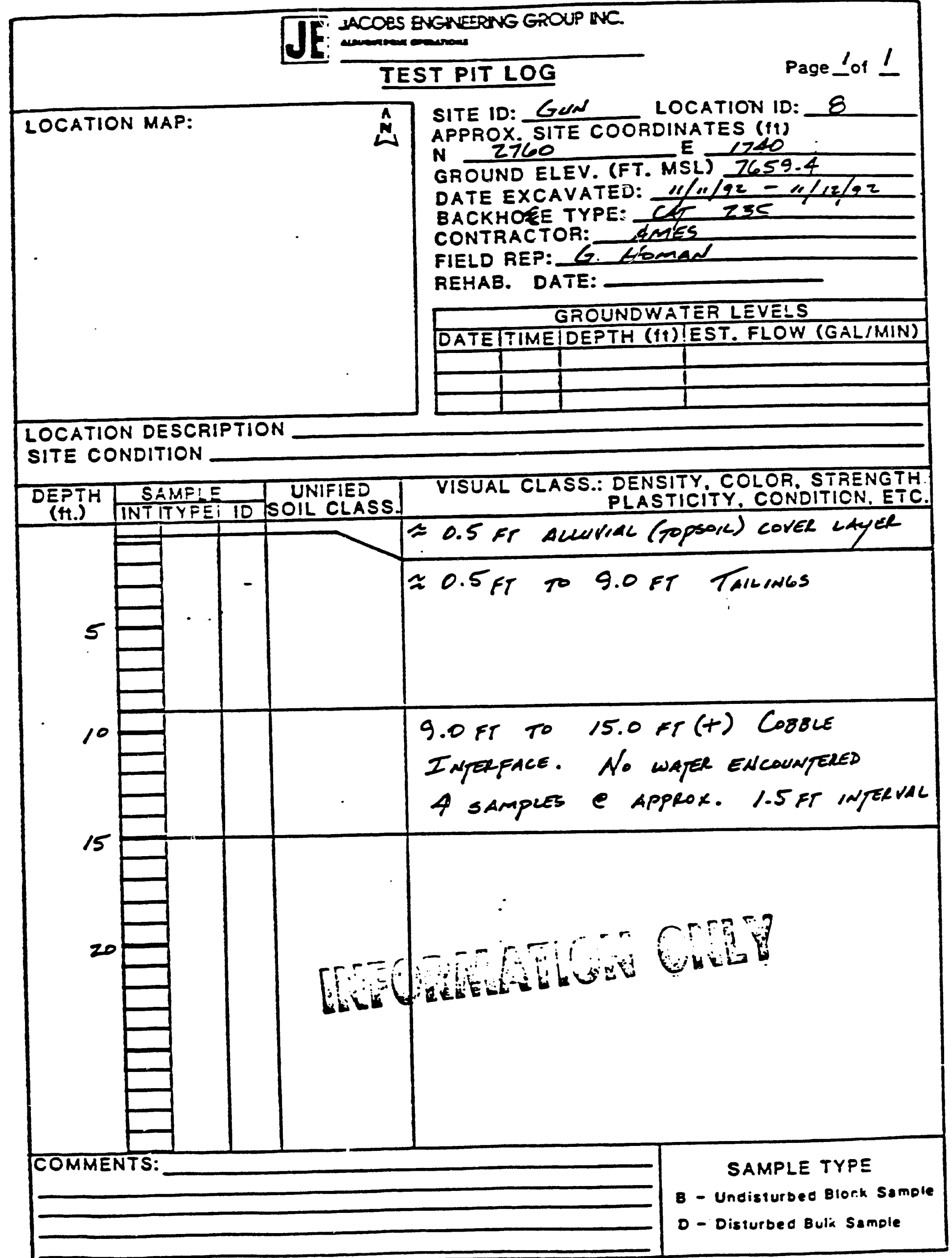




\section{COBBLES-TO-FINE TEST PIT DATA}

TEST PIT *:

8

IOCATION: N2760

E 1740

TAC TEST PIT: YES No $K$

CDH SAMPLES TO BE TAKEN BY MR-F/CWMFES: YES $\chi \underline{X}$ NO

SURFACE EIEVATION: 7659.4

DEPTH OF TAIINGS/COBBLES INTERFACE: $\quad 9 \%$

DEPTH OF GROUNDWATER: Ne GROWWDWATE GNCOUNFELED

SAMPLE INTERVAI: 4 samples e APPRox 1.5 fr infeerdi to 15 fT

COMMENTS:

SIGNATURE:

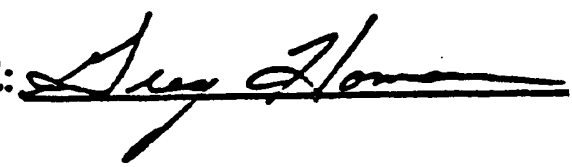

DATE:

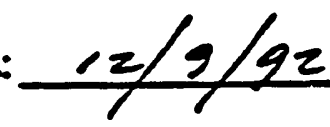

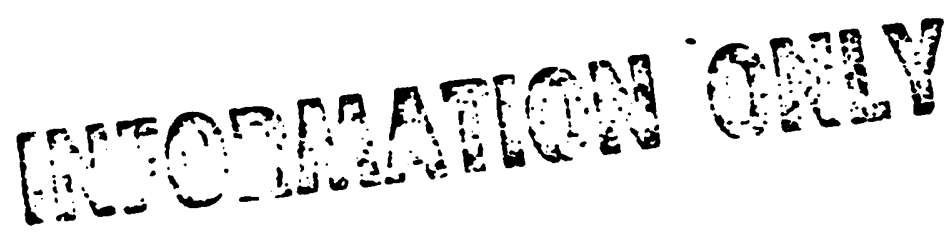




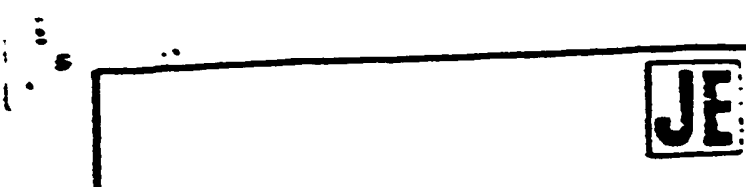

LOCATION MAP:

LACOBS ENGNEERTK GROUP NC

armonarom

TEST PIT LOG

Page I of 1

LOCATION ID: 9

LOCATION ID:

SITE ID: GUN

in

APPROX. SITE COORDINATES (I1)

N ZTES

GROUND ELEV. (FT. MSL) 7656.0

DATE EXCAVATED:

BACKHOKE TYPE:

CONTRACTOR:

ANES

FIELD REP:

REHAB. DATE:

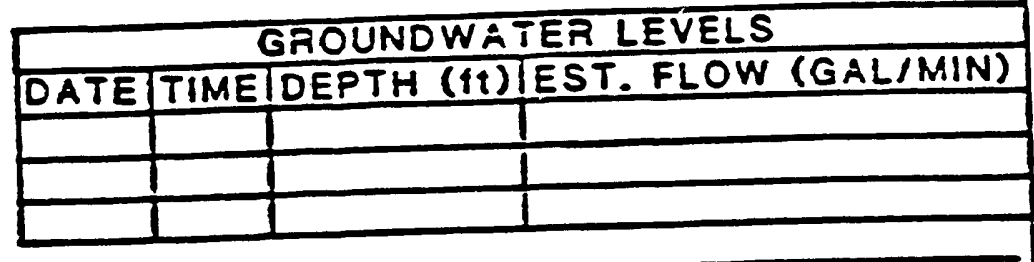

LOCATION DESCRIPTION

SITE CONDITION \begin{tabular}{c|c|c|} 
DEPTH & SAMPLE & UNIFIED \\
(HA.) & INTITYPEI ID & SCIL CLASS \\
\cline { 2 - 3 } &
\end{tabular}

VISUAL CLASS.: DENSITY. COLOR. STRENETH

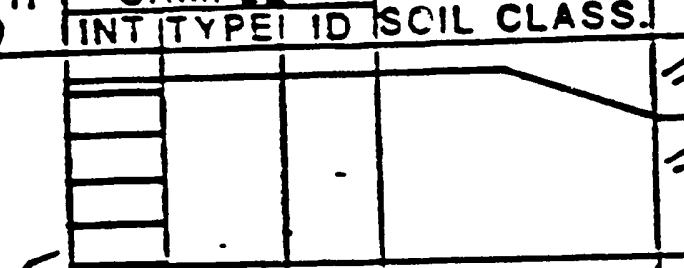

$0.5 \mathrm{ft}$ a curvial (TOPSOIL) of corel LAYER

$\approx 0.5 \mathrm{fr}$ to $5.0 \mathrm{fT}$ Talurados. Singy, SinTy SAND.

$\approx 5.0 \mathrm{FT} T 11.0 \mathrm{FT}$ SLIMES

\begin{tabular}{l} 
intertur \\
\hline 10 \\
\hline
\end{tabular}

${ }^{4} \mathrm{~T} 13.0$ FT.

15

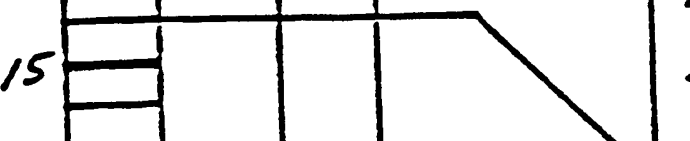

4 SAmpLES 2 APPROX. 1.0 FT InfERAL LAST SAMPLE $P$ AF.O FT.

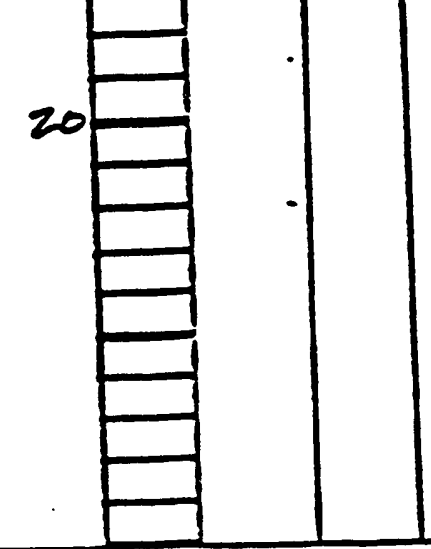

COMMENTS:

SAMPLE TYPE

B - Undisiurbec Blork Sample

D - Disiurbeo Bula Samole 
COBBLES-TO-FINE TEST PIT DATA

TEST PIT * 9

LOCATION: N 2745 E 2115

TAC TEST PIT: YES_ NO No $x$

DH SAMPLES TO BE TAKEN BY MR-F/CWMFES: YES No $x$ SURFACE ELEVATION: 7656.0

DEPTH OF TAIIINGS/COBBLES INTERFACE: $11.0 \mathrm{ET}$

DEPTH OF GROUNDWATER: $13.0 \mathrm{ET}$

SAMPLE INTERVAL: 4 samples $0 \approx 1.0 \mathrm{FT}$ interval. LAST sample COMMENTS:

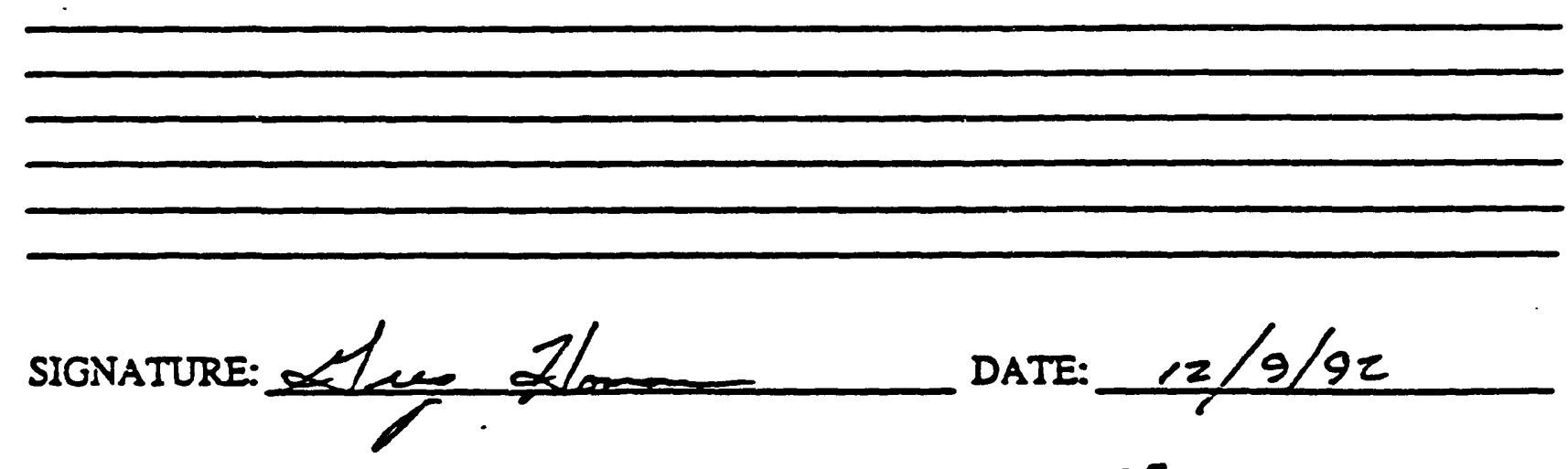




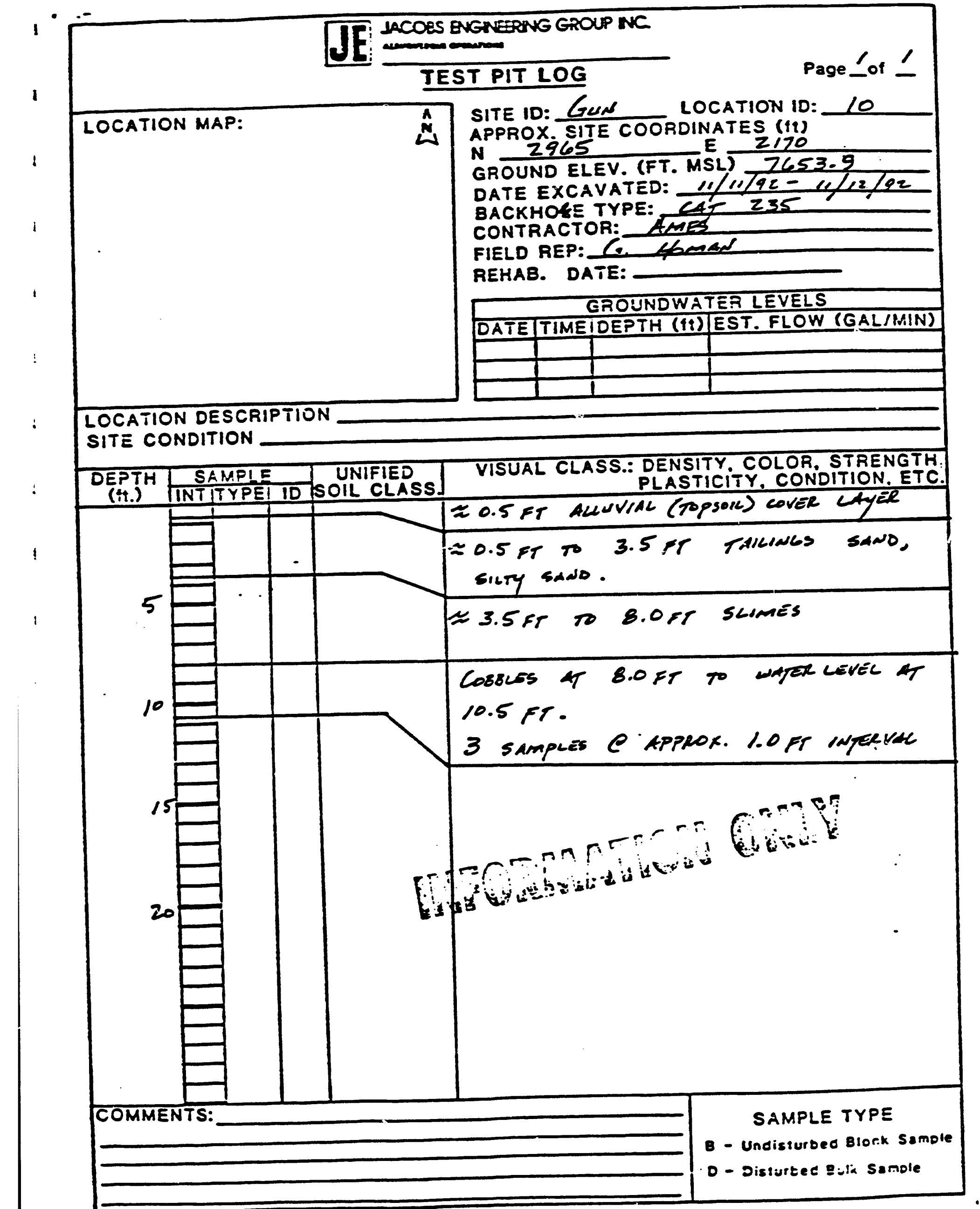


TEST PIT * 10

LOCATION: N 2965 E 2170

TAC TEST PIT: YES

No $x$

CDH SAMPIES TO BE TAKEN BY MK-F/CWMRES: YES $x$ NO

SURFACE EIEVATION: 7653.9

DEPTH OF TAIIINGS/COBELES INTERFACE:

8 EF

DEPTH OF GROUNDWATER: 10.5 ET

SAMPLE INIERVAI: 3 samples e APPROX. 1.0 FT ingeryal

COMOMENTS:

SIGNATURE: DATE: $12 / 9 / 92$

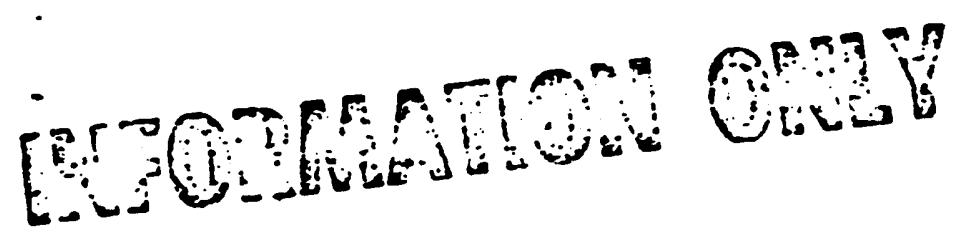




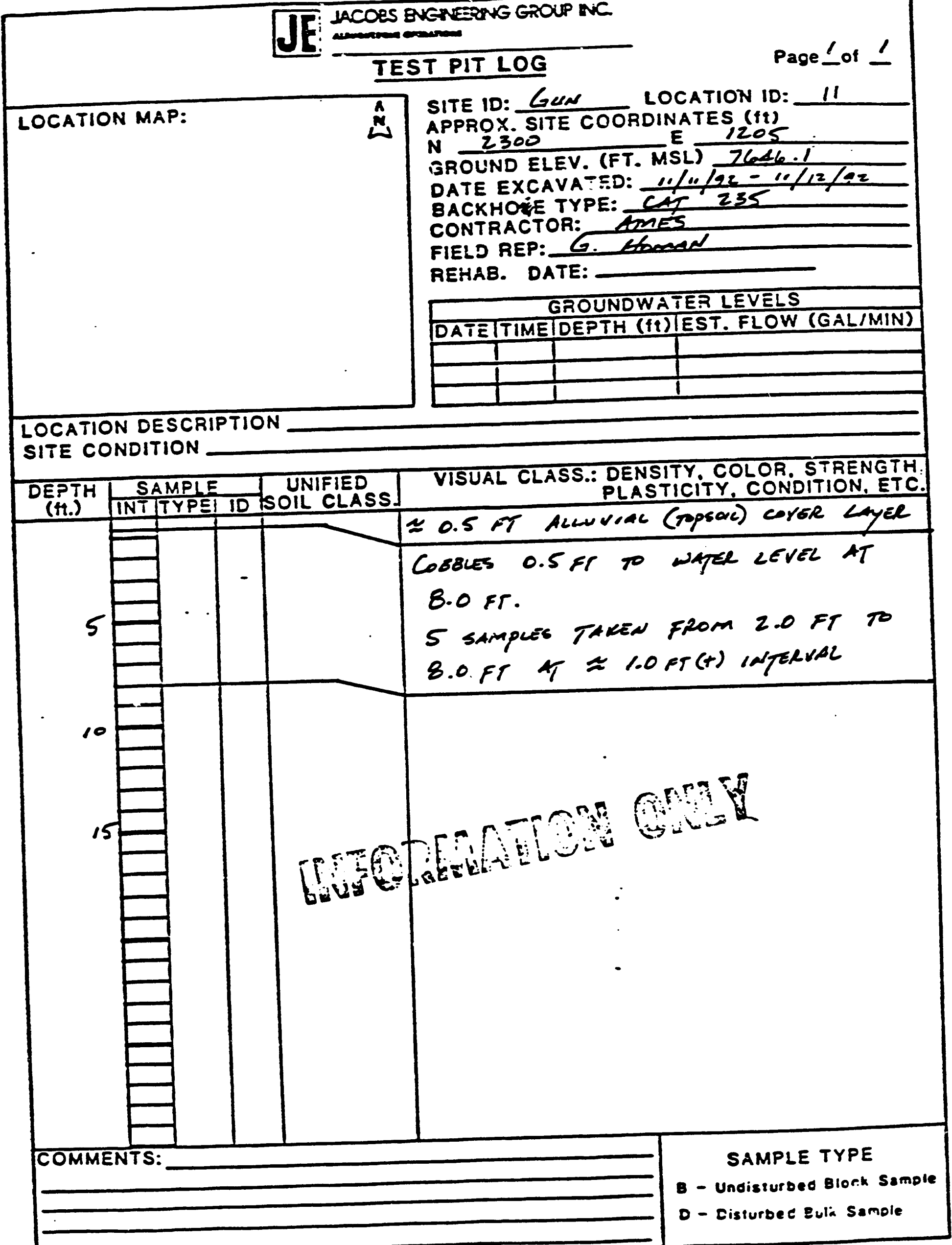




\section{COBBLES-TO-FINE TEST PIT DATA}

TEST FIT :

LOCATION: N_Z300 E 1205

TAC TEST PIT: YES__ NO_ $X$

CDH SAMPLES TO BE TAKEN BY MK-F/CWMFES: YES__ NO $\_\_$

SURFACE EIEVATION: 7646.1

DEPTH OF TAIINGS/COBBLES INTIRFACE: Ale coBbles, No TAlWNGS

DEPTH OF GROUNDWATER:

8 EF

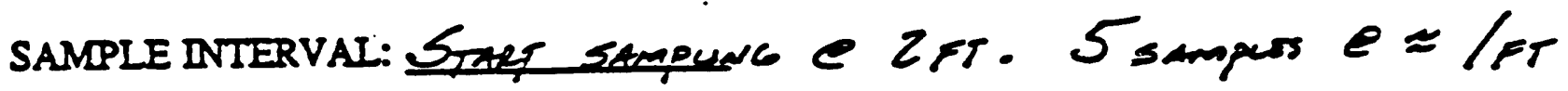
INTERVAL.

COMOMENTS:

SIGNATURE:

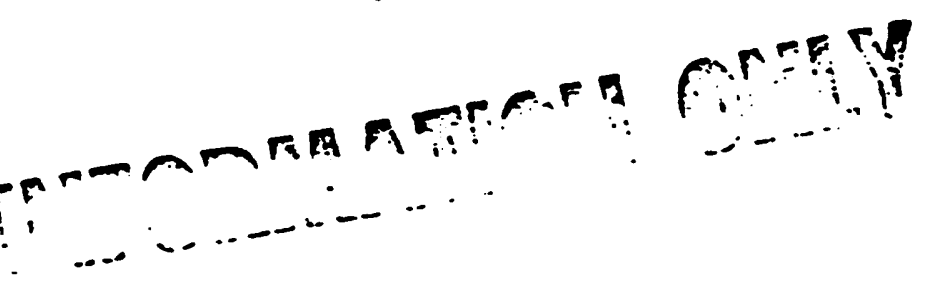




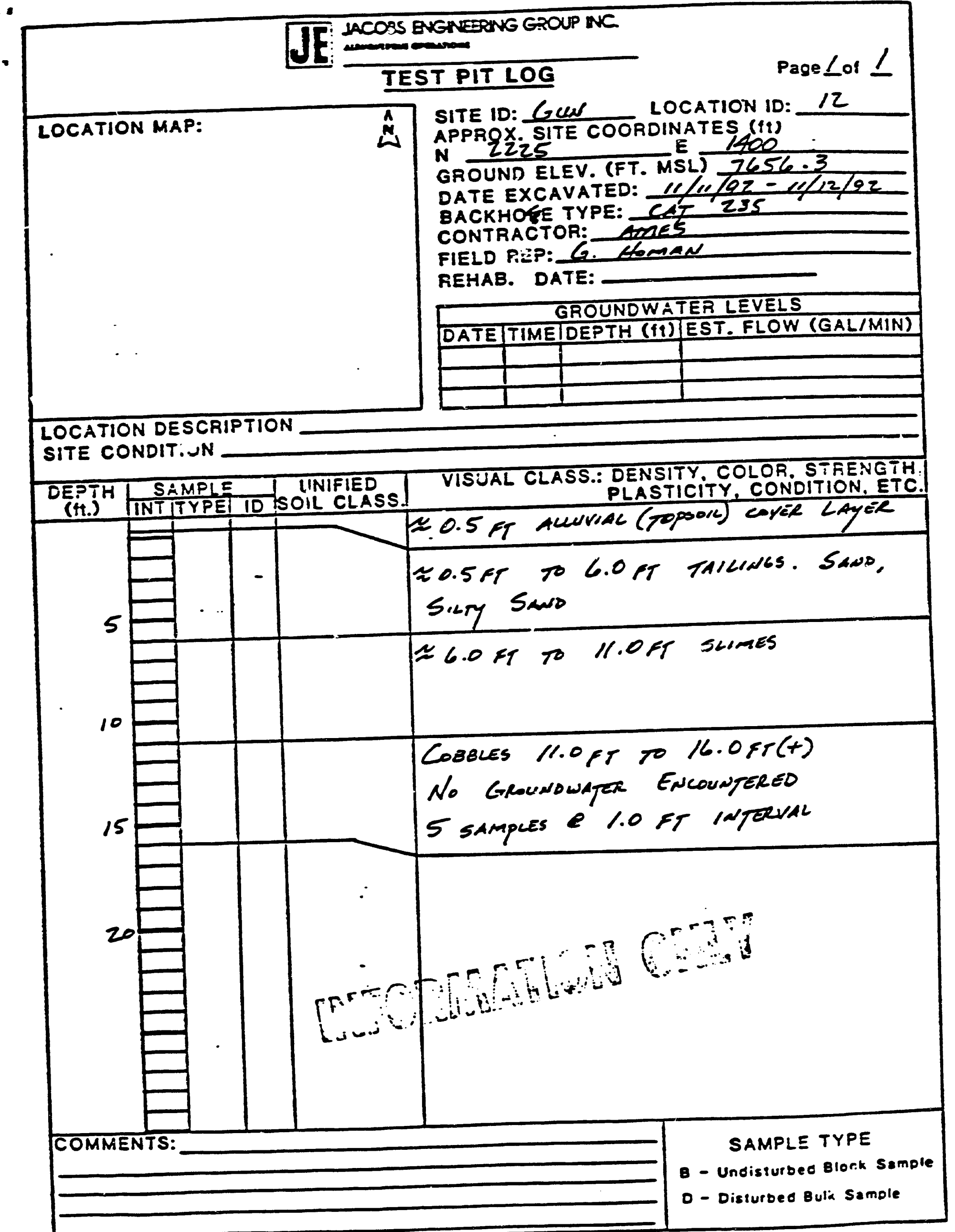




\section{COBBLES-TO-FINE TEST PIT DATA}

TEST PIT * 12

IOCATION: N_Z225 E 1400

TAC TEST PIT: YES__ NO

CDH SAMPLES TO BE TAKEN BY MK-F/CWMFES: YES $\_$NO

SURFACE EIEVATION: 7656.3

DEPTH OF TAIIINGS/COBBLES INTERFACE: $11.0 \mathrm{ET}$

DEPTH OF GROUNDWATER: Ne GRoundusfel EN counfereD

SAMPIE INTERVAI: 5 SAMPLES AT 1.0 pr IMTERAL

COMMENTS:

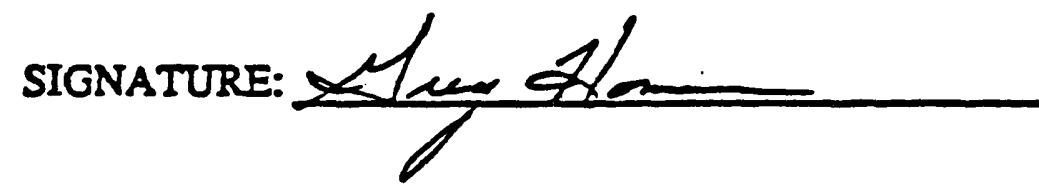

DATE: $12 / 10 / 22$

Guvi 2 - 


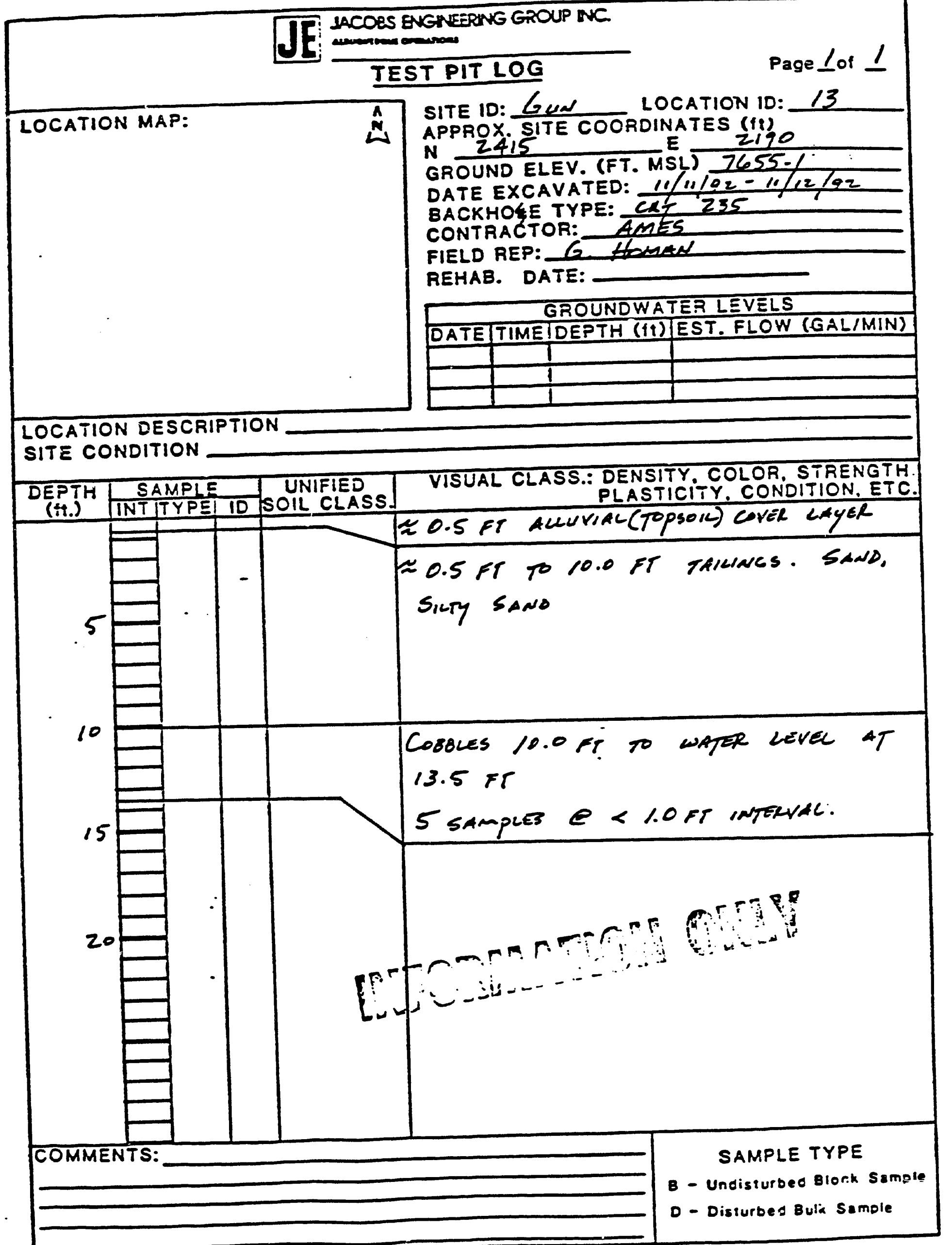


COBBLES-TO-FINE TEST PIT DATA

TEST PIT *: 13

LOCATION: H_ 2415 E 2190

THC TEST PIT: YES NO $x$

CDR SAMPLES TO BE TAKEN BY MR-F/CWMFES: YES__ NO_ $\_$_

SURFACE ELEVATION: $7655-1$

DEPTH OF TAIIINGS/COBBLES INTERFACE: $10.0 \mathrm{ET}$

DEPTH OF GROUNDWATER: $13.5 \mathrm{ET}$

SAMPLE INTERVAL: 5 SAMPLES $0<1.0 \mathrm{FT}$ INTERnAL

COMMENTS:

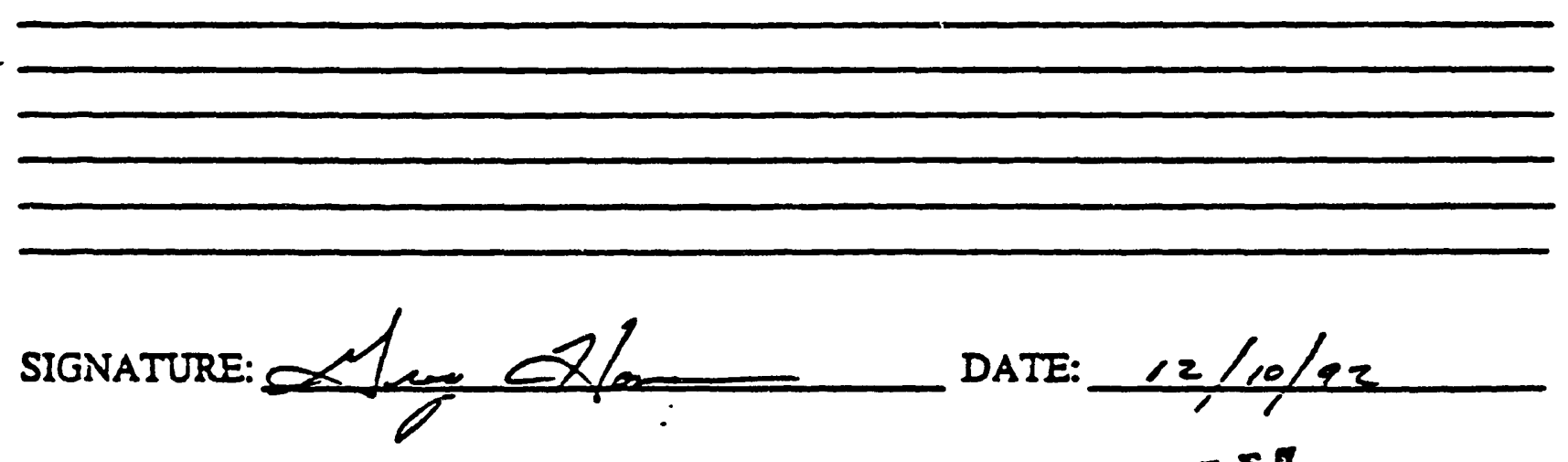

SIGNATURE: DATE: $\frac{12 / 10 / 22}{1}$

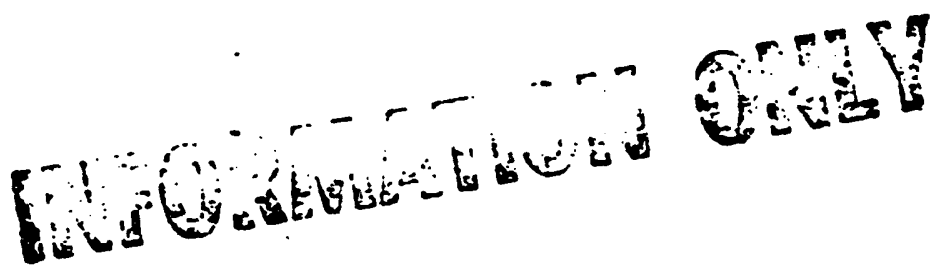




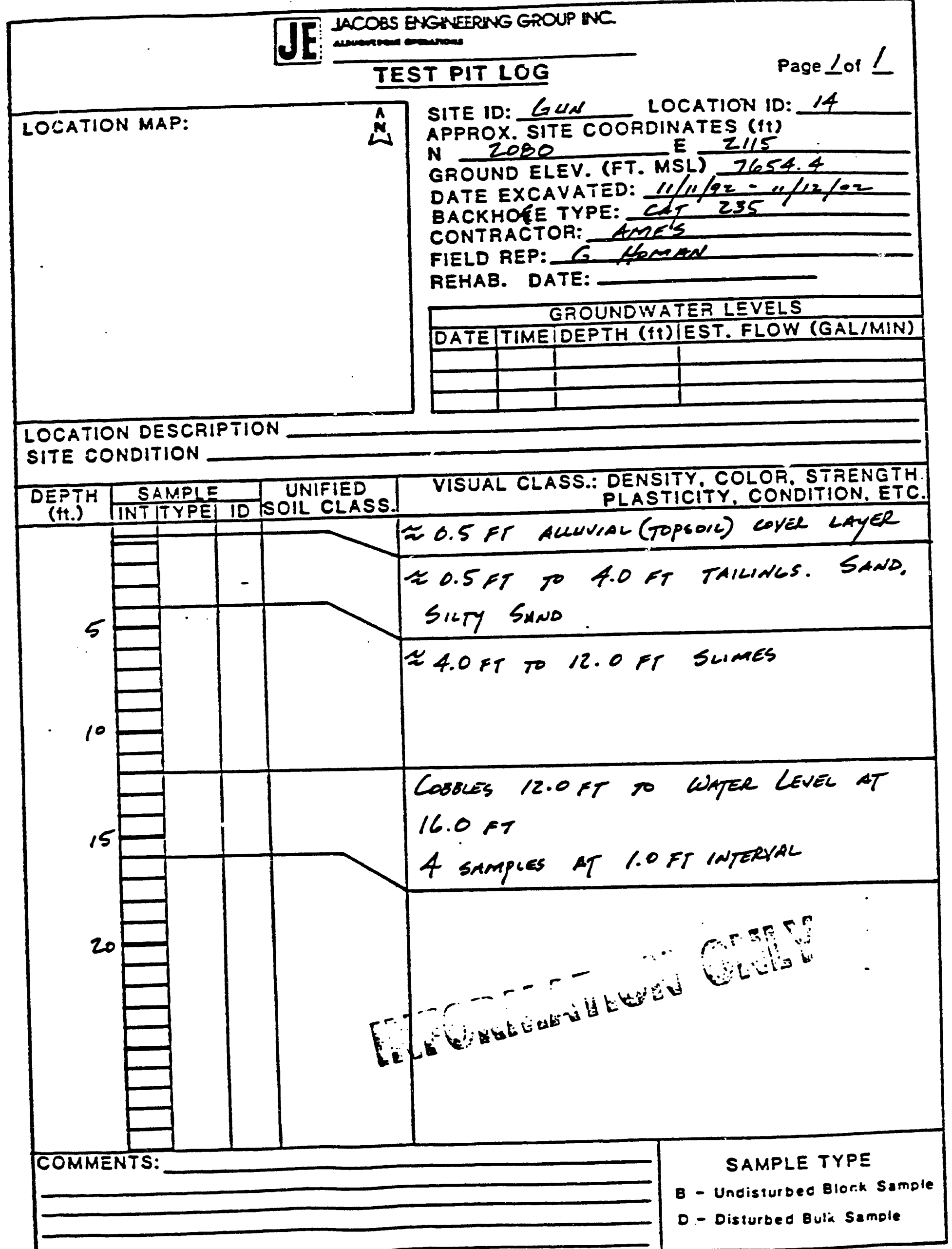


COBBLES-TO-FINE TEST PIT DATA

TEST PIT : 14

LOCATION: N_2080 EZ Z

FAC TEST PIT: YES No $x$

DH SAMPLES TO BE TAKEN BY MK-F/CWMFES: YES $\not \subset$ NO

SURFACE ELEVATION: 7654.4

DEPTH OF TAIINGS'COBBLES INTERFACE:

12.0 ET

DEPTH OF GROUNDWATER:

16. OEF

SAMPLE INTERVAI: \& sampLE Se $1.0 \mathrm{FT}$ interval

COMMENTS:

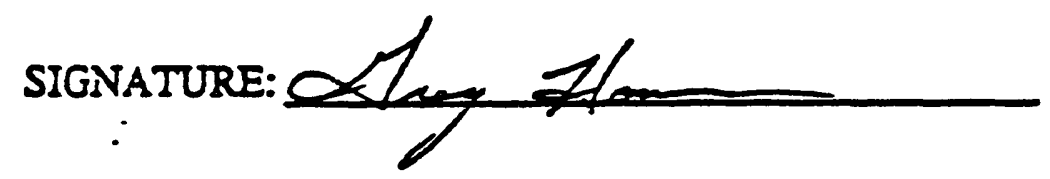

DATE: $\quad 2 / 10 / 02$ 


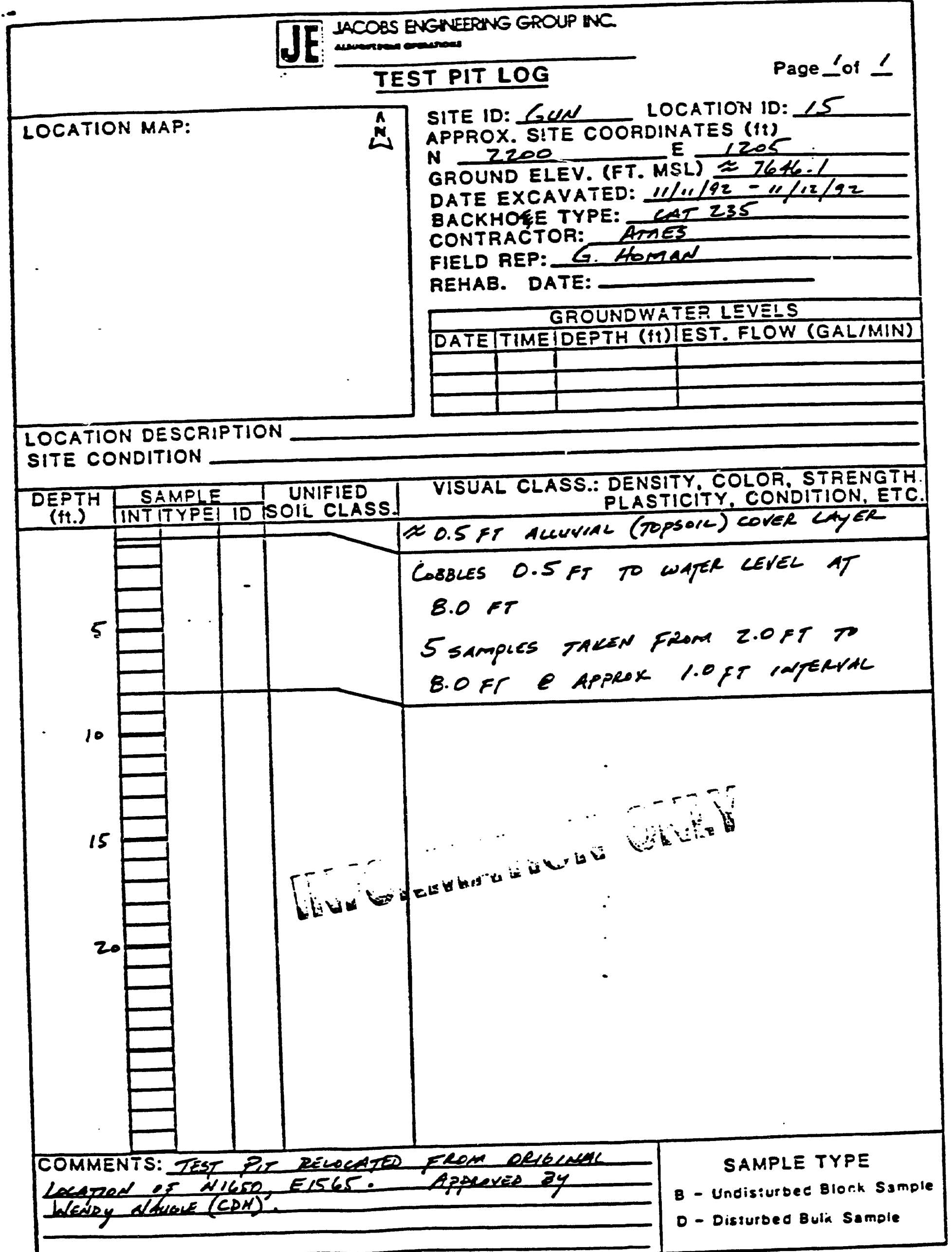




\section{COBBLES-TO-FINE TEST PIT DATA}

TEST PIT : 15

LOCATION: N_2200 E 1205

TAC TEST PIT: YES__ NO $\chi \chi$

CDH SAMPLES TO BE TAKEN BY MR-F/CWMIFES: YES__ NO

SURFACE ELEVATION: $\simeq 7646.1$

DEPTH OF TAIIINGS/COBBLES INTERFACE: Ak COBBLE, No Tallow

DEPTH OF GROUNDWATER: $\approx 8.0$ ET

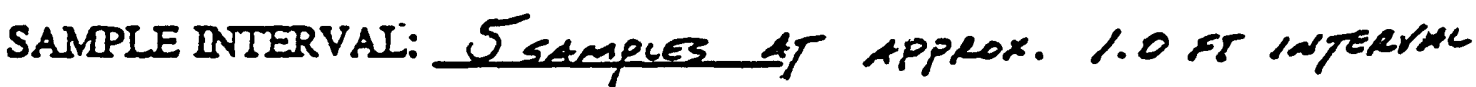

COMMENTS:

Devinal TEST PIT cochion was N1650, E1565. THE TEST

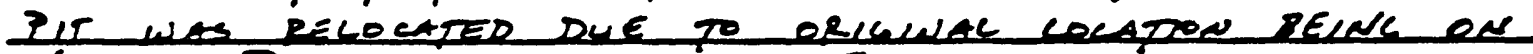

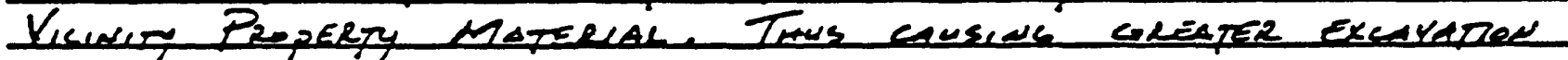

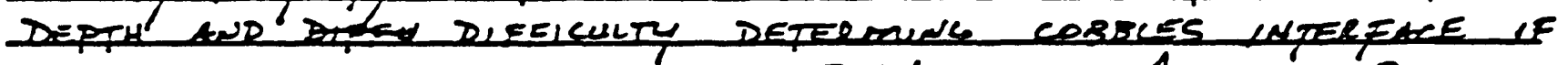

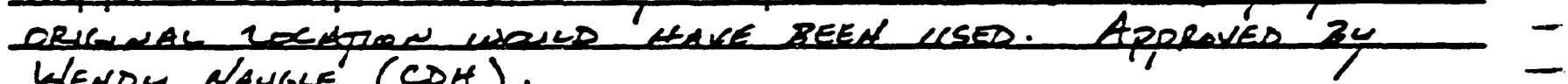
WeNOY NAUGE $(\mathrm{CDH})$.

SIGNTATURE:

DATE:

$12 / 10 / 12$

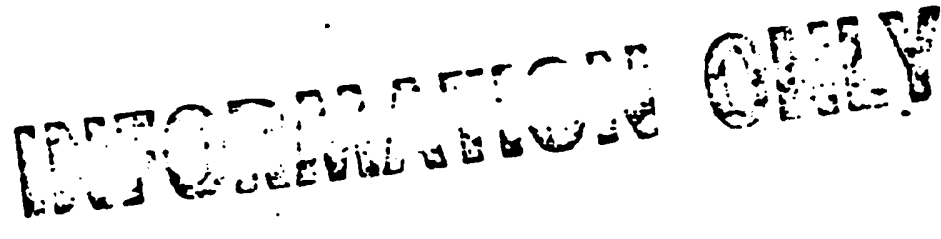




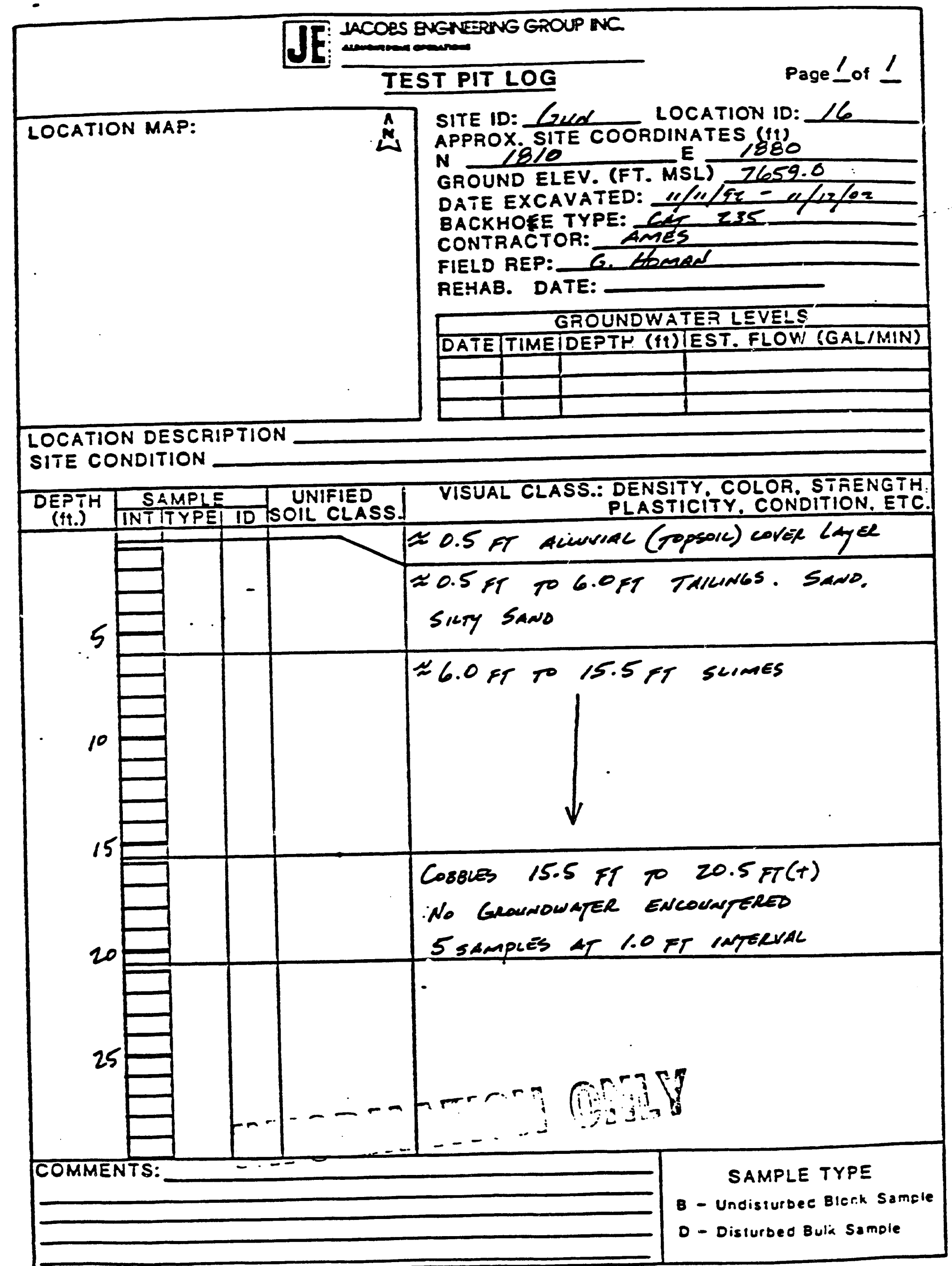




\section{COBBLES-TO-FINE TEST PI: DATA}

TEST PIT : 16

LOCATION: N 1810 E 1880

TAC TEST PIT: YES No $X$

CDH SAMPLES TO BE TAKEN BY MR-F/CWMAES: YES $\mathcal{X}$ NO

SURFACE EILVATION: 7659.0

DEPTH OF TAIIINGS/COBBLES INTERFACE: $15.5 \mathrm{FT}$

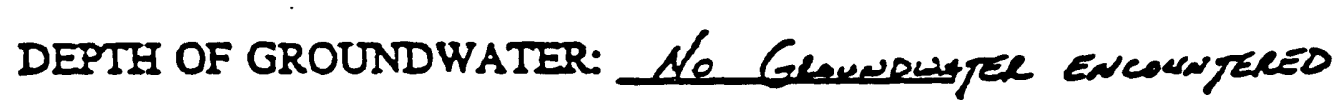

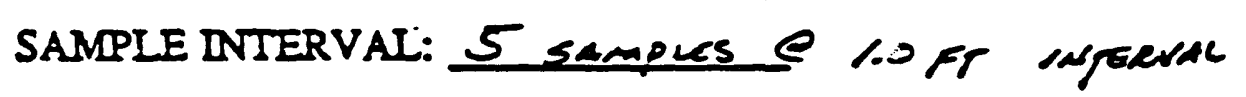

\section{COMMENTS:}

SIGNATURE:

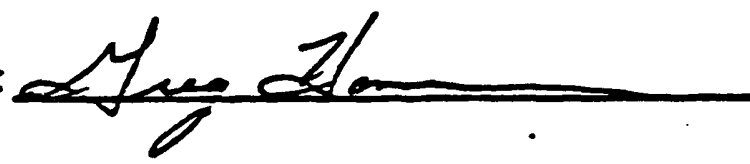

DATE: $12 / 10 / 92$

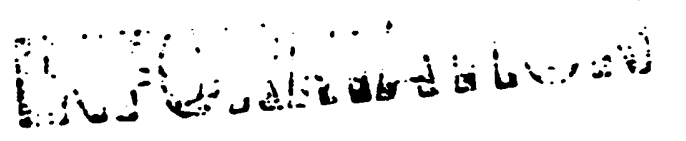

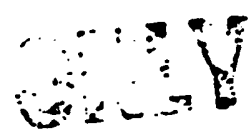




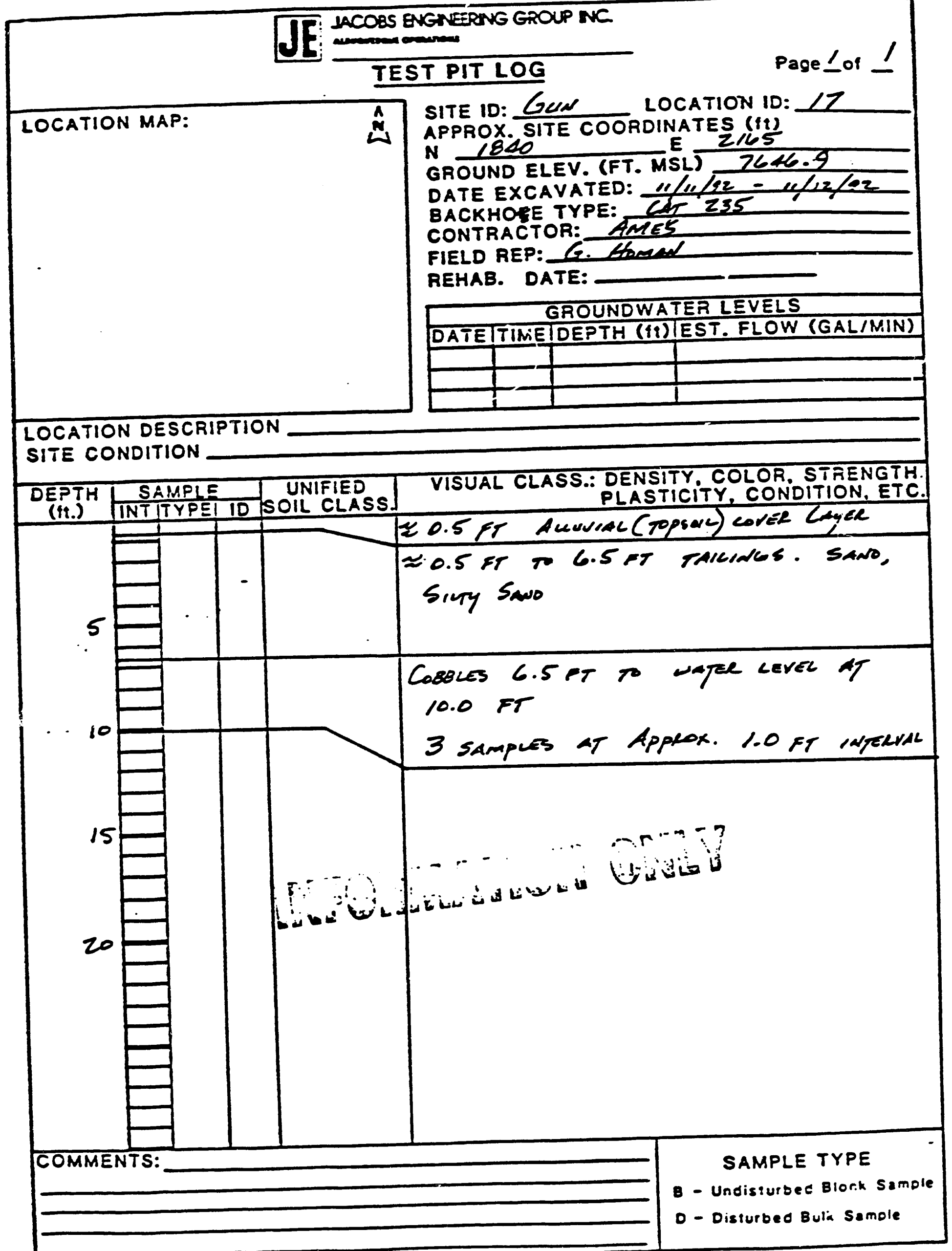


COBBLES-TO-FINE TEST PIT DATA

TEST PIT *

LOCATION: N 1E:40 E $2 / 65$

TAC TEST PIT: YES__ NO

CDH SAMPLES TO BE TAKEN BY MK-F/CWMFES: YES

No $x$

SURFACE ELEVATION: 7646.9

DEPTH OF TAIIINGS/COBBLES INTERFACE: $6.5 \mathrm{~F}$

DEPTH OF GROUNDWATER: $\approx 10.0 \mathrm{ES}$

SAMPLE INTERVAI: 3 sAmplese AFPRox. $1.0 \mathrm{gr}$ inferval

COMANENTS:

SIGNATURE: Nh 16 DATE:- $-22 / 9 / 92$

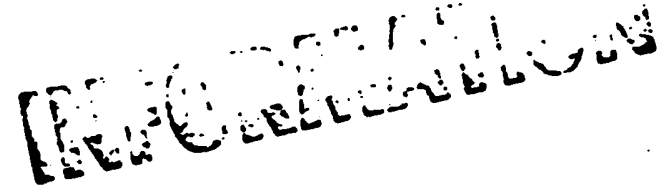




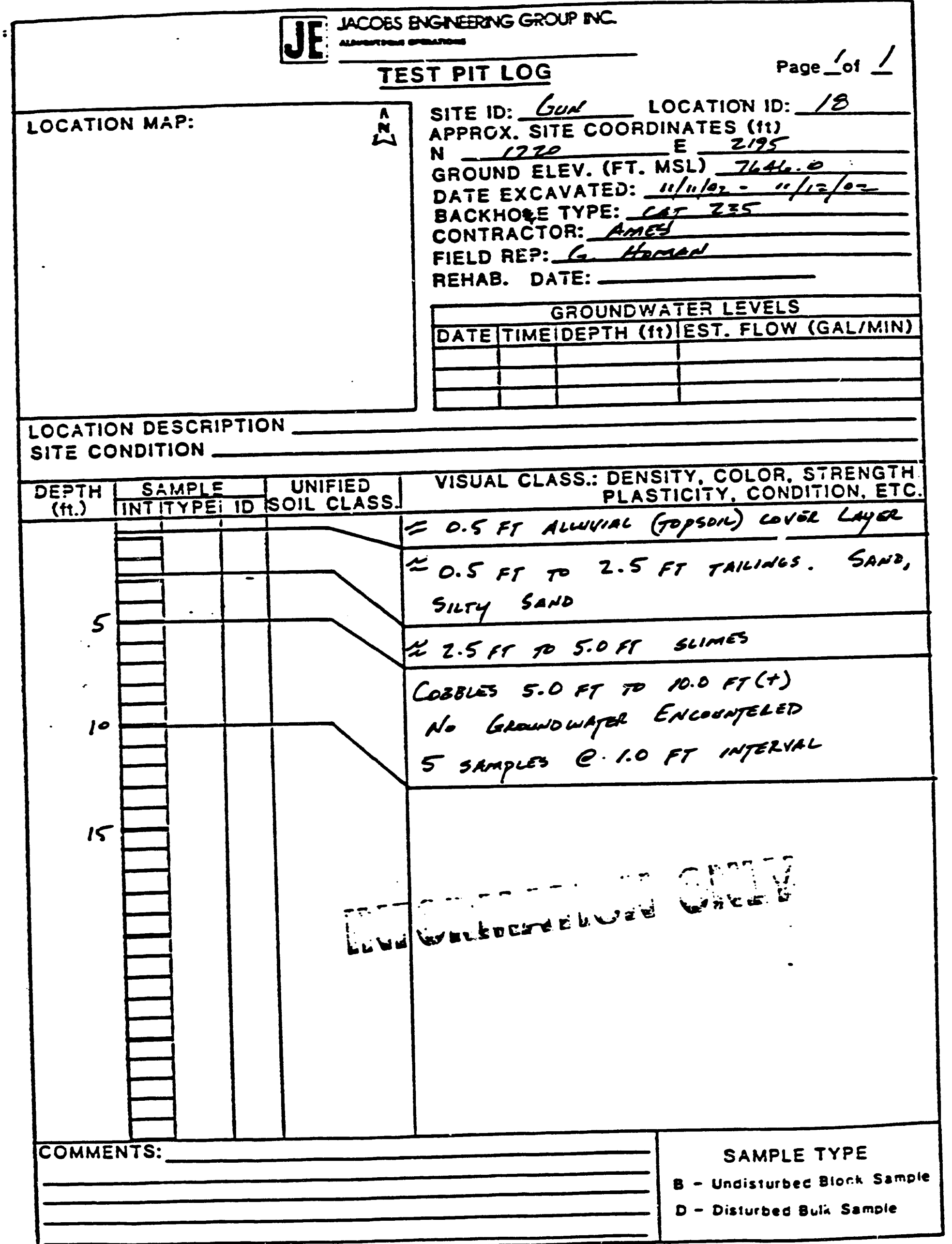


MK-:ERGUSON COMPANY

COBBLES-TO-FINE TEST PIT DATA

TEST PIT * 18

LOCATION: N_ $1720 \quad$ EZ Z195

THC TEST PIT: YES _ NO $X$

DH SAMPLES TO BE TAKEN BY MK-F/CWMFES: YES No $x$ SURFACE ELEVATION: 7646.0

DEPTH OF TAIINGS/COBBLES INTERFACE: 5.0 FT

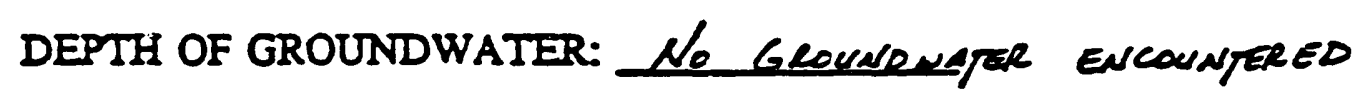

SAMPLE INTERVAL: 5 samples e 1.0 fT INTERVAL

COMMENTS:

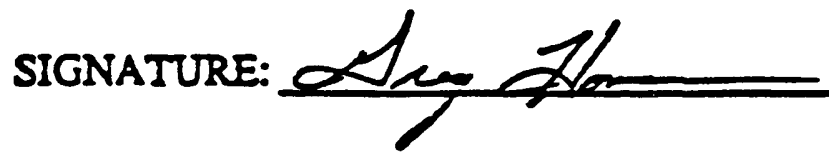
DATE: $12 / 10 / 12$

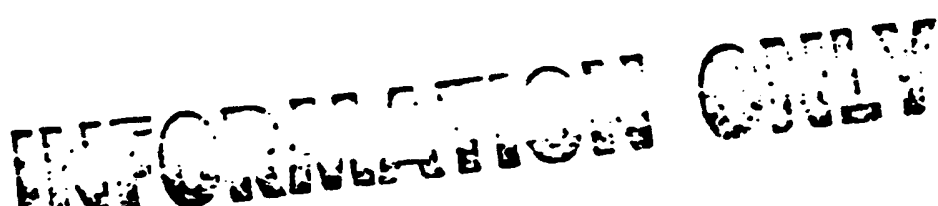




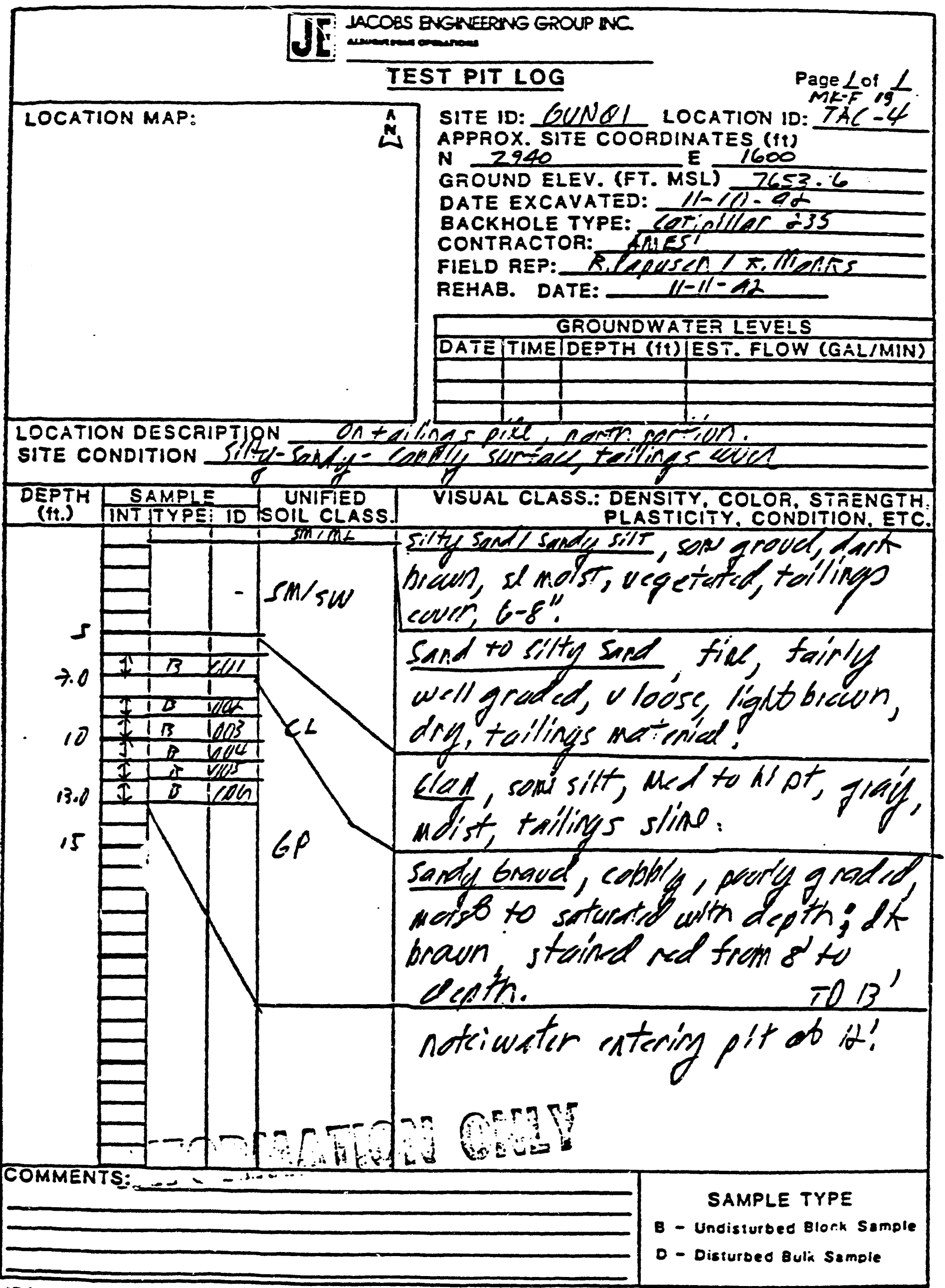


TF INCOSS ENGNESRNG GXOUP MR

SOIL SAMPLE INVENTORY

SITE ID: GWNOI

DATE: $\frac{\mu l i n l a z}{1}$

\section{LOCATION}

(BORING) ID: TAi -4

FIELD REP.: $\frac{\text { lingrake } / R \text {. Paausch }}{1}$
SAMPLE I.D.

$\frac{\frac{c 01}{0.2}}{\frac{c 03}{004}}$

DEPTH INT.

SAMPLE I.D.

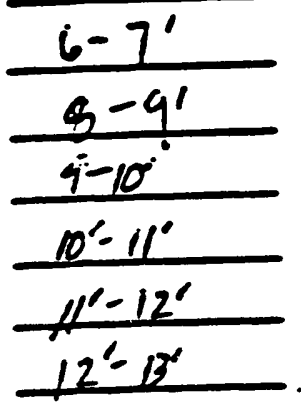

DEPTH INT.
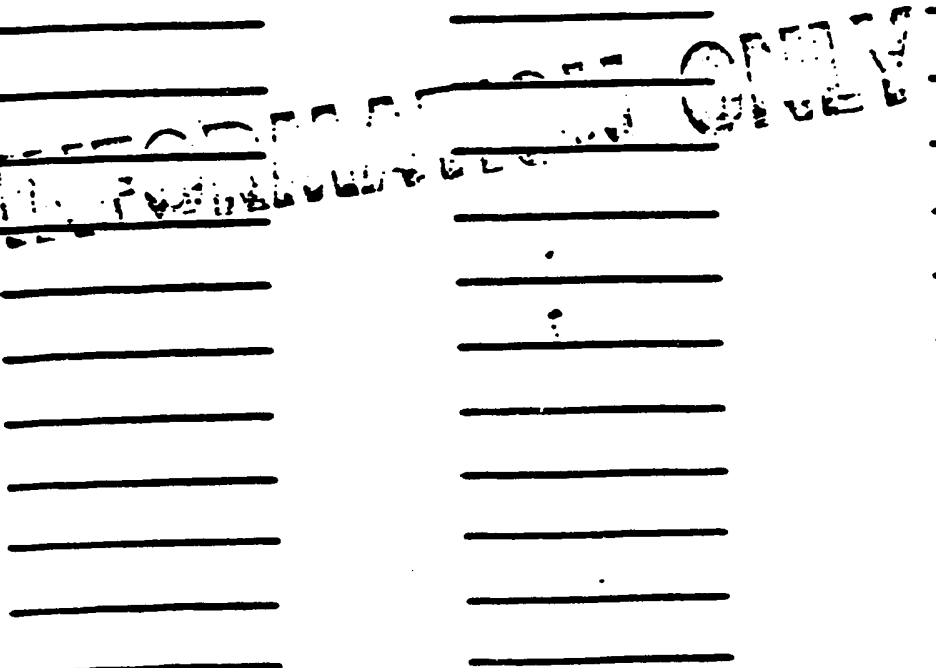
COBBLES-TO-FINE TEST PIT DATA

1

MK.FERGUSON COMPANY

I

COBBLESTO-FINE TEST PIT DATA

TEST PIT \#: $\frac{19}{\text { LOCATION: N } 2940}$ E 1600
LAC TEST PIT: YES $X$ NO
DH SAMPLES TO BE TAKEN BY MK-FICWMFES: YES NO X
SURFACE ELEVATION: 7653.6
DEPTH OF TAIIINGSICOBBLES INTERFACE: $\approx$ TET DEPTH OF GROUNDWATER: $1 Z$ ET

SAMPLE INTERVAL: 6 sAmpLES 0 loo FT INTERAA

COMMENTS:

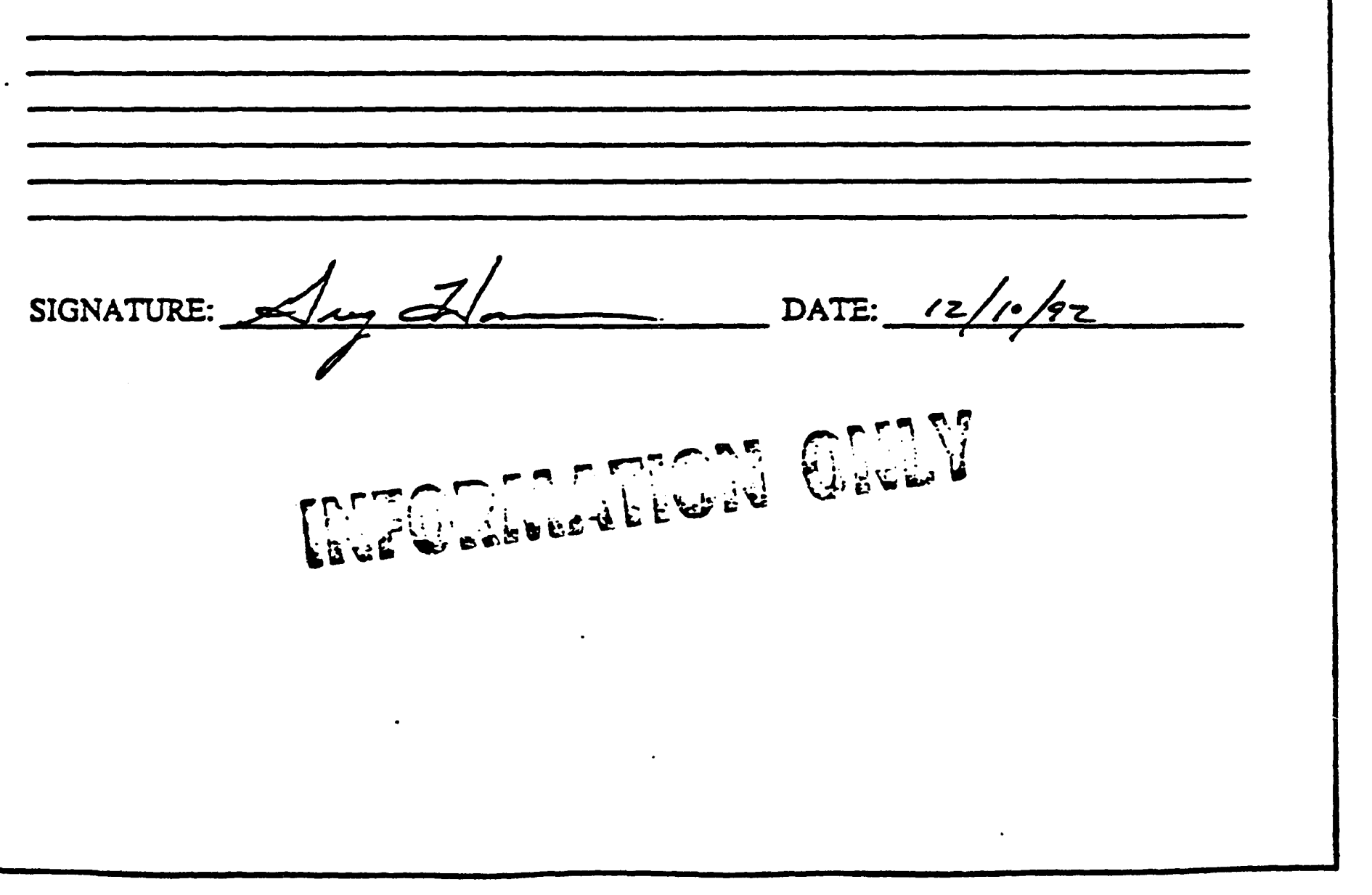




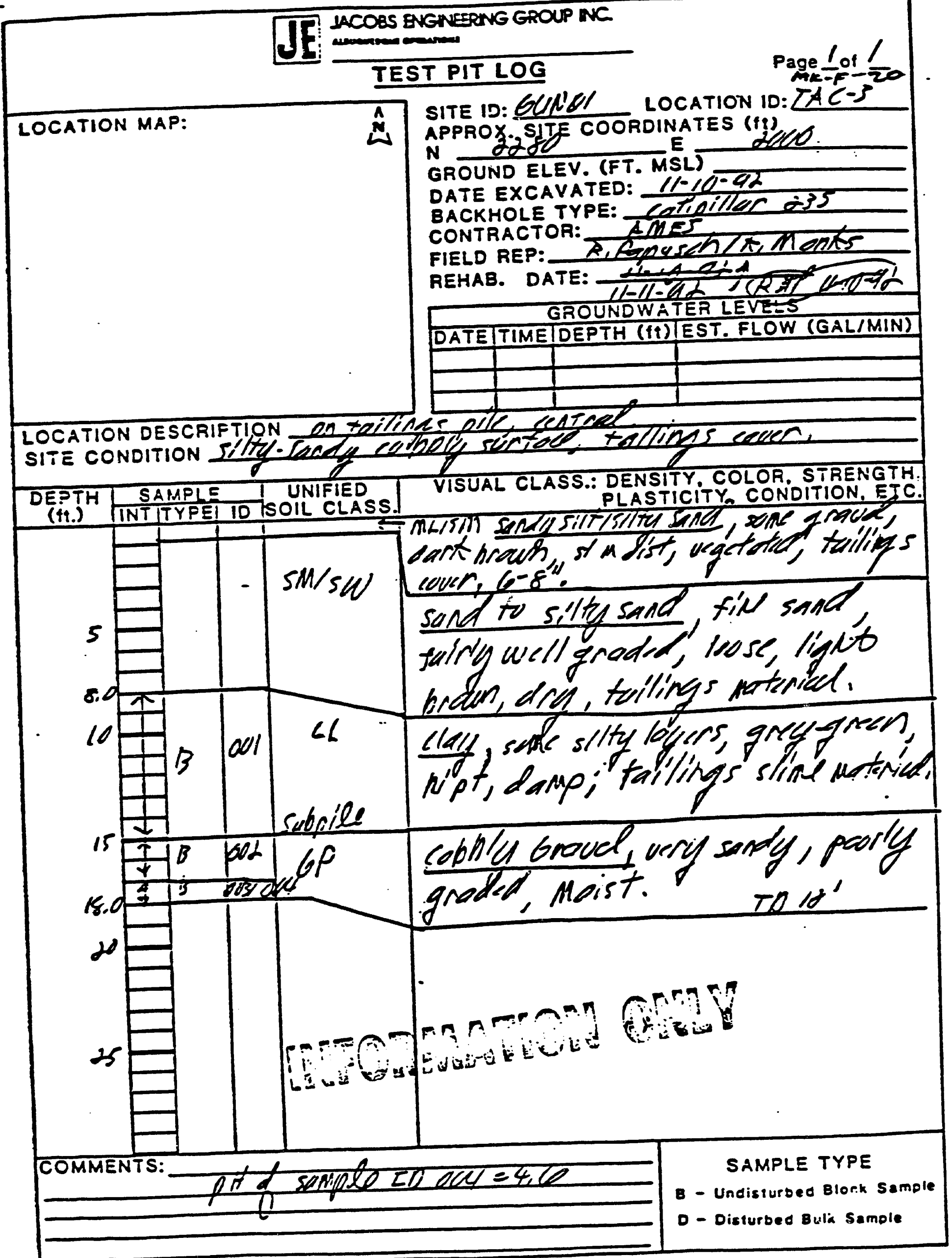




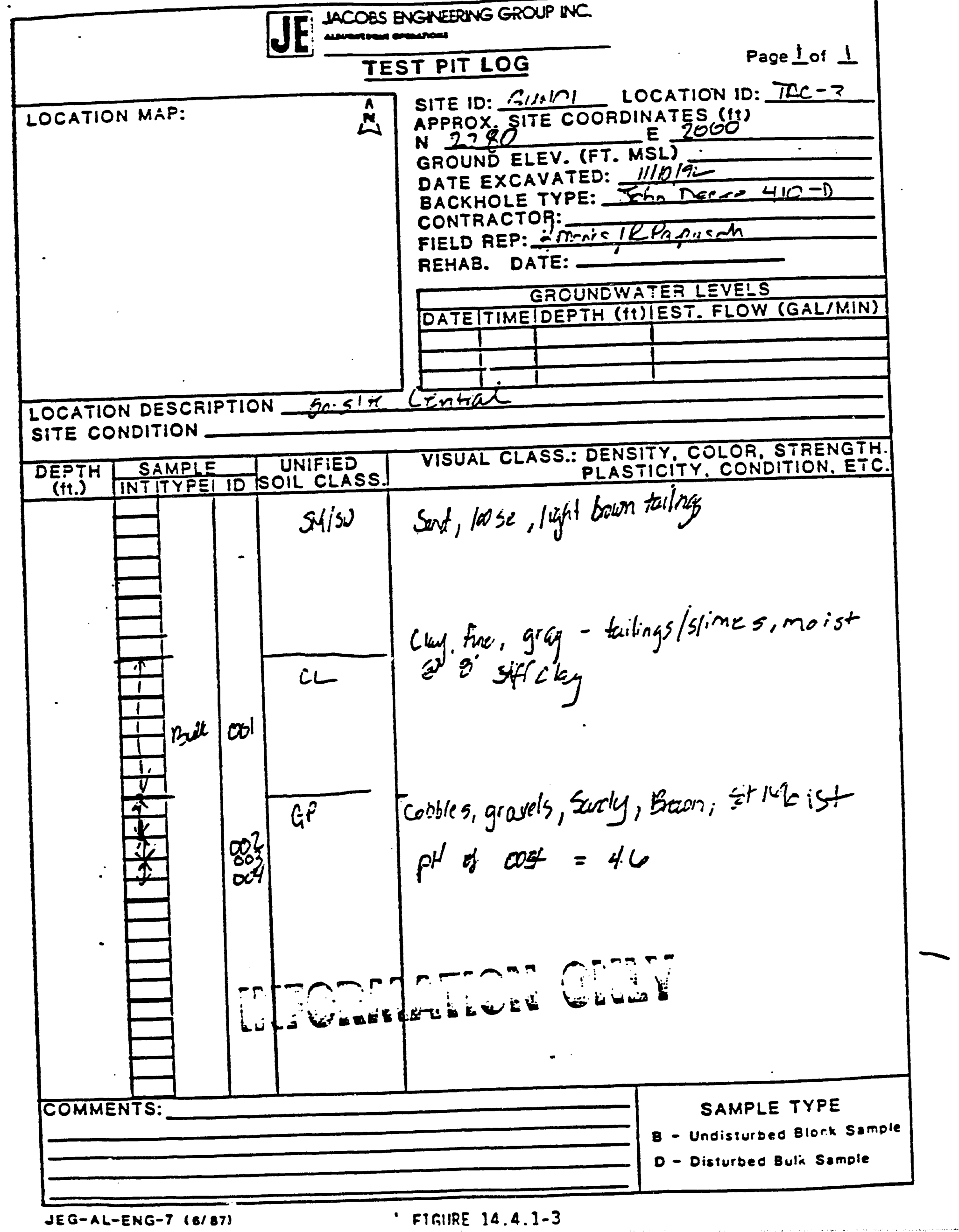


LCOSS ENGNESRNG GROUP AR

SOIL SAMPLE INVENTORY

SITE ID: $\stackrel{9}{9} \mathrm{H}$. 101

DATE: $\frac{11 / 10 / g}{16}$
LOCATION

(BORING) ID: TAC - 3

FIELD REP.: Kmonelepapienh

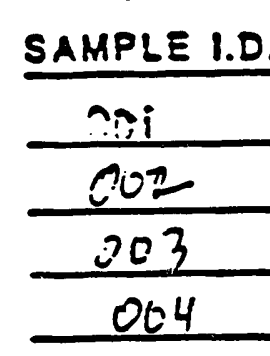

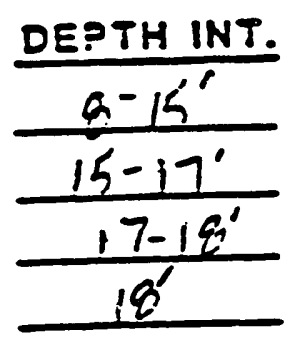

SAMPLE I.D.

DEPTH INT.

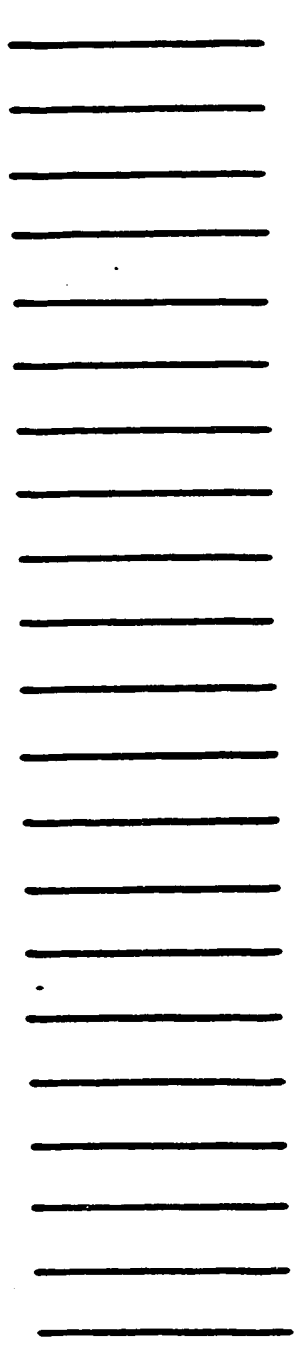




\section{COBBLESTO-FINE TEST PIT DATA}

TEST PIT *: ZO

LOCATION: N Z280 E Z2000

TAC TEST PIT: YES $\chi$ NO

CDH SAMPLES TO BE TAKEN BY MK-F/CWMFES: YES NO $x$ SURFACE EIIVVATION: 7657.4

DEDTH OF TAIINGS/COBBLES INIERFACE: $15.0 \mathrm{EF}$ DEPTH OF GROUNDWATER: $\approx 17$ FT T0 $18 \mathrm{FT}$ SAMPLE INTERVAI: BuLK SAMPU e BfT To 15 fF 3 samples $Q$ I FT unferoul

COMMEENTS:

SIGNATURE:

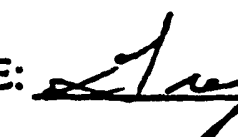

DATE: $12 / 10 / 92$

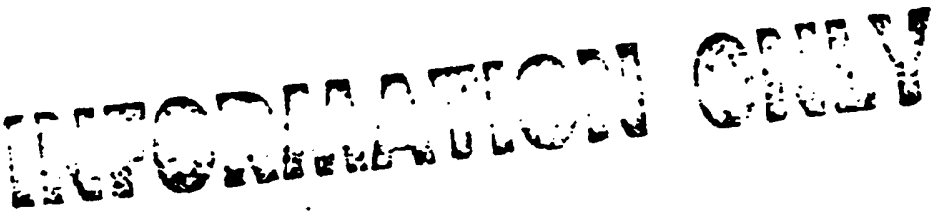




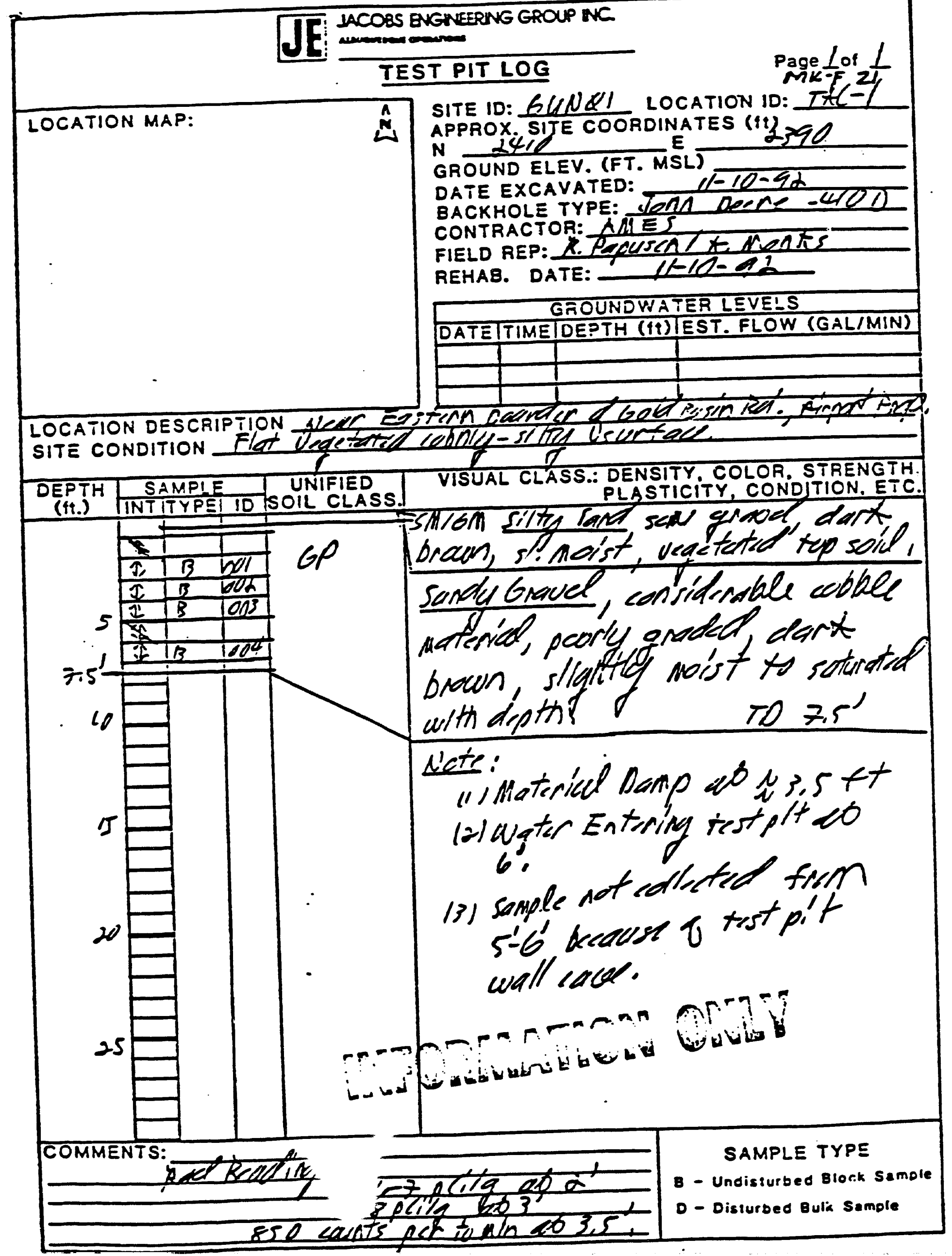


$\hat{M}$ SITE ID: GIINOI LOCATION ID: TAC.I

BACKHOLE TYPE:

CONTRACTOR:

FIELD REP: Kimimks RPanisch

REHAB. DATE:

\begin{tabular}{|c|} 
GRCUNDWATER LEVELS \\
\hline DATEITIMEIDEPTH (fI)IEST. FLOW (GAL/MIN)
\end{tabular}

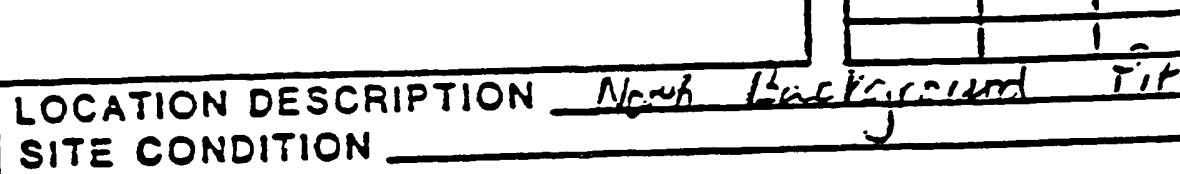

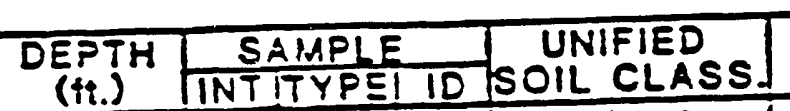

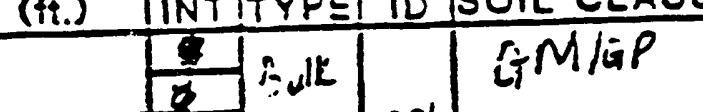

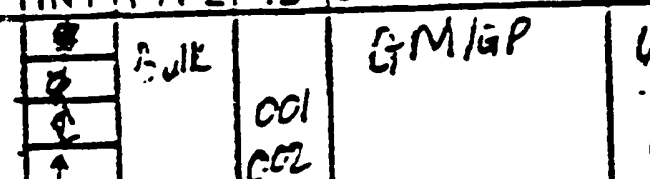

Ciible-Crovel, Sancin Slicing

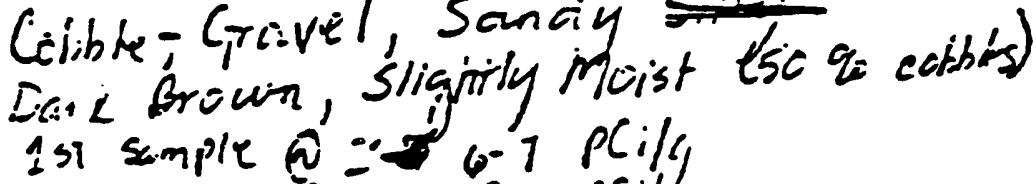

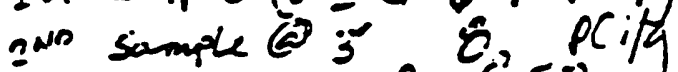
Damp at ₹ 3.5 if $\varepsilon 50$ iecints peitsin 6 urith iable $5-i$; sample iat ieltectid dive to caveCOMMENTS: 
ATIACHMIEN I Iraye $u$ -

$\checkmark$

TF: UCOQS ENGNESRNG GROUP NK.

JE

SOIL SAMPLE INVENTORY

SITE ID: EUNA!

LOCATION
(BORING) ID: TAC. -

DATE: $\frac{\| l \text { lokn }}{1}$

FIELD REP.:

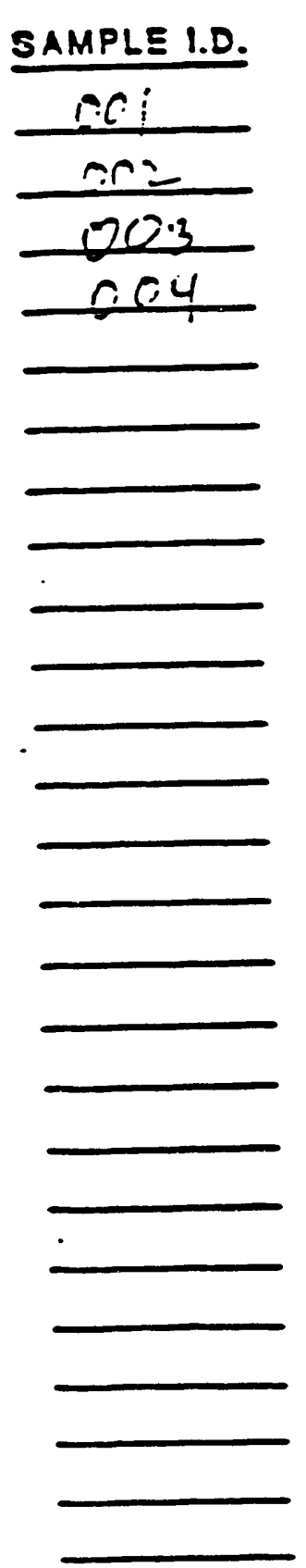

DEPTH INT.

SAMPLE 1.D.

DEPTH INT.
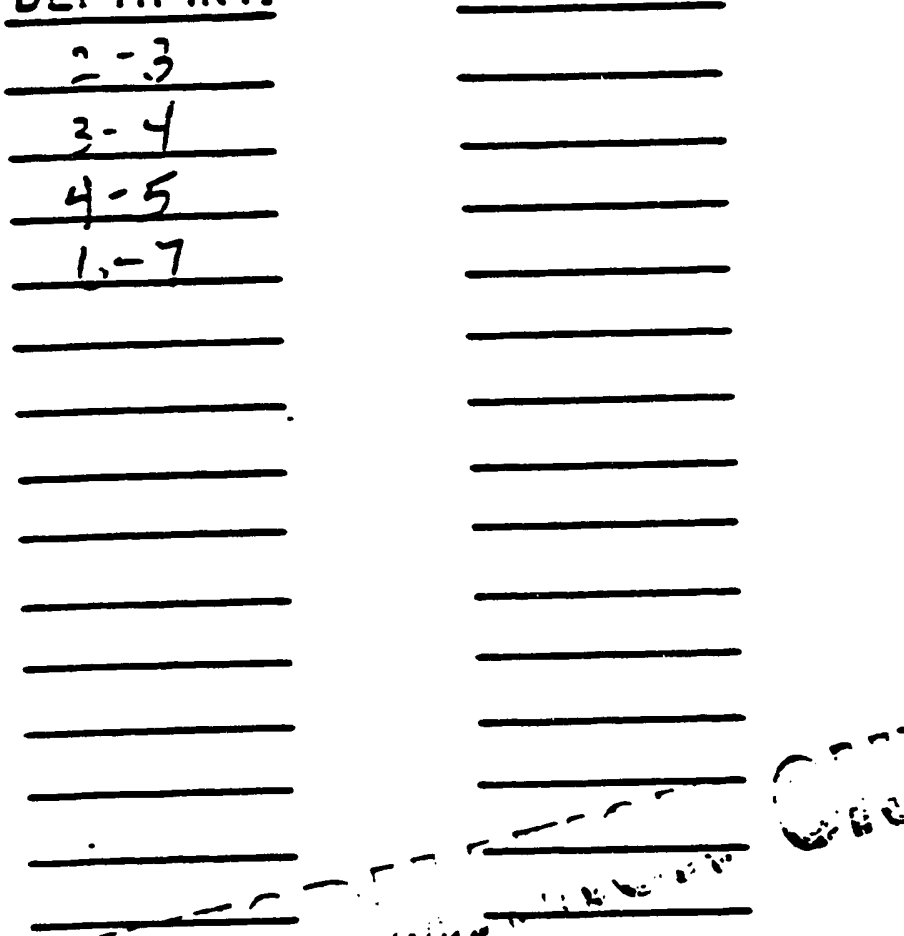

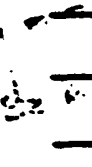
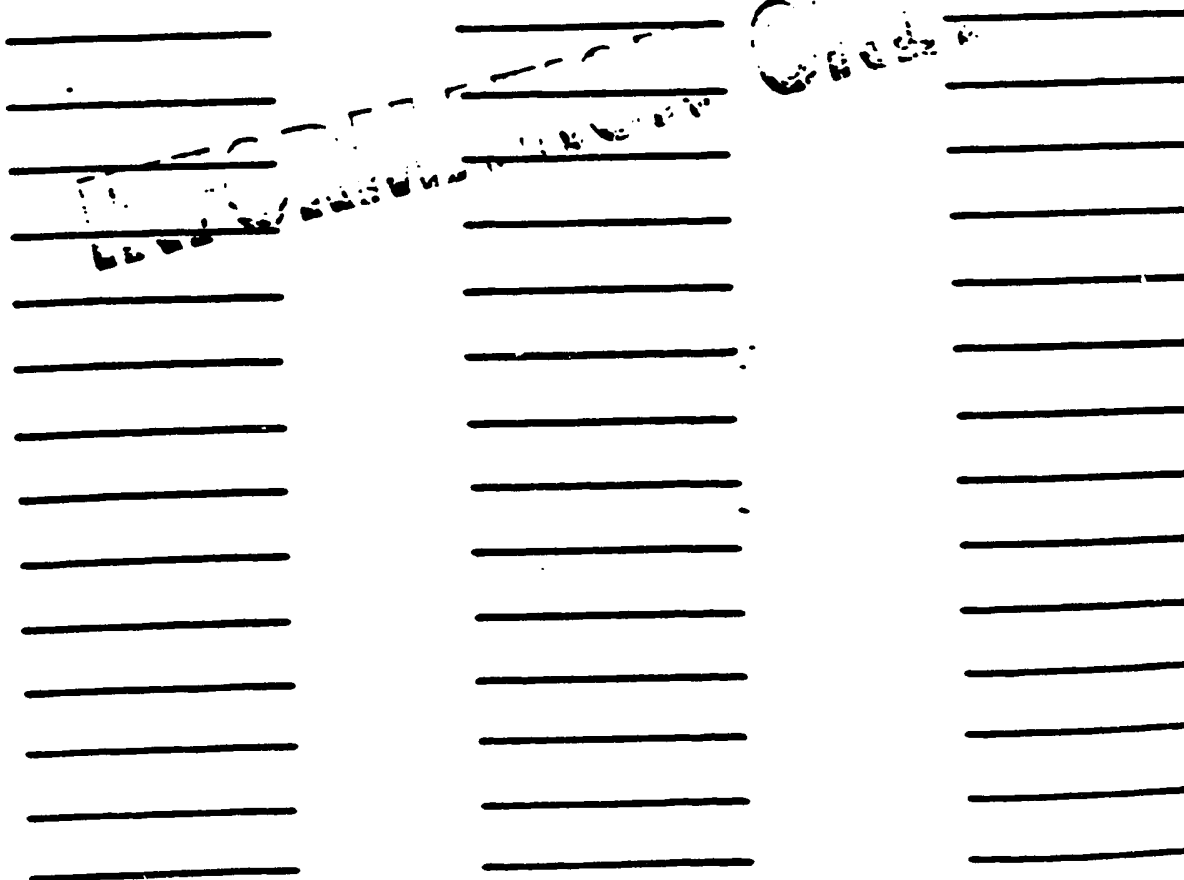
COBBLES-TO-FINE TEST PIT DATA

TEST PIT : 21

LOCATION: N Z410 E Z390

THC TEST PIT: YES $X$ NO

CDR SAMPLES TO BE TAKEN BY MK-F/CWMEFE: YES No $x$ SURFACE ELEVATION: 76.46 .7

DEPTH OF TAIINGS/COBBLES INTERFACE: No TaILINGS, ALL C.BBLES DEPTH OF GROUNDWATER: 6 FT

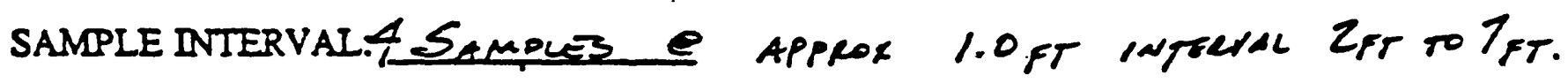

COMMENTS:

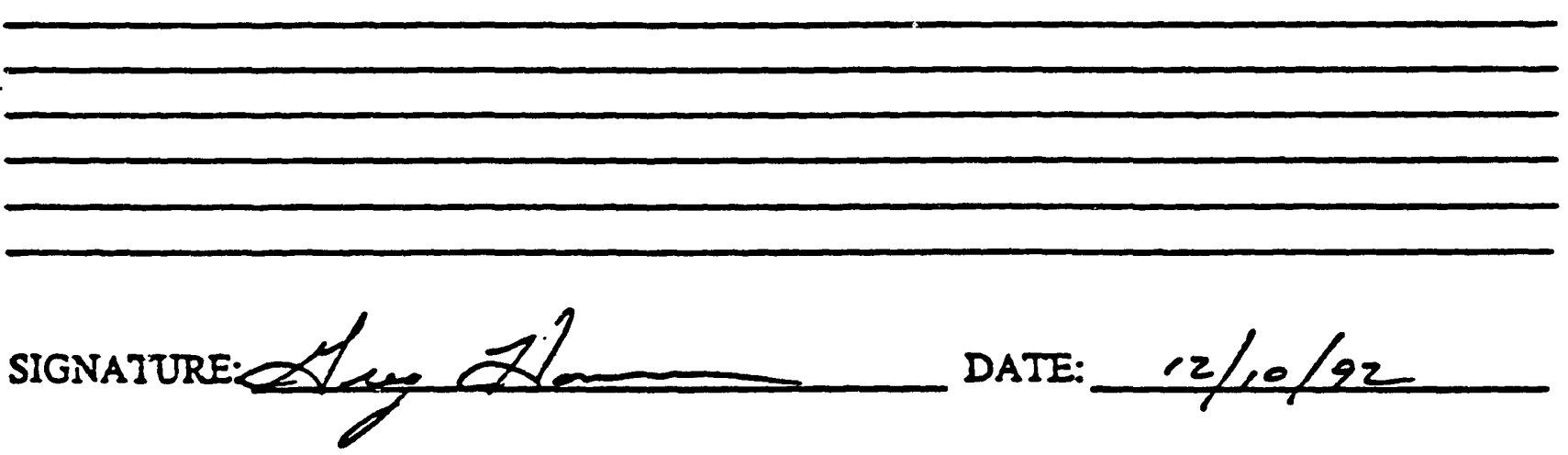




\section{TEST PIT LOG}

SITE ID: IUNEI

LOCATION ID: TAC-S

LCATION MAP:

APPROX. SITE COORDINATES (fI)

N 1880

E 2025

GROUND ELEV. (FT. MSL) 7655.5

DATE EXCAVATED: 11 lpte

BACKHOLE TYPE: Crik izL

CONTRACTOR:

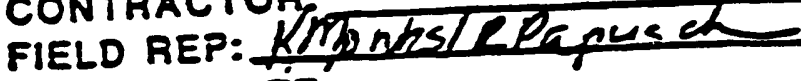

REHAB. DATE:

GROUNDWATER LEVELS

LOCATION DESCRIPTION

Antsit solith it

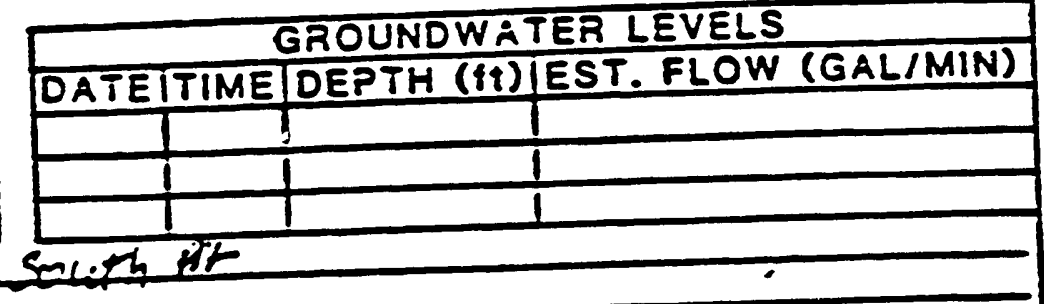

SITE CONDITION

DEPTH SRMPLE

(H.) INTITYPE ID SOIL CLASS VISUAL CLASS.: DENSITY, COLOR, STRENGTH:
PLASTICITY, CONDITION, ETC.

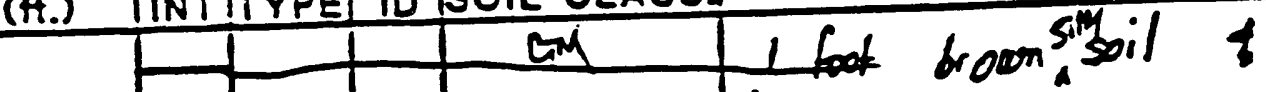

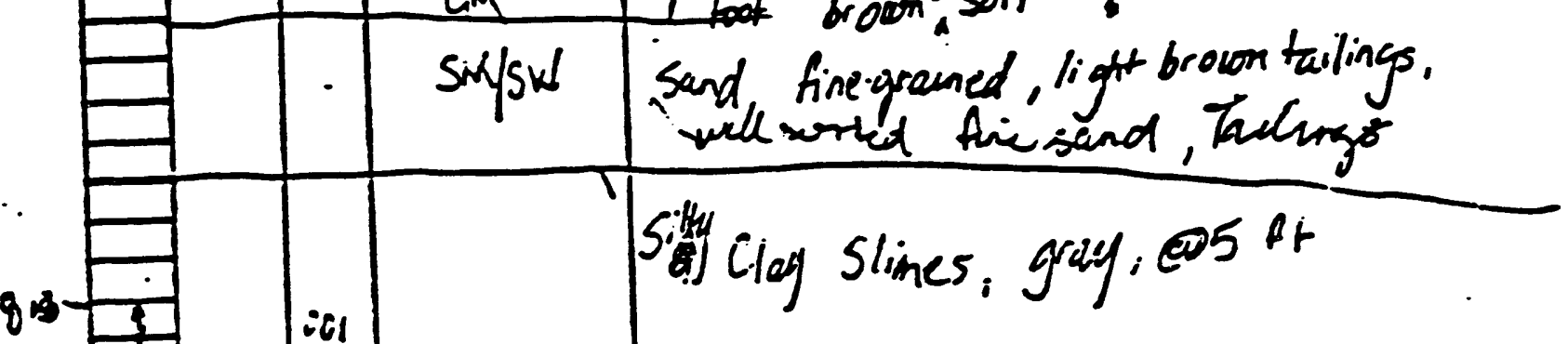

8

$+1$

in

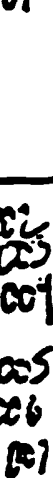

GTuivis, Coinhlis, sands, biciwn, moist

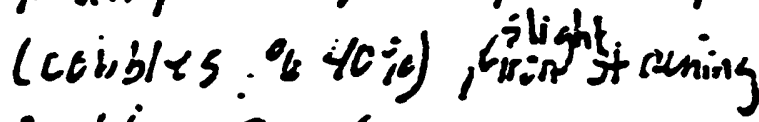

beilction $\overrightarrow{0}: ?^{\prime}$

watertable at $14, b$

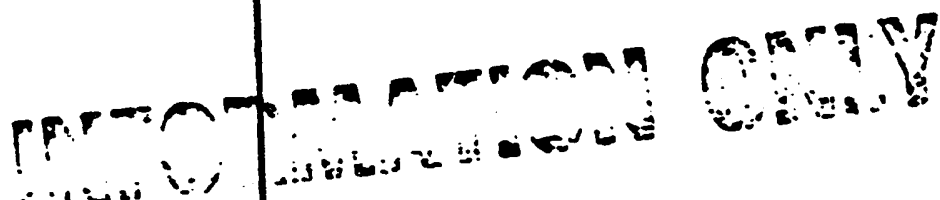

iviliop / Subsioil inter frice e $13^{\prime}$ 
ATTACHMENT 1 (Page 101

SOIL SAMPLE INVENTORY

LOCATION
(BORING) ID: TAE - S

SITE ID: $\frac{\text { iANNE! }}{4}$

DATE: $\frac{+1 / \ln 192}{1}$

FIELD REP:: Krents fo danusch

SAMPLE 1.0.
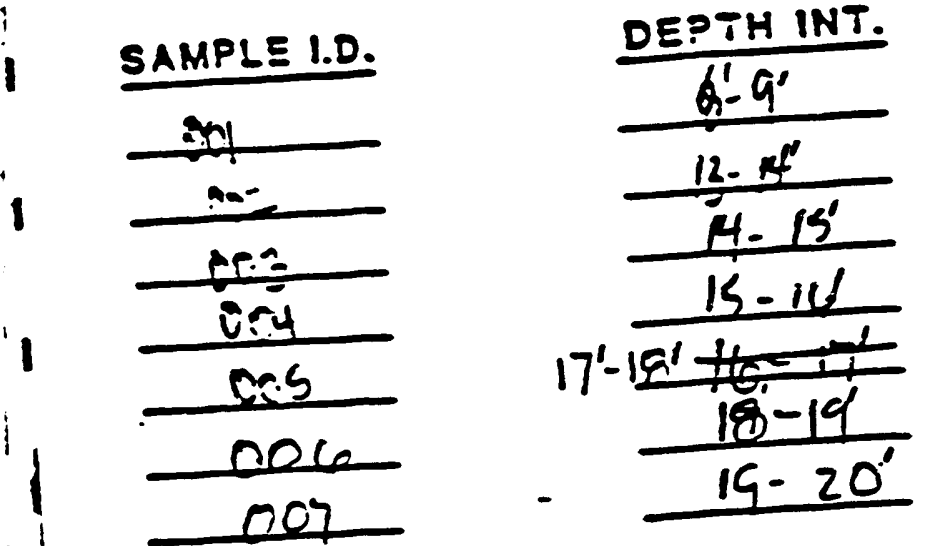

SAMPLE 1.D.

DEPTH INT.

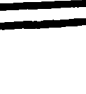

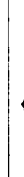
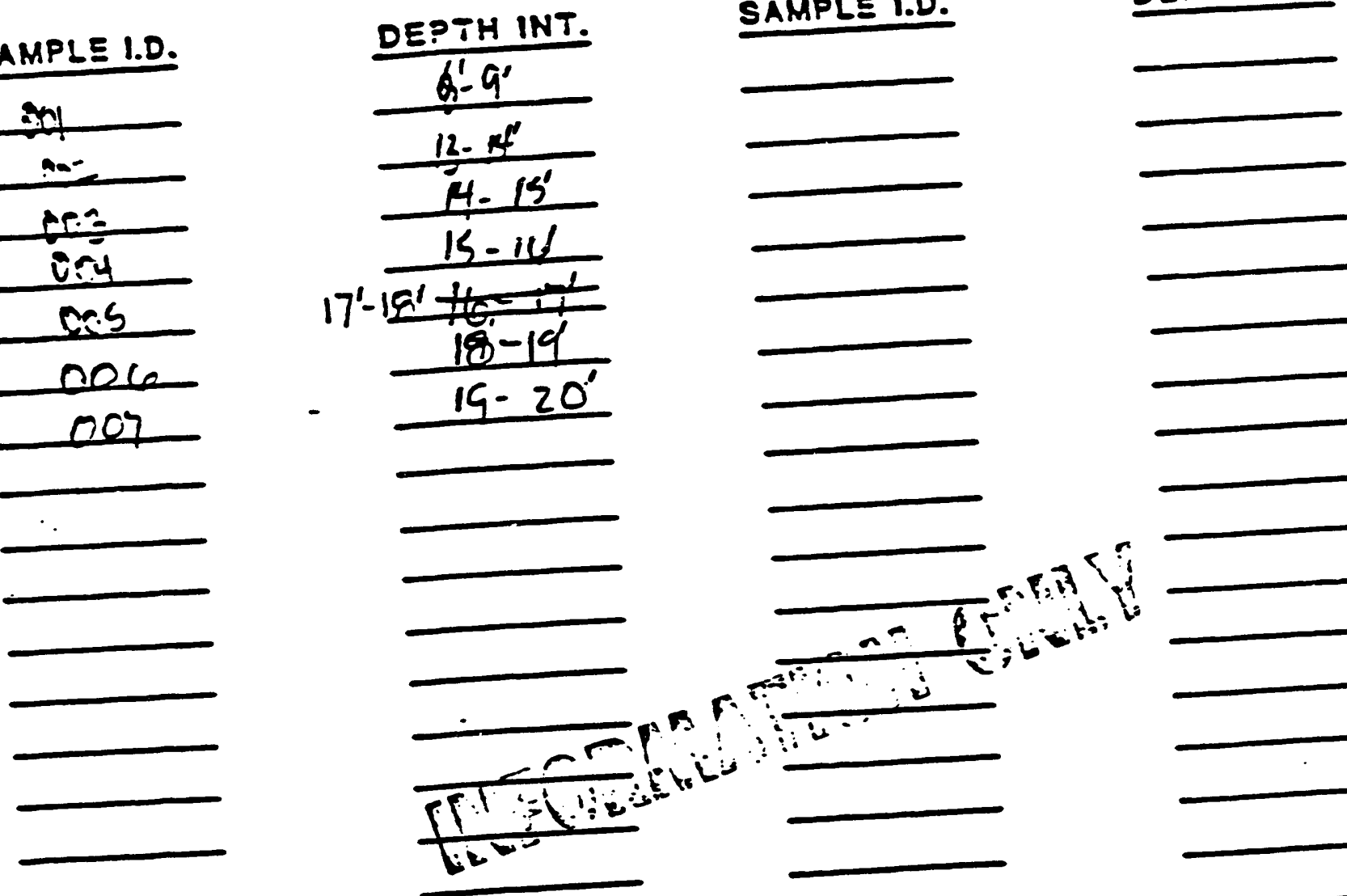


\section{COBBLES-TO-FINE TEST PIT DATA}

TEST PIT * ZZ ZZ

LOCATION: N_ 1880 E_L25

TAC TEST PIT: YES $\not$ NO

CDH SAMPLES TO BE TAKEN BY MK-F/CWMFES: YES

No $\underline{X}$

SURFACE ELEVATION: 7655.5

DEPTH OF TAIINGS/COBBLES INTERFACE: 13 ET

DEPTH OF GROUNDWATER: $19.0 \mathrm{ES}$

SAMPLE INTERVAL: 1 samples $e$ appeox. 1.0 fr infaeral

COMNENTS:

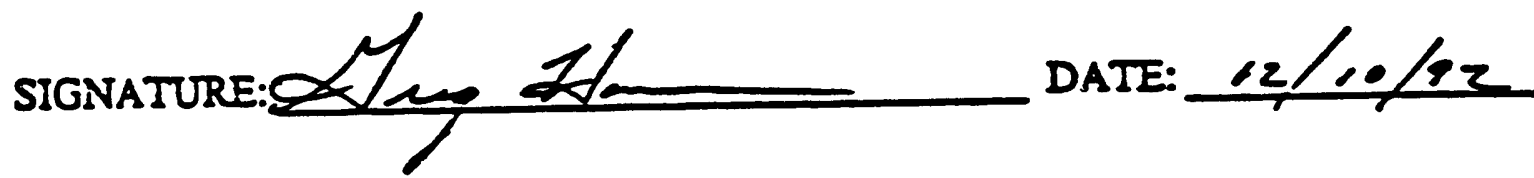

DATE: $12 / 10 / 82$ 


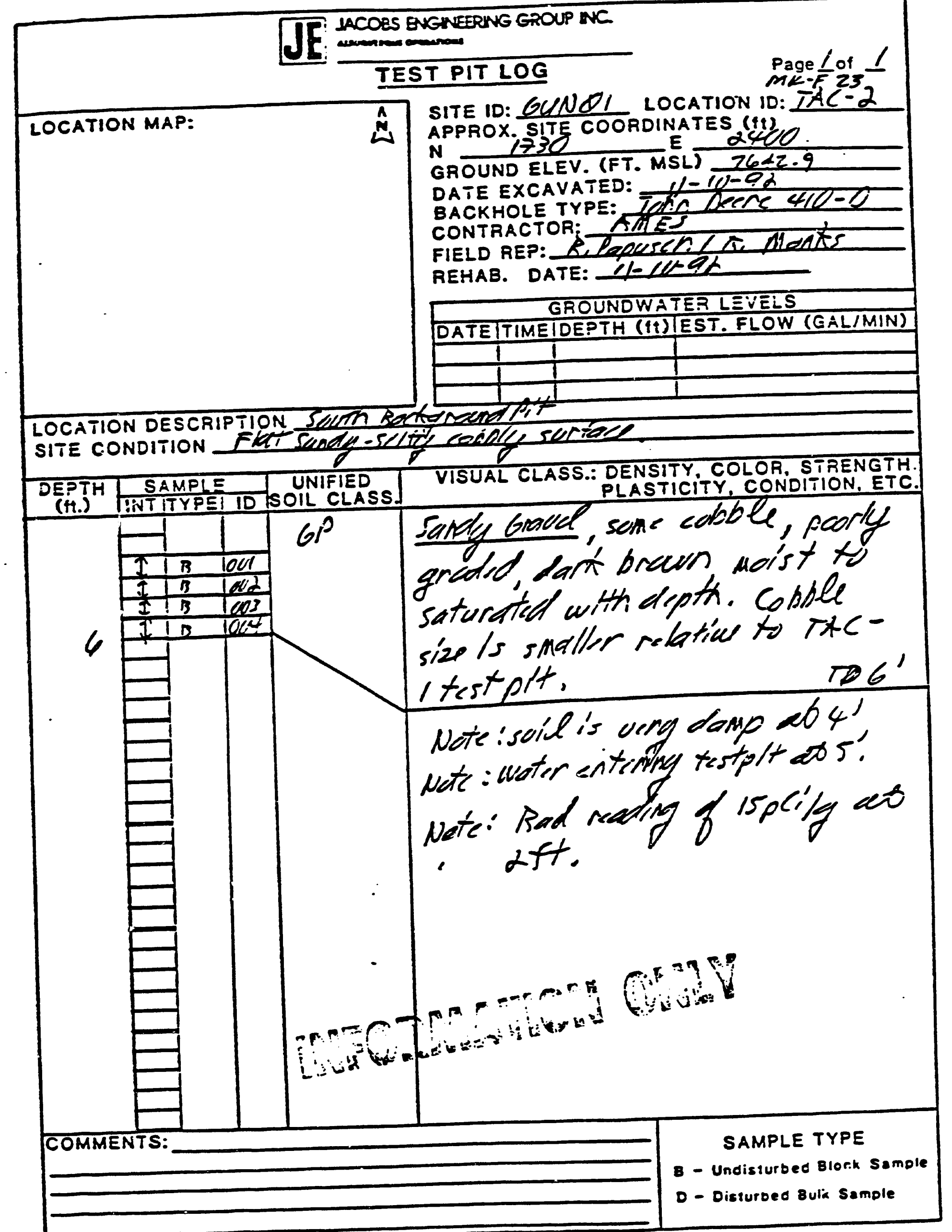




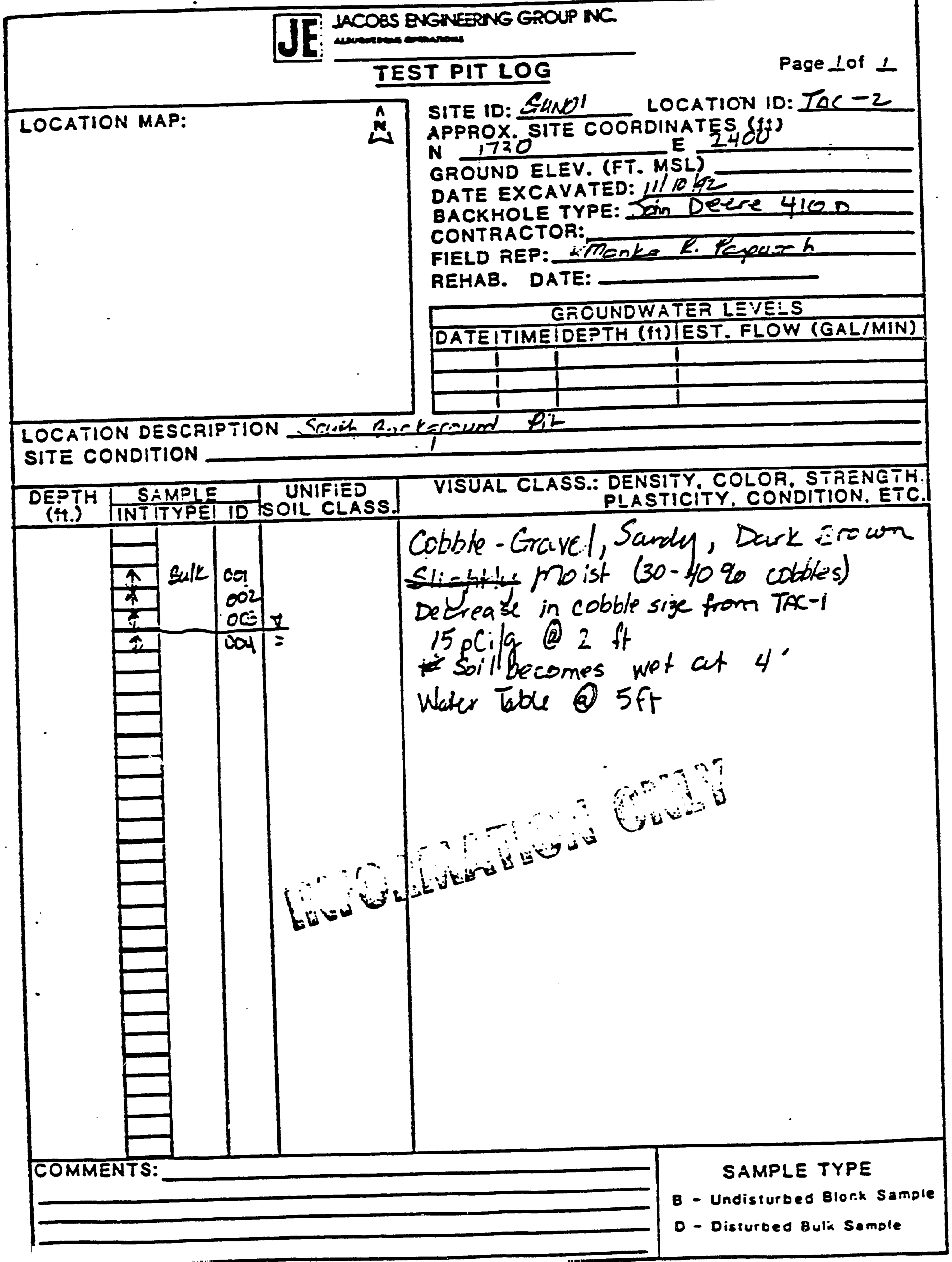


TF: JACOBS EVGNESRNG GROUP NR.

SOIL SAMPLE INVENTORY

SITE ID: GLUNI?:

DATE: $\frac{1110 / 42}{11}$
LOCATION TAC-2
(BORINE) ID:

FIELD REP:: R Pacises / KMone
SAMPLE I.D.

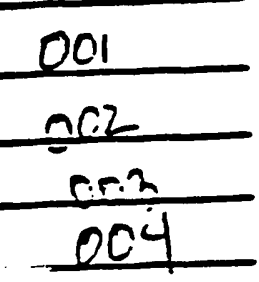

DEPTH INT.

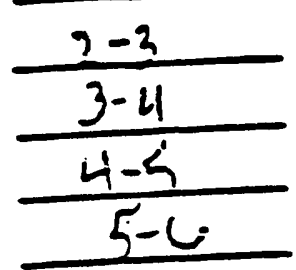

SAMPLE 1.0.

DEPTH INT. 
TEST PIT :

LOCATION: $\mathrm{N}$

1730

E 2400

TAC TEST PIT: YES_ $\not \underline{X}$ NO

CDF SAMPIES TO BE TAKEN BY MK-F/CWMFES: YES No $x$

SURFACE EIEVATION: 7642.9

DEPTH OF TAIIINGS/COBBLES INTERFACE: No pellewles ALC cooBCE DEPTH OF GROUNDWATER: 5.0 EF

SAMPLE INIERVAI: 4 SAMPLES \& $1.0 \mathrm{fT}$ infeRVAL FROM $2.0 \mathrm{fT}$ To $6.0 \%$

COMMENTS:

Bderinguns Tas ?IT

SIGNATURE:

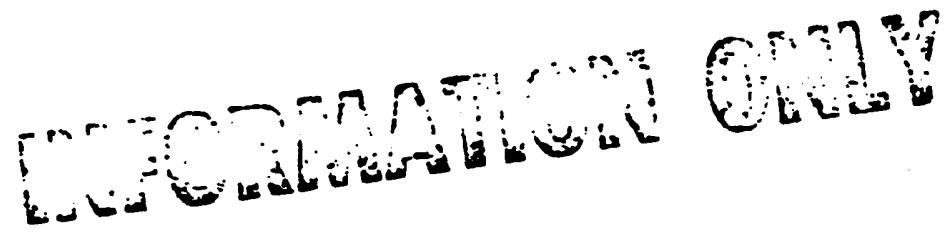


SIEVE ANALYSIS AND SUMMARY OF RADIOLOGICAL MEASUREMENTS: COMPOSITE COBBLY SOIL SAMPLES COBBLY SUBSOIL INVESTIGATION NOVEMBER 1992 
-ENGINEERS

AND

CONSTRUCTORS

Doug Gonzales

meroountens prince

ONE ERIBVIEW PLATA

CLEVLUNO. OM O U. SA ans

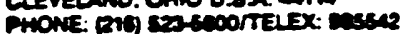

February 2, 1993

Mr. Steve Hemp

DOE Site Manager

U.S. Department of Energy

Uranium Mill Tailings Remedial Action Project Office

First National Bank Building

5301 Central Avenue N.E.

Suite 1700

Albuquerque, New Mexico 87108

SUBJECT: Cobbles to Fines - Gunnison, Colorado

REFERENCE: COATI aCt NO. DE-ACO4-83AI 18796

Dear Mr. Hemp:

Enclosed please find the radiological information developed for the subject test pits.

If you have any questions or comments, please contact Bob Hindman at 246-2557. sincerely.

MYTERYUSON COMPANY

Cache

C. R. Spencer

Construction Engineering Manager

CRS/RCH/mno

Attachment

cc: w/attachment :

พ. Naugle, CDH

oJ. Mckee. TAC/OMTRA
DEFY TO: IX FERGUSON COMPANY REMEDUL ACTIONS boo. $0 \times 1013$ ilvoverioue, nw mexico usa. into

$$
\begin{aligned}
& \text { 93-3050-097 } \\
& 845-5868
\end{aligned}
$$
contructonunTm Fmorect

$0723 \pi 2$ 
DATE: Jan. 20, 1993

TO: Jim Tumer

FROM: Ron Jacobs of for eaf LOCATION: Gunnison

SUBJECT: Cobbles-to-fines

Enclosed is the requested cobbles-to-fines information from the Gunnison site test pits.

If you have any further inquiries, please contact me.

$\mathrm{RDJ} / \mathrm{KD} / \mathrm{ts}$

pc John Innis 


\section{COBELES TO FINES CALCULATION SHEET}

l.

ine: $3050 / \mathrm{Gen}$

! 'sample/Erid I.D.
Fines

Vt Sample

a Bucker 20,45

N. Bucket

Nic Sample

$\frac{1.77}{12 . i^{5}}$

Idjusted

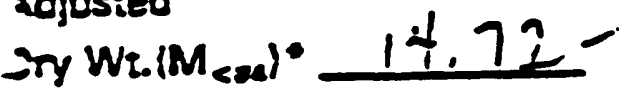

\section{Cobbles}

Wi. Sample

2 Bucker

Wr. Bucket

Wt. Sample

Adjusted

Dry $W_{\text {t. }}\left(M_{2 \text { sed }} \cdots 56.9 .4-\right.$
Date: $11 / 12 / 92^{\text {of }}$

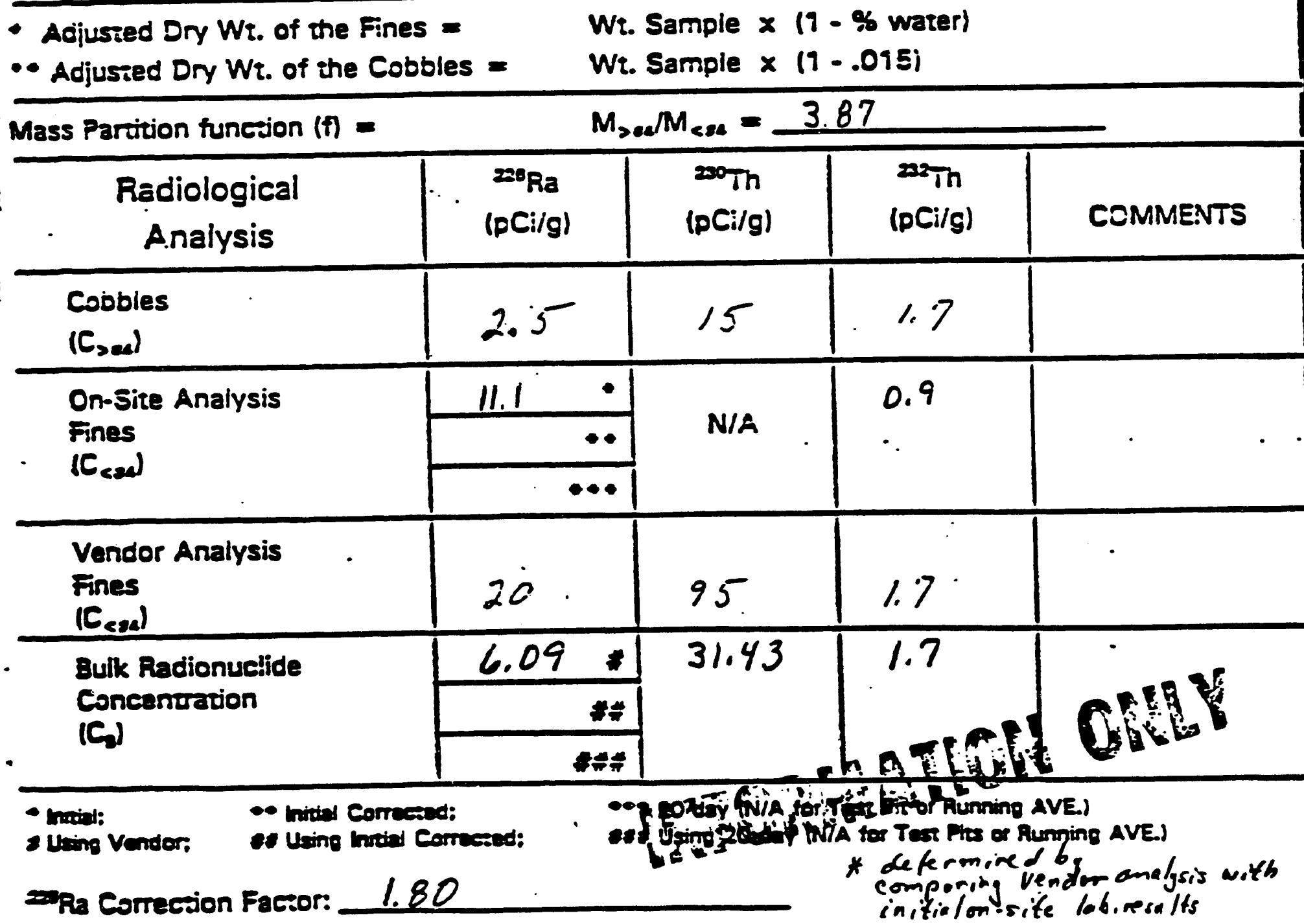


COBRLES TO FINES CALCULATION SHEET

ise: Givin Izaca)

umple/Grid I.D. Trer

Sheer of

Date: 11 inlan,

ines

\section{Sample}

\& Bucket 1s re

I. Bucket

it Sample

jijusied

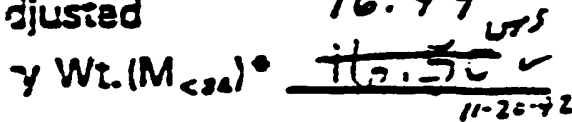

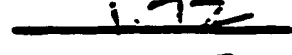

16.49

\section{Cobbles}

Wt. Sample

8 Bucket

Wt. Bucket

Wt. Sampile

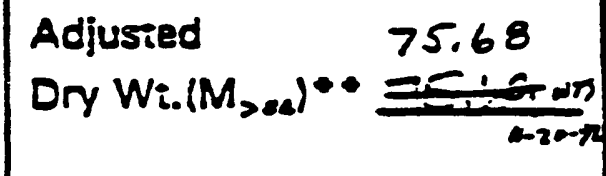
25
1.77

Moisture Content Fines

Wet Wt. Sample/Pan 616.5

Dry Wt. Sample/Pan 599.32

Tare Wt. Pan

18.22

17.2

Wt. Water"

Wt. Soil ${ }^{\bullet-\infty}$

Percent Water $(\bullet / \bullet \bullet)$

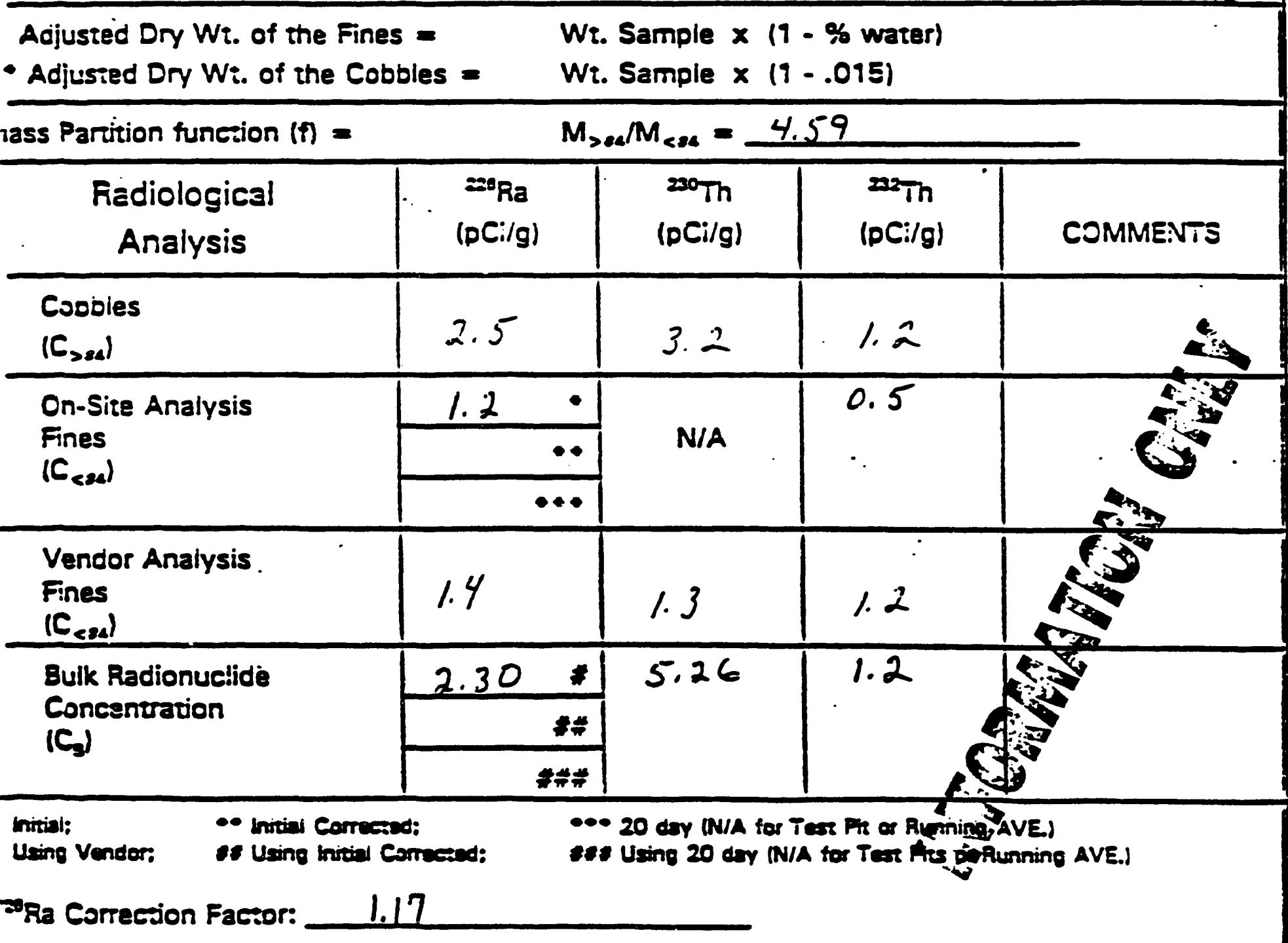

ieviewed By:

Date: 
COBBLES TO FINES EALCULATION SHEEI

ire: 3050$)$ (xin

1 ample/Grid 1.0. T/est tit it 3

Date: $\frac{1 / \sqrt{12 / 52}}{l}$

ines

it Sample

8 Bucker 32,43

it. Bucker

. Ir Sample

2.02

djusied

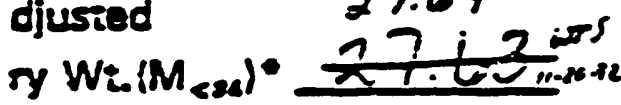

Cobbles

Wt. Sample

\& Bucket

Wt. Bucker

Wi. Sample 124,76

Adjusted

Dry $W_{t}\left(M_{2 e a}\right)^{\bullet+103.19}$
Moisture Content Fines

Wet Wt. Sample Pan S34.0.i)

Dry Wr. Sample!Pan

Tare WL. Pan

496.12

18.20

Wt. Water*

Wt. Soil *

Percert Water $(\bullet / *)$
43.68

477.92

Adjusted Dry Wi. of the Fines $=\quad$ Wi. Sample $\times(1-\%$ water $)$

- Adjusted Dry Wt. of the Cobbles $=$ Wt. Sample $\times(9-.015)$

!viass Partition funcsion $(f)=$

$M_{\text {sead }} / M_{\text {cas }}=3.73$

Radiological

Analysis

- $M_{\text {sea }} M_{\text {cas }}=3.73$

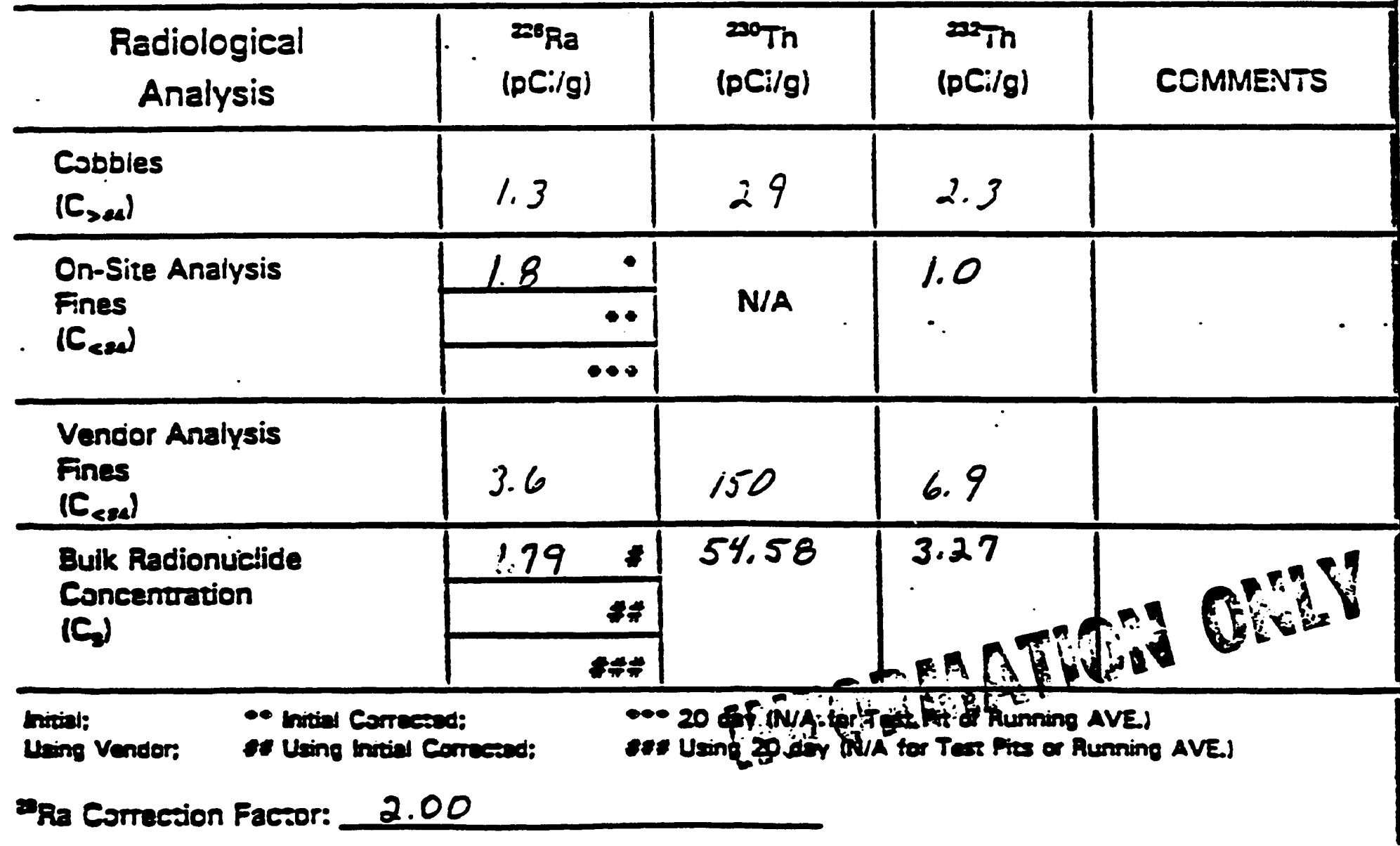

ieviewed By:

Date: 
Site:

sampie/Grid l.D. 1 Test $R_{i}^{-}+\ldots 4$
Sheer of Dare: $\frac{11 / \sqrt{1 / 52}}{1}$
Fines

$\mathrm{Nz}$ Sample

8 Bucket 38.83

iNt. Bucket

NL Sample

Adjusied

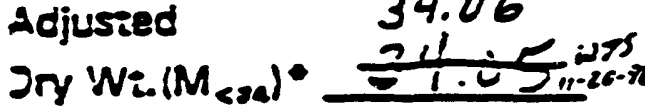

Cobbles

Wi. Sample

\&ucker $110,6,6$

Wt. Bucket

Wr. Sampie

Adjusted

Dry $W_{\text {t. }}\left(M_{>\text {ead }} \cdots 105.55\right.$

\section{Moisture Content Fines}

Wet Wi. Sample/Pan 5,2.49

Dry Wt. Sample/Pan 549,34

Tare Wt. Pan

Wt. Water"

$\frac{18.33}{.43 .10}$

Wr. Soil ••

Percent Water $(\bullet / \bullet \bullet)$

Wt. Sample $\times(9-\%$ water $)$

- Adjusied Dry Wt. of the Cobbles =

Wr. Samole $\times(9-.095)$

Mass Paŕtition tunction $(f)$ =

$M_{\text {sed }} M_{\text {Cse }}=3.10$

Fadiological

Analysis

Cotbies

(C) sea)

1.2

3. 7

(DCi/g)

(pC:/g)

(pCi/g)

$202 \mathrm{Th}$

(pCi/g)

COMMENTS

Cn-Site Analysis

Fines

(Cese)

Vendor Analysis

Fines

(C $C_{\text {sed }}$ )

0.3

Bulk Radionuclide

Concsmuation

(C)

$$
\text { ! }
$$

errain:

- Uring Vender.

zora Correcsion Facior:
- Iririal Comesad:

ot Uaing Intive Cormorad:

2.44

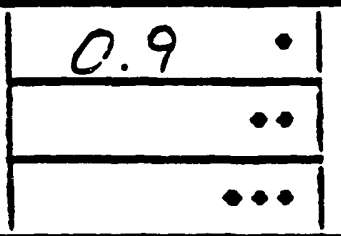

2.2

8. 7

N/A

0.7

0.8

\begin{tabular}{|r|}
\hline $1.44: 1$ \\
\hline
\end{tabular}

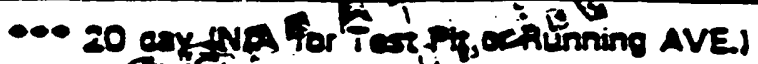

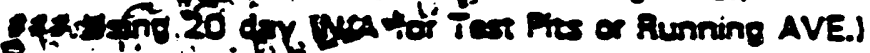
Exis 
1.

\section{COBBLES TO FINES CALCULATION SARET}

1

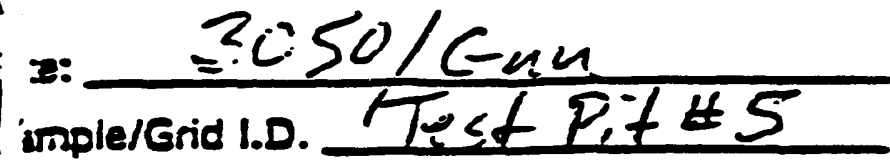
Date: $\frac{11 / 13 / 42}{2}$

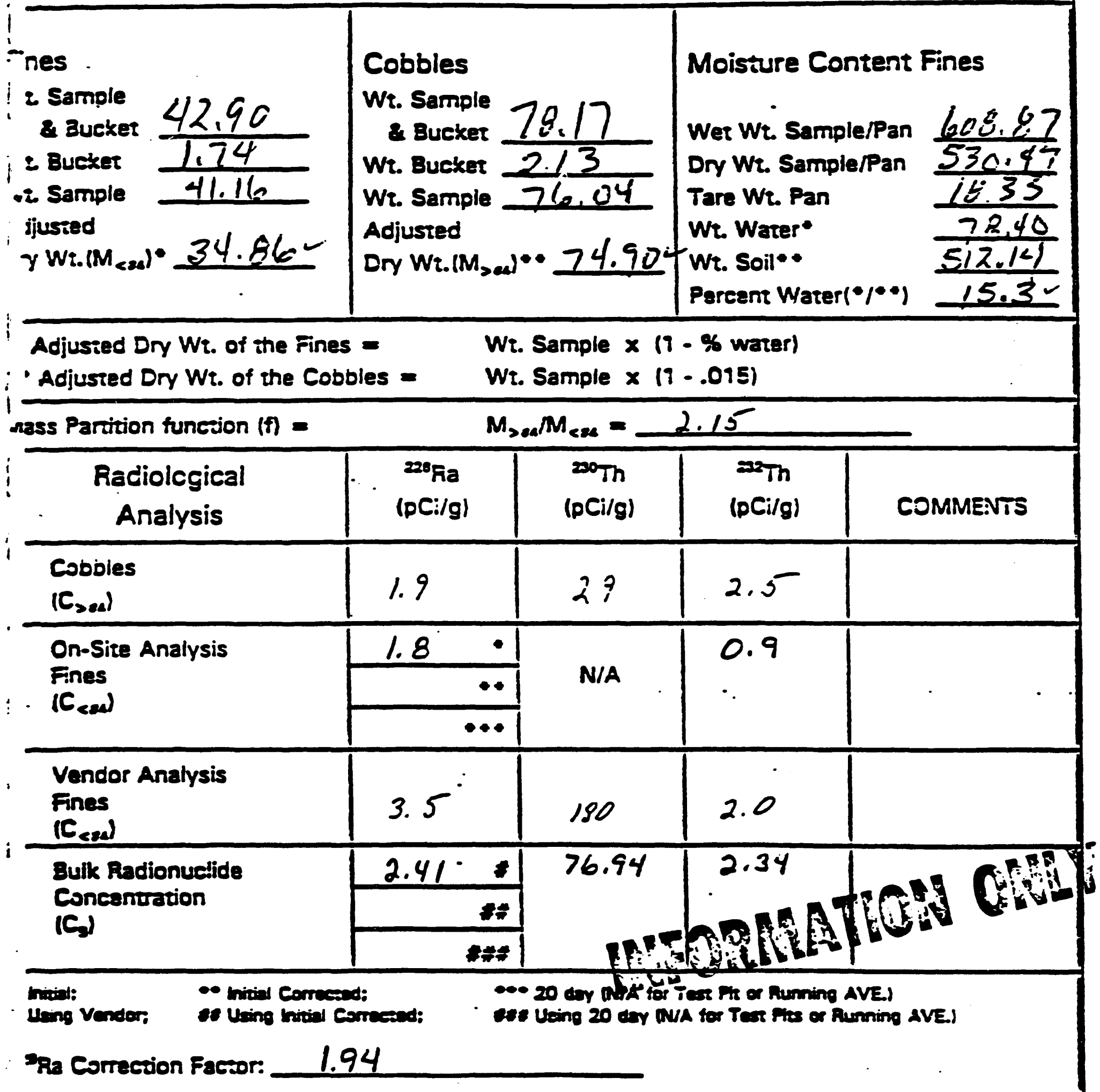

leviewed By:

Date: 


\section{COBBLES TO FINES CALCULATION SHEET}

site: $3050 / 6-2$ ample/Grid 1.0."Test P, t" Date: $\frac{11 / 12 / 92}{6}$

Fines

\section{it Samele}

8 3ucket 44.66

It. Bucker

Ii Sample

idiusted

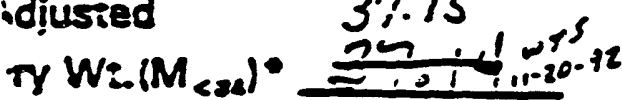

\section{Ccbbles}

Wt. Sarrole

2 Bucket $\mid 4 / .21$

Wt. Bucket

Wt. Sample

Adjusted

Dry $W_{t .}\left(M_{204}\right) \cdots 135.64$

\section{Moisture Content Fines}

Wet Wt. Sample/Pan 589.96

Dry Wt. Sample/Pan

Tare Wt. Pan

525165

Wt. Water"

Wr. Soil $*$.

Percant Water $(\bullet / \bullet$ )
18.19

27.28

504.49

$13.3=$

Adjusied Dry Wt. of the Fines =

Wr. Sample $\times(9-\%$ water $)$

- Adjusied Dry Wt. of the Cobbles =

Wi. Sample $\times 1 i-.015 i$

lass Partition funcsion $(f)=$

$M_{\text {soa }} M_{\text {cas }}=3.65$

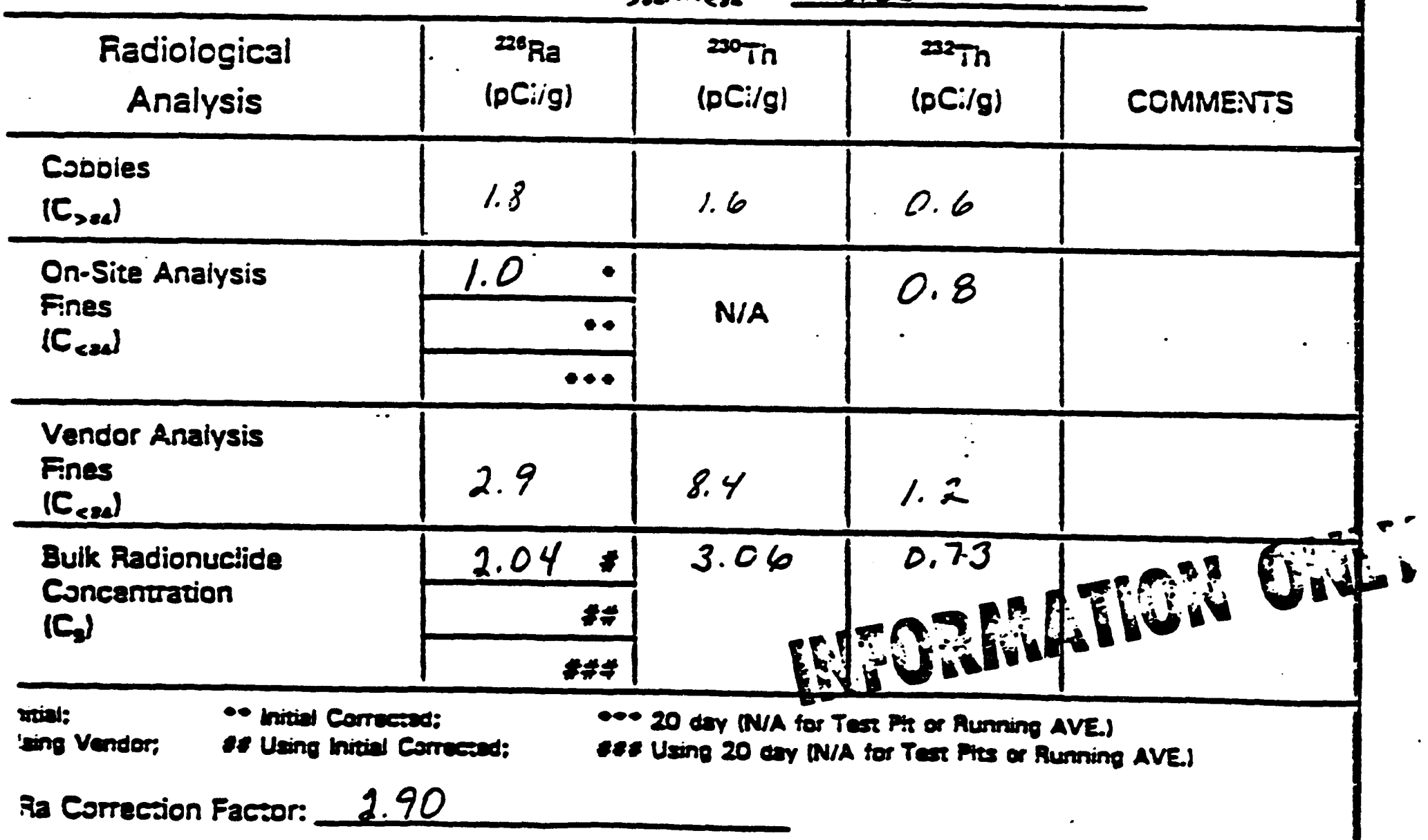

viewed By:

Date: 


\section{COBBLES TO FINES CALCULATION SHEET}

iite: $\quad 20501 \mathrm{Gun}$

ample/Gid 1.D. 'Test Rit \&7
Dare: $\frac{11 / 12 / 6}{1 / 1}$
Fines

Vz. Sarmple

8 sueier 44,52

Vi. Bucker

Nr. Sample

Idjusted

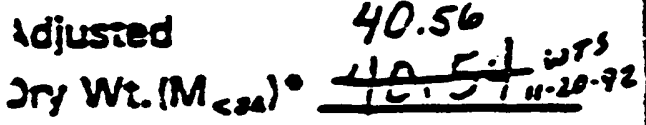

$\frac{\frac{17}{42.73}}{40.56}$

\section{Cobbles}

Wr. Sample

8 Bucket

W. Bucket

Wr. Sample

Acjusted

Dry Wr. M Masi"* 10775
Moisture Content Fines
Wer Wi. Sample.Pan 539.17

Dry Wt. Sample/Pan $\frac{513,28}{1,28}$

Tare Wt. Pan

Wt. Water"

$\frac{14.22}{25.89}$

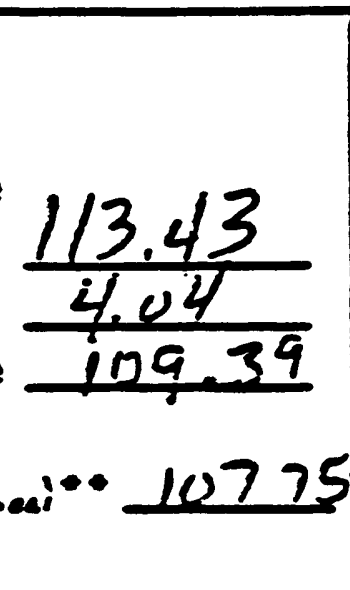

Wt. Soil"•

Parcent Water $(\bullet / \bullet)$

- Adjusied Dry Wr. of the Fines =

- Adjusied Dry Wt. of the Cobbles =

Mass Parvition funcsion (f) =

Fiadiological

Analysis

Copjoies

(C) >ee

1.9

Wr. Sample $\times(1-\%$ water $)$

Wi. Sample $\times(1-.015)$

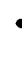

On-Site Analysis

Fines

(C ceal

\begin{tabular}{l}
\hline Vendor Analysis \\
Fines \\
(C ceal \\
\hline Bulk Radionuelide \\
Coneantration \\
(C)
\end{tabular}

- Livid:

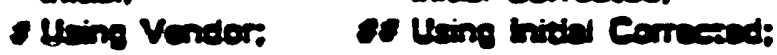

$M_{\text {sea }} M_{\text {ces }}=2.66$ 


\section{COBBLES TO FINES CALCULATION SHEET}

te: $3050 /$ Gin

ample/Grid 1.D. Test Pit 18
Date: $1 / 1 / 3 / 92$

\section{ines}

¿ Sample

8 Eucket

4. Bucket

It Sample

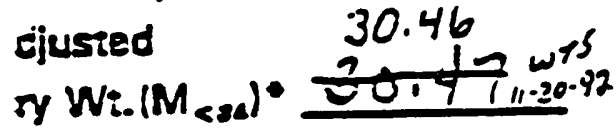

Cobbles

Wt. Sample

\& Bucker \& 5.15

Wit. Bucket

Wt. Sampie

1.74

Adjusied

Dry $W_{\text {t. }}\left(M_{\text {>aA }} \cdots\right.$ E2, 16
Moisture $C$ intent Fines

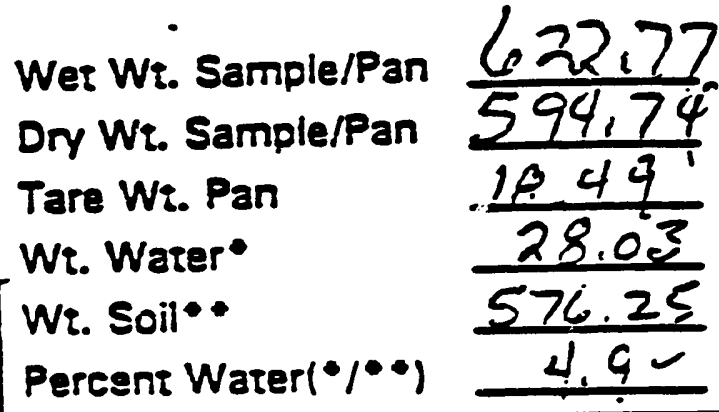

Adjusted Dry Wi. of the Fines =

- Adjusied Dry Wt. of the Cobbles =

lass Partition funcsion $(f)=$

Radiological

Analysis
WI. Sample $\times 19-\%$ water $)$

W. Sample $x(1 \cdot .015)$

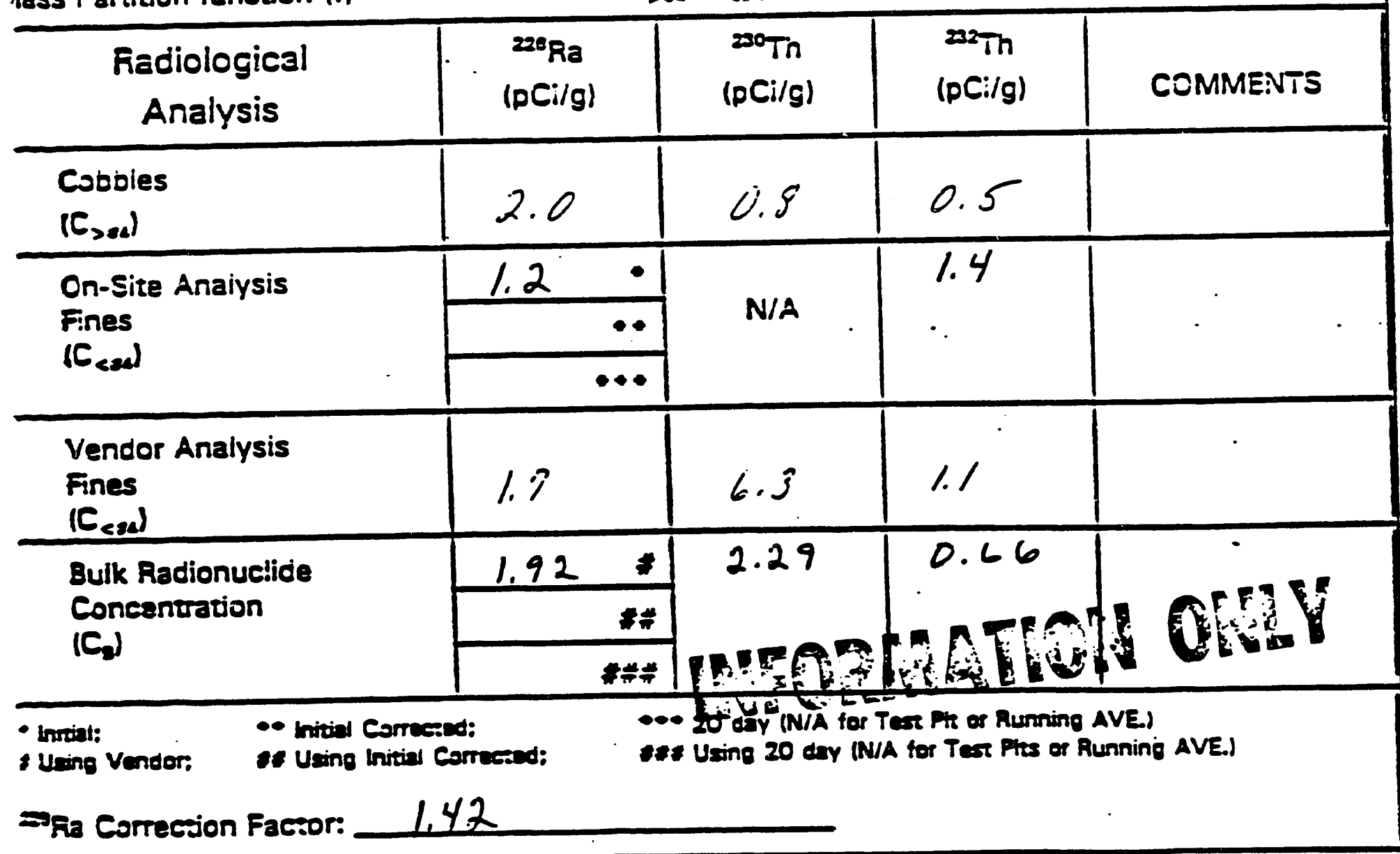

'eviewed By: 


\section{COBBLES TO FINES CALCULATION SHEET}

te: $3050 / 6$ en

i ample/Grid I.D. $\frac{1}{1} e^{2}+$ Pit $\# 9$
Date: $11 / 12 / 92$
Tines

: / Sample

8 Bucket

to. Bucker

it Sample

dịusted

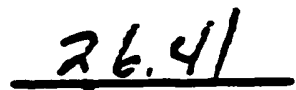

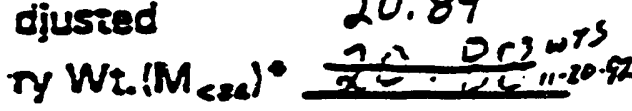

\section{Cobbles}

Wt. Sample

8 Bucker 47.67

Wt. Bucket

WL. Sample

Adjusted

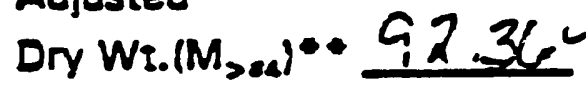

3,90

93,77

\section{Moisture Content Fines}

Tare Wt. Pan

Wt. Water"

Wt. Soii"•

Percent Water $(\bullet / \bullet \bullet)$ $\frac{498.65}{437.61}$

437.61

18,39

6046

419.22

14.42

Adjusied Dry Wi. of the Fines =

Wt. Sample $\times(1-\%$ water $)$

- Adjusted Dry Wt. of the Cobbles =

Wt. Sample $\times(9-.015)$

dass Partition function $(f)$ :

$M_{\text {sea }} M_{\text {cea }}=4,42$

Radiological

Analysis

$$
18=
$$

Trang

\begin{tabular}{|c|}
\hline $\begin{array}{l}\text { Cobpbles } \\
\left(C_{>e a}\right)\end{array}$ \\
\hline $\begin{array}{l}\text { On-Site Analysis } \\
\text { Fines } \\
\text { (C cad }\end{array}$ \\
\hline $\begin{array}{l}\text { Vendor Analysis } \\
\text { Fines } \\
\left.\text { (C } \mathrm{C}_{\text {ces }}\right)\end{array}$ \\
\hline $\begin{array}{l}\text { Bulk Radionuelide } \\
\text { Concentration } \\
\left(C_{\text {L }}\right)\end{array}$ \\
\hline
\end{tabular}

\begin{tabular}{|c|c|c|}
\hline $\begin{array}{c}228 \mathrm{Ra} \\
\text { (pCiig) }\end{array}$ & $\begin{array}{c}200 \mathrm{Th} \\
\text { (pCi/g) }\end{array}$ & $\begin{array}{l}22 \mathrm{Th} \\
\text { (pCi/g) }\end{array}$ \\
\hline
\end{tabular}

COMMENTS

3.0

$147 \mid$

2.9

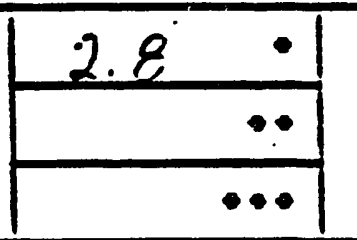

N/A

0.9

6.9

350

6.5

\begin{tabular}{|r|}
3.72 \\
\hline$*$ \\
\hline$*=1$
\end{tabular}

102.90

3.56

mixi:

- Mutial Correcad:

$\because 20$ doy ON/A for Tess Pto or Ruming AVE.J

: Uting Vondor: ex Using trital Comesad:

tes Uaing 20 dey mVA tor Ters Pres of Aunning AVE.)

Tha Correcsion Fassor: 2.46 


\section{COBBLES TO FINES CALCULATION SHEST}

Site: $\quad 30: 50 / t_{u+1}$

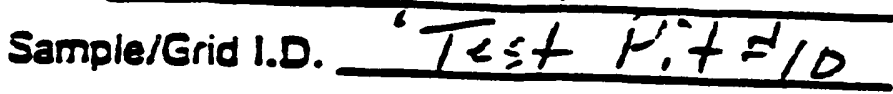

Date: $\frac{1 / \sqrt{12 / 92}}{1}$

Fines

'Ne Sample

\& Bucker 29.53

Wi Bucket

Ni Sample

Adjusied

$\frac{\frac{1.72}{270.1}}{22.25}$

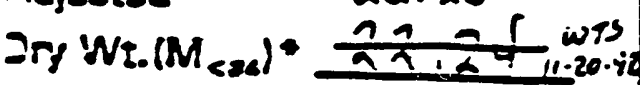

Cobbles

Wt. Sample

$\&$ Bucket

Wt. Bucket

Wt. Sample

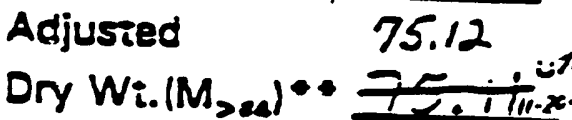

$\frac{76.39}{2.13}$
Moisture Content Fines

Wer Wt. Sample/Pan

Dry Wt. Sample/Pan

Tare Wi. Pan

Wt. Water"

WWt. Soil".

Percent Water $(\bullet / \bullet \bullet)$

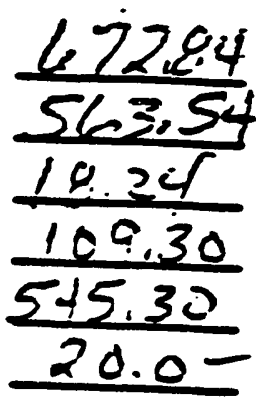

- Adjusted Dry Wt. of the Fines = - Adjusied Dry Wt. of the Cobbles =

Wt. Sample $\times 19-\%$ water $)$

Wi. Sample $\times(1-.015)$

lass Parition function (f) =

$M_{\text {sea }} M_{\text {CIS }}=3.38$

Racoiolocical

Analysis

\begin{tabular}{l}
\multicolumn{1}{c}{ Analysis } \\
\hline Cobbies \\
$\left(C_{\text {sea }}\right)$ \\
\hline Cn-Site Analysis \\
Fines \\
(C)sal \\
\hline Vendor Analysis \\
Fines \\
$\left(C_{\text {sea }}\right)$ \\
\hline Bulk Radionuclide \\
Concentration \\
$\left(C_{\text {s) }}\right)$
\end{tabular}

risoin:

laing Vendor.
- Initial Correosu:

- Using Initial Correced:
$2007 \mathrm{~h}$

(pCi/g)
$2227 h$

(pCi/g)

COMME:VTS

Ra Correcsion Factor:

2.19

sviewed By:

Date: 


\section{COBBLES TO FINES CALCULATION SHEET}

!

$\div$

i. impleiGrid I.D. Test Pit 11

Date: $\frac{11 / 12192}{1}$

Date: $\frac{111 / 2 / 92}{1}$

i

\section{- res}

i : Sample

8 Bucket

L Bucket

I Sample

ijussed

y $w_{i}\left(M_{\text {sad }}\right) 31.37$

\section{Cobbles}

Wt. Sample

2 Bucket

Wt. Bucket Wi. Sample Adjusied

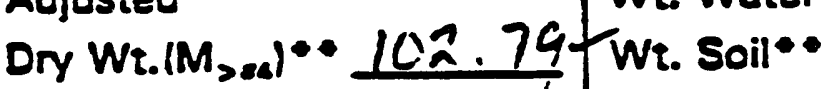

Percent Water $\left({ }^{\bullet} / \bullet\right)$
Moisture Content Fines

Wet Wt. Sample/Pan $\frac{579.17}{519.55}$ Dry Wt. Sample/Pan

Tare Wt. Pan

519.95 16,12 59.22 501.83

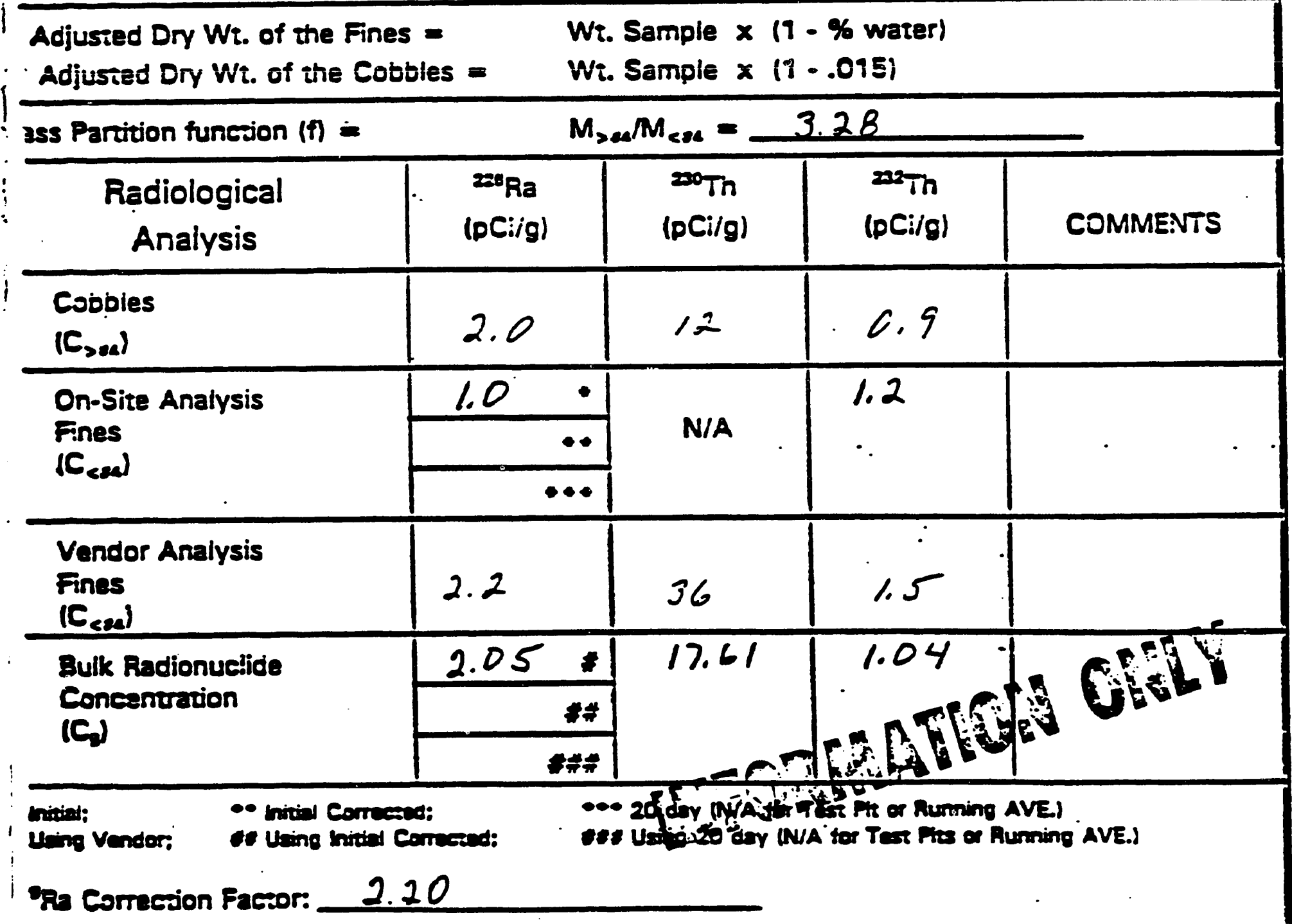




\section{COBBLES TO FINES CALCULATION SHEST}

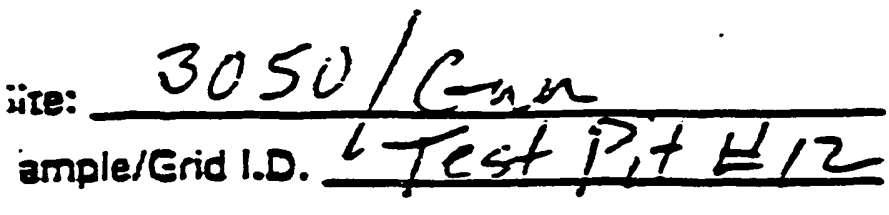

Date: $\frac{1111319}{1}$ of

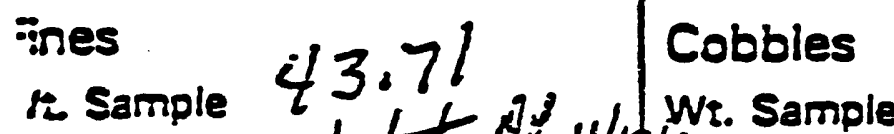

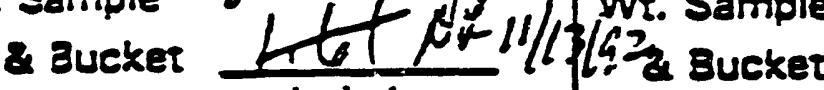

it. Bucket

At Sampie

dịusted

$$
\frac{\frac{1.61}{42.10}}{38.06}
$$

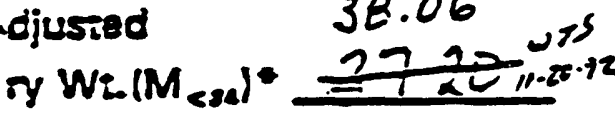

Adjusied
Wt. Bucket

Wr. Sample

Dry $W_{\text {t. }}\left(M_{>e d}\right)^{\cdots} 91.13^{-}$
Moisture Content Fines

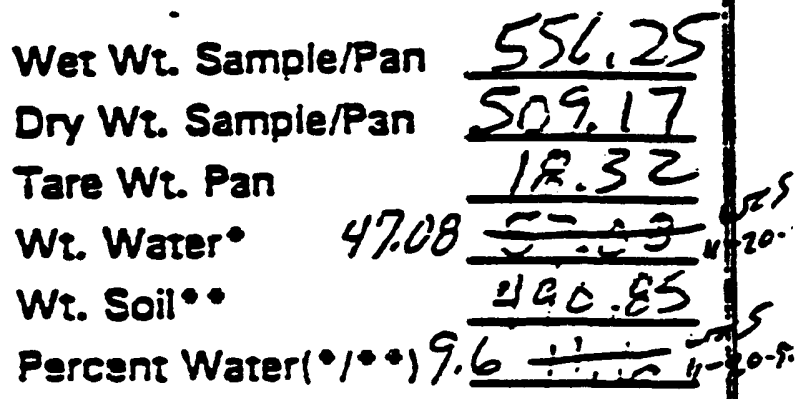

Adjusted Dry Wi. of the Fines =

- Adjusted Dry Wt. of the Cobbles =
Wq. Sample $\times 19-\%$ water $)$

Wt. Samole $\times(9-.015)$

iass Partition funcsion (f) =

$M_{\text {sea }} M_{\text {ses }}=2.39$

Radiological

Analysis

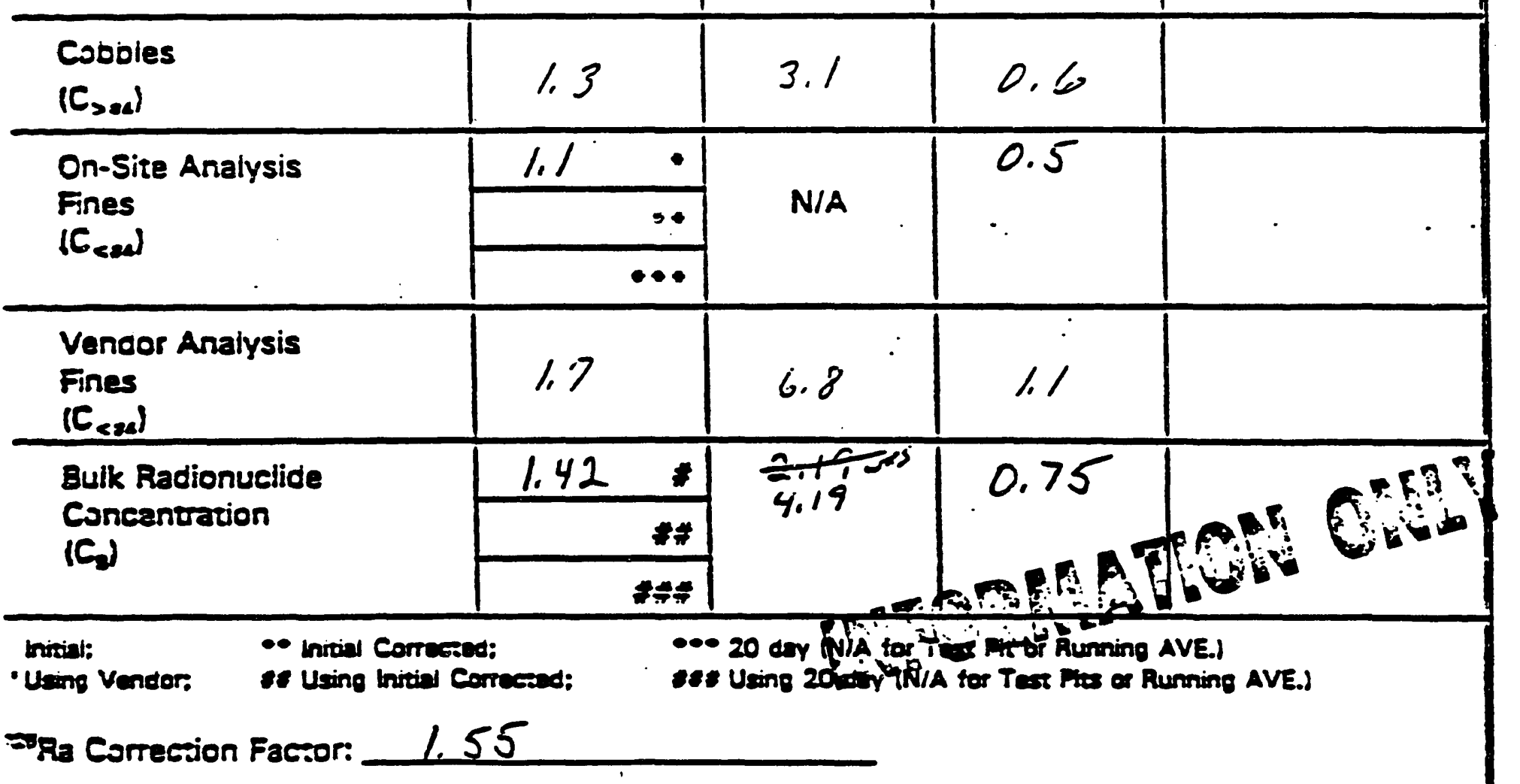

ieviewed By:

Date: 
1

\section{COBBLES TO FINES CALCULATION SHEE}

- $3050 / C_{41}$

ample/Erid 1.D. Test P. $t \notin 13$

Sheer

Date: of $1111^{2} / 6 ; 2$

\section{Fines}

It Sample \& Bucket

1.. Bucket

ir Sample ojusted or $W$ s.im<ad 39.41 '
Cobbles

Wr. Sample

\& Bucker $136.5 \%$

Wt. Bucket 4.212 Wr. Sample Adjusied

Dry $W_{\text {r. }}\left(M_{\text {sea }}\right.$ "* 130.294
Moisture Content Fines

$\begin{array}{ll}\text { Wet Wt. Sample/Pan } & \frac{546.52}{496.96} \\ \text { Dry Wt. Sample/Pan } & \frac{19.31}{49.36} \\ \text { Tare Wt. Pan } & \frac{178.65}{10.3} \\ \text { Wt. Water } & \\ \text { W. Soil** } & \end{array}$

Adjusied Dry Wt. of the Fines =

- Adjusted Dry Wt. of tite Cotbles =

dass Partition function $(f)=$

Radiological

Analysis
Wt. Sample $\times(9-\%$ water $)$

Wi. Sample $\times(1-.015)$

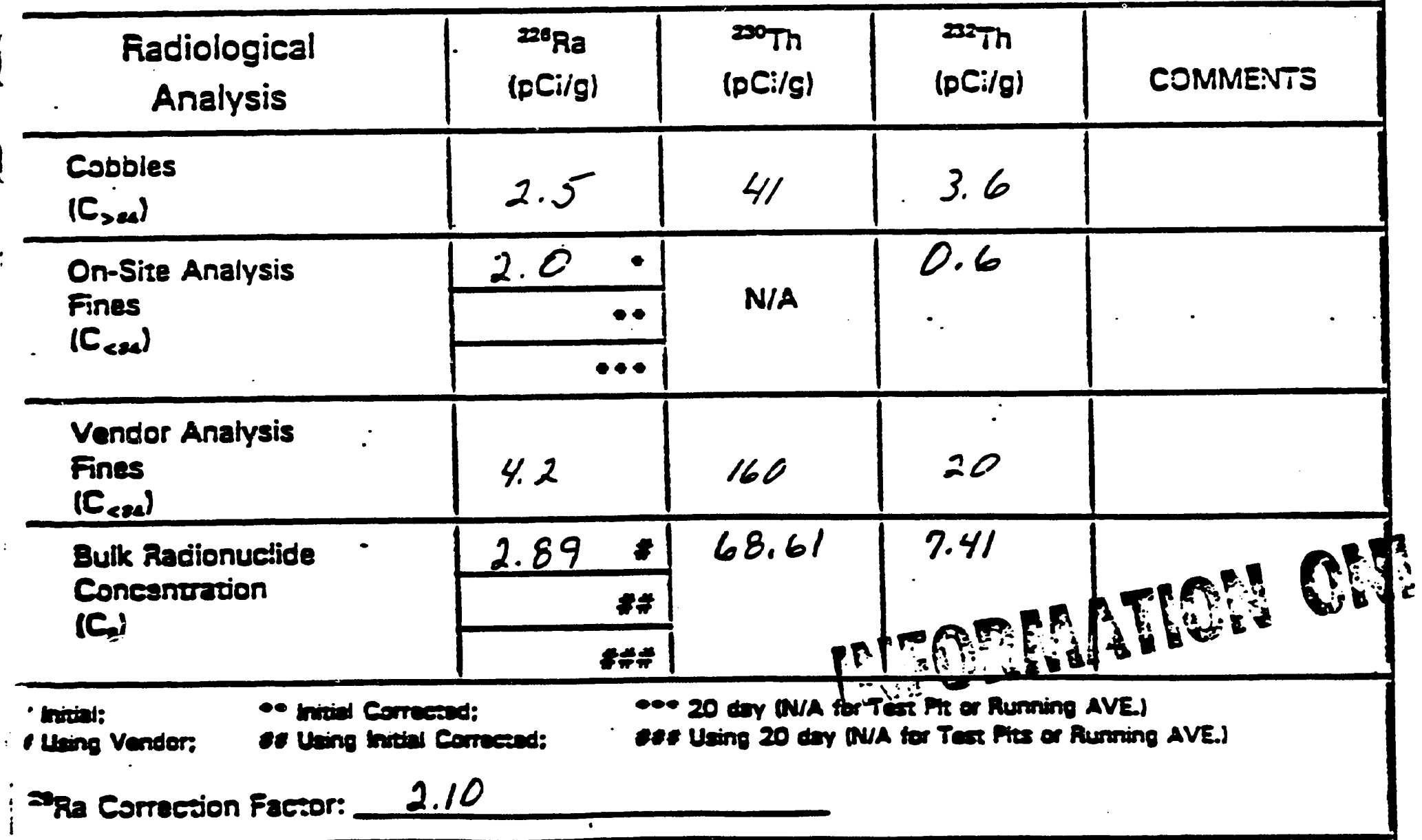




\section{COBBLES TO FINES CALCULATION SHEET}

ite: $3050 / E_{n+2}$ ample/Grid 1.0. ! $1257 k, \pm 14$
Date: $\frac{l l / 13 / s^{2} 2}{l}$ ines

is Sample

\& Bucker

it. Bucket

IL Sample

dijusted

55,09

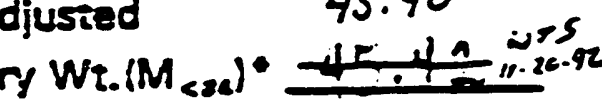

\section{Cobbles}

Wi. Sample

\& Bucket

Wt. Bucket

Wi. Sample

Adjusted
Dry Wt. $\left(M_{\text {>ea }} * * 47.45\right.$ Wt. Water"

Percent Water $(\bullet / \bullet \bullet)$
Moisture Content Fines

Wer wi. Sample/Pan $\frac{573.0}{501193}$

Dry Wr. Sample/Pan 541.93

Tare Wt. Pan 18,49 718 酸 493.41 $14.9 \%$

Adjusied Dry Wr. of the Fines =

Wi. Sample $\times(9-\%$ water $)$

- Adjusted Dry Wt. of the Cobbles =

Wi. Sample $\times(1-.015)$

lass Partition funcsion (f) :

$M_{\text {sea }} M_{\text {<se }}=0.94$

Radiological

Analysis

$$
(n)
$$

-

\begin{tabular}{l} 
Analysis \\
\hline Cobbies \\
$\left(C_{\text {sea }}\right)$ \\
\hline Cn-Site Analysis \\
Fines \\
$\left(C_{<s e}\right)$
\end{tabular}

\begin{tabular}{l} 
Vendor Analysis \\
Fines \\
(Ceeal \\
\hline Bulk Radionuclide \\
Concantration \\
(Cs)
\end{tabular}

- mriat:

8 Unino Vender.
- Initiad Comcond:

te Using tritivl Cornosad:
$2027 h$

(pC:/g)

(pC:/g)

(pC:/g)

1.5

13

1.2

0.7

N/A

$\bullet \bullet$

3. 9

160

3. 2

\begin{tabular}{|r|}
\hline $2.74 *$ \\
\hline$* *$ \\
\hline$*$
\end{tabular}

88.77

2.23

P.r.

COMMENTS

"Fa Correcsion Facior: 1.63 
GPMK-FEAGUSON COMPANY

CWM Federal Environmental Services, Inc.

COBBLES TO FINES CALCULATION SHEET

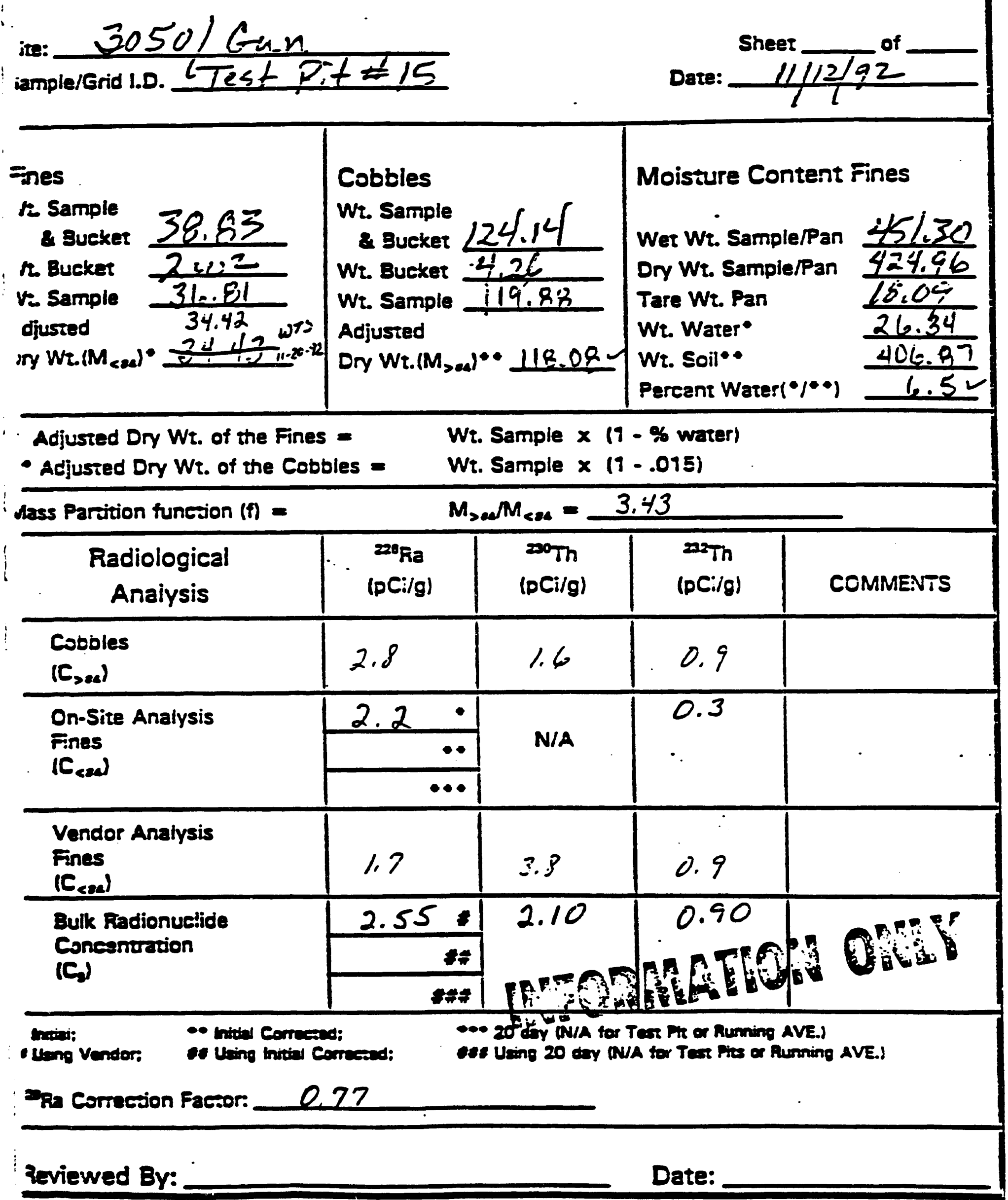




\section{COBBLES TO FINES CALCULATION SHEET}

Site: $3050 /$ Gur iample/Grid 1.D. Test Pit 16
Sheet of Date: $11 / 12 / 92$
Fines

$v_{i}$ Sample

8 Eucker

vi. Bucket

NL Sample

Adjusted

Jry We. $\left(M_{\text {csea }} * 41.62-\right.$

\section{Cobbles}

Wr. Sample

8. Bucker 140,24

Wt. Bucket 4.06

Wt. Sampie 136.18

Adjusted

Dry Wr. (M sad $\cdots 124.145$

\section{Moisture Content Fines}

Wet Wi. Sample/Pan 475.68

Dry Wt. Sampie/Pan

Tare Wt. Pan

Wt. Water"

Wt. Soil **

Percent Water $(\bullet / \bullet \bullet)$
454.37

14.32

25.31

436.05 $5.8=$

- Adjusted Dry Wr. or the Fines =

Wt. Sample $\times(7-\%$ water $)$

- Adjusted Dry Wi. of the Cobbles =

Wi. Sampie $\times(1-.015)$

Mass Partition funcsion (f) =

$M_{\text {sea }} M_{\text {ces }}=3.22$

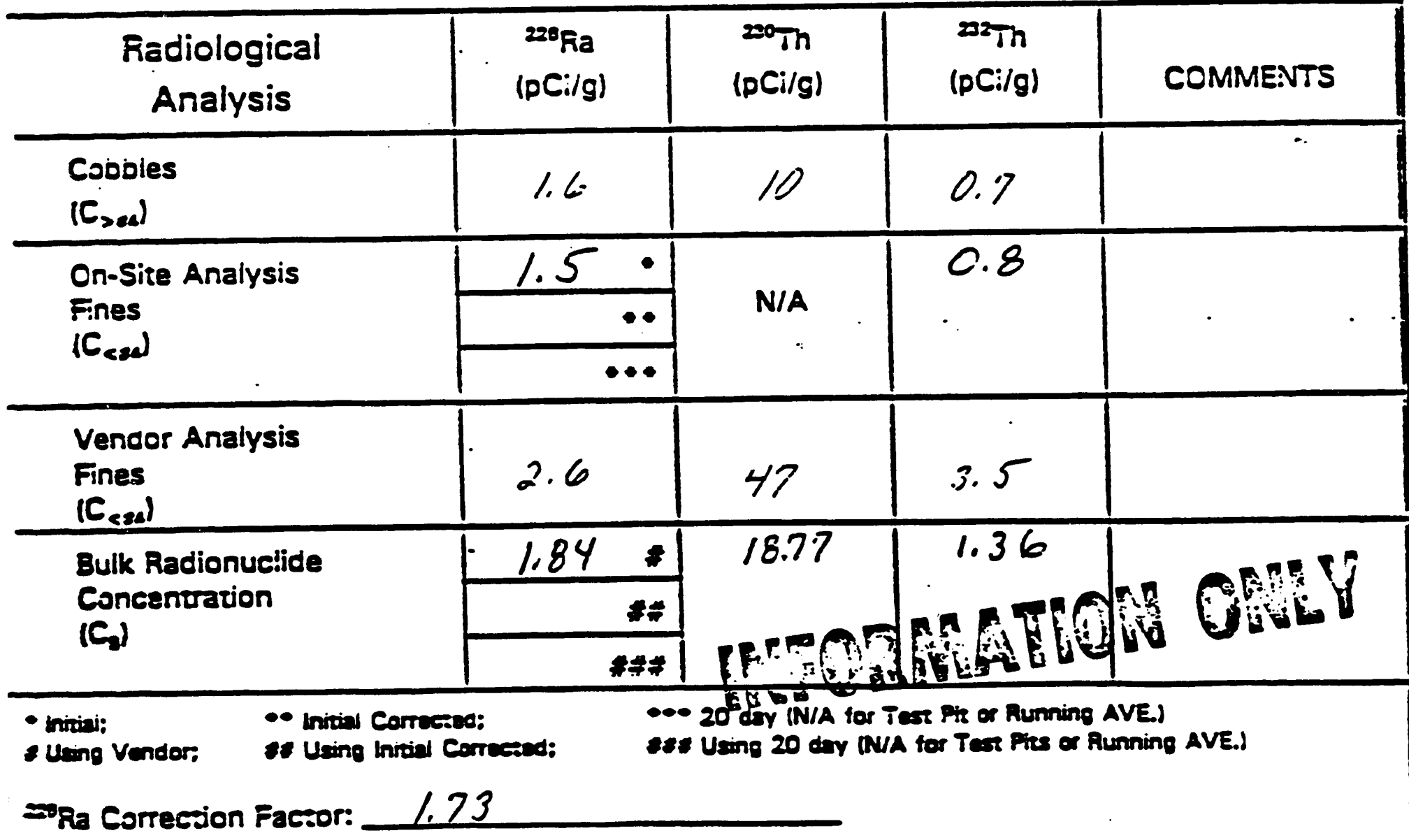

Reviewed By: 


\section{COBBLES TO FINES CALCULATION SHEET}

2: $\frac{3050 / \text { tani }}{T}$

jample/Grid 1.D. Test Pit \#/7

Date: $\frac{\text { Sheet } \frac{1 / 12 / 92}{1}}{1}$

Date: $\quad \frac{1 / 1 / 2 / 92}{1}$

Date: $\frac{\text { Sheet } / / 12 / 92}{1}$

Fines

fis Sample

\& Bucket

it. Bucket

Nin Sample

dịusied

ory $W_{i}\left(M_{\text {sod }}\right) 21.935$

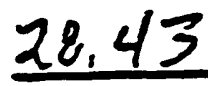

1.75

$2 \log$
Cobbles

1
Wi. Sample

8 Bucker 84.00

wt. Bucket $\hat{2}, 03$

Wi. Sample 81.97

Adjusted
Dry Wt. $\left(M_{2, e a}\right) * 80.74$
Moisture Content Fines

\begin{tabular}{|c|c|}
\hline $\begin{array}{l}\text { Wet Wt. Sample/Pan } \\
\text { Dry Wt. Sample/Pan }\end{array}$ & $\frac{\frac{566.02}{483.212}}{19.3}$ \\
\hline Wt. Water" & 8283 \\
\hline$W_{\tau}$. Soil $\bullet$ & 464,90 \\
\hline Percant Water $(\bullet / \bullet$ & 17.8 \\
\hline
\end{tabular}

- Adjusted Dry Wt. of the Fines =

- Adjusied Dry Wt. of the Cobbles =

Mass Parvition function (f) =

Radiological

Analysis
Wr. Sample $\times(9-\%$ water $)$

Wt. Sample $\times(9-.015)$

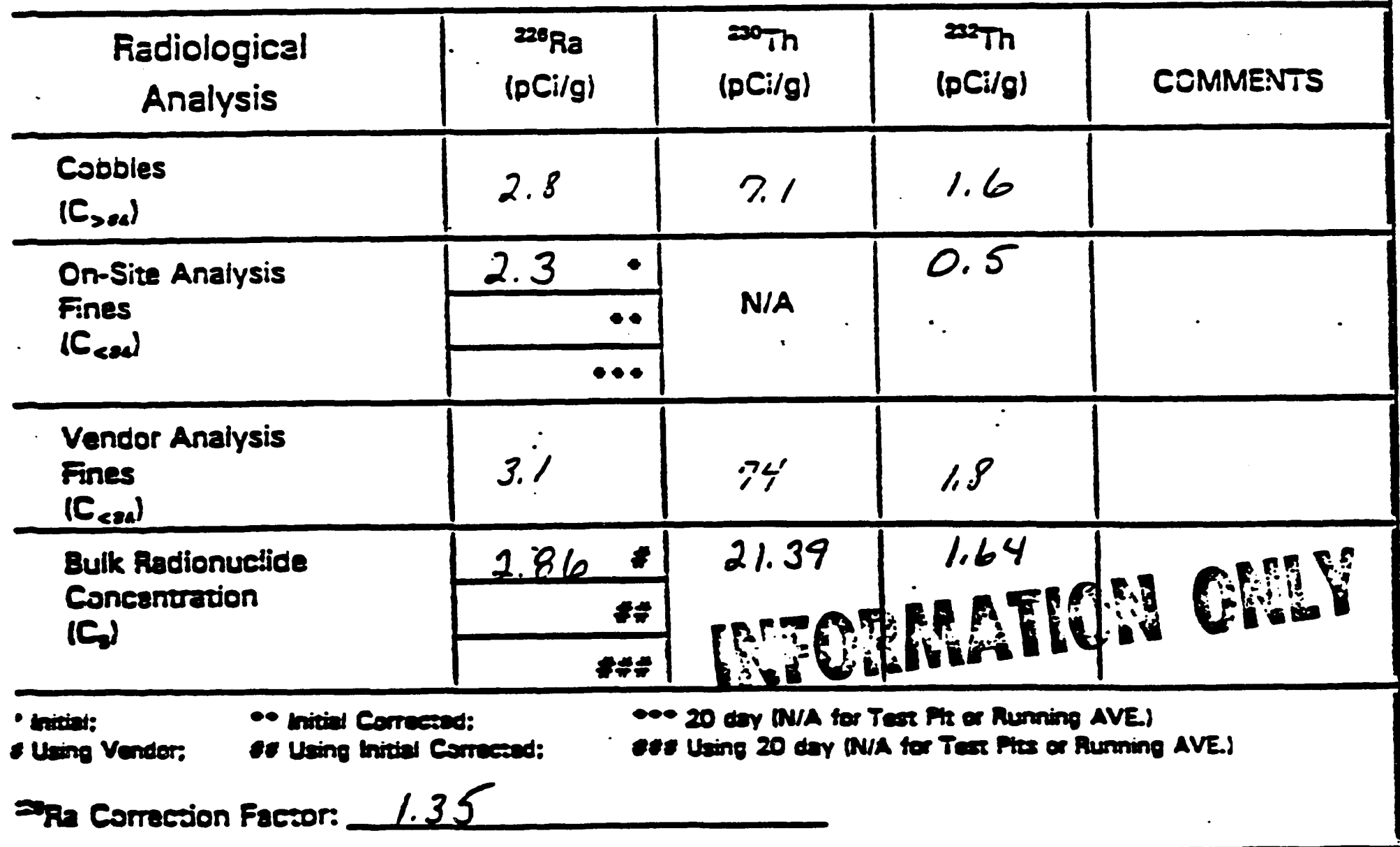

Reviewed By:

Date: 
ite: Gun: 13050

jample/Grid 1.D. Fes
Sheer of

Dare: 11 !1
Tines

It Sample

8 Bucker 2853

It Bueker

wis Sample

diusted

7.12

31.80

32.83

sy $W_{2}\left(M_{<8 a}\right)$ on

\section{Cobbles}

Wt. Sample

8 Bucker 138.90

Wt. Bucker 352

Wr. Sample $134.6 B$

Adjusied

Dry $W_{\text {t. }}\left(M_{>2 e a}\right) \cdots 13 \hat{a}, 66$
Moisture Content Fines

Wet Wr. Sample/Pan $\leq 27.810$ Dry W. Sample/Pan $\frac{47.3 .85}{18.785}$ Tare Wt. Pan We. Water"

Wt. Soil ${ }^{\bullet \bullet}$

18.24 tiction Percent Water $(* / *) 10.8+1$, $10.4-4$

- Acjusted Dry Wt. of the Fines =

Wi. Sample $\times(9-\%$ water $)$

- Adjusted Dry Wt. of the Cobbles =

Wi. Sample $\times(9-.015)$

Mass Partioion funcrion $(f)=$

$M_{\text {sea }} M_{\text {ces }}=4,04$

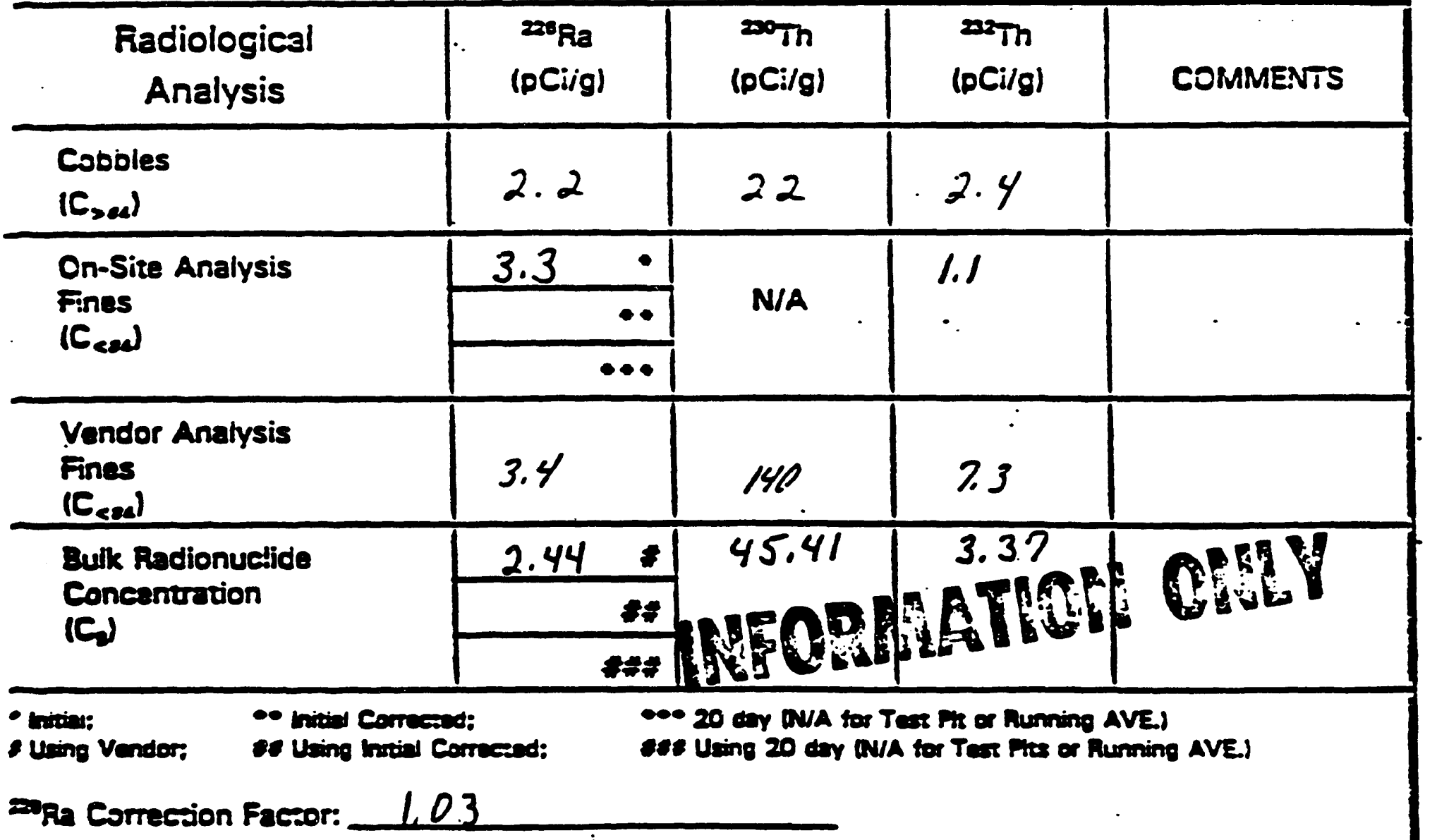

Rieviewed By: 


\section{COBBLES TO FINES CALCULATION SHEET}

ite: Ene /zoso

ample/Grid i.D. IE T In

Sheet of

Date: 111iiaz

ines

It. Sample

\& Bucker 34.20

ic Bucker 7.11

It sample $\frac{72.0 i}{210}$

cjusted

rg $W_{i}\left(M_{c o s}\right) \cdot 28,69$
Cobcles

Wi. Sampie

8 Bucker ad 21

Wt. Bucket 3.48

Wi. Sample 90.93

Adjusied

Dry Wi. (M zea $\cdots 89.57$
Moisture Content Fines

Wer Wi. Sample/Pan $\frac{546.9 \$}{496.72}$

Dry Wt. Sample/Pan 496.72

Tare Wt. Pan

We. Water"

Wi. Soil"•

Percent Water $(* / *)$
18.39

$5 n .23$

478.3 .3 10.5

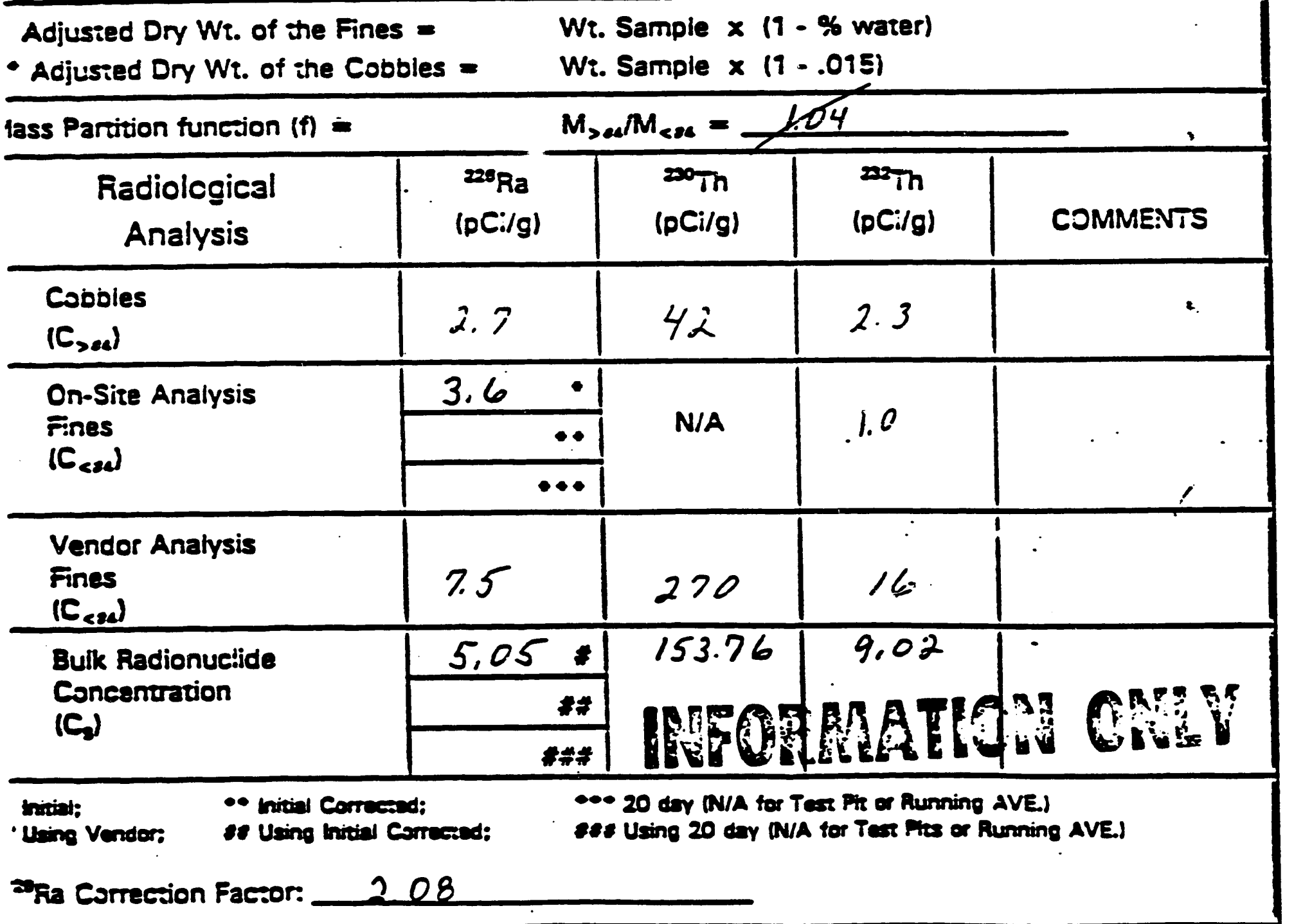

Zeviewed By:

Date: 


\section{COBELES TO fINES CALCULATION SHEET}

ite: $[$ and lensac

Sample/Grid l.D. Fes
Sheet of

Date: 11-in-a7 ines.

ort Sample

8 Bucker $39 . a b$ it Bucket

$N=$ Sample dijusted $\frac{\frac{1.72}{38.28}}{33.77}$
Cobbles

Wr. Sample

\& Bucker 138.55 Wt. Bucket 2.85 Wr. Sample $\frac{13 d .7 n}{132.68}$ Acjusted 132.68

\section{Moisture Content Fines}

Wer Wt. Sample/Pan hkas Dry W. Sample/Pan 552.64 Tare Wr. Pan Wt. Water" Wt. Soil"Percent Water $(\bullet \cdot \bullet)$
18.2 1.7 .31 5.34 .33 $11.7-$

- Adjusted Dry Wr. of the Fines =

Wr. Sample $\times(1-\%$ water $)$

- Adjusied Dry Wi. of the Cobbles $=$ B2.71W. Sample $\times(9-.015)$

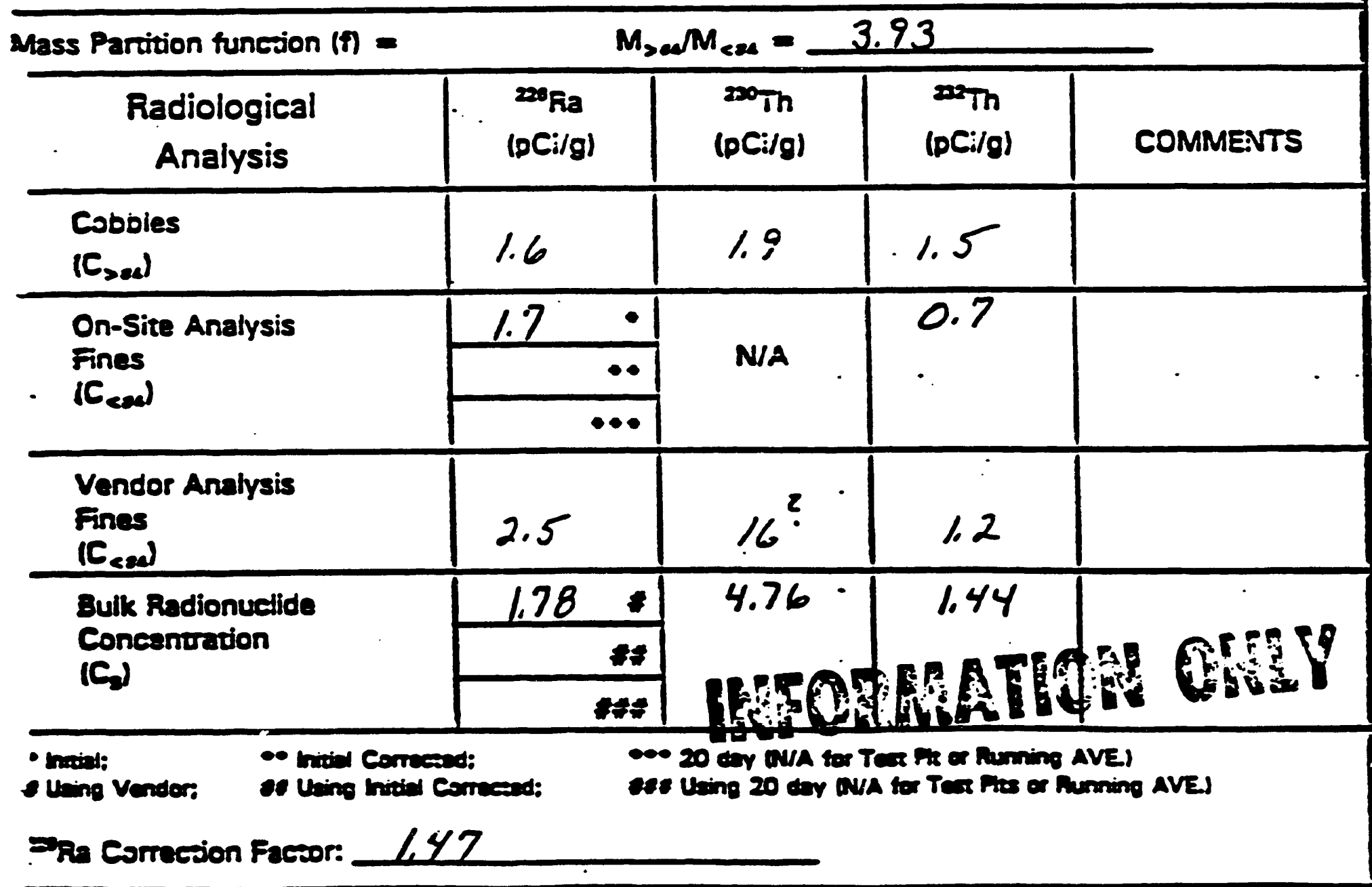

Reviewed By:

Date: 


\section{COBBLES TO FINES CALCULATION SHEET}

site: $\frac{\text { Gun/350 }}{\text { sample/Grid 1.0. Test TIt } 122}$

Sheer of

Date:

Fines

'NL Sample

2 Bucket

Wr. Bucker

Wr Sample

Adjusted

Dry $W_{\text {t. }}\left(M_{\text {cese }}\right.$ *

\section{Cobbles}

Wt. Sample

\& Bucker

Wr. Bucket

W:. Sample

Adjusied

Ery Wt. $\left(M_{>2 a}\right) \cdots 182.28$
Moisture Content Fines

\begin{tabular}{|c|c|}
\hline Wer Wt. Sart & 426.46 \\
\hline $\begin{array}{l}\text { Dry Wt. Sample/Pan } \\
\text { Tare Wt. Pan }\end{array}$ & $\frac{402.17}{18.31}$ \\
\hline Wt. Water" & 2429 \\
\hline It. Soil $\bullet$ & 81 \\
\hline ercent Water $(\bullet / \bullet$ & \\
\hline
\end{tabular}

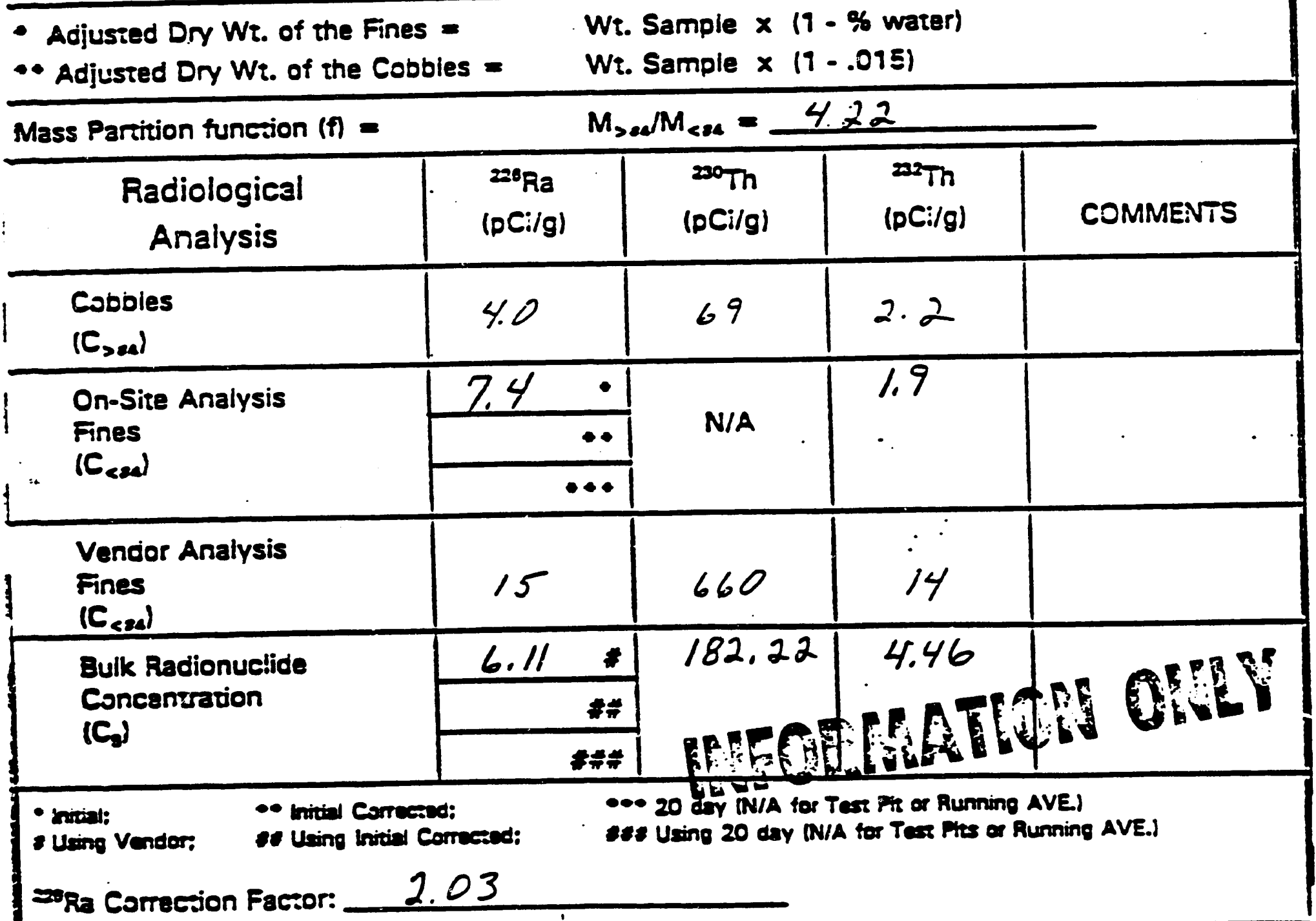

Reviewed By:

Date: 


\section{COBBLES TO FINES CALCULATION SHEET}

ire: Glul 3nso

mple/Grid 1.0. ISI
Sheet of

Date: $11-1 n 92$ änes.

- Sarmple

8 3ucket $4 . .75$

- Bucket

-i Sample jiusted s $W_{2}\left(M_{\text {seal }}\right) 2 E .295$
Cobbles

Wt. Sample

8 Bucker $117,6 a$

Wt. Bucket 3.85

Wt. Sample $112,8.4$

Adjusted

112.13

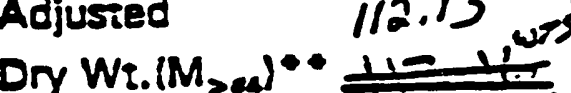

Moisture Content Fines

Wet Wr. Sample/Pan 673.72

Dry Wt. Sample/Pan $\lcm{610.87}$

Tare Wt. Pan

18.32

Wt. Water"

Wi. Soil *

Percent Water $(\bullet / \bullet \bullet)$ 82.316 592.55 13.47

Adjusted Dry Wt. of the Fines =

Wt. Sample $\times(9-\%$ water $)$

- Adjusted Dry Wt. of the Cobbles $=112.16$ Wt. Sample $\times(9-.015)$

rass Partition function $(f)=$ $M_{>a s} M_{\text {sea }}=2.93$

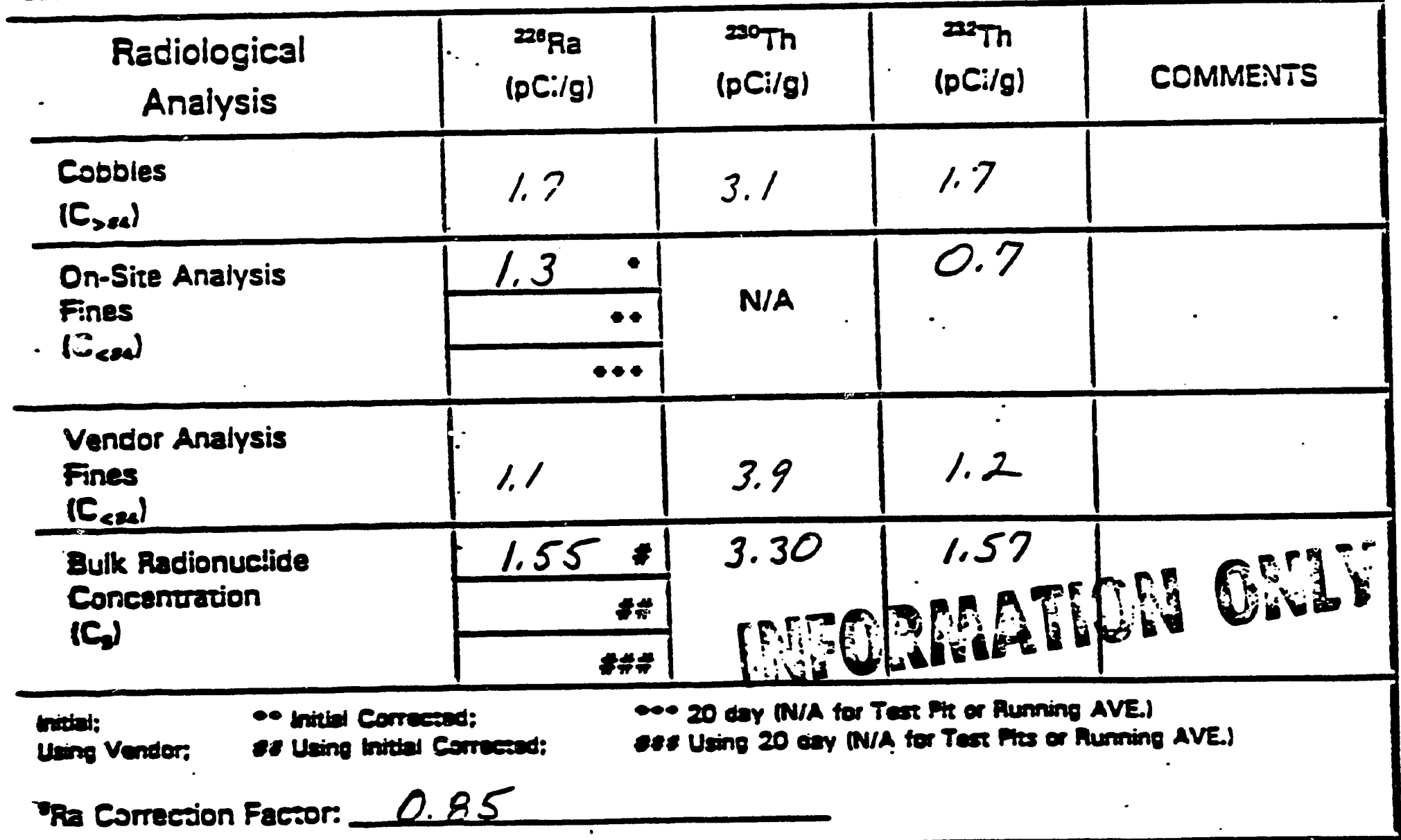




\section{COBBLES TO fINES CALCULATION SHEET}

Site:

Sheer of

jample/Grid I.D.

Date:

Fines

Vt Sample

8 Bucker

Vt. Bucket

Ve Sample

Adjusted

iny $W t .\left(M_{<a s}\right)^{*}$
Cobioies

Wt. Sample

2 Bucker

Wt. Bucket

Wt. Sample

Adjusied

Dry Wt.(M >ad $)^{\bullet \bullet}$

\section{Moisture Content Fines}

Wer Wt. Sample/Pan

Dry Wt. Sample/Pan

Tare Wt. Pan

Wt. Water"

Wt. Soil $\bullet$

Percent Water $(* \bullet \bullet)$

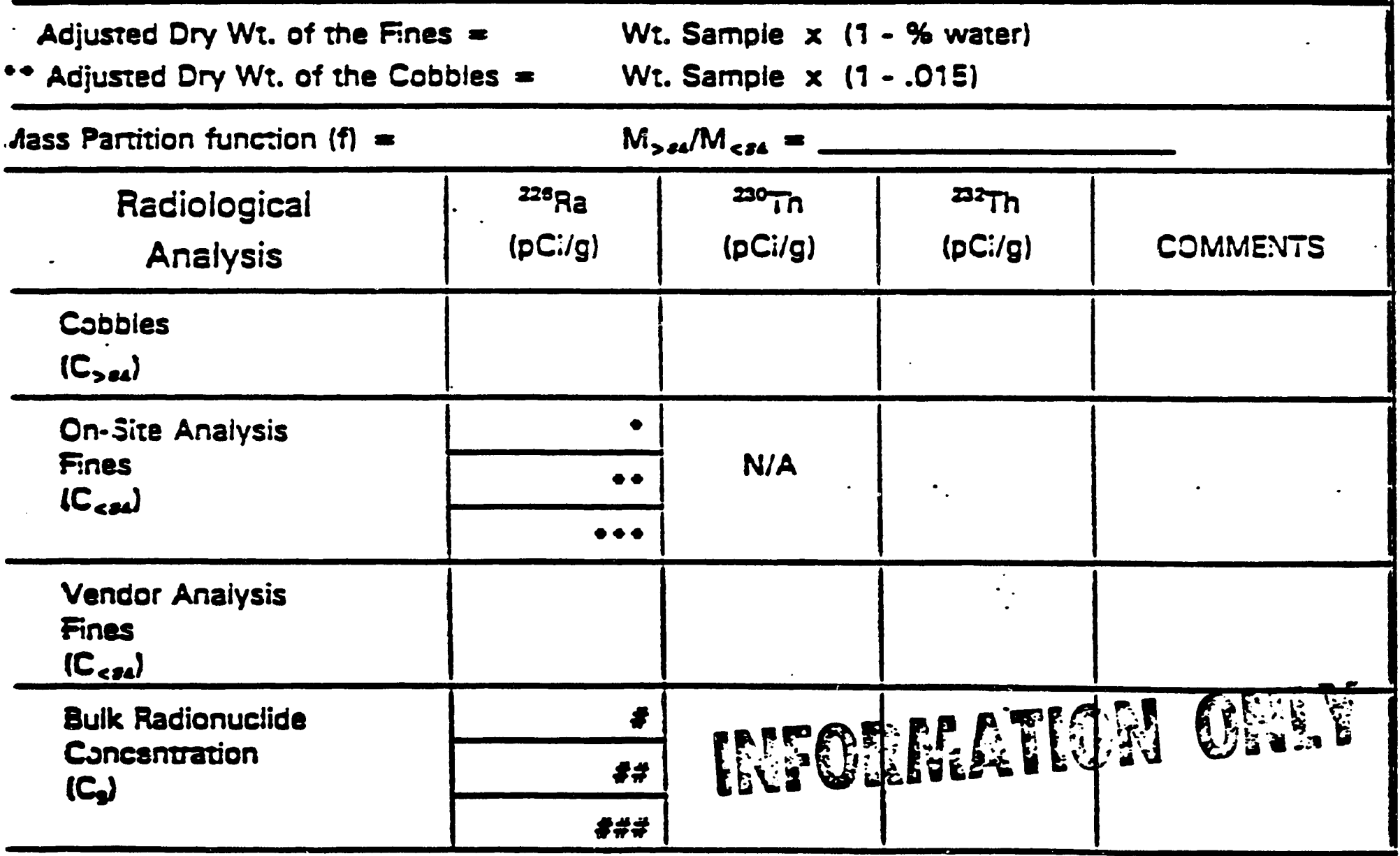

- Inicial:

- Inixial Corrosod:

Uning Vender.

-1 Uning hitial Com sad:
- 20 dey ONA for Tes Pit or Rumine AVEd

ate Using 20 dey (N/A tor Tar fies or Running AVE.J

Pra Correcsion Factor:

Feviewed By:

Date: 
VENDOR LABORATORY RADIOLOGICAL

MEASUREMENTS OF 1-FT DEPTH INCREMENT SOIL SAMPLES <\#4 MESH SIEVE COBBLY

SUBSOIL INVESTIGATION NOVEMBER 1992 


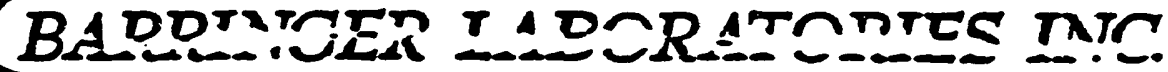

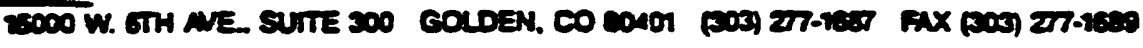

\title{
Ron Jacobs
}

Wi-Fezgusen

$1571 \mathrm{CR} 38$

Eus=ison, C? 89230

At:2:

Project: Gunnison

\author{
23-Dec-92 \\ T.ist P.t fines \\ 1 interzral \\ PO\$ 3050-511-10004\$058 \\ Received: 20-Nov-92
}

Icb: $225566 \mathrm{E}$

BARRINGER IABORATORTES

ANAIYTICAI RËPCRT PACXAGE

CASE MARRATIVE........................

ANAIYTICAI RESULTS $\ldots \ldots \ldots \ldots \ldots \ldots \ldots \ldots \ldots \ldots \ldots$. . . . .

QUAIITY CONTROI REPORT.......................

Status: Finel

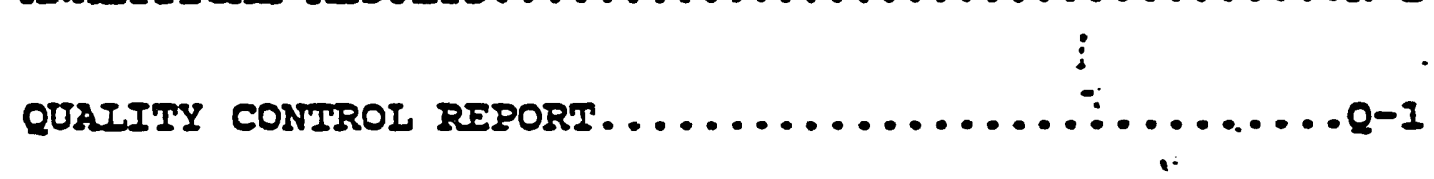

!
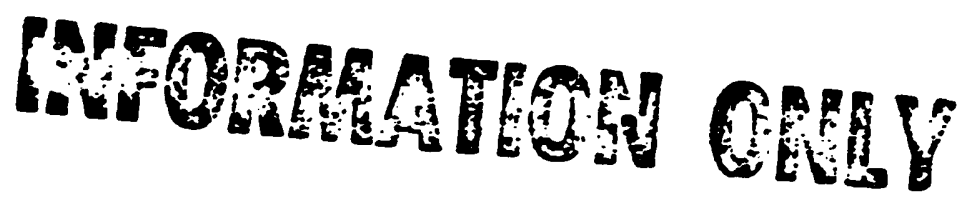


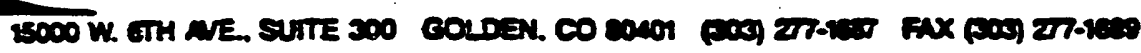

Ron Jacobs

XIR-Ferguson

$1571 \mathrm{CR} 38$

Gunnison, CO 81230

$$
\text { 23-DeC-92 }
$$

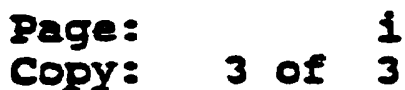

Attn:

Project: Gunnison

Job: S2E566E
Received: 20-Nov-92

PO $\$ 3050-511-10004 \$ 058$

SFAEYS: PMnai

CASE MARRATIVE

A total of 22 soil samples were received on 20-Nov-92. All were properly preserved and in good condition. As stated in the chain of custody, the samples were run for the following analyses: Ra-226, Th-230, Th-232 and 0 . Our procedures are summarized on the Quality Control Data sheet. There were no deviations to these procedures. All samples were extracted and analyzed within the proper holding times.

Quality control standards were run using standard EPA guidelines. A summary of that data is provided. The acceptance criteria for spikes and cuplicates is Iifteen percent. It is based on the relative percent deviation of the mean, taking the counting ersor into account for radiochemical analyses. All QC checks, Including duplicates, spikes, and blanks, passed.

It is possible that some Th-232 results may be biased high due to carzy over from Th-230 peak on the spectrum. Since Th-232 and Th-228 are general?y in equilibrium, the Th-228 results have been included in this report. This analysis delayed the report by one day.

signed:

.....t.ten

steven I. sincoes. Ph.

Director of Operations 


\section{BARRINGER LABORATORIES INC}

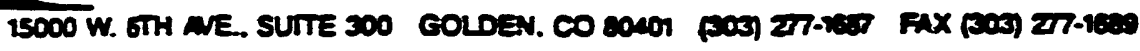

Ron Jacobs

IR-Ferguson

1571 CR 38

Gunnison, CO 81230

23-DeC-92

Page: R-1

Copy: 3 of 3
Atts:

Project: Gurnison

Job: $\quad 2255662$
Received: 20-Nov-92 09:30

PO : 3050-511-10004\$058

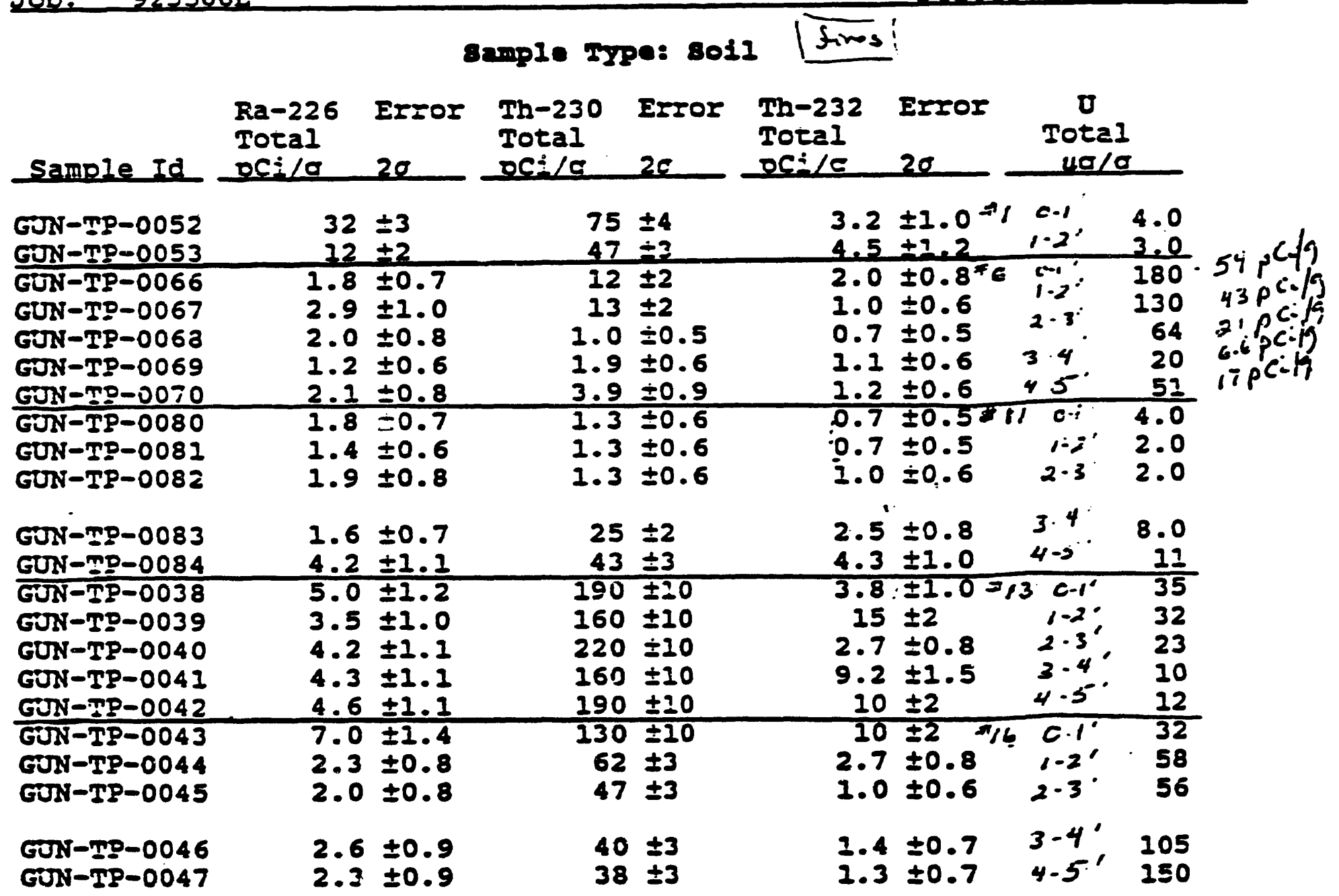

Th-228 ETrOI

Total

Sample Id pci/a 2r

GUN-TP-0052 $2.0 \pm 0.8$

GUN-TP-0053 $\quad 1.3 \pm 0.7$

GUN-TP-0066 $2.2 \pm 0.9$

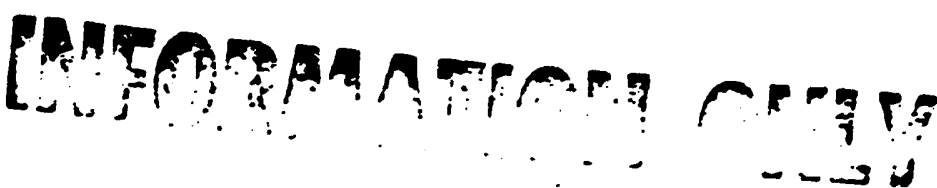




\section{BADDIATEEP IABORATORIES INC.}

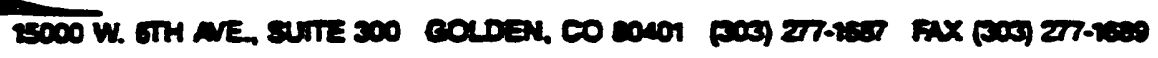

23-Dec-92

Ron Jacobs

IIR-Ferguson

1571 CR 38

Gunison, CO 81230

Aะเท:

Project: Gunnison

Received: 20-Nov-92 09:30

PO : 3050-511-10004 $\$ 058$
Page: $\quad R-2$

Cogy: 3 of: 3

Job: $\quad 925566 \mathrm{E}$

status:

Final

emple zype: soil

Th-228 Ersor

Total

Sample Id

ocio

GDN-TP-0067

GUN-TP-0068

GUN-TP-0069

GUN-TP-0070

GDN-TP-0080

GDN-TP-008I

GON-TP-0082

GDN-IP-0083

GUN-TP-0084

GUN-TP-0038 20

GON-TP-0039

GUN-TP-0040

GUN-TP-004I

GUN-TP-0042

GON-TP-0043

GNi-TP-0044

GUN-T2P-0045

ENI-IP-0046

GUI-IP-0047
$0.8 \pm 0.5$
$1.4 \pm 0.6$
$0.9 \pm 0.6$
$1.0 \pm 0.6$
$0.8 \pm 0.5$
$1.3 \pm 0.6$
$0.6 \pm 0.5$
$0.7 \pm 0.5$
$0.5 \pm 0.5$
$0.6 \pm 0.5$
$1.2 \pm 0.6$
$0.4 \pm 0.4$
$1.1 \pm 0.6$
$1.0 \pm 0.6$
$1.0 \pm 0.6$
$1.5 \pm 0.7$
$1.3 \pm 0.6$
$1.1 \pm 0.6$
$1.0 \pm 0.6$

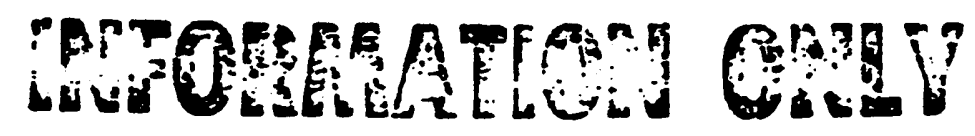




\section{BARRINGER LABORATORIES INC.}

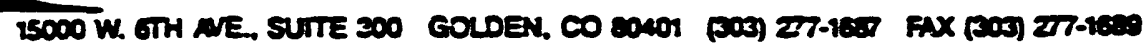

Ron Jacobs

MR-Ferguson

23-Dec-92

$1571 \mathrm{CR} 38$

Gunnison, CO 81230

Page: 3 of $e^{R-3}$

Att=2:

Project: Gunnison

Received: 20-ivov-92 09:30

PO *: 3050-511-10004 7058

Jab: Q25558E

status: Final

Abbreviations:

Parameters:

$\mathrm{Ra}-226$

Th-230

Th-232

U

Racium-226

: Thorium-230

: Thorium-232

: Uranium

$T h-228$

: Thorium-228

Dnits:

$p C \vdots / g$

20

$\mu \mathrm{g} / \mathrm{g}$

: picociries per gram

: Counting ersor at the $95 \%$ confidence level, $2 \sigma$

- micrograms per gram 
NQY) 20 IQS LABORATORY SERVICES AUTHORIZATION FORM raye $\rightarrow$ of 2 incy $\frac{11 / P / 92}{\text { Date }}$ $\frac{12,19,92}{\text { Need Date }}$ Urgent Prionity Standard

\begin{tabular}{|c|c|c|c|}
\hline nrisen, $C D$ & $\frac{3050-511-10004}{\text { P.0. Number }}$ & $\frac{0=8}{\text { Request No. }}$ & $\begin{array}{l}\text { ATTENTION LABORATORY: } \\
\text { An reports and invoices mast } \\
\text { Roferences PO and Request Nos. }\end{array}$ \\
\hline
\end{tabular}

of Samole: Water $\_$Soil Air Particulate Vegetation

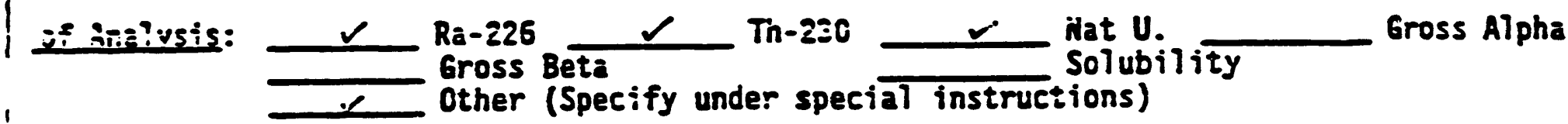
a) Instructions/Coments: awo anchuse for $T_{0}=33$. Phase secen iund poion.

\begin{tabular}{|c|c|c|c|}
\hline I.D. NO & $\begin{array}{l}\text { DATE } \\
\text { COLECTED }\end{array}$ & VOLUME & DESCRIPTICN \\
\hline$=T P .0052$ & $11-12-02$ & $443.2 a$ & $\therefore=0.100$ \\
\hline$-T P-O A E 2$ & $11-12-92$ & 62090 & $=1 \quad 1-2$ \\
\hline$=70.00 \mathrm{LL}$ & $11-12-92$ & $4460_{a}$ & $-1=n-1$ \\
\hline$=\pi$ - aph 7 & $11-12-92$ & 50550 & $=1, \quad 1-2$ \\
\hline L-TP- 9068 & $11-1=-0=$ & $5 i 5.00$ & \&6 $2-3$ \\
\hline$=>$ - D.012 & $11-1=-c_{2}$ & $\operatorname{lec} .9_{0}^{\circ}$ & $\Leftrightarrow 0^{2} 3-4$ \\
\hline$\frac{v-T P-0070}{1-T P-008 C}$ & $11-18-08$ & HLL.50 & $=1, \quad 4-5$ \\
\hline$\frac{1-T P-008 C}{1-T-1091}$ & $11-12-02$ & 546.00 & $\#=11 \quad 0-1$ \\
\hline 1-T0-napi & $11-12-92$ & 516.09 & $1-2$ \\
\hline \multicolumn{4}{|c|}{ CHAIN-OF-CUSTODY } \\
\hline \multicolumn{3}{|c|}{ 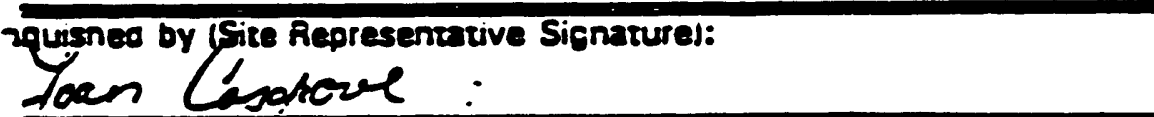 } & Datertime 21500 \\
\hline \multicolumn{3}{|c|}{ UPS 67/ 933-059 } & $\begin{array}{l}\text { Date/ing } \\
11 / 19 / 92\end{array} Q_{1500}$ \\
\hline \multicolumn{3}{|c|}{ 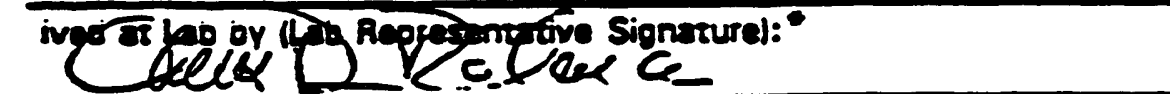 } & 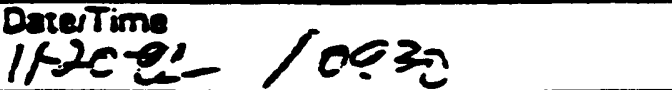 \\
\hline
\end{tabular}

ringer Chain-of-Custody as per comrace will be etrached

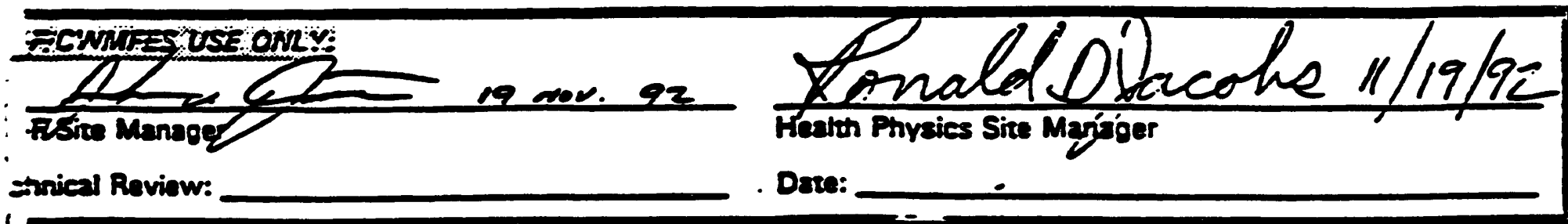

wo: Approved Vendor

For Radioanalvieal Services

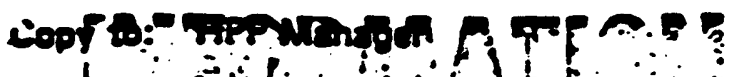
1 dut 


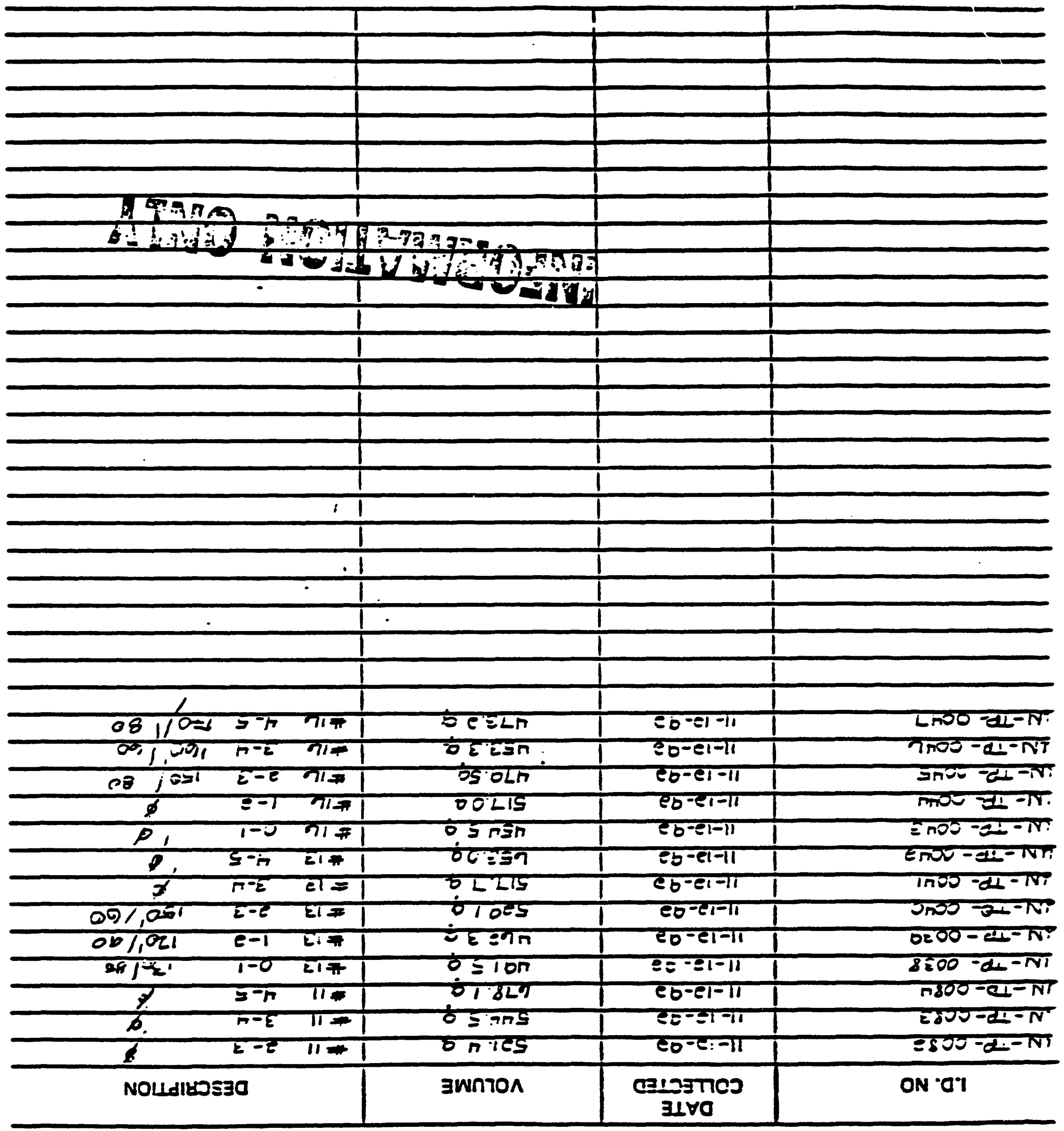

seounn $250 n$ bey/sequmn $0^{\circ} \mathrm{d}$

\$507त0001-115-CEOF

ट

$\gamma \mathscr{Q}, \cdots$

สิติ $02 \mathrm{nON}$

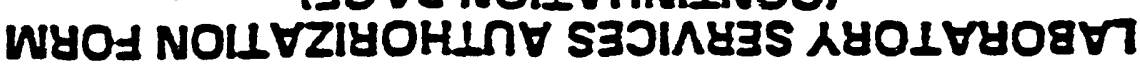

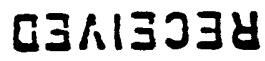

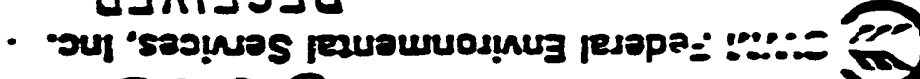

Ma $\triangle 6$ 


\section{BARRINGER LABORATORIES INC.}

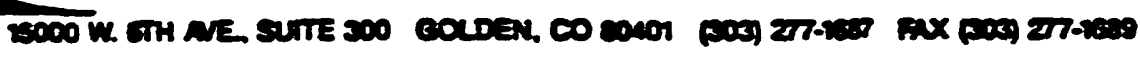

Ron Jacobs

IIR-Ferguson

1571 CR 38

Gunnison, CO 81230

23-DeC-92

Fise:
Copy: 3 of

At:=:

Project: Gunnison

Received: 20-Nov-92 09:30

PO : : 3050-511-10004\$058

Iob: 2255662

Status: Final

QUAIITYY CONTROL REPORT

sample Iype: 80i1

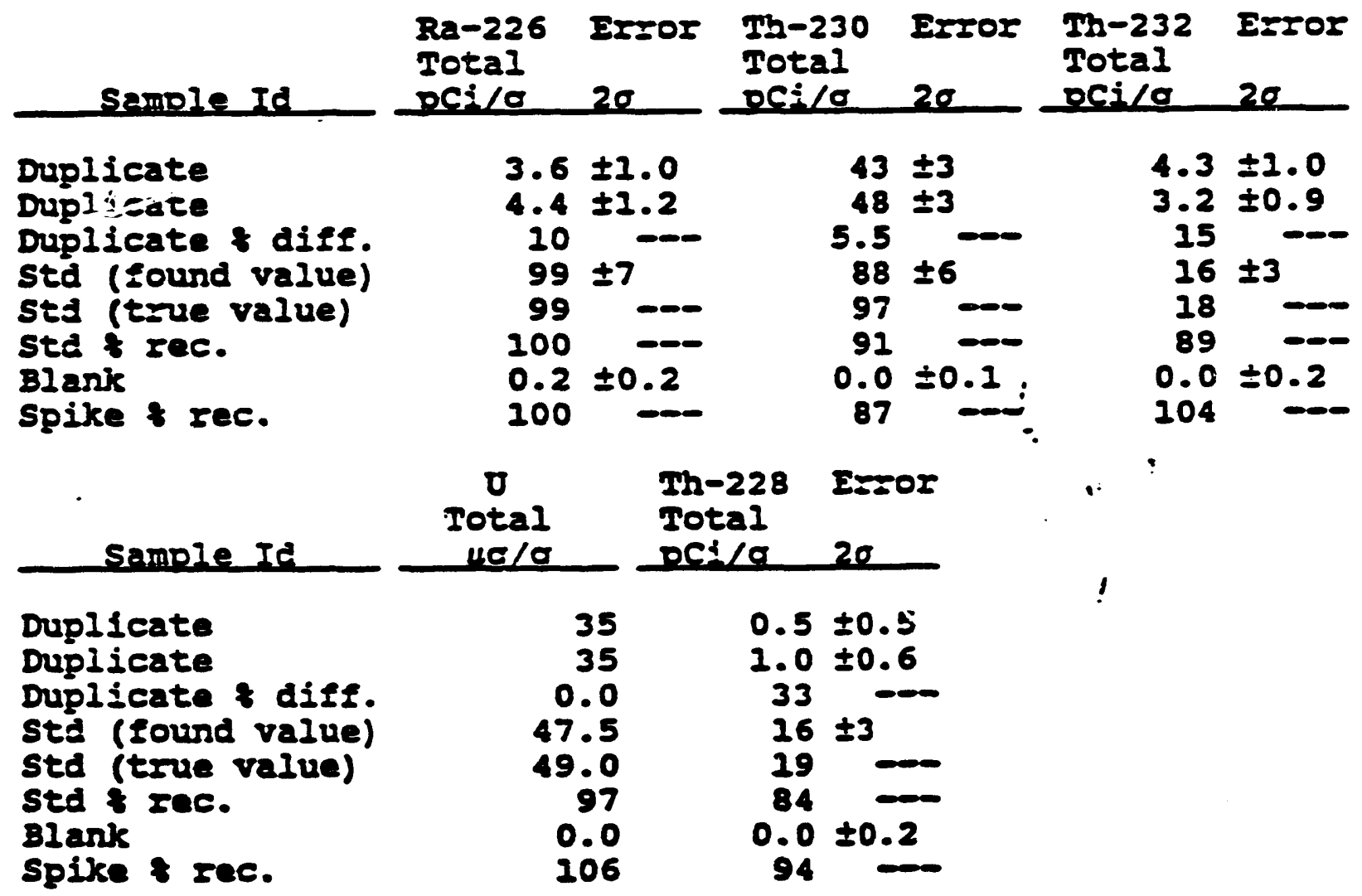

MOSWATES CW: 
Atta:

P=cject: Gurnison
Received: 20-Ncu-92 09:30 PO : 3050-512-10004\$058

Iab: $925566=$ status: Final QUAIFYY CONTROI REPGRT

\section{Abbreviations:}

\section{Parameters:}

$\begin{array}{ll}R e-226 & : \text { Radium-226 } \\ T h-230 & : \text { Thorium-230 } \\ T h-232 & : \text { Thorium-232 } \\ \tau & : \text { Uranium } \\ \text { Th-228 } & : \text { Thorium-228 }\end{array}$

\section{Units:}

pci/g

$2 \sigma$

$\mu \mathrm{g} / \mathrm{g}$
: picocuries per gram

: Counting error at the $95 \%$ confidence level, 20

: miczograms per gram 


\section{BARRINGER LABORATORIES INC.}

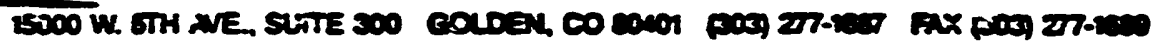

Ron Jacobs

ZRR-Ferguson

23-Dec-92

1571 CR 38

Gunnison, CO 81230

Page: $\quad 0-3$

Copy: 3 of 3

At:s:

Project: Gunnison

Received: 20-1100-92 09:30 PO : $3050-511-10004 \$ 058$

Job: $225566 \mathrm{E}$

statrs:

Final

QUALITY CONIROL REPORT

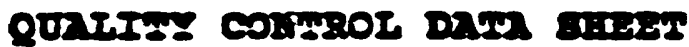

Received by: $j d r$

Via: UPS

Sample Container Type: 500ml mtl can

Sample Type: Soil

Preservative When Received: none

Additional Iab Preparation: 100 mesh

\begin{tabular}{|c|c|c|c|c|c|}
\hline Parameter & yethod & DII & $\begin{array}{l}\text { Pxase } \\
\text { rativiv }\end{array}$ & Inalyst & $\begin{array}{c}\text { Date (s) of } \\
\text { Analysis }\end{array}$ \\
\hline $\begin{array}{l}R a-226 \\
2 b-230 \\
T b-232 \\
0 \\
T b-232\end{array}$ & $\begin{array}{l}\text { SH-705 } \\
\text { USAEC } \\
\text { OSAEC } \\
\text { ASTM D2907 } \\
\text { USAEC }\end{array}$ & $\begin{array}{ll}0.3 & \mathrm{pci} / \mathrm{g} \\
0.4 \mathrm{pC} / \mathrm{g} \\
0.4 \mathrm{pCi} / \mathrm{g} \\
0.3 \mathrm{\mu g} / \mathrm{g} \\
0.4 \mathrm{pCi} / \mathrm{g}\end{array}$ & $\begin{array}{l}\text { none } \\
\text { none } \\
\text { none } \\
\text { none } \\
\text { none }\end{array}$ & $\begin{array}{l}\text { Pingilley } \\
\text { Ortiz } \\
\text { ortiz } \\
\text { Krox } \\
\text { Ortiz }\end{array}$ & $\begin{array}{l}12 / 10-12 / 15 \\
12 / 8-12 / 17 \\
12 / 8-12 / 17 \\
12 / 8-12 / 15 \\
12 / 8-12 / 21\end{array}$ \\
\hline
\end{tabular}

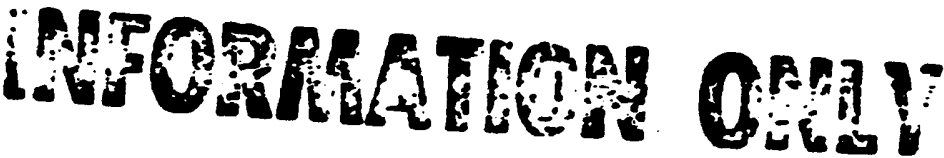




\section{BARRINGER LABORATORIES INC.}

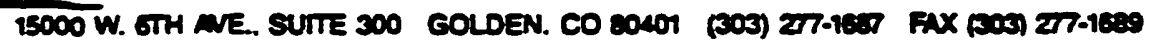

23-DeC-92

Ros Jacobs

MR-Ferguson

1571 CR 38

Gunnison, CO 81230

Pace:

Copy:

3 of 3

Atこว:

Pfoject: Gunnison

Received: 20-Nov-92 09:30

Job: $\quad 225566 \mathrm{E}$

PO : 3050-511-10004 \#058

Statis:

Final

QUAIITY CONTROL REPORT

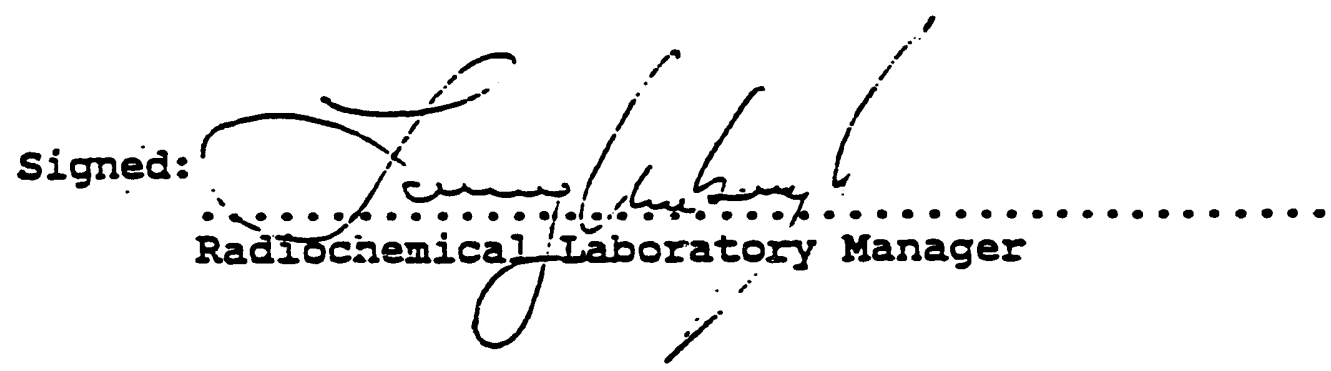

signed:

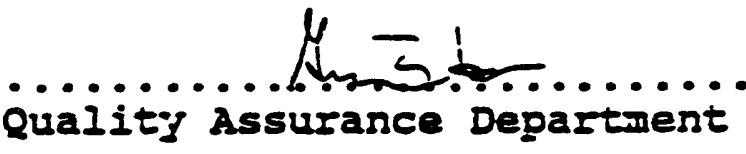

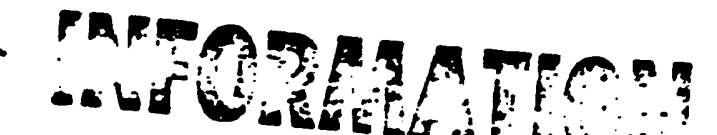

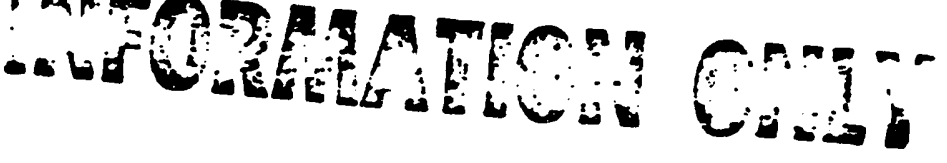




$$
\begin{aligned}
& \text { analysisfor }<44 \text { sieve } 14 \text { testpeits } \\
& \text { f fort Lifts }
\end{aligned}
$$

Helene Imalois

MR-FERGUSm (Eumien)

P. D. Bax 9136

Albuquergue, in 87119

Pogr: 1 of $\frac{1}{2}$

Attn:

Praject: Eumieon

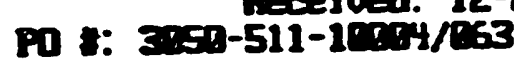

Jab: 9355352

Status: Final

Sople Iys: Soil

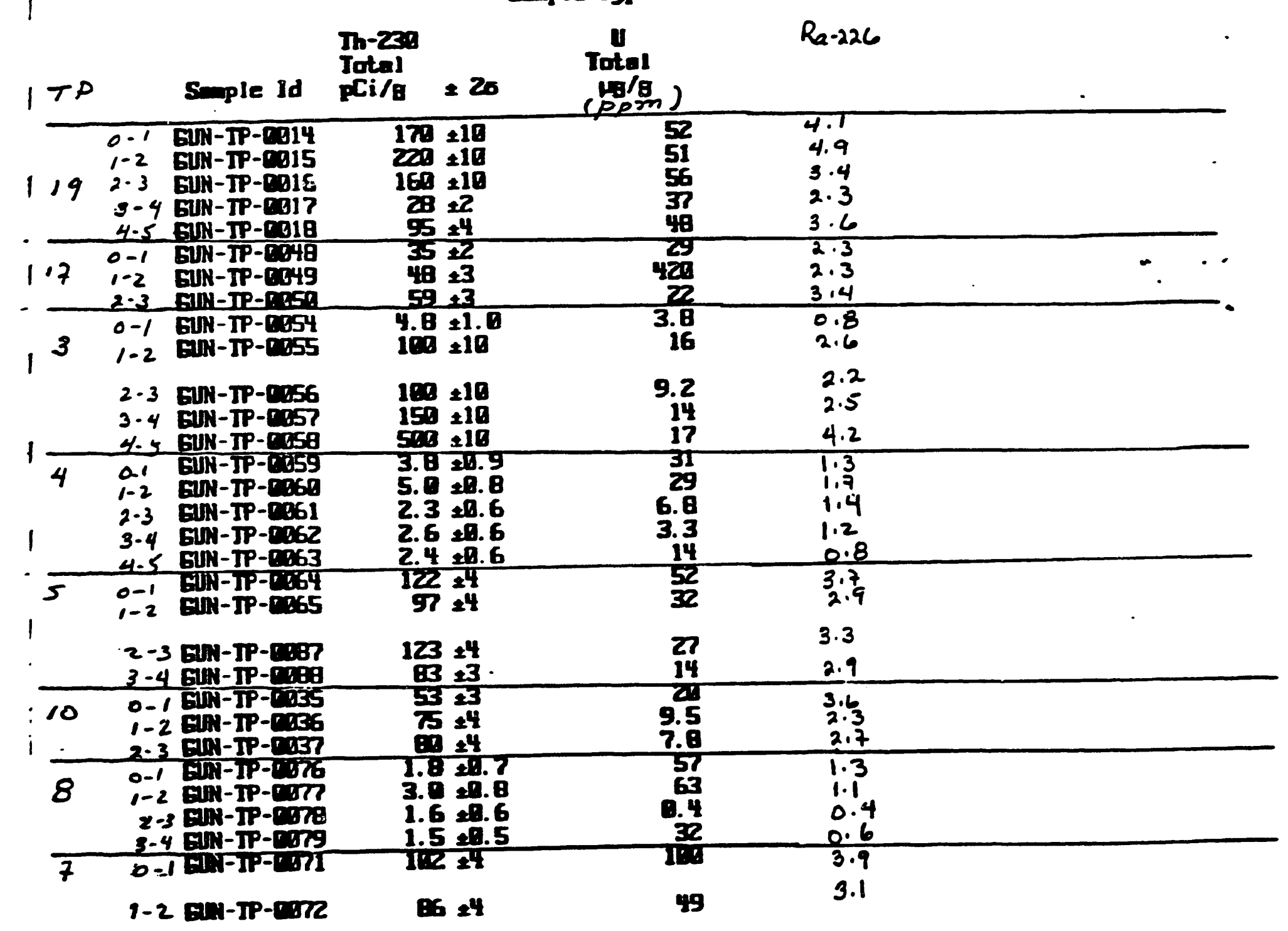




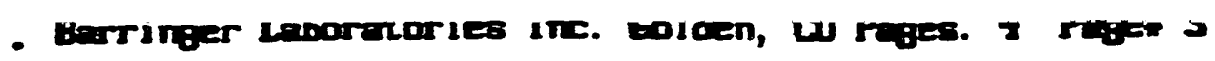

Helene Langlois

PK-FEPEUSO (Eumnicon)

P. D. Bax 9136

Aibuquerque, in 87119

Attr:

Prajat: Eumiom

Job: $536535 E$
9-Ays-93

Page: 1 of $\frac{2}{2}$
P0 5: 3:50-511-10.04/063

Stetus: Final

Sople Iype: Soil

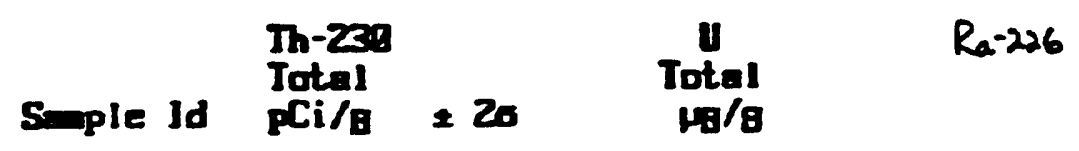

$7 \quad 2-3$ GHA-IP-COT3

3-4 EUN-TP-COT4

4-5 EUN-IP-DO75

\begin{tabular}{lll}
\hline 2 & $0-1$ & BUN-IP-COB5 \\
$1-2$ & ENN-IP-Co89
\end{tabular}

2-s EUN-TP-COB6

3-y EUN-TP-Coga

$4-5$ BUN-TP-COS1

Total

BUN-TP-LOSC

$1-2$ EUA-IP-DO93

$23 \pm 2$

$26 \pm 2$

Sit 43

$1.6 \pm 10.6$

2. $8 \pm 0.8$

$7.3 \pm 1.2$

$3.3 \pm 0.8$

6.1 \pm 1.1

$3.5 \pm 0.8$

o4 23

52

2.1

38

1.5

59

1.9

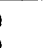

110

110

2-3 EUN-IP-DOS4

3.4 EUN-IP-COSS

$130 \pm 10$

$104 \pm 4$

51

1.0
0.9

1.1

b. 7

i. 1

$37-1,0$

$33 \quad 1.3$

$26 \quad 3.5$

$33 \quad 2.3$ 
Eelene Imalois

Ax-pEDEUSO (Eumieon)

P. 0. Bax 9136

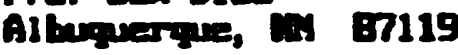

Attn:

Prajat: Prmaicon

lab: Tesgire 9-fy-93

Poge: i of 1

R-civad: 12-H-r-93 c9:45

Fo है: 3:30-511-10904/064

Steters: Final

\section{Sople Iype: Soil}

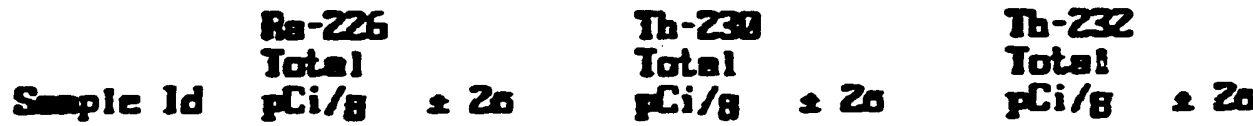

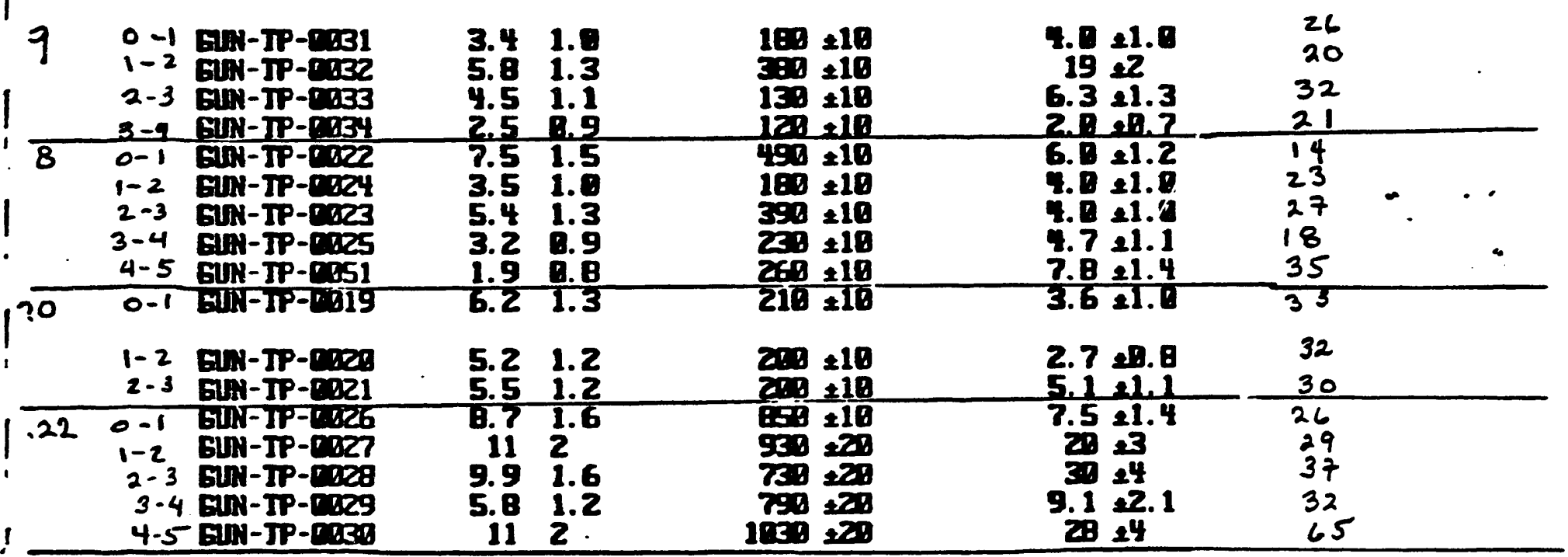

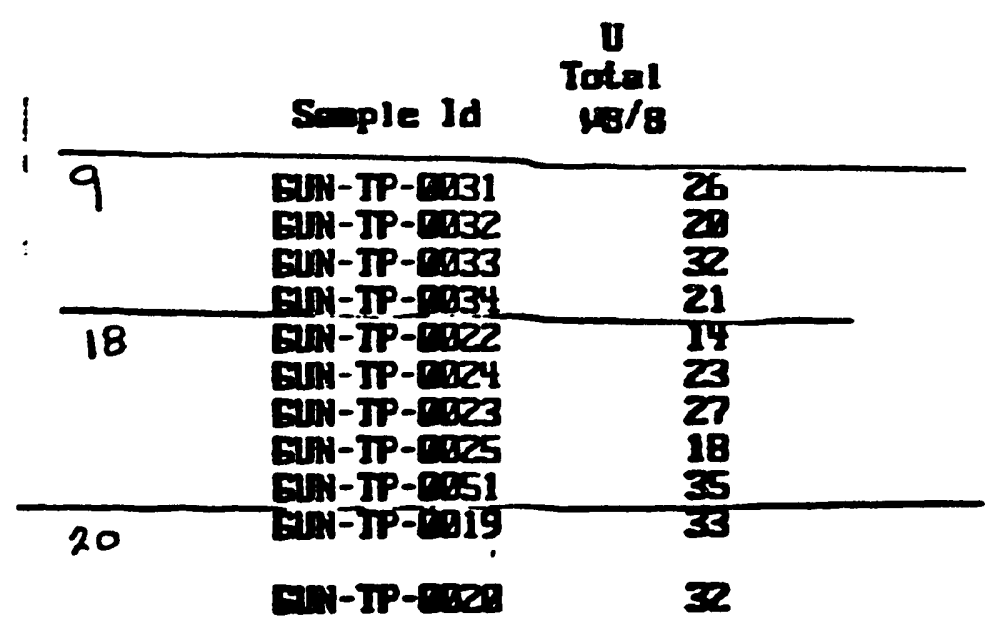


Helene Langlois

MK-FEREUST (Eumien)

P. 0. Box 9136

Albuquerpie, wis 87119

Attn:

Prajat: Eumieon

Job: $936540 \mathrm{c}$
9-Apr-93

Page: 1 of 2

P0 *: 3950-511-10094/R64

Status: Final

Sople Type: Sail

Seple Id $\begin{gathered}\text { U } \\ \text { Total } \\ \text { H/g }\end{gathered}$

BIN-TP-CD21

EIIT-TP-COZ6

EUN-TP-0.027

EUH-TP-DOZZ

ELN-TP-DDE9 


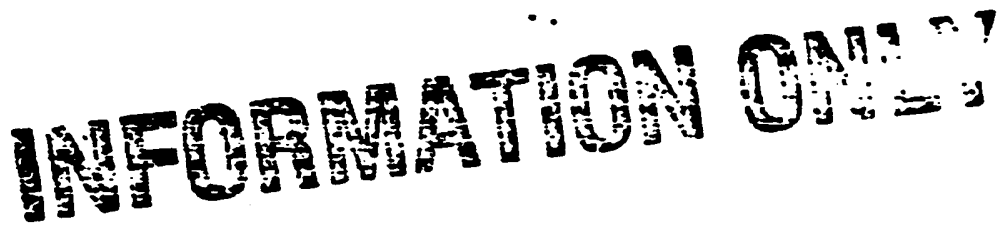

Ron Jacabs

MH-FEREUSON

1571 CR 38

Eurmison, on 81230

Attr:

Praject: Eumison

Job: gagstaE
9-Apr-95

Page: 2 of $\frac{1}{2}$

PD \#: 3050-511-10004/864

Recoived: 12-15ar-93 09: 出

Status: Fimal

Sample Type: Soil

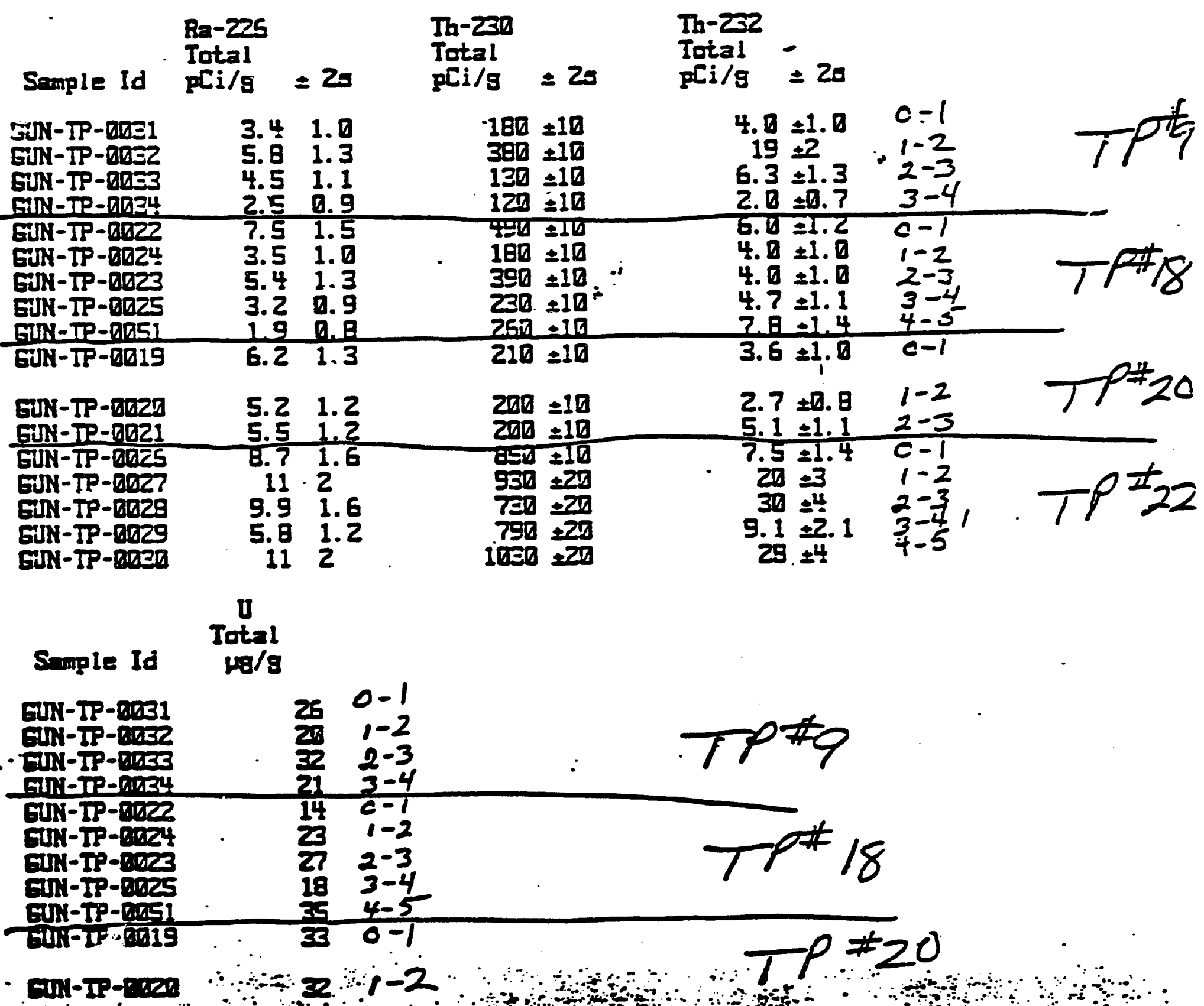

Ra-225

Iotal

3.41 .0

5.81 .3

4.51 .1

. 1.0

5.41 .3

$\begin{array}{lll}6.2 & 1.3\end{array}$

3.? 1.6

$11 \cdot 2$

1.6

.8 1.2

112
$4.0 \pm 1.0 \quad 0=$

$6.3 \pm 1.3 * 12-3$

2. $8=0.7 \quad 3-4$

$6.0=1.2 \quad c-1$

4. $011.0 \quad 1-2$

4. $0 \pm 1.0 \quad 2-3$

$4.7=1.1 \quad 3-4$

$3.6=1.8 \quad c-1$ $7.5 \pm 1.4 \quad c-1$

$20=3 \quad 1-2$

$30=4$

$1 \pm 2$.

$2-3$
$3-4$
$4-5$

TPI22 
Ron Jacobs

MR - FERGISOM

1571 CR 38

Eurmison, or 81230

Attr:

Praject: Eurmison

Jab: T2654taE
9-Apro-93

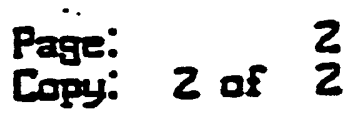

Received: 12-Mar-g3 00: 45

PD :: 3100-511-10004/064

\author{
Status: Finel
}

\section{Sample Iype: Sail}

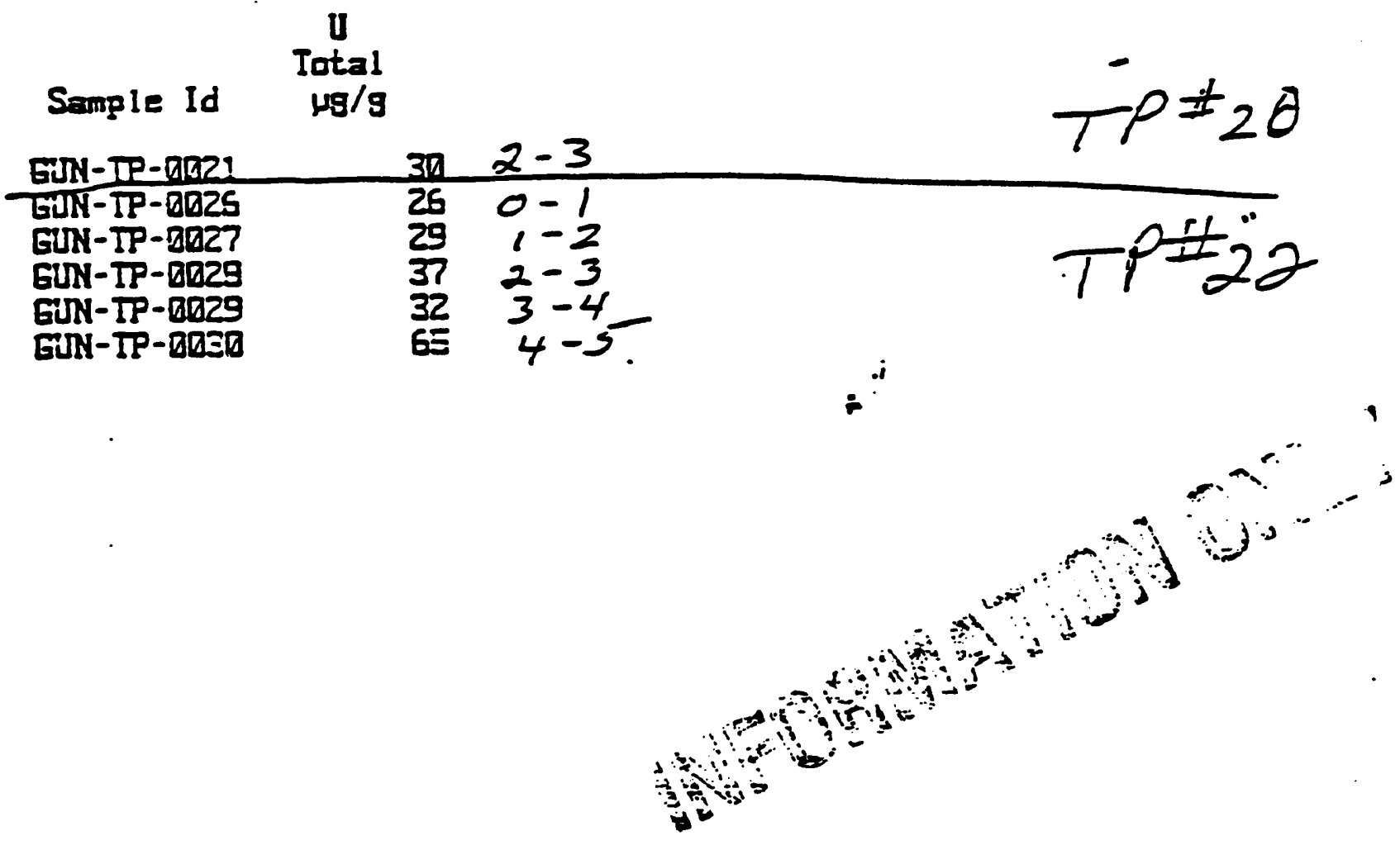

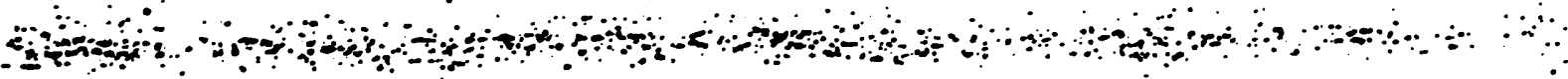

$=9$ - 


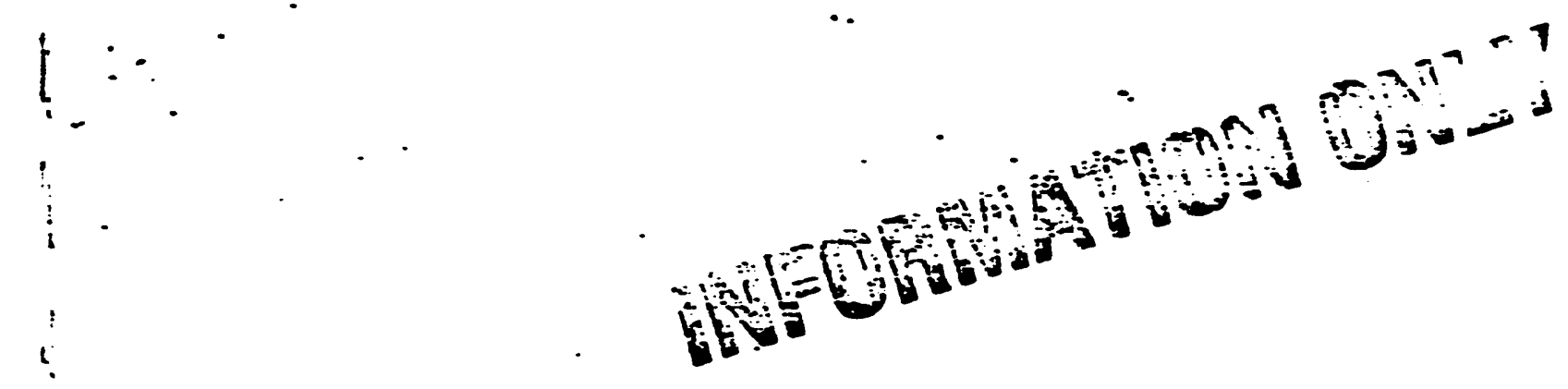

roinger Laburatmies Inc. Eolden, on Pages: 4 Page\# 4

Ron Jacobs

BR-FEREISIN

$1571 \mathrm{CR} 38$

Eumison, 00 B1230

Attr:

Project: E.mison

Job: TE6540E

Abbroviations:

Parameters:

$\begin{array}{ll}\text { Ra-226 } & : \text { Radium-225 } \\ \text { Th-230 } & \vdots \text { Thor ium-230 } \\ \text { Mh-232 } & \vdots \text { Thorium-232 } \\ \text { I } & \text { Hromium }\end{array}$

\section{9-Apr-93}

$\div \quad \begin{array}{ll}\text { Page: } & \\ \text { Copy: } & 3\end{array}$

PO \#: 3650-511-10004/06:

Status: Final

- Inits:

$\mathrm{pCi} / \mathrm{g}$
$\mathrm{Hg} / \mathrm{g}$

: picoluries per grom 
Finger Laboratories Inc. Golden, Dn Pages: 4 Page: 1

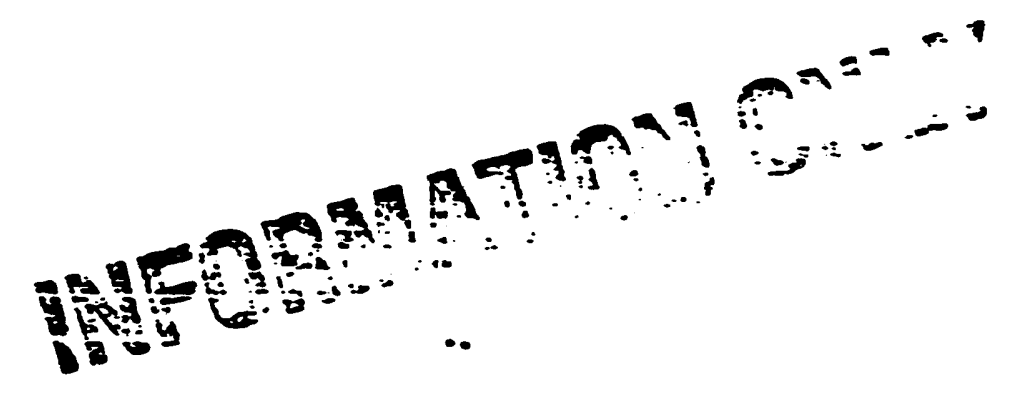

FAXED ALALUTICAL REPURT

FROM: Barringer Laboratories, Inc.

J0B : 93635

DATE: 9-Apr-93

IIME: $17: \Omega$

To : Ron Jacobs

MR-FEEEUSON

1571 CR 38

Eumison,

This is an electronically tramsferred fax of amalytical results (prel iminary or final, as designated on the roport). The data comtained in this report has been roviewed and apgroved by the laboratory mangers and $Q A / Q C$ Persorme1. The final report with all sppropriate signatures and $Q A / Q C$ will fallow by mil. We do not asseme any responsibilty far the fax transisaion. It

$\therefore$ is the client's reponsibility to notify us of any chambes in address and/or phome number.

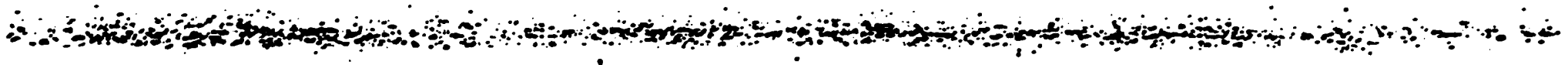
\% 
Ron Jacobs

MR-FEREISTI

$1571 \mathrm{CR} 38$

Eumison, 00181230

Attr:

Praject: Eumison

Job: 9365

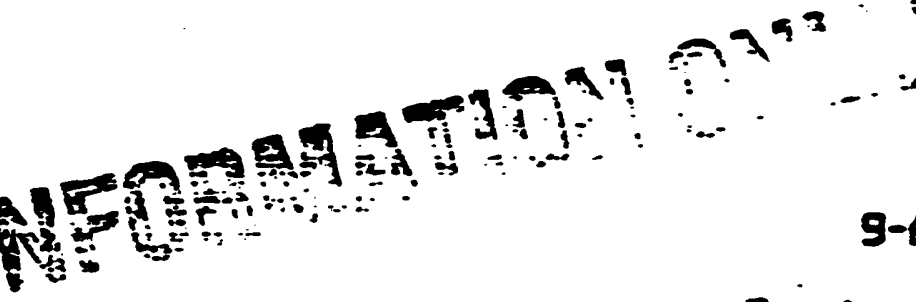

Prop:: 2 of $\frac{1}{2}$
PD F: 3050-511-10014/1863

Recived: 12-1wr-פ3 13:5

Stotus: Finel

Sumple Type: Sail

Ih-23b

Total

Somple Id $\mathrm{pCi} / \mathrm{g} \pm 20$

EIN-IP-RO14

ENN-IP-0015

EIN-IP-DQ16

EUN-TP-Q017

EIN-TP-0018

EUN-IP-8D48

EIN-IP-0049

ENI-IP-0050

EIN-IP-ninst

Ein-IP-005S

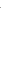

Eni-TP-Rose

Ein-IP-0057

EIN-TP-005a

EIN-TP-0.153

EUN-IP-006]

EUN-TP-0061

ENI-IP-0062

EII-IP-0063

GIN-IP-DDSH

Eni-IP-0.65

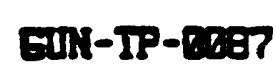

mini-TP-Gara

and-IP-en35

- - InI-IP-C.35

EU1:-TP-T.1257

aIN-IP-0.076

EnI-IP-D.977

Eni-IP-D.078

-III-IP-QT79

लाi-IP-iri

- FIR-IP-0.372
$170=10$

$200 \pm 10$

$160 \pm 10$

$28 \pm 2$

$95=4$

35 se

$48+3$

$50-3$

4. $8=1.0$

$100=10$

$100 \pm 10$

$150 \pm 10$

$590 \pm 10$

$3.8 \pm 0.5$

$5.0 \pm 0.8$

$2.3 \pm 1.6$

$2.6=0.6$

$2.4 \pm 0.6$

$122 \leq 4$

$97=4$

\section{3 난}

EB-3

$53-3$

to 2

8. 4

$1.8 \pm .7$

3.0 .9 .8

1.620 .6

$1.5=0.5$

I.R 24

is at:
0

Total

$\mu \mathrm{g} / \mathrm{g}$ ng $1-2$

$\begin{array}{ll}5 & 0-1 \\ 51 & 1-2 \\ 26 & 2-3\end{array} \quad T p \neq 1 \%$

37 3-4

48 4-5

${ }_{420}^{20} 1 .-2 \quad 7,7$

$22,2-3$

$16:-2$

9.2 2-3

$143-4$

$174-5$

$310-1$

$201-2$

6. 8 2-3

$3.33-4$

$14 \quad 4-5$

$32 \quad 0-1$

$27 \quad 2-3$

$14 \quad 3-4$

2. $0-1$

$9.5 \quad 8-2$

2. $2-3$

1.p?

$50-1$

63 1-2

D. $2-3$

$32 \quad 3-4$

I. 0 -

$$
7 P=7
$$

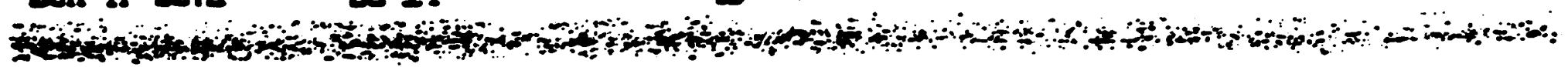

Fin 
Ron Jacabs

MK-FERGISTI

1571 CR 38

Eurmisen, 00 B1230

Attr:

Praject: Eumison

Job: 5365290
9-Apr-93

Page: 2 of $\frac{2}{2}$

Received: 12-Mar-g3 13:5

PD : $30=0-511-10004 / 863$

\section{Status: Final}

\section{Sample Type: Soil}

Th-250

Total

Sample Id

$\mathrm{p}[\mathrm{i} / \mathrm{s}=20$

ENR-TP-8073

ENN-IP-Q074

ENN-IP-6075

EIN-IP-DEES

EUN-IP-0089

EUN-IP-Q8BE6

ENN-IP-Q600

Gin-TP-gag1

EUN-IP-0002

EIN-IP-DB93

ENN-IP-6094

ENN-IP-DES5
$2 \Sigma \pm 2$

$25 \pm 2$

$54-3$

$1.6 \pm 1.6$

2. $8 \pm 0.8$

$7.3 \pm 1.2$

$3.3 \pm 0.8$

$6.1+1.1$

$3.5 \pm 0.8$

St \pm 3

$130=10$

$104=4$
0
Iotal
$\mu g / 9$

$\begin{array}{ll}25 & 2-3 \\ 33 & 3-4\end{array}$

$39 \quad 3-4$

$59 \quad 4-5$

$T p^{\Perp} 7$

IIV $0-1$

$1001-2$

$110 \quad 2-3$

$51: 4=4$

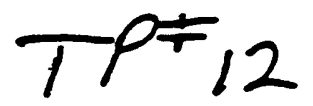

37 c-1

$33 \quad 1-2$

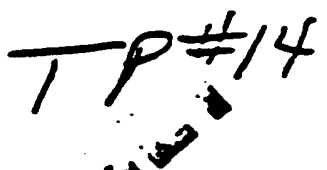


gur Laburatories Ine. Eolden, Do Pages: y Pagell i"

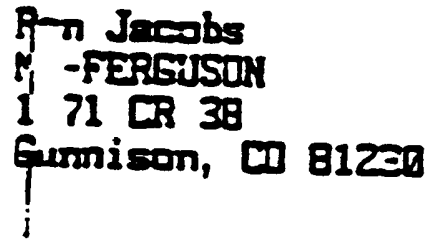

Attr:

F zijex: Eumison

Job: 536509

f'breviations:

F_rancter:

Th-220

U

Thorium-200
Uranium

\section{9-Apr-93}

Page:- 1 of 3

FO : 3650-511-10004/863

its:

$\mathrm{pCi} / \mathrm{g}$

$\mu \mathrm{g} / \mathrm{g}$ 
VENDOR LABORATORY RADIOLOGICAL

MEASUREMENTS: COMPOSITE SOIL SAMPLES $>44$ MESH SIEVE COBBLY SUBSOIL INVESTIGATION NOVEMBER 1992 
Ron Jacobs

IIR-Ferguson

$1571 \mathrm{CR} 38$

Gunaison, Co 81230

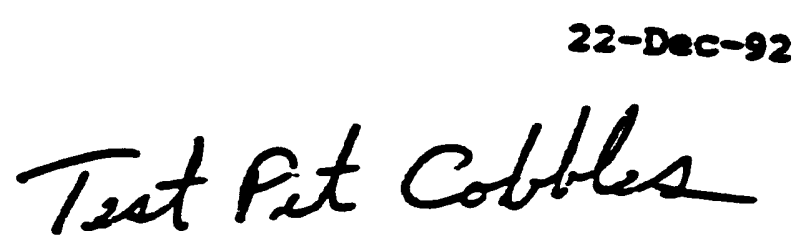

Attr:

Project: Gunnison

Received: 20-1100-9:

Tob: 9255715

P0\$ 3050-511-10004 \$057

statik: Fina:

BARRTNGER IABORATORIES

ANALYTICAI REPORT PACKAGE

CASE IARRATIVE.

ANALYTICAI KISULTS.

$R-1$

QUALITY CONTROL REPORT.

-2-1 

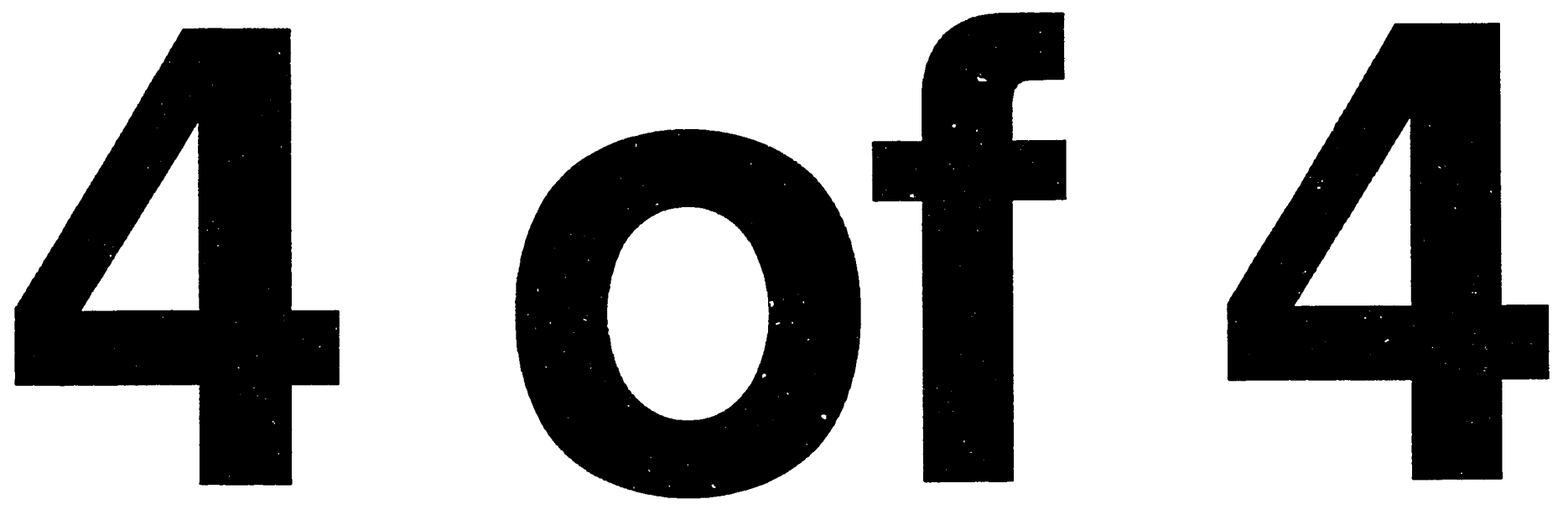


\section{BARRINGER LABORATORIES INC.}

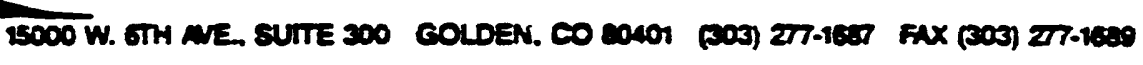

Ron Jacobs 2RK-Ferguson 22-DeC-92 $1571 \mathrm{CR} 38$ Gunnison, CO 81230

Page: Copy: 3 of $\frac{i}{3}$
Attr:

Project: - Gurrison
Received: 20-Nov-92 PO\# 3050-511-10004 \#057

Job: $925571 E$

Status: Final

\section{CASE NARRATIVE}

A total of 23 soil samples were received on 20-Nov-92. All were properly preserved and in good condition. As stated in the chain of custody, the samples were run for the following analyses: Ra-226, Th-230, Th-232 and 0 . Our procedures are summarized on the Quality Control Data sheet. There were no deviations to these procedures. All samples were extracted and analyzed within the proper holding times.

Quality control standards were ran using standard EPA guidelines. A summary of that data is provided. The acceptance criteria for spikes and duplicates is fifteen percent. It is based on the relative percent deviation of the mean, taking the counting error into account for radiochemical analyses. All QC checks, including duplicates, spikes, and. blasks, passed.

signed:

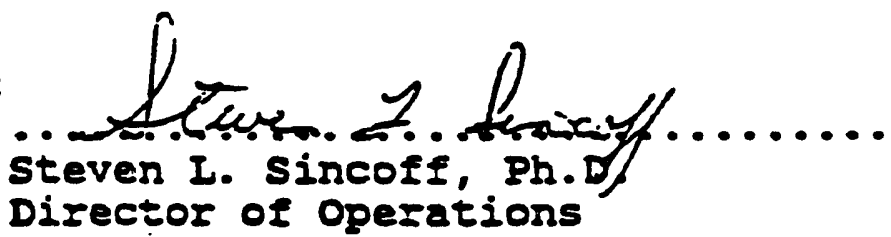

Director of Operations

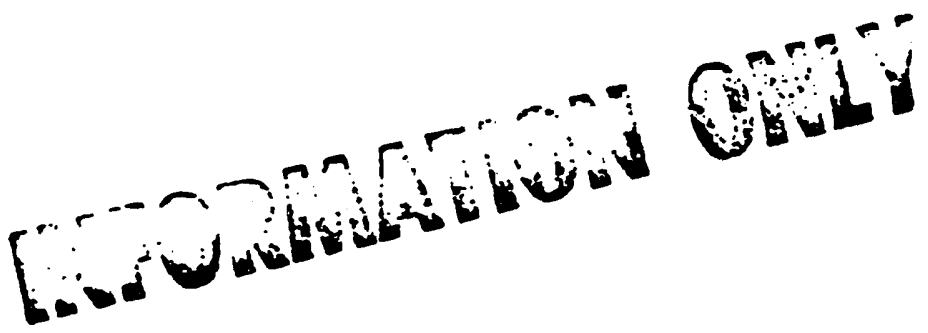




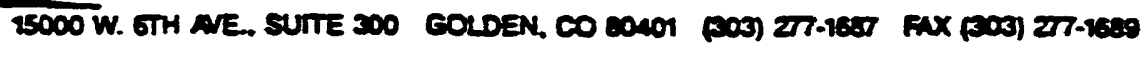

Ron Jacobs

MIR-Ferguson

22-Dec-92

1571 CR 38

Gungison, CO 81230

Attn:

Project: Gunnison

Page: $\quad R-1$

Copy: 3 of 3

cobbles

Received: 20-Nov-92 11:30 PO : 3050-511-10004 $\# 057$

Job: $\quad$ y255715

Status: Final

8ample zype: soil

Samcle Id

\begin{tabular}{|c|c|c|}
\hline $\begin{array}{l}\text { GUN-SS-01-CTF } \\
\text { GUN-SS-02-CTF } \\
\text { GUN-SS-03-CTF } \\
\text { GUN-SS-04-CTF } \\
\text { GUN-SS-05-CTF } \\
\text { GUN-SS-06-CTF } \\
\text { GUN-SS-07-CTF } \\
\text { GUN-SS-08-CTF } \\
\text { GUN-SS-09-CTF } \\
\text { GUN-SS-20-STF }\end{array}$ & $\begin{array}{l}11 \\
12 \\
12 \\
12 \\
12 \\
12 \\
12 \\
12 \\
12 \\
11\end{array}$ & $\begin{array}{l}\text { Bucket) } \\
\text { Buckets) } \\
\text { Buckets) } \\
\text { Buckets) } \\
\text { Buckets) } \\
\text { Buckets) } \\
\text { Buckets) } \\
\text { Buckets) } \\
\text { Buckets) } \\
\text { Bucket) }\end{array}$ \\
\hline $\begin{array}{l}\text { GUN-SS-11-CTF } \\
\text { GUN-SS-12-CTF } \\
\text { GUN-SS-13-CTF } \\
\text { GUN-SS-14-CTF } \\
\text { GUN-SS-15-CTF } \\
\text { GUN-SS-16-CTF } \\
\text { GUN-SS-17-CTF } \\
\text { GUN-SS-18-CTF } \\
\text { GUN-SS-19-CTF } \\
\text { GUN-SS-20-CTF }\end{array}$ & $\begin{array}{l}(2 \\
12 \\
(2 \\
(1 \\
(2 \\
(2 \\
12 \\
(2 \\
(2 \\
12\end{array}$ & $\begin{array}{l}\text { Buckets) } \\
\text { Buckets) } \\
\text { Buckets) } \\
\text { Bucket) } \\
\text { Buckets) } \\
\text { Buckets) } \\
\text { Buckets) } \\
\text { Buckets) } \\
\text { Buckets) } \\
\text { Buckets) }\end{array}$ \\
\hline $\begin{array}{l}\text { UN-SS-21-CTF } \\
\text { UN-SS-22-CTF } \\
\text { UN-SS-23-CTF }\end{array}$ & ( & $\begin{array}{l}\text { (s) } \\
\text { s) } \\
\text { s) }\end{array}$ \\
\hline
\end{tabular}

\begin{tabular}{llll} 
Ra-226 & Error & Th-230 & Exzor \\
Total & & Total & \\
DCi/a & 20 & DCi/a & 20 \\
\hline
\end{tabular}

$2.5 \pm 0.8$

$2.5 \pm 0.8$

$1.3 \pm 0.6$

$1.2 \pm 0.7$

$1.9 \pm 0.8$

$1.8 \pm 0.8$

$1.9 \pm 0.7$

$2.0 \pm 0.8$

$3.0 \pm 0.9$

$3.1 \pm 0.9$

$2.0 \pm 0.8$

$1.3 \pm 0.6$

$2.5 \pm 0.9$

$1.5 \pm 0.4$

$2.8 \pm 0.9$

$1.6 \pm 0.7$

$2.8 \pm 0.9$

$3.1 \pm 0.8$

$2.2 \pm 0.8$

$2.7 \pm 0.9$

$1.6 \pm 0.7$

$4.0 \pm 1.1$

$2.7 \pm 0.7$
$15 \pm 2$

$3.2 \pm 0.8$

$29 \pm 2$

$3.7 \pm 0.9$

$29 \pm 2$ ?

$1.6 \pm 0.6$

$7.5 \pm 1.2$

$0.8 \pm 0.5$.

$47 \pm 3$

$21 \pm 2$

$12 \pm 2$

$3.1 \pm 0.8$

$41 \pm 3$

$13 \pm 2$

$1.6 \pm 0.6$

$10 \pm 1$ ?

$7.1 \pm 1.2$

$41 \pm 3$

$22 \pm 2$

$42 \pm 3$

$1.9 \pm 0.6$

$69 \pm 4$

$3.1 \pm 0.8$
BK:

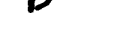

Th-232 Error

Total

icila 20

Iotal

$1.7 \pm 0.7$

$1.2 \pm 0.6$

2.2

1.9

$\oint K G$

GUN-5S-01-CTF (1 Bucket)
GUN-55-02-CTF (2 Buckets)

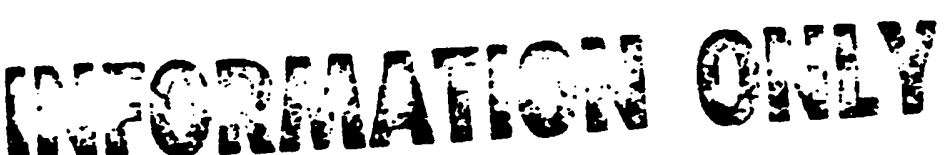

Meeting The Analytical Challenges Of A Changing World 
Ron Jacobs

MIK-Ferguson

1571 CR 35

GunRisorı, CO 81230

Atะת:

Project: Gunnison
22-Dec-92

Page: $\quad R-2$

Copy: 3 of 3

cobbles

Received: 20-Nov-92 11:30 PO : : 3050-511-10004 \#057

Job: $925571 \mathrm{E}$

status:

Final

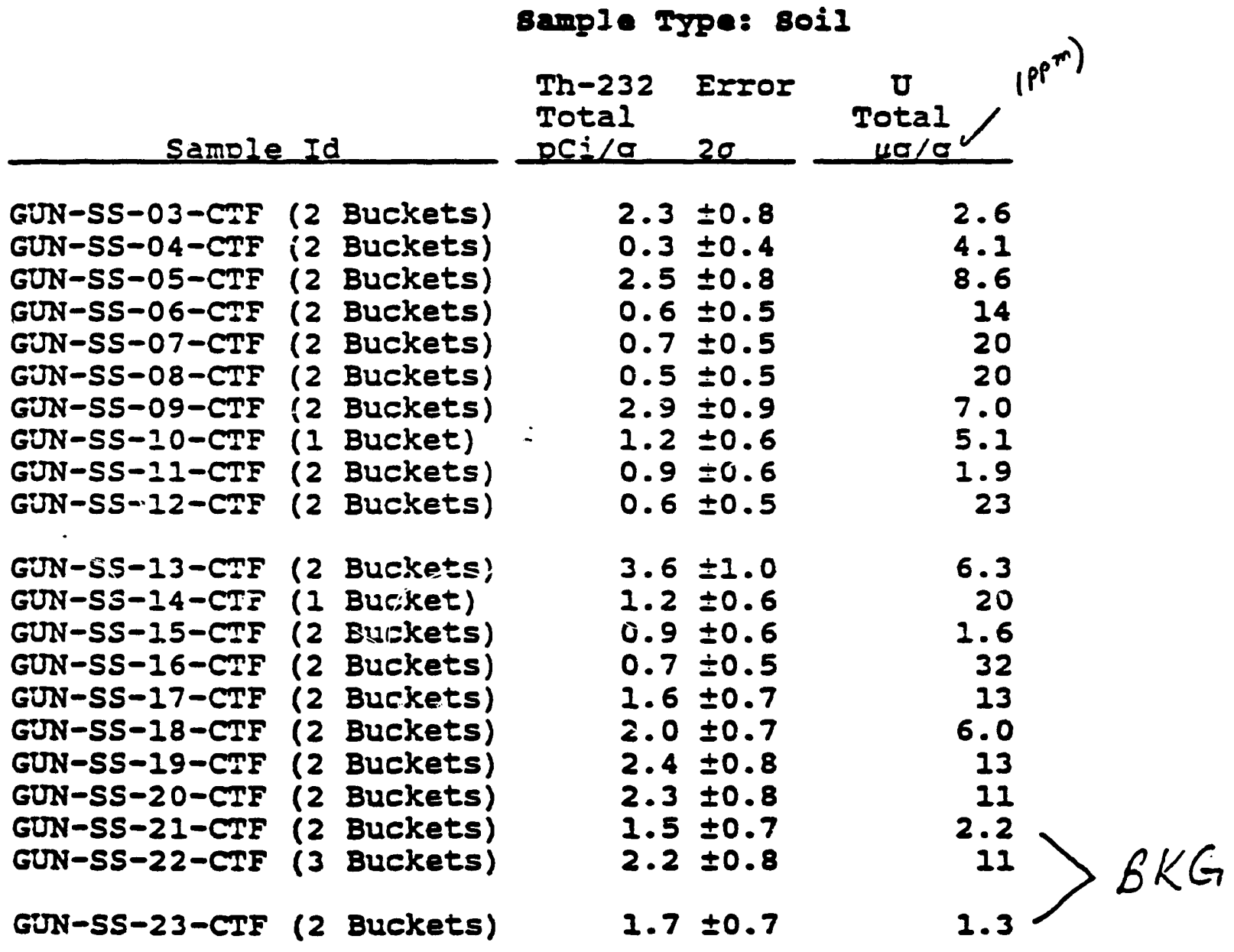

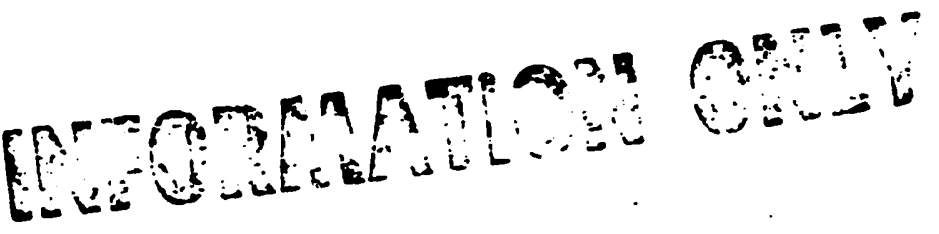




\section{BARRINGER LABORATORIES INC.}

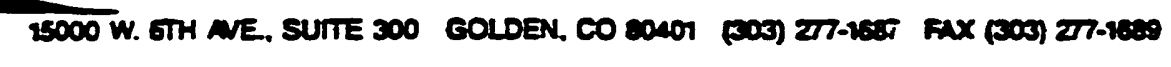

\section{Ron Jacobs}

MIR-Ferguson

1571 CR 38

Gunnison, CO 81230

22-Dec-92

Atธת:

Project: Gunnison

Received: 20-Nov-92 11:30 PO : 3050-511-10004\$057

Iob: $\quad 2255715$

status:

Final

$$
\text { QUAIITY CONTROL REPORT }
$$

\section{Bample Igpe: soil}

\section{Sample Id}

$\begin{array}{llll}\text { Ra-226 } & \text { Error } \begin{array}{ll}\text { Th-230 } \\ \text { Total }\end{array} & \text { Error } \\ \text { Total } & & \text { Totalo } & 20 \\ \text { DCi/a } & 20 & \text { DCis/a }\end{array}$

\section{Duplicate}

Duplicate

Duplicate diff.

Std (found value)

sta (true value)

Std \& rec.

Blank

Spike rec.

$1.4 \pm 1.9$
$0.4 \pm 1.6$
6.0
$102 \pm 7$
99
103
$0.0 \pm 0.1$
108

$41 \pm 3$

$53 \pm 3$

13

$101 \pm 6$

97 -

$104 \ldots$

$0.0 \pm 0.2$

$101 \ldots$

Page:
Copy: 3 of
$0-1$

Th-232 Error Total pci/o

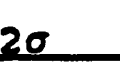

\section{Sample Id}

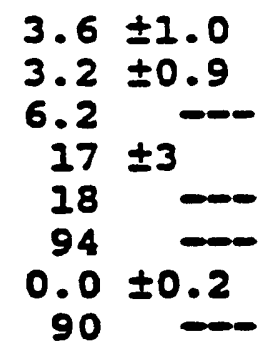

Duplicate

Duplicate

Duplicate o diff.

Sta (found value)

std (true value)

sta rec.

Blank

Spike \& rec.

6.0

Total $n \sigma / a$

6.0
6.2
1.6
44.4
49.0
91
0.0
103




\section{BARRINGER LABORATORIES INC.}

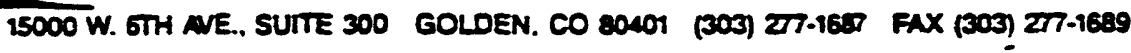

Ron Jacobs

MIK-Ferguson

1571 CR 38

Gunnison, CO 81230

22-Dec-92

Page:

Copy: 3 of 3

\section{Atニת:}

Project: Gunnison

Received: 20-Nov-92 11:30

PO : $3050-511-10004 \div 057$

Iob:

$925571 \mathrm{E}$

Status:

Final

QUAIITY CONTROI REPORT

Abkreviaticons:

\section{Parameters:}

$\mathrm{Ra}-226$

$T h-230$

Th-232

U

: Radium-226

: Thorium-230

: Thorium-232

: Uranium

\section{Units:}

$\mathrm{pci} / \mathrm{g}$

$2 \sigma$

$\mu \mathrm{g} / \mathrm{g}$

: picocuries per gram

: Counting ersor at the $95 \%$ confidence level, $2 \sigma$

: miczograms per gram

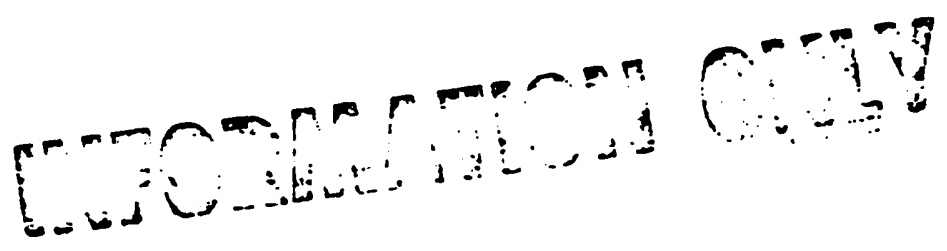


Ron Jacobs

MIR-Ferguson

22-Dec-92

1571 CR 38

Gursison, CO 81230

Page:

Copy:

Attr:

Project: Gunnison

Received: 20-Nov-92 11:30

Job: 2255715

status:

Final

QUAIITY CONTROI REPORT

QUATIHY COLHSOI DATA BEREm

Received by: jdr

Via: DSi Ine

Sample Container Type: 5 gal bucket

Sample Type: Soil

Preservative when Received: none

Additional Lab Preparation: none

\begin{tabular}{|c|c|c|c|c|c|}
\hline Parameter & Method & DIII & $\begin{array}{l}\text { Prese } \\
\text { vativ }\end{array}$ & e- Analyst & $\begin{array}{l}\text { Date (s) of } \\
\text { Analysis }\end{array}$ \\
\hline $\begin{array}{l}R z-226 \\
T h-230 \\
T h-232 \\
0\end{array}$ & $\begin{array}{l}\text { SM-705 } \\
\text { USAEC } \\
\text { DSAEC } \\
\text { ASTM D2907 }\end{array}$ & $\begin{array}{ll}0.3 & \mathrm{pCi} / \mathrm{g} \\
0.4 \mathrm{pCi} / \mathrm{g} \\
0.4 \mathrm{pCi} / \mathrm{g} \\
0.3 \mu \mathrm{g} / \mathrm{g}\end{array}$ & $\begin{array}{l}\text { none } \\
\text { none } \\
\text { none } \\
\text { none }\end{array}$ & $\begin{array}{l}\text { Preston } \\
\text { Ortiz } \\
\text { Ortiz } \\
\text { Rnox }\end{array}$ & $\begin{array}{l}12 / 17-12 / 22 \\
12 / 10-12 / 17 \\
12 / 10-12 / 17 \\
12 / 14-12 / 16\end{array}$ \\
\hline
\end{tabular}




\section{BARRINGER LABORATORIES INC.}

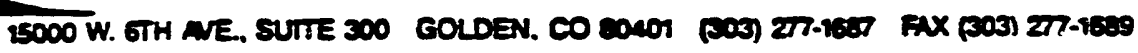

Ron Jacobs

IIR-Ferguson

22-DeC-92

1571 CR 38

Gunnison, CO 81230

Page: $R-3$

Copy: 3 of 3

Atニn:

Project: Gunnison

Received: 20-Nov-92 11:30

Iob: $225571 \mathrm{E}$

PO : 3050-511-100C4 $\$ 057$

\section{Abbreviations:}

\section{Parameters:}

$$
\begin{aligned}
& R a-226 \\
& T h-230 \\
& T h-232 \\
& 0
\end{aligned}
$$

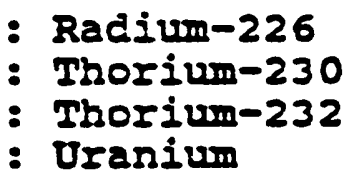

\section{Inits:}
$p c i / g$
20
: picocuries per gram
$\mu \mathrm{g} / \mathrm{g}$
: Counting error at the $95 \%$ confidence level, 20
: micrograms per gram 


\section{BARRINGER LABORATORIES INC.}

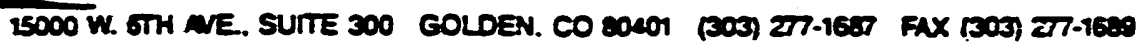

\section{Ron Jacobs}

IIR-Ferguson

1571 CR 38

Gunzison, CO 81230

22-Dec-92

Page:

Copy: 3 of 3

Attr:

Project: Gurnison

Received: 20-Nov-92 11:30

PO : 3050-511-10004\$057

Job: $\quad 925571 \mathrm{E}$

Statys:

Final

\section{QUAIITY CONTROL REPOR:}

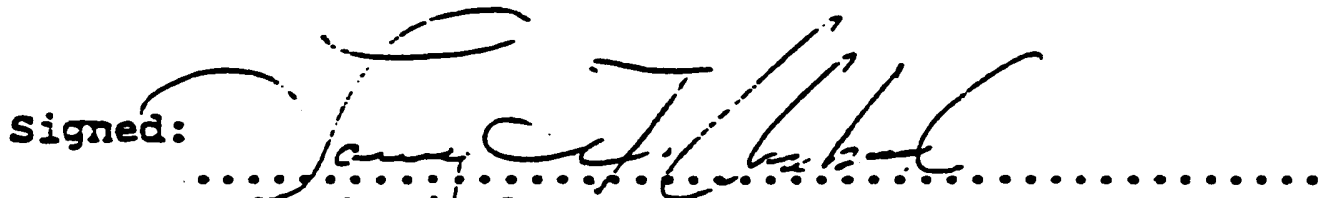

Radiochemical Iaboratory Manager

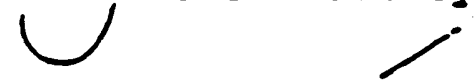

signed:

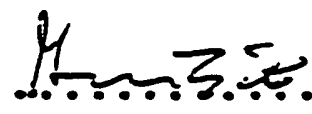

Quality Assurance Department 


\section{LABORATORY SERVICES AUTHORIZATION FORM}

Page 1 of 2

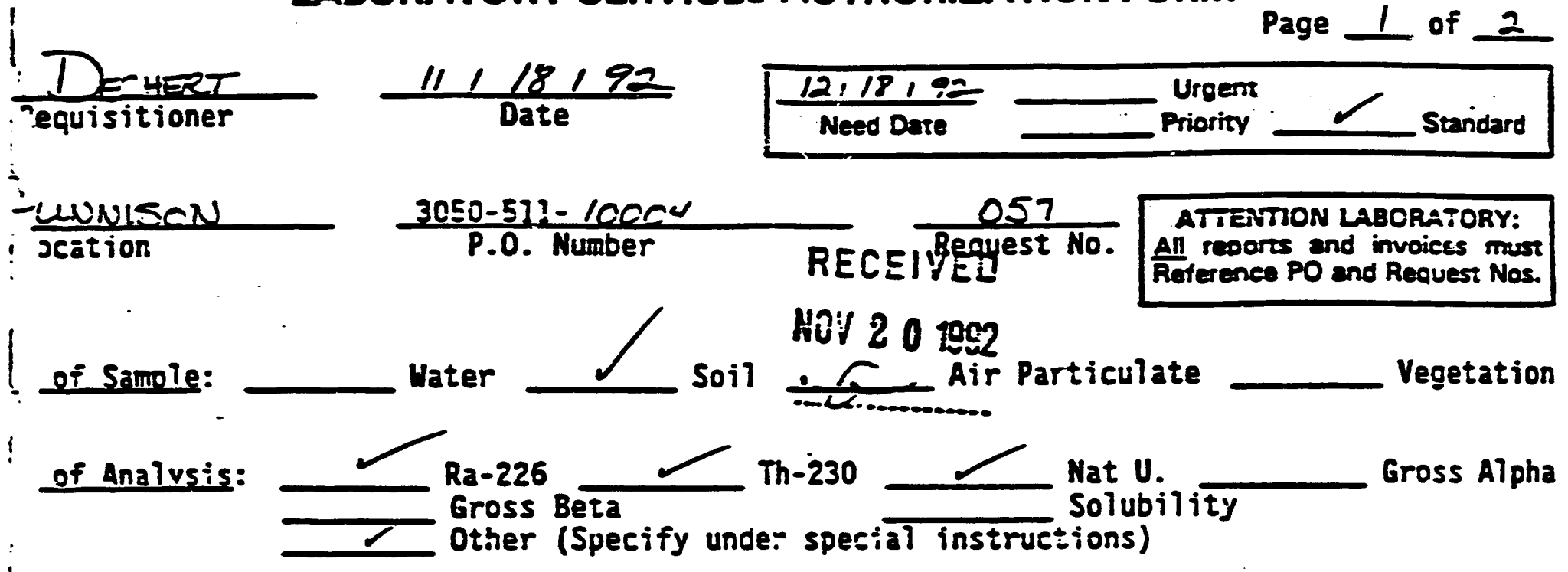

: ial Instructions/Comments: PLERSE CRUSH AND COMACSTTE ALL RUCKETS FROM

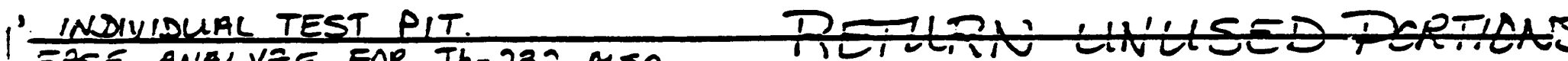
EASE ANALVEE FOR Th-23a ASA

I.D. NO DATE

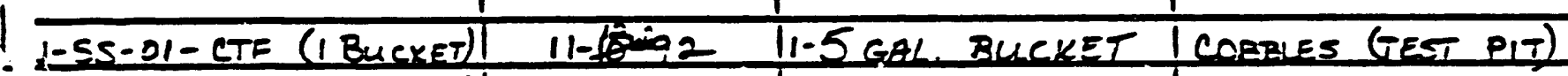
iN-SS-12-CTF (2 RUCKETS) | $11-10-92$ 2-5CAL. RUCKETS |TEST PIT 2 COBBIES 1-SS-03-CTFC 2 BUCYETSX $11-10-92$ 12-5CAL RUCKESS I TEST PIT 3 nABELES

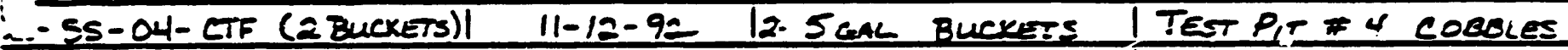

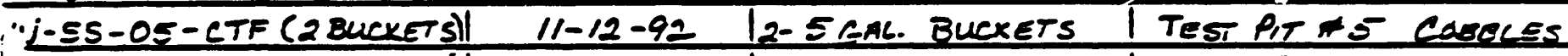
'-SS-06- CTF (a Buckets)l 11-12-92 12-5GAL. BUCKETS I TEST PIT $\% 6$ CONALSS

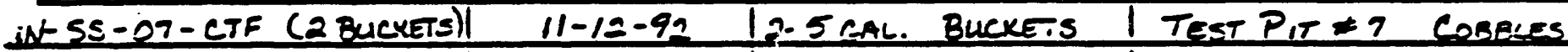
"1-SS-08-CTF (2 BUKKETS)I $11-12-92$ 12-5AR. BUCKETS I TEST PT \#8 CORRLS - 1-5S-09-CTF (2 BUCKETS) $11-12.92$ |2.5 GAL BuCXETS | TEST PIT $\# 9$ COBELES

\begin{tabular}{|c|c|}
\hline & CHAIN-OF-CUSTODY IF SEAL ON LD IS BREREN \\
\hline unquisneg by isite Representertve Signaturel: & $\mid \begin{array}{l}\text { Dareitine } \\
11-18-92: 1460\end{array}$ \\
\hline $2 / 20$ & $1 /-18-92$ \\
\hline
\end{tabular}

ortinger Crain-of-Custody as per contract will be arrached

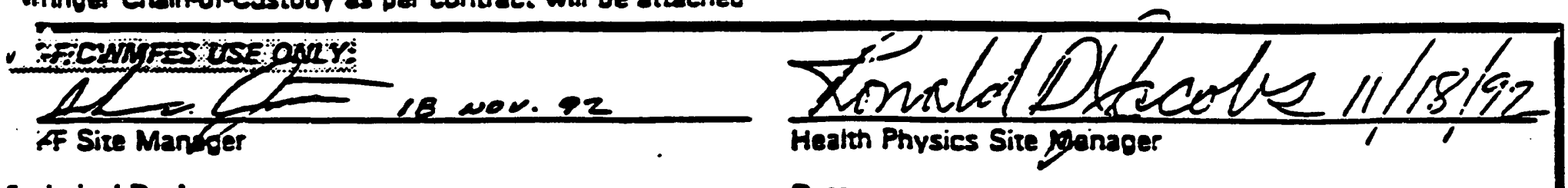

-echnical Roviow:

Drte:

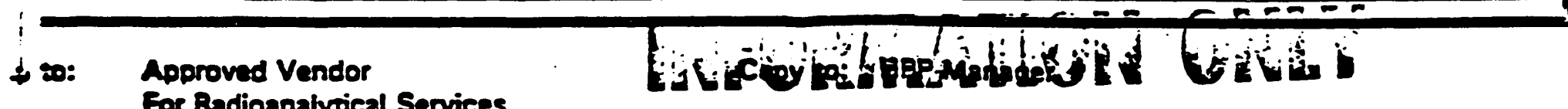
For Radioanalytical Services 


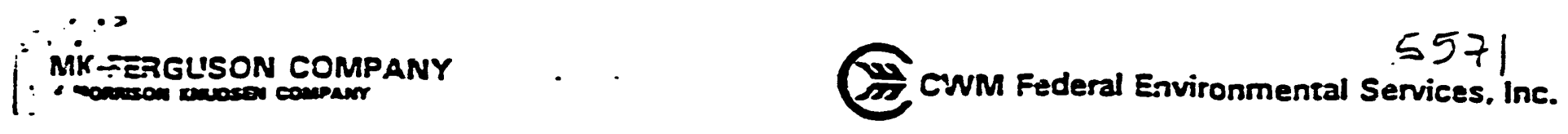

\section{LABORATORY SERVICES AUTHORIZATION FORM (CONTINUATION PAGE! C=! !E?}

$1:$

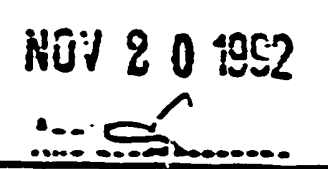

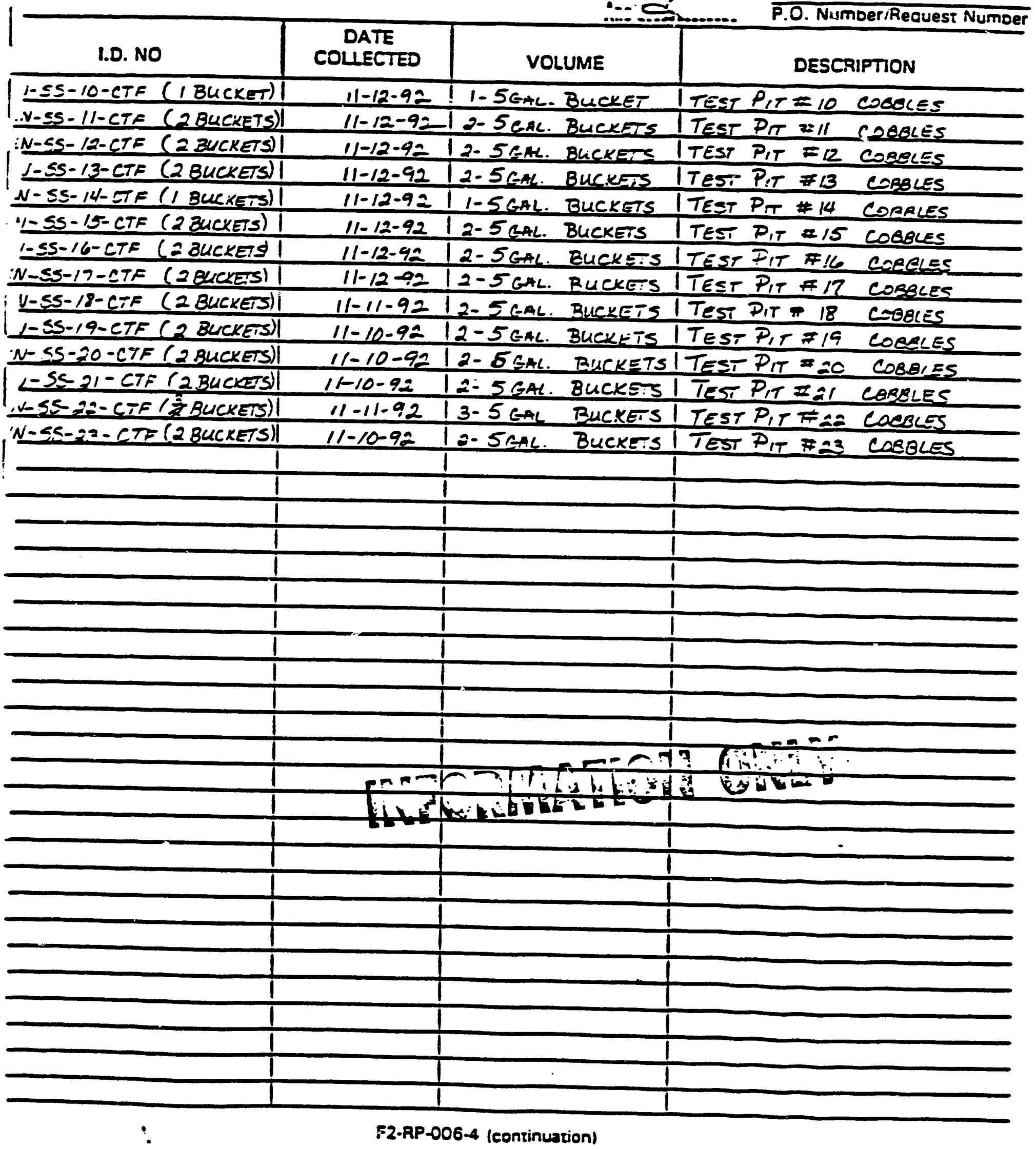


VENDOR LABORATORY RADIOLOGICAL

MEASUREMENTS: COMPOSITE SOIL SAMPLES <\#4

MESH SIEVE COBBLY SOIL INVESTIGATION

NOVEMBER 1992 
BARRINGER LABORATORIES INC.

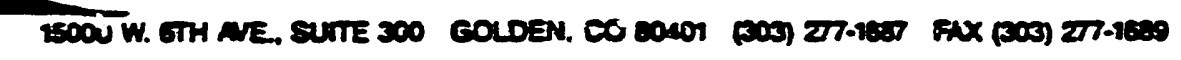

Ron Jacobs

ZMR-Ferguson

1571 CR 38

Gunnison, Co 81230

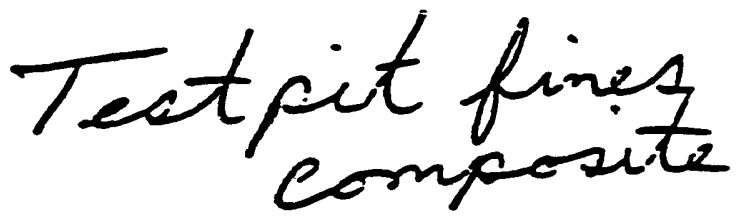

Attr:

Project: Gunnison

Recsived: 20-Nov-92

PO\$ 3050-511-10004 $\neq 059$

Job: $92555 \mathrm{E}$

Statys: Final

BARRINGER IABORATORIES

ANALYTICAI REPORT PACRAGE

CASE MARRATIVE. $\ldots \ldots \ldots \ldots \ldots \ldots \ldots \ldots \ldots \ldots \ldots$

ANAIYTICAI RESULTS.....................

QVAIITY CONTROL REPORT..................QQ

Ger 
Ron Jacobs

IIR-Ferguson

1571 CR 38

Gunnison, Co 81230

Attn:

Project: Gunnison
Received: 20-Nov-92

PO 3050-511-10004 \$059

Job: 9255675

status: Final

\section{CASE NARRATIVE}

A total of 23 Soil samples were received on 20-Nov-92. All were properly preserved and in good condition. As stated in the chain of custody, the samples were ran for the following analyses: Ra-226, Th-230, Th-232 and $v$. Our procedures are summarized on the Quality Control Data Sheet. There were no deviations to these procedures. All samples were extracted and analyzed within the proper holding times.

Quality control standards were run using standard EPA guidelines. A sumary of that data is provided. The acceptance criteria for spikes and duplicates is fifteen percent. It is based on the relative percent deviation of the mean, taking the counting error into account for radiochemical analyses. All QC checks, including duplicates, spikes, and blanks, passed.

It is possible that some Th-232 results may be biased high due to carry over from Th-230 peak on the spectrum. Since Th-232 and Th-228 are generally in equilibrium, the Th-228 results have been included in the report. This analysis delayed the report by one day.

Signed:

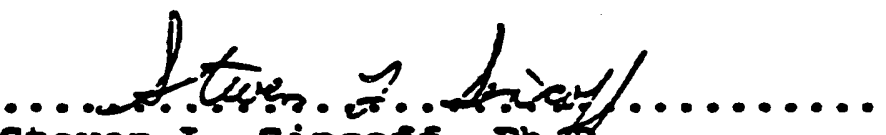

Steven I. Sincolf, Phob.

Director of Operations 
23-Dec-92

Ron Jacobs

IRT-Ferguson

1571 CR 38

Gurnison, CO 81230

AtẼ:

Project: Gurnison

Received: 20-Nov-92 09:30

PO : 3050-511-10004\$059

statis:

Final
Page:
Copy: 3 of

Job: $925567 \mathrm{E}$

\section{8amplo 5ype: 8011}

sample Id

GUN-SS-0524-01-CTF

GUN-SS-0525-02-C.F

GUN-SS-0526-03-CTF

GUN-SS-0527-04-CTF

GUN-5S-0528-05-CTF

GUN-SS-0529-06-CTF

GUN-5S-0530-07-CTF

GUN-SS-0531-08-CTF

GUN-5S-0532-09-CTF

GON-SS-0533-10-CTF

GON-SS-0534-11-CTF

GUN-SS-0535-12-CTF

GUN-5S-0536-13-CTF

GUN-SS-0537-14-CTF

GUN-5S-0538-15-CTE

GUN-5S-0539-16-CTF

GUN-5S-0540-17-CTF

GUN-5S-0541-18-CTF

GUN-5S-0542-19-CTF

GUN-5S-0543-20-CTE

GUN-5S-0544-21-CTF

GUN-5S-0545-22-CTF

GUN-5S-0546-23-CTE

Ra-226 Eszor
Total
Dcila 20

$20 \pm 2$

$1.4 \pm 0.7$

$3.6 \pm 1.0$

$2.2 \pm 0.8$

$3.5 \pm 1.0$

$2.9 \pm 1.0$

$4.0 \pm 1.0$

$1.7 \pm 0.7$

$6.9 \pm 1.3$

$3.5 \pm 1.0$

$2.2 \pm 0.8$

$1.7 \pm 0.7$

$4.2 \pm 1.1$

$3.9 \pm 1.1$

$1.7 \pm 0.7$

$2.6 \pm 0.8$

$3.1 \pm 1.1$

$6.7 \pm 1.3$

$3.4 \pm 1.1$

$7.5 \pm 1.4$

$2.5 \pm 0.9$

$15 \pm 2$

$1.1 \pm 0.6$

\begin{tabular}{ll} 
Th-230 & Error \\
Total & \\
oci $/ 0$ & 20 \\
\hline
\end{tabular}

$95 \pm 4$

$1.3 \pm 0.6$

$150 \pm 10$

$8.7 \pm 1.4$

$180 \pm 10$

$8.4 \pm 1.4$

$130 \pm 10$

$6.3 \pm 1.2$

$350 \pm 10$

$76 \pm 4$

$36 \pm 3$

$6.8 \pm 1.2$

$160 \pm 10$

$160 \pm 10$

$3.8 \pm 0.9$

$47 \pm 3$

$74 \pm 3$

$240 \pm 10$

$140 \pm 10$

$270 \pm 10$

$16 \pm 2$

$660 \pm 10$

$3.9 \pm 0.9$
Th-232 Exor Total ocila 20

$1.7 \pm 0.8$

$1.2 \pm 0.7 \quad$ GKG

$6.9 \div 1.4$

$0.8 \pm 0.6$

$2.0 \pm 0.8$

$1.2 \pm 0.7$

$2.8 \pm 0.9$

$1.1 \pm 0.6$

$6.5 \pm 1.4$

$2.3 \pm 0.8$

$1.5 \pm 0.7$

$1.1 \pm 0.6$

$20 \pm 2$

$3.2 \pm 1.0$

$0.9 \pm 0.6$

$3.5 \pm 1.0$

$1.8 \pm 0.7$

$7.3 \pm 1.3$

$7.3 \pm 1.3$

$16 \pm 2$

$1.2 \pm 0.6$

$14 \pm 2$

$1.2 \pm 0.6$

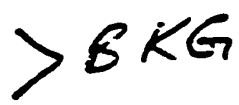

sample Id

GON-55-0524-01-CTF

GUN-S5-0525-02-CIF

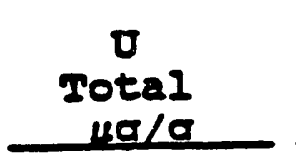

6.7

Ih-228 Exs0r

Total

ocile

20 


\section{BARRINGER LABORATORIES INC.}

15000 W. GTH AVE., SUITE 300 GOLDEN. CO 80401 (303) a77.162 FAX (303) 277.1689

Ror Jacobs

MRR-Ferguson

$1571 \mathrm{CR} 38$

Gunnison, CO 81230

Atたn:

Project: Gunnison

Received: 20-Nov-92 09:30

Job: $925567 \mathrm{E}$

status: Final

po \# : 3050-511-10004 \#059

Page: R-2 Copy: 3 of 3

\begin{tabular}{|c|c|c|c|}
\hline \multirow[b]{2}{*}{ sample Id } & \multicolumn{2}{|c|}{ Bample Type: } & \multirow{2}{*}{$\begin{array}{l}\text { soil } \\
\text { Error } \\
20\end{array}$} \\
\hline & $\begin{array}{c}\mathrm{J} \\
\text { Total } \\
\text { uc/a }\end{array}$ & $\begin{array}{l}\text { Th-228 } \\
\text { Total } \\
\text { pci } / a\end{array}$ & \\
\hline $\begin{array}{l}\text { GUN-SS-0526-03-CTF } \\
\text { GUN-SS-0527-04-CTF } \\
\text { GUN-SS-0528-05-CTF } \\
\text { GUN-SS-0529-06-CTF } \\
\text { GUN-SS-0530-07-CTF } \\
\text { GUN-SS-0531-08-CTF } \\
\text { GUN-SS-0532-09-CTF } \\
\text { GUN-SS-0533-10-CTF } \\
\text { GUN-SS-0534-11-CTF } \\
\text { GUN-SS-0535-12-CTF }\end{array}$ & $\begin{array}{r}15 \\
10 \\
26 \\
36 \\
2.9 \\
51 \\
33 \\
18 \\
6.7 \\
52\end{array}$ & $\begin{array}{l}1.2 \\
1.2 \\
1.8 \\
1.5 \\
1.7 \\
1.6 \\
1.2 \\
0.5 \\
1.3 \\
0.8\end{array}$ & $\begin{array}{l}=0.7 \\
\pm 0.7 \\
=0.8 \\
=0.7 \\
=0.8 \\
=0.8 \\
=0.7 \\
=0.5 \\
=0.7 \\
=0.6\end{array}$ \\
\hline $\begin{array}{l}\text { GUN-SS-0536-13-CTF } \\
\text { GUN-SS-0537-14-CTF } \\
\text { GUN-SS-0538-15-CTF } \\
\text { GUN-SS-0539-16-CTF } \\
\text { GUN-SS-0540-17-CTF } \\
\text { GUN-SS-0541-18-CTF } \\
\text { GUN-SS-0542-19-CIF } \\
\text { GUN-SS-0543-20-CTF } \\
\text { GUN-SS-0544-21-CTF } \\
\text { GUN-SS-0545-22-CTF }\end{array}$ & $\begin{array}{r}20 \\
29 \\
2.4 \\
86 \\
30 \\
32 \\
65 \\
41.0 \\
2.0 \\
36.0\end{array}$ & $\begin{array}{l}0.2 \\
1.8 \\
1.1 \\
1.0 \\
1.2 \\
1.3 \\
1.1 \\
1.4 \\
0.8 \\
1.8\end{array}$ & $\begin{array}{l} \pm 0.4 \\
=0.8 \\
\pm 0.6 \\
=0.6 \\
=0.6 \\
\pm 0.6 \\
=0.6 \\
=0.7 \\
\pm 0.6 \\
\pm 0.7\end{array}$ \\
\hline GUN-SS-0546-23-CTE & 2.0 & 1.0 & \pm 0.6 \\
\hline
\end{tabular}

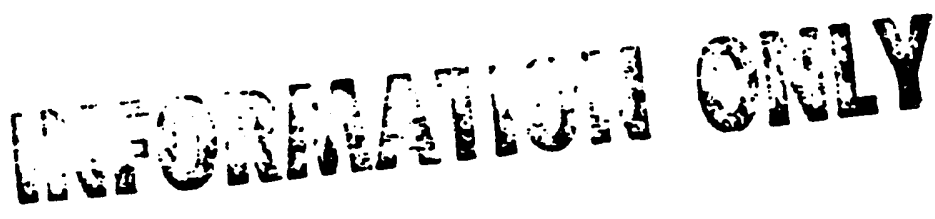




\section{BARRINGER LABORATORIES INC.}

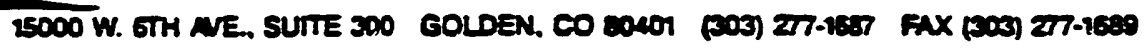

\section{Ron Jacobs}

IRK-Ferguson

23-Dec-92

1571 CR 38

GunRison, CO 81230

Atะa:

Project: Gurrison

Received: 20-Nov-92 09:30

Job: $925567 E$

Status:

Final

\section{Aboreviations:}

\section{Parameters:}

Ra-226

Th-230

Th-232

U

Th-228

\section{Dnits:}

$\mathrm{pCi} / \mathrm{g}$

20

$\mu g / g$
: Radium-226

: Thorium-23C

: Thorium-232

: Uranium

: Thorium-228

\section{PO : 3050-511-10004\$059}

Einal


Ron Jacobs

Bar-Ferguson

$1571 \mathrm{CR} 38$

Gunsison, Co 81230

Atะn:

Project: Gunnison

Job: - $925567 \mathrm{E}$
Received: 20-Nov-92 09:30 PO : : 3050-511-10004
23-Dec-92

Page: $\quad$ Q-1

Copy: 3 of 3

\begin{tabular}{|c|c|c|c|c|c|c|}
\hline \multirow[b]{2}{*}{ Sample Id } & \multicolumn{4}{|c|}{ QUAIITY CONTROL REPORT } & \multirow[b]{2}{*}{$\begin{array}{l}\text { Th-232 } \\
\text { TotaI } \\
\text { occi/a }\end{array}$} & \multirow[b]{2}{*}{$\begin{array}{l}\text { Ex=0r } \\
20\end{array}$} \\
\hline & $\begin{array}{l}\text { Ra-226 } \\
\text { Total } \\
\text { DCi/a }\end{array}$ & $\begin{array}{l}\text { Ez=OS } \\
20\end{array}$ & $\begin{array}{l}\text { Th-230 } \\
\text { Total } \\
\text { oci/a }\end{array}$ & $\begin{array}{l}\text { Error } \\
20\end{array}$ & & \\
\hline $\begin{array}{l}\text { Duplicate } \\
\text { Duplicate } \\
\text { Duplicate t diff. } \\
\text { std (found value) } \\
\text { Std (true value) } \\
\text { Std \& rec. } \\
\text { Blank } \\
\text { Spike \& rec. }\end{array}$ & $\begin{array}{r}9.3 \\
9.4 \\
0.5 \\
104 \\
99 \\
105 \\
0.1 \\
101\end{array}$ & $\begin{array}{l} \pm 1.5 \\
\pm 1.5 \\
\pm 7 \\
\\
\pm 0 .- \\
-\infty\end{array}$ & & $\begin{array}{l} \pm 0.9 \\
\pm 0.9 \\
\pm 6 \\
\pm 0.2 \\
-\end{array}$ & $\begin{array}{r}0 . \\
0 . \\
5 \\
1 \\
1 \\
10 \\
0 . \\
12\end{array}$ & $\begin{array}{l} \pm 0.6 \\
\pm 0.5 \\
\pm 3 \\
\pm 0.2 \\
=--\end{array}$ \\
\hline Sample Id & $\begin{array}{c}U \\
\text { Total } \\
\mu c / c\end{array}$ & $\begin{array}{l}\text { Th- } \\
\text { Tot } \\
\text { oCi }\end{array}$ & $\begin{array}{l}228 \\
21 \\
10\end{array}$ & & & \\
\hline $\begin{array}{l}\text { Duplicate } \\
\text { Duplicate } \\
\text { Duplicate t diff. } \\
\text { std (found value) } \\
\text { std (true value) } \\
\text { std \& rec. } \\
\text { Blank } \\
\text { Spike \& rec. }\end{array}$ & $\begin{array}{r}0 \\
47 \\
49 \\
0\end{array}$ & $\begin{array}{l}32 \\
32 \\
0 \\
6 \\
0 \\
7 \\
0 \\
0\end{array}$ & $\begin{array}{r}1.7 \pm 0 \\
1.0=0 \\
26 \\
20 \pm 3 \\
19 \\
105 \\
0.0 \pm 0 \\
91\end{array}$ & $\frac{8}{6}$ & & \\
\hline
\end{tabular}

Final

QUAIITY CONTROI REPORT 


\section{BARRINGER LABORATORIES INC.}

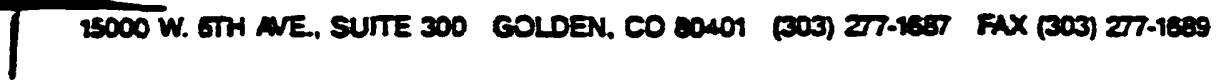

Ron Jacobs

MLR-Ferguson

1571 CR 38

Gunnison, Co 81230

Attת:

Project: Gunnison

Received: 20-Nov-92 09:30 PO : $3050-511-10004 \div 059$

Page: Q-2

Copy: 3 of 3

Iob:

9255675

status:

Final

\section{QUAIITY CONIROI REPORT}

abbreviations:

Parameters:

Ra-226

Th-230

Th-232

U

$T h-228$

: Radium-226

: Thorium-230

: Thorium-232

: Uranium

: Thorium-228

Units:

$\mathrm{pci} / \mathrm{g}$

20

$\mu \mathrm{g} / 9$

: picocuries per gram

: Counting error at the $95 \%$ confidence level, $2 \sigma=$

: micrograms per gram

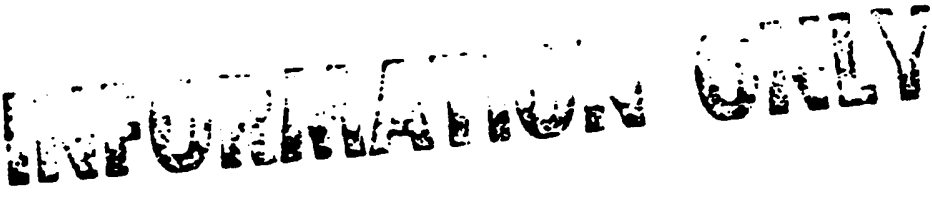




\section{BARRINGER LABORATORIES INC.}

25000 W. GTH ANE. SUTTE 300 GOLDEN, CO 80401 (303) 277.1687 FAX (303) 277-1689

Ron Jacobs

MIR-Ferguson

1571 CR 38

Gunnison, co 81230

23-Dec-92

Page:

Copy: 3 of 3

Attn:

Project: Gunnison

Received: 20-Nov-92 09:30

PO : 3050-511-10004

Job: 225567E

QUALITY CONTROL REPORT

status:

Fina?

QDALIMY CONHROL DATA SHEE!

Received by: jdz

Via: UPS

Sample Container Type: 500ml mtl can

Sample Type: Soil

Preservative When Received: none

Acditional Lab Preparation: 100 mesh

\begin{tabular}{|c|c|c|c|c|c|}
\hline Parameter & Method & DII & $\begin{array}{l}\text { Prese } \\
\text { vativ }\end{array}$ & - Analyst & $\begin{array}{c}\text { Date (s) of } \\
\text { Analysis }\end{array}$ \\
\hline $\begin{array}{l}R a-226 \\
T b-230 \\
T h-232 \\
U \\
T h-228\end{array}$ & $\begin{array}{l}\text { SM-705 } \\
\text { USAEC } \\
\text { USAEC } \\
\text { ASTM D2907 } \\
\text { USAEC }\end{array}$ & $\begin{array}{ll}0.3 & \mathrm{pCi} / \mathrm{g} \\
0.4 & \mathrm{pci} / \mathrm{g} \\
0.4 & \mathrm{pCi} / \mathrm{g} \\
0.3 & \mu \mathrm{g} / \mathrm{g} \\
0.4 \mathrm{pCi} / \mathrm{g}\end{array}$ & $\begin{array}{l}\text { none } \\
\text { none } \\
\text { none } \\
\text { none } \\
\text { none }\end{array}$ & $\begin{array}{l}\text { Pingilley } \\
\text { ortiz } \\
\text { ortiz } \\
\text { Rnox } \\
\text { ortiz }\end{array}$ & $\begin{array}{l}12 / 15-12 / 17 \\
12 / 9-12 / 17 \\
12 / 9-12 / 17 \\
12 / 9-12 / 8 \\
12 / 9-12 / 21\end{array}$ \\
\hline
\end{tabular}

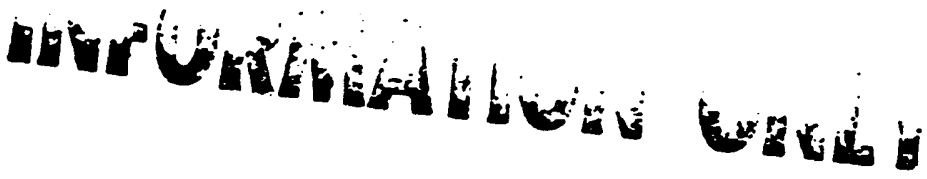




\section{BARRINGER LABORATORIES INC.}

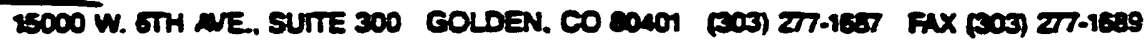

Ron Jacobs

MIR-Ferguson

1571 CR 38

Gunnison, CO 81230

23-Dec-92

Page:

Copy: 3 of 3

Attn:

Project: Gunsison

Received: 20-Nov-92 09:30 PO : 3050-511-10004\$059

Job: $\quad 225567 \varepsilon$

status:

Final

QDAIITY CONTROI REPORT

Signed:
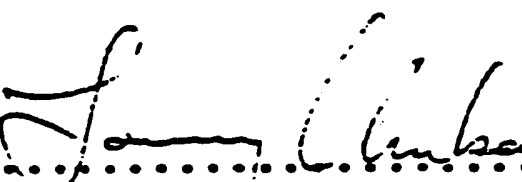

$\operatorname{lom}_{0 . . .0}$

Radiochemical faboratory Manager

Signed:

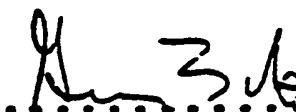

Quality Assurance Department

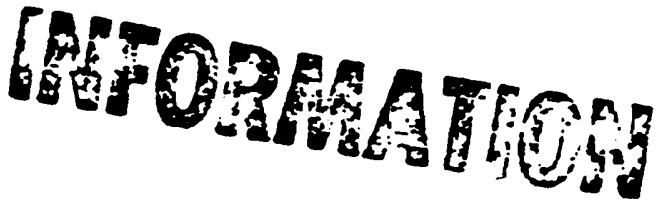


3. MK:FERUSON COMPANY

\section{LABORATORY SERVICES AUTHORIZATION FORM} (CONTINUAT:ON PAGE)
ז

CWM Federal Environmentah Sorviceschifc.

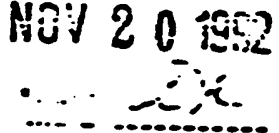

Page 20 of 2 $305 a-511-10004 / 059$ P.O. Number/Reaues: Number

I.D. NO

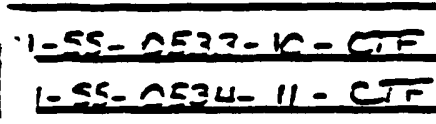
V-SS-ASEE-D-CEF

L-SS-ASZi. TA-CIF

1 - 55-0537- $11-C T F$

V-O-0538-15-CTE

$-35-C 520-112-C T E$

-55-C540-17-CT

$1.55-0541-18-C=$

$v$-ST-OEUE-10-CTF

$N-S-C D^{2}-2 C-C_{1}=$

' $N$ - SS-CSNH-21-CTF

1 - SS- CEUE-IE-CTE

$a l-65-A=d h-23-C$ if

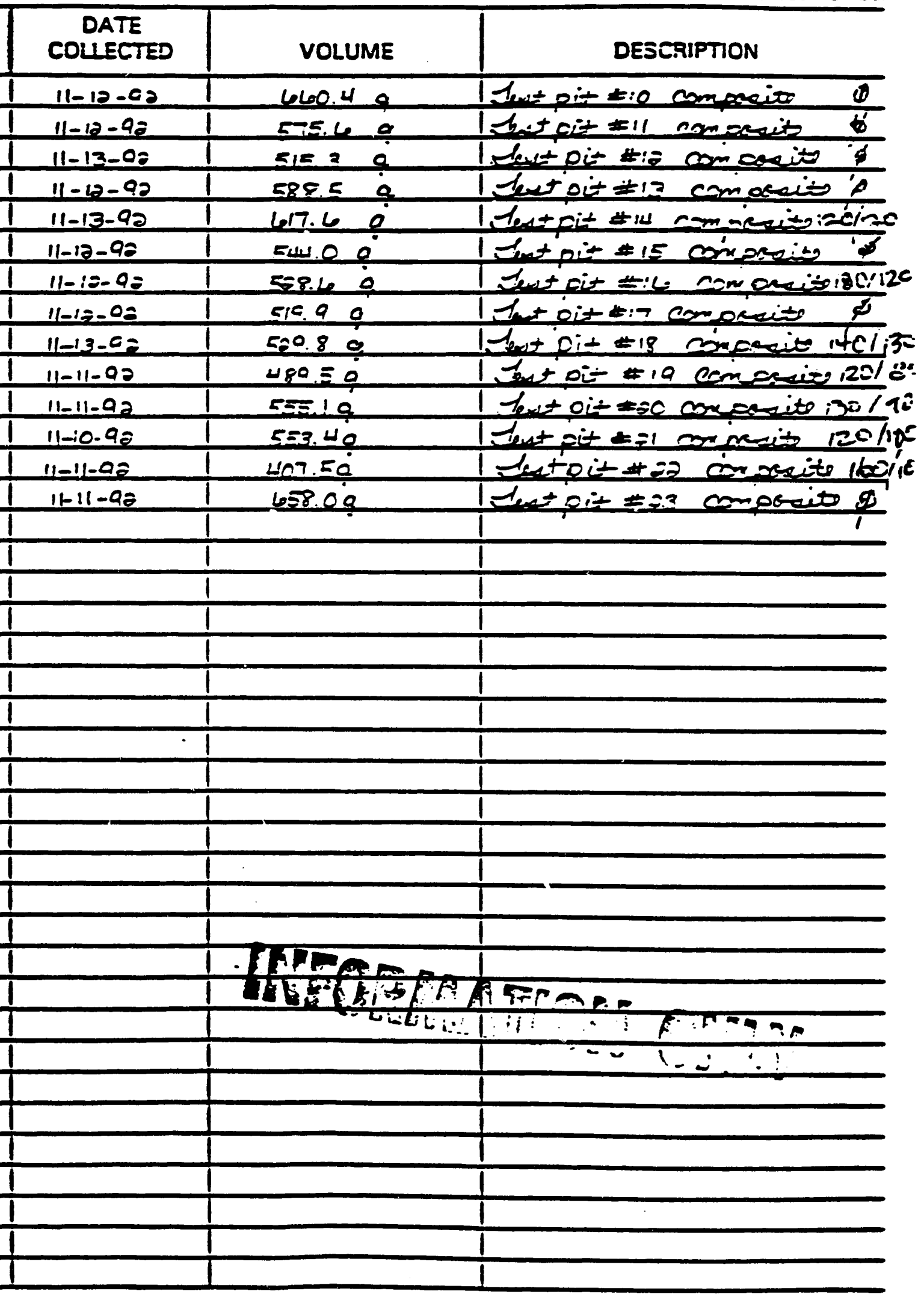

F2-AP-006-4 (continution) 
ON-SITE LABORATORY RADIOLOGICAL

MEASUREMENTS COBBLY SUBSOIL INVESTIGATION NOVEMBER 1992 
产

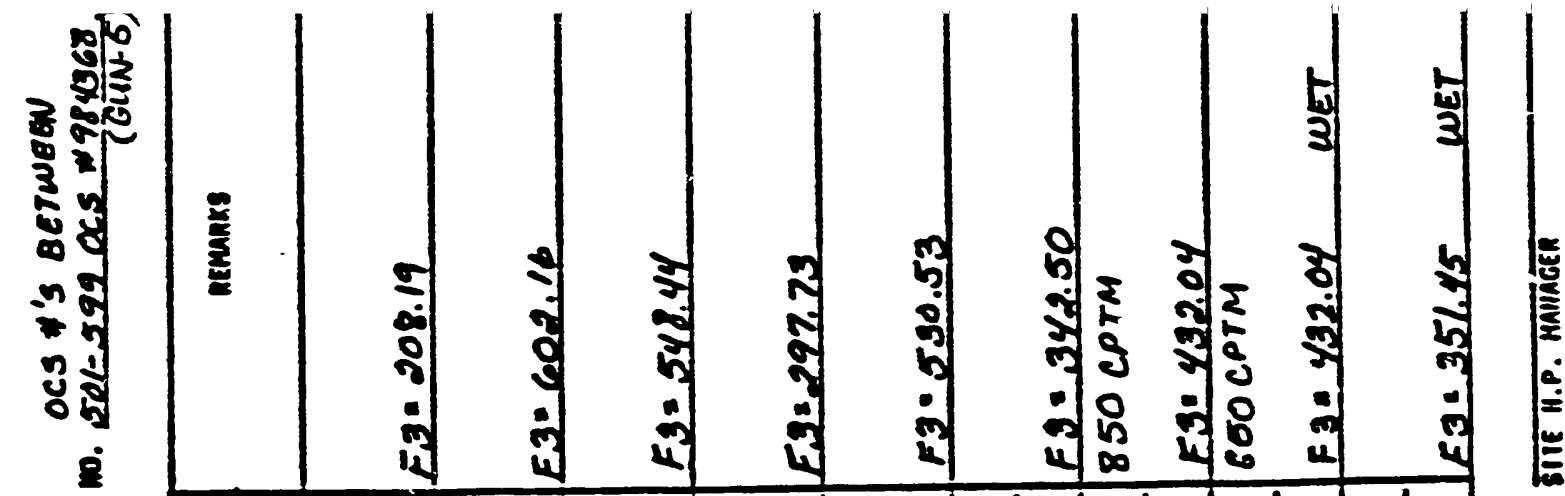

产

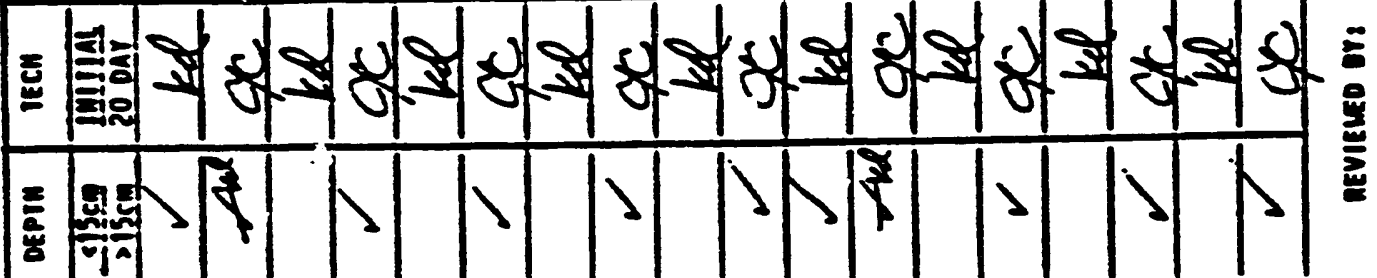

E.

管

$\stackrel{5}{\stackrel{2}{*}}$

$\frac{2}{2}$

$\left(-\frac{2}{5}\right.$

r.:-

a.

(6)

중

F.

$\theta$

ㄷ.

8 해해

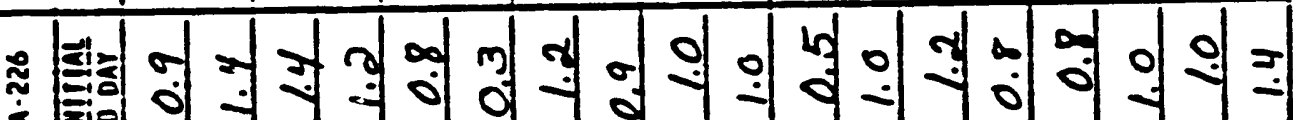

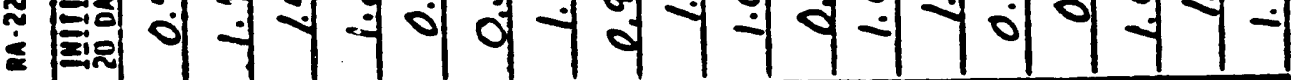

क जि

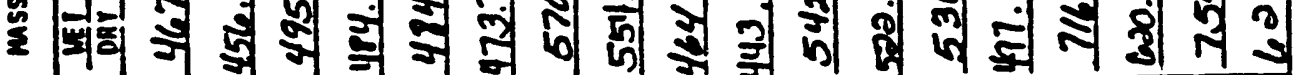

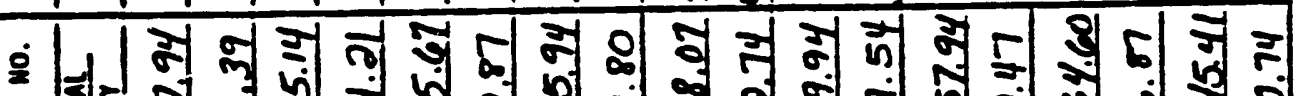

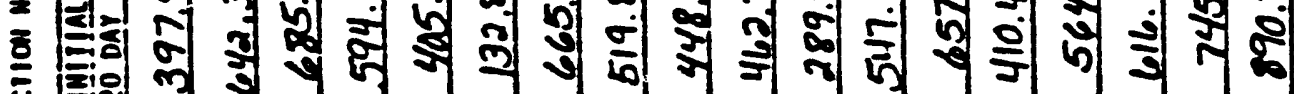

绶

in

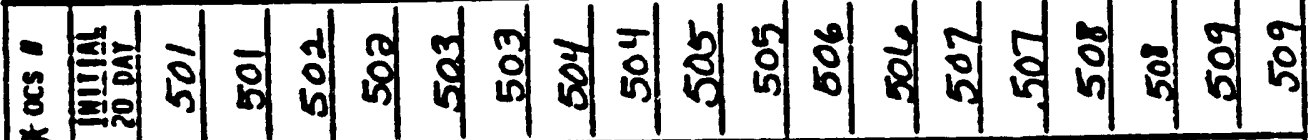

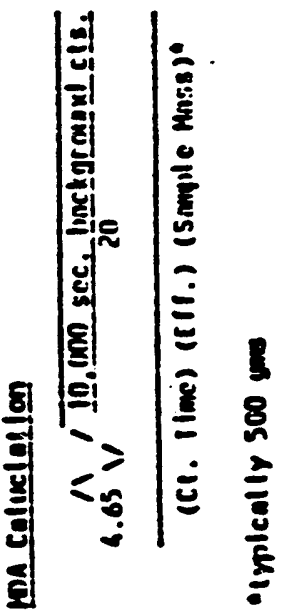

in

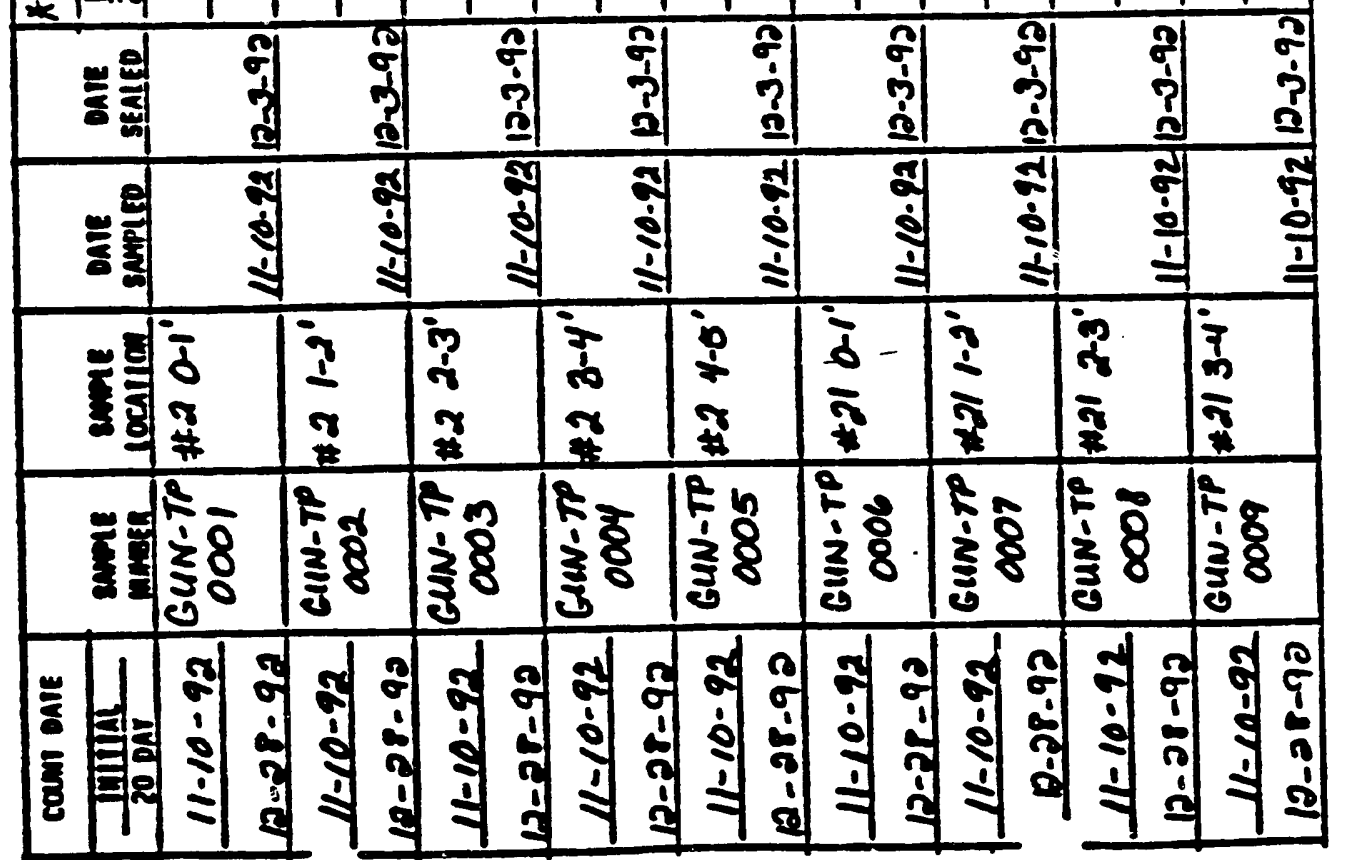

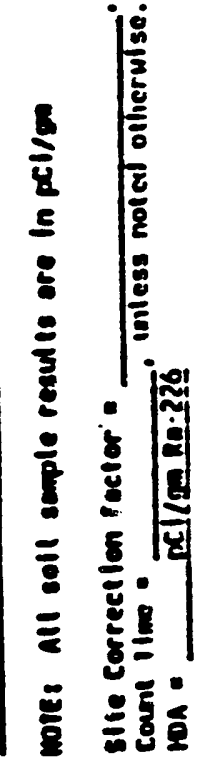


IIe mane Cunnison

ire anea $N / A$
PAnm

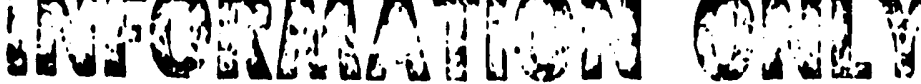

oCs Ht's oenveen sol-1 ocs senial No. 599 oCs 4984368 (4w

\begin{tabular}{|c|c|c|c|c|c|c|c|c|c|c|c|c|c|c|}
\hline calli oAIE & \multirow{4}{*}{ 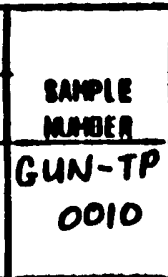 } & \multirow[b]{2}{*}{$\begin{array}{l}\text { smper } \\
\text { lochllos }\end{array}$} & \multirow[b]{2}{*}{$\begin{array}{l}\text { OAlE } \\
\text { SAMPIED }\end{array}$} & \multirow[b]{2}{*}{$\begin{array}{l}\text { DAIE } \\
\text { SEAIFD }\end{array}$} & \multirow{2}{*}{ 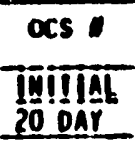 } & \multirow{2}{*}{ 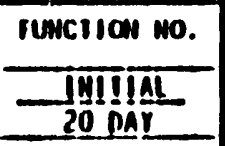 } & \multirow{2}{*}{$\begin{array}{l}\text { Mass } \\
\text { MEL } \\
D R Y \\
\end{array}$} & \multirow{2}{*}{ 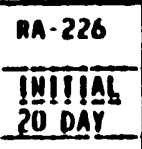 } & \multirow[b]{2}{*}{$\begin{array}{c}\alpha c \\
\text { SARPIE } \\
\end{array}$} & \multicolumn{2}{|c|}{ 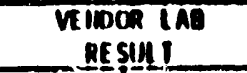 } & \multirow{2}{*}{ 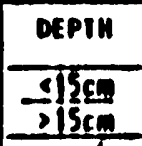 } & \multirow{2}{*}{ 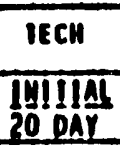 } & \multirow[t]{2}{*}{ aemanks } \\
\hline$\frac{\text { I.IIII }}{200 \text { ar }}$ & & & & & & & & & & Re. 2266 & ih-230 & & & \\
\hline $11-10-92$ & & $\$ 230.1^{\circ}$ & & & 510 & 437.40 & 560.0 & 0.8 & & & & & & \\
\hline$(2-2 p-92$ & & & $11-10.92$ & $0-3-92$ & 510 & 399.54 & 506.4 & 0.8 & & & & & & $E 3 \cdot 485.76$ \\
\hline $11-10-92$ & $\begin{array}{c}\text { GUN-TP } \\
\text { Doll }\end{array}$ & $1+231-2^{\prime}$ & & & 511 & 245.14 & 552.8 & 0.4 & & & & & & \\
\hline $12-28.92$ & & & $11-10 \cdot 92$ & $0-3-90$ & 511 & 621.14 & $511_{1} \cdot 5$ & 1.2 & & & & & & $E 3.602 .16$ \\
\hline $11-10-92$ & GUN-TP & th.23 $2.3^{\prime}$ & & & 512 & 381.14 & 633.2 & 0.6 & & & & & & \\
\hline $12-28-92$ & & & $11-10-92$ & $10-3-92$ & 512 & 254.20 & 547 & 0.5 & & & & & & $E 3.557 .39$ \\
\hline $11-10-92$ & GUN-TP & $4238-4^{\circ}$ & & & $5 / 3$ & 123.24 & 795.5 & 0.2 & & & & & & \\
\hline $12-28-92$ & & & $\mid 11-10$ & $0-3-92$ & 513 & 929.41 & 683 & 0.8 & & & & & & $E 32763.33$ \\
\hline $11-11-92$ & GUN-TP & 4190.19 & & & 601 & 1374.47 & 498.6 & -2.8 & & & & & & \\
\hline $12-21.92$ & 0014 & & $11-10-92$ & $0.3-92$ & 514 & 1721.68 & 472.1 & 4.1 & & & & & & $E 3: 335.54$ \\
\hline $11-11-92$ & GUN-TP & $\# 191-21$ & & & 502 & 1938.21 & 504.1 & 3.8 & & & & & & \\
\hline $12-28-92$ & 0015 & & $11-10 \cdot 92$ & $10.3-92$ & 515 & 2353.41 & 479.3 & 4. 2 & & & & & 4 & $E_{3}=405.17$ \\
\hline 11.11 .92 & GLIN-TP & $102-3^{\circ}$ & & & 503 & 1407.81 & 519.1 & 2.1 & & & & & & \\
\hline D-28-92 & & & $\mid 1-10-92$ & $10.3-92$ & 516 & 1695.01 & 491.7 & 3.4 & & & & & & $F 3=393.54$ \\
\hline $11-11-92$ & GuN-1P & $\# P 3-4^{\circ}$ & & & 504 & 730.71 & 494.1 & 1.5 & & & & & & \\
\hline $12-29-92$ & & & $11-10 \cdot 92$ & $\theta_{x} 2-92$ & 501 & 1037.67 & 446.9 & 2.3 & & & & & & E3-2kl.91 \\
\hline $11-11-92$ & $\begin{array}{c}\text { GUN-TP } \\
\text { OOII }\end{array}$ & $+194-5^{\prime}$ & & & 506 & 430.07 & 503.4 & 1.8 & & & & & & \\
\hline $12-29-92$ & 0010 & & $\mid 11-10-92$ & $10.3-92$ & 502 & 1011.08 .61 & 405.1 & 3.6 & & & & & $4 C$ & LE3 $=217.14$ \\
\hline
\end{tabular}

MEVIELED OY:

ma colucl ollon

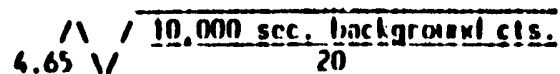

(Cl. Ilme) (Ell.) (Simple Hans)"

- inpicaller 300 goms 


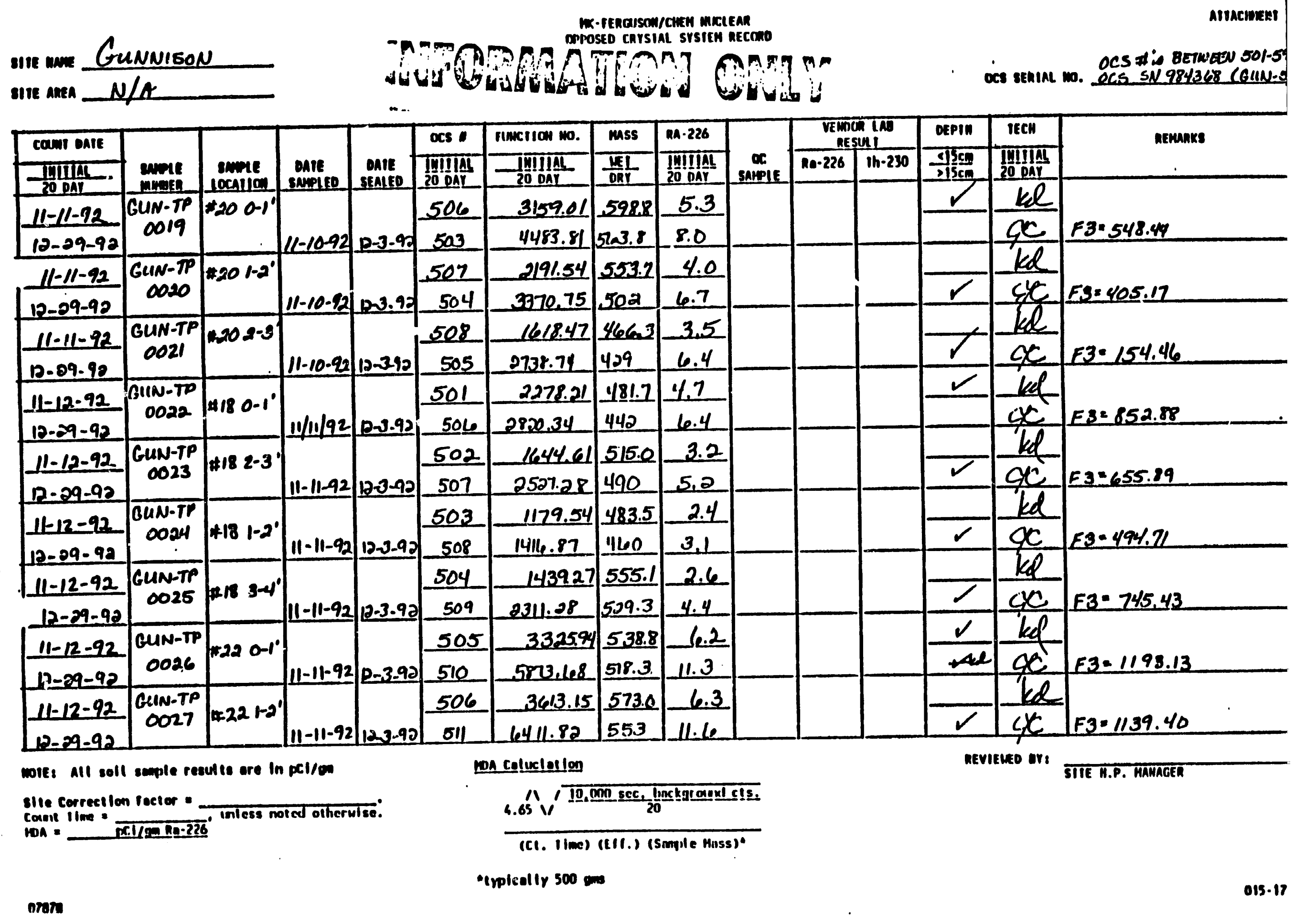



in $\operatorname{men} \frac{\text { Cunnison }}{N / A}$

counl oal

TIIIL

200AT - man

$11-12-92$ GuN-TP

$\frac{11-12-92}{1-29-92}$

$\frac{1-29-92}{\pi-12-92}$ GUN-TP

Do3s

$11-12-92$ GUN-TP

\begin{tabular}{l|l}
\hline $12-12-92$ & 0039 \\
\hline 11 CuN-TP
\end{tabular}

$11-12-92 \mid \begin{gathered}\text { Cun-T } \\ \text { DO40 }\end{gathered}$

$11-12-92$

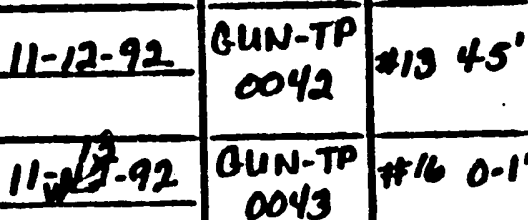

$11-13-92 \overbrace{\substack{\text { QuN-TP } \\ \text { 0044 }}}^{1-21}$

11-13-92 GUN-TP 0045

OrEs All soll semple results ore in pacl/on

I1e Correction lector -

ount lime - inless noted otherulse.

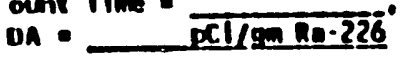

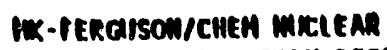

oproseo carsial srsich accono

\begin{tabular}{|c|c|c|c|c|c|}
\hline & & focs " & runciloul no. & miss & nA. 226 \\
\hline $\begin{array}{l}\text { onle } \\
\text { sumpise }\end{array}$ & $\begin{array}{c}\text { OAfIt } \\
\text { SEAlEe }\end{array}$ & $\begin{array}{l}7911111 \\
20 \text { oar } \\
\end{array}$ & $\frac{111111 \mathrm{AI}}{20 \mathrm{OAI}}$ & $\frac{\mathrm{M} L}{\text { onr }}$ & $\frac{10 ! 111}{200 A 7}$ \\
\hline \multirow[b]{2}{*}{$11-12-92$} & \multirow{3}{*}{$\mid 0-3-2$} & 516 & 1348.62 & 1676.6 & 2.0 \\
\hline & & 521 & 1552.87 & 577.1 & 2.7 \\
\hline \multirow[b]{2}{*}{$11-12-92$} & & 517 & 1089.41 & 491.5 & 2.2 \\
\hline & & & & & \\
\hline \multirow[b]{2}{*}{$11-12-92$} & & 518 & 1291.27 & 462.3 & 2.8 \\
\hline & & & & & \\
\hline \multirow[b]{2}{*}{$11-12-92$} & & 519 & 1305.14 & 529.1 & 2.5 \\
\hline & & & & & \\
\hline \multirow{4}{*}{$11-12-92$} & & 520 & 452.07 & 517.7 & 0.9 \\
\hline & & & & & \\
\hline & & 521 & 938.47 & 653.0 & 1.4 \\
\hline & & & & & \\
\hline \multirow{4}{*}{$1-10=92$} & & 501 & 1582.47 & 4545 & 3.5 \\
\hline & & & $0,1<$ al & 15170 & 10 \\
\hline & & 602 & 790.41 & $\frac{510.0}{1}$ & 1.0 \\
\hline & & & & & \\
\hline $11-12-92$ & & 603 & 530.21 & 479.5 & 1.1 \\
\hline
\end{tabular}

En coluctolion

(C). Ilma) (Eil.) (Snmile Mnss)"

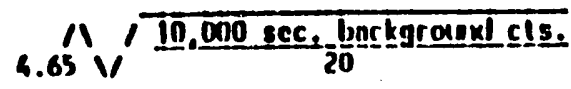

Arracment

ocs * D BeTwELN GOI-E

ocs senial mo. OCs sa 9 R4368 (BLN-;

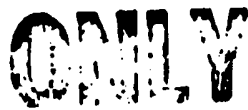

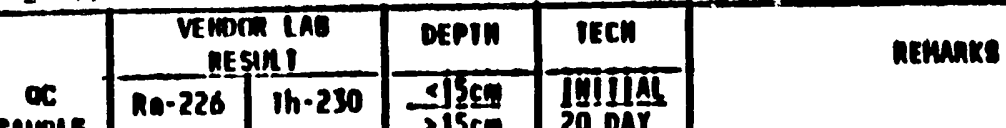

riscm

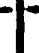

$\left.\frac{V}{V}\right|_{E 3.396 .22} ^{E 32.252 .96}$

$F 3-208,19$

$E 3 \cdot 378.31$

$E 3.575 .80$

$F 3 \cdot 378.31$

$F 3=423.08$

$F 3=351.45$

$F 3=763.33$

MEVIELED AY

SIIE II.P. HAHAGER 
$r$

I num Gunnlison

s anea N N/A areaciurent?

MK - IEnGISOW/r.MEH MELIEAR

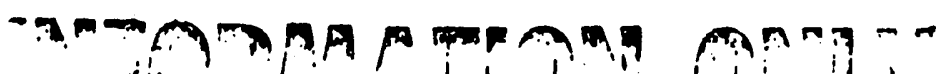

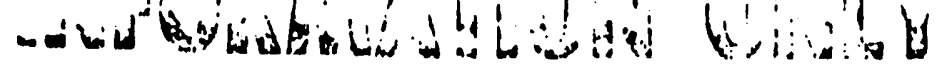

tocs \#'s BeTween Gorocs senial mo. 599 ecs sng8436

ca्dN:

\begin{tabular}{|c|c|c|c|c|}
\hline LaWI DAIE & & & & \\
\hline$\frac{\text { IIIIIAL }}{200 A 1}$ & 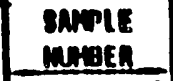 & $\begin{array}{c}\text { sarre } \\
\text { Locallon }\end{array}$ & $\begin{array}{l}\text { DAIE } \\
\text { SAMPLED }\end{array}$ & $\begin{array}{l}\text { OAIE } \\
\text { SEALED }\end{array}$ \\
\hline $11-13-92$ & $\begin{array}{c}\text { GUN-TP } \\
\text { O046 }\end{array}$ & $2+163-4$ & $11-12.92$ & \\
\hline$\pi-13.92$ & $\begin{array}{r}\text { GIIN-TP } \\
0047\end{array}$ & $\$ 16 \quad 4-5^{\circ}$ & $11-12-92$ & \\
\hline$\frac{11-13-92}{12-29-92}$ & $\begin{array}{c}\text { GUN-TP } \\
\text { Do4f }\end{array}$ & $=170.1$ & $11-12-92$ & $12-4-92$ \\
\hline$\frac{11-13-92}{1-29-92}$ & $\begin{array}{c}\text { GUN-TT } \\
0049\end{array}$ & $+17\left(-2^{\prime}\right.$ & $11-12-92$ & $\mid a-4-90$ \\
\hline$\frac{11-13 \cdot 92}{1-29-92}$ & $\begin{array}{c}\text { GUN-TP } \\
0050\end{array}$ & $10172-3^{\circ}$ & $11-12-92$ & $0-4-92$ \\
\hline$\frac{11-13-92}{12-29-92}$ & $\begin{array}{c}\text { GuN-TP } \\
0051\end{array}$ & $\neq 1845^{\circ}$ & $11-12-9 ?$ & $D-4-98$ \\
\hline $11-13-92$ & $\underset{0052}{\text { GUN-TP }}$ & $\begin{array}{lll}1 & 0 & 0\end{array}$ & $11-12-92$ & \\
\hline $11-13-92$ & $\begin{array}{c}\text { GUN-TP } \\
0053\end{array}$ & $1+1-2^{\prime}$ & $11-12-92$ & \\
\hline$\frac{11-13-92}{12-29-90}$ & $\begin{array}{c}\text { CUN-TP } \\
0054\end{array}$ & \#3 $0-1^{\prime}$ & $11-12-9 t$ & \\
\hline
\end{tabular}

IE, All soll somple results ore In pxl/on

1. Correctlon factor :

nelt Ilme.

in: xilgana-226

\begin{tabular}{|c|c|c|c|c|}
\hline Jocs & rincrioul no. & Muss & $R A-226$ & \\
\hline 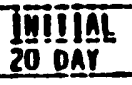 & - MIIIIAL & $\frac{\text { RLL }}{\text { DPR }}$ & $\begin{array}{l}\text { TMIIIAI } \\
20 \text { Dar }\end{array}$ & $\begin{array}{c}\text { OC } \\
\text { SARPIE }\end{array}$ \\
\hline 504 & 538.71 & 453,3 & 1.2 & \\
\hline 505 & 529.41 & 473.2 & 1.1 & \\
\hline 506 & 722.42 & 438.9 & 1.6 & \\
\hline 582 & 934.74 & 405.7 & 2.3 & \\
\hline 507 & 1014.47 & 525.2 & 1.9 & \\
\hline 523 & 1034,21 & 447 & 2.3 & \\
\hline 508 & 890.21 & 527.5 & 1.2 & \\
\hline 524 & 1477.14 & 432.7 & 3.4 & \\
\hline 509 & 729.14 & 470.0 & 1.6 & \\
\hline 605 & 1495.54 & $411 . .3$ & 3.16 & \\
\hline 510 & 7068.89 & 443.2 & 15.9 & \\
\hline 511 & 4727.28 & 630.8 & 7.5 & \\
\hline 512 & 309.40 & 429.9 & 0.7 & \\
\hline 526 & 3.11 .14 & 409.5 & 0.8 & \\
\hline
\end{tabular}

ena coluclesion

4 10.000 sec. Inckground cts.

(CL. I Ime) (Ell.) (Simple Mnss)"

- inically 500 gnos

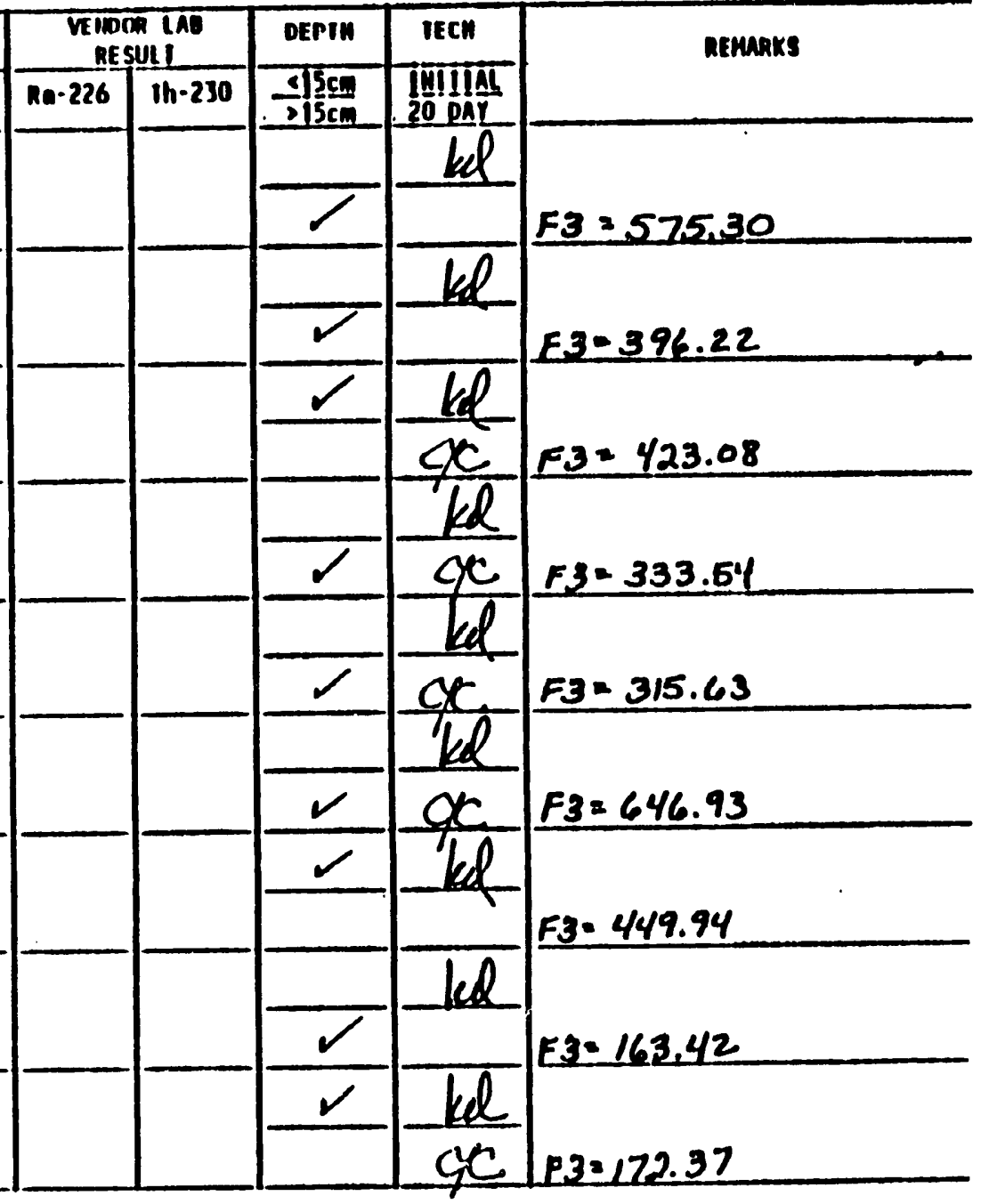

REVIELED UY: 
$=\frac{\text { GunNison }}{N / k}$

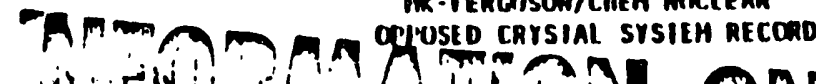

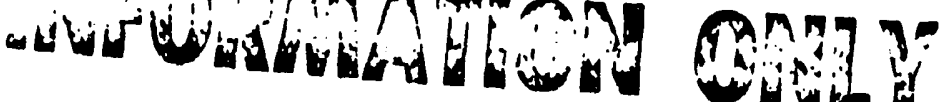

HOCs *'s GCTwUton 60i-

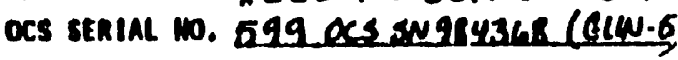

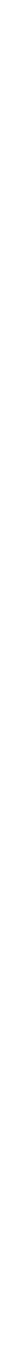

. All soll somple results ore in pcilom

Correctlon inctor

11 Ilwa : inless moled olherwlse.

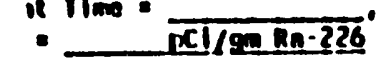

Ha coluclation

$\int_{4.65}^{\prime} / 10.000 \mathrm{sec}, \frac{\text { bon! kgronkl cls. }}{20}$

(Cl. IIme) (EII.) (Somple Hinss)"
REVIECED UY

STIE M.P. MAMAGER 
II mon GunNison

ie amen $N / t$

\begin{tabular}{|c|c|c|c|c|c|c|c|c|c|}
\hline coWI DAIE & & & & & focs: & BnMCIIOH MO. & Mass & $R A-226$ & \\
\hline$\frac{111110}{20} \frac{1}{p A r}$ & 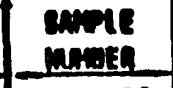 & $\begin{array}{l}\text { same } \\
\text { locallon }\end{array}$ & $\begin{array}{l}\text { DAle } \\
\text { seneplete } \\
\end{array}$ & $\begin{array}{l}\text { OAIR } \\
\text { SEALED }\end{array}$ & Ty!nII & - 20 Dal & $\frac{\text { NEL }}{\text { DRY }}$ & III!! & $\begin{array}{c}\text { oc } \\
\text { sarpie }\end{array}$ \\
\hline$\frac{11-16-92}{p-30-90}$ & $\begin{array}{r}\text { GUN-TP } \\
0073\end{array}$ & $\$ 72-3^{\prime}$ & 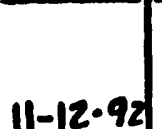 & & $\frac{5 / 2}{504}$ & $\frac{950.42}{1183.01}$ & $\frac{577.7}{551.2}$ & $\frac{1.6}{2.1}$ & \\
\hline$\pi-16-92$ & $\begin{array}{r}\text { CUN-TP } \\
0074\end{array}$ & $173-4^{\circ}$ & & & $5 / 3$ & 837.14 & $51: 0.7$ & 1.5 & \\
\hline $12-30-92$ & & & $11-1$ & 12-4-90 & 605 & 796.87 & 526.1 & 1.5 & \\
\hline $11-16 \cdot 92$ & $\begin{array}{c}\text { GUN-TP } \\
0075\end{array}$ & 147 4-5 & & & 514 & 713.41 & 499.5 & 1.4 & \\
\hline $12-30-90$ & & & $11-1$ & $12-4.92$ & 506 & 916.61 & 472.2 & 1.9 & \\
\hline $11-16-92$ & GLN-TP & $=80-1^{\prime}$ & & & $5 / 5$ & 421.67 & 400.1 & 1.1 & \\
\hline $2-30-92$ & & & $11-1$ & 98 & 507 & 505.94 & 379.7 & 1.3 & \\
\hline $11-16-92$ & GUN-TP & $1 \neq 8 \quad 1-2^{\circ}$ & & & 516 & 620.61 & 409.2 & 1.5 & \\
\hline $12-30-92$ & & & & $0-4-92$ & 508 & 446.47 & 388.3 & 1.1 & \\
\hline $11-16-92$ & GIIN-TP & In 2-3 & & & 517 & 477.40 & 426.3 & 1.1 & \\
\hline $12-3$ & & & 101 & $2-4-92$ & 509 & 178.73 & 408.8 & 0.4 & \\
\hline $16-92$ & $\begin{array}{c}G u N-T P \\
0079\end{array}$ & $=8 \quad 3-4^{\prime}$ & & & 518 & 517.41 & 446.0 & 1.2 & \\
\hline & & & & $10.4-90$ & 510 & 245.40 & 425.3 & 0.6 & \\
\hline $11-16-92$ & $\begin{array}{c}\text { GUN-TP } \\
\text { D080 }\end{array}$ & All $0-1^{0}$ & & & 519 & 61.67 & 546.0 & 0.1 & \\
\hline & & & $11-12-92$ & & & & & & \\
\hline $11-16-92$ & 0081 & $p+11-2^{0}$ & & & 520 & 355.00 & 516.0 & 0.1 & \\
\hline
\end{tabular}

DiEs All soll semple results ore in pel/on

lie correction fector.

oull ilue : Inless noted otherwlse.

DA.

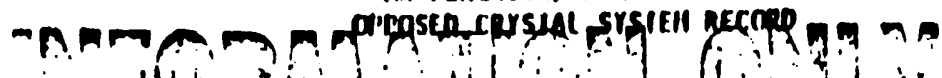

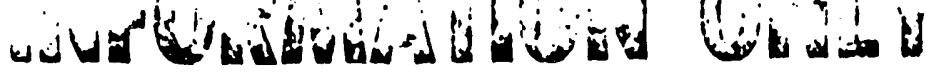

* OCs F's Benuben bo. ocs senial mo. G94 DCS SN 984368 (GW

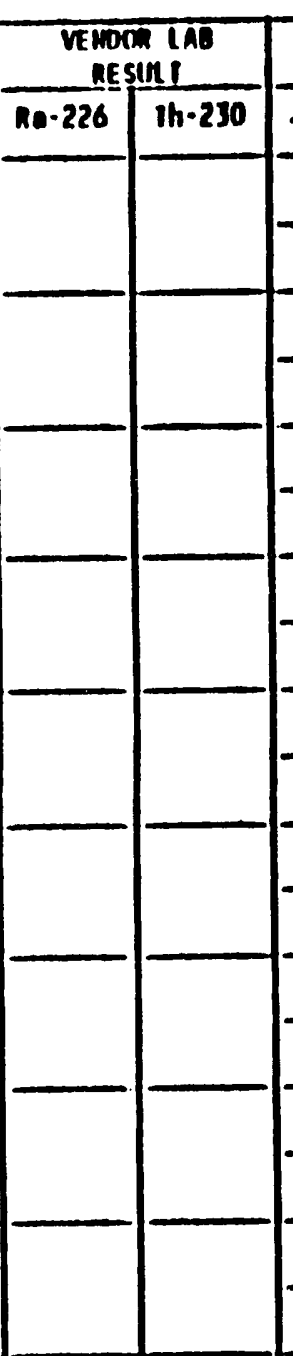

ton coliecletlon

$$
4.65 \mathrm{~V} \frac{10.000 \text { sec, bonkgroind cls }}{20}
$$

(C.1. IIme) (Ell.) (Snmple Mnssian. 
Cunnison

men $N / A$

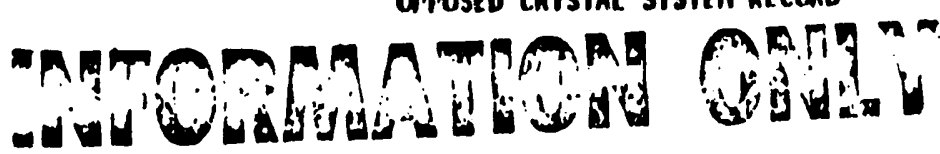

NOCS A's BGTween 601-

OCS SERIAL WO. G99 QCS SN Q843SA (AUN-5

\begin{tabular}{|c|c|c|c|c|c|c|c|c|c|}
\hline MI DAIE & \multirow[b]{2}{*}{$\begin{array}{l}\text { SAMPLE } \\
\text { MUPOER } \\
\end{array}$} & \multirow[b]{2}{*}{$\begin{array}{c}\text { Samik } \\
\text { LOCallon } \\
\end{array}$} & \multirow[b]{2}{*}{$\begin{array}{l}\text { DAIE } \\
\text { SAMPLED }\end{array}$} & \multirow[b]{2}{*}{$\begin{array}{c}\text { OAIE } \\
\text { SEALED }\end{array}$} & \multirow{2}{*}{ 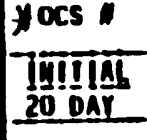 } & \multirow{2}{*}{ 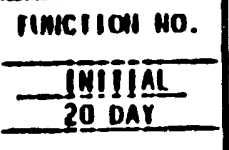 } & \multirow{2}{*}{ MASS } & \multirow{2}{*}{$\begin{array}{l}\text { RA-226 } \\
\text { iu!!IIII } \\
20 \text { DAY }\end{array}$} & \multirow[b]{2}{*}{$\begin{array}{c}\text { oc } \\
\text { saimie }\end{array}$} \\
\hline$\prod_{0,1}$ & & & & & & & & & \\
\hline$-116-92$ & $\begin{array}{c}\text { cun-TP } \\
0082\end{array}$ & $\$ 112.3^{\prime}$ & $11-12-$ & & 521 & 237.14 & 521.4 & 0.6 & \\
\hline$\sqrt{6-42}$ & $\begin{array}{c}\text { CUN-TP } \\
0083\end{array}$ & ifll $3-4^{\circ}$ & & & 522 & 300.02 & 5445 & 0.6 & \\
\hline$-16-92$ & $\begin{array}{c}\text { GLN-TP } \\
0084\end{array}$ & $\# 114-5^{\circ}$ & & & 523 & 720.01 & 678.1 & 1.1 & \\
\hline $16-92$ & $\begin{array}{c}\text { GUN-TP } \\
0085\end{array}$ & & & & 524 & 119.80 & 500.5 & 0.2 & \\
\hline $30-92$ & & & $11-12-92$ & $10-4-92$ & 511 & 466.74 & 442 & 1.1 & \\
\hline$-16-92$ & $\begin{array}{c}\text { GUN-TP } \\
0086\end{array}$ & $1223^{\prime}$ & & & 525 & 450.74 & 506.5 & 0.9 & \\
\hline 30.0 & & & $11-12-92$ & 10.4 .92 & 512 & 479.50 & 465 & 1.0 & \\
\hline & $\left.\right|_{0087}$ & $15523^{\circ}$ & & & 526 & 221.40 & .367 .1 & 0.9 & \\
\hline $30-92$ & & & $11-12-92$ & $12-4-92$ & 513 & 1049.41 & 313.7 & 3.3 & \\
\hline$-16-92$ & $\begin{array}{c}\text { GUN-TP } \\
0088\end{array}$ & $153-x^{\circ}$ & & & 527 & 573.67 & 531.1 & 1.1 & \\
\hline 12-30-9: & & & $11-12-92$ & $13-4-92$ & 514 & 1311.01 & ycer.te & 2.9 & \\
\hline$=11-92$ & GIIN-TP & t. $121-2^{\circ}$ & & & 528 & 441.94 & 448.3 & 0.9 & \\
\hline$-30-92$ & & & $11-12.92$ & $0-4-92$ & 515 & 3116.60 & 424.4 & 0.7 & \\
\hline & GUN-TP & $-123-4^{\prime}$ & & & 529 & 458.20 & 519.4 & 0.9 & \\
\hline$=30$. & & Tro & $11-12-92$ & $12-4-92$ & 516 & 445.40 & 487.1 & 0.9 & \\
\hline
\end{tabular}

\& All soll somple results ore in pelion

Correction iector =

Itme: Iilless noted otherwlse.

- PCI/2ana-226
LA Coluclation

$$
{ }_{4.65}^{\prime} V^{\prime} \frac{10.000 \text { sec. Gackgroind cls. }}{20}
$$

(Cl. Ilime) (EII.) (Smple Hn:s)"

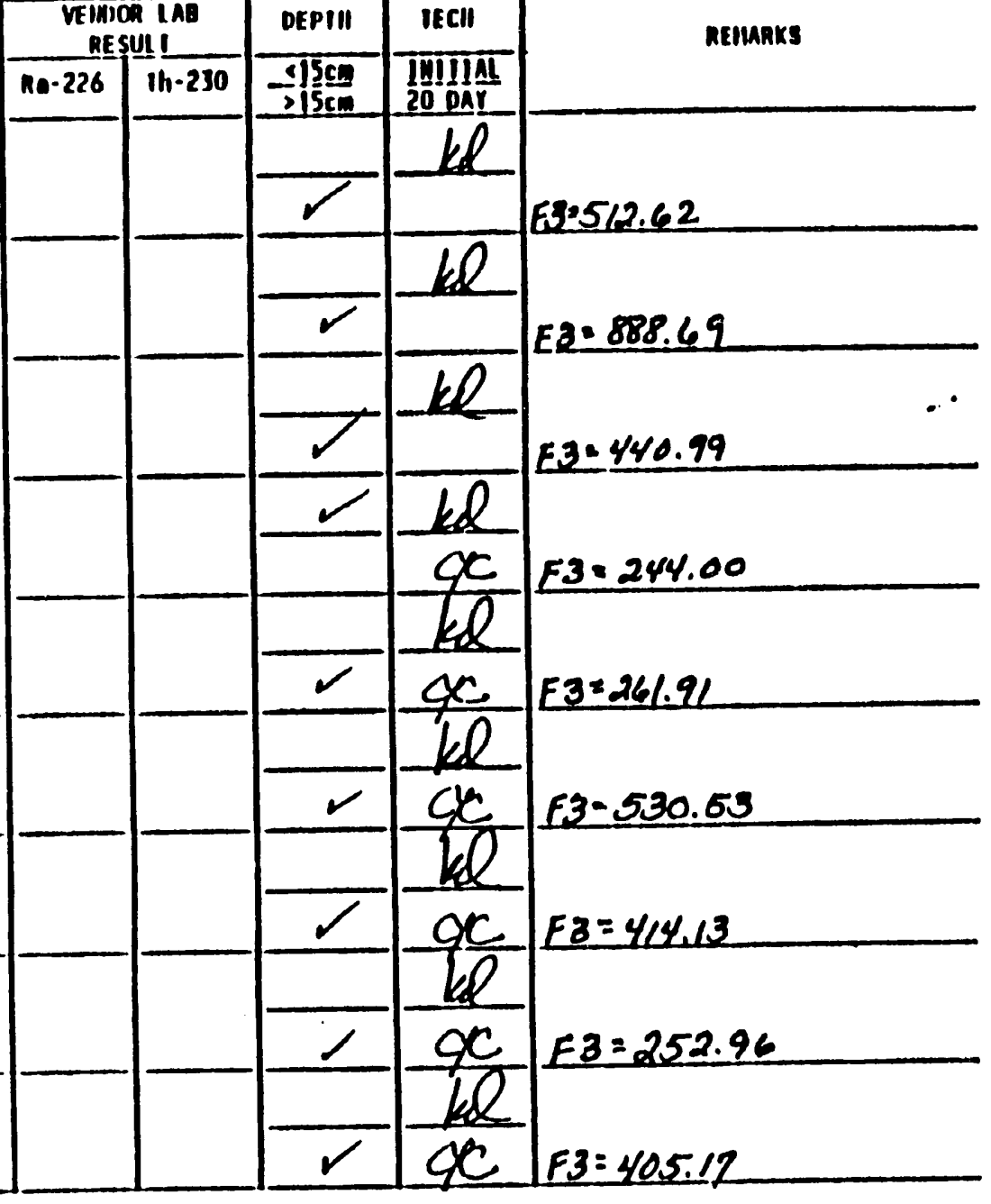

REVIELED OY 
Ire-iencasom/ciren mictean

\section{Gune TUNISON}

: amen N/A
: OrIUSEd carsial srsien Recono tarbarimanivit com
Altacingent $?$

Hocs is BetweEnsol. OCS BERIAL Do. 599 OCS SN984368 (GLN

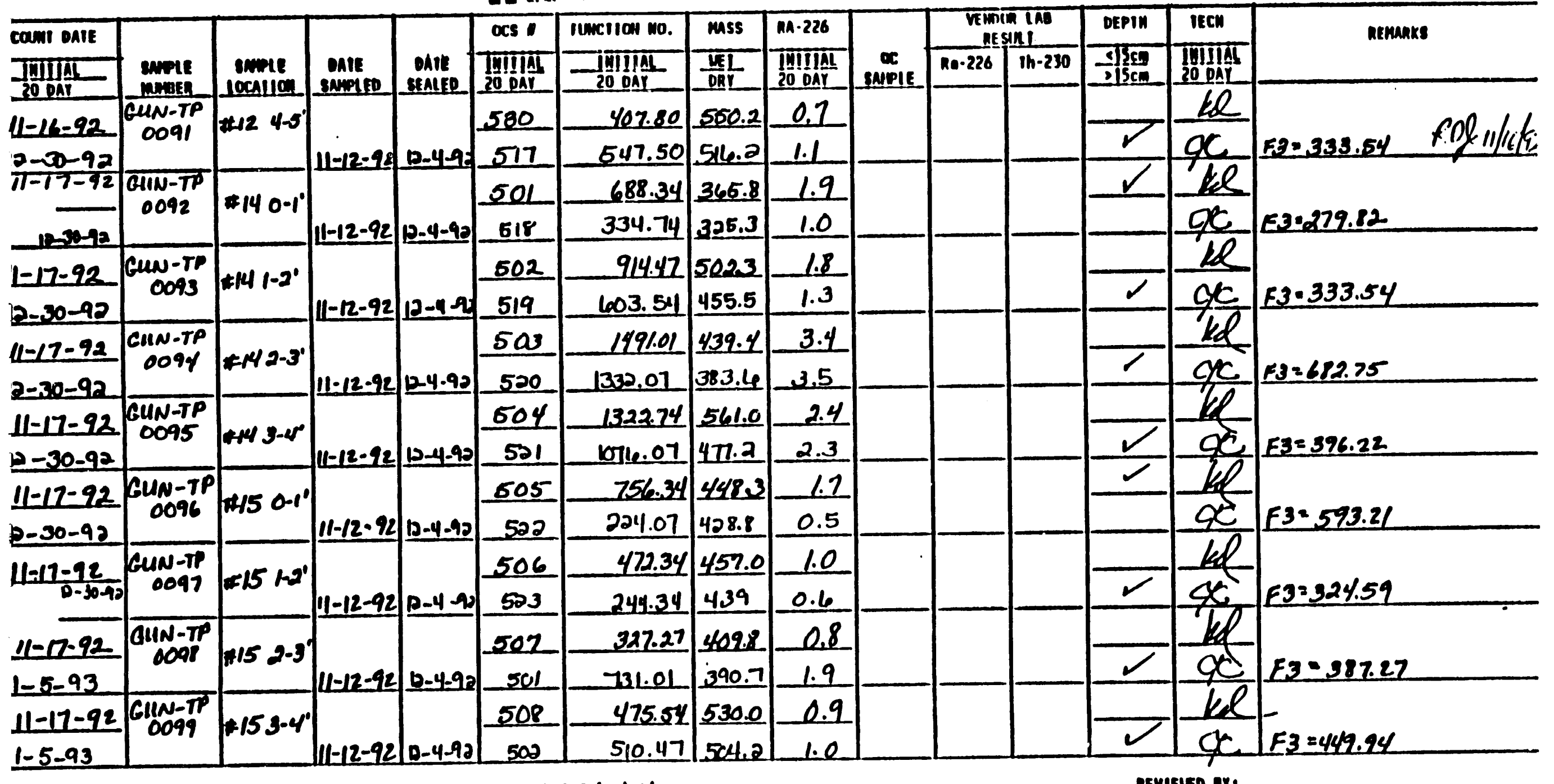

IIEs All soll scople resulte ore In petion

Ile correct Ion Pector

ine lim - inless noted othermtse.

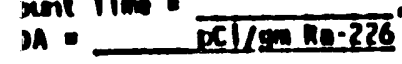

in coluclat ion

$4.65 / \frac{10.1000 \text { sec, bockgrog!nl cls. }}{20}$

(Cl. Ilime) (ECl.) (5maile Unss)"

- Imicalle 500 mo 
$r$

man Gusuresen

anen $N$ L/P

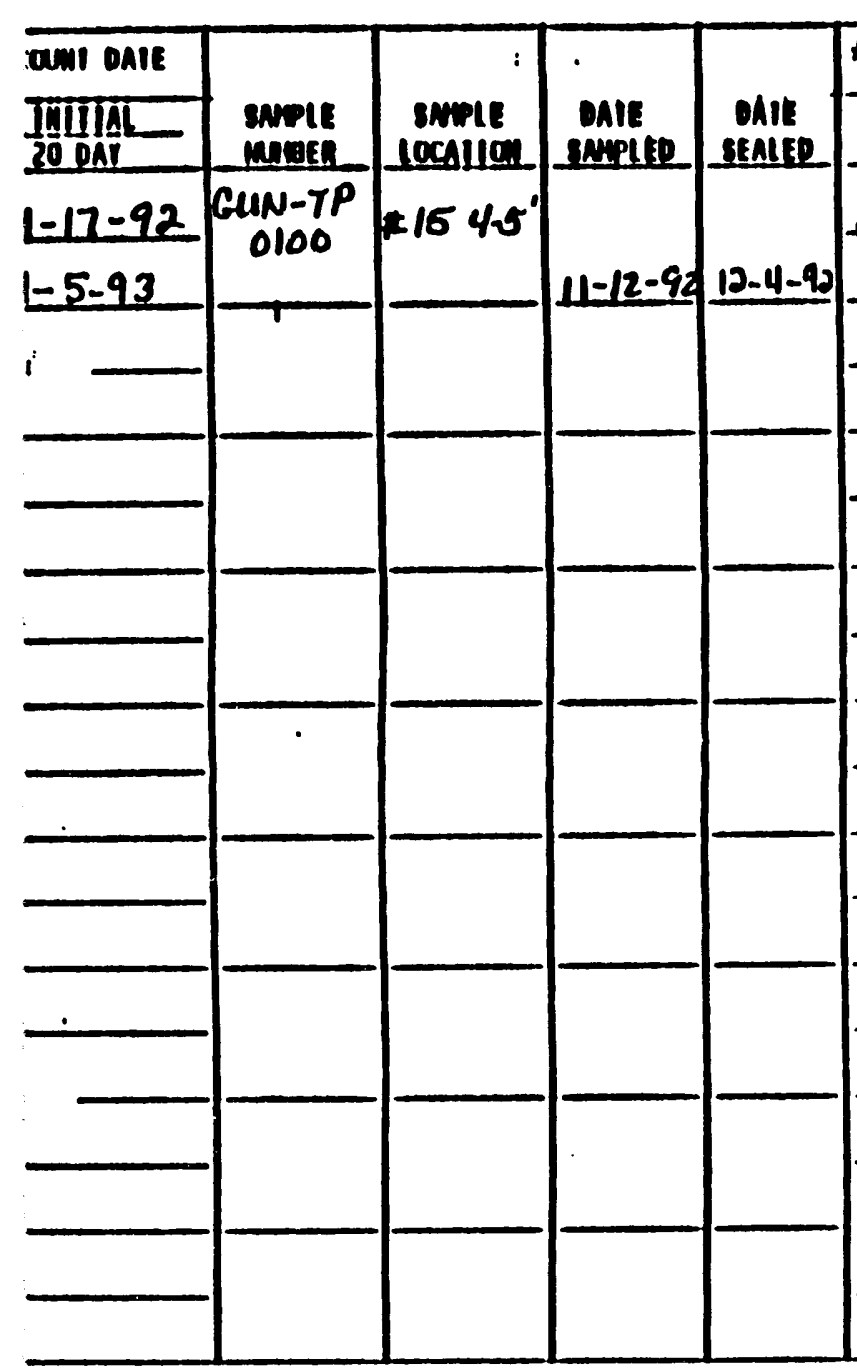

EI All soll sample results ore In pclion

- Correction inclor.

ill lime "

ill $\sqrt{x / 1800-2 ? 6}$
F!

IIT- IERCASOM/CHEN MM:I EAM OrIUSED CRYSIAL SYSIEN RECLAD

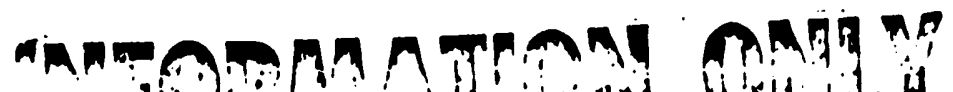

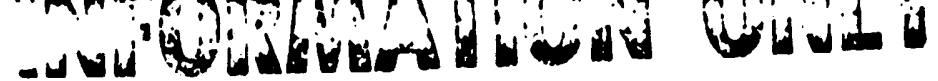
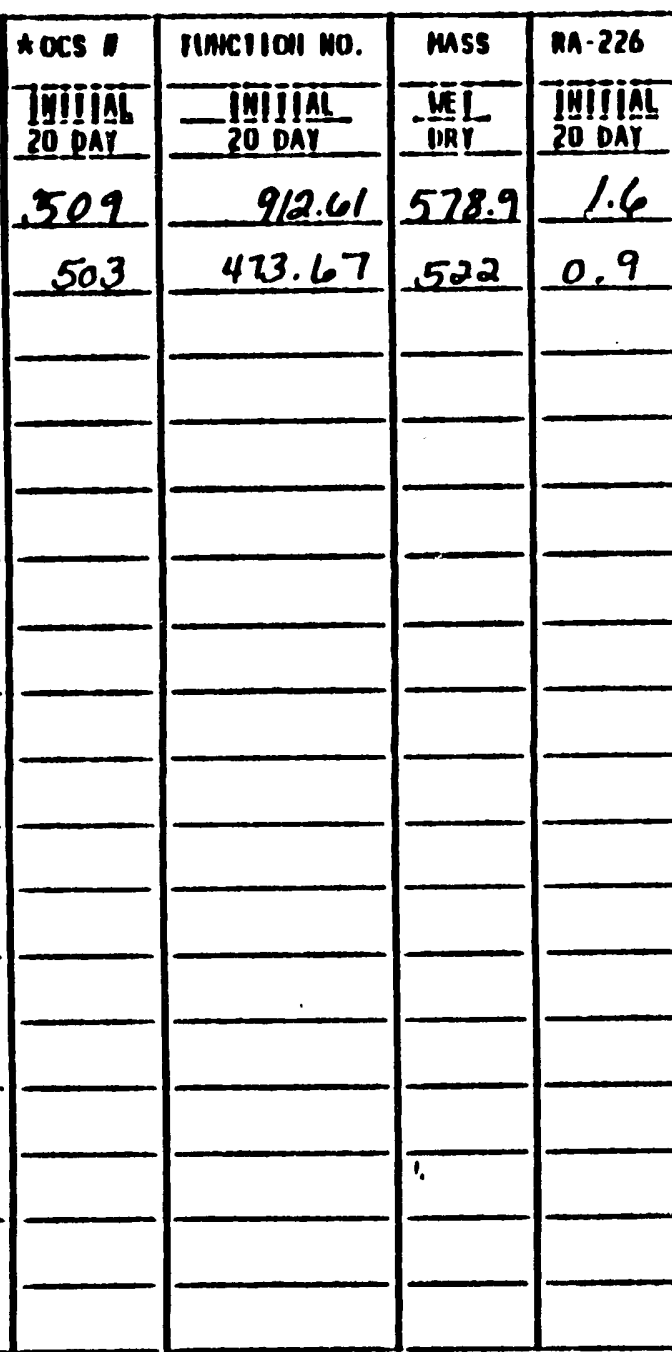

un colucl allon

$6.65 v^{\prime \frac{10.000 \mathrm{sec}_{1} \text { bog Egronex c!s. }}{20}}$

(CI. II Ime) (Ell.) (Somple Mass)"

inpicaller sion gno
OCS SEALAL NO. 599 OCS
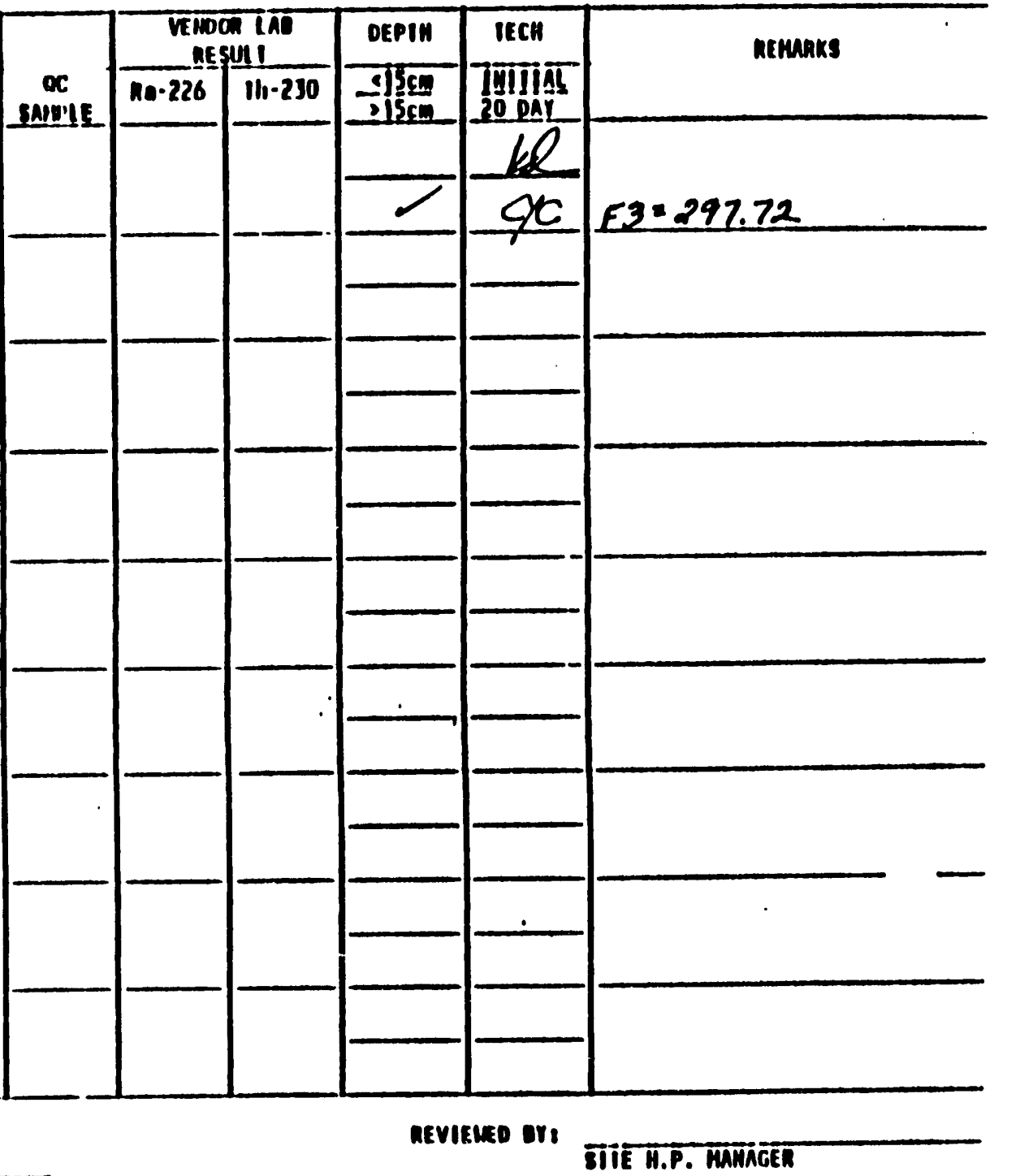

REVIELED OV:

AllacinewI

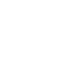




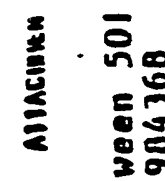

후

- ำำ

旁

- 01

og日⿸厂二⿺乚一匕

Onn

:

है

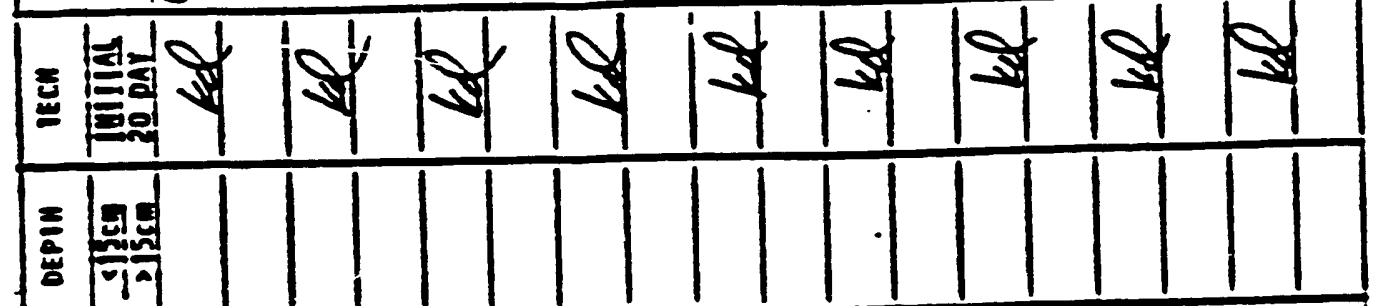

E

F

$5 \mid \approx$

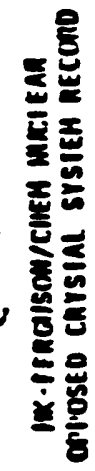

$$
\text { d }
$$

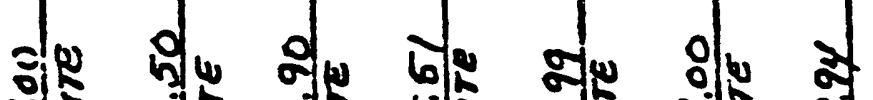

莫

$\lim _{0 \rightarrow 0}$

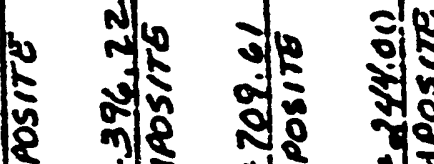

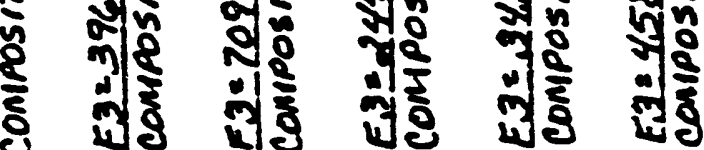

ำ ㅇำ

is

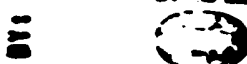

兽

in

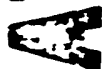

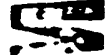

$\therefore$

$\therefore \rightarrow$

$\because 3$

$\therefore=$

$c \therefore$

83

₹

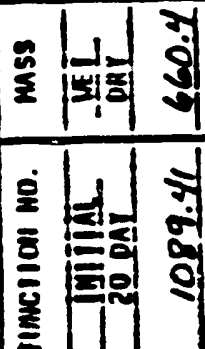

จ

2.

if

भी $\mid$ ๆ $\mid$ भิ $\mid$ के

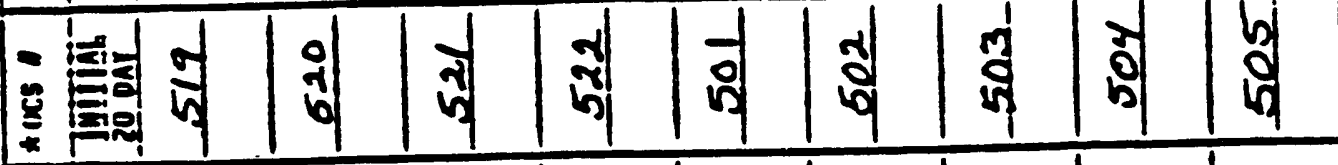

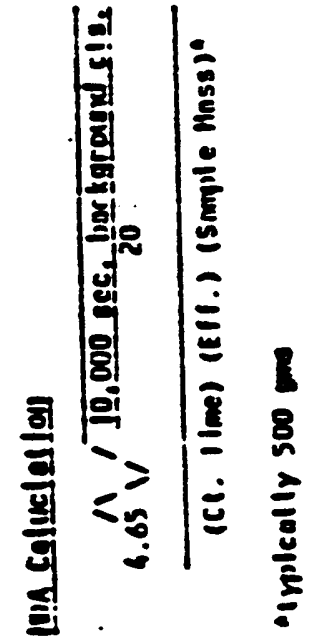

2. 14

讋

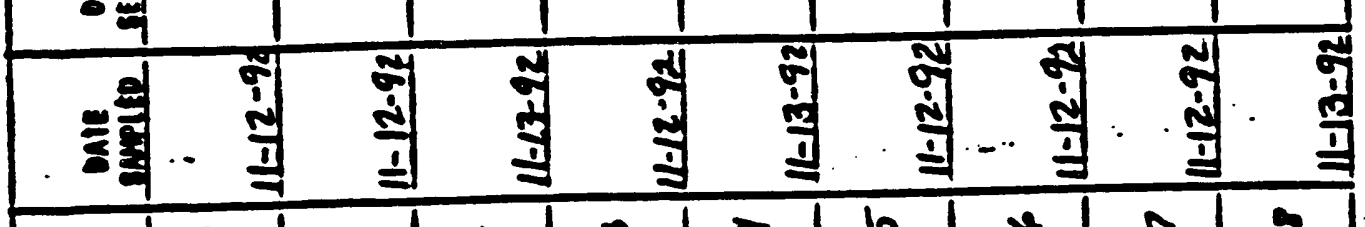

날

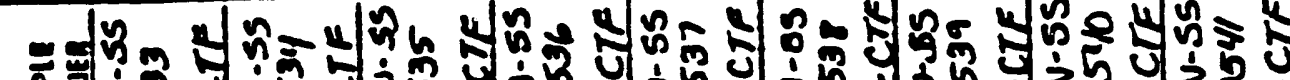

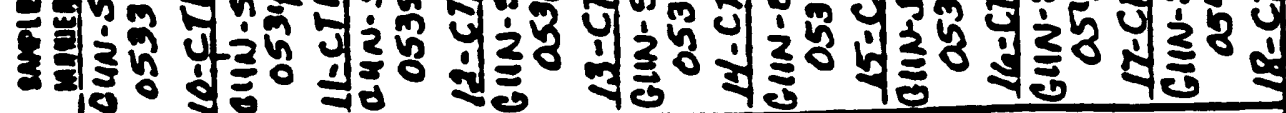

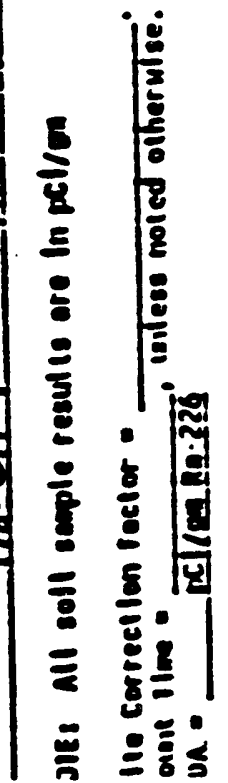




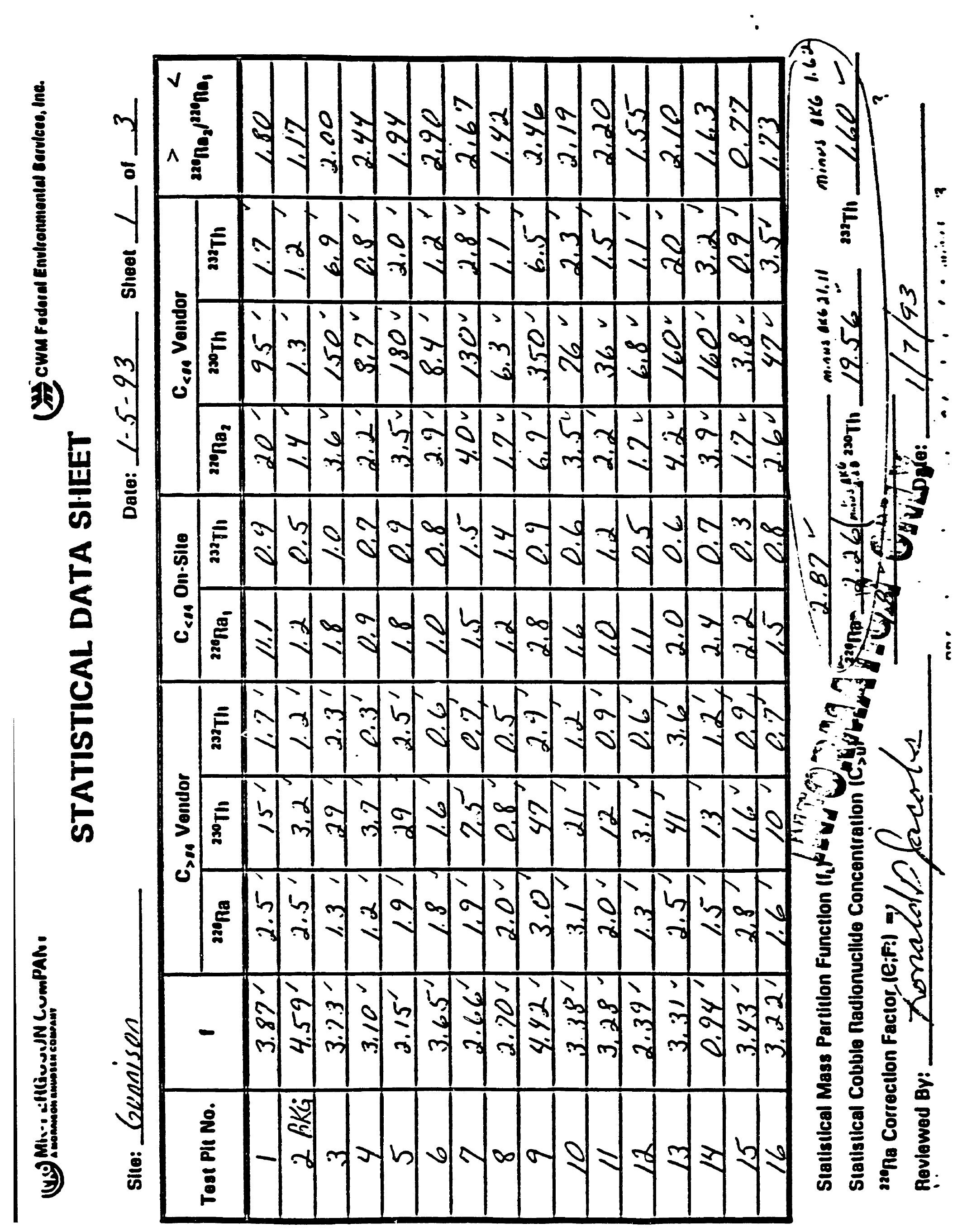


(140) Mn-rengugoN GunPAicr

STATISTICAL DATA SHIEET

Sho: Gwacisen

Date: $1-5 \cdot 23$

sheot 2 of 3

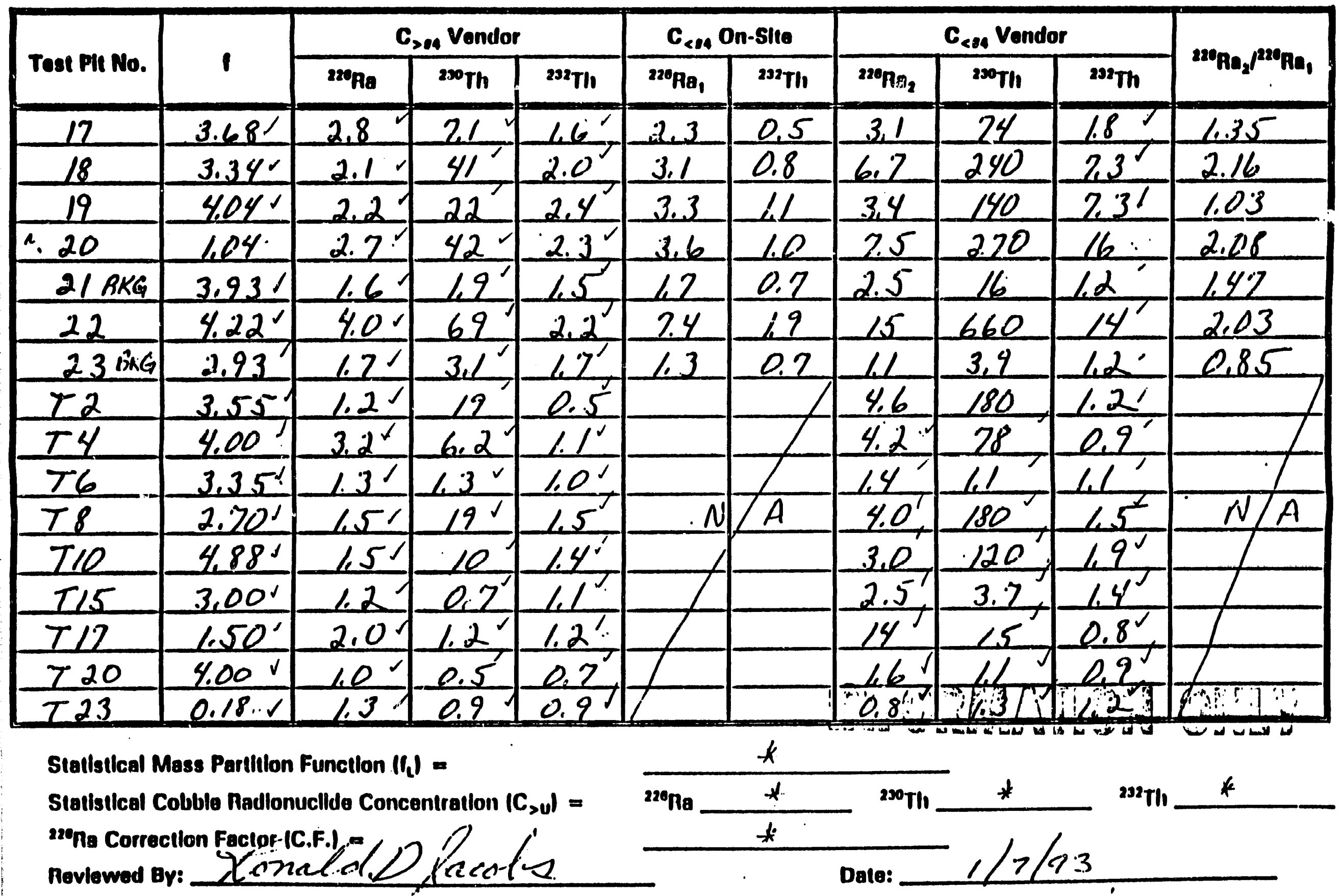


sile: Guancion

STATISTICAL DATA SHEET

CWM Fodoral Environmontal serivices, Ine.

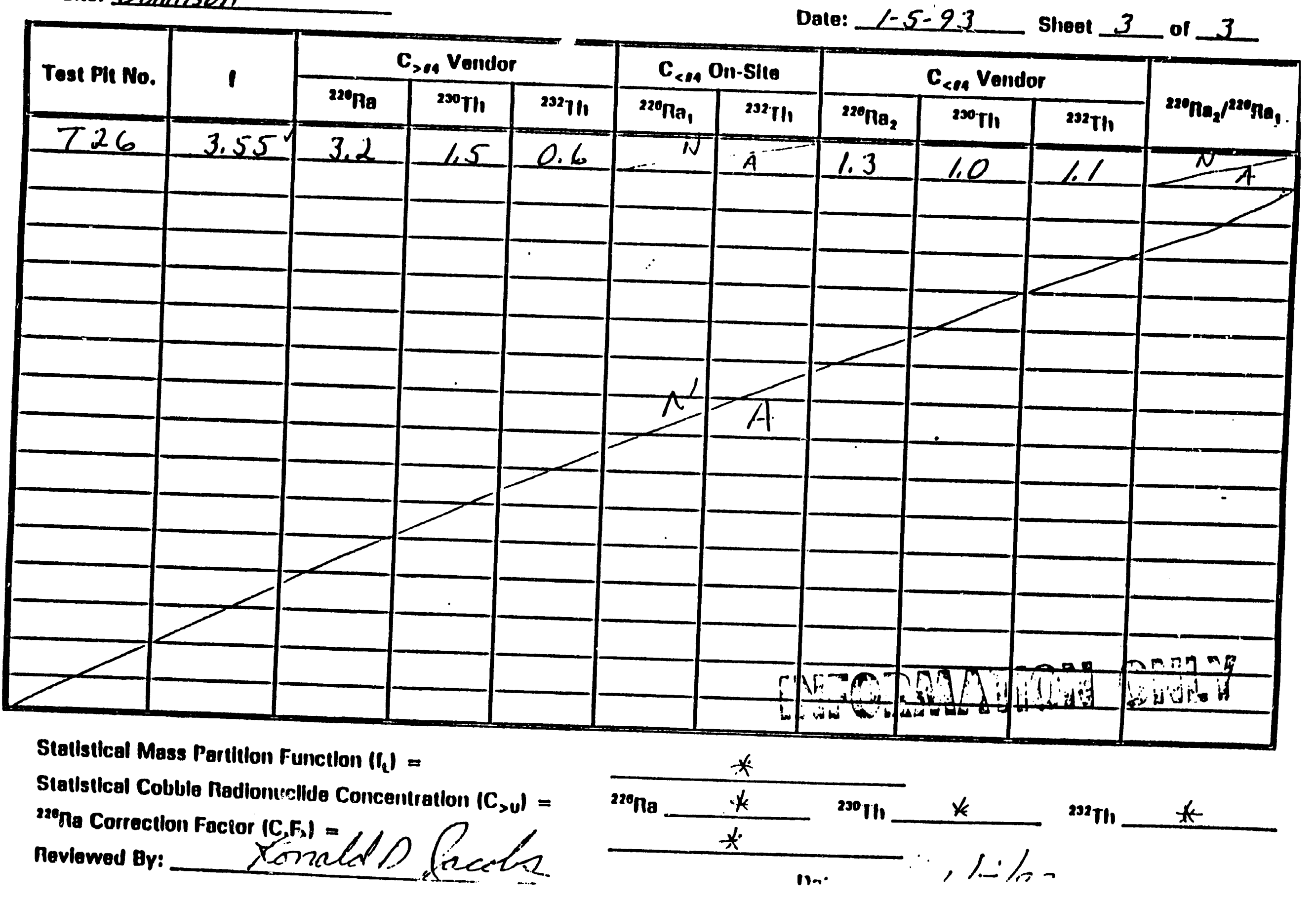



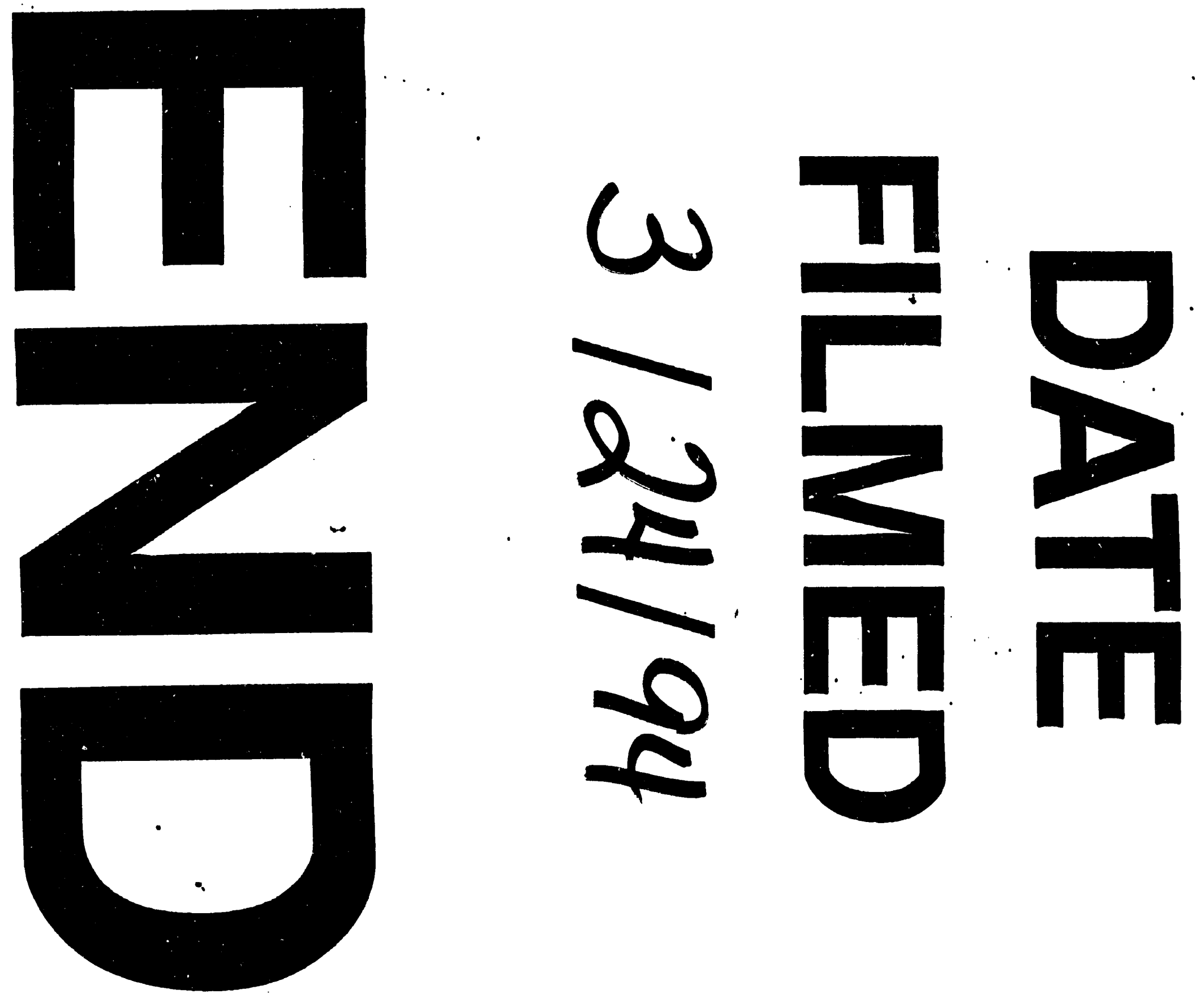